University of Rhode Island

DigitalCommons@URI

Open Access Dissertations

1997

\title{
A Quantitative and Qualitative Assessment of Adolescent HIV- Risk Predictors
}

Michelle A. Lang

University of Rhode Island

Follow this and additional works at: https://digitalcommons.uri.edu/oa_diss

\section{Recommended Citation}

Lang, Michelle A., "A Quantitative and Qualitative Assessment of Adolescent HIV-Risk Predictors" (1997). Open Access Dissertations. Paper 984.

https://digitalcommons.uri.edu/oa_diss/984

This Dissertation is brought to you for free and open access by DigitalCommons@URI. It has been accepted for inclusion in Open Access Dissertations by an authorized administrator of DigitalCommons@URI. For more information, please contact digitalcommons-group@uri.edu. 


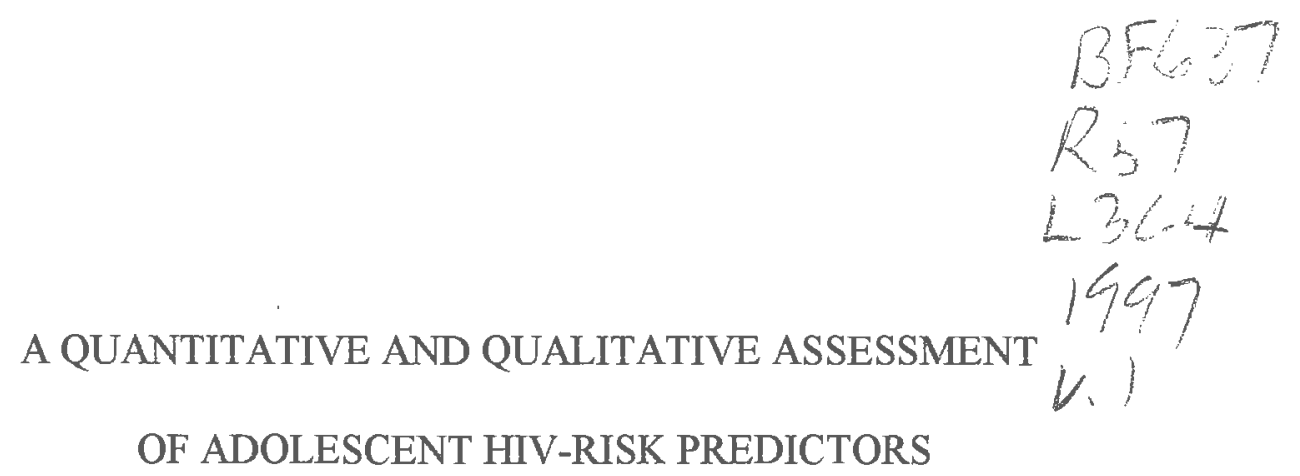

BY

MICHELLE A. LANG

A DISSERTATION SUBMITTED IN PARTIAL FULFILLMENT OF THE REQUIREMENTS FOR THE DEGREE OF DOCTOR OF PHILOSOPHY

IN

PSYCHOLOGY

$$
3460 \%, 2
$$

UNIVERSITY OF RHODE ISLAND 


\begin{abstract}
HIV-risk was assessed, and staged by the Transtheoretical Model, among 239 adolescents, ages 13-19 (mean age 15), with a 163-item self-administered questionnaire, supplemented with qualitative data from 28 focus groups. Thirteen psychometrically reliable scales were isolated and validated with 457 youth pooled from previous samples: factual, transmission, and perceived AIDS knowledge, misconceptions, perceived susceptibility, self-efficacy, homophobia, substance use and abuse, communication assertiveness, condom use assertiveness, and risk histories for self and partner(s). AIDS risk was defined by frequency of unprotected occasions and number of intercourse partners. Sexual activity was indicated by 54 percent of the sample. Young men reported significantly lower sexual onset age than women (13 and 14, respectively), and significantly more lifetime partners ( 5 and 3 , respectively). Utilizing a multivariate approach, gender-specific risk profiles were identified, and interaction effects noted
\end{abstract} between gender and sexual activity for risk variables. Sexually active women had higher AIDS knowledge with fewer misconceptions than men, and men had higher perceived susceptibility, homophobia, substance use and abuse, self-risk histories, and assertiveness than women. High misconceptions, perceived susceptibility, substance use and abuse, partner- and self-risk histories, and low condom assertiveness were significant risk factors for sexually active men. High perceived susceptibility, partner- and self-risk histories, and low self-efficacy, assertiveness for communication and condom use were significant risk factors for sexually active women. High homophobia was predictive of risk for the group. Youth typically did not view their sexual activities as risky. Among sexually-active youth, 
three-quarters reported substance use prior to sex; 95 percent reported "unsafe" activity, with inconsistent or no condom use; and, 39 percent of those "unsafe" considered themselves to be at "no risk" for HIV contagion. Self-estimates of behavior change state for current and predicted condom use were inaccurate. Based on adjustments, less than half of sexually active youth would be in advanced stages of behavior change in six months. Focus group participants discussed AIDS awareness, assertiveness complexities, informational resources, risk perceptions, gender roles, societal repercussions of the AIDS pandemic, and intervention suggestions. Prediction of adolescent risk and implications for effectiveness of HIV educational intervention and prevention programs are discussed. 


\section{Acknowledgments}

Thanks to Lisa Harlow, Patricia Morokoff and Kathryn Quina, principal and co-investigators of the Women's Health Project, for financial support under National Institute of Mental Health Grant MH47233. This endeavor could not have been accomplished without their aid. After receiving the photocopying bill, I recall considering bankruptcy. As always, I am especially grateful to my major professor, Kathryn Quina, for her encouragement in my work with youth, patience with excessive, lengthy analyses, editorial expertise with my run-on sentences, and allowing me "just one more" extension for submission. Eighteen times. Thanks also to Kat for taking coffee away from me when my blood levels became toxic, and I experienced acute manic episodes. With delusions. Those times Kat was instrumental in convincing me that multicollinearity and homogeneity of variance-covariance matrices were abstract concepts and were not "out to get me," and that I did not have to study for the $F$-test. Not once did she suggest Haldol. Thanks to my primary care physician, John DiOrio, who also did not suggest Haldol in the midst of my incoherent rambling, and instead, gave me a few more medical cites to incorporate. I am grateful to my core and extended committee, Allan Berman, Richard Sullivan, Denise DeZolt, Albert Lott, and Wilfred Dvorak, for reading this work and not having me mysteriously "eliminated" given the length. Although I think I'm being followed. I am also indebted to Gary Burkholder for his contribution and feedback to the questionnaire, despite numerous revisions, word by word. Thanks to Gary, I am now addicted to caffeine. Along those lines I give thanks to creators of caffeine, Visine, toast, and various hair products that hide gray. Thanks also to Bob Houghtaling and Amy Hockman, Administrative Staff for the 1994 Youth-to-Youth Eastern Conference, for allowing me to 
become involved with participants. Mere words alone cannot describe my experiences spending 3 days with 350 teenagers. I am grateful to Denise DeZolt for her influence and assistance in acquiring high school participation. After lengthy preparation for the first school board and losing the sample, Denise was truly an emotional support. As she was when the second sample was lost. And third. I was rather pessimistic, and considered never leaving the "quiet room." Thanks to Jonathan Lieberman and faculty at Griswold High School in Jewett City, Connecticut for their patience and assistance in data collection. Especially for not reneging at the last minute. Thank you also to my assistants and group co-facilitators, Jim Quinlan and Selene Angier, who helped enormously in data collection, and served as highly competent translators of teenage jargon. Although I am still not confident that I fully comprehend what the term "butt-..." ...never mind. I don't want to know. Thanks also to Roy Arnold, John Degnan, Bonnie Orlowski, and Gary Breault, who covered "just one work shift" for me. Every week. For a few months. I am appreciative to Brown University computer repair people, who diligently recovered valuable data reviving my computer when it spontaneously "blew up." Twice. To all my friends, thanks, for patience and tolerance with my lack of social behavior, neglected self-care, and hibernation. I think they're still my friends. Then again, nobody calls anymore. Finally, special thanks to my feline companions, being most helpful, unobtrusively sitting in the center of whatever, pointing out cursor location, providing pre-staple holes, and timely retrieving printouts. So timely, printing was incomplete. Without them sitting on the continuous feed pile, I would have never conserved on so much paper. Nevertheless, I think I'll keep them. They faithfully stood by me during the acute, neglected self-care phase. It's been a real... experience. 


\section{Table of Contents}

Epidemiology .

The Present Investigation.

Identifiable Trends Increasing Adolescent Risk . . . . . . . . . . . . . . . . 6

Heterosexual Transmission. . . . . . . . . . . . . . . . . . . 13

Adolescent Risk Predictors. . . . . . . . . . . . . . . . . . . . . . . 16

Cognitive . . . . . . . . . . . . . . . . . . 16

Attitudinal . . . . . . . . . . . . . . . . . 18

Behavioral . . . . . . . . . . . . . . . . . . . . 21

Gender Differentials in Risk Behavior . . . . . . . . . . . . . . . . . 27

The Transtheoretical Model: Stages of Behavior Change. . . . . . . . . . . . 33

Qualitative Research. . . . . . . . . . . . . . . . . . . . . 34

Overview . . . . . . . . . . . . . . . . . . 35

Research Hypotheses . . . . . . . . . . . . . . . . . . . . . 37

Method

Participants. . . . . . . . . . . . . . . . . . . . . . . . 38

Measures. . . . . . . . . . . . . . . . . . . . 39

Demographics . . . . . . . . . . . . . . . . . . . 40

Cognitive Measures . . . . . . . . . . . . . . . . . . . . . . . . 40

Attitudinal Measures. . . . . . . . . . . . . . . . . . . . . . . . . . 41

Behavioral Measures. . . . . . . . . . . . . . . . . . . 43

Psychosocial Measures . . . . . . . . . . . . . . . . . . . . . 47

Codebook . . . . . . . . . . . . . . . . 48

Focus Group Semi-Structured Interviews $\quad$. . . . . . . . . . . . . . 48

Procedure . . . . . . . . . . . . . . . . . . . . . . . . 49

Informed Consent . . . . . . . . . . . . . . . . . . 52

Confidentiality. . . . . . . . . . . . . . . . . . . . . . 53 
Risks Involved.

Results

Demographics .

Quantitative Results: Scale Construction

Procedure. . . . . . . . . . . . . . 58

Cognitive Measures . . . . . . . . . . . . . . . . . . . . . . . . 59

Attitudinal Measures. . . . . . . . . . . . . . . . . . . 60

Behavioral Measures. . . . . . . . . . . . . . . . . . 61

Psychosocial Measures. . . . . . . . . . . . . . . . . . . . 65

Scale Construction Across Demographic Variables . . . . . . . . . . . . . 68

Age . . . . . . . . . . . . . . . . . . . . . . . 73

Geographic Location . . . . . . . . . . . . . . . . . 74

Ethnicity. . . . . . . . . . . . . . . . . . . . . . 75

Socioeconomic Status . . . . . . . . . . . . . . . . . 76

Factor Stability Synopsis . . . . . . . . . . . . . . . . . . . . . . . 77

Quantitative Results: Risk Predictors and Group Differences

Sexual Experience . . . . . . . . . . . . . . . . . . . . . 79

Substance Use and Abuse Correlates with Sexual Behavior . . . . . . . . . . 81

Self and Partner AIDS Risk Estimates . . . . . . . . . . . . . . . . . . . . . 82

Risk Analyses . . . . . . . . . . . . . . . . . . . . . . . . . . . . . . . . . . 84

Sexual Assertiveness Predictors . . . . . . . . . . . . . . . . . . . 84

AIDS Risk Predictors . . . . . . . . . . . . . . . . . . . 86

Predictors of Self-rated Level of AIDS Risk . . . . . . . . . . . . . 89

Predictors Contributing to Accurate and Inaccurate

Levels of Self-rated AIDS Risk . . . . . . . . . . . . . . . . . . . 91 
Differential Risk Profiles by Gender and Sexual Activity . . . . . . . . . 95

Cognitive . . . . . . . . . . . . . . . . . . . . . 96

Attitudinal . . . . . . . . . . . . . . . . . . . . . 97

Behavioral . . . . . . . . . . . . . . . . . . . . . 98

Sexually Active Youth . . . . . . . . . . . . . . . . . . . . . 100

Sexually Nonactive Youth . . . . . . . . . . . . . . . . 101

Safe Versus Unsafe Behavior . . . . . . . . . . . . . . . 103

Sexually Active Versus Nonactive . . . . . . . . . . . . . 104

Transtheoretical Stages of Behavior Change . . . . . . . . . . . . . 105

Sexually Active Participants . . . . . . . . . . . . . . . . . . . . 106

Gender Differences . . . . . . . . . . . . . . . . . . 111

Predicted Condom Use Among the Sexually Nonactive Participants . . 113

Qualitative Results: Focus Groups

Conference Youth Focus Groups: A Pilot Study . . . . . . . . . . . . 114

Session One . . . . . . . . . . . . . . . . . . . . . . 115

Session Two . . . . . . . . . . . . . . . . . . . . 116

Session Three . . . . . . . . . . . . . . . . . . . . 116

Session Four . . . . . . . . . . . . . . . . . . . . . 117

Session Five

Session Six . . . . . . . . . . . . . . . . . . . . . . . . . . . . . 118

School Youth Participation . . . . . . . . . . . . . . . . . . . . . . 119

School Youth Focus Groups . . . . . . . . . . . . . . . . . . . 120

Anonymous Questionnaire Feedback . . . . . . . . . . . . 123

Focus Group Topic One: AIDS Research. . . . . . . . . . . . . . 125

Survey Discussion . . . . . . . . . . . . . . . . . . 125

Suggestions for AIDS Prevention Strategies . . . . . . . . . . . 127

Focus Group Topic Two: The Impact of the AIDS Pandemic . . . . . . . 132

Age Group . . . . . . . . . . . . . . . . . 132 
Location. . . . . . . . . . . . . . . 134

Populations Afflicted

Homophobia . . . . . . . . . . . . . . . . . . . . 135

Scenario One: A Student . . . . . . . . . . . . . . . . . . . 138

Scenario Two: A Best Friend. . . . . . . . . . . . . . . . . 139

Scenario Three: A Sibling . . . . . . . . . . . . . . . 140

Scenario Four: A Parent . . . . . . . . . . . . . . . . . . 140

Focus Group Topic Three: Societal Dilemmas . . . . . . . . . . . . . 141

Insurance Companies . . . . . . . . . . . . . . . . . 141

Segregation. . . . . . . . . . . . . . . . . . . . . 143

Pregnant Women . . . . . . . . . . . . . . . . . . . 146

Focus Group Topic Four: Teenage Risk . . . . . . . . . . . . . . . . 149

Risk Situations . . . . . . . . . . . . . . . . . . . . . . . 149

Scenario One: Meeting Friends . . . . . . . . . . . . . . . . . . 149

Scenario Two: Meeting Friends with Alcohol . . . . . . . . 150

Scenario Three: Meeting Friends with Other Drugs . . . . . . 150

Scenario Four: Having Sex . . . . . . . . . . . . . . . . . 151

Popularity . . . . . . . . . . . . . . . . . . . . . 152

Attractiveness . . . . . . . . . . . . . . . . . . . . 153

Informational Resources . . . . . . . . . . . . . . . . 154

Assertiveness . . . . . . . . . . . . . . . . . . . . 156

Scenario One: Partner Pressure . . . . . . . . . . . . . . 156

Scenario Two: Threat to the Relationship. . . . . . . . . . 157

Scenario Three: Negotiation . . . . . . . . . . . . . . 158

Scenario Four: Emotional Coercion . . . . . . . . . . . . . 159

Developing Assertiveness Capabilities . . . . . . . . . . . . . . . . . . 160

Other Topics Raised . . . . . . . . . . . . . . . . . . . . . . . . 163

Questions Raised . . . . . . . . . . . . . . . . . . . . . . 165

Factual Inquiries . . . . . . . . . . . . . . . . . . . . 166

AIDS Transmission Inquiries . . . . . . . . . . . . . . . 167 


\section{Discussion}

Overview . . . . . . . . . . 168

Scale Construction . . . . . . . . . . . . . . . . . . . . . . . 169

Age . . . . . . . . . . . . . . . . . . . . . . . 169

Geographic Locale . . . . . . . . . . . . . . . . . . 171

Ethnicity. . . . . . . . . . . . . . . . . . . . . 172

Socioeconomic Status . . . . . . . . . . . . . . . . . 173

Deviations from the Hypothesized Subscale Structure . . . . . . . 175

Summary . . . . . . . . . . . . . . . . . . . . . 175

Questionnaire Feedback. . . . . . . . . . . . . . . 176

Quantitative Findings and Qualitative Supports . . . . . . . . . . . . 177

Hypothesis One: Predictors of Sexual Assertiveness . . . . . . . 178

Hypothesis Two: AIDS Risk Predictors Among the Sexually Active . . 192

Hypothesis Three: Participants' Estimated AIDS Risk Level . . . . 203

Hypothesis Four: Gender Differences in AIDS Risk Predictors . . . . 208

Hypothesis Five: Sexually Active Versus Nonactive Group Differences . 214

Interaction Effects Among Risk Predictors . . . . . . . . . . 217

Stages of Change for Current and Predicted Condom Use . . . . . . 219

Risk Variables in the Context of Stages of Change for Condom Use . . 221

Gender Differences in Stages of Change for Condom Use . . . . . . 223

Other Qualitative Topics Investigated . . . . . . . . . . . . . . . 224

Psychosocial Dilemmas . . . . . . . . . . . . . . . . . 225

Adolescents' Suggestions . . . . . . . . . . . . . . . . . 228

Suggested Intervention and Prevention Strategies . . . . . . . . . . . 231

The Educational Domain . . . . . . . . . . . . . . . . 232

Promoting Abstinence Versus Safe Sexual Behaviors . . . . . . . . 234

Empowerment. . . . . . . . . . . . . . . . . . . . 238

Skills-Building. . . . . . . . . . . . . . . . . . . . 239

Future Research Directions . . . . . . . . . . . . . . . . . . 242 
Study Limitations. . . . . . . . . . . . . . . . . . . . . . . . 244

Conclusions . . . . . . . . . . . . . . . . . . . . . . . 247

References . . . . . . . . . . . . . . . . . . . . 251

Appendices . . . . . . . . . . . . . . . . . . . . . . . . . . . . 388

Bibliography . . . . . . . . . . . . . . . . . . . . . . . 430 


\section{Tables and Figures}

Table 1: Demographic Profiles for Conference Youth and School Youth by Gender

Table 2: Exploratory Factor Analysis Pattern Matrix and Factor Correlation Matrix for Cognitive Subscales Utilizing 4-Sample Pooled Data

Table 3: Confirmatory Factor Analysis Pattern Matrix and Factor Correlation Matrix for Cognitive Subscales Utilizing a Random 50 Percent Sample from 4-Sample Pooled Data

Table 4: Response Frequencies for Cognitive Scale Items 303

Table 5: Exploratory Factor Analysis Pattern Matrix and Factor Correlation Matrix for Attitudinal Subscales Utilizing 4-Sample Pooled Data . 304

Table 6: Confirmatory Factor Analysis Pattern Matrix and Factor Correlation Matrix for Attitudinal Subscales Utilizing a Random 50 Percent Sample from 4-Sample Pooled Data.

Table 7: Response Frequencies for Attitudinal Scale Items

Table 8: Exploratory Factor Analysis Pattern Matrix and Factor Correlation Matrix for Substance Use and Abuse Behavior Subscales Utilizing 4-Sample Pooled Data of Substance Using Adolescents

Table 9: Confirmatory Factor Analysis Pattern Matrix and Factor Correlation Matrix for Substance Use and Abuse Behavior Subscales Utilizing a Random 50 Percent Sample from 4-Sample

Pooled Data of Substance Using Adolescents.

Table 10: Exploratory Factor Analysis Pattern Matrix and Factor Correlation Matrix for Sexual Assertiveness Behavior Subscales Utilizing 4-Sample Pooled Data of Sexually Active Adolescents

Table 11: Confirmatory Factor Analysis Pattern Matrix and Factor Correlation Matrix for Sexual Assertiveness Behavior Subscales Utilizing a Random 50 Percent Sample from 4-Sample Pooled Data of Sexually Active Adolescents .

Table 12: Exploratory Factor Analysis Pattern Matrix and Factor Correlation Matrix for Behavior Risk History Subscales Utilizing 4-Sample Pooled Data of Sexually Active Adolescents 
Table 13: Confirmatory Factor Analysis Pattern Matrix and Factor Correlation Matrix for Behavior Risk History Subscales Utilizing

a Random 50 Percent Sample from 4-Sample

Pooled Data of Sexually Active Adolescents .

Table 14: Exploratory Factor Analysis Pattern Matrix and Factor Correlation Matrix for Three Major Areas of Behavior Utilizing 4-Sample Pooled Data

Table 15: Confirmatory Factor Analysis Pattern Matrix and Factor Correlation Matrix for Three Major Areas of Behavior Utilizing

a Random 50 Percent Sample from 4-Sample Pooled Data

Table 16: Response Frequencies for Behavior Scale Items .

Table 17: Exploratory Factor Analysis Pattern Matrix and Factor Correlation Matrix for Environment Scales Utilizing 4-Sample Pooled Data.

Table 18: Confirmatory Factor Analysis Pattern Matrix and Factor Correlation Matrix for Environment Subscales Utilizing a Random 50 Percent Sample from 4-Sample Pooled Data .

Table 19: Response Frequencies for Environment Scale Items

Table 20: Exploratory Factor Analysis Pattern Matrix and Factor Correlation Matrix for School Education Subscales Utilizing 4-Sample Pooled Data

Table 21: Confirmatory Factor Analysis Pattern Matrix and Factor Correlation Matrix for School Education Subscales Utilizing a Random 50 Percent Sample from 4-Sample Pooled Data .

Table 22: Exploratory Factor Analysis Pattern Matrix and Factor Correlation Matrix for Home Education Subscales Utilizing 4-Sample Pooled Data

Table 23: Confirmatory Factor Analysis Pattern Matrix and Factor Correlation Matrix for Home Education Subscales Utilizing a Random 50 Percent Sample from 4-Sample Pooled Data .

Table 24: Exploratory Factor Analysis Pattern Matrix and Factor Correlation Matrix for Peer Education Subscales Utilizing 4-Sample Pooled Data 
Table 25: Confirmatory Factor Analysis Pattern Matrix and Factor Correlation Matrix for Peer Education Subscales Utilizing a Random 50 Percent Sample from 4-Sample Pooled Data .

Table 26: Exploratory Factor Analysis Pattern Matrix and Factor Correlation Matrix for Three Major Areas of Education Utilizing 4-Sample Pooled Data

Table 27: Confirmatory Factor Analysis Pattern Matrix and Factor Correlation Matrix for Three Major Areas for Education Utilizing a Random 50 Percent Sample from 4-Sample Pooled Data .

Table 28: Response Frequencies for Education Scale Items

Table 29: Group Comparisons by Demographic and Risk Variables Across Four Samples

Table 30: Subscale Intercorrelation Table for Current and Pooled Samples

Table 31: Summary Table of 88 Confirmatory Factor Analyses for Scale and Subscale Structures for Differences on Dichotomized Demographic Variables

Table 32: Descriptive Statistics for Subscale Variables by

Gender and Sexual Activity

Table 33: Age of Sexual Debut by Gender

Reported by the Sexually Active Participants .

Table 34: Participants' Estimated Risk for AIDS by Gender, Sexual Activity and Condom Use

Table 35: Multiple Regression $\beta$ Weights, and $s r^{2}$ Predictors of Sexual Assertiveness Among all Participants by Gender

Table 36: Multiple Regression $\beta$ Weights, and $s r^{2}$ Predictors of

AIDS Risk by Cognitive, Attitudinal and Behavioral Area, Among the Sexually Active Young Men

Table 37: Multiple Regression $\beta$ Weights, and $s r^{2}$ Predictors of AIDS Risk by Cognitive, Attitudinal and Behavioral Area, Among the Sexually Active Young Women 
Table 38: Multiple Regression $\beta$ Weights, and $s r^{2}$ Predictors of

Participants' Self-rated Level of AIDS Risk by

Cognitive, Attitudinal and Behavioral Area,

Among the Sexually Active Young Men

Table 39: Multiple Regression $\beta$ Weights, and $s r^{2}$ Predictors of

Participants' Self-rated Level of AIDS Risk by

Cognitive, Attitudinal and Behavioral Area,

Among the Sexually Active Young Women

Table 40: Multiple Regression $\beta$ Weights, and $s r^{2}$ Predictors of Participants' Self-rated level of AIDS Risk by Cognitive, Attitudinal and Behavioral Area, Among the Sexually Active Participants Reporting an Accurate Level of "Unsafe" Risk.

Table 41: MANOVA and Post Hoc ANOVA Results Across

Cognitive, Attitudinal and Behavioral Areas by Gender and Accuracy Versus Underestimation in Participants'

Self-rated Level of AIDS Risk Among the Sexually Active "Unsafe" Participants

Table 42: MANOVA Interactions, Simple Effects, and Main Effects for Gender and Sexual Activity

Table 43: MANOVA and Post Hoc ANOVA Results Across Cognitive, Attitudinal and Behavioral Areas by Gender Among the Sexually Active Participants

Table 44: MANOVA and Post Hoc ANOVA Results Across Cognitive, Attitudinal and Behavioral Areas by Gender Among the Sexually Nonactive Participants

Table 45: MANOVA and Post Hoc ANOVA Results Across Cognitive, Attitudinal and Behavioral Areas by Safe Versus Unsafe Sexual Activity Among the Sexually Active Participants

Table 46: MANOVA and Post Hoc ANOVA Results Across

Cognitive, Attitudinal and Behavioral Areas by Sexual Activity .

Table 47: Current and Predicted Condom Use by Transtheoretical Model Staging Among the Sexually Active Adolescents by Gender . 
Table 48: Sexually Active Adolescents' Reported Transtheoretical Model

Stage for Current Condom Use Compared to

Reported Sexual Behavior.

Table 49: Current Condom Use by Transtheoretical Model Staging

Comparisons by Cognitive, Attitudinal and Behavioral

Risk Variables Across the Sexually Active Participants

Table 50: Predicted Future Condom Use by Transtheoretical Model Staging Comparisons by Cognitive, Attitudinal and Behavioral Risk Variables Across the Sexually Active Young Men

Table 51: Predicted Future Condom Use by Transtheoretical Model Staging

Comparisons by Cognitive, Attitudinal and Behavioral

Risk Variables Across the Sexually Active Young Women

Table 52: Predicted Condom Use by Transtheoretical Model Staging

Among the Sexually Nonactive Adolescents by Gender

Table 53: Focus Group Tally for Study Participation with School Sample . . . 368

Table 54: Focus Group Demographics and Participant Feedback

for School Youth Sample . . . . . . . . . . . . . . . . 369

Table 55: Survey Feedback Checklist Responses for School Youth Sample . . 370

Figure 1: Questionnaire Scale and Subscale Breakdown by Exploratory and Confirmatory Factor Analyses Results

Figure 2: Sample Characteristics by Gender, Sexual Activity

(Active Versus Nonactive), and Condom Use Behavior

("Safe" Consistent Use, or "Unsafe" No Use or Inconsistent Use) . 372

Figure 3: Mean Scores for Dependent Variables by Gender

Among the Sexually Active "Unsafe"

(Inconsistent or No Condom Use) Participants

Figure 4: Mean Scores for Dependent Variables by Self-rated

Level of AIDS Risk Among the Sexually Active "Unsafe"

(Inconsistent or No Condom Use) Participants

Figure 5: 2X2 MANOVA Interaction for Gender and Sexual Activity

with Dependent Variable: Misconceptions 
Figure 6: 2X2 MANOVA Interaction for Gender and Sexual Activity with Dependent Variable: Perceived Susceptibility for Risk . . . 376

Figure 7: 2X2 MANOVA Interaction for Gender and Sexual Activity with Dependent Variable: Homophobia.

Figure 8: 2X2 MANOVA Interaction for Gender and Sexual Activity with Dependent Variable: Sexual Assertiveness Partner Communication (Actual Versus Predicted) . 378

Figure 9: 2X2 MANOVA Interaction for Gender and Sexual Activity with Dependent Variable: Sexual Assertiveness Condom Use (Actual Versus Predicted)

Figure 10: 2X2 MANOVA Interaction for Gender and Sexual Activity with Dependent Variable: Substance Use .

Figure 11: 2X2 MANOVA Interaction for Gender and Sexual Activity with Dependent Variable: Substance Abuse

Figure 12: 2X2 MANOVA Interaction for Gender and Sexual Activity with Dependent Variable: Self Risk History

Figure 13: Mean Scores for Dependent Variables for

Sexually Active Youth by Gender

Figure 14: Mean Scores for Dependent Variables for Sexually Nonactive Youth by Gender 384

Figure 15: Mean Scores for Dependent Variables by Sexual Active

(Actual Versus Predicted Behavioral)

Figure 16: Sexually Active Adolescents' Reported Current and

Six-Month Predicted Condom Use by

Transtheoretical Model Stage .

Figure 17: Sexually Active Adolescents' Current and

Six-Month Predicted Condom Use Adjusted by

Reported Sexual Behavior by Transtheoretical Model Stage . 


\section{Appendices}

Appendix A: Demographics . . . . . . . . . . . . . . . . . . . . 388

Appendix B: Cognitive Subscales . . . . . . . . . . . . . . . . . 389

Appendix C: Attitudinal Subscales. . . . . . . . . . . . . . . . . . 390

Appendix D: Behavioral Subscales . . . . . . . . . . . . . . . 392

Appendix E: Psychosocial Subscales . . . . . . . . . . . . . . . 397

Appendix F: Codebook . . . . . . . . . . . . . . . . . . . 400

Appendix G: Focus Group Feedback Sheets and

Semi-Structured Interview Questions . . . . . . . . . 415

Appendix H: Passive Consent (Youth-to-Youth) . . . . . . . . . . . 418

Appendix I: Parent Cover Letter (Griswold) . . . . . . . . . . . . 420

Appendix J: Parent Consent (Griswold) . . . . . . . . . . . . . . . 422

Appendix K: Informational Card (Griswold) . . . . . . . . . . . . . 424

Appendix L: Participant Cover Letter . . . . . . . . . . . . . . 425

Appendix M: Participant Assent (Youth-to-Youth) . . . . . . . . . . . . 426

Appendix N: Participant Assent (Griswold). . . . . . . . . . . . . . . 428 


\title{
A Quantitative and Qualitative Assessment
}

\author{
of Adolescent HIV-Risk Predictors
}

\section{Epidemiology}

To date, it is estimated that well over one million Americans are infected with Human Immunodeficiency Virus (HIV) (U.S. Department of Health and Human Services, 1993, the World Health Organization [WHO], 1995b). Currently, the Centers for Disease Control and Prevention (CDC), (1994a) estimates that one in every 250 persons in the U.S. alone is infected with HIV. At the close of $1994,426,978$ confirmed cases of Acquired Immunodeficiency Syndrome (AIDS) were reported in the U.S., while another 270,870 individuals had already lost their lives (CDC, 1994a). By the end of 1995, there were over a half million AIDS cases in the U.S., and 62 percent were reported as having died (CDC, 1995e).

Worldwide, an estimated 5,000 persons were newly infected each day a few years ago (CDC, 1993i), with more recent estimates at 6,000 per day (CDC, 1994j). By the close of 1993, the World Health Organization (WHO) reported 611,689 known ADDS cases worldwide, with estimated figures of actual AIDS and HIV cases being, 2.5 million, and 14 million, respectively (WHO, 1993a), a 100-fold increase since first identified in 1981. Total number of AIDS cases from the start of the pandemic to the end of 1994 reveal 1,025,073 
reported infected individuals; however, the WHO (1995b) estimates four times that figure to be more accurate. More recent estimates declare 18 million adults and 1.5 million children infected with HIV, resulting in approximately 4.5 million AIDS cases in the world (WHO, 1995a, 1995b). At the turn of the century, the WHO predicts that there will be a cumulative total of 40 million HIV cases, and 10 million adult AIDS cases worldwide (“AIDS Plague," 1993; WHO, 1993a).

More alarming are estimates that half of the 14 million people with HIV, worldwide, were infected between the ages of 15 and 24 years (Hein, Dell, Futterman, RotheramBorus, \& Shaffer, 1995), with young women among this age group being the hardest hit (WHO, 1993e). Worldwide, young women under age 25 account for nearly 30 percent of female AIDS cases, and young men for approximately 15 percent of male cases (WHO, 1994a). By the year 2000, over 13 million women will have been infected and four million of them will have died (WHO, 1994a).

In the U.S., over one quarter of all people with full-blown AIDS (a T-helper lymphocyte cell count of $<200$ per unit of blood) are between the ages of 20 and 29 -yearsold (CDC, 1994a). Locally, in Rhode Island, 21 percent of the total AIDS cases are in the 20 to 29-year-old category; as well as 28 percent of the HIV positive cases (Rhode Island Department of Health, 1994). The long latency period of HIV, prior to AIDS conversion, seemingly suppresses symptoms that may not be apparent until young adulthood (Boyer \& Kegeles, 1991; Brooks-Gunn, Boyer, \& Hein, 1988; Brooks-Gunn \& Furstenberg, 1990; Hernandez \& Smith, 1990; Joseph, 1991; Millstein, 1990; Schwarcz \& Rutherford, 1989; Slater, 1989; Vermund, et al., 1989; Wilcox, 1990), which ultimately masks the true number 
of infected adolescents (DiClemente, 1990). Therefore, the current figures of AIDS cases among adolescents are misleading, since many young adults categorized in older age groups most likely had acquired HIV infection during the adolescent years; and, consequently, were not diagnosed until years later. Although the current figures for adolescents appear to be comparatively small, the rate is still doubling each year (Boyer \& Kegeles, 1991; BrooksGunn, Boyer, \& Hein, 1988; Kelly \& St. Lawrence, 1988). Researchers note much evidence that adolescents are moving toward becoming one of the highest risk groups for contracting HIV (D’Angelo, Brown, English, Hein, \& Remafedi, 1994; Hein, 1991c; Janke, 1989; Kipke, Futterman, \& Hein, 1990; Kolbe, 1990; Takanishi, 1993), particularly via heterosexual exposure (Hein, 1991a; Poppen \& Reisen, 1994; van de Wigert \& Padian, 1993; WHO, 1993d, 1994a). Seroprevalence has increased at alarming rates among teenaged Americans in the military (Burke, et al., 1990) as well as the Job Corps (Hein, 1991b).

Although the psychosocial impact of the pandemic is only recently coming to the forefront, the national death rates left a impact on several subpopulations. In 1984, AIDS was the tenth leading cause of death among 15 to 24 -year-olds, while three years later, the AIDS mortality rate ranked sixth among this age group (Hein, 1992; Kilbourne, Buehler, \& Rogers, 1990). By the close of 1991, AIDS ranked third in cause of death among persons aged 25 to 44 (CDC, 1993c, 1993j), and in 1992 ranked number one among men in this age group (CDC, 1993i). By 1993, AIDS became the leading cause of death in the U.S. among individuals in this latter age group, both genders and all ethnic groups combined (CDC, 1994k, 19941, 1996). 
Other findings indicate that increases in HIV and AIDS cases for teenagers (Hein, 1991a, 1991c; WHO, 1994a), as well as for women (Hankins, 1993; Ledergerber, von Overbeck, Egger, \& Luthy, 1994), are resulting from heterosexual transmission, a mode of HIV infection that has increased from three to ten percent in the U.S. (CDC, 1993e, 1995e). Cumulative data collected in Rhode Island are consistent with national statistics on heterosexual transmission rates for women, at 32 percent; however, not so with ethnicity, with a preponderance of infection noted among Caucasian women (Carpenter, et al., 1991). Laumann, et al. (1989) provide evidence that the CDC may be underestimating the AIDS prevalence among higher socioeconomic, Caucasian population, and overestimating prevalence in minority populations.

Other data from the CDC (1994b, 1995d) show indications of increasing adolescent AIDS risk: one in every nine teenage women will get pregnant, with an estimated 95 percent of such pregnancies being unintended; two-thirds of the estimated 12 million who acquire a sexually transmitted disease (STD) are under the age of 25 , and each year 3 million teenagers are infected with STDs. Further, researchers have determined that those with a history of STD infection are especially prone to HIV transmission due to physiological susceptibilities (Hein, Dell, Futterman, Rotheram-Borus, \& Shaffer, 1995; Kalichman, 1995, p. 45; Otten, Zaidi, Peterman, Rolfs, \& Witte, 1994; Quinn, et al., 1988; Wagner, et al., 1994; Wynder, 1994). Other data show young women to be especially at risk, as infection among them is increasing rapidly (CDC, 1995a; Stine, 1995, p. 207). The CDC (1994b) reports the overall ratio of HIV to AIDS among adolescent men, 13 to 19 years of age, is $8: 1$, and a ratio much higher for female counterparts, at $21: 1$. 
The absence of data for teens regarding sexual behavior patterns, preferences, attitudes, and potential HIV-risk predictors (DiClemente, 1993; Gardner \& Wilcox, 1993; Millstein, 1990; Nelkin, Willis, \& Parris, 1991) has had, and will continue to have potential negative implications in the development of age-appropriate prevention strategies. Further, with the increased prevalence of HIV and AIDS cases, community, public health and education officials may greatly benefit from accurate estimates of adolescent risk behavior. The present study is an expansion of previous research (Lang, 1994; Lang, Harlow, Quina, \&, Morokoff, 1994; Lang, Quina, Harlow, \&, Morokoff, 1994) seeking further understanding of HIV-risking behaviors in youth by using a set of predictor variables based on several conceptual frameworks developed with varied populations in the existing literature. Suggested potential factors, together with risk behaviors empirically noted among youth, constitute the variables of interest. Reliability and validity of potential risk factors with cognitive, attitudinal, behavioral, psychosocial and psychoeducational constructs are further explored in continuing effort to develop a comprehensive assessment of adolescent risk. The investigation attempts to examine whether such risk predictors are evident in a large, socioeconomically and ethnically diverse adolescent population from the Eastern United States attending a youth conference, and a regional high school population in Jewett City, Connecticut. The study will further seek to determine whether there are gender differences in risk predictors and risky behaviors. Utilizing the Transtheoretical Model (see Prochaska \& DiClemente, 1983, 1984; Prochaska, DiClemente, \& Norcross, 1992; Prochaska, et al., 1994), the project also examines the process of adopting safe and 
healthy behavior by assessing stages of change in current and predicted adolescent behaviors pertaining to condom use. Finally, the study seeks to supplement this quantitative investigation with qualitative data utilizing focus group methodology, with open-ended inquiry regarding survey content (i.e., readability and truthfulness), and adolescent feedback and concerns regarding AIDS research and suggestions for prevention/intervention strategies.

It is believed that identification of risk predictors is a crucial first step towards future development of age and gender appropriate prevention and intervention strategies. A multivariate approach will be utilized to identify youth risk factors and assess their prevalence and possible correlates.

Identifiable Trends Increasing Adolescent Risk

Studies among young adults indicate considerable acceptance of premarital sexual behavior (Cates, 1991; Katz, Mills, Singh, \& Best, 1995; Mason, 1989; Moore \& Stief, 1991; Rubinson \& deRubertis, 1991), typically being a spur-of-the-moment decision (Zelnik \& Shah, 1983). Based on longitudinal studies in the 1970's (1971, 1976, and 1979), DiClemente $(1989 ; 1990)$ identified two trends that increase the likelihood of adolescent exposure. The first trend across that decade was earlier initiation of sexual intercourse, with adolescents below the age of 14 showing the greatest increase. The second trend was an increase in number of sexual partners. Lang (1994) found evidence supporting these findings, with age of sexual initiation averaging 13.5 , and teens reporting an average of two sex partners by age 16 . Other studies with adolescents confirm these and other trends of sexual activity and higher rates of pregnancy and STDs than their predecessors (Belfer, 
Krener, \& Miller, 1988; Hingson, Strunin, \& Berlin, 1990; Hofferth, Kahn, \& Baldwin, 1987). Researchers have observed such trends of declining age of sexual debut and increase in sex partners occurring over the past two to three decades (Gayle \& D'Angelo, 1991; Graham, 1994; Hofferth, Kahn, \& Baldwin, 1987; Kahn, Kalsbeek, \& Hofferth, 1988; O'Reilly \& Aral, 1985; Zelnick \& Kantner, 1977, 1980).

There is ample evidence in the literature for these patterns of sexual activity. It has been estimated that 50 percent of adolescent women have had sexual intercourse in their adolescent years, and that two-thirds of those women use ineffective birth control methods or none at all (Millstein, 1990); and one out of six has had more than four partners (Belfer, Krener, \& Miller, 1988). Nationwide, 53 percent of high school students in grades nine through twelve reported having had sexual intercourse during their lifetime, 18.8 percent with four or more partners (CDC, 1995b, 1995c); with young men significantly more likely to have sex at an earlier age, and have more lifetime partners, than young women (CDC, 1993b; Epstein, Dusenbury, Botvin, \& Diaz, 1995; Ford, Rubinstein, \& Norris, 1994; Klassen, Williams, \& Levitt, 1989; p. 161; Leigh, Morrison, Trocki, \& Temple, 1994; Maxwell, Bastani, \& Yan, 1995). Boyer and Kegeles (1991) report the average age of first sexual intercourse is 16-years-old; however, in some urban areas the average has been documented as young as 12-years-old (Clark, Zabin, \& Hardy, 1984). Other accounts of sexual activity of adolescent women in urban areas reveal that 60 percent of young Caucasian women and 80 percent of young African-American women have experienced intercourse by the age of 19 (Lewis, Battistich, \& Schaps, 1990). Sixteen-year-old Caucasian women constitute the greatest increase for sexual activity (DiClemente, 1990). 
Locally, Paxson and Quina (1989) surveyed a sample of college students and found 68 percent of freshman woman were active, and 69 percent of those women were not using birth control. In another Rhode Island sample of a much younger age, it was found that 25 percent of surveyed seventh and eighth graders (mean age 13.2) were sexually active, and 53 percent of the sexually active young men and 40 percent of the sexually active young women had had more than five sexual encounters (Brown, DiClemente, \& Beausoleil, 1992). Sonestein, Pleck and Ku (1989) indicated that among their sample, one-third of the 15-year-old young men were sexually active, one-half of the 16-year-olds, two-thirds of the 17-year-olds, and 86 percent of the sample at 19 years-old. Adolescents involved with the juvenile justice system were found to have extremely low mean ages of first intercourse, typically around age 12, and high pregnancy rates (DiClemente, 1991; Melchert \& Burnett, 1990).

To date, there is little indication that the epidemic proportions of AIDS in itself has been an influential factor in delaying the onset of sexual behavior among adolescents (Kelly \& Murphy, 1992), or successful in promoting abstinence (Hein, 1993). For example, Strunin and Hingson (1987) found that 70 percent of their adolescent sample were sexually active. Only 15 percent reported a change in their sexual behavior for fear of contracting AIDS, and of that percentage, a mere one in five altered their behavior in effective ways. Similarly, in Roscoe and Kruger's (1990) study, only one-third of their adolescent sample disclosed altered sexual behavior for fear of AIDS. In a Philadelphia sample, only one in ten adolescents reported refraining from sex because of AIDS (Brooks-Gunn \& Furstenberg, 1990). Additionally, a considerable number of infected adolescents are not 
aware of their condition, and consequently do not consider themselves at a high risk (Joseph, 1991). Numerous studies have shown that consistent condom use in adolescents remains rare due to a common perception of invulnerability to AIDS (e.g., Baldwin \& Baldwin, 1988b; Bell, Feraios, \& Bryan, 1990; Burger, 1988; Catania, Kegeles, \& Coates, 1990; Quadrel, Fischhoff, \& Davis, 1993). A perception of invulnerability that also promotes sexual and drug experimentation, a well-documented risk factor (Aggleton, 1991; Barnard \& McKeganey, 1990; Boyer \& Kegeles, 1991; Joseph, 1991; Hochhauser, 1989; Manoff, Gayle, Mays, \& Rogers, 1989; Moore \& Rosenthal, 1991a; Reulbach, 1991; Segest, Mygind, Harris, \& Bay, 1991; Sonenstein, Pleck, \& Ku, 1989; Strunin, 1991; Surgeon General of U.S. Public Health Service, 1987). Given the exponential nature of the spread of infection and the frequency of unprotected adolescent intercourse, the potential for spread via heterosexual contact within this age group is most alarming.

Current data suggest that condoms can substantially decrease AIDS risk during vaginal intercourse (CDC, 1993f; Boyer \& Kegeles, 1991; Conant, Hardy, Sernatinger, Spicer, \& Levy, 1986; Kaemingk \& Bootzin, 1990; Reiss \& Leik, 1989; Romanowski \& Piper, 1988; WHO, 1993f). In fact, Fineberg (1988) has developed a mathematical model that predicts that consistent condom use could prevent nearly half of the sexually transmitted HIV infections in persons with one sexual partner and over half of HIV infections in persons with multiple partners. Yet many studies indicate adolescent condom use remains unequivocally low (Caron, Davis, Halteman, \& Stickle, 1993; Catania, et al., 1992; Jaffe, Seehaus, Wagner, \& Leadbeater, 1988; Leite, Buocompagno, Leite, Mergulhao, \& Battistoni, 1995; Lewis, Battistich, \& Schaps, 1990) and sexual activity high 
(Becker \& Joseph, 1988; Boyer \& Kegeles, 1991; Brooks-Gunn \& Furstenberg, 1990;

Friedman, 1994; Gayle, et al., 1990; Hausser \& Michaud, 1994; Levy, et al., 1994; Seidman \& Rieder, 1994).

In one study, adolescents reported very little condom use (Kegeles, Adler, \& Irwin, 1988), although they valued condom use highly as a prevention method against STDs.

Other studies have found that young women who use alcohol and other drugs, in particular, are less likely to negotiate condom use (Flanigan, McLean, Hall, \& Propp, 1990; Harvey \& Spigner, 1995; Millstein, Moscicki, \& Broering, 1994; Orr, et al., 1992), although substance use deters safe sexual behavior for both genders (Aruffo, Gottlieb, Webb, \& Neville, 1994; Hingson, Strunin, Berlin, \& Heeren, 1990; Koopman, Rosario, \& Rotheram-Borus, 1994; Lowry, et al., 1994; Rotheram-Borus, et al., 1994; Strunin \& Hingson, 1993). In a Massachusetts study, Strunin and Hingson (1987) found that only two percent of adolescents in their sample used effective prevention behaviors against AIDS. Further, younger teens, 12 and 13 years of age, are less likely to use condoms and more likely to use ineffective methods of birth control than are 14-year-olds (Nguyet, Maheux, Béland, \& Pica, 1994).

Apart from these individual studies, clear evidence of unprotected sex is provided with the national figures for teenage pregnancies and STDs. In the U.S. alone, there are between 1 and 1.2 million teenage pregnancies each year, and one in seven adolescents has a STD (CDC, 1993k; Belfer, Krener, \& Miller, 1988; Boyer \& Kegeles, 1991). Nearly onethird of premarital sexually active adolescents have had at least one pregnancy (Dawson, 1986). While STD rates among older age groups decreased from 1981 to 1991, rates 
among adolescents, specifically for gonorrhea, have increased or remained unchanged (CDC, 1993g). Further, the CDC (1993h) indicated that data collected by the United States Bureau of the Census during 1990 showed Rhode Island as one of six states with an increase of more than 10 percent in teenage pregnancy rates from 1980 to $1990(87.7$ per 1000 women aged 15 to 19 years in Rhode Island, during 1990). World reports of STDs show the highest incidence among those aged 20 to 24 , followed closely by those aged 15 to 19 (WHO, 1989, cited in Aggleton, 1991). Also noteworthy is the fact that STD rates in the U.S. remain higher than most Western European countries (Brooks-Gunn \& Furstenberg, 1990).

Attitudes and behaviors that are commonly accepted as high-risk for HIV infection have been extensively noted among sexually active members of the adolescent population (Belfer, Krener, \& Miller, 1988; Boyer \& Kegeles, 1991; Hein, 1988; Irwin \& Millstein, 1986; Lewis, Battistich, \& Schaps, 1990; Livingston, 1991). These behaviors include substance use and abuse (Coates, 1990; Johnson, 1991), particularly with alcohol (Pulford, 1991) and marijuana (Keller, et al., 1991); intravenous drug use (Barnard \& McKeganey, 1990; Kelly \& Murphy, 1992; McCoy \& Khoury, 1990; Mondanaro, 1987; Schuster, 1988; Schwarcz \& Rutherford, 1989; Stiffman \& Earls, 1990); drug experimentation (D'Angelo, Getson, Luban, \& Gayle, 1991); sexual behavior with multiple partners and/or sexual promiscuity (D’Angelo, 1994; Durbin, et al., 1993; Romanowski \& Piper, 1988; Stuber, 1990); frequent unprotected sex (Rotheram-Borus \& Koopman, 1991); infrequent or lack of condom use (Kegeles, Adler, \& Irwin, 1988); negative attitudes toward condoms (Morrison, 1985); lack of knowledge about risk factors (Crawford \& Robinson, 1991; 
DiClemente, Boyer, \& Mills, 1987; Kegeles, Adler, \& Irwin, 1989; Strunin \& Hingson, 1987); misconceptions of AIDS transmission (DiClemente, Boyer, \& Morales, 1988; Helgerson, Petersen, \& The AIDS Education Study Group, 1988; Sandberg, RotheramBorus, Bradley, \& Martin, 1988); perceived invulnerability to HIV infection (Baldwin \& Baldwin, 1988b; Bell, Feraios, \& Bryan, 1990; Bing, et al., 1990; Boyer \& Kegeles, 1991; Eiser, Eiser, \& Lang, 1990; Gladis, Michela, Walter, \& Vaughan, 1992; Price, Desmond, Hallinan, \& Griffin, 1988; Siegel \& Gibson, 1988; Zimet, et al., 1991); underestimating riskiness of one's sexual practices (Bauman \& Siegel, 1987); impulsive behavior (Brown, DiClemente, \& Park, 1992; Strunin, 1991); apathy and denial of risk (Barling \& Moore, 1990); lack of self-efficacy (Bandura, 1977, 1990); low social conformity (Stein, Newcomb, \& Bentler, 1994); unconventionality (Donovan, Jessor, \& Costa, 1991); sensation seeking (Arnett, 1990); acting-out behavior (Piercy, Trepper, \& Jurich, 1993); adhering to negative peer norms (Basen-Engquist \& Parcel, 1992; Billy \& Udry, 1985); and, external or chance locus of control (Brown, Nassau, \& Levy, 1990; Janz \& Becker, 1984; Kelly, et al., 1990, 1993; Larzelere \& Mulaik, 1977; Long, Williamson, Gaynor, \& Clark, 1988; Prewitt, 1989; Price-Greathouse \& Trice, 1986; Strickland, 1978; Trice \& Price-Greathouse, 1987). DiClemente (1992) adds, "While each behavior singularly increases the probability for HIV infection, they are more often reported in combination, further elevating an adolescent's risk for infection" (p. 325).

The importance of acquiring informational, practical, and social supports has been noted in the literature with HIV-testing and assistance with seroconversion (Folkman, Chesney, Cooke, Boccellari, \& Collette, 1994; Hays, Turner, \& Coates, 1992); however, 
most adolescents do not seek social support or health services, allowing the consequences of high risk status to remain unnoticed (Hein, 1988; Melton, 1989). Therefore, many infected adolescents are not aware of their serostatus, thus increasing the likelihood of infecting others. More disturbing is that nearly 20 percent of two adolescent samples responded that they would consider suicide if they tested HIV seropositive (Goodman \& Cohall, 1989; Mansfield, Conroy, Emans, \& Woods, 1993), suggesting a lower likelihood of having status revealed due to potentially severe negative emotional reactions.

\section{Heterosexual Transmission}

Early notions promoted inappropriate perceptions of the AIDS epidemic as one which afflicts primarily homosexuals and intravenous drug users; hence, many heterosexuals erroneously felt immune from AIDS. The critical factor for AIDS infection was identified as membership in a specific group, and individual behaviors were de-emphasized (Bing, et al., 1990; Kain, 1989; Lui, Darrow, \& Rutherford, 1988; Slater, 1989). The focus on risk groups was, and still is, pertinent to the understanding and targeting of more effective prevention efforts, since risk behaviors for homosexuals and heterosexuals do not greatly differ (Kelly \& St. Lawrence, 1988). Today, investigators know that any form of unprotected intercourse within any group is associated with increased exposure risk.

Unmarried heterosexuals are placing themselves at risk for HIV or other STDs, as researchers found a substantial increase and high prevalence for multiple partners, and low condom use, and associate such risk behavior and number of sexual partners with a greater probability of infection (Dolcini, Coates, Catania, Kegeles, \& Hauck, 1995). 
Nevertheless, many adolescents do not feel vulnerable to AIDS because they continue to see AIDS as a homosexual male disease (Mickler, 1993; Westerman \& Davidson, 1993); have little or no concern about HIV/AIDS, or fail to personalize the threat of HIV infection (Rosenthal \& Shepherd, 1993); or attribute low personal risk to a lower prevalence of AIDS in rural communities (DiClemente, Brown, Beausoleil, \& Lodico, 1993). It can not be assumed that the sexual knowledge, attitudes and behaviors among mature individuals will pertain to other age groups (Osborn, 1991). Moreover, Johnston (1993) discusses a flawed educational system which produces "informed adolescents" who continue to exhibit a gap between factual knowledge and actual sexual behavior.

Worldwide, heterosexual activity is the primary route of HIV infection (Mann, Tarantola, \& Netter, 1992, p. 33; U.S. Department of Health and Human Services, 1993). A large National Institute of Mental Health-funded nationwide survey of HIV-related risk factors in the general heterosexual population revealed that between 15 and 31 percent of heterosexuals reported at least one HIV-risk factor (U.S. Department of Health and Human Services, 1993). Further, AIDS cases in the U.S. attributed to heterosexual contact show a 21 percent increase from 1990 to 1991, exceeding all other exposure categories for the adolescent age group (CDC, 1994a), with evidence of rapidly increasing figures for infection via heterosexual transmission for youth (CDC, 1995e). However, it has been noted that the CDC's hierarchical method of classifying infection could be concealing even more cases of heterosexual transmission (Corea, 1993, p. 255) and exaggerating infection via intravenous drug use (Lauritsen, 1993). 
Heterosexually acquired AIDS increased 130 percent from 1992 to 1993, partially resulting from expansion of the surveillance definition of AIDS. However, the largest increases occurred among persons aged 13 to 19 , and 20 to 24 -years, with a greater proportion of infection among young women (CDC, 1994f). Infection via heterosexual route continued upward, increasing 54 percent from 1993 to mid-year 1995 (CDC, 1995f). Cross-sectional "serosurveys" conducted in 130 clinics in 24 U.S. cities with nearly 80,000 teenagers found that rates of infection among young women were higher than young men, and most teenagers with risk information reported heterosexual activity as their only potential risk exposure to HIV (Sweeney, Lindegren, Buehler, Onorato, \& Janssen, 1995). The highest proportion of cases associated with heterosexual contact during 1993 were in the South (42 percent) and Northeast (31 percent) (CDC, 1994d). In New York City, which accounts for 20 percent of AIDS cases among 13 to 21-year-olds, proportionately, heterosexual contact has been the most reported transmission route by adolescents (Hein, $1990,1992)$.

Kalichman $(1996$, p. 95) identified five factors contributing to the rise in heterosexual infection: (1) less tendency to take precautions; (2) resistance against using condoms; (3) beliefs that AIDS is a disease affecting only drug users and gay men; (4) the perception that AIDS "can't happen to me;" and, (5) the sheer numbers of infected people unknowingly spreading the virus. As the numbers of infected heterosexual individuals increase, the incidence and prevalence of heterosexual infection and transmission will also rise in consequence. A common misconception is the rationale that if one is not most at risk, one is not at a risk at all; although a more accurate, but less adopted view is the notion 
that every sexually active person faces some risk depending on their behavior and their partner.

\section{Adolescent Risk Predictors}

Specific risk predictors examined in the present study were extracted from past studies that yielded contrasting or contradictory results among variables with adolescents or had been previously documented to be related to high risk behavior. The study examines the following variables: (1) cognitive components of AIDS knowledge, (factual AIDS knowledge, transmission knowledge, perceived AIDS knowledge, and commonly held misconceptions); (2) attitudinal components (condom use efficacy, perceived susceptibility for risk, homophobia, and fear of AIDS); and (3) behavioral components (substance use, substance abuse, sexual assertiveness for both condom use and partner communication, and risk histories for both partner(s) and self, based on past sexual behavior). The rationale for investigation of these three areas are discussed below.

Cognitive. Studies examining AIDS knowledge and attitudes among adolescents have generally been varied, often with contradictory findings. However, significant gaps in HIV/AIDS knowledge, particularly with transmission and awareness of preventive recourse, have been identified (Brown, DiClemente, \& Beausoleil, 1992; DiClemente, 1989; Piercy, Trepper, \& Jurich, 1993; Sandberg, Rotheram-Borus, Bradley, \& Martin, 1988; Singh, Zemitzsch, Ellis, Best, \& Singh, 1994), as well as a disparity of AIDS knowledge between Caucasian, African-American, and Latino adolescents (Bing, et al., 1990; Crawford \& Robinson, 1991). Brown and colleagues (1992) found that adolescents who were less knowledgeable of AIDS were less fearful, with riskier attitudes and behavior when 
compared with their peers. Yet, in other studies, high level of sexual risk contrasted with moderately high knowledge and positive beliefs of self control for AIDS prevention (Fergusson, Lynskey, \& Horwood, 1995; Roscoe \& Kruger, 1990; Rotheram-Borus \& Koopman, 1991). Bell, Feraios and Bryan (1990) emphasize that one must know how AIDS is transmitted before protective behaviors can be implemented; however, they found that "seemingly responsible" young men knew less than their risk-taking counterparts of how AIDS was transmitted sexually, which is a direct risk if sexually active. Other researchers also indicated that one-third of their adolescent sample did not relate the risk of contracting AIDS with unprotected sexual intercourse (Dusenbury, Botvin, Baker, \& Laurence, 1991; McDermott, Hawkins, Moore, \& Cittadino, 1987). More recently, researchers reported that AIDS knowledge scores for junior high school students had decreased from 1989 to 1991, while it increased for senior high students from 1988 to 1991 (Zimet, et al., 1993), suggesting such information may be more beneficial if dispersed at younger ages.

Additional research endeavors regarding adolescents have found relationships between high knowledge and associated beliefs of less susceptibility than most others of acquiring AIDS despite high risk behavior (Adame, Taylor-Nicholson, Wang, \& Abbas, 1991; Keller, et al., 1991; Moore \& Rosenthal, 1991a), or inaccurate self-perceptions of serial monogamy as being completely safe (Hernandez \& Smith, 1990; Overby \& Kegeles, 1994); or risk behavior associated with disinterest in AIDS education (Henderson, Weinman, \& Smith, 1994). However, numerous studies have shown a relatively high degree of AIDS knowledge but identified several areas of confusion, especially in 
transmission and prevention of infection (DiClemente, Boyer, \& Mills, 1987; DiClemente, Zorn, \& Temoshok, 1986, 1987; Dusenbury, Botvin, Baker, \& Laurence, 1991; Livingston, 1991; McDermott, Hawkins, Moore, \& Cittadino, 1987; Memon, 1990; Ndeki, Klepp, Seha, \& Leshabari, 1994; Ponton, DiClemente, \& McKenna, 1991; Price, Desmond, \& Kakulka, 1985), distinguishing safe from less-safe behaviors (Rotheram-Borus, Becker, Koopman, \& Kaplan, 1991), and misunderstanding of the seriousness of AIDS (DiClemente, Zorn, \& Temoshok, 1987; Eiser, Eiser, \& Lang, 1990; Longshore, 1990; Petosa \& Wessinger, 1990). Discrepancies and conflicting messages about sexuality from various sources, for example, parents, school and media, appear to complicate and mislead adolescents regarding their sexual decisions and behavior (Brooks-Gunn \& Furstenberg, 1990).

Attitudinal. The impact of attitudinal characteristics on AIDS-risk behavior has been widely explored; however, the majority of the studies have focused on only limited populations, generally excluding adolescents. Since self-efficacy has been associated with an individual's beliefs of self-control in behavior and motivation (Bandura, 1977, 1990), it appears to be a viable factor in predicting AIDS risking behaviors, particular to condom use. Bandura $(1977,1990,1992)$ has noted the influential role of self-efficacy as a predictor of the accuracy of personal risk estimation, or perceived risk, and of self-control over risk behaviors, and has suggested it may be linked to sex risk behaviors (see also Langer, Zimmerman, Warheit, \& Duncan, 1993; O'Leary, 1985; Weinstein \& Nicolich, 1993; Zimet, et al., 1992; van der Pligt \& Richard, 1994). For instance, for adolescent women, high self-efficacy has been associated with taking precautionary measures (Richard 
\& van der Pligt, 1991), and with condom use for older women (Kegeles, Adler, \& Irwin, 1989). In a more recent investigation, self-efficacy expectations were found to be important determinants of intended condom use (Schaalma, Kok, \& Peters, 1993). In California, incarcerated adolescents in a detention facility showed lower self-efficacy for AIDS prevention, lower perceived personal threat of AIDS and lower perceived norms of safe sex practices when compared to adolescent students from urban public high schools, suburban private schools and adolescent members of a gay youth organization (Nader, Wexler, Patterson, McKusick, \& Coates, 1989).

In one focus group study, researchers found that adolescents' self-efficacy was actually lower than had been indicated on previous questionnaire ratings, and although many of the teens reported a moderate level, the result may have been affected by the belief that AIDS is preventable (Sandberg, et al., 1988). Another study reported associations between knowledge, self-efficacy and perceived risk for AIDS suggesting that AIDS knowledge may be linked with self-efficacy, where self-efficacy and self-control were found to be significantly related to AIDS knowledge (Rotheram-Borus, Becker, Koopman, \& Kaplan, 1991). Similarly, Rotheram-Borus and colleagues (1991) also surmised that AIDS knowledge may be an influential factor in the development of coping skills to adequately handle risk situations and instrumental in the accurate perception in self-efficacy and selfcontrol.

Perceived personal susceptibility, or conversely perceived invulnerability for AIDS is also noted as being a widespread factor with adolescents and risk behaviors (Hochhauser, 1989), and has been evident even with highly promiscuous heterosexual individuals who are 
knowledgeable of AIDS risk and still minimize their level of susceptibility (Hernandez \& Smith, 1990; Leishman, 1987). Additionally, one investigation found that sexually active adolescents in grades six through eight who had sex with partners they did not know well, did not perceive themselves at a greater risk than those who knew their partners (Dolcini, et al., 1988, cited in Boyer \& Kegeles, 1991). Similarly, a study with New York City adolescents reported that those who engaged in the highest sexual risk behaviors perceived themselves to be at a low risk (Reuben, Hein, \& Drucker, 1988, cited in Boyer \& Kegeles, 1991). Livingston (1991) postulated that often in risk perception studies, individuals underestimate familiar risks and tend to overestimate unfamiliar risks that may be involuntary, invisible or catastrophic. Nevertheless, it seems evident that possession of positive, realistic attitudes towards safe sex practices and the belief that one can avoid HIV infection are practical factors in AIDS risk prediction among adolescents and may be critical to effective education for reducing risky behavior.

Negative attitudes and fear towards gay and lesbian individuals were found to be associated with higher HIV-risk behavior among older adolescents in a recent URI study (Burkholder, 1994). Burkholder surmised this finding to be associated with high risk behavior on the part of heterosexual individuals assuming that AIDS is a "gay disease," erroneously believing that precautions are not necessary regarding their sexual behavior. Westerman and Davidson (1993) also found that many adolescents felt invulnerable to HIV infections because they still viewed AIDS as a "gay disease." Further, those who were most prejudiced against homosexuals, were five times more willing to engage in indiscriminate unprotected sexual behavior, than the least prejudiced group; and among young men, the 
effect was greater (Westerman \& Davidson, 1993). Efforts to educate the public about AIDS, as a means to combat prejudice against persons with AIDS and stereotyping certain "risk groups," have been based on the assumption that factual AIDS knowledge and prejudice are inversely related. Negative attitudes toward people with AIDS were inversely related to AIDS knowledge in some studies (Landefeld, Chren, Shega, Speroff, \& McGuire, 1988; Peruga \& Celentano, 1993; Shafer \& Boyer, 1991); and only slightly so in another (Witt, 1989). Other researchers found that among men and women at low risk for AIDS in San Francisco, New York and London, fear of AIDS and anti-gay attitudes were negatively correlated with knowledge about AIDS (Temoshok, Sweet, \& Zich, 1987). These researchers were unclear whether fear and prejudice, which were highly intercorrelated $(r=$ .64), promote ignorance, or whether ignorance increases fear and prejudice. Nevertheless, the absence of recent data on the correlation of homophobia, fear of AIDS, and risk behavior suggest further examination would be beneficial with a younger population, especially when attitudes may still be forming.

Behavioral. Numerous studies that have investigated risk behavior of young people have concluded, with a strong emphasis, a need to further examine behavioral risk factors associated with the prevalence of AIDS. In particular, these studies have examined factors such as: sexual activity, contraceptive use, and substance abuse (Boyer \& Kegeles, 1991; DiClemente, 1990; Millstein, 1990; Osborn, 1988, 1991; Richard \& van der Pligt, 1991; Strunin, 1991); maladaptive behaviors relating to substance use (Clapper \& Lipsitt, 1991; Frances \& Miller, 1991; Grant \& Johnstone, 1990; McCoy, McKay, Hermanns, \& Lai, 1990; Molgaard, Nakamura, Hovell, \& Elder, 1988; Niven, 1987; Plant, 1990; Pulford, 
1991; Stall, Coates, \& Hoff, 1988; Walter, Vaughn, \& Cohall, 1991; Windle, 1989); multiple partners or sexual promiscuity (Romanowski \& Piper, 1988; Stuber, 1990); frequent unprotected sex (Rotheram-Borus \& Koopman, 1991); infrequent or lack of condom use (Kegeles, Adler, \& Irwin, 1988); and sexual assertiveness (Franzini, Sideman, Dexter, \& Elder, 1991; Kelly \& St. Lawrence, 1990). Only six years ago, it was estimated that one in four teens were vulnerable to high-risk behaviors (The Carnegie Council on Adolescent Development, 1989, cited in Johnson, 1991); this estimate in itself justifies a need to examine present trends in the adolescent population to diminish further spread of infection.

A number of studies have indicated a high prevalence of sexual activity in the teenage years. For example, 64 percent of the college women in the Harlow, et al. (1993) study containing nearly 500 participants, reported their age at first intercourse as being between 15 and 17 years, and another 8 percent reported an age less than 15-years-old. The Rotheram-Borus, et al. (1991) sample of delinquent $(n=60)$ and non-delinquent $(n=$ 57) adolescent young men also yielded a high proportion who were sexually active at young ages. Seventeen percent of the young delinquent men and 29 percent of the young nondelinquent men reported age at first intercourse between 14 and 17 years, and an astonishing 30 percent of the delinquent men and 61 percent of the non-delinquent men disclosed an age of 13 years or younger. In another investigation, 407 inner-city adolescents were examined five years ago for HIV-relevant behaviors, and revealed mean ages for onset of sexual activity as 13.3 years for a clinic sample, and 13.8 years of age for a school sample (Keller, et al., 1991). A large majority (71 percent) of the adolescent 
subjects in the Adame et al. (1991) study $(n=226)$ also indicated having engaged in sexual intercourse in their adolescent years, where 68 percent were between the ages of 16 and 17 , and 17 percent reported being 15 years or younger. Teens in a Copenhagen study also revealed an average age of 14.6 for sexual debut (Segest, Mygind, Harris, \& Bay, 1991), with a slightly higher average in Norway, at 15.4 years (Kraft, Rise, \& Traeen, 1990).

National figures provided by Hein (1988) show that approximately half of all adolescents have had sexual intercourse by the age of 19-years-old; however, more recent national data on youth between the ages of 14 through 19 , found sexual activity among 45.4 and 70.1 percent of adolescents in school, and out of school, respectively (CDC, 1994e). In a national telephone survey, 43.4 and 81.7 percent of youth between the ages of 14 through 17 years, and 18 through 21 years, respectively, reported they were sexually active (CDC, 1994g). Other risk behaviors for youth are also evident. For instance, in San Francisco, 21 percent of sexually active adolescent women and 22 percent of the adolescent men in a sample of nearly 500 participants reported receptive or insertive anal intercourse (Broering, Moscicki, Millstein, Policar, \& Irwin, 1989). Similar findings of potential risk involving unprotected sexual activity, especially at early ages were found in a studies among incarcerated teens (DiClemente, 1991; Magura, Kang, \& Shapiro, 1994; Morrison, Baker, \& Gillmore, 1994; Rickman, et al., 1994), and homeless, adolescent runaways (Goodman \& Berecochea, 1994; Rosenthal, Moore, \& Buzwell, 1994; Rotheram-Borus, et al., 1992).

Typically, only about 15 percent of teenagers reported that having sex the first time was planned (Brooks-Gunn \& Furstenberg, 1990), and consistent condom use has been shown to be low among sexually active adolescent samples. Many reports on condom use 
have produced very low frequencies: fewer than 10 percent of a sexually active adolescent sample attending health clinics in San Francisco (Kegeles, Adler, \& Irwin, 1988), 10 percent of young men and 4 percent of young women attending STD clinics in California (Maxwell, Bastani, \& Yan, 1995); 23.2 percent in a sample of adolescent men (Keller, et al., 1991); 21 percent in a sample of adolescent gay men (Rotheram-Borus \& Koopman, 1991); 10 percent of sexual active teens in a Massachusetts telephone survey (Strunin \& Hingson, 1987); 12 percent of sexually active 14-year-old girls (Brooks-Gunn \& Furstenberg, 1990); 28 percent with casual partners and 18 percent with regular partners for Australian teens (Moore \& Rosenthal, 1991a); and only 13 percent of adolescent men who consumed alcohol prior to their first sexual intercourse experience (Robertson \& Plant, 1988).

Moore and Rosenthal (1991a) suggest that a common notion among adolescent justification for not using condoms is associated with a flawed perception of condoms being unnecessary when involved in serial monogamous relationships. Many teenagers, however, reported the reasons for unprotected sex were unplanned intercourse and firm beliefs that they would not conceive (Brooks-Gunn \& Furstenberg, 1990). Hingson and Strunin (1992) found that very few adolescents report discussing condoms prior to intercourse. These researchers surmised that adolescents may fear that discussion may raise questions about fidelity, moral values with the expectation to have sex, or that sex partners may think that they are infected with HIV already. Additionally, condom use and sexual behaviors have been associated with attitudes and supportive norms of particular populations (Catania, Coates, \& Kegeles, 1994). However, researchers found that with a young high school sample, teens will engage in sexual intercourse if the opportunity presents itself, with or 
without a condom and irrespective of their attitudes and norms regarding condom use (Fisher, Fisher, \& Rye, 1995). Demb (1990) interviewed African-American, inner-city adolescent women about condom use and STDs and found a high resistance to condom use with this population. Catania, et al. (1989a) examined the psychosocial correlates of condom use and multiple partners with sexually active heterosexual adolescent women who attended a family planning clinic and found condom use to be relatively low, 27 percent. Of interest in this last study was the relevance of sexual assertiveness in communication with the primary partner. The willingness to request partners to use condoms and enjoyment of condom use were significantly correlated with more frequent use (Catania, et al., 1989a).

It appears that substance use, especially prior to sexual activity, increases the likelihood of high-risk behaviors (Clapper \& Lipsitt, 1991; Grant \& Johnstone, 1990; MacGregor, 1988; Stall, McKusick, Wiley, Coates, \& Ostrow, 1986). Even moderate amounts of alcohol or marijuana have been predictors in high risk behaviors (Keller, et al., 1991), interfering with the "ability or willingness" to use condoms (Millstein, 1990; Remafedi, 1988), and causing disinhibition and impaired judgment in sexual situations (Kelly \& St. Lawrence, 1988). Perhaps the most convincing evidence is provided by Robertson and Plant (1988), who found that age of first alcohol use and age of first sexual intercourse were highly correlated ( $r=.58$ for young men; $r=.79$ for young women), with the majority reporting alcohol use prior to their first sexual encounter (58 and 48 percent for young men and young women, respectively). The importance of substance use prior to first intercourse was evident in other studies, associated with lower likelihood of condom use (e.g., Flanigan, 1990; Leigh, Schafer, \& Temple, 1995; McLean \& Flanigan, 1993; 
Newton, 1995, p. 98), and with less planning before first intercourse (McLean \& Flanigan, 1993). Similar results were found with Norwegian adolescents: 28 percent of the young women and 43 percent of the young men reported alcohol use before first intercourse, with nearly one-half of those teens not using condoms (Kraft, Rise, \& Traeen, 1990). BrooksGunn and Furstenberg (1990) suggest that adolescents tend to be highly influenced by situational factors, exemplified by having sex while drinking. On two Southeast college campuses surveying 18 to 21 year-old students, 35 percent of men and 7 percent of the women reported substance use on a date, enough to impair their judgment, one or more times a month (Hernandez \& Smith, 1990).

Sexual assertiveness is another important behavioral factor. Some heterosexual adolescents may recognize the risk but are unable to negotiate safe behavior with their partners (Brooks-Gunn \& Furstenberg, 1990). Rosenthal, Moore, and Flynn (1991) found that sexually-active adolescent men showed more confidence than young women in their ability to assert their sexual needs but were less confident in their ability to decline sexual demands. Overall, the ability to say "no" in sexual situations was the sole predictor of safe sexual behavior for both genders. Kelly, St. Lawrence, Hood and Brasfield (1989) found that sexual assertiveness training, together with self-affirming social supports, were successful in reducing high-risk sexual behavior (defined as the ability to refuse sexual coercions) among gay men. A change that was maintained at an eight-month follow-up. In one sexual assertiveness training experiment, young university students who participated in behavioral training sessions with verbal reinforcement, role playing, corrective feedback and behavior shaping, showed more assertive behavior than controls who did not receive the 
training (Franzini, Sideman, Dexter, \& Elder, 1990). Additionally, gender differences in assertiveness were indicated in the Franzini, et al. experimental group, with young men being more assertive in requesting condom use in sexual situations and young women more assertive in asking about partner's history at the post-intervention measure.

\section{Gender Differentials in Risk Behavior}

The investigation of possible gender differences in AIDS-risk behavior is also a particular concern in this study, since very little research in this area has focused on adolescent women. Of the handful of investigations evaluating young women, one examined African-American, inner-city, young women (Demb, 1990); two studied adolescent women attending a health clinic (Catania, et al., 1989a; Rickert, Gottlieb, \& Jay, 1990); and one surveyed 69 sexually active teenaged women to see if they recalled receiving a brochure on AIDS and whether they read it and discussed it with someone (Kamachi, Irwin, Shafer, \& Wong, 1990). Overall, investigations of HIV-risk prevention, etiology, treatment, and policy research for women and adolescents is meager; nearly ignoring women completely, categorizing adolescents as "others," and mainly concentrating on gay, and to some extent, heterosexual intravenous drug-using men (Anderson, et al., 1991; Baum \& Nesselhof, 1988).

It may be important to identify potential risk predictors separately for young men and young women in order to design the most appropriate intervention and prevention strategies. There is evidence that young women face a significantly higher biological susceptibility to HIV infection than men or older women. Based on risk models of infection probabilities, 76 percent of young women were found to have higher risk of infection (one 
chance in 100,000) compared with only three percent of young men (Smith, McGraw, Crawford, Costa, \& McKinlay, 1993). Smith, et al. (1993) attributed this gender risk differential as greater prevalence of HIV among potential sex partners of young women, as well as to more efficient transmission of the virus from men-to-women than vice versa (see also, Curran, et al., 1988; Healy \& Coleman, 1989; Hearst \& Hulley, 1988). Other researchers note the greater odds of man-to-woman HIV transmission than woman-to-man due to a host of physiological factors (The European Study Group, 1992; Friedman \& Klein, 1987; Padian, et al., 1987; Padian, Shiboski, \& Jewell, 1991). Infection from woman-to-man is estimated to be anywhere from twice to twenty times less likely than the reverse (The European Study Group, 1992; Laga, et al., 1989; Padian, Shiboski, \& Jewell, 1991; Rehmet, et al., 1992; WHO, 1994a). Further, as a receptive partner, women are more vulnerable to infection than men because they have a larger mucosal surface exposed during sexual intercourse; and moreover, semen contains a far higher concentration of HIV than vaginal fluid (WHO, 1993d). Additionally, ethnicity has been noted in the literature as possibly having increased relevance in infection rates, as recent researchers have found a higher women-to-man ratio for infection among African-Americans, than the reverse (Brunswick, et al., 1993). Adolescent women have greater odds of becoming infected than older premenopausal women due to biological susceptibilities, such as limited vaginal secretions and thinness of vaginal tissue lining in the developing cervix (Brooks-Gunn, 1988; Cates, 1991; Ostergard, 1977; McGrath, Strasburger, \& Cushing, 1994; Moscicki, Winkler, Irwin, \& Schachter, 1989; WHO, 1993e). 
Overall, early intervention regarding such skills as sexual assertiveness, sexual control issues, partner communication and decision-making may be highly beneficial, but the needs may vary according to gender and other circumstances. Findings are discussed below regarding gender roles and power differentials in the context of heterosexual relationships, attitudes and barriers toward condom use, and sexual assertiveness, which may provide some insight into areas where potential gender risk differentials exist.

Gender role expectations undoubtedly play a significant part in many relationships between men and women. This is probably more so with adolescents since teens are easily socially influenced, especially by peers (Sears, 1986). The dominance of men over women in heterosexual relationships is nearly worldwide, and this dominance is likely to permeate the area of sexual decision-making, specifically regarding protective behavior (WHO, 1993c). The power dynamics that operate in sexual relationships may not encourage women to open a dialogue about protection, let alone to insist on it. In most cultures, negotiation has not been an area in which women have been encouraged to communicate, and/or negotiate, thus silencing women in sexual matters (WHO, 1993c). Addressing power differentials in relationships and their potential correlation to various HIV-risk behaviors, is key to understanding women's interpersonal power in leading healthy lifestyles (Quina, Harlow, Morokoff, \& Saxon, in press). Fullilove, Fullilove, Haynes, and Gross (1990) found effects of traditional gender roles African-American women, where sexual communication difficulties were aggravated by an imbalance of power. For instance, the notion that men were allowed by tradition to have sexual freedom was evident; however, the reverse scenario for women did not hold true. 
Barling and Moore (1990) report that girls tend to be more compliant toward social norms, which presents difficulty in the initiation of condom use since this task has been traditionally seen as the man's responsibility. These outcomes, like other reported gender differences and the continuing prevalence of gender role stereotypes (e.g., Broverman, Vogel, Broverman, Clarkson, \& Rosenkrantz, 1972; Gilligan, 1979, 1982; Miller, 1986; Stern, 1990) are assumed to reflect the differential socialization and intrapersonal processes for young women. Although it is assumed that men and women are relatively equal in their potentialities for risk behavior, actual behaviors may differ widely as a function of personal choice, the behavior of others, situational contexts and socialization differences. Cultural values and norms, peer pressure and customs within adolescent groups often dictate gender role behavior which may indirectly influence risk behavior (e.g., Airhihenbuwa, DiClemente, Wingood, \& Lowe, 1992; Damond, Breuer, \& Pharr, 1993; Dancy, 1991; Jemmott \& Jemmott, 1991; Kalichman \& Coley, 1995; Kalichman, Kelly, Hunter, Murphy, \& Tyler, 1993; Schinke, Botvin, Orlandi, Schilling, \& Gordon, 1990; Stevenson \& Davis, 1994; Stevenson, Gay, \& Josar, 1995; Wingood \& DiClemente, 1992).

Presumably, variables such as self-efficacy, sexual assertiveness, substance abuse and sexual behavior may vary according to gender; however, there are limited data to substantiate the assumption. This assumption is made by the observance that often, within a cultural context, mixed messages are conveyed regarding the acceptability of sexual and contraceptive experiences for adolescents (Brooks-Gunn \& Furstenberg, 1990), which may have a direct reflection in behavior. Self-efficacy and sexual assertiveness for initiating and negotiating condom use and for partner communication have yielded significant gender 
differences, with young men exceeding young women (Lang, 1994). Women may face a dilemma when responsible acts of safe sex conflict with maintaining their relationship. For example, adolescent girls are more likely to stay with their male partner who is injecting drugs in order to help their partner stop using, than are young men in the reverse situation having a female intravenous drug using partner (Barnard \& McKeganey, 1990). Data suggest that when women revealed HIV infection to male partners, the partners were more likely to become angry and withdraw, or eventually leave the relationship (Simoni, et al., 1995).

Sexually active young women have been found to have less partner communication assertiveness than their male counterparts (Lang, 1994). This finding coincides with past research which has postulated that young women may require specific assistance in assertiveness toward partner communication, and specific skills of how to use a condom (Barling \& Moore, 1990). The ability to discuss with a potential partner about his or her sexual and drug activities is undoubtedly a risk factor to be considered. However, research has shown that inquiry may only provide an illusory sense of safety, as most men would lie about their sexual history to gain sex (Cochran \& Mays, 1990; Keeling, 1989). Therefore young women with a strong sense of personal efficacy and communication skills may be likely to engage in unprotected intercourse, unless sufficient assertiveness skills for condom use are acquired.

Data on gender differences in attitudes regarding condom use among adolescents, however, are rather mixed. For instance, adolescent women may hesitate more than young men to buy and carry condoms, for fear of negative comments (Barnard \& McKeganey, 
1990; Rickert, Jay, Gottlieb, \& Bridges, 1989; Sacco, Levine, Reed, \& Thompson, 1991); or may even express an "anti-condom" sentiment (Demb, 1990). Among young men, condom use is inhibited by concerns about embarrassment, reduced sexual pleasure, ignorance about how to use them properly, and fear that using condoms will permanently interfere with fertility (Pleck, Sonenstein, \& Ku, 1991; WHO, 1994a), while young women believed condoms were inconvenient to use (Kegeles, Adler, \& Irwin, 1989). Despite being cognizant of potentially high risk in unprotected sexual activity, young men are more likely to place relatively low values on such outcomes compared to perceived positive value of immediate sexual gratification (Pendergrast, DuRant, \& Gaillard, 1992). In another study, young men were more likely to place sexual precautionary responsibilities (e.g., condoms and birth control) on their partners (Moore \& Rosenthal, 1991b).

Fear of AIDS was found to be related to risk behavior, where among young women, those who reported behavior change had more fear of AIDS (Goodman \& Kohall, 1989). Young women 8 to 16-years-old express a significantly greater fear of AIDS than their male counterparts (King \& Gullone, 1990; Wilkins \& Lewis, 1993), which suggests that young women may eventually bear the burden of promoting safe sex with their partners. Worry, or fear of AIDS, was hypothesized to predict behavior change in another study, but different patterns for women and men were found. In this study, more extensive sexual behavior histories were significant predictors of worry for young women, while perceptions of personal vulnerability and homophobia were significant worry predictors for young men (Cochran \& Peplau, 1991). When compared with nonminority women, minority women 
report less concern about AIDS and estimate their personal risk to be lower (Kalichman, Hunter, \& Kelly, 1992).

Much of the literature cites substance use as being associated with unsafe behavior, particularly reduced condom use (e.g., Aruffo, Gottlieb, Webb, \& Neville, 1994; Koopman, Rosario, \& Rotheram-Borus, 1994; Lowry, et al., 1994; Rotheram-Borus, et al., 1994). Stall (1988) noted significant gender differences with drug use, typically with young men being 2.3 times more likely to use alcohol and 8.4 times more likely to use "other drugs" prior to sexual activity, than young women. Condom use behavior and attitudes among youth appear to be gender related (Barnard \& McKeganey, 1990); therefore, it would make sense to direct specific educational efforts to young men and young women appropriately, with specific emphasis on empowering young women in areas of assertiveness.

\section{The Transtheoretical Model: Stages of Behavior Change}

The Transtheoretical Model (Prochaska, 1979, 1984; Prochaska \& DiClemente, 1983,1984 ), or stages of behavioral change model, describes behavior change as a gradual, continuous, and dynamic process, and has been successfully applied to a variety of behaviors such as smoking cessation (DiClemente, et al., 1991), weight loss (O'Connell \& Velicer, 1988), sexual and HIV-related behavior change including condom use, self-efficacy and decisional balance (CDC, 1993i; Bowen, \& Trotter, 1995; Freeman, Cohn, Corby, \& Wood, 1991; Galavotti, et al., 1995; Grimley, Harlow, Morokoff, \& Quina, 1992; Grimley, Riley, Bellis, \& Prochaska, 1993; Pino, 1993; Prochaska, et al., 1990, 1994; Redding, 1993; Redding, Rossi, Velicer, \& Prochaska, 1989; Redding, \& Rossi, 1993a, 1993b; Schnell, Galavotti, \& O'Reilly, 1993). Individuals do not move directly from old behaviors to new 
ones, according to the Transtheoretical Model, but rather progress through a sequence of four discrete stages: Precontemplation (no intention to change behavior, unawareness of risk or decision not to change); Contemplation (formed intentions of change with minimal, specific plans to change in near future); Action (behavior change has been initiated relatively recently); and Maintenance (behavior change has been maintained consistently for an extended period of time). The pace of movement through stages varies greatly from individual to individual, as is the vulnerability to relapse, regressing back through stages. In terms of intervention, the model's view is that prevention be targeted to the stage the individual is in, to facilitate progressive movement through more desirable stages. Limited research has been conducted in the area of behavior change progression in adolescent sexual behavior, suggesting an examination of staging variables with potential risk variables may be instrumental for appropriate, effective intervention and prevention applications.

\section{Qualitative Research}

In a previous study (Lang, 1994), handwritten feedback provided by adolescent participants in the "additional comments" section, provided an awareness that self-report, paper and pencil surveys may not always capture the information desired. For example, one adolescent provided separate explanations in the margins for many of her answers to the knowledge inquiries, while another discussed risk behavior of a close friend. Many elaborated further about specific relationships, dilemmas, strategies they have undertaken for safe sex, and/or provided feedback on the ease or difficulty responding to the survey questions. Others asked questions about AIDS indicating some misconceptions continued to prevail. Due to the anonymous nature of the previous study, such individual questions 
could not be answered, as it was impossible to determine which study participant made the inquiry. The current study began with awareness that teens had much more to contribute beyond fixed survey answers, therefore the decision to use informal focus groups was made to supplement the teens' quantitative responses.

Many investigators note the value of qualitative strategies such as focus groups which can yield information not available to researchers through other methods (Fullilove, Fullilove, Haynes, \& Gross, 1990; Manning, Balson, Brenberg, \& Moore, 1989; Patton, 1991). For instance, focus group methodology has been useful in the exploration of sensitive issues such as sexual behavior and decision making (Desvousges \& Frey, 1989; deVries, Weijts, Dijkstra, \& Kok, 1992; Fullilove, Fullilove, \& Haynes, 1990; Joseph, et al., 1984; Kline, Kline, \& Oken, 1992; Morgan \& Krueger, 1993; Steckler, McLeroy, Goodman, Bird, \& McCormick, 1992); the impact of poverty on and HIV-risk (Nyamathi \& Vasquez, 1989); and the evaluation and refinement of survey instruments (Fuller, Edwards, Vorakiphokatorn, \& Sermski, 1990; Morgan \& Krueger, 1993; O'Brien, 1990). Additionally, anonymity and confidentiality of focus group responses can be easily maintained by moderating self-disclosure on sensitive topics and reducing respondents' potential uneasiness by having youth begin statements with, "I know someone who..." (Morgan \& Krueger, 1993).

\section{Overview}

Special emphasis must be placed on the identification of adolescents as an at-risk population. Given available adolescent statistics and the fact that AIDS is presently an incurable, fatal disease, emphasis must be placed on correctly identifying behavior and 
attitude predictors. Since many adolescents appear to lack sufficient AIDS knowledge, carry several misconceptions, and most importantly, engage in a myriad of high risk activities that increase their likelihood of contracting HIV and spreading it further, they clearly represent a vulnerable, at-risk population.

Pilot research (Lang, 1994) demonstrated a need for more accurate assessment with risk predictors, especially in the area of alcohol use, substance abuse, and gender differences in sexual assertiveness skills for condom use and partner communication. Moreover, the added comments provided by participants in the Lang (1994) study indicated a need to direct attention beyond traditional paper-and-pencil instruments in order to adequately address the concerns adolescents may have regarding HIV/AIDS research, intervention and prevention strategies. The present study sought to expand on previous research by assessing a diverse population of youth conference goers from diverse socio-economic and ethnical backgrounds and an intact group of high school students from a middle-class, rural setting in Connecticut, utilizing a revised survey instrument, and supplemental qualitative assessment in the form of informal focus groups.

In addressing this need, the present investigation focused on: (1) further development of a psychometrically sound instrument for adolescent risk assessment utilizing a questionnaire previously piloted with a sample of over 200 teens (Lang, 1994); (2) examination of possible correlates among knowledge, attitudes and risk behavior; (3) identification of gender differences among potential risk predictors; and (4) supplementing quantitative survey data with qualitative methodology derived from small focus groups with teens. 


\section{Research Hypotheses}

In this investigation, it is hypothesized that:

(1) a higher potential risk, as defined by the combined components of sexual assertiveness, (predicted or actual) for condom use and partner communication, will be associated with lower AIDS knowledge and higher AIDS misconceptions, higher perceived susceptibility for AIDS risk, higher homophobia, and higher substance use and substance abuse, among both the sexually active and nonactive youth, across both genders;

(2) among those teens who are sexually active, a higher risk for AIDS, as defined by number of unprotected intercourse occasions and number of intercourse partners, will be associated with lower AIDS knowledge and higher misconceptions, lower condom use efficacy, lower sexual assertiveness, higher perceived susceptibility for risk, higher self and partner-risk histories, and higher substance use and abuse, across both genders;

(3) higher levels of risk, as estimated by each participant, will be associated with lower AIDS knowledge and higher AIDS misconceptions, lower condom use efficacy, lower sexual assertiveness (actual or estimated), higher perceived susceptibility for risk, higher homophobia, and higher substance use and abuse, among the sexually active and nonactive participants, across both genders;

(4) gender differences will be found between the sexually active young men and women on behaviorial components, where young men will have higher incidences of substance use and abuse, higher sexual assertiveness, and consequently, a higher risk for AIDS, as defined by number of unprotected intercourse occasions and number of different intercourse partners, and lower partner-risk than the sexually active young women; 
(5) differences between the sexually active and nonactive adolescents will be found across the cognitive, attitudinal and behavior variables where the sexually active young men and young women will have:

(A) lower measures of AIDS knowledge and higher AIDS misconceptions, than the nonactive adolescents;

(B) lower condom use efficacy, higher perceived susceptibility for risk, and higher homophobia than the sexually nonactive adolescents' estimated self-attitudes or predicted behavior of the same; and

(C) higher substance use and abuse, and lower measures of sexual assertiveness, than the nonactive adolescents' actual or predicted behavior of the same.

\section{Method}

\section{Participants}

All adolescent participants between the ages of 13 through 19 attending the 1994 Eastern States Regional Youth-to-Youth Conference (July 18-20, 1994) at the Roger Williams University, Bristol, Rhode Island were invited to participate in this study ("Conference Youth"). The annual Youth-to-Youth program emphasizes peer leadership, drug education and information, personal growth and healthy life skills, positive programs and drug-free fun activities, attracting youth from the Eastern United States, Jamaica, Bermuda, and Cayman Islands. Workshops include discussion and role playing exercises in teaching social skills and decision-making strategies in the prevention of AIDS/HIV/STDs 
and drug use. Participation is funded by various community facilities, schools, youth groups, or privately by parents. Some participants are former substance users/abusers, who are funded by various mental health and residential programs. Participants must provide a parent consent for conference participation as well as pay a fee for the 3-day conference for housing, meals, conference materials, and recreational events.

Approximately 300 adolescents attended the 1994 Youth-to-Youth conference, 56 percent were young women, and 44 percent young men. Conference Youth represented a diverse population with approximately 40 percent of the entire group being either AfricanAmerican, Asian-American, Hispanic-American, Native-American or of island descent. Approximately 51 percent of the participants were from Rhode Island, Massachusetts or Connecticut, while the remaining half were from a variety of areas in the Eastern United States, or Bermuda, Jamaica or the Cayman Islands.

All junior and senior high students $(n=956)$ enrolled at Griswold High School in Jewett City, Connecticut during the Fall, 1994 semester were also invited to participate in the study ("School Youth"). The Griswold High School population for the 1994-95 school year had equal numbers of men and women, representing primarily European-Americans (94 percent), from a middle class, rural area, and were between the ages of 12 and 18 .

\section{Measures}

Five components were measured in the 163-item questionnaire: (1) general demographics and background information; (2) cognitive; (3) attitudinal; (4) behavior risk; and (5) educational and environmental, explored by another researcher (Burkholder, 1994). Several subscales were included under each component of interest. 
During a previous investigation of 192 heterosexual adolescents (Lang, 1994), scale items were factor analyzed within four general areas of interest: AIDS locus of control, cognitive, attitudinal and behavioral. Subscales were developed with items which loaded significantly on one factor only with a conservative cut of .40 for inclusion of a variable on a factor. The reliability of each scale and all subscales was determined using Cronbach's Alpha $(\alpha)$. Since the alpha coefficient obtained for the AIDS Locus of Control Scale originally included in the first investigation was found to be low $(\alpha=.55)$; and, the internal and external Locus of Control subscale factors were highly correlated with the two sexual assertiveness subscale factors in the behavior scale (ranging from .21 to -.52 ), this scale was dropped in the current investigation.

Demographics. Gender, age, geographic location, ethnicity, marital status, religion, religiosity, living situation and parental level of education make up the background demographics (see Appendix A).

Cognitive Measures. The cognitive component consisted of a compilation of items derived from previously developed scales to assess AIDS knowledge for ages 14 to 18 (DiClemente, Zom, \& Temoshok, 1986; DiClemente, Boyer, \& Morales, 1988) and ages 11 to 16 (Siegel, Larazus, Krashovsky, Durbin, \& Chesney, 1991). Originally, a pilot study was conducted in the Spring of 1993 prior to the Lang (1994) study with the new scale utilizing 20 members of the targeted age group which confirmed readability and produced a few minor changes to the original 4-point Likert scale. The original scale, composed of "definitely" and "probably" degrees of true and false, was changed to degrees of "yes" and "no," to eliminate "feelings of taking an exam," which was voiced by the pilot participants. 
A fifth choice, "not sure" was added, as several suggested its inclusion. The pilot scale was comprised of 29 items providing an assessment of both (1) factual AIDS knowledge and (2) knowledge of AIDS transmission.

Nine items were removed from the Lang (1994) analyses which were answered correctly by 85 to 92 percent of the sample to reduce the skewness and ceiling effects of the knowledge component. After all inconsistent and complex items were removed with the factor analysis, 11 items remained loading on two factors: Factual AIDS Knowledge and Transmission/treatment Knowledge. The two-subscale solution accounted for 49 percent of test variance with a fairly high reliability for entire scale $(\alpha=.77)$, as well as for each subscale (Factual Knowledge, $\alpha=.78$, and Transmission/treatment Knowledge, $\alpha=.75$ ).

In the current study, eight new items were added to the cognitive scale in order to assess more thoroughly knowledge of AIDS transmission, as well as participants' perceived knowledge of AIDS. The additional items developed by a co-investigator (Burkholder, 1994) were included in a previous study (Lang, 1994). Commonly held misconceptions about HIV/AIDS have been noted to prevail within the adolescent population (e.g., Lang, 1994), and constituted a separate hypothesized scale in the current study in order to reduce error in factor structure and ease interpretation. The 19 cognitive items and the hypothesized four-subscale structure (Factual AIDS Knowledge, Transmission Knowledge, Perceived AIDS Knowledge and Misconceptions) are presented in Appendix B, with items 13 through 19 being the new additions.

Attitudinal Measures. Attitudinal components were originally divided into two subscales comprised of a total of 14 items, Condom Use Efficacy, and Perceived Risk for 
AIDS, both taken from The URI Health and Lifestyle Survey (Harlow, Quina, \& Morokoff, 1991). A seven-item scale was constructed for assessment of Condom Use Efficacy (Harlow, Quina, \& Morokoff, 1991; Prochaska, et al., 1992; Sorenson, Gibson, Heitzmann, Dumontet, \& Acampora, 1988), and seven items measuring Perceived Risk for AIDS from the Harlow, et al. (1991) survey.

Factor analyses, however, revealed that the 14 items comprising the attitudinal scales successfully loaded on three subscale factors rather than the originally hypothesized two, with no inconsistent or complex items. The original subscale, Condom Use Efficacy, remained intact with all 7 item loading highly $(\alpha=.92)$, and two separate factors were formed with the remaining 7 items. The constructs associated with each of the remaining factors were identified as Perceived Risk for AIDS composed of 5 items $(\alpha=.77)$, and Perceived Self-efficacy for AIDS Prevention with 2 items $(\alpha=.82)$. The new factor, Perceived Self-efficacy for AIDS Prevention, seemed to be a self-efficacy measure for global avoidance, while the Condom Use Efficacy subscale is situationally specific to condom use. Total reliability for the Attitudinal Scale was .73.

In the current study, 14 items reflecting Homophobia and Fear of AIDS were added to the attitudinal scale for a more in-depth examination of youths' attitudes about AIDS and sexual behavior. These items were developed by a co-investigator to explore the relationship of homophobia and AIDS risk with a slightly older adolescent population (Burkholder, 1994; Lott, 1991). With the addition of these two subscales, it was hypothesized that the original Perceived Risk for AIDS subscale, or more accurately labeled, Perceived Susceptibility for Risk, would remain intact, revealing a total of four 
subscales: Perceived Susceptibility for Risk, Condom Use Efficacy, Homophobia and Fear of AIDS.

The Perceived Susceptibility for Risk subscale is a susceptibility estimation based on global indicators of one's ability to avoid "risky" situations and/or "risky" partners and ability to protect oneself in reference to past encounters. Use of this subscale may be helpful in pinpointing adolescents' attitudes toward personalizing their own potential risk behavior. More particularly, youth identified perceived invulnerabilities, or risk denial, based on inconsistencies or discrepancies with their reported sexual behavior, as well as their responses from the single item that had them rate themselves for their own level of risk on a 5-point scale.

Condom Use Efficacy measured condom use in specific situational contexts (e.g. pressured, angered partner) and internal affective states (e.g. depression, fear, anger). Homophobia and Fear of AIDS are represented by items stereotyping HIV/AIDS with specific populations and associated fears of exposure based on such stereotypes, respectively. The 26 items comprising the Attitude Scale and the hypothesized foursubscale structure are listed in Appendix C.

Behavioral Measures. Originally, the behavioral components were measured by four subscales: (1) Substance Use (alcohol and other drugs), (2) Substance Abuse, (3) Sexual Assertiveness, and (4) AIDS Risk by sex history, sexual behavior, condom use, and a single item self-evaluation of current risk. Alpha coefficients were found to be relatively high for all the behavior subscales: Substance Use $(\alpha=.90)$, Potential Substance Abuse $(\alpha=.89)$, 
Serious Substance Abuse $(\alpha=.82)$, Sexual Assertiveness Partner Communication $(\alpha=$ .94), and Sexual Assertiveness Condom Use $(\alpha=.85)$.

Pertaining to substance use and abuse, seven items were selected from the Harlow, et al. (1991) survey to measure the same with minor adjustments to the questions referring to drugs, collapsing hard substances into one question. The Substance Abuse scale composed of 16 items was also taken from the Harlow, et al. (1991) survey with no adjustments made. In the previous study (Lang, 1994) the 23 original items comprising the substance use/abuse portion of the Behavioral Scale remained intact with two interpretable subscale factors, having a full scale reliability of .82 . However, one item from the Substance Abuse subscale was adopted in the Substance Use factor having a high loading of .74. The remaining Substance Abuse items were further divided into two factors which were interpreted as Potential Substance Abuse and Serious Substance Abuse, in the Lang (1994) study. In the current study, Substance Use measured amount and frequency of substance use over the past year, alcohol and other drugs inclusive, while Substance Abuse reflects frequency of problem areas indicating potential abuse (e.g., while under the influence: drove a car, arrested, tried to kill self, etc.).

Pertaining to sexual assertiveness, ten items were selected as the subscale components developed by Quina, et al. (1990) originally measuring sexual initiation, refusal for sex and birth control. The selected items focus primarily on (1) initiating condom use, and (2) partner communication (ability to discuss partner history and protective methods). Slight revisions were made including removing the sexual initiation component of sexual assertiveness, thus creating a more suitable scale for the intended younger population. In 
the current study, Sexual Assertiveness Condom Use is more specific to ability to insist on, negotiate and follow through with condom use if personally desired, while Sexual Assertiveness Partner Communication measured the participant's ability to discuss and/or inquire about various sexual risks of their partner (e.g., partner's past partners, STDs, needle drug use).

Pertaining to sexual experience, measures of the participant's sexual history, behavior and condom use were also adapted from the Harlow, et al. (1991) survey with the removal of extremely sensitive sections regarding childhood sexual abuse and detailed items of explicit sexual acts. Minor adjustments were made in the wording for gender-neutral item content. Twenty items were used that pertain to sexual history and condom use with an additional eight-item matrix measuring sexual risk which focuses on the frequency of unprotected intercourse during the past year. A single item referent to condom use at last intercourse has been established as a poor surrogate for assessing HIV-risk because it does not consider either consistency of use, number or partners, or degree of exposure (Nangle \& Hansen, 1993; Smith, McGraw, Crawford, Costa, \& McKinlay, 1993). Use of a behavior matrix is deemed more appropriate. One additional item was adopted from the Kelly, et al. (1990) study which had the participants estimate their level of risk on a 5-point Likert scale from "no risk" to "high risk." The "sex partner" definition taken from the above-mentioned Harlow, et al. survey was also retained in the current questionnaire, but slight modifications were made for a more subtle description (see top of Appendix D). The decision to include such a definition was based on past research findings that have indicated measurement error due to terminology and question structure, particularly with the adolescent population. 
Measurement error has been noted due to confusion in terminology pertaining to specific sexual activities; therefore, the usefulness of providing definitions has been emphasized as a way to reduce such misconceptions and to increase scale validity (Catania, Gibson, Chitwood, \& Coates, 1990). Careful consideration was given to the format and rewording so as not to promote a feeling of inadequacy or to identify individuals who have never been sexually active (e.g., by completing the survey rather quickly). Thus, by having sexually nonactive youth complete the entire survey, predicting behavior in areas where behavior has not occurred, their sexual activity status remained confidential to other nearby participants. Additionally, the questionnaire was designed in a sequence format in which less sensitive, potentially uncomfortable questions precede the more sensitive inquiries regarding substance use and sexual behavior, which is noted in the literature as most desirable arrangement to reduce uneasiness (Stanton, Black, Keane, \& Feigelman, 1990).

Pertaining to additions to the behavioral component, sexual behavior together with substance use was examined. Although, the relationship between substance use and sexual behavior risk was examined in the previous study (Lang, 1994), and an interpretive flaw in the self-report measure design with these two variables was discovered. Measures of overall substance use and measures of unprotected intercourse were collected, and the relationship between these two variables was examined. However, the design did not include the frequency of alcohol use or drugs in conjunction with sexual activity, more specifically, whether alcohol or drugs were used prior to sexual encounters. Three specific questions were added inquiring about frequencies of drinking or drug use prior to sex, in order to determine more accurately the relationship between substance use/abuse and 
likelihood of condom use. Potential risks secondary to sexual behavior in the current study, were divided into two new subscales reflecting risk behavior on the part of the participant as well as for the participants' sex partner(s). A new composite, Self-risk History, is an indicator of potential AIDS risk based on sexual history, including length of involvement with current partner, age of sexual onset, number of lifetime partners, number of partners in the past year, and substance use prior to sex. Similarly, Partner-risk History measured risk of exposure given participant's past sex partners and partners' sexual behavior (e.g., certainty of exposure of past partner(s), estimates of number of partners most recent partner has had, needle-using and/or HIV-positive partners, etc.). The 60 items comprising the Behavior Scale, and the six hypothesized subscale structure (Sexual Assertiveness Partner Communication, Sexual Assertiveness Condom Use, Substance Use, Substance Abuse, Self-risk History, and Partner-risk History) are listed in Appendix D.

Initially ten parents of adolescent children volunteered in the Spring of 1993 to review the questionnaire in the Lang (1994) study, for questionable content matter if administered to the intended target group. Slight changes were made with reference to the "sex partner" description as a result. As mentioned above, the original version in the Harlow, et al. (1991) survey was revised to a more subtle wording. All ten agreed that the present material was appropriate and non-threatening, and believed that possible increased knowledge of personal behavior habits and AIDS awareness would be beneficial and would not be harmful to their child; especially given anonymity of individual responses.

Psychosocial Measures. Additional subscales were also included in the present survey regarding AIDS education and environmental aspects of AIDS, as well as their 
potential influence on behavior. These items, together with the Homophobia and Fear of AIDS subscales were developed for future analyses by a co-investigator in the Experimental Psychology program at URI (Burkholder, 1994). These additional items were factor analyzed for scale structure purposes only, and were not explored further in the current study. The 46 additional psychosocial items and hypothesized four-subscale structure are listed in Appendix E.

Codebook. A codebook was prepared for subscale ranges and scoring for the IBM version of the Statistical Package for the Social Sciences personal computer version 4.0 ("SPSS PC+") (Norusis, 1990). Codebook items are presented in scale and subscale clusters reflecting the results of complete exploratory and confirmatory factor analyses in the current study, more fully detailed in sections to follow. Scale adjustments and reverse coding are included (see Appendix F).

Focus Group Semi-Structured Interviews. Feedback checklists regarding both the survey instrument and the focus group process were provided to each group attendee, and semi-structured questions were prepared in advance covering several areas of discussion (see Appendix G). Feedback adjectives represent equally negative and positive selection choices with one neutral item. Focus group topics included: AIDS research (survey feedback, and suggestions for AIDS prevention strategies); the scope of the AIDS pandemic (adolescents' perceptions of age groups, geographical locations and populations afflicted, and homophobia); societal dilemmas (insurance issues, segregation, and pregnancy); and risk pertaining to teenagers (risk situations, resources for information, and assertiveness). 


\section{Procedure}

Approximately 1,250 youth (Conference and School Youth) between the ages of 12 and 19 were asked to anonymously complete a 163 -item paper-and-pencil survey, measuring AIDS knowledge, attitude, psychosocial influence, behavior risk and general demographic information. Completed surveys were returned in designated areas that were conducive to anonymity.

During the Summer of 1994 (for Conference Youth) and Fall semester of 1994 (for School Youth), adolescents were provided an overview of the investigation either verbally from the primary investigator, verbally from class instructors, visually by school-based televised announcements, video promotions, posters, and/or through typed informative handouts. Information included a general overview regarding "an investigation of teenage health and lifestyle behaviors," using an anonymous paper-and-pencil survey which emphasized confidentiality of the participants. School Youth were offered an optional discussion period afterward, while Conference Youth participated in discussion groups as part of the conference program. Interested potential candidates were requested to acquire parental consent prior to participation. Adolescents and parents or guardians were provided with a brief description of the study along with an explanation of the potential benefits of participation. Benefits included: (1) increased knowledge of ADS-related issues; (2) increased awareness of one's own behavior; and (3) providing the researcher with valuable information with regard to potential adolescent HIV-risk behaviors.

Six half-hour focus groups were undertaken with the Conference Youth as part of the conference program, with the primary investigator as group leader and a 17 -year-old 
Caucasian man as co-facilitator. These six sessions consisted of the same nine adolescents, six young women and three young men, ranging in age between 13 and 15 years (mean age: 14). Conference Youth group attendees were primarily Caucasian, with one member of Hispanic origin, and were from varied backgrounds ranging from affluent to one member in foster care with a sexual abuse history. Half of the group members were from Rhode Island, while the remaining participants represented Pennsylvania, New York, and New Hampshire. These six half-hour focus groups served as a pilot study for relevant topics areas to explore with the School Youth population later in the same year, while simultaneously conforming to the Youth Conference discussion format.

Twenty-two informal focus groups comprised of two to ten individuals from the School Youth population met with the researcher after survey completion for approximately one hour, to acquire feedback about the survey and to discuss any concerns the adolescents may have had about HIV/AIDS. Discussion group opportunities were open to all survey participants who would like to attend. With permission from group attendees, the researcher, as group leader, and a 23-year-old Caucasian woman, as co-facilitator, compiled notes of the responses during discussion periods.

Focus group participants were allowed to deviate from the prepared question format if the material discussed was relevant to AIDS and/or AIDS research. The role of the group leader was primarily to facilitate conversation, as all information discussed within the group as a whole, and any ideas raised, conclusions drawn, etc., are strongly indicative of the behaviors and social conditions that characterize the adolescents' community (WHO, 1993c). This method allowed the group leader to assess the circumstances, needs and 
prevalence of educational gaps in the participants' prior knowledge and experience.

Questions were answered and misconceptions corrected by the group leader only at the close of each focus group in order to allow other participants an opportunity to respond to their peers. Misconceptions raised in the group were addressed tactfully, by selecting some accurate portion of a participant's statement prior to correction and affirming its truth, or by offering portions of the statement to the group to discuss.

Conference Youth participated in half hour focus groups with the current investigator as part of their conference agenda twice per day for the 3-day period. Information and topics presented from Conference Youth constituted a pilot study for areas requiring a more comprehensive assessment from which the semi-structured interview questions were developed. Volunteers were recruited from the School Youth survey participant pool to partake in more in-depth informal discussions about HIV/AIDS, and reflect the majority of the qualitative results presented herein. Self-disclosure was moderated by instructing participants to answer questions in general, with encouragement not to disclose whether any personal or sensitive information pertained to themselves, or someone they know, by beginning their responses with, "I know someone who...".

Two separate $\$ 50$ cash bonus incentives were offered to School Youth for participation, one for survey participants and another for focus group participants, with the potential for any individual to win $\$ 100$. Similarly, incentives for Conference Youth participants were awarded; however, at the Conference Administrator's request, two $\$ 50$ gift certificates for the Youth Conference gift store were offered, rather than cash. All youth were given bonus tickets for participation. Winners were drawn at the closing 
ceremonies at the Youth Conference for Conference Youth, and in December, 1994, in the main office with office administrators as witnesses for School Youth.

Informed Consent. Passive consent forms were utilized for Conference Youth since parental consent for conference attendance was previously obtained (see Appendix H). Parents of Conference Youth received consent forms in registration packets, and were directed to return the form at the registration table if they did not want their child to participate in the study. No Conference Youth parent objected to their child's participation.

A cover letter to parents of School Youth (see Appendix I) was provided with parental consent forms (see Appendix J) prior to administering the questionnaire. Only those adolescents returning parental consent were allowed to participate, unless the adolescent was 18 or older. Informational packets for parents which included cover letter, consent forms, and telephone numbers for further resource information (see Appendix K) were distributed to all School Youth, along with an informational flyer, during homeroom periods in September, 1994, and again during lunch hours the following week for those who did not receive or misplaced their parent packets. Signed parental consent forms were returned to the main office or directly to the researcher on prearranged survey dates.

The letter assured confidentiality of the child's survey and focus group participation, and anonymity of the child's survey responses, and emphasized the possible benefits that could be derived from the study. A similar cover letter was provided to all participants (see Appendix L) before completing the survey. Participants were provided informed assent forms (see Appendix M for Conference Youth, and Appendix N for School Youth) prior to survey completion and focus group participation. Informed consent and assent forms 
reassured parents and participants of strict anonymity of survey responses, confidentiality of participation, confidentiality of focus group discussion to the extent possible, and that all responses would be used for psychological research purposes only and would be reported in grouped data which would not reveal specifics regarding any individual. Additionally, all youth received resource numbers for further information either on their assent form (Conference Youth), or on informational resource cards (School Youth) distributed at both survey and focus group periods.

Confidentiality. Confidentiality was further guarded by having informed consent and assent forms collected separately from the surveys prior to survey participation. Further, no information was obtained that could identify any particular individual on the survey itself. Precautions were taken to ensure that identities of participants were kept in strict confidence, as only the principal investigator would have the acquired consent and assent forms. Names in the sample pool derived from the consent and assent forms are kept in a locked file drawer in room 6 of the Social Sciences Research Center, CPRC Building in Kingston.

Parents were assured that only group data would be analyzed and that no individual profiles would be available. Precaution was also undertaken to ensure that focus group discussions remained confidential to the extent possible. Focus group participants were encouraged to speak about "somebody they know" in order to put each attendee at ease about discussing personal matters or sensitive issues and to moderate self-disclosure.

Risks Involved. Minimal risk was present in the study due to the sensitivity of some of the questions concerning sexual experience, attitudes, and use of alcohol or drugs. 
Telephone numbers for assistance and informational resources were provided to all participants and their parents, including a toll-free URI number, in the event they wanted to talk to someone or to ask any questions that may arise from participation in this research project. Additional resources and informational brochures from RI Project AIDS and the HIV/AIDS Information Lines of Eastern Connecticut were offered to parents and participants upon request. Further opportunities to discuss any concerns were offered to participants individually or within a group format after survey completion and after focus group participation. Additionally, the principal investigator offered parents of potential participants individual or group opportunities to have their questions answered, allow them to review the survey, or discuss any concerns they may have had regarding the study. Finally, the primary investigator was available during the entire course of the three-day adolescent conference for Conference Youth and their parents; and, a toll-free, 800 number was installed for School Youth and their parents, for questions and issues regarding this study.

One Conference Youth parent utilized the researcher for informational resources, and several Conference Youth participants inquired about HIV/AIDS transmission and were provided verbal information and pamphlet resources. Two School Youth parents utilized the toll-free number in a positive manner, inquiring for additional information about how their child may be involved in the study. Finally, at the request of the School Social Worker, the primary investigator returned to speak with two School Youth students (brothers) to provide educational resources and support, as they had recently lost a relative to AIDS. 


\section{Analytic Procedures}

Data analyses for quantitative survey data were performed using the SPSS PC+ statistical program, version 4.0 for IBM personal computers (Norusis, 1990). Exploratory and confirmatory factor analysis were conducted to examine the structure and confirm the orthogonality between the major scales (Cognitive, Attitudinal, Behavioral and Psychosocial) and 14 subscales (Factual AIDS Knowledge, Transmission AIDS Knowledge, Perceived AIDS Knowledge, Misconceptions, Perceived Susceptibility of Risk, Condom Use Efficacy, Homophobia, Fear of AIDS, Substance Use, Substance Abuse, Sexual Assertiveness Partner Communication, Sexual Assertiveness Condom Use, Self-risk History, and Partner-risk History, as well as Psychosocial variables consisting of Environmental and Educational Influence). Cronbach's alpha was calculated for each final scale to assess the internal reliability, and individual scale composite scores were computed by summing loadings $(\geq 40)$.

Descriptive statistics were computed for means and standard deviations for demographics on all subscale composites. Additional descriptive statistics were computed for gender comparisons, and for comparisons of sexually active and nonactive subgroups, across all scales and subscales. Descriptive statistics were also computed for present and predicted stages of change for condom use among the sexually active subgroup, by gender.

Standard multiple regression analyses ("MRAs") were utilized to examine the significance of various predictor variables for potential risk with sexually nonactive, AIDS risk for sexually active, and all participants' own risk estimates. One-way Multivariate Analyses of Variance ("MANOVAs") and follow-up Analyses of Variances ("ANOVAs") 
were performed to determine whether group differences exist between genders and between the sexually active and nonactive adolescents, across all areas of investigation. Since researchers have questioned many studies correlating substance use and/or abuse with highrisk sexual behavior (e.g., Leigh \& Stall, 1993), particularly if measures address whether the individual used substances prior to engaging in risky behavior (Leigh, 1990; Martin, 1990; Valdiserri, et al., 1988), analyses were undertaken to examine such behaviors.

Qualitative data analyses include response frequencies by gender and age where possible, in relation to quantitative findings, as well as knowledge gaps indicated by frequent comments and/or inquiries, and misconceptions reported by focus group attendees.

\section{Results}

\section{Demographics}

Of the 300 Conference Youth, 130 completed surveys, yielding a 43 percent participation rate. Participation among School Youth was considerably lower, with I10 of 956 completing questionnaires for a participation rate of 12 percent. Thus, the overall participation was 19 percent $(240 / 1256)$. Only one survey was deemed unusable, reducing the Conference Youth sample to 129. Demographic information separated by gender is provided for the 239 completed surveys in Table 1. Marital status has been eliminated from the demographic profile as all participants reported having "single" status.

Insert Table 1 about here 
Of the 239 participants, 157 (66 percent) were young women, and 82 (34 percent) were young men. Both samples had an approximate two-third to one-third ratio of young women to young men participants. Significant differences were found between the two samples for age $(\underline{F}(1,237)=32.96, p<.001)$, with the mean age of Conference Youth being one year greater than the School Youth (15.5 versus 14.5 years, respectively); and correspondingly grade, being one year higher $(E(1,237)=31.59, p<.001),(10.2$ versus 9.0 , respectively $)$. A significant difference between the two samples was also evident for ethnicity $(\underline{F}(1,237)=$ $21.50, p<.001)$, revealing the School Youth as less ethnically diverse (97 percent Caucasian) than the Conference Youth sample (71 percent Caucasian). Conference Youth reported being slightly more religious and/or spiritual on a 5-point scale (mean score 3.1 ; "somewhat") than School Youth counterparts (mean score 2.7 "slightly to somewhat") $(E(1,237)=6.42, p<.01)$. Although a significant difference was not found in mother's education between the two samples (most reporting "high school graduate" to "some college"), a significant difference was apparent in father's education, with Conference Youth reporting higher education for their fathers ("some college") in comparison to School Youths" reported educational level of their fathers ("high school graduate" to "some college") $(\underline{E}(1,237)=13.72, p<.001)$. Nearly 70 percent of participants in each sample reported currently residing with both parents at the time of surveying, with approximately one quarter of participants from each sample reporting that they were living with their mother only, showing no significant difference between groups.

Given the significant findings of differences between groups with age (grade inclusive), geographic locale, ethnicity, and socio-economic status (as estimated by parents' 
education), further confirmatory factor analyses of the scales were undertaken with dichotomized subgroups, discussed more fully in a later section.

\section{Quantitative Results: Scale Construction}

\section{Procedure}

In order to increase the power of analyses, data were pooled from the four samples of adolescents who were administered the instrument. The two samples from the current study (Conference Youth (1994) and School Youth) and two samples from the Lang (1994) investigation, Conference Youth (1993) and Community Youth, were used for scale construction purposes only. The latter two samples are more fully described elsewhere (Lang, 1994), and generally represent similar populations to counterparts in the present investigation. Mean substitution was utilized for missing data for new items not offered to earlier Lang (1994) participants, and a 4-group comparison was computed on final scale/subscale composition following exploratory and confirmatory factor analyses results.

Items were factor analyzed with principal components extraction, utilizing a 4sample pool of 457 adolescents within four general areas of interest: Cognitive, Attitude, Behavior, and Psychosocial (Environment and Education). Principal components analysis was utilized initially with both orthogonal and oblique rotation. Given the high intercorrelation between subscales (above .30 in the majority of the cases), the oblique method of rotation was justified to reduce error (Tabachnick \& Fidell, 1989, p. 636). All 457 participants were included in each exploratory factor analysis unless stated otherwise, and each was followed by a confirmatory factor run with a random computer-generated sample 
consisting of 50 percent of the original sample. Subscales were developed with items which loaded significantly on one factor only with a conservative cut of $\geq .40$ for inclusion of a variable on a factor. Alpha coefficients on full scale and individual subscale components were calculated for determining scale/subscale reliability, utilizing the full sample pool when exploratory and confirmatory were found to be consistent. Response frequencies are provided within each section for the current sample only (Conference and School Youth); response frequencies have been provided elsewhere for the earlier two samples (Lang, 1994). All subscale composite scores were converted to a 5-point scale for ease of interpretation, with a higher score indicating endorsement of a subscale.

Cognitive Measures. The four hypothesized subscales constituting the Cognitive Scale remained intact, consisting of six items measuring Factual AIDS Knowledge ( $\alpha=$ .74); four items measuring Transmission AIDS Knowledge $(\alpha=.94)$; three items measuring Perceived AIDS Knowledge ( $\alpha=.85$ ); and six items measuring Misconceptions $(\alpha=.79)$. Total scale reliability was .81 with 61 percent of the variance accounted for in the 4-factor solution. As indicated in Table 2 (exploratory) and Table 3 (confirmatory) nearly all items presented with very strong loadings, with one item exception ("You can have AIDS and not know it."), rendering an acceptable .40. All items delineated clear factor structures having neither inconsistent nor complex item loadings. See Tables 2 and 3 for pattern matrices and factor correlation matrices for exploratory and confirmatory subscale structures.

Insert Tables $2 \& 3$ about here 
Response frequencies for the Cognitive Scale items are provided in Table 4 for the current $(n=239)$ sample participants only.

Insert Table 4 about here

Attitudinal Measures. The factor analysis run for the Attitudinal Scale rendered a 3factor solution, rather than the hypothesized 4-factor solution. Five items measured Perceived Susceptibility for Risk $(\alpha=73$ ); seven items measured Condom Use Efficacy ( $\alpha$ $=.92)$; and fourteen items measured Homophobia $(\alpha=.91)$. The two hypothesized Fear of AIDS items loaded significantly on the Homophobia subscale with loadings $\geq .51$ in both exploratory and confirmatory runs (see Tables 5 and 6 ). Due to clearly consistent loadings, these two items were retained as indicators relating in some manner to homophobia. Correlations between the two items in the hypothesized Fear of AIDS subscale, and the remaining 12 items in the hypothesized Homophobia subscale were significantly high for all sexually active young men $(r=.65, p<.001)$; all sexually active young women $(r=.52$, $p<.001)$; all sexually nonactive young men $(r=.54, p<.001)$; and, all sexually nonactive young women $(r=.52, p<.001)$. Implications of these high correlations are discussed in a later section. The combined 14 items are hereinafter referred to as a measure of Homophobia, although two items within this scale have no specific reference to sexual orientation and are more indicative of Fear of AIDS.

For the Attitudinal Scale, the 3-factor solution explained 54 percent of the variance, with a full scale reliability of .83 , and no inconsistent nor complex items were found. Tables 
5 and 6 show pattern matrices and factor correlation matrices for exploratory and confirmatory subscale structures.

Insert Tables $5 \& 6$ about here

Response frequencies for the Attitudinal Scale items are provided in Table 7 for the current $(n=239)$ sample participants only.

Insert Table 7 about here

Behavioral Measures. The hypothesized 6-factor solution for the Behavioral Scale rendered uninterpretable results when all items were included in the initial exploratory analyses, whether with free runs or forced factors. Subsequent factor analyses partitioning subscale areas into three areas, Substance Use/Abuse, Sexual Assertiveness, and Behavior Risk History, improved pattern matrix interpretation; however, numerous items remained inconsistent and/or complex. It was postulated that the dichotomous nature of the participant's reported behavior (e.g., substance use vs. nonuse; and/or sexually active vs. nonactive) may affect the factor analyses in the process of grouping items into interpretable subscales. Thus various subsample selections with substance users and sexually active youth were fully explored, and found to greatly enhance interpretation within each of the three above-mentioned areas for subscale construction. Factor analyses with Substance Use and Abuse utilized only the participants indicating substance use within the four samples, 
while Sexual Assertiveness and Behavior Risk History analyses utilized only those participants who indicated sexual activity among the four samples. Finally, exploratory and confirmatory analyses were conducted with final subscale composite scores, first with all subjects, and secondly with a random 50 percent sample, to confirm subscale independence. With only the substance-using adolescents in the data pool $(n=308)$, exploratory factor analysis rendered a 2-factor solution, with eight items measuring Substance Use ( $\alpha=$ .87 ), and 15 items measuring Substance Abuse $(\alpha=.90)$. One item ("I drove a car after having alcohol or drugs") was found to be complex, loading on both factors; however, in the confirmatory factor analysis, the interscale difference improved, thus the item was retained (see Tables 8 and 9). The 2-factor solution accounted for 40 percent of the variance with a full scale reliability of .90 . Tables 8 and 9 show matrices and factor correlation matrices for exploratory and confirmatory subscale structures.

Insert Tables $8 \& 9$ about here

Utilizing only sexually active youth $(n=268)$ in analyses, the Sexual Assertiveness subscales remained intact, rendering a 2-factor solution, with five items measuring Partner Communication Assertivenss $(\alpha=.93)$, and five for Condom Use Assertiveness $(\alpha=.85)$. No items were found to be complex or inconsistent in the 2-factor solution, explaining 73 percent of the variance. Full scale reliability was .92 . Tables 10 and 11 show pattern matrices and factor correlation matrices for exploratory and confirmatory subscale structures. 
Again, utilizing only sexually active youth in analyses, Behavior Risk History was examined for both self and partner(s). Age of first intercourse was converted to a 5-point scale based on sample distribution for subscale inclusion. Specifically, ages were distributed around the mean age of 14 with lower ages receiving a higher score of five, and higher ages receiving a lower score of one. With all 17 hypothesized items included in the analysis, the first exploratory factor run was uninterpretable, revealing three inconsistent and complex items. Two items ("Do you use condoms?" and "In the next six months, do you plan to start always using condoms?") proved highly complex, and inconsistent, loading on both factors. Given the behavior these items seek to examine, it is believed these items would affect both self and one's partner, and quite possibly that gender may influence condom use or intended condom use. Thus, these items were removed from subsequent factor analysis runs and examined independently rather than within a subscale. One additional item, ("If you were to make a guess, how much risk do you have for getting HIV/AIDS at this time in your life?") was found to be both complex and inconsistent, with high loadings on both Self- and Partner-risk History subscales. Considering past findings (Lang, 1994) that this particular item was highly inconsistent with youths' reported sexual behavior, more particularly overestimating or underestimating risk in comparison to reported behavior over the past year, this item was removed from analyses and examined independently. 
The remaining 14 items were subjected to further exploratory factor analysis, rendering interpretable results with a 2 -factor solution accounting for 39 percent of the variance. Seven items were found to survey Self-risk History $(\alpha=.93)$, and seven items to measure Partner-risk History $(\alpha=.68)$, with a full Behavior Risk History scale reliability at 75. As indicated in Table 12, two items in the Partner-risk History subscale had fair factor loadings at .29 , one slightly improving in the confirmatory factor run (see Table 13). Despite not meeting the conservative $\geq .40$ cut, these three loadings were clearly not complex (having differences $>.2$ between subscales), and improved slightly in subsequent runs, justifying a decision to retain them. Additionally, it is believed that estimating risk behavior of one's partner(s) may prove difficult (see supportive qualitative section of this study), which may partially explain complexities of individual items attempting to measure Partner-risk History, further justifying inclusion of fair, but minimally acceptable loadings.

Insert Tables $12 \& 13$ about here

In order to ascertain whether each of the three subareas within the behavior domain were truly independent subscale measures, and to justify separating the factor analyses in the hypothesized three areas, further analyses were conducted. Composite scores were calculated and subjected to final exploratory and confirmatory factor analyses utilizing all participants, and a random 50 percent sample. Table 14 (exploratory) and Table 15 (confirmatory) depict the independence of the three behavior areas, with a high full scale 
reliability $(\alpha=.88)$ when all youth were included in analyses. The three sub-factor solution accounted for 84 percent of the variance revealing no inconsistent nor complex scales.

Insert Tables $14 \& 15$ about here

Response frequencies for the Behavior Scale items are provided in Table 16 for the current $(n=239)$ sample participants only.

Insert Tables 16 about here

Psychosocial Measures. For purposes of future questionnaire interpretation, the remaining Burkholder (1994) items, deemed "psychosocial," were subjected to factor analysis; however, were excluded from multivariate AIDS risk analyses in this study. Two psychosocial areas were examined: Environment and Education. Twenty-six Environment items were subjected to exploratory and confirmatory factor analyses, showing a 4-factor solution: Peer Risk ( $\alpha=.69$, eight items), AIDS Proximity ( $\alpha=.82$, eight items), Media Resources ( $\alpha=.77$, five items), and Media Influence ( $\alpha=.95$, five items). The last three subscales proved to be sound with high, consistent loadings. Four items in the Peer Risk subscale had minimal loadings of .30 but were retained (see Tables 17 and 18). One item ("Most of my friends have had sex") may be complex, loading on both Peer Risk and AIDS Proximity subscales, and may reflect overlap. Nevertheless, the loading improved in the confirmatory factor run and was retained. The 4-factor solution accounted for 54 percent 
of the variance with full scale reliability of .80 . Tables 17 and 18 show pattern matrices and factor correlation matrices for exploratory and confirmatory subscale structures.

Insert Tables $17 \& 18$ about here

Response frequencies for the Environment Scale items are provided in Table 19 for the current $(n=239)$ sample participants only.

Insert Table 19 about here

Factor analyses of the remaining 21 Education items produced uninterpretable results with both free run and forced factors. Interpretation difficulties may be due to the fact that many items measuring education largely focus on school-based knowledge, with minor attention on other areas using only one or a small number of items. Items were separated into three areas of education for further analyses: School Education, Home Education, and Peer Education. Interpretation is cautioned with the last two areas until further scale development, as they may be unstable given the small number of items constituting "a scale."

The 13 School Education items factored into a 2-factor solution, accounting for 44 percent of the variance, with a full scale reliability of .82 . Eight items measure School Education $(\alpha=.72)$, while five items measure School Influence $(\alpha=.75)$, with neither 
inconsistent nor complex items. Tables 20 and 21 show pattern matrices and factor correlation matrices for exploratory and confirmatory subscale structures.

Insert Tables $20 \& 21$ about here

A 2-factor solution accounting for 70 percent of the variance best explains Home Education items, two measure Parent Education and Influence $(\alpha=.68)$, and two Sibling Education and Influence $(\alpha=.69)$. Full scale reliability was fairly low $(\alpha=.66)$, likely due to a small number of indicators. Tables 22 and 23 show pattern matrices and factor correlation matrices for exploratory and confirmatory subscale structures.

Insert Tables $22 \& 23$ about here

The remaining four Peer Education items rendered a 2-factor solution with one item measuring Peer Education ( $\alpha=n / a$, single item), and three items examining Peer Influence $(\alpha=.74)$. The 2 -factor solution accounted for 75 percent of the variance, with a full scale reliability of .68 , having no inconsistent or complex loadings. Tables 24 and 25 show pattern matrices and factor correlation matrices for exploratory and confirmatory subscale structures.

Insert Tables $24 \& 25$ about here 
The independence of the Educational subscales was examined in order to justify separating the factor analyses in the hypothesized three areas. Composite scores were calculated and subjected to final exploratory and confirmatory factor analyses utilizing all participants, and a random 50 percent sample. Table 26 (exploratory) and Table 27 (confirmatory) depict the independence of the three educational areas. This 3-factor solution accounts for 72 percent of the variance with a full scale reliability of .81 , finding no inconsistent or complex items.

Insert Tables $26 \& 27$ about here

Response frequencies for the Education Scale items are provided in Table 28 for the current $(n=239)$ sample participants only.

Insert Table 28 about here

\section{Scale Construction Across Demographic Variables}

Group differences were explored with both demographic and composite score variables across all four samples of youth in order to justify pooling data for scale construction analyses. More specifically, in-depth analyses were undertaken to explore whether the questionnaire subscale structures remained stable across various demographic subpopulations for generalizing construct interpretation. MANOVAs and post hoc ANOVAs were conducted utilizing the Pillai's trace criterion, as Pillai's is most robust 
against violations of assumptions and has a more conservative measure of strength of association than Wilks' Lambda (Olson, 1979). Use of the conservative Scheffé test identified specific group differences. As indicated in Table 29, results show significant group differences for: age $(F(3,453)=25.60, p<.001)$, with Conference ' 93 Youth exceeding all other groups by Scheffé $(.01)$ criteria; in geographic location $(F(3,453)=$ $166.81, p<.001)$, with significant differences among all group dyad combinations except between Community Youth and Conference ' 93 Youth; in ethnicity $(\underline{F}(3,453)=8.63$, $p<.001$ ), with significantly less diversity among School Youth than all other groups; in grade $(F(3,453)=18.09, p<.001)$, with Conference '93 Youth one year ahead of all other groups; in religiosity $(\underline{F}(3,453)=3.76, p<.01$; no two groups significantly differing $)$; and lastly, in socioeconomic status, based on mother's $(E(3,452)=3.62, p<.01)$, and father's $(\underline{F}(3,448)=9.61, p<.001)$ education, with only the latter having significant group differences showing School Youth significantly lower than both Conference '93 and Conference '94 Youth.

Insert Table 29 about here

Also included in Table 29 are group differences across subscale composite scores and reported sexual behavior, although the demographic differences between the samples are the focus for scale development. Mean scores and standard deviations are reported for all subscales based on a 5-point scale, with five indicating the highest endorsement of a scale. Items reporting sexual behavior represent actual figures or percentages with the exception 
of the "Sex Risk" composite and logarithmic transformation ("Log Sex Risk"), both of which were variables created to assess risk, more fully explained in later analyses.

Group differences were also examined utilizing only the 2-sample population for further justification of pooling data for later analyses. As with the 4-sample data pool, significant demographic differences were found in the 2-sample data pool for: age $(\underline{E}(1$, $237)=32.96, p<.001)$; geographic location $(\underline{F}(1,237)=73.72, p<.001)$; ethnicity $(\underline{F}(1$, $237)=31.50, p<.001)$; grade $(\underline{F}(1,237)=31.59, p<.001)$; religiosity $(\underline{F}(1,237)=6.42$, $p<.01)$; and socioeconomic status, based on father's education $(F(1,234)=13.72, p<.001)$. Of interest, only five subscales were identified as having significant differences between the 2-sample data pool: Misconceptions $(\underline{F}(1,237)=32.01, p<.001)$; Self-risk History $(\underline{F}(1$, $237)=6.30, p<.01)$; Peer Risk $(\underline{F}(1,237)=13.19, p<.001)$; Sibling Influence $(\underline{F}(1,237)=$ $22.51, p<.001)$; and Peer Influence $(\underline{F}(1,237)=30.53, p<.001)$.

Given the diversity of characteristics within both the 2- and 4-sample data pools, confirmation of questionnaire structure stability over various subgroups appeared necessary to assess strengths and weaknesses of the measure.

First, subscale intercorrelations were calculated for both the 4-sample pool and the 2-sample pool under investigation across all subscale composite scores. Table 30 indicates subscale correlations for the 4-sample pooled data set (above the diagonal in bold type) and the current 2-sample pool (below the diagonal).

Insert Table 30 about here 
Significant subscale intercorrelations are reported at the $(p<.001)$ and $(p<.01)$ levels, justifying oblique rotation in the factor analyses, as many variables appear to overlap (see Table 30).

A series of confirmatory factor analyses were conducted for each of the demographic variables on which the 4-sample population differed. Given the small numbers of adolescents within various subpopulations (e.g., ethnic groups, specific ages, etc.), demographic variables were dichotomized based on post hoc analyses with the conservative Scheffé test. As post hoc results for age revealed adolescents younger than 15.3 years showing the greatest difference, age was dichotomized into two groups: adolescents younger than 15 years $(n=146)$, and adolescents 15 years and older $(n=311)$. Geographic location was also dichotomized for confirmatory analyses, with the majority of adolescents being from Rhode Island, Connecticut and Massachusetts $(n=382)$ versus "other" (New Hampshire, New Jersey, Maine, New York, Pennsylvania, Ohio, Delaware, Vermont, Bermuda, Jamaica, and Cayman Islands) $(n=75)$. Ethnicity was divided by Caucasian $(n=$ 372) and "other" (African-American, Asian-American, Hispanic-Americans, and "other") (n $=85$ ). Grade differences were eliminated in further confirmatory factor analyses, as this variable was subsumed within the age variable, and would reflect redundant analyses. Father's education was used as the socioeconomic index, as mother's education was nonsignificant in post hoc Scheffé findings among all groups. Socioeconomic estimate was dichotomized with "lower-to-middle class" (father's education being high school graduate or less) versus "middle-to-upper class" (father's education greater than high school 
graduate). Religiosity was eliminated from further analyses as individual group differences were nonsignificant in post hoc calculations.

A series of eleven confirmatory factor analyses were conducted in each subscale area: Cognitive, Attitudinal, Behavioral/Substance, Behavioral/Assertiveness, Behavioral/Risk History, Behavioral/Full Scale, Environment, Education/School, Education/Home, Education/Peers, Education/Full Scale, for each of the four dichotomized demographic variables: Age, Geographic Location, Ethnicity, and Socioeconomic Estimate. Thus, a total of 88 confirmatory factor analyses were conducted to determine scale and subscale strengths and weaknesses. As with the original factor analyses, various subpopulations were drawn for the Behavioral Scales (e.g., substance-using and sexually active subgroups). Alpha coefficients were calculated with each new subsample to determine the inter-item reliability. Concordance between the new factor(s) among subsample populations and factors developed with all participants were determined by the number of items varying from the original structure. If the factors in demographic analyses matched the factors found with the entire sample, they were deemed concordant. If the factors were found to be discordant, the varying item(s) were totaled and classified as inconsistent (not loading on any factor) or complex (loading on more than one factor). Finally, a consistency percentage was calculated based on the number of items in the confirmatory analyses with separate subsamples matching the original factor structure utilizing all participants. Table 31 depicts a summary of the total 88 confirmatory factory analyses for scale and subscale structures for similarities and differences on dichotomized demographic variables. 
Age. For the age variable, the concordance was relatively consistent. For adolescents of all ages, the Cognitive Scale remained intact with 100 percent consistency among all subscales. The Attitude Scale remained in agreement with adolescents 15 years and older, showing 96.2 to 100 percent consistency rates for subscales and an overall 96.2 percent full scale consistency. Attitude, for younger teens, was moderately consistent at 76.9 percent for the full scale. Within that domain, Condom Use Efficacy showed a stable 100 percent consistency rate, while Perceived Susceptibility for Risk and Homophobia were slightly less stable at 60.0 and 71.4 percent, respectively. The Behavioral Scale remained intact with a full scale consistency rate of 83.0 percent for youth 15 and older, and 87.2 percent for those younger. Substance Use (75.0 percent) and Substance Abuse (73.9 percent) subscales remained moderately concordant with a full scale consistency of 73.9 for older teens, and much more stable for younger teens, ranging from 93.3 to 100 percent. Assertiveness among the older population remained at 100 percent consistency, while reaching an acceptable 80 percent for younger teens. The Sexual Assertiveness for Condom Use subscale appeared to be problematic for sexually active youth under age 15 , showing only a 60.0 percent consistency rate. The Behavior Risk History subscales were similar for both age groups, ranging from 71.4 to 85.7 percent consistency, with Partnerrisk History at the lower end of the continuum for both groups. 
The Environment Scale remained moderately consistent with the older teens ( 88.5 percent consistency) as well as with younger participants ( 92.3 percent). AIDS Proximity, Media Resources, and Media Influence all retained 100 percent consistency with both age groups while the Peer Risk measure fell much lower at 75.0 and 62.5 percent for younger and older groups, respectively. The Education Scale, still under development, portrayed several inconsistencies, having consistency rates ranging from 25.0 to 80 percent in the school domain for both groups. Older teens showed higher subscale agreement for School Education and School Influence than the younger group. However, interpretation of these latter subscales should be cautioned due to the limited number of items included in subscales weakening the power and interpretation of the construct.

Overall, the questionnaire proved to have acceptable 84.9 and 87.8 percent consistency rates with the entire sample for younger and older teens, respectively.

Geographic Location. Geographic location posed less difficulty in interpretation than age in confirmatory analyses. The Cognitive Scales were 100 percent consistent as might be expected given the majority of the sample were located in Rhode Island, Massachusetts or Connecticut. Scale construction utilizing adolescents from other areas revealed slight inconsistency with Factual AIDS Knowledge, at an acceptable 83.3 percent consistency rate; and an overall Cognitive Scale rate of 94.7 percent. Conversely, the Attitude Scale had higher consistency rates among teens from other areas, ranging from 85.7 to 100 percent. For local teens, the Homophobia scale was most inconsistent among attitude variables, with 71.4 agreement, versus 100 percent with teens from other areas. Behaviorally, Substance Use and Abuse were relatively concordant across both samples 
with the exception that Substance Abuse with teens from other areas showed a 73.3 percent consistency rate in comparison to 86.7 percent with local counterparts. Assertiveness measures remained intact, with local youth maintaining 100 percent agreement across all subscales, while agreement ranged from 80 to 100 percent with youth from other areas. The Behavioral Risk History subscales also sustained 100 percent consistency rates across all subscales for local youth, but fared moderately well with youth from other areas ranging from 71.4 to 85.7 percent consistency.

As with the age differential, Peer Risk was moderately consistent within the Environmental Scale for both geographic locales, each revealing a 75.0 percent consistency rate. Full Scale consistency as well as the Media subscales remained highly consistency ranging from 92.3 to 100 percent. The Education Scale remained intact with local youth, but was found to be less stable with youth from other areas, particularly with items pertaining to school. The School Education subscale consistency rate was found to be 69.2 percent consistent while School Influence was a weak 50.0 percent.

In its entirety, the questionnaire maintained a 92.8 percent consistency rate with local youth, and an acceptable 88.5 percent among youth from other areas.

Ethnicity. The Cognitive Scale established its stability with the Caucasian youth with 100 percent consistency rates across all subscales. The full Cognitive Scale maintained an 89.5 percent consistency rate with youth of color, with particular difficulty posed by Transmission AIDS Knowledge subscale at 75.0 percent consistency. The Attitudinal Scale also fared well with Caucasian youth, ranging from 93.6 to 100 percent consistency, while moderate rates were obtained with youth of color. Perceived Susceptibility for Risk was 
especially low at 60.0 percent, and Homophobia at 78.6 percent, for the latter group.

Results here also must be cautioned given the small number of adolescents of color $(n=85)$ included in the analyses. Substance Use and Abuse were found to be relatively stable between groups, less so for Substance Abuse at 73.3 percent for Caucasian youth, and 66.7 percent for youth of color. The Assertiveness subscales remained intact for Caucasians at 100 percent consistency, and 80 percent for youth of color. Condom Use Assertiveness for the latter group was fairly low with a 60 percent consistency rate. Behavior Risk History subscales were more favorable for youth of color ranging from 85.7 to 100 percent.

Among these, the Partner-risk History subscale revealed the greatest discrepancy, with 71.4 percent consistency for Caucasians versus 100 percent for youth of color.

As with other demographic variables, the Peer Risk subscale achieved the lowest consistency rate among both samples on the Environment Scale, falling at 75.0 percent for Caucasians and 87.5 percent for youth of color. The Education Scale also rendered low consistency rates for school based education and influence with 69.5 and 62.5 percent consistency rates, respectively for Caucasians, and 71.4 and 62.5 percent for youth of color. Given the small number of items in the remaining education subscales, interpretation remains difficult.

All scale items inclusive, consistency rates were moderately high for Caucasian youth at 89.2 percent and similar for youth of color at 84.9 percent.

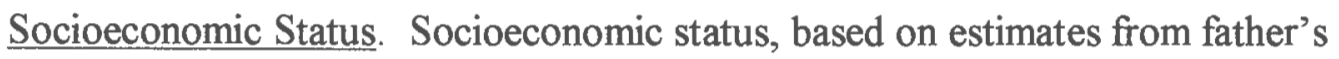
education level, appeared to have little influence over response structure, with a few exceptions. The Cognitive Scale remained unaltered with both lower-to-middle and middle- 
to-upper classes at 100 percent agreement. The Attitudinal Scale, however, was less consistent with lower-to-middle class youth at a consistency rate of 64.3 versus 92.9 for middle-to-upper class counterparts. On Behavioral Scales, Substance Use and Abuse were fairly consistent between groups ranging from 87.5 to 91.3 consistency. For the Assertiveness subscales, with the lower-to-middle class youth had a much lower consistency rate than the middle-to-upper class youth, at 60 and 100 percent, respectively. The Behavioral Risk History subscales, however, reflect more stability among lower socioeconomic youth ranging from 85.7 to 100 percent while the middle-to-upper class youth reveal a 71.4 percent consistency rate for both Self and Partner-risk Histories.

The Environment Scale revealed similar findings among both groups with Peer Risk at 75 percent as the lowest consistency rate for both classes. For the Education Scale, middle-to-upper class youth had high consistency rates ranging from 87.5 to 100 percent, while lower-to-middle class group revealed consistency rates as low at 50 percent for School Education.

Overall consistency for the entire questionnaire revealed the middle-to-upper class youth responses as being more consistent with the entire sample at 92.8 percent versus an acceptable 87.8 percent for lower-to-middle class teens.

\section{Factor Stability Synopsis}

As the current investigation is exploring only cognitive, attitudinal and behavioral aspects of AIDS risk, the stability, consistency and reliability of these particular measures are the primary focus. Utilizing a 70 percent consistency rate criterion in this synopsis, relatively few areas are cautioned for interpretation, and are outlined below. 
The current questionnaire poses some difficulties with youth under age 15 with some subscales, particularly with Perceived Susceptibility for Risk and Sexual Assertiveness for Condom Use being only moderately stable constructs. Conversely, subscale constructs present as relatively stable with youth 15 years and older. Geographic locale had little influence on the construct formation across all areas. Perceived Susceptibility for Risk, Substance Abuse and Sexual Assertiveness Condom Use were all moderately affected by ethnicity, showing greater inconsistency with youth of color in the analyses. Homophobia and Sexual Assertiveness for Condom Use were problematic measures for youth from lower-to-middle socioeconomic classes, but fairly stable with middle-to-upper class adolescents.

Figure 1 depicts the breakdown of the five major scale areas and subscales as a result of exploratory and confirmatory factor analyses.

Insert Figure 1 about here

Table 32 reflects mean scores and standard deviations for all subscale variables by gender and sexual activity. All subscale composite scores have been calculated on a 5-point scale, with a score of five indicating the highest endorsement of a subscale.

Insert Table 32 about here 


\section{Quantitative Results: Risk Predictors and Group Differences}

The following section is a comprehensive exploration of AIDS risk predictors and group differences by sexual activity and gender utilizing constructs described above within the cognitive, attitudinal and behavioral domains. Only the current 2-sample data pool described earlier (Conference Youth and School Youth) are utilized in following analyses.

\section{$\underline{\text { Sexual Experience }}$}

Of the 239 participants in the current study, 128 (53.6 percent) of the sample indicated sexual activity. For the young men, 63 (76.8 percent) reported sexual activity, while 65 (41.4 percent) of young women indicated the same. The majority (95.3 percent) of the sexually active teens indicated "unsafe" behavior, with inconsistent or no condom use during the past year. By gender, 92.3 percent of sexually active young women, and 98.4 percent of active young men, reported unsafe sexual activity. Figure 2 depicts the characteristics of the entire sample by gender, sexual activity and condom use (safe versus unsafe) behavior.

Insert Figure 2 about here

The mean age of the sexually active subsample was 14.97 years $(\underline{S D}=1.28)$ for sexually active young men, and 15.57 years $(\underline{S D}=1.54)$ for sexually active young women. Mean age for the sexually nonactive subsample was 14.26 years $(\underline{S D}=1.45)$ for nonactive young men, and 14.76 years $(\underline{S D}=1.59)$ for nonactive young women. All of the sexually active 
young women and 98.4 percent of the active young men reported being heterosexual, with one young man indicating bisexual orientation.

A significant majority of the active young men reported being involved in their current or most recent relationship for one year or longer ( 73.0 percent), in comparison to 40 percent of the young women $(\underline{F}(1,126)=12.41, p<.001)$. Young women reported the average relationship length for their current partner being between six months to a year, compared to young men's reports exceeding one year. Mean age of sexual onset was significantly younger for men $(\underline{M}=13, \underline{S D}=1.6)$ than women $(\underline{M}=14.1, \underline{S D}=1.4),(\underline{F}(1$, $126)=16.12, p<.001$ ). Table 33 indicates reported age of sexual debut by gender.

Insert Table 33 about here

Sexually active young men reported having significantly more sex partners $(\underline{M}=4.48, \underline{S D}=$ $3.53)$ in their lifetimes than sexually active young women $(\underline{M}=2.94, \underline{S D}=2.86)(\underline{F}(1,126)$ $=7.36, p<.05)$. This finding held true for number of partners in the past year as well, $(\underline{F}(1$, $126)=9.13, p<.01)$, with young men reporting an average of $2.76(\underline{S D}=2.49)$ partners versus $1.69(\underline{S D}=1.38)$ for young women. Active young women reported significantly higher estimates for number of sex partners their most recent partner had had $(\underline{F}(1,126)=$ $5.31, p<.05)$, estimating an average $3.46(\underline{S D}=3.29)$ compared to young men's average estimate of $2.29(\underline{S D}=2.39)$. Both genders reported equal certainty in their estimates of the number of partners their most recent partner had had, with the average response being "very sure." Although not significant, the frequency distributions varied greatly regarding 
certainty within this area, with 46.2 percent of the young women reporting "absolutely sure," and 29.2 percent claiming to be "very sure," compared to 27.0 and 42.9 percent for male counterparts, respectively.

\section{Substance Use and Abuse Correlates with Sexual Behavior}

For sexually nonactive youth, 48.6 percent $(n=54)$ reported substance use, and 32.4 percent $(n=36)$ were categorized as substance abusers based on their responses. Comparatively, 78.9 percent $(n=101)$ of sexually active youth reported substance use, with 67.2 percent $(n=86)$ considered abusers of substances. Other correlates of substance use and sexual activity were found: 33 percent of sexually active youth reported they had engaged in sexual activity on one or more occasions during the past year, which they believed they wouldn't have engaged in if they had not used substances.

Analyses of sexually active substance users $(n=101)$ showed 75.2 percent $(n=76)$ as reportedly using alcohol prior to having sex during the past year; with three-quarters $(n=$ 75) reporting "sometimes," "half the time," or "usually." Over one-quarter of this subgroup also reported cannabis use prior to sex $(28.7$ percent, $n=29)$, most reporting "sometimes" $(n=24)$. Specific to inconsistent or no condom use, 98 of the 101 sexually active substance users ( 97.0 percent), were participating in "unsafe" sexual activity by this standard.

Regarding more intense substance use with potential problematic behavior, or abuse, the 86 identified substance abusers who were also sexually active were examined for correlates. The majority ( 83.7 percent, $n=72$ ) reported using alcohol prior to having sex in 
the past year; most reporting high frequencies ("sometimes," "half the time," or "usually"). Additionally, 32.6 percent $(n=28)$ of these teens reported using cannabis before sex.

Significant differences were found between genders for substance use prior to sexual behavior. More young men (44.4 percent) reported alcohol use prior to sex "sometimes," than women ( 33.8 percent), and fewer (25.4 percent) reported "never" than women (55.4 percent $)(E(1,126)=16.65, p<.001)$. During the past year, 50.8 percent of young men reported having 1 to 2 drinks prior to sexual encounters, compared to 41.5 percent of women $(\underline{F}(1,126)=17.84, p<.001)$. Cannabis use was also significantly more frequent for young men prior to sex than for women $(\underline{F}(1,126)=6.02, p<.05)$; with 22.2 percent reporting use "sometimes," versus 16.9 percent for women.

\section{Self and Partner AIDS Risk Estimates}

Both genders reported equally their certainty of not being exposed to AIDS, with an average response being "very sure," among sexually active youth. More young women (43.1 percent) responded "absolutely sure," than men (30.2 percent), although not statistically significant. Similarly, the average response for certainty as to their partner(s) exposure, was "very sure," for both genders. Response distributions were similar in higher certainty ranges, with 40.0 percent of young women responding "absolutely sure," 29.2 percent "very sure," and 20.0 percent "slightly sure," versus $30.2,41.3$ and 22.2 percent, respectively, for sexually active men.

Regarding testing, 87.3 percent $(n=55)$ of sexually active young men and 78.5 percent $(n=51)$ of women had never undergone HIV/AIDS testing. For young men, 11.1 percent $(n=7)$ tested negative, versus 20.0 percent $(n=13)$ for women, and one man and 
one woman reported they tested positive for HIV. The majority of sexually active youth, reported never having had a needle-using (96.9 percent, $n=124)$, or an HIV-positive (98.4 percent, $n=126$ ) sex partner.

Sexually active participants' self-rated risk estimates did not significantly differ between genders, with both genders reporting an average of being at "slight" risk. Interestingly, incidence of over- and underestimating personal risk for AIDS persisted as it did in recent findings (Lang, 1994). Sixty-six percent of the participants portrayed accurate perceptions of risk when compared with their sexual behavior, while 34 percent indicated inaccuracies either by over- or underestimating their AIDS risk level. Within the entire sample, two percent were considered "safe" or consistent condom users, while 31 percent considered "unsafe," with inconsistent or no condom use, rated themselves at a level other than "no risk," (slight, some, moderate or high). Thirty-three percent were sexually nonactive and rated themselves at "no risk." These three groups comprised the "accurate" group. The remaining 34 percent represented overestimates (14 percent) and underestimates (20 percent) of AIDS risk. One percent of the entire sample were "safe" sexually active youth who overestimated their risk, conservatively rating themselves at "slight" or "some" risk, and 13 percent were sexually nonactive, also overestimating their risk by rating themselves at "slight," "some" "moderate," or "high" risk for AIDS. Of most concern is the remaining 20 percent of the entire sample (or 38.5 percent of the sexually active "unsafe" youth), who considered themselves to be at "no risk" for AIDS. Table 34 depicts participants' estimated risk for AIDS by gender, sexual activity and condom use behavior. 
Risk Analyses

To examine predictors of potential risk, standard MRAs were computed, with post hoc evaluation on independent predictors using Larzelere and Muliak's (1977) conservative $F$ test. The Larzelere and Mulaik (1977) formula (see also Tabachnick \& Fidell, 1989, $p$. 155) accounts for the high likelihood of Type I error when utilizing several a posteriori tests, and controls accordingly with a conservative $F$ test to retain Type I error rate below $\alpha$ for all combinations of predictor or independent variables (IVs). Post hoc significances are indicated on bivariate correlates with the dependent variable (DV), since the Larzelere and Mulaik formula utilizes the bivariate correlation among variables in the calculation of $F$.

The standardized residual histogram and normal probability plot of residuals was utilized to confirm assumptions of normality, linearity and homoscedasticity between the predicted DV scores and errors of prediction for each MRA. Outliers among cases were identified through the standardized residual z-score listing $( \pm 3.00)$ and standardized residual histogram. Inspection of the correlation matrix and significant Beta $(\beta)$ weights confirmed presence or absence of suppressor variables. Unless otherwise indicated, each MRA met assumptions of normality, linearity and homoscedasticity, with neither outliers nor suppressor variables present.

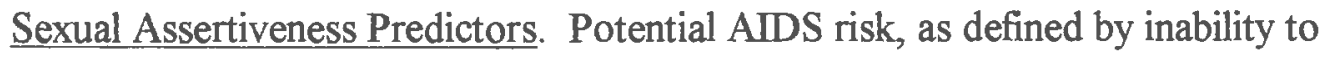
assert oneself sexually, was examined for young men and women. Sexually active teens 
reported assertiveness skills with their most recent partner, while nonactive youth predicted behavior. The DV, potential AIDS risk, was comprised of a composite score of the two Sexual Assertiveness subscales (estimated or actual condom use together with estimated or actual ability to communicate with partner(s), with low Sexual Assertiveness indicating a potential risk for AIDS). Eight predictor variables were entered simultaneously: Factual AIDS Knowledge, Transmission Knowledge, Perceived AIDS Knowledge, Misconceptions, Perceived Susceptibility for Risk, Homophobia, Substance Use, and Substance Abuse.

In order to determine whether predictors of potential risk were influenced by gender, separate MRAs were conducted. For young men, only high Perceived AIDS Knowledge was a significant predictor of high Sexual Assertiveness $(\underline{R}=.58 ; \underline{F}(8,73)=$ $4.70, p<.001)$, with 34 percent of the DV variance explained $\left(\underline{R}^{2}=.34\right.$, Adjusted $\left.\underline{R}^{2}=.27\right)$. Positive Perceived AIDS Knowledge $(\beta=.32, p<.05)$, contributed 10 percent of explained variance. For young women, low Misconceptions $(\beta=-.17, p<.05)$, low Perceived Susceptibility for Risk $(\beta=-.16, p<.05)$, and low Substance Use $(\beta=-.43, p<.001)$, and Abuse $(\beta=-.25, p<.001)$, were significant predictors of high Sexual Assertiveness $(\underline{R}=.56$; $\underline{F}(8,148)=8.26, p<.001)$, with 31 percent of the DV variance explained $\left(\underline{R}^{2}=.31\right.$, Adjusted $\underline{R}^{2}=.27$ ). As shown in Table 35, Substance Use and Abuse contributed most to the explained variance (11 and 10 percent, respectively), while Misconceptions and Perceived Susceptibility for Risk contributed an additional combined 6 percent.

Insert Table 35 about here 
Post hoc analyses were nonsignificant for women; however, low AIDS Misconceptions was significant for men $(r=-.44 ; \underline{F}(8,73)=2.19, p<.05)$. Thus, for young men, perceiving their knowledge for AIDS as high and having low Misconceptions are significant predictors of assertiveness. For young women, having little or no AIDS Misconceptions, low Perceived Susceptibility for Risk, and no reported Substance Use or Abuse, contribute to the same.

AIDS Risk Predictors. Thirteen predictors were used to examine their relations to the DV, Sex Risk, among the 128 sexually active participants: Factual AIDS Knowledge, Transmission AIDS Knowledge, Perceived AIDS Knowledge, Misconceptions, Perceived Susceptibility for Risk, Condom Use Efficacy, Homophobia, Sexual Assertiveness Partner Communication, Sexual Assertiveness Condom Use, Substance Use, Substance Abuse, Partner-risk History and Self-risk History. The Sex Risk index utilized in a previous study (Kelly, et al., 1990) was computed by multiplying the number of unprotected intercourse occasions during the past year by the number of different intercourse partners during the past year. This computational index has been justified by Kelly, et al. (1990) in defining, under most circumstances, the level of risk for AIDS as a function of both how many times an individual engages in a risky practice and number of different sexual partners.

Evaluation of assumptions led first to the deletion of six identified outliers (5 young men and one young woman) reporting a Sex Risk composite score range of 600 to 3480 . These extreme cases, with $z$-scores ranging from +3.00 to +4.00 , were deleted from the MRA analysis to reduce their impact on the regression solution, lowering the sample size from 128 to 122 . Moderate improvement in the distribution was evidenced with the 
deletion of outliers and nontransformed scores; however, a significant improvement was realized with logarithmic transformation of the Sex Risk variable ("Log Sex Risk"), and was the method of choice. In order to increase power in the analyses given the small $n$ with the sexually active subsample, three MRAs were run separately within each of the three domains of interest: Cognitive, Attitudinal and Behavioral subscale variables.

To permit examination of whether gender differences exist within the sexually active subgroup for AIDS risk as defined by the (Log) Sex Risk variable, separate analyses were undertaken for each gender. For sexually active men, the Cognitive MRA was significant $(\underline{R}=.40 ; \underline{F}(4,58)=2.83, p<.05)$, with 16 percent of DV variance explained $\left(\underline{R}^{2}=.16\right.$, Adjusted $\left.\underline{R}^{2}=.11\right)$. High Misconceptions $(\beta=.43 ; p<.01)$ significantly predicted Sex Risk, contributing 15 percent to explained variance. The Attitudinal MRA was also significant, $(\underline{R}=.52 ; \underline{F}(3,59)=7.17, p<.001)$, with 27 percent of the variance explained $\left(\underline{R}^{2}=.27\right.$, Adjusted $\left.\underline{R}^{2}=.23\right)$. High Perceived Susceptibility for Risk $(\beta=.44 ; p<.001)$ significantly predicted Sex Risk, contributing 15 percent of the variance. Behaviorally, for sexually active men, high Partner- $(\beta=.30 ; p<.01)$ and Self-risk Histories $(\beta=.67 ; p<.001)$ significantly predicted Sex Risk $(\underline{R}=.82 ; \underline{F}(6,56)=19.35, p<.001)$, with 68 percent of the DV variance explained $\left(\underline{R}^{2}=.68\right.$, Adjusted $\left.\underline{R}^{2}=.64\right)$, contributing 28 and 12 percent to the shared variance, respectively. Post analyses showed low Condom Use Sexual Assertiveness $(r=-.48 ; \underline{F}(6,56)=4.34, p<.01)$, high Substance Use $(r=.54 ; \underline{F}(6,56)=5.97, p<.01)$, and high Substance Abuse $(r=.40 ; \underline{F}(6,56)=2.76, p<.05)$ as significant predictors of Sex Risk for sexually active men. Table 36 depicts results of the three MRAs for Sex Risk predictors sexually active men. 
For sexually active young men, high Misconceptions, Perceived Susceptibility for Risk, Substance Use and Substance Abuse, Self- and Partner-risk Histories, and low Condom Use Assertiveness contribute to AIDS risk as defined by the Sex Risk variable.

For sexually active women, the Cognitive MRA was nonsignificant. High Perceived Susceptibility for Risk $(\beta=.32 ; p<.01)$ and low Condom Use Efficacy $(\beta=-.38 ; p<.001)$ significantly predicted Sex Risk in the Attitudinal MRA $(\underline{R}=.55 ; \underline{F}(3,61)=8.87, p<.001)$, with 30 percent of the variance $\left(\underline{R}^{2}=.30\right.$, Adjusted $\left.\underline{R}^{2}=.27\right)$ (Perceived Susceptibility for Risk contributed 9 percent while Condom Use Efficacy accounted for 21 percent). The Behavioral MRA was significant, showing low Partner Communication Assertiveness ( $\beta=$ $-.26 ; p<.05)$ and low Condom Use Assertiveness $(\beta=-.45 ; p<.01)$ as predictors of Sex Risk $(\underline{R}=.62 ; \underline{F}(3,58)=6.05, p<.001)$, with 39 percent of the variance explained $\left(\underline{R}^{2}=.39\right.$, Adjusted $\underline{R}^{2}=.32$ ). While Partner Communication accounted for only 2 percent of the variance, 15 percent was attributed to Condom Use Assertiveness. Post hoc analyses found both high Partner-risk History $(r=.45 ; \underline{F}(6,58)=3.81, p<.01)$ and high Self-risk History $(r$ $=.45 ; \underline{F}(6,58)=3.81, p<.01)$, as significant contributors to Sex Risk, with 6 and 2 percent of the shared variance. Table 37 depicts the 3-area MRA results for sexually active women.

Insert Table 37 about here 
Overall, for sexually active young women, information in the form of knowledge is noncontributory to Sex Risk, while high Perceived Susceptibility for Risk, Partner- and Self-risk Histories, and low Condom Use Efficacy and Sexual Assertiveness (Partner Communication and Condom Use) skills are significant predictors of the same.

Interestingly, analyses with the sexually active youth as a group, inclusive of both genders, revealed similar significant risk predictors distributed among genders, with exception of one additional variable regarding attitude. Within an Attitudinal MRA utilizing all sexually active youth, Homophobia was also found to be significant, $(\underline{R}=.50 ; \underline{F}(3,124)$ $=14.07, p<.001)$, with 25 percent of the DV variance explained $\left(\underline{R}^{2}=.25\right.$, Adjusted $\underline{R}^{2}=$ .24). Post hoc analysis found high Homophobia $(r=.31 ; \underline{F}(3,124)=3.27, p<.05)$ as a significant predictor of Sex Risk for sexually active adolescents, but not independently for either gender when analyzed separately.

Predictors of Self-rated Level of AIDS Risk. Examination was undertaken to determine which factors, if any, contributed to adolescents' self-rated level of AIDS risk. All 13 IVs (Factual AIDS Knowledge, Transmission AIDS Knowledge, Perceived AIDS Knowledge, Misconceptions, Perceived Susceptibility for Risk, Condom Use Efficacy, Homophobia, Sexual Assertiveness Partner Communication, Sexual Assertiveness Condom Use, Substance Use, Substance Abuse, Partner-risk History, and Self-risk History) were simultaneously entered as predictors of self-rated AIDS risk.

In order to determine whether predictors differed by gender among the sexually active subgroup, separate analyses were conducted. Separate MRAs by area (Cognitive, Attitudinal and Behavioral) were necessary to maintain power in the analyses given the 
small $n$ designs. For sexually active young men, both the Cognitive and Attitudinal MRAs were nonsignificant; however, the Behavioral MRA produced one significant predictor $(\underline{R}=$ $.48 ; \underline{F}(6,56)=2.84, p<.05)$, explaining 23 percent of the $\mathrm{DV}$ variance $\left(\underline{R}^{2}=.23\right.$, Adjusted $\left.\underline{R}^{2}=.15\right)$. As indicated in Table 38 , only low Substance Abuse $(\beta=-.39 ; p<.01)$, with 3 percent of the variance significantly contributed to sexually active young men's self-rated level of AIDS risk. Post hoc analyses were nonsignificant.

Insert Table 38 about here

For the sexually active young women, the Cognitive and Attitudinal MRAs also were nonsignificant, and initially one variable emerged as significant in the Behavioral MRA $(\underline{R}=.61 ; \underline{F}(6,58)=5.70, p<.001)$, explaining 37 percent of the $\mathrm{DV}$ variance $\left(\underline{R}^{2}=.37\right.$, Adjusted $\left.\underline{R}^{2}=.31\right)$. As shown in Table 39 , only positive Partner-risk History $(\beta=.28$; $p<.05)$ contributed to the young women's self-rated risk level.

Insert Table 39 about here

However, post hoc analyses revealed positive Substance Use $(r=.50 ; \underline{F}(6,58)=5.00$, $p<.01)$, positive Substance Abuse $(r=.48 ; E(6,58)=4.49, p<.01)$, and positive Self-risk History $(r=.43 ; \underline{F}(6,58)=3.40, p<.01)$, as significant factors in sexually active young women's self-rated AIDS risk level. 
Utilizing all participants, sexually active and nonactive, both genders, an additional predictor surfaced. As sufficient power was realized with a larger $n$ design, one overall MRA was utilized which produced significant results $(\underline{R}=.46 ; \underline{F}(13,225)=4.65, p<.001)$, with 21 percent of the DV variance $\left(\underline{R}^{2}=.21\right.$, Adjusted $\left.\underline{R}^{2}=.17\right)$. As found with sexually active teens, high Substance Use $(\beta=.27 ; p<.01)$, and high Partner-risk History $(\beta=.31$; $p<.01)$ were found to be significant contributors to adolescents' self-rated level of AIDS risk; however, low Homophobia $(\beta=-.15 ; p<.05)$, was added as a significant predictor. Post hoc analyses revealed Self-risk History also as a significant contributor $(r=.31 ; \underline{F}(13$, $225)=1.84, p<.05$ ). Thus, Homophobia may have been overshadowed as a significant predictor of adolescents' self-rated level of risk with the individual analyses of sexually active youth by gender, due to small sample sizes.

Nevertheless, interpretation of predictors of adolescents' self-rated level of AIDS risk appears to be confounded with issues of accuracy and over- and underestimating risk requiring a more in-depth analysis.

Predictors Contributing to Accurate and Inaccurate Levels of Self-rated AIDS Risk. The accuracy and/or inaccuracy of adolescents' self-rated level of AIDS risk may be influential in examining which determinants are significant in their risk selection process. Based on the comparison of self-rated level of AIDS risk and behavioral reports, participants were dichotomized into "accurate estimates" and "inaccurate estimates" of risk levels. Those individuals in the accurate group consisted of: (1) sexually nonactive youth rating themselves at "no risk;" (2) sexually active youth reporting consistent condom use and rating themselves at "no risk;" and (3) sexually active youth reporting inconsistent or no 
condom use rating themselves at a level higher than "no risk." The inaccurate group was subdivided into: (1) sexually nonactive teens overestimating their risk, ranking themselves at a level higher than "no risk;" (2) sexually active teens reporting consistent condom use, overestimating their risk level above "no risk;" and, (3) sexually active teens reporting inconsistent or no condom use and ranking themselves at "no risk," or "underestimaters." Of primary interest are relevant predictors of risk levels and comparisons between the third groups in each category, with special emphasis on the underestimaters. Analyses were tailored to seek out which factors contributed to adolescents' self-rated level of AIDS risk, more specifically the "unsafe, underestimaters," as this group is most likely in need of intervention efforts; and to assess how they differed from the "unsafe, accurate estimators."

Since it is not possible to conduct a MRA with the desired "unsafe, underestimaters" due to lack of DV variability (all responses were "no risk"), MRAs were calculated with the "accurate" counterpart. All 13 IVs utilized with MRAs in the previous section were simultaneously entered as predictors of self-rated AIDS risk as the DV, separately by Cognitive, Attitudinal and Behavioral areas to increase power. Second, a $2 \mathrm{X} 2$ (gender X underestimate/accurate) MANOVA was calculated for group comparison, with the same 13 variables.

For sexually active adolescents who were considered "unsafe," and rated themselves accordingly (accurately), two of the three domains investigated, Cognitive and Attitudinal, were found to be significant. As shown in Table 40, low Misconceptions $(\beta=-.42 ; p<.001)$ significantly predicted their accurate self-rated risk level $(\underline{R}=.41 ; \underline{F}(4,70)=3.48, p<.01)$, explaining 17 percent of the DV variance $\left(\underline{R}^{2}=.17\right.$, Adjusted $\left.\underline{R}^{2}=.12\right)$, and independently 
contributing 13 percent of that variance. Low Homophobia $(\beta=-.37 ; p<.01)$ significantly predicted the same in the Attitudinal MRA $(\underline{R}=.32 ; \underline{F}(3,71)=2.76, p<.05)$, with 10 percent of the DV variance explained $\left(\underline{R}^{2}=.10\right.$, Adjusted $\left.\underline{R}^{2}=.07\right)$, and independently contributing the entire 10 percent of the variance. The Behavioral MRA, as well as the post hoc analyses, were nonsignificant.

Insert Table 40 about here

Having few or no AIDS misconceptions and low homophobia appear as significant contributors to accurate, self-rated AIDS risk levels among sexually active youth with unsafe sex practices.

Next, analyses were conducted to examine contributors of self-rated risk for youth with inaccurate ratings, particularly underestimation of AIDS risk. Utilizing gender and self-rated level of risk (accuracy versus underestimation) as IVs, group differences were explored with a 2X2 MANOVA and post hoc ANOVA with all 13 subscales as DVs.

Evaluation of assumptions of normality, linearity, and multicollinearity were satisfactory and no outliers were identified. In order to meet the assumption of variance-covariance matrices due to unequal $n$ 's for both IVs, the SPSS PC + method of adjustment for unequal $n(\mathrm{METHOD}=\mathrm{SSTYPE}(\mathrm{UNIQUE}))$ was utilized in computation. Examination of results from Box's $M$ test, which is very sensitive to departures from normality, revealed the homogeneity-of-dispersion-matrices assumption as satisfactory. Pillai's trace criterion was used instead of Wilks' Lambda to evaluate multivariate significance, as it is the most robust 
in instances of violated assumptions (Olson, 1979). Although the $F$ distributions were similar among the criteria, the measure of strength of association is more conservation with use of Pillai's trace.

No gender X self-rated risk level interaction was found in the MANOVA; however, significant main effects were found for both gender (Pillai's $F(13,106)=9.48, p<.001$ ) and self-rated level of risk (Pillai's $\underline{F}(13,106)=3.27, p<.001$ ), with relatively strong and moderate associations between IVs and combined DVs evident for both gender $\left(\eta^{2}=.54\right)$ and self-rated level of risk $\left(\eta^{2}=.29\right)$, respectively. Findings were inconsistent with MRA findings above, in many respects. Differences in Homophobia were found nonsignificant between accurate self-raters and underestimaters. Further, although there was a significant difference between the accurate group and underestimaters for Misconceptions $(p<.05)$, the accurate group $(\underline{M}=2.50, \underline{S D}=1.20)$ had significantly higher Misconceptions than underestimaters $(\underline{M}=2.03, \underline{S D}=1.01)$. Table 41 reveals MANOVA and post hoc ANOVA results for gender and self-rated level of risk among sexually active "unsafe" participants.

\section{Insert Table 41 about here}

With respect to gender among sexually active "unsafe" teens, young women had significantly higher Factual AIDS Knowledge $(\underline{F}(1,118)=12.17, p<.001)$, Perceived AIDS Knowledge $(\underline{F}(1,118)=12.70, p<.001)$ and lower Misconceptions $(\underline{F}(1,118)=18.08$, $p<.001)$ than men. Young men had significantly higher Perceived Susceptibility for Risk $(\underline{F}(1,118)=30.71, p<.001)$, Homophobia $(\underline{F}(1,118)=30.64, p<.001)$, Sexual 
Assertiveness Partner Communication $(\underline{F}(1,118)=16.31, p<.001)$, Sexual Assertiveness

Condom Use $(\underline{F}(1,118)=6.02, p<.01)$, Substance Use $(\underline{F}(1,118)=8.25, p<.01)$,

Substance Abuse $(\underline{F}(1,118)=12.52, p<.001)$ and Self-risk History $(\underline{F}(1,118)=22.14$, $p<.001$ ) than women (see Table 41 for means and standard deviations). Figure 3 depicts mean scores for DVs by gender among sexually active "unsafe" youth.

Insert Figure 3 about here

With regard to self-rated level of risk among sexually active "unsafe" participants, accurate youth had significantly higher Misconceptions $(\underline{F}(1,118)=3.92, p<.05)$, Perceived Susceptibility for Risk $(\underline{F}(1,118)=5.97, p<.01)$, Substance Use $(\underline{F}(1,118)=19.47$, $p<.001)$, Substance Abuse $(\underline{F}(1,118)=4.09, p<.05)$, Partner-risk History $(\underline{F}(1,118)=$ $25.72, p<.001)$ and Self-risk History $(\underline{F}(1,118)=26.84, p<.001)$; and lower Sexual Assertiveness Condom Use $(E(1,118)=4.94, p<.05)$ than inaccurate underestimaters (see Table 41 for means and standard deviations). Figure 4 illustrates the differential DV mean scores by self-rated level of AIDS risk among the sexually active "unsafe" participants.

Insert Figure 4 about here

Differential Risk Profiles by Gender and Sexual Activity

A 2X2 MANOVA was conducted to examine differential risk profiles by gender and sexual activity across all participants. All 13 DVs were included in three MANOVAs, 
separated by Cognitive, Attitudinal and Behavioral areas in order to maintain sufficient power in the analyses. Factual AIDS Knowledge, Transmission AIDS Knowledge, Perceived AIDS Knowledge, Misconceptions, Perceived Susceptibility for Risk, Condom Use Efficacy, Homophobia, Sexual Assertiveness Partner Communication, Sexual Assertiveness Condom Use, Substance Use, Substance Abuse, Partner-risk History, and Self-risk History, constituted the risk variables of interest in this analysis. Evaluation of assumptions of normality, linearity, and multicollinearity were satisfactory and no outliers among any variables were identified. Due to unequal $n$ 's, adjustment was made in statistical computation, as described earlier, in order to meet the assumption of variancecovariance matrices. Additionally, an examination of Box's $M$ test results revealed the homogeneity-of-dispersion-matrices assumption as satisfactorily met.

Overall, significant interactions were found in all three domains. For reference, Table 42 shows the means and standard deviations for all subgroups analyzed and discussed more fully below. Main effects are included in Table 42 for the interested reader, although the central foci in the reported results are the significant interactions.

Insert Table 42 about here

Cognitive. A significant interaction was found between gender and sexual activity for the Cognitive MANOVA (Pillai's $\underline{F}(4,232)=4.15, p<.01$ ), although a relatively weak association between the IVs and combined DVs was present $\left(\eta^{2}=.07\right)$. Only one Cognitive DV, Misconceptions, was significant $(\underline{F}(1,235)=16.23, p<.001)$, showing 
sexually active young men with the highest Misconceptions, and their nonactive counterparts at the lowest (see Table 42 for means and standard deviations). Young women have significantly greater Misconceptions when sexually active than when nonactive. Figure 5 depicts the effects of gender $\mathrm{X}$ sexual activity on the variable, Misconceptions.

Insert Figure 5 about here

Attitudinal. The Attitudinal MANOVA also rendered a significant interaction (Pillai's $\underline{F}(3,233)=6.00, p<.001)$, also with a relatively weak association between the IVs and combined DVs present $\left(\eta^{2}=.07\right)$. Gender and sexual activity were found to have significant interaction effects for both Perceived Susceptibility for Risk $(\underline{F}(1,235)=4.74$, $p<.05)$ and Homophobia $(\underline{F}(1,235)=12.89, p<.001)$. Perceived Susceptibility for Risk appears to be significantly higher among the young men than young women, sexually active or not (see Table 42 for means and standard deviations); and significantly higher for sexually active teens than nonactive counterparts by gender. Figure 6 indicates the significant gender X sexual activity interaction for Perceived Susceptibility for Risk.

Insert Figure 6 about here

With regard to the Homophobia variable, young women appeared to be fairly consistent, whether sexually active or nonactive, in having low reported homophobic indicators. 
However, sexually active young men were found to be at the highest for Homophobia, in contrast both to their nonactive male counterparts and to sexually active young women. Figure 7 displays the gender $\mathrm{X}$ sexual activity interaction for Homophobia.

Insert Figure 7 about here

Behavioral. The Behavioral MANOVA also produced a significant gender $\mathrm{X}$ sexual activity interaction (Pillai's $\underline{F}(6,230)=11.19, p<.001$ ), with a moderate association between the IVs and combined DVs present $\left(\eta^{2}=.23\right)$. Four of the five DVs had significant interactions: Sexual Assertiveness Partner Communication $(\underline{F}(1,235)=24.23$, $p<.001)$, Sexual Assertiveness Condom Use $(\underline{F}(1,235)=13.04, p<.001)$, Substance Use $(\underline{F}(1,235)=4.21, p<.05)$, Substance Abuse $(\underline{F}(1,235)=6.30, p<.01)$, and Self-risk History $(\underline{F}(1,235)=17.45, p<.001)$.

Sexual Assertiveness Partner Communication was generally perceived as higher by the nonactive subgroup, who offered their predicted responses, than the actual behavior reported by sexually active teens (see Table 42 for means and standard deviations). Nonactive young women predicted their assertiveness behavior to be highest, however the opposite occurred for the active women. Sexually active young men reported higher assertiveness for partner communication than active young women. A similar pattern emerged for Sexual Assertiveness Condom Use among subpopulations. Figures 8 and 9 depict the gender X sexual activity interaction for Sexual Assertiveness Partner Communication (Figure 8) and Condom Use (Figure 9). 
Similar patterns emerged for Substance Use and Substance Abuse, with both genders within the sexually nonactive population reporting significantly less use and abuse than sexually active counterparts (see Table 42 for means and standard deviations). For sexually active adolescents, young men reported significantly more substance use and substance abuse than young women. Figures 10 and 11 depict gender $\mathrm{X}$ sexual activity interaction for Substance Use (Figure 10) and Substance Abuse (Figure 11).

Insert Figures $10 \& 11$ about here

Finally, a significant interaction between gender and sexual activity was also found for Self-risk History. Although the sexually nonactive teens, both men and women, reported low Self-risk History, this pattern changed with sexual activity (see Table 42 for means and standard deviations). Sexually active young men reported a significantly higher Self-risk History than sexually active young women. Figure 12 shows the gender $\mathrm{X}$ sexual activity interaction for Self-risk History.

Insert Figure 12 about here 
Sexually Active Youth. Further MANOVA analyses utilizing only the sexually active subgroup were undertaken to examine gender differences among the 13 DVs within this subpopulation. Results of evaluations of the assumptions of normality of sampling distributions, homogeneity of variance-covariance matrices, linearity, and multicollinearity were satisfactory. Computations were adjusted for unequal $n$ 's, and separate analyses were undertaken in the three areas of interest to maintain sufficient power.

With use of Pillai's criterion, the Cognitive MANOVA was significant (Pillai's $\underline{F}(4$, $123)=8.39, p<.001)$, with a moderate association between the IV and combined DVs found $\left(\eta^{2}=.21\right)$. As depicted in Table 43, three of the four Cognitive components were significantly different for gender: Factual AIDS Knowledge $(\underline{F(1,126)}=12.89, p<.001)$, Perceived AIDS Knowledge $(\underline{F}(1,126)=10.75, p<.001)$, and Misconceptions $(\underline{F}(1,126)=$ $24.05, p<.001)$.

Insert Table 43 about here

Sexually active young women had significantly higher Factual AIDS Knowledge, and perceived their AIDS knowledge as significantly higher than sexually active young men; and young men had significantly higher Misconceptions than young women (see Table 43 for means and standard deviations).

The Attitudinal MANOVA also rendered significant results (Pillai's $\underline{E}(3,124)=$ $21.68, p<.001)$, with a moderately high association between the IV and combined DVs found $\left(\eta^{2}=.34\right)$. Perceived Susceptibility for Risk $(\underline{F}(1,126)=39.96, p<.001)$, and 
Homophobia $(\underline{F}(1,126)=41.95, p<.001)$ were significantly different between genders. Sexually active men reported significantly higher Perceived Susceptibility for Risk and Homophobia than female counterparts (see Table 43 for means and standard deviations).

Behaviorally, nearly all the DVs were found to be significantly different between genders (Pillai's $\underline{F}(6,121)=13.21, p<.001$ ), with moderately strong associations between the IV and combined DVs $\left(\eta^{2}=.40\right)$. Sexual Assertiveness Partner Communication ( $F(1$, $126)=6.50, p<.01)$, Sexual Assertiveness Condom Use $(\underline{F}(1,126)=21.14, p<.001)$, Substance Use $(\underline{F}(1,126)=12.59, p<.001)$, Substance Abuse $(\underline{F}(1,126)=13.54, p<.001)$, and Self-risk History $(\underline{F}(1,126)=6.50, p<.001)$ significantly differed between the sexually active young men and women. Young men reported significantly higher Sexual Assertiveness for both Partner Communication and Condom Use than young women, and reported higher Substance Use, Substance Abuse, and Self-risk History than young women (see Table 43 for means and standard deviations). One-way ANOVAs also reealed that sexually active young men had a significantly higher Sex Risk composite score $(\underline{F}(1,126)=$ $6.30, p<.01$ ), and significantly higher logarithmically transformed Sex Risk composite score ("Log Sex Risk") than young women $(\underline{F}(1,126)=3.71, p<.05)$.

Figure 13 pictorially depicts DV mean scores for sexually active youth by gender.

Insert Figure 13 about here

Sexually Nonactive Youth. Similar MANOVA analyses were conducted for nonactive youth for potential gender differences among the 13 DVs. Results of evaluations 
of the assumptions of normality of sampling distributions, homogeneity of variancecovariance matrices, linearity, and multicollinearity were satisfactory. Computations were adjusted for unequal $n$ 's, and analyses were undertaken in the three areas of interest to maintain sufficient power.

Gender was nonsignificant in Cognitive analyses. The Attitudinal MANOVA rendered significant results (Pillai's $\underline{F}(3,107)=2.87, p<.05$ ), with a low association between the IV and combined DVs $\left(\eta^{2}=.06\right)$. Perceived Susceptibility for Risk $(\underline{F}(1,109)$ $=6.44, p<.01)$ and Condom Use Efficacy $(\underline{F}(1,109)=3.73, p<.05)$ showed significant gender differences. Nonactive young men perceived their susceptibility for risk as significantly higher than nonactive women, while nonactive women predicted their Condom Use Efficacy to be significantly higher than nonactive young men's. Table 44 shows MANOVA and post hoc results across all three domains for nonactive youth by gender.

Insert Table 44 about here

The Behavioral MANOVA was also significant (Pillai's $\underline{F}(5,105)=3.32, p<.01$ ), with a low association between the IV and combined DVs $\left(\eta^{2}=.07\right)$. Sexually nonactive women predicted their Sexual Assertiveness abilities to be significantly higher than that predicted by nonactive men for both Partner Communication $(\underline{F}(1,109)=12.28, p<.001)$ and Condom Use $(\underline{F}(1,109)=8.50, p<.01)$ (see Table 44 for means and standard deviations).

Figure 14 graphically displays DV mean scores for nonactive youth by gender. 
Insert Figure 14 about here

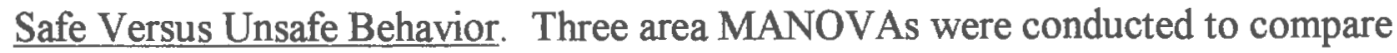
sexually active participants with safe and unsafe sexual practices, defined by consistent versus no condom use. Although assumptions of linearity and multicollinearity were satisfactory and no outliers were identified, the assumption of homogeneity-of-dispersionmatrices was violated in each MANOVA analysis; therefore, the results from these analyses should be treated with caution. Additionally, in order to meet the assumption of variancecovariance matrices, similar calculation adjustments were made in the statistical analyses as mentioned with previous MANOVA analyses; however, the extreme differences for unequal $n$ 's in these analyses also caution interpretation of the results that follow.

In comparisons of safe versus unsafe sexually active adolescents, both the Cognitive and Behavioral MANOVAs rendered nonsignificant results. The Attitudinal MANOVA, however, approached significance (Pillai's $E(3,124)=2.78, p<.05$ ) with a low association between the IV and combined DVs $\left(\eta^{2}=.06\right)$. Table 45 lists means and standard deviations for the comparison groups, and MANOVA and post hoc analyses.

Insert Table 45 about here

The consistent condom users, or safe adolescents, reported significantly lower Perceived Susceptibility for Risk $(\underline{F}(3,126)=5.08, p<.05)$ and Homophobia $(\underline{F}(3,126)=4.18$, 
$p<.05)$, and significantly higher Condom Use Efficacy $(E(3,126)=4.99, p<.05)$, than their unsafe counterparts (see Table 45 for means and standard deviations).

Sexually Active Versus Nonactive. Three area MANOVAs comparing sexually active and nonactive youth across the $13 \mathrm{DVs}$ were conducted. Evaluations of the assumptions of normality of sampling distributions, homogeneity of variance-covariance matrices, linearity, and multicollinearity were satisfactory.

MANOVAs comparing the sexually active youth to the sexually nonactive group across both genders reveal significant differences in the Cognitive domain (Pillai's $\underline{F}(4,234)$ $=7.70, p<.001)$, with a low association between the IV and combined DVs $\left(\eta^{2}=.12\right)$. Nonactive youth report significantly higher Factual AIDS Knowledge $(\underline{F}(1,237)=4.38$, $p<.05)$ and significantly lower Misconceptions $(\underline{F}(1,237)=4.38, p<.05)$ than sexually active counterparts. Table 46 shows MANOVA and post hoc results of gender differences by sexual activity.

Insert Table 46 about here

The Attitudinal MANOVA produced significant findings for all three DVs (Pillai's $\underline{F}(3,235)=15.62, p<.001)$ with a moderate association between the IV and combined DVs $\left(\eta^{2}=.17\right)$. The sexually active youth perceived their susceptibility for risk as significantly higher than nonactive youth $(E(1,237)=37.18, p<.001)$, had significantly higher Homophobia than nonactive teens $(\underline{F}(1,237)=17.20, p<.001)$, and had lower Condom Use 
Efficacy than that predicted by nonactive youth $(\underline{F}(1,237)=17.48, p<.001)$ (see Table 46 for means and standard deviations).

Behaviorally, significant differences were found for all DVs between the sexually active and nonactive adolescents (Pillai's $\underline{F}(6,232)=176.64, p<.001$ ) with a strong association between the IV and combined DVs $\left(\eta^{2}=.82\right)$. Nonactive youth predicted their assertiveness skills to be significantly higher than reported behavior of the sexually active group for Partner Communication $(\underline{F}(1,237)=121.75, p<.001)$ and Condom Use $(\underline{F}(1$, $237)=92.10, p<.001)$ (see Table 46 for means and standard deviations). Sexually active youth had significantly higher Substance Use $(\underline{F}(1,237)=56.36, p<.001)$, significantly higher Substance Abuse $(\underline{F}(1,237)=29.47, p<.001)$, significantly higher Partner-risk History $(\underline{F}(1,237)=255.34, p<.001)$, and significantly higher Self-risk History $(E(1,237)=$ $724.65, p<.001)$ than nonactive adolescents.

Figure 15 depicts mean scores for DVs between sexually active and nonactive teens.

Insert Figure 15 about here

Transtheoretical Stages of Behavior Change

Adolescent responses were examined for staging with current (indicative of behavior during the past year of completing the questionnaire) and future (for the six month period following completion of the questionnaire) condom use among the sexually active group. Response categories for current condom use were paired with four Transtheoretical Model stages (see, Prochaska \& DiClemente, 1983, 1984; Prochaska, DiClemente, \& Norcross, 
1992; Prochaska, et al., 1994): Precontemplation ("Never use condoms"); Contemplation ("Sometimes use condoms, but not always"); Action ("Started always using condoms less than one year ago"); and Maintenance ("Started always using condoms over one year ago"). Similarly, predicted condom use responses for "the next six months" were also staged accordingly: Precontemplation ("No plans for future use"); Contemplation ("Maybe sometimes, but not always"); Action ("Plan to start using condoms"); and Maintenance (“Already use condoms").

Sexually Active Participants. Examining staging variables with sexually active youth revealed that 27.1 percent $(n=33)$ of the adolescents considered "unsafe," reported their stage of behavior change inaccurately. These youth perceived their current condom use behavior to fall within Action or Maintenance stages, but given their reported sexual behavior in the past 12 months, would more accurately be categorized as Contemplation or, in some cases, Precontemplation (no condom use). Nevertheless some accuracies were noted. Of the six safe sexually active teens, four considered themselves currently in the Maintenance stage, and two in the Action stage. Six months from questionnaire completion date, five of the six consistent condom users predicted they would be in Maintenance, with one remaining in Action. Table 47 lists response frequencies for current and predicted condom use behavior for sexually active youth by gender.

Insert Table 47 about here 
Totals were adjusted to correct for the above-mentioned inaccuracies, by restaging according to "unsafe" (inconsistent condom use) or "safe" (consistent condom use) behavior reported in the behavior matrix. Unsafe was recoded as Precontemplation/ Contemplation, and safe as Action/Maintenance. When totals were adjusted, 95.3 percent $(n=122)$ of the sexually active youth would fall in the Precontemplation or Contemplation stages, with the remaining 4.7 percent $(n=6)$ considered within Action or Maintenance.

In order to pinpoint inaccuracies more precisely, sexually active youth were then categorized into three groups based on condom use reported in the behavior matrix: always, sometimes, or never (using condoms in the past year). Comparing adolescents' reported condom use in the behavior matrix to their responses for present condom use staging, inconsistencies were noted. As shown in Table 48, 27 of the 63 sexually active young men ( 42.9 percent), and 25 of the 65 sexually active young women ( 38.5 percent) placed themselves in current condom use stages that were more advanced than their reported sexual behavior reflected during the past year. Thus, aside from the 27.1 percent misclassifying themselves mentioned above, an additional 14.8 percent $(n=19)$ erroneously classified themselves in Contemplation (or sometimes using condoms), when they reported no condom use during the past year in matrix format, more accurately reflecting the stage of Precontemplation. Therefore a total of $40.6(n=52)$ staged themselves inaccurately by self-report.

Insert Table 48 about here 
An attempt to estimate accurate staging of the sexually active adolescents was generated based on the Transtheoretical Model's progressive stages of change. Specifically, individuals advance through the stages of change sequentially, and are not likely to bypass stages in the process, especially in a short span of six months. Using the figures provided in Table 48, stages were adjusted to the reported sexual behavior during the past year. For the most part, individuals reporting inconsistent condom use ("sometimes") and staging themselves in the Action or Maintenance stages were moved to Contemplation, and individuals reporting no condom use ("never") were reclassified in Precontemplation, which more accurately reflected their sexual behavior patterns over the past year. Similarly, stage estimates were adjusted for predicted condom use in the next six months, without skipping ahead more than one stage. As depicted in Figure 16, original staging indicators for current and predicted condom use reported by adolescents revealed a substantial increase in the most adaptive stages, Action and Maintenance.

Insert Figure 16 about here

However, when staging was adjusted based on adolescents' sexual behavior reports over the past year for both current and predicted condom use, the graph portrays a less than optimistic view for safe behavior patterns (see Figure 17).

Insert Figure 17 about here 
Although Figure 17 may represent a more realistic view of adolescent sexual behavior, it should be noted that these figures were also estimated by downgrading stages based on sexual behavior reported in a matrix format for the past year. Since it is impossible to determine whether information obtained in single-item inquiries or figures reported in the behavior matrix account for such inaccuracies, further analyses with staging variables utilized teens' reported stages. Inaccuracies noted in comparing Figures 16 and 17 are provided for cautionary interpretation of differential staging findings with sexually active youth, provided below.

Analyses were undertaken to examine whether AIDS risk variables differed by stage of change (IVs) with the sexually active participants among all 13 subscales as DVs. Attempts to include gender with a factorial 2X4 MANOVA (gender X stage) rendered nonsignificant interaction results in all three domains, Cognitive, Attitudinal, and Behavioral risk for both current and predicted condom use staging. Significant main effects for gender were noted, however, only for predicted condom use staging $(E(1,126)=4.35, p<.05)$. Thus analyses were conducted with sexually active youth, inclusive of both genders for current condom use staging; while separate analyses by gender were conduced for predicted condom use staging in order to preserve power in the analyses and examine differential stage progression among variables. All assumptions were satisfactory for MANOVA analyses with no outliers identified. Post hoc ANOVAs were conducted as well as conservative Scheffé tests utilizing a stringent alpha level at .01 , for identifying specific stage differences for risk. 
The Cognitive MANOVA for current condom use rendered significant findings (Pillai's $\underline{F}(12,369)=2.96, p<.001)$, with a fairly strong association between IV, or staging variable, and combined DVs $\left(\eta^{2}=.26\right)$. Table 49 reports means, standard deviations, MANOVA and post hoc results for AIDS risk variables across Transtheoretical stages.

Insert Table 49 about here

Significant group differences were realized for Transmission AIDS Knowledge $(\underline{F}(3,124)=$ $2.64, p<.05)$, and Misconceptions $(\underline{F}(3,124)=7.44, p<.001)$. Post hoc Scheffé tests reveal that no two staging groups differed significantly for Transmission AIDS Knowledge; however, the Maintenance group significantly differed from the Contemplation group with Misconceptions. Youth at the Maintenance stage for current condom use had significantly lower Misconceptions than youth in the Contemplation stage (see Table 49 for means and standard deviation comparisons).

The Attitudinal MANOVA examining differential stages for current condom use was also significant (Pillai's $\underline{F}(9,372)=4.11, p<.001$ ), with a moderately strong association between the staging variable, and combined DVs $\left(\eta^{2}=.27\right)$. Both Condom Use Efficacy $(\underline{F}(3,124)=11.00, p<.001)$ and Homophobia $(\underline{F}(3,124)=3.41, p<.05)$ significantly differed by stage, although post hoc Scheffé analyses found only Condom Use Efficacy having significant differences. Youth in the Maintenance stage for current condom use reported significantly higher Condom Use Efficacy than youth in the Contemplation stage (see Table 49 for means and standard deviations). 
The Behavioral MANOVA was also significant (Pillai's $\underline{F}(18,363)=2.33, p<.01$ ), with a fairly strong association between the staging variable and combined DVs $\left(\eta^{2}=.31\right)$. Sexual Assertiveness Partner Communication $(\underline{F}(3,124)=2.92, p<.05)$, Sexual Assertiveness Condom Use $(\underline{F}(3,124)=5.19, p<.01)$, and Self-risk History $(\underline{F}(3,124)=$ $4.28, p<.01)$ differed significantly by stage. Scheffé analyses found only Sexual Assertiveness Condom Use to have significant differences between the Maintenance and Contemplation stages, with the former group reporting higher assertiveness skills than the latter (see Table 49 for means and standard deviations).

One way ANOVA analyses for the Sex Risk composite score and its logarithmically transformed equivalent, Log Sex Risk, found significant group differences for Log Sex Risk $(\underline{F}(3,124)=5.83, p<.01)$. Post hoc Scheffé analyses found significant group differences between the Precontemplaters and Contemplaters for current condom use, with the more advanced stage, Contemplation, having a higher Log Sex Risk composite score (see Table 49 for means and standard deviations).

Gender Differences. As mentioned above, gender differences were not found in the current condom use domain, thus gender differential analyses were conducted for only predicted condom use with staging variables among the 13 subscale risk variables (DVs). A factorial 2X4 MANOVA (gender X stage) rendered nonsignificant interaction results in all three domains, Cognitive, Attitudinal, and Behavioral risk, thus separate MANOVA analyses were performed for the sexually active young men and young women. All assumptions were satisfactory for MANOVA analyses with no identified outliers. 
As shown in Table 50, the Cognitive MANOVA was significant for the sexually active young men (Pillai's $\underline{F}(12,174)=1.85, p<.05)$, with a fairly strong association between the staging variable and combined DVs $\left(\eta^{2}=.34\right)$ noted. Transmission AIDS Knowledge $(\underline{F}(3,59)=3.64, p<.05)$ and Misconceptions $(\underline{F}(3,59)=3.32, p<.05)$ were significantly different between stages for predicted condom use; however post hoc Scheffé test failed to distinguish specific group differences between the four stages (see Table 50 for means and standard deviations).

Insert Table 50 about here

The Attitudinal MANOVA was also significant for sexually active young men (Pillai's $\underline{F}(9,177)=2.45, p<.01)$, with a fairly strong association between the staging variable and combined DVs $\left(\eta^{2}=.33\right)$. Only Condom Use Efficacy revealed significance between the groups $(\underline{F}(3,59)=5.26, p<.01)$ with the Scheffé tests showing youth in the Maintenance stage as having significantly higher Condom Use Efficacy than youth in the Contemplation stage (see Table 50 for means and standard deviations).

For the sexually active young men, the Behavioral MANOVA was also significant (Pillai's $\underline{F}(18,168)=2.43, p<.01)$, with a very strong association between the staging variable and combined DVs $\left(\eta^{2}=.62\right)$ noted. Only Partner-risk History was significant $(E(3,59)=3.74, p<.01)$, with nonsignificant post hoc results for group distinction.

While both the Cognitive and Behavioral MANOVAs were nonsignificant for sexually active young women, the Attitudinal analyses revealed significant group differences 
(Pillai's $\underline{F}(9,183)=1.94, p<.05)$, with a moderate association between the staging variable and combined DVs $\left(\eta^{2}=.26\right)$. Depicted in Table 51 , as with sexually active young men, only Condom Use Efficacy was significant $(\underline{F(3,61)}=4.27, p<.01)$, for sexually active young women.

Insert Table 51 about here

The post hoc Scheffé test revealed young women in the Maintenance group had significantly higher Condom Use Efficacy than counterparts in the Action group (see Table 51 for means and standard deviations).

Predicted Condom Use Among the Sexually Nonactive Participants. Responses from the sexually nonactive youth regarding their predicted condom use and associated staging are listed in Table 52.

Insert Table 52 about here

Fifteen of the 111 sexually nonactive youth predict they will become sexually active within the next six months, and report their behavior would be in the Precontemplation or Contemplation stages for predicted condom use. The majority ( 86.5 percent) report no plans for sexual activity within the next six months. 


\section{Qualitative Results: Focus Groups}

\section{Conference Youth Focus Groups: A Pilot Study}

Given the structured time frame of the Youth-to-Youth Conference, and limited opportunities to conduct in-depth focus groups, the six half-hour sessions for the nine Conference Youth participants comprised a pilot study for further exploration. In this manner, the Youth-to-Youth Conference "family group" guidelines and structure were maintained while simultaneously exploring areas related to adolescent perceptions of HIVrisk. Ultimately, these initial six sessions provided the information necessary to produce the semi-structured format for more extensive investigation at a later date with the School Youth (see Appendix G).

Nine youth, six young women and three young men, ranging from 13 to 15 years in age, met twice daily with the primary investigator and a 17 -year-old young man as cofacilitator. Sessions were comprised of games to learn names, establish trust, and resist peer pressures for drug use and unwanted sex, and followed by open discussions on particular topics. Brief descriptors of participants that were informally gathered are listed below, although the names have been changed for confidentiality:

Cathy: 13-year-old, Caucasian, young woman from New England, affluent family background, listens attentively, enjoys sailing

Christine: 15-year-old, Caucasian, young woman from New England, middle class socio-economic background, talkative, cooperative, often challenged young men in discussions

Clark: 14-year-old Caucasian, young man from the Northeast, lower middle class socio-economic background, very dramatic in attention seeking, attempted to "out-do" participants, group quickly ignored tactics for attention (e.g. tripping over furniture, etc.) 
Patrick: 14-year-old, Caucasian, young man from the Northeast, middle class socio-economic background, befriended Clark, giggled often and cracked numerous jokes, somewhat disruptive

Leon: 14-year-old, young man of Hispanic descent, from New England, low socio-economic background and currently in foster care, biological parents divorced, small build, often sulked and withdrawn, uncooperative with games, drug and sexual abuse history

Amy: 14-year-old, Caucasian young woman from New England, upper middle class socio-economic background, timid at first but easily warmed up to group after first session, not very talkative

Shelbi: 14-year-old, Caucasian, young woman from the Northeast, affluent background, appeared and presented as slightly more mature than her chronological age, fairly talkative

Megan: 14-year-old, Caucasian, young woman from New England, middle class socio-economic background, slightly overweight and timid, emotional about conference ending

Tara: 14-year-old, Caucasian, young woman from New England, affluent background, appeared and presented much older than chronological age

Session One: Youth participated in games to get acquainted and discussed

difficulties adolescents often face. Four teens mentioned suicide of a friend or family member, six experienced peer pressure to have sex, and all nine had frequent peer pressure for drug experimentation. With reference to peer pressure to have sex and condom use, participants mentioned the following:

Christine: "Almost everyone I know talks about sex, like yeah, they had sex; but sometimes you don't know if they're just bragging to be popular or something."

Clark: "I never heard anybody talk about condoms, nobody brings it up where we're talking... they probably don't care or something like that." Patrick: "Yeah, it seems like they're making you feel like a 'dweeb' if you say you never did it [have sex]." 
Session Two: During the second session, teens participated in games related to resisting peer pressure. Discussion ensued regarding adolescent issues related to drug use and sex. One group member confessed to socializing with older youth and having tried "all" drugs. Two others joined in stating that they had also tried drugs, but were challenged by the group as to whether they were bragging or telling the truth. These two youth quickly withdrew from the conversation when inquired about specific drug reactions by the adolescent claiming to "do the most drugs."

Leon: "Your parents can tell, like you can't fool 'em, by the eyes, by the black dot in the eyes..."

Clark: "I did it [tried drugs] and wished while I was doing it that I didn't. Like it felt too weird, all the time while I was doing it I wished I didn't... But I don't remember a lot of stuff... I was with my girlfriend, she did it too, I dunno what happened, she kissed me I think."

Patrick: "Yeah, I felt that way too."

Leon: "It's like time stood still, like a minute was like a million hours... yeah, I forget what happened a lot of times, and I was always with a girl if I wanted to be.. the first time I did it [had sex] I was smoking [marijuana] and it [had sex] just happened."

Session Three: The third session continued with peer pressure topics, games to assist in youth saying, "No," and role playing assertiveness skills. Discussion regarding what may occur when adolescents say, "No," how they are perceived, and drug accessibility followed.

Amy: "My school is boring, nothing happens there with drugs, we're all really boring I guess."

Shelbi: "Mine too, but I know someone, my best friend's friend, who does drugs and brags about a bunch of boyfriends... she makes me feel bad sometimes, like I'm not good enough to have a boyfriend because I don't want to have sex. I think you have to be ready, and she makes fun of me." Cathy: "I know someone like that, she's really popular in my school. Like she's very pretty, she's probably got about 25 boyfriends, um..., and lots of girls want to be like her, um..., sometimes I do too, but I don't know how." 
Patrick: "I know someone like that, her name is K---. I was with her once, but nothing happened..., she wouldn't even let me kiss her, and a bunch of guys like made fun of me because of it. So I like sometimes would pretend that we did it [had sex], so they wouldn't laugh at me."

Session Four: Continued role playing ensued to resist peer pressure in various situations, with open discussion regarding substance use following.

Megan: "I tried wine once at Christmas time and, like gross, I don't like the taste of it."

Tara: "I like beer. I know it's bad, and I hate to say it, but I like the taste of it."

Christine: "Once I was at a party my [older] brother had and they had tons of beer and I had some. I felt like really, really silly and laughed a lot, and I was dancing with one of my brother's friends, he was really cute. But later he tried to get me into my bedroom. [Pauses.] I dunno what would have happened if my brother wasn't there, he got really mad, started yelling and smacking him. I felt really bad after that."

Leon: "I tried it all. Now I just say, 'No,' I just don't care. Once I even had a guy pull out a knife, but all my friends told him to just leave me alone. Then he just said, 'Okay, cool,' when he saw all my friends with me."

Patrick: "Yeah, sometimes the older gangs in my school try to force you to do it [drugs and/or alcohol], you just want to run away as fast as you can, but sometimes you just can't."

Session Five: Discussion revolved around difficulties teenagers have regarding sexual activity, peer pressure, and knowledge and questions about AIDS. Four participants reported misconceptions, primarily labeling HIV “a gay disease." Approximately half did not realize that HIV can be acquired heterosexually; however the young women appeared to be more knowledgeable on the topic than the young men.

Clark: "I don't know any gay people."

Tara: "You don't have to be gay to get AIDS you know..."

Clark: "Yeah, gays and druggies get AIDS, I don't have to worry..."

Tara: "You can get AIDS from sex too, you don't have to be gay, you dork!"

[Group is silent] 
Participants were highly inquisitive regarding transmission routes, prevalence rates among their age group and protective measures. Two young women interjected often, responding, "I knew that," while others remained silent or inquired further.

Clark: "So I could get AIDS even though I'm not gay?"

Patrick: "Whoa! No way!"

Tara: "If you have sex with a lot of different people, you're like just asking for it."

Amy: "Come on you guys, you didn't know that?"

Shelbi: "Even I knew that."

Session Six: During the final session, the majority of the time was spent with reviewing lessons learned during the course of the entire conference. Youth eagerly volunteered information they each thought was most important.

Clark: "I don't think sex is a good idea, I was thinking about it, I'm afraid of AIDS."

Amy: "I think I'll wait until I get married..."

Cathy: "I don't need to be popular that way..."

Tara: "You could use a condom..."

Patrick: "I think everyone should always use a condom..."

Shelbi: "I would, or I'd tell him, "No way..."

Clark: "I'm still really afraid of catching it, I never thought about that, I mean I really thought it was mostly gay guys that get it."

Christine: “I don't think I'll drink beer anymore if my brother has another party. I'll probably just go out of the house or something. You never know what you might do, I mean when you drink and stuff." Leon: "I already learned a long time ago, I don't like the headaches [referring to hangovers]. I once had one that lasted for a week. It's just not worth it."

Megan: "After hearing your stuff, I don't think anyone could make me drink, plus it's gross tasting anyway."

Prior to closing ceremonies and "good-byes," all participants were offered an opportunity to inquire further about any misconceptions or questions they had together as a group or privately with the group leader. Participants felt fairly knowledgeable and did not raise any relevant issues. 


\section{School Youth Participation}

Prior to conducting focus groups with School Youth, an informal tally was conducted through homeroom and first periods for School Youth to determine rationale for nonparticipation two days before the last of the seven survey dates. Since focus group participants were comprised of volunteers from youth completing the survey, the informal tally was an additional effort to recruit last minute participants if conditions permitted. Additionally, it allowed youth who opted not to participate an opportunity to state why, and provided further qualitative information, or at a minimum, offered a creative outlet.

Anonymous tally sheets consisted of a checklist format with four questions: gender, grade, age and five options regarding participation: (1) did or will participate; (2) would have participated if there was a better class time; (3) did not want to participate; (4) parents did not give permission to participate, and (5) other. Only 11 homeroom teachers did not return the tally sheet packets, yielding a 63 percent return rate, where 600 of the 956 students returned the tally sheets. Table 53 reveals the results of the participation tally.

Insert Table 53 about here

Among tally respondents, it appears that 14 students indicated that they "will participate" on the last survey date, but did not. Also, 20 percent would have participated if there was a better class time, 42 percent did not want to participate, five percent did not receive parental permission, and 20 percent stated "other" reasons. The most frequent among

\footnotetext{
1 Because some participants indicated more than one reason for this item, this total percentage exceeds 100 percent.
} 
"other" responses were: "forgot about it," indicated by 36 students, "didn't know about it," (29 students), and "never got the time" (eight students). Twenty-four teens reported "other" responses such as "I was absent," "I'm busy that day," "lost" or "didn't get permission form," "didn't ask parents," or "parents didn't read," or "parents (or teacher) lost form." Nine indicated "other" responses of "I don't like AIDS," "I don't really care," "I wasn't ready to participate," or "I don't know," and two stated the prize money wasn't adequate. The remaining ten reported creative "other" responses, such as "my cousin is a gay transvestite and asked me not to participate," "I want to be a guy [inclusive with a feminine name], I'm gay," "I am a homosapian," "I'm having a sex change," "not smart enough," "my pet amadillo [sic] died," "threw it on the floor," "it got trashed," "surgery on my brain," and one is best not repeated.

\section{School Youth Focus Groups}

Of 110 survey participants, 80 signed up, 23 did not attend, and 4 neglecting to enroll decided to attend, yielding a sample of 61 participants. Focus groups were held on three separate days, with each day having a different semi-structured discussion outline. Since School Youth had the option to attend up to three focus group sessions, once on each of the three dates, some overlap is present in feedback checklist information. Overall, 61 participants comprised the focus group attendees, with some attending one, two or three sessions.

To reduce uneasiness and establish rapport, questions started with general areas and moved to more specific topics regarding behavior. Topics included: (1) AIDS Research (survey feedback, understandable, sections liked or disliked, suggested areas for inclusion, 
perceptions of response honesty, the value of survey research, and suggested methods of AIDS prevention for teenagers); (2) Scope of the AIDS Pandemic (ages afflicted, local prevalence rates, populations afflicted and homophobia); (3) Societal Dilemmas (problems for insurance companies, issues with segregating infected persons, and rights of infected pregnant women); and, (4) Teenage Risk Situations (availability of information, risk situations on a continuum and sexual assertiveness scenarios). Although a semi-structured format was followed, students were encouraged to discuss differences of opinion, bring up relevant issues and ask questions. All incorrect statements or misperceptions were corrected at the close of each group in order to allow group participants to discuss or debate issues with minimal interference by the leader. The unobtrusive method was utilized to examine the extent of knowledge before providing correct information. All groups were prefaced with an introduction: (1) thanking participants for attending; (2) emphasizing no "right" or "wrong" answers, (3) the importance of confidentiality with material discussed in group, (4) minimizing self-disclosure by having participants speak in generalities or precede comments with "I know someone who..."; and (5) obtaining permission to take notes.

All School Youth participants completed an anonymous demographic checklist inquiring about gender, ethnicity, age, grade, number of groups attended, and a 14-item adjective feedback checklist regarding both the survey and group attended. Although the average group attendance was about six, on three instances only one student attended; however, the remaining 19 groups had adequate attendance. Table 54 provides a focus group summary of demographics across the 3-day period for School Youth, as well as feedback from the 14-item adjective checklist. 
As shown in Table 54, checklist feedback was fairly positive. The "other" category included a mixed reaction: 22 teens reported positive comments as: "pretty awesome," "good idea for teens," "will come again," "liked to discuss it," "got a lot of questions answered," "glad I could express my feelings," "educational," "learned a lot," "you asked good questions," "pretty cool," "very informative," and "thank you." Four teens reported: "good, but I sort of held back," "would have liked it better if I was with people in my own age group," "weird," and "very hard."

Focus group participants also provided anonymous feedback regarding the questionnaire instrument in a checklist format, as well as within group discussion on the same topic. Many adolescents reported positive feedback for the questionnaire itself, as indicated in Table 55. Adolescents indicated in written form "other" feedback responses which included: "didn't apply to me," "uncomfortable," "weird," "asked a lot about my friends," "personal," or "uneasy to answer, "while eight students had more positive "other" indicators: "survey good for kids," "educational," "a good idea," and "informative."

Insert Table 55 about here

It should be noted that since focus group dates took place on three prearranged days within a 4-week period after survey completion, and many participants attended on more than one 
occasion, adolescents attending groups on the last day were not asked for survey feedback

to take into account recall considerations and to reduce response redundancy. Thus Table

55 reflects adolescents' feedback from group participants on days one and two only.

Anonymous Questionnaire Feedback. All survey participants, both Conference and

School Youth, had the opportunity to provide anonymous feedback regarding the questionnaire in a "comments" section at the end of the document. No Conference Youth participant provided comments, which may have reflected the limited time span these teens had to complete the questionnaire and the full schedule involved in the 3-day conference.

School Youth, however, provided ample feedback, listed below.

$\mathrm{YW}(13) \mathrm{SN}:{ }^{2}$ "My mom is gay so I don't have a problem with gay people."

YW(13)SN: "I think it was a kind of confusing test, but I'm glad I could help. Thank you!"

YW(13)SN: "Thank you for allowing kids our age to be able to take the survey!"

YM(13)SN: "Very long, fairly hard, takes a long time to do."

YW(15)SN: "This is a very interesting test."

YW(13)SN: "Some of the questions are kind of personal. But it was a good idea. I think there should have been separate surveys, one for junior high, and one for senior high."

YW(14)SN: "I want to know more a lot more about AIDS, because there are a lot of times when I want to have sex with someone I really love. I know if I do, I could get AIDS. That's what I'm afraid of. But I always think about having sex. I am to [sic] afraid to talk it over with my mom because I don't know if she'll give me a huge lecture. She told me never to be afraid to talk to her, but I am still."

YM(14)SN: "I think this survey was greatly put together. It did not make me feel uneasy."

YW(13)SA: "I think this was a good survey. Very interesting."

YW(13)SA: "I think this was a very good idea!"

YW(15)SA: "I think people (teachers) should talk about relationships

with people also. Like a teenager getting involved with an adult. I wish people would spend more time on that."

2 Demographic information obtained from questionnaire: YW=Young Woman; YM=Young Man; number in parenthesis indicates age of respondent; $\mathrm{SN}=$ Sexually Nonactive, $\mathrm{SA}=$ Sexually Active. 
YW(13)SN: "I don't know what this survey is going to prove, but I think it was well organized and I hope you learn a lot from it. Thanks!" YW(15)SN: "Questions \#108-113 [peer risk] were hard to answer because my friends are not sexually active."

YW(13)SA: "I feel very sure of all of all my answers."

YM(17)SN: "Question \#138 [sexual orientation] confused me, when I read it I thought of it as if I was going to have sex what type of partner would I prefer."

YW(13)SN: "This survey was very personal but I did not mind. I'm a healthy person and not planning to have sex until I'm married. I have not drank or taken drugs and not planning to. This was very helpful to my thoughts of the future. Don't worry you will see me in the three classes [focus groups]. I'm looking forward to them. Thank you for bringing up this test, it will change many people's lives and how they think on the HIV/AIDS subject."

YW(15)SA: "I'm very glad I could help with the survey!"

YW(15)SA: "I've been with the same person for 2 years. He was a virgin before me. He and I enjoy sex together and do it frequently even though I'm 9 1/2 months pregnant. ${ }^{3}$ I've been very careful about who I sleep with in my life and I get tested about every 6 months."

YW(16)SN: "Questions about my friends were difficult to answer, because I don't have sex and my friends don't either."

YW(16)SN: "It was a good survey, but I don't have anything to do with sex, and I don't want to."

YW(13)SN: "I think this test was very helpful. It let me learn that I will have safe sex. When I am married is probably the first time I will want to have sex. This test was very personal but it taught me many things as I filled it out. I have never had a drink and I have never done drugs and I hope to keep it that way! Thank you for your enrichment on this subject. Thanks!"

YW(13)SN: "Are they going to find a cure for AIDS?"

YM(13)SA: "I don't know what oral sex is. I have had sex but it is too hard for me to talk about so some of the questions I just put none."

YM(17)SA: "I think the questions were very right to the point and it's [sic] makes me wonder how kids or teenagers can even think about using and doing drugs without having a few screws loose upstairs. It is very sad to know that our teenagers are so bad and make so many stupid decisions for themselves that we have to have a survey like this to find out how bad this young world really is. I've learned over the last 5 months that know [sic] matter how much you think you know someone or love someone you can never be to [sic] sure about everything they tell you no matter about sex or just themselves. Thank you!"

3 Visually apparent. 
YW(15)SN: "It wasn't as bad as I thought the survey would be. I have had boyfriends but I told them I am thinking of waiting to [sic] I get married."

YW(13)SN: "Do they have a cure for the AIDS virus or HIV and when will they find it?"

YW(16)SA: "Very good survey, and I hope you get all the information you need."

YW(13)SN: "HIV is an easy thing to get. I never want to have sex. This survey has taught me a lot of things that I didn't know."

YM(13)SA: "This survey was fine, but a lot of it was about sex like it was a sex survey, I know it was also about AIDS."

YW(14)SA: "The survey was very interesting and helpful."

YW(15)SA: "The definition of 'sex partner' was strange to me. I thought a sex partner is someone who you've had sex with."

YW(16)SA: "Sometimes as strong as a person may be, sex gets tricky with so many feelings involved. You want to please the person your [sic] with but also do what's right for you. You should also ask questions \#152-154 [substance use with sexual behavior] in the way of -- was your partner under the influence?"

YW(13)SN: "I think this survey was a great idea. Now others can really know how much they know about AIDS."

\section{Focus Group Topic One: AIDS Research}

Survey Discussion. There were no indications of comprehension difficulties with the survey, many stated the survey was understandable, "okay," "fine" or "easy." Additionally, nearly every group participant mentioned that the anonymity of the questionnaire had eased uncomfortable feelings or awkwardness, and ensured honesty of responses:

YW(13)4: "Anonymous was a good way because people would probably just put what their friends would put or they would just like lie about their answers if it wasn't anonymous."

YM(14): “... it wouldn't be good to have people put their names on it... they might be afraid like someone might like make fun of them." YW(13): “... they might feel uncomfortable, but they don't have to put their names on it, so it's ok." YW(14): "... most people would be honest if it was anonymous." YW(14): "I knew it was confidential, like no one would know my answers, so that was good."

${ }^{4}$ Information regarding gender and age were noted during discussion groups on feedback sheets. 
YW(16): “... I think it's kind of necessary to ask those kinds of questions."

YW(13): "I think anonymous was good, like it was really helpful."

YW(13): “... it's good not to put your name on it... and it's good to tell

why you need to find out about it, that it's important."

YW(15): "I think you would have got a lot of different answers if it wasn't anonymous..."

Suggestions for survey revisions were few. Eight students stated that they did not like answering questions about friends' behaviors because they "didn't know," and one 13-yearold young woman disliked homophobia questions. One 14-year-old young woman suggested that survey-takers list all the facts they know about HIV/AIDS. Another 16year-old young woman stated that questions regarding substance use prior to sex were relevant, “ ... but more should have been asked if their partner was under the influence... that could help determine what the answer would be like during the whole situation..."

Although the project was promoted as a study on adolescent health, many students were aware the topic was relevant to AIDS from information provided from teachers. It should be noted that originally, the global "health" reference was intended to increase participation by reducing uneasiness (e.g., Gwadz \& Rotheram-Borus, 1992), along these lines, and approximately 10 percent of focus group participants believed others may have participated but felt it was irrelevant to them. Nevertheless, several students reported personal benefits from completing the survey:

YW(13): "It's hard to answer some questions when you didn't do anything, but I like the fact, that well, you know, you think of what you might do in that situation..."

YW(14): “... it was good, because it made you think."

YW(16): "The survey was good like because you have so many classes that you start to get like a little burnt out, and a lot of people just start to like not paying attention because it's just the same stuff over and over again, and you're just not as interested as if it were like when you're all by yourself and you have the survey in front of you and you have to answer 
questions, and it kind of makes you think more than sitting and spacing out in a class."

YW(13): "I think it's good to have kids predict behavior, because then you can think about it."

YW(13): “... I thought that the questions about education were good, like 'could you answer questions about AIDS,' or like teach someone about AIDS and give them the right answer, and stuff like that were really good because it made you think about what I knew, and it made me think a lot about what I would do... because there were a lot of questions like testing your knowledge and there were some things that I thought I knew, but I didn't."

YM(13): "I think you should give it to parents."

YW(13): "I did a report on AIDS and I think it helped but not as much as I thought, I mean I didn't know that much about it even after the report, but I think I learned a lot when I took the survey."

Seventy-one percent of the group participants agreed that surveys can help researchers learn more about AIDS, while the remaining individuals did not comment one way or another.

Most students responded that surveys "were a good idea," and some added that "researchers can find out what you have to teach them by their answers," "it helps them have teenagers' opinions," and that can be beneficial in predicting risk, and prevention and intervention for teens.

Suggestions for AIDS Prevention Strategies. Ample suggestions were provided when participants were asked for suggestions for AIDS prevention strategies for teenagers. The most popular response (discussed by 66 percent of the students) was "teaching" or "providing information," coupled with comments about health education at younger ages, and the relevance of having same gender teachers.

YW(13): “... show them how and talk to them about having AIDS, tell them about the different ways they can protect themselves..."

YM(14): "... teach them the correct ways to try harder not to get it..." YM(14): "Yeah, like tell them the truth, like I hear a lot about like you can get it from kissing, or like it's just a gay disease..."

YW(13): "I'd go classroom to classroom and tell them about it..."

YW(15): "Teach them what to do not to get it." 
YW(14): "But no one listens to teachers, they're [students] just bored, so they don't listen, and they do things anyway... but to add humor is a good way to get people to listen."

YW(15)YM(13): "I just think they should have an optional class so like someone could go if they want, and didn't have to... yeah, if you want to learn about sex."

YW(14)YW(15): "You could talk to people."

YW(13): "Yeah, just ask them what they do know first."

YM(14): ".. have discussion groups... yeah, use the 'I know someone who...' idea."

YW(13)YW(14)YW(15): “... could have groups like this for people who want to know more about it."

YW(16): "I don't think educating people is good anymore, because I know, like even though I know a lot of stuff about it, but I don't know if people would actually do the right thing, I mean when it comes right down to it, it's the person's own decision."

YW(13): “... when people just don't know about some things, and they should somehow have classes that transfer over for adults in schools and for people who have kids with HIV or AIDS."

YM(14): "Yeah, I think parents are telling the wrong things sometimes, just to scare us."

YW(13): "... yeah, parents should be taught too, like if they had more knowledge, then they probably like would not worry, and like I think we should try to get things to tell parents about it, and have them go to maybe like a class, then they wouldn't be so afraid to have like the kids know about the same things."

Discussions about health class with different gender teachers and the importance of

knowledge at an early age occurred in several groups, typical of the following excerpts.

Several participants indicated that learning about safe behaviors does not promote sexual experimentation.

YM(13): "Right now the seventh grade, I know even the sixth graders, a lot of people didn't like get all the information on AIDS, and yet like we're still getting a chance to fill out the survey and these discussion groups, like they're real good, but I think a lot of them are still being ignored because... AIDS was barely touched upon and we're not getting a lot of information. But different people mature at different rates and they're willing to try new things and they've had sex unprotected or something, or drugs... a lot of people are trying drugs out of curiosity..."

YM(14): "Yeah, it's not even taught to younger kids, we learned about biology and stuff about our bodies, but nothing about AIDS until health 
class in like high school. We should have talked about it a lot earlier, like before junior high but a lot of people think that it's too early. They should teach stuff like that way before high school."

YW(16): "I think that even if people aren't sexually active, they should still learn about it, because a lot of people just don't know if they are going to be sexually active, they can't say 'well I'm 16' or whatever, and they might get pressured into it, and they really don't realize it but maybe they will become sexually active, or maybe they really don't want to be but it just happens..."

Group Leader: "Do you think teachers talking about sexual issues might give the students the impression that it's okay to go out and do it?" YW(16): "I think that they have the choice anyway, and it just helps them to know how to like protect themselves if they choose to."

YW(13): "No, it doesn't influence them, it just gives them knowledge about AIDS."

YM(13): "It's much better than learning stupid stuff, or like things that you like find out later are wrong, and then you just don't believe them [parents] anymore."

YW(13) "... the teachers should just ask the kids if they know about it, because I mean kids don't mind talking about it but I mean like having boys and girls together for some things are not good..., they laughed and it felt weird."

YM(13) "Yeah, we just got out of health and we had to learn the reproductive system and all that, and we had a lady teacher, and the class was all boys, so like she was always running around because the boys were laughing. Well probably half of them felt uncomfortable having a lady teacher, when the only time we basically ever really learned about the stuff was when we had another man..."

YM(13): "There's like a certain sense that like if certain information is given to people like sometimes that if you give too much information and people think they like know it all, that they're like going to go out and probably like try sex and whatever and like they know it and they're not going to get AIDS and everything, but then if you don't tell them anything, like they could be really in worse trouble because they probably will go out and try it anyway and like won't know what to do, like you know, protection and stuff."

YW(13): "Yeah, it's worse not to tell kids."

YW(13): "Yeah much worse not to tell them."

The second most popular suggestion for AIDS prevention for teens, discussed by 31 percent of the group participants, was having a "real" person with AIDS talk to them.

Interestingly, many youth placed much emphasis on the term "real" in their descriptors. 
YW(14): "I think like bringing in real life people so that people like can see a real person with AIDS might change their minds about stuff like sex, and make them think a lot more. I was at another school once, and they brought in people, and before they came in all of us had no idea about it, and we all thought it was mostly a gay thing."

YW(14)YM(13): “... maybe get a real person with AIDS tell them..." YW(13): "... you could have person with AIDS come and talk to a class, but they might be afraid... they say they wouldn't be afraid..., but they back away."

YW(14): “... learn about it from their friends, peer teachers, or a peer with AIDS, ...someone our own age would be good... a credible person, like Magic Johnson, ... everyone would listen."

YW(13): " $\ldots$ have a person with AIDS talk to them, a real person, so like they could know what it was really like, like maybe Magic Johnson or someone like that."

Group Leader: "Would it have to be a popular person? What about a stranger?"

YW(13): "A stranger would be good... but a teenager would be better, because like everyone would just say, 'oh they're so young, they have so much more life left, how could that happen to them?' ...because a lot of teenagers think, 'oh, I'm young it would never happen to me." YW(14): " $\ldots$ if they weren't the same age as us then I mean, they would have to be the same age."

YM(14): “... show them a teenager with AIDS, a real teenager, otherwise they just don't care."

YW(15): "... seeing someone with it, would be scary."

YW(15): “... maybe I'd feel differently if I knew someone, or I met someone with AIDS."

YW(15): " $\ldots$ it would hit me certainly if I thought I could be that way, like it could be me..."

YW(13): "... like if they had a classmate that has AIDS, bring them in to talk, so they could like see this person, and say 'oh wow,' like kind of the Ryan White story."

YW(15): "... maybe take them to a hospital and show them a person with AIDS."

YW(14): “... I mean suddenly your whole viewpoint on that would change, people would start thinking, well what did this person do to get AIDS? And well, am I doing the same thing?"

YW(14): “... you're back to that old scenario that you won't get AIDS unless you are gay, so if you brought someone that was heterosexual, I mean you have to teach people that you can get it if you are heterosexual... I mean then they would say, 'Wow, I could really get it.' They'd say, 'yeah, it could happen to me, I think I should slow down." YW(15): "Well the first time [I met a person with AIDS] it was kind of weird, cause when I found out he had AIDS I just stared at him, and 
couldn't believe it because he looked just like everybody else, he didn't look sick at all. Once you realize, like you know, that you could be with someone with it who looks totally normal, you realize that you could get it."

Approximately one-quarter of the group participants mentioned the distribution of condoms

as a suggested method of AIDS prevention for teens.

YW(13): "Buy a lot of condoms and make a lot of brochures and give them to kids and schools."

YM(13): “... run condom commercials... and they should open a condom store 24-hours a day."

YW(13): "Probably, putting a [condom] vending machine in the bathroom, all the bathrooms."

YW(14): "If they were free or in a vending machine, it would be less embarrassing... more people would get them in a machine, or use them like if they didn't cost money."

YW(15): "I think they should hand out condoms in school or have dispensers in bathrooms because I know a lot of people that have had sex and they didn't use a condom, and they said they were embarrassed to go to the drugstore, and if there had been a dispenser in school, then, you know, then when no one was in the bathroom they could have just got one then."

YW(14)YW(15) “... high school definitely [for condom dispensers] and of the junior high people could just come over and get one anyway."

YW(14): "Teenagers just don't care... they wouldn't use a condom if a woman on was birth control... why should they if she's taking the pill?... or maybe she can't, maybe her boyfriend won't agree to it [using a condom], maybe she thinks it could never happen to her, but mostly because nobody ever really thinks about it."

YW(14): "She might not want to [use a condom] because probably because the guy she is with, she like probably thinks oh well, he really looks healthy, he probably doesn't have AIDS... Yeah, I was sitting with a guy once and I found out that he has AIDS, and I was like, no way, he looks so normal, I couldn't believe it!"

Socialization issues arose on a few occasions regarding gender responsibility with condom use, typical of the dialogue below:

YM(13): “... teach people about AIDS... and give them condoms so that they have them... give them free condoms at school... or have condom machines in bathrooms."

Group leader: "In the ladies' room and men's room?" 
YM(13): "Well, they [girls] don't need them."

YM(13): "It's kind of the guy's responsibility."

YM(13): "Why would girls carry one around? They shouldn't have to."

YW(15): "No, they should have one, just in case the guy doesn't bring one or something, or he doesn't want to go get one, there won't be any excuse then not to use one."

Approximately 13 percent suggested prevention methods utilizing the media with

pamphlets, newspaper, billboards, television, books, radio and movies.

YM(13): "... give them pamphlets... you could put a big ad in the newspaper that says, 'have safe sex' or billboards... but some people won't do the right thing even if they know the right thing... you can't really help them if they don't care."

YW(14): "I have Magic Johnson's book, I think that's a good way..." YW(13): "Maybe put some real life stuff in a real story on TV or movies that like would make it really interesting... it would be more believable." YW(13): "Yeah, they just sort of slip AIDS into the movies, but there's never really a movie about AIDS, it's like slipped in later on because people don't like to hear it. That's why I really like the Ryan White story better, it was more real, and less boring... the kids really want it, because it's less boring when it's real."

YW(13)YW(14)YW(15): “... could have TV and radio shows..." YW(13)YW(14): "Do a promotion or something on TV... on MTV, like a teenager with AIDS..."

Ten percent of the respondents discussed other prevention suggestions such as finding a cure, giving counseling, mandatory testing, giving people more self-esteem "to know what to do," and one 14-year-old young man thought that giving young men a vasectomy would reduce the spread of AIDS.

\section{Focus Group Topic Two: The Impact of the AIDS Pandemic}

Age Group. Group participants were asked which age group they felt AIDS was most a problem for, and why. Ninety-one percent stated "teenagers," and the remainder replied "little kids," "college kids," "people in their 20s" and "possibly 30s." Excerpts are listed below: 
YM(13): "College kids... maybe high school, maybe teenagers... because they use drugs, they have gangs... they're like smoking and having sex." YM(14): "Little kids and then teenagers... like little kids cut themselves up like doing blood brothers and stuff... teenagers are a big problem because like they are the ones that are having babies, so it's a big problem for them."

YW(13): “... because a lot of teenagers are sexually active at earlier ages and most people don't check to see if their partner has AIDS or a sexually transmitted disease."

YW(13): “... because a lot of teenagers are having sex and all that, and the statistics show that more and more are getting sick and killed by it."

YW(13): " ... more peer pressure to have sex... if you want your friends to think you're cool, like it's cool to have sex."

YW(14): "Probably because of all the sex, there's a lot of it, I guess it's supposed to be that way if you want to be popular."

YW(15): “... because they're fooling around or whatever more than those who are married."

YW(15): "They're fooling around more."

YM(13): “. . because they're the party animals."

YW(13): " ... too much drinking and stuff."

YW(15): “... they're experimenting."

YW(13): "... they're sexually active and drinking."

YW(15): "... too much sex, and they're more active."

YW(14): "... too much unprotected sex."

YW(14): "They're curious... experiment too much"

YW(14): "I think in general they don't realize, well they don't think it could happen to them, they think it would never happen to them."

YW(14): " ... sex is supposed to be good but it's good if women have assertiveness to use a condom."

YW(13): “... probably the older kids... well because a lot of 15 -year-olds, or a lot of teenagers want to be cool and everything, and think it's cool to have sex, they are more popular because they brag a lot, and they are usually the leaders, so they're cool... there's a lot of pressure to like, you know, [to have sex] on younger kids."

YW(13): "Well, I know a lot of people, like our age [13] that already had sex..."

YW(13): “... there are things like to do to protect yourself like with a condom, but like a condom is not like an everyday thing, so they're [teenagers] not thinking about it, like they're not thinking well if I'm doing it I could be taking my own life in my hands and like risking it, but you're not thinking about that. But though, I know people like when they were going to have sex they're not thinking about using condoms, or about AIDS, they're not thinking about it." 
Location. Teens were asked if they thought AIDS was a problem in Connecticut.

Slightly less than half stated "yes," or "it is everywhere," yet misperceptions were evident:

YW(13): "No it's not really a problem in Connecticut..."

YW(13): "It's like a problem, over there."

YM(13): “...there's no gay people in Connecticut!"

YM(13): "My mother says we don't have to worry here [in Connecticut]."

YW(13): "No, I mean right now I think certain places it's a real problem in like California and New York, like in California, I'm sure there are people there that have AIDS and New York probably has some people with AIDS."

YM(13): “... well, if you live in the country, you probably won't get it... [because] in the country like there are no gangs, and no drug dealers... and I haven't heard a lot about it in Connecticut."

YW(13): "No... maybe."

YW(13): "I don't know anybody with it and I think a lot of people don't know anybody with it here."

YW(14): "I think it's becoming a problem in Connecticut, but it isn't right now."

One dialogue revealed fear of discussion regarding AIDS and that a few students thought

there might be some safe location in the world:

YW(15): “... stuff like that scares people and this is a small community, and you don't want stuff like that to get around, because then everyone might get real scared."

YW(15): "There's no way anyone would say anything."

YM(14): "Yeah, my parents say real stupid things, and don't want to talk about it."

YM(14): "Yeah, they would just try to ignore it."

YM(13): "Yeah but, what about Alaska, is it a problem there?"

YW(15): "Yeah, I'm going there."

YW(15): "Well, yeah, is it like a problem everywhere? How about like-Africa?"

YW(15): "Africa is where it all started!"

YW(15): "Of course it's in Africa, more than everywhere else!"

YM(13): "How about Brazil? Or like Siberia?"

Populations Afflicted. In the first focus group, the issue of homosexuality arose, and five of the nine participants indicated that they didn't know that heterosexuals could be 
infected. About 75 percent of all participants verbally indicated that AIDS was "a problem for people in general," that it was not a "gay disease," yet some said, "... most people today think it's a gay disease," of the remaining 25 percent, some nodded in agreement, others appeared to be unsure. In one dialogue, two young women discussed a recent television program and the Bible:

YW(13): "I heard a show on TV the other day and how um.., how only gay people get AIDS, it was like a talk show or something, and that's what they were telling people that only gay people can get AIDS, and like there was this guy and he didn't like gay people so when people started getting AIDS, he was saying that like they were all gay people and everything." Group leader: "Which show was that?" YW(13): "Rikki Lake... I don't think that that is true, and I don't think that they should have a show like that with that title because if you were just flipping the channel and go by it, you might get the wrong idea or whatever, listening to him talk about it. You can get AIDS and not be gay, because anyone can get it and anyone can give it."

YW(15): "I don't think that it's right like because I don't understand how they can say that on that show because like it's probably not true and probably a lot of people will hear it."

YW(13): "I'm saying like God said it shouldn't be so, because in the Bible it says that people, that people shouldn't be gay and everything, that they shouldn't like and something about um..., only gay people have bad things happen to them, it says that in the Bible...that being gay is bad and they're the cause of all bad."

Homophobia. Degrees of homophobia were assessed by inquiring of participants their perceptions of homosexuals, how, if at all they were "different," stereotypical identifiers and predicted behavior in various scenarios. The majority indicated that gay people are not different, however some stated otherwise reflecting attitudes of themselves or others:

YW(13): "Maybe the way they act or look."

YW(13): “... maybe like they won't wanna be like friends with the opposite sex because I don't know, because they're gay."

YW(13): "It feels different [to be around gay people]." 
YW(16): "They're not, but other people might think they are... some people think that something is wrong with them."

YM(15): "Some people think that they [homosexuals] all have AIDS, and stuff like that."

YM(14): "They're not [different] ...it doesn't really effect their life unless people choose to make fun of them..." [the same student who later stated that it would make him nauseous in given scenarios.]

A 13-year-old young woman expressed belief that homosexuality could be a psychological, or a difference "mentally," but could not elaborate beyond reference to a television show:

YW(13): "It's not really physical or anything, but it could be mentally, a mental problem sometimes... like they act differently some of them." Group leader: "It could be a mental difference?"

YW(13): "Yeah, like---, like guys like there's a show called, 'My Socalled Life,' and there's this guy in it, and he talks like a girl and he acts like a girl and stuff, and like, like that's because like he's gay, and so sometimes it's like hard to tell but they're a little different but otherwise they're not different from everybody else."

Perceptions of stereotypic behavior were assessed by inquiring whether people could tell if someone was a homosexual without them stating so. Generally, most youth stated that they usually could not identify a gay person, although some revealed stereotypic identifiers:

YW(13): "I wouldn't be able to tell but if they were trying to like make a point of it, and like acting like they were gay, or something, I would get a little bit suspicious if they were... like if they were hanging around, like say it was a girl, like hanging around girls and really acting weird..."

YM(14): "Well like sometimes, it's like stereotypical but like on TV and stuff, like sometimes like, they have like they have all lesbians that are all manly and stuff."

YW(15): "Like sometimes you can tell, you know, I mean you can't be positive but like sometimes like the way they act you would know... just the way they talk."

YM(15): "The girls are more manly."

YW(15): "The way they act around people of their own sex."

YM(14): "Well, you can't sometimes, it depends how physical they are with it, you know, and how they express theirselves in the real world outside of the home, you know... they would be out, you know, kissing and hugging with their lover and everything else, out of the home, holding hands..." 
YW(13): “... the way they walk, they swing their hips back and forth and everything."

YW(13): "Maybe, sometimes, guys are very feminine and girls are very masculine, but it's like not, well anyone can be like more feminine or more masculine."

YW(13): "Sometimes, like sometimes like they way they walk."

YW(13): "Like if they are a man, yeah I mean the way they put their hands and everything, and if you see two of them together, I mean, you know they are."

YW(13): "Like they had a gay man come in, and I guess it was like last year and he talked to us, like the class, and one kid asked like 'how come every time you move your hand, your like wrist flips?' and he said that it wasn't true, but like every time he like moved his hand his wrist always flipped, so you could know if their wrist always flipped... I can always tell if a man is gay."

Teens were asked to respond to what, if anything, would be different if someone in their school was gay, and how might they feel. Changes in perceptions, attitude and affective response were measured on a continuum of "closeness," with scenarios ranging from a student to a parent. Despite the fact that most youth stated that homosexuals were not different from heterosexuals (other than choice in partners), perceptions, attitude and affect changed when global inquiries were rephrased in individual situational contexts. For instance, 10 older teens said nothing would change, and remained consistent throughout all scenarios, and may have influenced younger students' responses. Yet, consistent with quantitative results provided to the school with age comparisons, many groups consisting of primarily younger teens revealed a higher sense of uncomfortableness as the scenario presented individuals that were closer to them. Interestingly, the descriptor "weird," was often used, and further probings for a concrete description yielded, "I can't explain it," or "I don't know." Perceptions that homosexuals could not control their behavior, and that heterosexuals were in danger of being molested or raped by a homosexual were evident: 
YW(13): “... I'd try to stay away from them because I think I'd feel weird about people like that because I do know of like two people in our grade that are and um..., like I never go near them anyways but I know that's probably wrong."

YW(13): "Well, I kind of think it's, well like it depends, like I wouldn't feel weird if it was like an adult then I mean like I would be too young anyway."

YW(13): "I would feel a little bit weird about it but I wouldn't ignore them completely or I wouldn't like be really quiet to them... I wouldn't be ignorant."

YW(13): "I wouldn't mind them, I wouldn't like try to stay away from them, and I wouldn't like make a point of like walking with them, but if they were like just like showing it like around like telling everybody, not like just saying they're gay but like keep on saying it like making it more than what it should be then I'd get annoyed."

YM(13): "Well, if it was in my grade it would be weird because everyone would look and see if it was them, and wondering if it was them or them, or who it was."

YW(15): "No, as long as they didn't make a pass at me."

YM(13): "Well, like if I thought that S--- was gay, well, but like, I just like might stay away... well I'd still be friends with him but..., because like it scares me to like, you know, because like, I wouldn't sleep over his house or anything because like he might rape me."

YW(15): "Well, it's most likely that an [a homosexual] adult would make a pass than a kid..."

YM(14): "I would feel a little bit uncomfortable... because you don't know what they're going to do... it'll be difficult to not have them look at you like, you know, 'oh I think you are real cute,' and all that... I wouldn't want them to like be hitting on me."

YW(13): "I have an aunt who... is like that... and you kind of feel uncomfortable because she gives really big, sloppy kisses..."

YM(14): “ $\ldots$ and I'm sure they might be thinking, 'I don't want my baby being around you because you're gay.'... like they might give them a bad influence or something."

YW(13): "I would feel different. I never confronted a gay person before so... I would not ignore them or be like, I don't know, rude, I wouldn't like be rude to them, but I would like just feel different... it's hard to explain... it's just like it would be different if I knew them all of my life and then all of a sudden I found out that they were gay. I don't know. They would stand out in a crowd and nobody else would like them so how would I treat them if nobody else liked them?" 
YW(15): "Well I seem to feel a lot different because I don't know how they might have been judging me the whole time and I would be like scared, you know like judging me like if they liked me..."

YW(13): "I would try not to be like grossed out, but then I wouldn't treat them mean or anything, I would still be nice to them, but it would be like, you know, different."

YW(13): "Probably, I mean, you might feel different but if they were like a friend, you would try to keep them friends unless they like did something really weird or whatever."

YW(15): "It kinda freaks me out."

YM(13): "If I didn't know the person I wouldn't be too worried but I mean, if... maybe if the person was a little closer than I thought... if it were a best friend, it would be different... like the way you act around them... maybe what you say."

\section{Scenario Two: A Best Friend}

YW(16): "Their sex preference doesn't change the person."

YM(13): "I can't explain it... well, he would be a friend, I would still care about them... I'd try."

YW(15): "As long as you made it clear to them that you weren't gay."

YW(13): "Well it kind of depends on whether it was a boy or a girl... I

mean you could be friends with them but you would have to watch out and make sure they didn't make a move on you."

YW(13): "Everyone would make fun of them."

YW(13): "It would be kind of confusing."

YW(13): "Yeah, if it was a girl, you wouldn't know if they were going to come on to you ..."

YW(13): "Well people would think that if I kept hanging around her that I might be gay, so I wouldn't like... I would just like tell her and like still be her friend but 'it's the community that doesn't like you and not me' and everything."

YW(13): "Well if the kid's parents were gay, I wouldn't feel different about the person because the kid wouldn't be gay just because the parents might be, so it wouldn't change anything under that."

YW(13): "I wouldn't go over their house! ...because you would never know what they were going to do to you!"

YW(15): "They just have a different sexual preference, it doesn't mean they're going to molest you..."

YW(13): “... you don't know how gay they are, I mean some people are like obsessed because they're gay and so they should be like separate... yeah, like they're obsessed with like their own kind." 
As situational contexts changed, youth confirmed that with homosexuality, a higher degree

of uncomfortableness was associated with a same-gender relative in family scenarios.

\section{Scenario Three: A Sibling}

YW(13): “... like if they liked you, or if they liked one of your friends, it would like feel really weird."

YW(13): "It like wouldn't really matter, because you know there's like no way they'd like you or something, or do anything that would make you uncomfortable for you..."

YW(13): "I don't think that it should matter, but it would feel kind of like a little weird, like if you were there and like I would feel a little bit uncomfortable..."

YM(13): "Well if my grandparents found out, it'd be like on that show, on 'Wings' there's like, Roy..., well Roy has a son and the son is just as big as him and he was gay and he told Roy and they kept fighting and fighting--he was yelling."

YM(13): "I don't think my sister would make a pass at me anyway... but I wouldn't allow them in my room... they might make a pass."

YW(15): “... they're probably not interested in you anyway, and so you should treat them..., well they're still part of the family."

YM(14): “... your family isn't gonna hit on you. Nothing [would be different] because you wouldn't have to worry about anything because they're family..."

YW(13): "I would feel really strange... because just seeing them together gives me the chills, just seeing like two men or two women together... I don't know, it doesn't really bother me, it's just so different, I don't know, like I can't imagine my brother or anything, it would just be weird." YW(13): "I would feel like really strange like if it were my sister, but if it were my brother, I wouldn't feel like that bad because, I mean, he wouldn't feel anything about me, well it would bother me a little if it were my brother, but like it would just kind of gross me out and everything, but if it was a sister and I mean it's like we're both girls and it would just feel uncomfortable."

$\mathrm{YM}(13)$ : 'I'd let my parents know what's happening, but it's her choice not mine."

\section{Scenario Four: A Parent}

YW(13): "It would be different because like, I mean it would be weird just seeing them, I don't know, it would be just weird, I don't know, like if you were going to live with them."

YW(15): “... I would just feel like I would want to be by myself because I would feel like my parents betrayed me." 
YW(13): "I'd be embarrassed at first... I would like probably feel real uncomfortable because I don't know any gay people but later on I would probably get used to it and I would probably not mind..."

YM(13): "I'd totally like, would do really bad in school so I would have to like go to a different school, and then I'd do bad there so then I would have to go to like a foster home... I wouldn't want to see my parents."

YW(13): "I would have to think about that one... because I've been with my mom a long time."

YW(15): “... I would feel very uncomfortable... if it was my dad, I

wouldn't mind because he's gay... but if it were my mom, I would just feel very uncomfortable about it."

YM(13): "I'd go with the straight one... you think that the gay one would marry and you'd have two dads or two moms or whatever, that would be scary..."

YM(14): "I would be uncomfortable with it... I would ask my mom not to talk about it or ask my dad not to talk about it in front of me.. it would make me sick... because! you teach your children, I don't even want to know because it makes me nauseous just to think of it, you know. It's all right and everything, just keep me out of it, you know."

YW(13): “... my friends wouldn't want to come over or anything anymore... if it's like your parents then it's different, but if it's like somebody else's parents it would be like a lot different. But then if it happens to me but then, like it's never going to happen to me, but then like if it does, it's like kind of, it's like really weird..."

YW(13): "Very uncomfortable."

YW(13) "I'd go with the straight one."

YM(14) "I would too!"

YW(15): "I'd have to be in that situation to know."

YW(13): "I would feel really uncomfortable."

YW(13): "I would be very... I would be very upset, I don't know. I would change completely if it were one of them... I probably wouldn't accept, like if it..., like the girlfriend or boyfriend, because I would... like... feel very uncomfortable."

YW(13): “... I know I would like go with the other parent automatically.

But like if the court would allow me to, I would just go with another relative because I would just not be able to see my parent like... being gay."

\section{Focus Group Topic Three: Societal Dilemmas}

Insurance Companies. In order to promote awareness of how AIDS can affect all individuals directly or indirectly three social issues were raised for discussion or debate. 
The first societal dilemma posed to the students was the cost of insuring infected individuals. The dilemma was introduced to students explaining that since medications permit longer life expectancy for HIV and AIDS infected individuals, insurance companies may attempt to drop infected persons, or raise premiums for policyholders to cover costs. Nearly all replied that it was "unfair" or that insurance companies "shouldn't have the right" to drop infected people. However, when the roles were reversed and students were asked to imagine themselves as a policyholder paying higher premiums or as a president of a company about to go bankrupt, several suggestions and debates ensued.

YW(13): “...I think it's mean to drop a person with AIDS, I mean it wasn't their fault that they got AIDS, they didn't plan to get AIDS on purpose, and they need the benefits the most... I would think that those insurance companies should just go bankrupt because at least they could say, 'at least we tried, we didn't just drop them."'

YW(13): "I think maybe we should have two different kinds of insurance companies, like a low cost for people with AIDS, and a high cost for like rich people that can afford like high prices, and just have something for other people that can't afford like high prices."

YM(13): “... it's not fair... probably have to make people pay more [premiums], or maybe just raise it for the rich and lower for poor." YW(13): "They should be treated just like everybody else, no matter what it costs."

YM(14): “... it's not fair... I guess you could raise the rates..."

YM(14): “... if they got a job they should be able to deduct it from their paycheck or something, and if they don't have a job, maybe the state could help out."

YW(14): "If you're dying anyway, it shouldn't really matter. Like they couldn't really just throw you out in the street if you're dying or anything like that. I mean you could go to a hospital or something, so they shouldn't have to worry about it."

YW(14): “... they should still help them, it doesn't matter about the money."

YW(13): “... if I had the money, I'd just pay it, but if I didn't have the money, I guess I'd be mad."

YW(15): "... maybe they [insurance companies] should limit the time that they help them or something, like start out with full coverage, and then start dropping off, like a limited time of coverage... like a partial thing..., or like I'll pay half you pay the other half." 
YW(15): "What about all that money in research, why can't we take some of that money and put it into funding for people with AIDS... you can't spend all the money on trying to find a cure."

$\mathrm{YM}(14)$ : “... just recommend other insurance companies or find somewhere else for them to get help."

YM(14): "Maybe they could get two insurance companies, and both could like split it."

Segregation. The second dilemma presented to students was the issue of segregating people with AIDS to reduce the spread of infection as attempted in other countries. Teens were asked to provide good and bad points to that solution. Most professed segregation as "unfair" or a "bad idea," and stated that a good point was reduction in the spread of AIDS. However, some students added to that or stated otherwise:

YW(13): "I don't think it's right because we know how to not get it so it shouldn't be a problem to us."

YW(13): "I think that someone like you maybe should go down there and like teach them because they might not have the education that we have up here."

YM(13): "I think it's a good idea!"

YM(14): "That's what my parents think, put 'em on an island,' put gay people far away."

YM(13): “... families get separated... and some may not have it and might be put there by mistake or on purpose... and a good point might be that it won't spread... well, it might keep them from getting blood transfusions from people that have it... and it will, I think, it will like help people with AIDS because it will like protect them from other people from killing them... but it would be different because you know the person." YM(13): “... people that got it, it's their fault... except for like people who got it from like blood transfusions... it's their own fault! Because they didn't protect themselves, so yeah, it's their fault... so they should be isolated... because if they got AIDS they could pass it..."

YM(13): “... I wouldn't want to watch them suffer anyway, so I would just say 'goodbye' now."

YW(13): "I think they should definitely be separated."

YM(14): "They should be in there!"

YM(14): "The disease could spread faster because they were with each other..., it could progress a lot faster in their bodies, you know." 
YW(13): "They couldn't spread it, they couldn't give it to somebody else... but you would be held up in one area for a long time and you don't have a life anymore..."

YW(13): "It's not a good idea... maybe one good point might be that they can be tested and stuff without having any problems because they're all like being like--, treated altogether..."

YW(13): "Yeah, if they're not friends with people within that area then they don't have anybody to talk to about their feelings and everything. And people outside there's problems for them because they don't know how it feels..."

YW(16): "I think no matter who it was... isolating him or her from everybody else ...that's wrong."

YW(16): "We can take care of their problems without hiding them, we start stashing them away it's not going to solve anything... we're just scared of it, so we're just going to like push them in a corner and not worry about it."

YW(15): " $\ldots$ at the same time all these people that are scared of it are going out and having sex with a lot of people you know, wait till it comes their way... they probably think that they won't get it."

YW(16): "Nobody thinks that anything is going to happen to them."

YW(15): " ... could stop the spread of AIDS, but it would be hard to get everybody..."

YW(13): “... it's not fair... it's just like having a cold... but it's worse... it could kill you."

YM(13): "It would be like concentration camps like we think because they're different they're being isolated, well maybe that might help the spread of it but they're not getting all their rights."

YW(13): “... it's totally unfair to like lock them up, and they can't even see their families before they die, but I'm glad that the rates are going down, but people have a choice to check to see if they have AIDS or not, so it's like their fault, it's not like the country's fault, they have a choice to check to see if they have AIDS."

YW(14): "... most people know how they got AIDS in the first place, but it's spreading anyway, so it might be a good idea to get away from people with it, but even though I don't like them it's not fair."

Questions were posed later to find out whether solution answers changed in hypothetical situations if friends or family members would have to be segregated due to infection.

Generally older students had consistent opinions, while younger students who were in favor of the idea with strangers, stated that they would "protest, " "kidnap," "fight for" or "hide" 
friends or family members. In some groups, teens debated "fault" in infection, insisting that people should know the status of a partner beforehand, and all should be honest with their HIV-status.

YW(14): "Well, it's partially their fault so maybe they should be in a separate town or place."

YW(14): "I don't think they should do that because it might not be their fault, like I think a partner with AIDS should tell the other person that they have it... they should be responsible about it, and tell a partner if they have it."

YW(15): “... and if he loves you, he should tell you."

Group leader: "Do you think that an infected person would be honest about their status?"

YW(14): "Probably not. But he should be."

YW(15): "No."

YW(15): "They'd just lie."

YW(14): "He'd definitely lie."

YW(15): "When it comes down to sex, they guy will just lie."

YW(15): “... but if they have it, they just don't care, and they're angry, so why should they tell anyone... there should be a rule or something." YW(15): “... they should have a piece of paper as evidence... or some little thing that you can carry around to find out right away whether someone has it or not..."

Confusion about HIV-testing procedures was revealed in several groups. For instance,

nearly all students suggested testing as an adequate safeguard; however, none of the students were aware that HIV had to be in the body for six months before appearing on a test result.

YW(13): "...they have a choice to check to see if they have AIDS." Group leader: "Okay that's brings up an interesting point. What if somebody is about to become intimate, and one asks, 'Do you have AIDS, do you have any sexually transmitted diseases' what do you think the other person is going to say?"

YW(13): "No."

YW(15): "Yeah right. Like he's going to say he's got something!"

YW(13): "No. If he says that he does then she won't have sex, so he'll just say, "no.""

YM(14): "Guys just want to have sex."

Group leader: "But that person checked, and asked..." 
YW(13): "Then she should just say we have to get checked out for every sexually transmitted disease before we can do anything."

YW(13): "Go in for a blood test or something."

[group discussion regarding six month latency for accurate blood test results]

YW(13): “ $\ldots$ if they were really in love then it wouldn't matter, they should wait then and check again in six months if that's what they have to do."

Pregnant Women. The final societal dilemma presented to students was that of pregnant women's rights versus rights of the unborn. It was explained to students that findings indicate that certain medication given to infected pregnant women greatly reduce the likelihood that the unborn child will be infected. Given this information, students were asked whether pregnant women should be forced to be tested and take medication.

Responses to this dilemma were most interesting. Several held firm convictions either way regarding forcing a pregnant woman to be tested and taking medication; however, inquiring whether society should be most concerned with rights of the woman or unborn child, many disregarded their previous stance and changed their response. Often inconsistencies were rationalized as presented in the following dialogue excerpts:

YW(15): "Yeah... she should be forced." [3 other young women agree]

YW(15): "She should be [forced]" [2 other young women agree]

YW(15): "No, she should be forced, and she just should."

Group leader: "What about rights? Should society be most concerned about the rights of the pregnant woman or the rights of the unborn baby?" YW(15): "The woman's." [unanimous]

YW(16): "Women's rights!"

YW(15): "Definitely."

YW(15): "Yeah, she should be forced..." [3 other young women agree] YM(13): "Yeah." [one other young man agrees]

[Later in same group after discussing positive and negative consequences of testing]

Group leader: "So does the woman have the right to refuse to be tested?" 
YW(15): "Yeah, it's her body, maybe she doesn't want to know if she has it, because then she doesn't have to worry."

YM(15): "I think it should be their own free will... it's the woman's choice."

YW(13): "Women have a right for their own bodies and stuff." YW(16): "I don't think so, I think that with all the AIDS in the world, it should be like just a normal think, if you get pregnant, you get tested." YW(15): "I think any mother who cares a little bit about their child would want to take the drug..."

YW(15): “...it's her decision."

YW(13): “...definitely her decision, yeah."

YW(15): "I think it should be required but if they can't afford it they don't have to pay for it."

Group leader: "What about rights? Should society be most concerned about the rights of the pregnant woman or the rights of the unborn baby?" YM(15): "The baby's" [unanimous]

Group leader: "So then should society force the mother to be tested and take the medication if they are protecting the unborn baby's rights?"

YM(15): "Oh, yeah, wait a minute."

YW(13): "No, it should be up to her."

YW(13): "No, she shouldn't be forced... I think that it should be their choice though, because I mean it's their baby that their raising."

YM(14): “... I think that every woman should be tested free..., if they wanna be tested..."

YW(13): "Well that also depends on them though, I mean if everyone goes around and says, 'It's not going to happen to me,' then she might think her baby won't have AIDS."

YM(14): "Yeah but what if it does happen to them..."

YW(13): "Well then it's not the doctor's fault then, it's hers for not getting tested."

YW(13): "I think they should be tested, but not forced."

YW(13): “... they shouldn't be forced but have a choice and everything... it's their choice."

Group leader: "What about rights? Should society be most concerned about the rights of the pregnant woman or the rights of the unborn baby?" YW(13): "The baby's." [unanimous]

Group leader: "Then should society make women get tested and take medication for the baby?"

YW(13): "I would." 
YW(13): "Uh..., well..."

YM(14): "No. Well, yes. I don't know."

Group leader: "So then would there be a choice for the woman?"

YW(13): [Shrugs shoulders]

YW(13): “... if she had it and was getting like really, really sick and she was pregnant, I think she should be forced to take the medicine for the baby, but if she wasn't really that sick and it was like the very beginning of the disease then I think she should have a choice."

Group leader: "What if she doesn't want to be tested, she doesn't want to know?"

YW(13): "I think that she should still be forced to do it because she needs to know what, if she does have AIDS, I think she needs to know if she should take the medicine or not for the baby."

YW(13): "No, it's their choice, if they want their kid to be born with AIDS or if they want their baby to have a chance..."

YM(13): "I don't think they have that choice really, because in a way, like they do have AIDS and they're not going to get the test and everything, that's just like taking another life..."

YW(13): "If you were tested and found out you had it, you might like turn real negative... I'm sure that everybody always thinks, 'Oh it would never happen to $\mathrm{me}^{\prime} . . . "$

YW(13): "Well it depends on how old the person is too that's carrying the baby, like if she is really young or something then it would probably be the doctor's choice, but if it's like an old woman then should could like have the test, but it could be like her choice."

YM(14): "There shouldn't be a choice, you should just have to take it. I mean you have a choice if you don't have the baby and don't get an AIDS test but if you're going to have the baby you should have to get the test automatically."

YW(13): “... you know how you have to go for a check-up and everything because you're pregnant, well I think that maybe the doctor, like instead of asking you, should just do it on everybody."

YM(14): "Well you could like take a vote, you know, and have the state do that and if everybody says like 'yeah' then she should like automatically be tested if she didn't already."

YW(13): 'Like if she doesn't want it or stuff like that, they should just do it like without telling her, like for the baby, and just tell her it's another test..."

Group leader: "Does that violate her rights?" 
YM(13): "Yeah... well wait, I think it would be wrong, but it would be better... it would be [wrong] because of their rights and the constitution." YW(13): "Well, not really, because if she's pregnant then that's the baby's rights."

YW(13): "She probably might not want to be tested because she thinks, 'oh that could never happen to me,' and she might just be scared."

\section{Focus Group Topic Four: Teenage Risk}

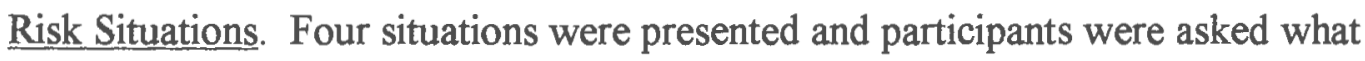
risks, if any, teens would be faced with in each predicament. Youth were urged to think of what "most" teens would do, and that it was not necessary to disclose what they would do personally. "Risk" remained undefined in order to investigate what teens would present as their perceptions of risk. Scenarios began relatively mild, sneaking out to meet friends against parents' will, and progressed to more potentially "risky" situations. The second and third scenarios involved sneaking out of the house to meet friends who had alcohol, or thirdly drugs. Last, teens were asked what risks were involved if parents were out and a boyfriend or girlfriend were alone and decided to become intimate.

\section{Scenario One: Meeting Friends}

YM(13): "They'd go, they wouldn't think about it." YW(15): "Well, it depends on how much you want to go out too... it depends on who your parents are, and like how they are... like some parents don't care..."

YM(13): "Yeah, it depends on whether you don't care what your parents said."

YW(15)YW(16): "Wouldn't take much... not much at all."

YM(14)YM(15): "If the kid really wants to go, then they'll go, no matter what the parents say."

YM(14): "It depends on whether they're goody-goody or not... I mean it wouldn't take me much, if I wanted to go out, I'm going."

YW(13): "It depends on how old that person is, well not really how old but how close they are to that person... if they are really like a best friend, then they might like say um, 'like you do this,' or they would like bribe you or like threaten you and stuff so that you would go..."

YW(13): "Not risky. Most teenagers would just go." 
YW(15): "Well if it were someone they really like, they'd go."

YW(15): "Would depend on if it were a guy, or a girl for a guy."

YM(13): "Yeah, I guess if it was like one of your good friends."

YW(15): " $\ldots$ if it was a boyfriend, they would be right out there!"

YM(13): "For a girlfriend? They'd go right through the glass! ...would be right out there!"

YM(13) "They'd go for it... a little risky ...but like it would be okay if no one would know."

\section{Scenario Two: Meeting Friends with Alcohol}

$\mathrm{YM}(13)$ : "Well, they'd probably let them in, but if they were going to use alcohol, I'd kick them out."

YM(13): "I would do it."

YM(14): “... if it was a party I wouldn't, but if it was only a couple of people and a case of beer, I might."

YW(15): “... well like if the person was planning it that night and they just had beer, I would."

YM(15): "It's more risky than the window one... but most teenagers would let them in... well, not if they're like totally against drinking alcohol, like they don't drink, but they'd probably still let them in just to party and fool around."

YW(15): "It's more risky than jumping out the window, because if you get caught going out with your friends, like sneaking out is a lot less bigger issue than getting caught with alcohol..."

YW(13): "I would turn off the lights and lock the door... I wouldn't let them in..."

YW(13): "You could have the alcohol, but not take any, and then like I saw lots of TV shows and everything like that where they did like have that and like they cleaned up like perfect before the parents got home an the parents never found out."

YW(13): “... they would think about it, and if the kids put like pressure on them, I still think that they would think about it."

YW(13): "Yeah, they would probably do it at somebody else's house but it's different when you're in your own house."

YM(13): "Yeah, I know quite a few people who would go with no problem at all."

\section{Scenario Three: Meeting Friends with Other Drugs}

YM(13): "I'd probably kick them out."

YW(15)YM(14): "That's riskier."

YM(13): "That's risky..., well, it depends on what kind of drugs, if it's heroine or something..." 
YM(13): “... because you wouldn't want to do like opium... you'd have the police all over you..."

YW(15): "Yeah, when it's just like pot, then that's different, it's okay."

YM(13): "Yeah, then [if marijuana] we probably would."

YW(15): "I wouldn't let them in my house."

YW(13)YM(14): "Very risky... at lot of peer pressure."

YW(13): "I don't think that they'd go."

YW(13): "Not in my own home... if they really want to do it for some reason, they should like do it, like I don't know, like in the streets or something like far away from people."

YW(13)YW(15)YM(13): "Yeah... I know a lot of people that would go."

Although AIDS was brought up as a risk by some participants within the fourth scenario, it

was never brought up as a first concern, usually being secondary to pregnancy.

\section{Scenario Five: Having Sex}

YM(14): "They'd really be in trouble if their parents came home!"

YW(14): "That's risky, like she could get pregnant for sure."

YW(13): "It kind of depends on how well they know the person or depends on how long they've been together, like being together for a long time, and if they already had it."

YW(14): "She might get pregnant."

YM(14): "Whoa! Right."

YW(13): "Yeah, they could like get AIDS or the girl could get pregnant, especially like if it were a teenager, like most teenagers might not feel responsibility for a child, and so if they were using like birth control and stuff, you know."

YM(13): "Risky, yeah, but like they'd just do it most likely."

YW(15): "Well, it would be up to them, maybe the guy or girl said they weren't ready, and if they were drinking they might not be able to handle it, like the pressure to do it and everything."

YM(15): "Very high [risk] because there's no adult supervision and there's no one to really stop you from doing it if they're out until late or whatever."

YW(16): "Very risky, they'll just end up doing it if the parents aren't there."

YM(14): "Yeah, it's risky."

YW(15): "I know a lot of people that would just do it..."

YM(13): "Well, for the girl, there's a risk that she'll get pregnant."

YW(13): " $\ldots$ but they might think it [pregnancy] would never happen to them."

YM(13): "Yeah, and if they had sex, they could get AIDS."

YW(13): "It depends on if the parents decide to come home early..." 
YW(13): "They probably would [have sex] ... if they really liked each other... they probably would."

In order to assess decision-making and problem-solving processes, youth were then asked what would make that person decide it was okay to have sex. Concern for disease was low:

YM(13): "I don't know."

YM(13): "If they both want to like, if you're in love or whatever."

YW(15): "Teenagers think that they're in love all the time, they're always in love."

YW(15)YM(14): "Well, if both of them want to, they're just going to have sex."

YM(13): "Well if they're drunk or whatever... they'll say they're in love."

YW(15): "They wouldn't know what's going on if they were drunk."

YW(15): "If they're prepared."

YM(14): "A persuasive partner."

YW(13): "Someone they're interested in a lot... well, like mostly that they trusted, if they trust that person a lot because then that person would say, 'you could trust me' so then they might do it."

YW(13)YM(14): "Peer pressure."

YW(13): "Well like if they really did love the person and they were like around in the last year of high school and they wanted to do it with that person, just that."

YM(13): "Hormones."

YW(15): "How much they liked the person."

YW(15): "Morals."

YM(13): "Pressure [from partner]."

YW(13): “... if you don't like your parents or something and you're not allowed to go out... you might do it to annoy your parents if they didn't want you to go out with that person."

As popularity and attractiveness were assessed, it appeared that popularity was important for younger teens in the decision to have sexual relations, but not necessarily so for older teens.

\section{Popularity:}

YW(13): "For some people it might, because they think that 'if I have sexual intercourse with them that it would make me popular too." YW(13): “... yeah, because like if it's a popular person then they could be saying like 'well, I'm so popular so, if I want it, then I can get it."' 
YW(13): "But I think that if that person were popular then, especially if they were really popular, then the person that is popular might tell them that, 'oh yeah, she doesn't like sex,' or something like that and they might make her really unpopular."

YW(15)YM(13): "No [popularity doesn't make a difference]."

YM(13)YM(14): "Well yes."

YM(13): "Well if you had no friends it would be I guess."

YW(15): "No... because then the guy would like go and spread rumors."

YM(14): "Not always."

YW(13): "Well if they had sex then it would be passed all around school if the guy or the girl was popular... they'd probably say 'yes' because like the person is popular and they want to be popular too so they probably would."

YW(13)YW(14): "Yeah, probably... it probably would make a difference."

YW(15): "No, it doesn't make a difference."

YW(15): "It probably would if they wanted to."

YW(15): "It depends on how much that person likes the other person, but it probably would."

YM(13): "They'd probably end up getting more attention."

YW(15): “ ... there might be some pressure... you might like to become popular so you might do it."

YW(15): “Yeah, because if you don't they'll just talk about you and make you look like a total idiot, everyone believes what they [popular people] say, so, you know."

YW(15): "But if you do, they could call you 'loose,' so it goes both ways."

YW(15)YW(16): "Yeah, that's right."

YW(15): "I think that it's worse for girls worse than for guys, girls don't talk about guys like that."

YM(14): "That's what you think probably!"

YW(15): "Well, I haven't been in any locker rooms."

YW(16): "I know guys talk about girls all the time."

YW(15): "Guys go around doing all these girls and they say that the girls are pigs. I've never heard a girl make fun of guys for sleeping around. I never heard of it happening."

\section{Attractiveness:}

YM(13): "No, I don't think so."

YM(13): "That helps!"

YM(14): "Most people would do it." 
YW(15): "Well that might be the reason why they go with some cute guy, because he's cute, or a girl probably because she's popular or pretty."

YW(15): "It depends on the person I think, depends on their morals."

YW(16): "If they want to have sex, they will, or if they're against it, their not just gonna jump in bed just because they're cute."

YW(15)YW(16): "Yeah, it makes a difference."

YW(13): "Probably does."

YW(13): “I kinda of think that, well I don't really think that it makes that big of a difference though, because if they don't know the person they can try to get to know them before they have sex, so whatever, but really for here there's a lot of peer pressure around sex."

YW(15)YW(16): "They wouldn't do much thinking."

Informational Resources. Youth were asked what their best sources of information

for sex and AIDS education were for one-to-one discussions. Ease of talking with friends and parents were discussed, as well as who an ideal individual would be for the task. Not surprisingly, most indicated that friends were preferable over parents for such discussions.

Most stated that the topic was difficult to bring up to parents, kids would be embarrassed, or that parents "don't understand." Typical comments about friends are listed below.

YM(13): "Yeah... friends are easier to talk to because they understand what you're going through, and parents don't."

YW(13): "Close friends, yeah."

YW(15): "Yeah, all I know is that on the high school side that's all you hear."

YW(16): "That's like the only topic!"

YW(15): "Yeah, the everyday cafeteria talk."

YW(16): "If you just need someone to talk to, you could always just go to you friends. I think it's better than your parents."

YM(13): “ $\ldots$ your friends aren't going to squeal, like especially if you told them you had sex or something they're not going to go running around spreading it everywhere."

YW(13): "Yeah, they're going through the same thing."

YW(15): "They understand you better."

Most youth stated that parents were the ideal people to talk to about sexual issues or AIDS, however, some teens had preferred other individuals.

YW(15): "Counselor." 
YW(13)YW(15): "Friends."

YW(13): "Teachers or counselors, for a question on AIDS."

YW(15): "Someone who will give them good information."

YM(13): "Counselors, sex educators."

YW(15): "Someone who is your own gender probably."

YW(15): "Someone that would understand."

YW(15): "They should be able to talk to their parents, but a lot of them can't."

YW(15): "My mom blew up on me, when I tried to talk to her about it." YM(13): "My parents make up stuff."

YW(13): "Probably parents would be like the best but most teenagers don't have enough courage because they like think that they might like get in trouble if they talk to their parents about it [sex]... it's better to talk to friends."

Teens discussed whether they thought parents talk to their children about AIDS. Most said, "no;" however one interesting dialogue developed regarding sex education in a large group:

YW(15): "Some do."

YW(13): "If they're good parents they should."

YW(13): "...they might say something about precautions or like 'be careful, '..."

YM(13): "No, they let the kids learn on their own."

YW(13): "Only unless they ask or something."

YW(15): "Well it depends, like if the kid asks a question they might want to answer it but with no great details."

YW(14)YW(15): "You eventually learn about it, but then it might be too late."

YM(15): "I didn't have sex ed until ninth grade and there were kids up there who were 12-years-old that were getting pregnant. That's too late, waiting until ninth grade."

Group leader: "What's a good age for sex education?"

YW(14): "Right before they're a teenager."

YW(15)YW(16)YM(14): "Yeah."

YW(14): "I'd say, ten."

YW(16): "When they're young."

YM(15): "Just telling them to start thinking about it when they're young."

YW(16): "Well not even that but just understanding what it is."

YW(15): "If anything you're trying to prevent it by telling them."

YW(16): "I think a lot of times they try to scare them away from it [sex] with all these diseases..."

YM(15): "Yeah."

YW(16): “... and if anything they're trying to prevent it. And people bring up that thing about condoms in schools, they're just trying to prevent 
people from getting diseases, I think. They're not trying to say, 'here's some condoms, go have sex.' If you tell them in the classroom where they can be serious about it and actually learn, I think it's better than five years later when you learn it from your friends and then say, 'oh, this is great."' YW(15): "A lot of people are really not going to stop us because we have our own minds. If they pass out condoms, or don't pass out condoms, we're still going to do it. So they know they can't stop us, they're just trying to protect us."

Assertiveness. Finally sexual assertiveness was assessed utilizing four scenarios with gender-neutral inquiries. Decision-making responses were elicited for people in difficult sexual situations. In each case, youth were asked how the person could handle it, what they could do, or say. Role playing strategies were implemented or at a minimum discussed if time did not permit. The first scenario presented was a person being pressured by their partner to have sex. Teens were asked next what they would say to a friend with a similar issue who was concerned about losing their partner. The last two situations involved two consenting individuals, however when presented with a condom, one responds, "no way," or in the last scenario, states, "if you really love me, we wouldn't have to use that."

In some cases it is interesting to observe students' selection of pronouns in their responses to gender-neutral questions. Also during one group, a 15-year-old discloses a personal experience that "everyone knows." She states she was forced, but "not raped," thus her perception of rape meant to be "beaten," and "force," for her is now "normal." When approached on an individual basis, this young woman felt it wasn't necessary to talk to someone, that she had dealt with it already; however, previously during the group session she stated, "I think we should have a group talking about sexual assault... definitely."

\section{Scenario One: Partner Pressure}

YW(13)YW(15): "Talk to him about it... if they don't like they're attitude they could just leave." 
YW(15): "It depends because like in that situation, you'd think you'd be able to say something, but like, you know, when you're in the situation, you can't."

YW(13): “... if they don't have a good enough relationship to talk about it anyway, then they shouldn't be together in the first place."

YW(13)YM(13): "Just tell him that they don't want to do it, and see if they could just be friends..."

YW(13)YW(15)YM(13): "Say that they'll leave... or just say, 'no'..."

YW(15): "Say that they don't feel like it."

YW(13): “... say 'I'm not ready."

YW(13): "Yeah, I'm physically ready, but not psychologically."

YW(13): "Say, 'I'm too young."

YW(13)YM(13)YW(15): "Leave."

YW(13): “... if they got angry, then obviously they don't care enough for you or for your feelings."

YW(15): “... if you weren't scared or whatever, or more nervous, you could think clearly."

YW(15): "Lie."

YW(15): "If they won't wait until you're ready, then they're not worth it."

YW(15): "Tell them how you feel about it, like why you don't want to, like supposed you don't like them a lot, and that's just how you feel."

\section{Scenario Two: Threat to the Relationship}

YW(15): "Well, some of the information you might say, just might not work though when you think of it, because like when they're in that position they're really not going to be thinking really..."

YM(13): “... try to find out if they're being pressured, then try to like tell them like indirectly like give them ways like how to build up their esteem..."

YW(13)YW(15): “ $\ldots$ not to worry, they're not worthwhile."

YW(13): “... ask them if it's really worth it."

YW(13): "Ask them if their health is more important than true love."

YW(13): "... tell them maybe later on in the relationship... but not now, no way."

YW(14): “... tell her to do what she wants to do... make up her decision beforehand and stick with it."

$\mathrm{YM}(13)$ : "If her boyfriend or whoever, her partner is like pressuring her, that's not good, they [the girl] obviously don't want to just have sex, and he really doesn't want to have a relationship."

YM(13): " ... tell the friend, like you could do something else like maybe have them go out on a date or something that is not really going to put her in that situation in the end... go out like with a couple of friends to a movie or something." 
YW(13): “... just have to do what they feel is right."

YW(15): "... tell them what you would do."

YW(15): "Yeah but that is bothering me because you could tell them that, but no matter how much you say that, no matter how much I say to myself, if--, if I was them saying certain things, if I weren't in that like certain situation, I wouldn't..."

YW(15): “ $\ldots$ it just wouldn't work."

YW(15): "Yeah, it just wouldn't work... yeah, no matter how much you're taught, it's never the same situation, it's like one where you really can't really get out of it."

YW(13): “... tell them what the risk is, or like you won't be his girlfriend anymore, like he wouldn't be worth it anymore."

YM(14): "If they really liked you then they'd really understand."

YM(14): "If they're pressuring you, then pressure them."

YW(13): "Yeah, you could make other conversation, like having a baby, or you don't want to have AIDS, or something..."

YM(13): "He's probably just going out like to have sex."

YW(15): "Dump them."

YW(15): "Get rid of him."

YW(15): “... it's like you think you could when you're in that situation, and someone just says, 'get rid of him,' because you know what it feels like..."

YW(15): “... I haven't been in that situation..."

YW(15): “... just wait, it will happen... maybe they just had different morals growing up, I don't know, it's just that when I was-- I don't care what other people are going to say, so I'm not going to say, 'I know someone who...' so it's just like I'm just saying everyone knows anyways, I got my virginity taken from me when I was young, not like really young, I wasn't raped or anything, I was like 12 or 13 , I wasn't raped, I was like forced, but not like beaten or anything, everybody knows that. So it kind of takes away from what I kind of think of now, because I was so young then, so it's just like, if somebody's forcing you, it's like pretty much normal, because I can pretty much just think about it. So if my friends come up to me and tell me actually, I never had that situation, but if they ever did, I'd--- I don't know what I'd say, but I'd probably if they really liked them, not just say, 'just get rid of him,' I'd think about it more... I'm just saying, that like I wouldn't say just get rid of the guy because there's more to the feelings."

\section{Scenario Three: Negotiation}

YW(13): "Dump him... he's not worth it."

YW(13): “... try to talk to him... you could say what the consequences

are... you could get AIDS."

YM(13): “... or pregnancy." 
YW(14)YW(15)YW(16)YM(13): “Just say, 'if you don't use it, then no way...' no condom, no sex."

YW(14): “... you can't let that person control you."

YW(15): “... tell them they were afraid of getting a STD, or that they were afraid of getting pregnant. And that if they did get pregnant then they'd have to help take care of the baby. Usually guys are afraid of that... yeah, guys usually don't want to take care of kids till they're 30 or something. They just want to have fun...."

YW(13): "... leave and come back later if he gets mean..."

YW(13)YM(15): "Say, 'No way,' to them."

YW(13): "... the person who wants to use the condom should use it, and tell the person who doesn't want to use it, "too bad, you don't get sex." YW(13): "Yeah, if he really cared he would use it."

YW(15): "If they didn't use a condom, then have them use some other form of birth control."

YW(15): "Ah, excuse me! Excuse me! ...that's something that's not going to stop you from getting AIDS. Exclamation point, exclamation point, exclamation point!"

YW(15): "There's a new thing now, the female condom, and it's kind of like the male condom..."

YW(15): "I don't think I'd use it..."

YW(15): "I don't know if I'd use it."

YW(15): "Let the males use it---, it's their job." [ 3 other young women agree]

YW(15): "I think to both of those questions there's two perfect answers, like if your boyfriend won't wait for you, then dump him, and if he won't wear a condom then drop him or don't let him pick you up, those are like two perfect answers, but they're never gonna happen really, except on TV."

YW(15): “Yeah you wouldn't want that to happen in your life, but it probably won't happen, I mean you have to think what is he gonna think...”

YW(15): “... and you also have to think, what if he has AIDS? Or what if I get pregnant, or you know? So you also have to think about yourself, not just about him."

YW(15): “... it depends on the situation because it's not gonna happen, because you'll think to yourself I'll always use a condom when I have sex, but then when you're there, you don't."

YW(15)YW(16): "Yeah."

\section{Scenario Four: Emotional Coercion}

YM(15): "I'd tell them from the start that like you would want to wait, so you know."

YW(13): “... it's not worth it, leave them." 
YW(13): "Say, 'I'm making you use that because I do love you' ...maybe that person doesn't love them that much."

YW(13): “...wouldn't be much different, because if they really love you then you would use it."

YW(13): “... tell him the risks, like if he had AIDS all the things that could happen."

YM(14): "Just say, 'No' if they didn't want to... or 'no condom, no sex." YW(13)YM(14): "Say, 'If you really love me, you'd understand."

YW(13): "You could tell them that it's protecting both of us."

YW(13): "... you could say to them, "Well I really want to have you as a boyfriend or girlfriend, but like I want you to be healthy and not to die or anything." "

YW(16): “... if it's a one night stand it's a different situation than if it's a long relationship I think, like if you know a lot about the person... I mean if you are with someone, and you were with them for a couple of years or something, then you should pretty much know a lot about them."

YM(14): "They should always be prepared [have a condom]."

Developing Assertiveness Capabilities. Next, assertiveness and role playing were

defined more fully and discussed in groups. Several participants were experienced in

playing out "skits" with peer pressure regarding refusing coercions for drug use; however,

teens had never tried the same with other decision-making scenarios. Some teens provided

their views on how sexual assertiveness could be acquired.

YM(13): “... movies, video cassettes that have it like all set up so that you can't get out, like when they're being pressured into it... I remember we did that I think in fifth or fourth grade health class, because like we had health like as a special, and we'd like get a little skit or we'd get the topic or we could make up our own and like act it out... a lot of it was on drugs... it's pretty good, I mean, not only do you learn from it, but other people that are watching are learning from it."

YW(13): "Teach them."

YW(13): "I think it has to do with self-esteem."

YW(15): "Give them more confidence, yeah..., like self-esteem."

YM(13): "Yeah, give them compliments and stuff like that, what their doing and their strengths."

YW(14): “... yeah, like having more self-esteem could help you do stuff better, because you would like know you could do it."

YW(15): "I know that [self-esteem] would help me!"

YW(15): "Friends, like in discussion groups, talking with people who really know what it's like... people that have been in those situations, but 
had no assertiveness... maybe they could have classes in school that teach you... maybe parents... but I don't think most kids can talk to their parents about those things."

YW(13): “... talk to mom and dad, or like a counselor or someone they feel comfortable with..."

YM(14): "Talk to brothers and sisters, because sometimes when you tell something like that to your mom or dad they might just not give the right answers."

YW(13): “ $\ldots$ in my experience, my mom and dad would not help me, and just say, 'no,' altogether, I mean I would never be able to talk to my mom about that... I don't think they really know that much of what you might be going through, and then you just have one more person that is pressuring you about what you're doing and that doesn't help at all... I don't know anybody who could talk to their parents like that."

YM(14): "No, I wouldn't either... I think friends are easier to talk to because they grew up with the same stuff you're growing up with, the drugs, the alcohol, the sex and AIDS."

YW(13): " $\ldots$ when you get older and in high school, especially when you're a girl, like, your parents might be, like not have the reality that you might be doing it which changes what they might say."

YW(13) "They could give education, teach in classes in school..."

YW(13): "Yeah, talk about it."

YM(14): "Education in the home... parents should bring it up."

YM(14): "Yeah, parents should, but... like they never do."

While discussing assertiveness, some dialogues returned to the issues of condom distribution and popularity:

YW(15): "I strongly feel that if there are condoms there, like available to us, then they would be used like many percents more..."

YW(15) "... yeah, people are so scared to buy them."

YW(15): "... nobody wants to go in and buy them."

YW(15): "Nope, no one would."

YW(15): "No way."

YW(15): “... most people already know this, this guy in my health class went into a drugstore... he wasn't planning on buying anything, and suddenly this old lady comes flying down the isle and says, 'What are you doing! You're too young to be looking at stuff like that!' and starts screaming..."

YM(13): "Yeah, I mean because they're going to do it anyway, like so why not have it protected?"

YW(15): "Yeah, I just think they should just hand them out at school." YW(15): "Leave them in the bathroom or something, so no one is embarrassed." 
YW(15): "Yeah, a dispenser!"

YW(15): "Definitely!"

YW(15): "Oh dear God, yes!"

YM(13): "I don't think that people should be judged if they're too young or not, because if they are going to have sex at least they can have it protected, then you can't say, 'well you're too young' and then he goes out and gets some girl pregnant or gets a disease, that's what's gonna happen."

YW(15): "In our country you're raised to think that sex is a dirty thing and not to do it, so when you get in our position, you just have to keep it a secret and stuff like that. We're taught that it is bad."

YW(15): "Yeah, it's bad."

YW(15): "Naughty."

YW(15): "And dirty."

YW(15): "And sleazy."

YW(13): "Real bad."

YW(16): "I think that a lot of them end up doing anyway and regretting it, and most of the people I know regret the first time anyway, whether it be that they were too young or they didn't want to be with that person, so it's hard to tell someone what to do, I mean if they're friends and everything, they should wait, but when it comes down to it, I think it is that person's decision, or if they're being pressured, they end up doing it anyway." YW(13): “... if they're doing it for like popularity or anything, they could just say, 'no' ...if they didn't it would just be their fault."

YW(16) [to YW(13)]: “... I don't really think it should be their fault, I think that you, well until you're in that situation you wouldn't understand how it feels or when you're with someone and you like them a lot and you may not want to do it, and they do, it's not popularity or anything like that, I think it's just you don't know what else to do."

YW(13): "But I think that if you really like someone, you should like talk about those things right away, and tell them how you feel about it, that you're not ready, and then they'll just know, and then that situation like won't happen..."

YM(14): "Sometimes it is for popularity."

YW(13): "Yeah, they're cool."

YW(16): "And then they just get a reputation."

YW(16): "Slut."

YM(14): “... they can't stop doing it cause they got to live up to the reputation, it's a no win situation, if they live up to it they're called a 'slut' and if they don't live up to it they're called 'a tease."'

YW(16): "Well, people I've known that have gotten that reputation, eventually they kind of resort out things, and slow down." 
YW(13): "There are some people in our grade [7th] now that already have that reputation and you don't know who to believe because you don't know if they already did it, like some people say they have, or like if they really didn't like they're just making it up just to be popular."

YW(16): "I just wanted to say that there's really no peer pressure with like seniors, there's so many like different groups and it's not like cool or uncool, and there's not just different kinds of cliques... and there's the people that get that reputation but they just have that reputation they're not like cool or uncool or people really want to hang around with them. In high school, I guess we don't have like set things like that... it's not labeled or anything, well yeah, they're labeled 'slut' or something, but it's not like you would want to be in that group of people, it's like an individual thing if you're called that, there's not like a group of them."

Group leader: "Why do you think people would want to do that in junior high?"

YW(13): "To feel wanted. To feel that way with one person or to feel like wanted with the people in that group, or people in your class. I have a couple of friends like that say they did it, and one of them would never talk to me because I'm not cool, like they won't even talk to us now. So I don't even think she was a real good friend anyway."

\section{Other Topics Raised}

Other topics were raised ranging from parasuicide attempts of friends to local prevalence rates. Some youth made inquiries during group discussions, while others asked the group leader privately. One 13-year-old asked how she should act around a lesbian:

YW(13): "Should you act differently around a family member, cause like, nobody has told you that but like your dad did or something and he told you that she's a lesbian, how should you act in front of her, because it feels, like different, kind of weird... so how do you act?"'

Two 13-year-old young women each had a friend with a recent parasuicide attempt and asked numerous questions about what to do, act, and how to help. Another 13-year-old young woman discussed a family member who is doing drugs and inquired what to do. A 15-year-old young women commented about a conversation on the bus, where someone mentioned that they knew an HIV-infected student. 
YW(15): "Our school is like, really bad like, I say that if one person in our school has HIV, within the next 5 years, everybody will have it. Yeah, it's really bad because like everyone is sleeping with everyone's boyfriends and all that stuff... like that [bus conversation referring to one student being infected] really scared me, I was like, I better think twice..."

YM(14): "... it would make you slow down a little bit."

YW(15): "I don't think that NFA [another local high school] would have, I don't know, I think they would be worse than us... because we're so, um, so--, Griswold isn't any bigger, Griswold is in the country, and everybody is like hicks and, and..."

YW(15): "I would think NFA would have more [people with HIV/AIDS]..."

YW(15): “... yeah, but all the guys here have no respect for the girls and that kind of stuff... some guy hit me yesterday, and I was like, 'how old do you have to be to hit a girl?' Then I ignored him for a while and said, 'going on nine?"'

YW(15): “That was weird, because I never had any questions, I thought I knew all about it, like this person I know, and how not to get it, how you get it and all that stuff, and then when we got talking about people who actually had it in my school, I was like--, I thought of so many things, that I really don't know..."

YM(14): “... I didn't know that either, not in a rural area, it like freaks you out."

YW(15): "They don't know it's [AIDS] is here."

YM(14): "They all sleep around, but they just don't say it."

Group leader: "Does anyone know how many AIDS cases there are in Connecticut?"

YM(14): "Forty to fifty?"

[Discussion continues answering questions on stats]

In a moderate sized group of six composed primarily of 15 -year-old young women and one young man, two discussed a student whom one nicknamed, "Joe Stud:"

YW(15): “... everybody loves him but you can't like, well, because he's Joe Stud, well he's got a couple of zillion girls that like him, and he's had several hundred sex partners but everybody goes with him, you know, because he's so popular... he's very strong, and he's got a reputation as being a rapist, but there's still a zillion girls that want to go with him." Group leader: "A rapist? Why would girls want to go with a rapist?" YW(15): “... they don't want to believe it... because he's hot, that's all there is to it, his body is really hot."

Group leader: "If Joe has so many partners, is sounds like he's putting himself at risk..., what if he became infected, what sort of effect would it have on people?" 
YW(15) “... everybody would listen, everybody looks up to him."

Group leader: "A rapist?"

YW(15): "Yeah, because like his word means something to most people, it's like for some people it [HIV] would be a reality."

Group leader: "Why wouldn't it be the opposite, why wouldn't people be worried to be with him?"

YW(15): "They don't want to believe it... he's like um..., very persuasive, you know, he'll make you feel very special."

YW(15): “... yeah, you don't want to believe it... so you end up going with him anyway."

[three others agree]

Group leader: "Do you think that going out with a Joe Stud is a risky situation?"

YW(15): "No... well, it's not her fault if he wants to..."

YM(14): "... well if he has a reputation as being a rapist then it is...,"

YW(15): “... well, yeah but...,"

YM(14): “... she should realize that."

Another 16-year-old young woman retold a story about a "friend of a friend" who went to

Florida on vacation, met a guy, and had a sexual relationship. Upon returning home, he

presented her with an envelope to open on the plane which said, "welcome to the wonderful world of AIDS." Group discussion turned to possible anger and revenge involving infected individuals, legal dilemmas with the prosecution of deliberate infection or withholding HIV positive status, difficulties using protection, the responsibility of revealing positive serostatus to partners, and returned to the possibility that infected partners may not be truthful in order to gain sex.

\section{Questions Raised}

Younger teens raised a substantial number of questions regarding AIDS, while older participants generally inquired only of specifics regarding testing procedures. Four groups consisting of a total of approximately 30 individuals became involved with discussion of gender differences with transmission. One-third were aware of women contracting HIV 
easier than men; however, no participant knew why. Consequently, some misconceptions among young men had to be clarified. More particularly, where some young men held the belief that HIV could not be contracted by a man from a woman, but the reverse was true.

Overall, whether saliva or mosquitoes can transmit AIDS were most frequently asked.

\section{Factual Inquiries:}

YW(14)YM(13): "How did it get here?"

YW(13)YW(13): "Can you get rid of HIV before it becomes AIDS?"

YW(13): "What's the difference between HIV and AIDS?"

YW(13): "Once you have HIV, does it automatically turn to AIDS?"

YW(13): "How can you tell if you have it?"

YW(13): "How good is the test, like is it always right, or can it be wrong sometimes?"

YW(13): "Is it mostly gay people that have it?"

YW(13): "Are condoms safe?"

YW(13): "When you get a physical, do they do an AIDS test?"

YW(13): "So who was the very first person who got it?"

YM(14): "How long has AIDS been around?"

YM(13): "Where did it come from?"

YM(13): "How many researchers are there?"

YM(13): "How long will they [people with AIDS] live?"

YW(13): "When you are born with AIDS don't you like live like a long time?"

YM(13): "Do you think they'll find a cure?"

YM(13): "Who do you think is the biggest group, like at what age is the most people that have it?"

YM(13): "Do you know anybody that has it?"

YW(13)YW(14): "They had a cure in this movie on TV, is that true?"

YW(13): "How come, why is it all of a sudden it's [AIDS is] more popular, it's like a big thing now?"

YM(13): "Could we like separate people who have it so that like other people don't get it?"

YW(13): "Do you think that the information like that we get in school and everything about AIDS, do you think that it will like, that the cases will ever go down where we're starting to prevent it?'

YM(14): "It's not really AIDS that kills them it's all other things you get because of AIDS right?"

YM(14): "So AIDS can't survive a long time out in the open?"

YM(13): "What would happen if you had two infected partners?

YM(13): "How many people in the U.S. have AIDS?" 


\section{AIDS Transmission Inquiries:}

YW(13)YW(13)YM(13)YM(13)YM(13)YM(14): "Can you like get it through like mosquito bites?" "Can mosquitoes give you AIDS?" YM(14): "My sister said you can get AIDS from a mosquito, is that true?"

YW(13)YM(14)YM(14): "Can you get it if you kiss someone?"

YM(13): "Can you get AIDS from French kissing?"

YW(13)YM(13): "Can you get it from like spit, or like if you kiss someone?"

YW(13) "Do they know if you can get AIDS from, you know, spit?"

YW(13): "Can you get AIDS from saliva?"

YW(13): "Someone said that girls can get it easier than boys, is that true?"

YM(13): "Yeah, guys can't get it from girls, but girls can get it from guys. Guys only get it from guys, right?"

YW(13): "Can you like get it [HIV] from doing the blood test?"

YM(13): "Can you get it from a cut?"

YW(13): "Why do they tell you not to sit on public toilets? Can you get AIDS from toilets? My mom thinks so."

YW(13): "If someone has sex with someone with AIDS do they automatically get it?"

YW(13): "Could you get it if you never fooled around and never used drugs?"

YM(13): "Can you be born with it?"

YM(14)YM(14): "What are the chances of a pregnant woman with AIDS giving it to the baby?"

YM(14): " $\ldots$ if HIV is what the [pregnant] mother has instead of AIDS, like HIV, would the baby get it?" ... Is there a chance that the baby wouldn't like get it?" "If the baby has AIDS when it's born how long does it have to live?"

YW(13): "Do people that do drugs and needles and stuff, like will they automatically get AIDS?"

YM(14): "So how do people get AIDS from the dentist?"

YM(14): "If you like brush your teeth or like if you cut your elbow or something and it bleeds and there's like dried up blood, can you get it from that?"

$\mathrm{YM}(13) \mathrm{YM}(13)$ ): "You can't get AIDS from oral sex right, not if you spit, only if you swallow."

$\mathrm{YM}(13)$ : "What if guys got like neutered or whatever you call it [a vasectomy?] ...yeah, can they still transfer AIDS that way?" 
Overall, focus group youth reacted positively to the discussion process, even in the frequent cases where younger and older teens debated based on experience or inexperience. Youth were very open to discussing topics in group format, although a few approached the primary investigator in private to clear misconceptions.

\section{Discussion}

\section{Overview}

In an effort to place the quantitative results found in this study in appropriate context, elaboration regarding the strengths and weaknesses of the measures analyzed precedes discussion of hypotheses with quantitative and qualitative findings. Aside from analyses of the questionnaire construction, quantitative analyses examined predictors of AIDS risk in the cognitive, attitudinal, and behavioral domains, as well as whether differences existed between the genders and between those who chose to be sexual active versus those who are not. It is important to reiterate that focus groups are not intended to test hypotheses, nor produce generalizable data (Basch, 1987; Calder, 1977; Manoff, 1985; Morgan, 1993), but were utilized in the present study to supplement quantitative findings. Briefly, qualitative topics attempted to examine several areas, such as, What is the general level of awareness about AIDS? Where do teens acquire information regarding HIV and AIDS, and are such resources correct and reliable? Can youth identify, and do they engage in, behaviors that may result in HIV infection? Can teens identify and utilize refusal skills for avoiding risky situations? What are the perceptions of gender roles? Can adolescents accurately perceive their own risk of being infected with HIV? Are adolescents 
aware of societal repercussions of the AIDS pandemic, and what are their reactions and solutions? What suggestions do teenagers have for intervention and prevention?

Following scale construction comments, qualitative findings are discussed in conjunction with quantitative results, with separate emphasis on discussion of societal dilemmas and adolescent suggestions for intervention and prevention. Discussion ensues with suggested interventions, future directions for further adolescent research, and limitations of the current study.

\section{Scale Construction}

Reliability and validity of potential risk factors comprised of cognitive, attitudinal, behavioral, environmental and psychoeducational constructs were explored in the current study in the continued development of a comprehensive measure of adolescent risk. The consistency and reliability of the constructs under investigation across various adolescent subpopulations appear relatively stable, although some areas and specific measures are cautioned in interpretation. Potential explanations are provided below for noted scale construction differences among demographic variables. Constructs were analyzed with four dichotomized demographic variables (age, geographic location, ethnicity, and socioeconomic status) for their stability or inconsistency as compared to the overall construct formation found utilizing the entire sample and computer generated random samples.

Age. Regarding age, overall, the questionnaire proved to be stable for ages of adolescents, although minor difficulties were found with youth under age 15 with measures of attitude and behavior, and problems arose with measuring estimated partner-risk history 
among all ages. Nevertheless, it is not surprising that estimating the risk of one's partner(s) was problematic, and not differentiated by age. Focus group participants voiced difficulties providing information about their partners given numerous complexities, situational contexts, and reliability or truthfulness of information obtained from a potential partner. The most noticeable construct pitfalls for younger teens were realized in measuring both perceived susceptibility for risk and condom use assertiveness, both being the least stable constructs. Additionally, measuring homophobia was found to be only moderately stable for the younger group. Given that adolescents may be only beginning to form their opinions and attitudes prior to age 15 , these inconsistencies might be expected, in combination with the nature of younger teens' limited experiences, sexual or otherwise, given their young age. Specific to perceived risk susceptibility, overestimating and underestimating personal risk has been noted elsewhere in this study and in others (e.g., Lang, 1994; Stanton, Black, Kaljee, \& Ricardo, 1993), and it is possible that inaccurate perceptions occur more frequently with among younger adolescents than older, suggesting further area of study. Moderate instability was noted with measures of substance use and abuse for older teens, whereas younger adolescents had more stable constructs in this area. As only substance using teens were utilized in the analyses, it is possible that younger teens are more consistent in reporting use, whereas older youth may choose to conceal or perhaps deny use and abuse of substances.

Environmental measures remained stable with respect to age, with the exception of estimates of peer risk, which were unstable for older youth, and improved slightly for youth under age 15. As with the partner-risk measure, youth voiced difficulties reporting behavior 
of peers due to extensive complexities and unknowns involved in estimating behavior and reactions of others. Such confounds would likely contribute to scale inconsistencies. The educational measures portrayed low stability among young teens improving with age for school-based education and influence. Such subscale instability may simply be reflective of age and years of exposure to school environment. For instance, during focus group conversations many younger teens expressed dissatisfaction with the health curriculum, more particularly, receiving nothing more than limited basic biology information in earlier years. Nevertheless, interpretation of these latter educational subscales is cautioned due to limited number of items, weakening the strength of the constructs.

Geographic Locale. Geographic locale had little influence on the construct formation across all areas with minor differentials noted. Consistency rates were slightly lower for local youth in the attitude measure of homophobia in comparison to youth residing outside the Rhode Island, Massachusetts and Connecticut area. Given that a large majority of adolescents were obtained from a rural area in Connecticut, who voiced homophobic attitudes during discussion groups, it is likely that local teens may appear to possess more homophobic tendencies in comparison to youth from other areas. On the other hand, the small number of adolescents from other areas $(n=75)$ may have serious implications in statistical analyses in itself, with unstable results as a consequence.

Behaviorally, substance use and abuse were relatively stable across both samples with slight less stability noted among teens from other areas in measures of substance abuse in comparison to local counterparts. This finding may be reflective of sample characteristics as many of the out-of-area adolescents were participants of youth conferences which 
focused on reducing risk behavior. Moreover, many youth conference goers disclosed former substance use in discussion groups, and voiced hesitancy in revealing relapse occurrences, feeling they would be judged as a "failure." As with other subpopulations, measuring estimates of partner-risk histories proved to be only moderately stable with youth from outside areas, but very stable among local youth. Since the majority of local youth were high school teens, one explanation may be that these local teens possessed much more firm convictions of their estimates of their partner(s) sexual behavior histories, as they were dating school peers and had more informants for reports of promiscuity, or faithfulness, as the case may be.

Environmental measures remained consistent, again, with minor difficulties in measuring peer risk across both groups. Educational measures were found to be less stable with youth from other areas, particularly with items pertaining to school. School education and influence were found to be considerably weak measures among youth from other areas, which may reflect differences in educational systems from various locations (e.g. central United States, Bermuda, Jamaica, Cayman Islands).

Ethnicity. With regard to ethnicity, measures of perceived susceptibility for risk, substance abuse and condom use assertiveness were moderately unstable, with greater psychometric variability among scales for youth of color. Interpretation of results here also must be cautioned given the small number of adolescents of color $(n=85)$ included in the analyses, which may be affecting statistical procedures. However, one explanation for the differential finding with ethnicity for perceived susceptibility for risk may be the demographic profiles of populations most afflicted by HIV and AIDS, which has been noted 
to affect misperceptions (McCaig, Hardy, \& Winn, 1991). Youth of color may be in conflict with estimating their own risk given the typical "invulnerability," commonly experienced by adolescents, together with statistics of higher incidence of infection among people of color. The weakness of the substance abuse measure for youth of color could be due to personal characteristics and histories common among conference youth. Conference youth were much more ethnically diverse, were likely to be former substance users, and several reported resistance in revealing or admitting to using substances at all.

Assertiveness for condom use among youth of color also posed problems for interpretation, with measure instability in comparison to Caucasian youth, who showed strong stability in subscale responses. Interestingly, the partner-risk history measure proved highly consistent among youth of color, while Caucasian counterparts were only moderately stable. These findings of differential measurement difficulties among ethnic groups may be confounded by level of confidence in assertiveness abilities as well as cultural expectations, norms and social roles, and may need to be explored more fully with adequate sample sizes of various ethnic groups.

Environmental subscales remained consistent across both ethnic groups with slightly less stability measuring peer risk among Caucasian youth. Educational measures also rendered low consistency rates for school based education and influence with both ethnic samples, and may reflect distinctly different educational systems. However, as mentioned above, these subscales are still under investigation rendering cautionary interpretation.

Socioeconomic Status. In exploring socioeconomic variability, the cognitive measures remained stable; however, homophobia and condom use assertiveness were 
problematic constructs for youth from lower-to-middle socioeconomic class, but stable with middle-to-upper class adolescents. Again, this may suggest an area of further study, revealing differential levels of homophobia across socioeconomic levels of youth. Although not particular to attitude and behavior, one study found misconceptions more common among the less educated and in areas where AIDS was more prevalent (McCaig, Hardy, \& Winn, 1991), which may be areas that are associated with lower socioeconomic levels. The condom use assertiveness measure was also found to be unstable among lower-to-middle class youth while very strong among middle-to-upper class youth. Interestingly, risk history, for both self and partner, proved to be very stable constructs among lower socioeconomic youth, and slightly less so for middle-to-upper class youth. Beyond numerous confounds noted among focus group participants, it is unclear what particular factors influence adolescents' confidence in risk estimates for themselves or their partners with regard to socioeconomic status. However, conducive to AIDS risk for oneself as well as for respective partners, these measures of potential risk via sexual history could be exemplary of further inconsistencies between perceptions and reported behavior, noted elsewhere in this study. Therefore it is likely that greater instability in measures of risk containing items regarding partners' behavior are associated with a more realistic perception of one's inability to be accurate given the number of unknowns. Conversely, greater stability estimates may exemplify a faithfulness in partner communication, or perceived invulnerability or denial of true risk probabilities.

The environmental measures revealed similar findings among both socioeconomic groups with minor difficulties measuring peer risk for both groups. For the educational 
measures, middle-to-upper class youth had higher measure stabilities in comparison to lower-to-middle class teens for school-based education and influence. Again, these low consistency rates may be explained by various educational systems, but are more likely due to the instability of these scales still under development.

Deviations from the Hypothesized Subscale Structure. One subscale deviation was noted with measuring fear of AIDS and homophobia. Statistical analyses failed to distinguish these two subscales as divergent measures, and rather clustered them together within one construct. Further, strong associations were discovered within all subpopulations by gender and sexual activity between the two hypothesized constructs (ranging from $r=.52$ to $r=.65$ ). Since causation and directionality cannot be determined, more particularly, whether fear of AIDS promotes homophobia or whether negative attitudes towards gays creates a fear of AIDS, interpretation is cautioned. It is possible, however, that further development of the subscale measuring fear of AIDS is warranted, especially due to the small number of items (two) in the present scale, which consequently may have seriously affected statistical results.

Summary. Overall the current questionnaire poses some interpretation difficulties within the cognitive, attitudinal and behavioral domains under investigation in this study. The following summarizes strengths and weaknesses: (1) for youth under age 15, cautions are recommended in interpreting perceived susceptibility for risk and condom use assertiveness, both proving to be only moderately stable constructs for this subpopulation; (2) geographic locale had little influence on the construct formation across all areas; (3) measures of perceived susceptibility for risk, substance abuse and condom use assertiveness 
were moderately affected by ethnicity, showing greater inconsistency among youth of color; (4) measures of homophobia and condom use assertiveness are problematic constructs for youth from lower-to-middle socioeconomic class, but stable among middle-to-upper class teens; and (5) the homophobia subscale consists of items hypothesized as measuring fear of AIDS, which could not be statistically separated, although a strong association between both hypothesized measures exist among all subsamples of gender and sexual activity.

Questionnaire Feedback. Typically adolescents provided positive feedback regarding the questionnaire, anonymously and within discussion groups. Anonymously, adolescents divulged positive outcomes with increased awareness of their own behavior as well as potential correlates of risky behavior. Many thanked the researcher for the opportunity to be involved. One individual suggested the inclusion of questions regarding partner's substance use prior to sexual activity, together with the same of the survey-taker. Knowledge of a partner's condition prior to sexual activity may be helpful in some respects. However inclusion of these data will likely pose other associated inquiries, such as unwanted, forced or coerced sexual behavior, and/or submission due to the influence of substances. Several ethical concerns are associated with collecting this type of data with young adolescents, although important in designing appropriate intervention and prevention strategies.

Feedback from focus groups was also positive, with most reporting the survey to be "interesting," "important," "worthwhile," and "helpful," and many reporting they learned a lot. Discussants appreciated the fact that the survey responses were anonymous, and felt that teenagers would probably not be truthful in their answers if it had not been. Sexually 
nonactive youth felt predicting their behavior was helpful in identifying potential factors of risk in various contexts and situations, “...I think it's good to have kids predict behavior, because then you can think about it." Nonactive youth also indicated that predicting their behavior assisted in keeping their sexual activity status confidential, because they "didn't finish fast... and had to think about it." Only one percent reported the survey was upsetting, however in focus groups, they elaborated that the topic of AIDS was upsetting, moreso than the questionnaire itself.

\section{Quantitative Findings and Qualitative Supports}

Sexual activity findings among adolescents in the current study appear to follow trends found in studies conducted with youth in the United States. For instance, a 1990 national CDC survey showed that 54 percent of U.S. teens in grades nine through twelve had engaged in sexual intercourse (CDC, 1992). In the 1990 survey, the CDC found that one in eight ninth graders reported having four or more sex partners, and this proportion increased by grade, reaching one in four among twelfth graders. Other data indicate that sexual activity among adolescents has increased steadily since the 1970s (e.g., Brooks-Gunn \& Furstenberg, 1989; CDC, 1991; DiClemente, 1989, 1990; Sonenstein, Pleck, \& Ku, 1989; Zelnick \& Kantner, 1980). Following suit, nearly 54 percent of the adolescents in the current study indicated sexual activity, with a higher percentage of young men ( 77 percent) than young women ( 41 percent). The mean age of sexual debut in this study, 13.6 years, was consistent with findings in a similar study (Lang, 1994). Consistent with studies mentioned above, sexually active youth in the current study reported having three and four lifetime sex partners (for young women and men, respectively), which may be cause for 
concern since the average age of this subsample was 15 years. Researchers, educators and parents must be mindful that many adolescents will continue to engage in sexual activity despite the promotion of abstinence, therefore it becomes essential that youth receive the message that they must practice safer sex and use condoms properly and consistently.

\section{Hypothesis One: Predictors of Estimated or Actual Sexual Assertiveness.}

Predictors of higher potential HIV-risk, defined as having little or no sexual assertiveness for both condom use and partner communication, were investigated in the first hypothesis. Analyses included both sexually active youth who reported actual behavior as well as sexually nonactive youth who estimated their assertiveness skills. For young women, as hypothesized, having high AIDS misconceptions, substance use and substance abuse and high perceived susceptibility for risk, were significantly associated with potential risk. For young men, low perceived AIDS knowledge and high AIDS misconceptions were associated with low sexual assertiveness abilities. Two components of AIDS knowledge (factual and transmission) and homophobia were not found to be significant predictors of sexual assertiveness for either gender.

These results show that more variables may be relevant for young women's sexual assertiveness skills than for young men's. Young men may perceive that they already know all there is to know about AIDS, and believe that alone is sufficient for having the skills to assert oneself in intimate settings. However, three salient points should be emphasized.

First, gender comparisons revealed that both genders had perceived their AIDS knowledge as fairly high, with nearly perfect scores, with young women being slightly, but significantly, higher than young men. Yet as a predictor of assertiveness skills, this factor 
was only significant for young men. Further, although young men seem to utilize their perceived knowledge base as predictive of assertiveness skills, gender comparisons showed that young women, in fact, had significantly higher factual AIDS knowledge and significantly lower AIDS misconceptions than young men, thus being more accurate in their perceptions of their AIDS knowledge base. Therefore it appears that young men are taking into account neither their attitude nor their behavior with regard to assertiveness skills, and consider only faulty perceptions of their AIDS knowledge, which is likely to be lower than their partner's knowledge base (in a heterosexual dyad). One explanation may be reference to "know-it-all" teenagers, who have been noted in the literature as individuals who simultaneously believe in the importance of safe sex behaviors while expressing doubt about the viability of some safe sex behaviors (Stevenson, Davis, Weber, Weiman, \& Abdul-Kabir, 1995).

Second, for young women, variables in all three areas (cognitive, attitudinal, and behavioral) were found to be significant predictors of high sexual assertiveness skills, possibly showing a more prudent observation of what might contribute to having strong assertiveness skills. The sexual assertiveness analyses appear to be more comprehensive of contributory factors in several domains for young women than for young men. More particularly, the women had lower AIDS misconceptions, lower perceived susceptibility for risk, and lower substance use and abuse than young men, which were associated with significantly higher assertiveness skills than young men. Moreover, assertiveness skills for young women were very high, near perfect scores, but only in mid-range for young men. 
The reader is reminded that both the sexually active and nonactive youth were included in these analyses, leading to the third point of emphasis.

Third, and likely most relevant in the consideration of sexual assertiveness predictors, are the disproportionate numbers of nonactive young women to nonactive young men included in the analyses. Since there was almost a 1:5 ratio (19:92) of nonactive young men to young women, and since nonactive youth predicted their assertiveness skills in comparison to reported actual behavior of sexually active teens, the gender comparison results may be greatly influenced by having a higher frequency of faultless, nearly perfect scores predicted by nonactive young women, just by virtue of the sampling distribution. Hence the quantitative results may be seriously confounded, rendering difficulty in interpretation. Thus the qualitative findings may better provide some insight in predictive variables to sexual assertiveness skills.

Discussants mentioned several variables associated with assertiveness in focus groups. Issues related to situational and emotional constraints, potential negative consequences, trust and honesty, and gender role expectations are outlined below. Additional related topics included condom availability and distribution, external pressures to engage in sexual activity, perceived invulnerability, misinformation and availability of informants.

Although personal disclosure was discouraged, focus group participants in most cases divulged indicators of sexual activity or nonactivity, and freely debated with one another. Sexually nonactive women reported consistencies with their questionnaire responses, claiming, or rather predicting, an ease of partner communication for unwanted 
sex, simply telling their partner, "I'm not ready," "just get rid of him," or asking the partner to leave or just saying, "No." However, sexually active young women emphasized difficulties of generalizing sexual assertiveness because, "it depends on the situation." Similarly, sexually active women made a salient point about what they thought they might do prior to being in a situation requiring assertiveness abilities, and what they actually did, "you'd think you'd be able to say something, but you can't," "no matter how much you're taught, it's never the same situation, it's like one where you really can't really get out of it." Young women added the emotional aspect and importance placed on maintaining the relationship, “just wait, it will happen..., if they really liked them..., I wouldn’t say just get rid of the guy because there's more to the feelings." Additionally, some indications of low assertiveness negotiation skills were evident with young women, especially in a dilemma of potentially losing a partner, "well until you're in that situation you wouldn't understand how it feels or when you're with someone you like a lot and you may not want to do it, and they do... I think it's just you don't know what else to do." Interestingly, most of the young men remained quiet during these discussions despite subtle prompting by the group leader to contribute feedback to the group.

Young women were clearly more concerned with their partner's reaction to their assertiveness or potential assertiveness, and the possibility of angering or losing their partner as a result, than young men with their respective partners. One young woman emphasized the difficulty she faces in the comments section of the questionnaire, "Sometimes as strong as a person may be, sex gets tricky with so many feelings involved. You want to please the person your [sic] with but also do what's right for you." Clearly 
many of the young women involved in the current study were experiencing much difficulty with becoming assertive early in their relationship histories. Inability or difficulty discussing sexuality and sexual behavior has been associated with not using condoms (MacDonald, et al., 1990), but is not limited to local youth, as it has been noted to be prevalent worldwide (WHO, 1993b). Boldero, et al. (1992) found that the degree of communication with a sexual partner greatly influenced condom use, and that those who communicated directly with partners about condom use had a stronger intention for use, and were more likely to use one. Nevertheless, focus group findings were consistent with the literature, stating that women are hesitant to raise the topic of condom use, as it may raise doubts about infidelity, question traditional male prerogative in sexual matters (WHO, 1993b), question moral values with expectation to have sex, create an assumption that one is already infected (Hingson \& Strunin, 1992), and/or generate physical or psychological abuse (WHO, 1993c). Particularly with older women, the WHO (1993b) indicated that attempted negotiation concerning condom use destabilizes sexual power roles, and has the potential to contribute to emotional coercion and physical abuse. Combined with masculine culture of machismo and stereotyped norms for sexual behavior for men and women, lack of assertiveness encourages risky behavior and perpetuates the spread of HIV. Researchers note that unless women are empowered to negotiate safe sex practices, the decision whether to use condoms or not will be left to the man, which ultimately may become a decision of life or death (du Guerny \& Sjöberg, 1992).

The issue of honesty in a partner's response was typically not addressed by group participants with reference to partner communication about STDs and sexual behavior 
history. Often the group leader would introduce the topic into discussion with a gender neutral situation. Typically, participants, whether known to be sexually active or nonactive, responded that partners would not be truthful, and more interestingly, attributed a male gender to the perpetrator in their response, "if he says that he does [have a STD] then she won't have sex, so he'll just say, 'No," "He'd definitely lie," "When it comes down to sex, the guy will just lie," "Yeah right, like he's going to say he's got something!" Nevertheless, individuals of all ages have been noted to have great difficulty asking their sexual partners about past sexual practices, and further, inaccurate responses from casual potential partners undermine this strategy (Cates, 1990). Ignorance of a sex partner's sexual and drug activities is undoubtedly a risk factor to be considered, as group participants acknowledged. Yet participants did not account for truthfulness in a partner's response until probed further. Consistent with the literature (e.g., Cochran \& Mays, 1990; Keeling, 1989), respondents later realized inquiry may only provide an illusory sense of safety, as most men would lie about their sexual history to gain sex.

Both genders expressed sentiments that condom use was "the male's job," and most young women responded negatively to potential use of a female condom. Typically, young men stated young women would not carry a condom, because, "girls don't need them," “it's kind of the guy's responsibility," although older teenaged women seemed to believe women should carry condoms, “just in case the guy doesn't bring one... or he doesn't want to go get one." Again, the seemingly sexually active young women disputed the firm convictions of the apparent nonactive young women in the perceived ease of insisting on condom use, “there's two perfect answers, like if your boyfriend won't wait for you, then dump him, and 
if he won't wear a condom then drop him... but they're never gonna happen really, except on TV." Again, concern over one's partner's feelings was significant for young women, "you have to think what is he gonna think," as was the importance of the situation, "if it's a one night stand it's a different situation than if it's a long relationship" However, in the situational context of a "long term" relationship, which most often was identified as serial monogamy ranging from one week to two years for many focus group participants, young women felt a sense of trust for their partners in the "safety" of serial monogamy, claiming condom use was not necessary, "if you were with them for a couple of years or something, then you should pretty much know a lot about them." Yet effective contraception has been associated with partner support (Whitley, 1990), thus the length of the relationship may be relevant to acquiring trust, support, and hence safety. This distinction between regular and casual partners has been noted by other researchers to occur more often with young women than young men; and, with the belief that restricting sexual association to only regular partners is safe practice (Carroll, 1991; Crawford, Turtle, \& Kippax, 1990; Moore \& Rosenthal, 1991). Further, among adolescents, particularly young men, have been noted to be generally less monogamous than young women (Sonenstein, Pleck, \& Ku, 1991).

The majority of youth voiced positive sentiments regarding condom distribution, due to a sense of embarrassment in purchasing them, and claimed they would be more likely to use condoms if freely available. Embarrassment about condom purchase has been noted elsewhere as an impediment to using condoms (MacDonald, et al., 1990). In most cases, discussions about embarrassment associated with purchasing condoms were led by the young men, again with young women prefacing that it was the "man's job." However, this 
finding is contrary to the literature, where young men were found to be less embarrassed than young women about buying condoms (Helweg-Larsen \& Collins, 1994). Yet both genders supported distribution of condoms, particularly in school. Typical were statements like, "I think they should hand out condoms in school or have dispensers in bathrooms because I know a lot of people that have had sex and they didn't use a condom, ...they were embarrassed to go to the drugstore." Other young men added, "because they're going to do it any, like so why not have it protected?," "because if they are going to have sex at least they can have it protected... you can't say 'well you're too young' and then he goes out and gets some girl pregnant or gets a disease, that what's gonna happen." Youth of both genders added that teenagers "were going to do it anyway," that is, have sex, despite promotion of abstinence; several made facial gestures, grimaced or rolled their eyes discussing the fruitlessness of such promotion. Further, many focus group participants believed that teaching teens safe sex practices and providing condoms were not promoting sexual behavior: "people bring up that thing about condoms in schools, they're just trying to prevent people from getting diseases, I think. They're not trying to say, 'here's some condoms, go have sex." "But then if you don't tell them anything, like they could be really in worse trouble because they probably will go out and try it [sex] anyway and won't know what to do" Or as one sexually active 15-year-old young woman stated, "I strongly feel that if there are condoms there, like available to use, then they would be used like many percents more."

Issues such as peer or partner pressure, and/or popularity, were associated with sexual activity; however, an age differential was quickly recognized. Interestingly, engaging 
in sexual activity among junior high youth was associated with popularity moreso than for the older adolescents. As one 13-year-old young man put it, "if you want your friends to think you're cool, like it's cool to have sex." Young junior high school women agreed, one 13-year-old stated, "a lot of teenagers want to be cool and everything, and think it's cool to have sex, they are more popular because they brag a lot, and they are usually the leaders, so they're cool... there's a lot of pressure... on younger kids." Older teenaged women disagreed, “with seniors, it's not like cool or uncool... it's not labeled or anything, well yeah, they're labeled 'slut' or something, but it's not like you would want to be in that group of people." During discussions, it appeared as though peer and partner pressure appeared salient for all ages, "you might like to become popular so you might do it," "but really for here there's a lot of peer pressure around sex."

When the contrasting issue of "sex for popularity" was addressed further, teens in upper grades voiced an overall sense of individuality, while junior high youth, particularly young women, responded that they had sex, "to feel wanted... to feel that way with one person or to feel like wanted with the people in that group, or people in your class." It seemed that the importance of "belonging" was sought through sexual behavior by junior high youth of both genders, who had to deal with peer pressures for popularity, more often than senior high students. Senior high students, particularly young women, seemed to acquire a sense of belongingness in the mere association with peer groups with little need of utilizing sex as a means for popularity. Acquiring a "reputation," with descriptors such as 'slut' and 'tease,' were more common among senior high students, whereas popularity was associated with sexual activity with junior high youth. 
Jessor and Jessor (1975) found that sexually active youth differed from nonactive youth in having higher independence, lower values for achievement, greater social criticism and tolerance for deviance, and greater peer influence for problem behavior. Additionally, results were stronger for high school youth than college, and for young women than young men (Jessor \& Jessor, 1975). Similar findings were evident with group reactions in the current study, especially among young women with conformity and peer influence. In another study, nonactive teens anticipated a significantly greater amount of "feeling better" after first intercourse than sexually active teens reported actually feeling (Langer, Zimmerman, \& Katz, 1995). Further, among sexually active youth in the Langer, et al. (1995) study, "feeling better" was associated significantly with being male and having a greater number of sex partners. For nonactive youth, "feeling better" was significantly associated with placing less value on postponement of sex and being highly influenced by peers. The sentiments expressed by focus group participants in the present research appear to match these findings, and also support past research, where correlates of adolescent sexual behavior included curiosity, need for love, and peer pressure (Cullari \& Mikus, 1990). Nevertheless, further examination is needed on potential age and gender differences in teenage pressures to engage in sexual activity, as questions remain unanswered, such as the frequency and intensity of such external pressures, individual abilities to deal with such pressures, and the meaning and importance of group conformity.

Some participants claimed that the risks associated with AIDS were simply not thought about, emphasizing invulnerability along with a passive outlook among young women regarding assertiveness: “Teenagers just don't care... they wouldn't use a condom 
if a woman was on birth control, why should they if she's taking the pill?... or maybe she can't maybe her boyfriend won't agree to it [condom use], maybe she thinks it could never happen to her, but mostly because nobody ever really thinks about it." Barling and Moore (1990) have postulated that young women may require specific assistance in assertiveness toward partner communication, and specific skills of how to use a condom. Developing such skills may be instrumental in having adolescents be more cognizant of potential risks. Another 13-year-old young woman added, "I know people like when they were going to have sex they're not thinking about using condoms, or about AIDS, they're not thinking about it." These comments are consistent with research noting adolescents' sense of invulnerability to HIV infection (Baldwin \& Baldwin, 1988a; Bell, Feraios, \& Bryan, 1990: Bing, et al., 1990; Boyer \& Kegeles, 1991; Eiser, Eiser, \& Lang, 1990; Whalen, et al., 1994; Zimet, et al., 1991). Further, individuals who lack a sense of perceived vulnerability are unlikely to change biologically driven or socially appropriate behavior (Fineberg, 1988), which appears to be prevalent among youth.

Simple knowledge gaps may very well coincide with perceptions of invulnerability. Many youth believed AIDS was "not a problem in the country," "not really a problem in Connecticut," with another citing figures for AIDS cases in Connecticut as being "forty to fifty." One youth believed he could "get away from AIDS," by moving to Africa. Extensive knowledge gaps were evident with participants' inquiries at the close of group sessions. Of most concern were beliefs that there was a cure, how to get rid of it once infected, and questions about transmission through mosquito bites, kissing, saliva, and public toilets. Aside from private inquiries, evidence of AIDS misconceptions were noted in 
dialogue, as well. Several teens believed that if one looks healthy, they could not be infected, "She might not want to [use a condom] probably because the guy she is with... he really looks healthy, he probably doesn't have AIDS. Yeah, I was sitting with a guy once and I found out that he has AIDS, and I was like, no way, he looks so normal, I couldn't believe it!" Most provided descriptions of infected individuals, "like in the movies," and believed unless visually apparent otherwise, one is healthy. During a discussion about condom use assertiveness, another 15-year-old young woman talked of using "some other form of birth control," if the man refused to use a condom, not realizing the implications of contracting a STD, being concerned only with preventing pregnancy. One boisterous, 14year-old young man believed he had the solution to the AIDS pandemic, which was to give all men vasectomies, and some of the younger participants seriously entertained this idea as a viable solution. This latter point raises questions about youths' knowledge not only with AIDS transmission, but also regarding basic physiology, moral social issues and human rights. Other researchers have noted the "healthy looking" myth as being one difficult to dispel, and found youth still held beliefs that birth control pills provide protection against HIV infection (Anderson, et al., 1990), or AIDS is transmitted by insects bites (Gallup \& Gallup, 1988).

Many participants stated their solution was to keep sex a secret because, "we're taught that it is bad," "naughty," "dirty," and "sleazy." This appeared to be an impediment for youth to discussing matters with others, most especially adults. Typically adolescents stated they would discuss issues with peers rather than adults; however, many revealed that their peers often provided incorrect information. The success of intervention and 
prevention strategies utilizing peer educators may depend highly on adolescents' comfort and preference in discussing personal matters with peers. Feedback from focus group participants suggests informed peer educators may be useful in assertiveness training, particularly with role playing, not only by providing youth with educated informants, but more importantly, by reducing the spread of misinformation. The importance of peer education and the trust associated with prevention messages furnished by group peers, as well as the credibility of peer educators who are themselves at risk or infected, have been noted for teens (WHO, 1992; Rickert, Jay, \& Gottlieb, 1991).

Summarizing findings with the first hypothesis is difficult, as predictors of sexual assertiveness appear to be plagued with numerous confounds. With that in mind, clearly the unidimensional measures of sexual assertiveness utilized in this study did not adequately address some of the specific confounds adolescents brought to light. However some key points may be worth exploring more thoroughly in future research.

First, sexual assertiveness, or sexual negotiation skills, may be dependent on numerous situational and contextual factors. Sexually active young women, in particular, identified various degrees and intensity of emotions, and attachment to their partner, which impose on preference and ability to utilize assertiveness skills. Second, sexually active young women typically place a higher value on maintaining the relationship than on their own welfare, and often view sexual assertiveness behavior as a potential threat to the relationship. Third, assertiveness with regard to partner communication is often moot, as partners who are infected or have positive risk histories, are likely to be untruthful in their responses. Fourth, consistent with the literature, gender role expectations tend to interfere 
with sexual assertiveness, suggesting the need to empower young women to be able to resist pressure for unwanted sex, and negotiate safe sex behavior. Fifth, for adolescents, potential gender and age differentials exist with frequency and intensity of external pressures to engage in sexual activity, which may interfere with implementing assertiveness skills. Particularly for younger teens, the need to be popular, to feel wanted, or have a sense of belongingness may be associated with engaging in sexual activity at very young ages. Lastly, although it has been noted that knowledge alone is not sufficient to change behavior (Baldwin \& Baldwin, 1988a; Baldwin, Whiteley, \& Baldwin, 1990; Basch, 1989; Brandt, 1988; Brown \& Fritz, 1988; Clarke, Abram, \& Monteiro, 1990; DiClemente, Forrest, \& Mickler, 1990; Keller, Schleifer, Bartlett, \& Johnson, 1988; Ku, Sonenstein, \& Pleck, 1992; Stiffman, Earls, Dore, \& Cunningham, 1992; Rosenthal, Moore, \& Brumen, 1990; Skinner \& Ross, 1994), adolescent misconceptions, knowledge gaps and perceived invulnerability may be at least partially associated with barriers to sexual assertiveness abilities. Recently, other researchers have paid particular attention to human sexuality research on similar underlying factors, specifically, the importance of condom use in affectionate versus casual relationships, sexual communication and coercion, substance use in relation to sexual intercourse, and sexual schema and scripts relevant to HIV-risk (Kelly \& Kalichman, 1995).

With regard to sexually nonactive youth, it may be impossible to have these individuals accurately predict their sexual behavior because of their inexperience and their inability to: (1) appreciate the emotional aspects of an intimate context; (2) comprehend the potential interaction effects of their behavior with their partner's; (3) integrate potential 
external pressures with their future behavioral responses; and (4) consider possible negative consequences of implementing positive sexual assertiveness skills.

Keeping these confounds in mind, a summary of the quantitative findings for the first hypothesis is provided. For young women, the first hypothesis was partially supported; significant predictors of risk, indicated by low sexual assertiveness include: (1) high AIDS misconceptions; (2) high perceived susceptibility for risk; and (3) high substance use and abuse. For young men, low assertiveness was predicted by: (1) low perceived AIDS knowledge; and (2) high misconceptions. Although hypothesized, homophobia, factual and transmission AIDS knowledge were nonsignificant predictors of sexual assertiveness.

Hypothesis Two: AIDS Risk Predictors Among the Sexually Active. Predictors of AIDS risk, among the sexually active teens as defined by the number of sex partners and unprotected intercourse occasions, were examined in the second hypothesis. The hypothesis of higher risk with lower AIDS knowledge was only partially realized in analyses, with significant findings on only one cognitive component: high AIDS misconceptions. However, as hypothesized, nearly all other variables were found to be significant predictors of risk for at least one gender.

Separate gender analyses found significant risk predictors for sexually active young men to be high AIDS misconceptions, perceived susceptibility for risk, substance use and abuse, partner and self-risk histories, and low sexual assertiveness for condom use. For sexually active young women none of the four cognitive components were found to be significant predictors of AIDS risk, while high perceived susceptibility for risk, partner and self-risk histories, and low situational condom use efficacy and sexual assertiveness for both 
condom use and partner communication were significant. High homophobia was a significant predictor of AIDS risk with group analyses, but not for either gender in separate calculations, which may have been a consequence of small sample sizes.

Gender differences on these predictor variables were found particularly with higher misconceptions and substance use and abuse for sexually active young men, but not for women; and, low situational condom use efficacy and partner communication assertiveness for sexually active young women, but not for men. Pertaining to AIDS risk predictors, both genders perceived their susceptibility for risk to be high, had low condom use assertiveness skills, and reported high risk histories for both themselves and their partners. Individual risk variables, qualitative feedback and implications for gender differences are discussed more fully below.

Regarding AIDS misconceptions, several fallacies prevailed. On the questionnaire, for instance, over half of the entire sample were not sure or believed a new vaccine had been developed which can prevent AIDS, and over half were not sure or believed mosquitoes could transmit AIDS (see Table 4). Additionally, over one-quarter of the sample believed they could avoid AIDS by having sex with healthy looking people, and over one-quarter believed that only gay people and needle-users can get AIDS. Many studies continue to find misconceptions among adolescents (e.g., Bertrand, Brown, Kinzonzi, Mansilu, \& Djunghu, 1992; DiClemente, Forrest, \& Mickler, 1990; DuRant, Ashworth, Newman, \& Baillard, 1992; Fennell \& Beyrer, 1989; Stevenson \& Stevenson, 1990; Sugerman, Hergenroeder, Chacko, \& Parcel, 1991) as well as among adults, especially with regard to transmission (Hardy, 1990). Since sexually nonactive youth reported a 
significantly lower average of misconceptions in comparison to sexually active youth, it is clear that the majority of youth reporting inaccuracies were among the latter group. Similarly, focus group participants confirmed continued prevalence of AIDS misconceptions, as mentioned above in discussion of the first hypothesis. Since sexually active young men had the highest misconceptions of all subgroups by gender and sexual activity, it is not surprising that this factor would be a notable predictor of risk for them. These findings are consistent with many studies in the literature showing that areas of confusion, especially in transmission and prevention of infection, continue to prevail among youth (e.g., Livingston, 1991; Memon, 1990; Ponton, DiClemente, \& McKenna, 1991).

High perceived susceptibility for risk, or inability to avoid risky situations, was a significant predictor of AIDS for both sexually active young men and women. In questionnaire format, nearly half of the entire sample were not sure or thought they were probably or definitely exposed to AIDS, one quarter were not definitely sure they could do things to help protect themselves from getting AIDS, and one third were not positive they had not had sex with someone who could have given them AIDS (see Table 7). Since these latter calculations included both sexually active and nonactive youth, the percentages are likely to be much higher when accounting for only sexually active teens. Acknowledgment of their perceived susceptibility to risk is contrary to some recent research. Teenagers typically minimize their perceived risk of experimental and occasional involvement in healththreatening activities, more so than adults; and, teenagers are less optimistic about avoiding illness than their parents (Cohn, MacFarlane, Yanez, \& Imai, 1995). Other researchers recently found that heterosexual college students who are more "future oriented," were 
more likely to use HIV-risk reducing methods such as inquiring about a partner's sexual history, and delaying or abstaining from sex, as opposed to those were who more "present time oriented," having a greater propensity for immediate gratification (Rothspan \& Read, 1996). Thus, many adolescents in the present study may fall in the latter category, partially explaining high susceptibility, minimizing the same, and associated risky behavior.

Comments from focus group participants shed some light on the breadth and depth of risk perceptions.

Feedback from progressive "risk" (purposely undefined) situations in focus group discussions revealed that most adolescents would "risk" sneaking out of the house to meet friends against parents' wishes, without much hesitation. But sneaking out to meet friends with alcohol was a dilemma for approximately half of the younger, junior high participants, but not for older teens, representing a relatively mild "risk." Adding other drugs to the scenario posed a fairly high level of "risk" for junior high youth, although many rationalized specific types of drugs would be acceptable, as long as the meeting place was not in their own home. At this point, most adolescents defined "risk" by likelihood of getting caught by parents, and being punished as a consequence. Last, participants provided feedback on "risks" involved if a teenager was home alone with a partner and they decided to become intimate. Most admitted this scenario was "most risky," and again focused on issues of getting caught by parents, length of relationship prior to sex, peer and partner pressure, pregnancy and AIDS, but stated teens, "would probably have sex anyway." Further, pregnancy was cited more frequently as a risk and concern, than AIDS. With regard to assessment of decision-making influences to engage in sexual activity, concern for 
contagion was hardly mentioned. Rather teens expressed: love or "degree of like," substance use, persuasion, peer pressure, and trust. Only when probed further by the group leader, did youth mention risk of HIV infection, but stated, "most [teens] don't think about condoms." These and other references teens voiced (e.g., condoms not needed when woman is using oral contraceptives), support findings that condoms are often viewed as contraceptives, and not prophylactics among youth (Noell, et al., 1993; Maticka-Tyndale, 1991).

Overall, youth verbally supported quantitative findings of adolescents having high perceived susceptibility for risk, or perhaps inability, or little desire to avoid risk situations. Although the risk susceptibility appeared to be "perceived" in focus groups, it was not in the conventional sense. That is, personal risk seemed to be overshadowed by "being caught by parents," and consequently, punished; whereas, risk for disease, and possible death secondary to substance use or unprotected sex, seemed to be hidden in perceptions of invulnerability. This adolescent personal invulnerability, and minimizing level of susceptibility appears to be consistent with the literature (e.g., Burger \& Burns, 1988; Hernandez \& Smith, 1990; Hochhauser, 1989; Leishman, 1987). These findings may support Rothspan and Read's (1996) “present time oriented" classification mentioned above, with teens focusing on immediate gratification, being less concerned with future consequences of their behavior. Further, "future oriented" consequences, if acknowledged at all, are short-term associated with punishment, whereas longer term consequences are not recognized. Other researchers found cruising, or driving around with no particular destination as a noteworthy in significantly increasing the likelihood of sexual activity 
(Alexander, et al., 1989). Such activities were not explored in the current study, and may provide valuable data regarding adolescents' perceptions and perceived abilities to handle various situational risk situations appropriately.

Supporting previous findings (Lang, 1994), substance use and abuse were significant predictors of risk for sexually active young men, who reported higher levels of substance use and abuse than their female counterparts. Other researchers have found substance use lower among young women than young men (Vanderschmidt, Lang, Knight-Williams, \& Vanderschmidt, 1993). Specific to substance abuse, 14 percent of the entire sample (active and nonactive, both genders) reported they had thought about or tried to kill themselves secondary to drinking or drug use in the past year; and 14 percent had experienced blackouts in the past year (see Table 16). Among the sexually active, 33 percent indicated at least one occasion of having sex that they wouldn't have engaged in, if they were sober in the last year. Again, the significant differences between sexually active and nonactive youth in reports of substance use and abuse leads to the conclusion that the majority of heavy users with possible serious implications were among the sexually active. Additionally, three-quarters of sexually active substance users reported using alcohol prior to having sex during the past year, increasing their likelihood of unprotected sex. This was deduced by examining reports of sexually active substance users, where 97 percent were having sex with inconsistent or no condom use, and three-quarters of those youth admitted to using alcohol prior to having sex in the past year. This evidence supports other findings that substance use, especially prior to sexual activity, will increase the likelihood of high-risk behaviors (e.g., Clapper \& Lipsitt, 1991; Grant \& Johnstone, 1990). Other researchers also 
suggest that having a greater number of sex partners and intoxication preceding sexual activity are likely predictors of vulnerability to risk behavior relapse (Kelly, St. Lawrence, \& Brasfield, 1991).

Focus group feedback provided supplemental data with regard to using substances in conjunction with sexual activity, with disinhibition and impaired judgment, as well as persuasion tactics, "they wouldn't know what's going on if they were drunk," "well if they're drunk... they'll just say they're in love." Additionally, group participants identified teenagers as a potential risk group for AIDS because, "they're experimenting," "too much drinking and stuff," and "they're sexually active and drinking." Thus substance use not only reduces disinhibition thereby increasing high risk behaviors (Kelly \& St. Lawrence, 1988), but may also increase the likelihood of untruthfulness with potential persuasion and pressure to have a partner succumb to sexual activity.

The significance of homophobia was evident as a predictor of AIDS with the sexually active group as a whole, but disappeared with separate gender analyses. One explanation may be decreased power in the analyses attributable to smaller sample sizes. Nevertheless, further support for recent research associating high risk behavior of heterosexuals with the assumption that AIDS is a "gay disease" (Burkholder, 1994) was evident in both quantitative and qualitative findings. Item analyses with the entire sample found that half of the respondents reported they would not like it if a person with AIDS was in their gym class; nearly three-quarters reported they would feel nervous in a group of gay people; and over three-quarters reported they would feel uncomfortable if they saw same genders holding hands (see Table 7). Most of these responses were clearly from sexually 
active youth since these teens had significantly higher indicators of homophobia than nonactive youth.

In focus groups, youth discussed perceptions about gay people, stereotypic behavior, and potential changes in attitude within four scenarios ranging in interpersonal distance. The first scenario involved a student, second a best friend, third a sibling, and finally a parent. Although well over two-thirds of the participants revealed no indications of homophobia when speaking in generalities, as scenarios became more personal, or "closer to home," many contradicted earlier statements, and in some cases, retracted them altogether. Some inaccuracies were evident, describing stereotypical behavior they viewed on television, the way they "act," "walk," and "talk." Approximately three-quarters of focus group participants stated that AIDS was not a "gay disease;" however, at least a dozen individuals were surprised to find out heterosexuals could be infected: "Yeah, gays and druggies get AIDS from needles, I don't have to worry." Several believed AIDS was not "really a problem in Connecticut," that "Connecticut didn't have gay people," and that AIDS is more a problem "over there." Where "over there," was defined as other states, "probably New York and California." One 13-year-old preached of religion, the Bible, and implications of AIDS in a small group setting, "God said it shouldn't be so, because in the Bible it says that people shouldn't be gay... only gay people have bad things happen to them, it says that in the Bible..., being gay is bad and they're the cause of all bad."

Changes in perceptions, attitude and affective responses were evident in scenario discussions, as responses became increasing abstract with the common descriptor of "weird" repeated, with an inability to elaborate further. With reference to reactions to a gay 
peer in school, many stated they would attempt to avoid the person, afraid others may assume they were also homosexual if seen with them. Nevertheless, most stated they would be cordial, but distant. Gray and Saracino (1991) found similar reactions with college students who reported inconsistencies between accepting attitudes toward people with AIDS and personal involvement. Some youth had perceptions that homosexuals could not control their behavior, and feared being in danger of molestation or rape, "I wouldn't sleep over his house or anything because like he might rape me," "you don't know what they're going to do, I wouldn't want them hitting on me." In the case of a best friend, a sense of uncomfortableness was common, however, most stated they would "try" to remain friends. Interestingly, when the scenario moved to a sibling, some adolescents were still concerned about siblings "making a pass," or "hitting" on them. Increased uncomfortableness was associated with a perceived threat to their safety, believing homosexuals as being "obsessed" with sex. In the final scenario, having a gay parent, many adolescents expressed feelings of embarrassment, betrayal, nausea, and expectation of moving away with "the straight one."

Significant risk histories for both self and partner(s) were also predictive of AIDS risk among sexually active young men and women. Both genders reported moderate certainty of not being exposed to AIDS, and moderate certainty that their partner(s) had not been exposed. However, active young women reported significantly higher estimates of number of sex partners their most recent partner had had, than young men ( 3 versus 2 , respectively). Partner-risk history was fairly equal between the genders. Pertaining to selfrisk history, it is likely that an early sexual debut would contribute to an increase in sexual 
activity and number of partners, and thus a greater risk; this is supported in the literature (e.g., DuRant, Sanders, Jay, \& Levinson, 1988; Ekstrand \& Coates, 1990). DiClemente, et al. (1992) found that adolescents with a history of three or more sex partners were half as likely to use condoms consistently. In the current study, young men had onset of sexual activity approximately one year earlier than women (age 13 versus 14, respectively), and had an average of one more lifetime sex partner than young women (4 versus 3 , respectively). Another study found that young men not only had significantly more sex partners than young women, they more often stated that they "definitely intended" to have multiple intercourse partners ( 25 versus 2 percent, respectively; Walter, et al., 1993). Also contributing to self-risk history was frequency of substance use prior to sex, which, as mentioned above, was reported by the majority of sexually active youth. More sexually active men reported having one or two drinks prior to sex, than young women (51 versus 42 percent, respectively), contributing to self-risk for AIDS.

Low situational condom use efficacy was found to be a significant predictor of AIDS risk for sexually active young women, but not for men. Whether young women are depressed, angry, feeling pressured to have sex, have been using substances, etc., may have little or no bearing on condom use efficacy if the decision to use condoms is solely placed on their partners, for reasons indicated in the sexual assertiveness discussion in hypothesis one above. Several women stated in focus groups that condoms were "the male's responsibility," supporting other findings that young women tend to be more compliant toward societal norms traditionally placing precautionary responsibilities on young men, 
particularly buying, carrying, and initiating condom use (Barling \& Moore, 1990; Moore \& Rosenthal, 1991a).

Low sexual assertiveness for condom use was found to be a significant predictor of risk for both genders. For sexually active young men one explanation may be a lack of desire to use condoms, since young women are generally reporting compliance to their partner's sentiments. This would make sense given mean scores found in the current study. Although sexually active young men surpassed women in assertiveness skills for condom use, the mean response was disconcerting, being slightly less than "usually" for young men and "about half the time," for young women. Gender aside, the significance of low assertiveness skills for condom use predicting AIDS risk is apparent, as frequent unprotected sex has been identified as a behavioral risk factor in numerous studies of teenage sexual behavior (e.g., Boyer \& Kegeles, 1991; DiClemente, 1990; Rotheram-Borus \& Koopman, 1991).

Finally, low sexual assertiveness for partner communication appeared as a significant predictor of AIDS risk for sexually active women, but not for male counterparts. Given young women's hesitancy to discuss sexual matters with their partners (at least in the context of heterosexual dyads), or to risk negative consequences of potentially disrupting the relationship, empowerment for self-protection seems relevant to reduce AIDS risk among young women (see discussion of hypothesis one).

In summary, the second hypothesis was supported with sexually active youth, and partially supported with each gender. For sexually active youth, AIDS risk, as defined by a greater number of sexual partners combined with a greater number of unprotected sex 
occasions, was predicted by: (1) lower AIDS knowledge, particularly having higher misconceptions about AIDS; (2) higher perceived susceptibility for AIDS risk, or inability to avoid risky situations; (3) lower situational condom use efficacy; (4) higher homophobia; (4) higher use and abuse of substances; (5) higher risk histories for both self and their partner(s); and, (6) lower sexual assertiveness, particularly for condom use abilities.

Individual examination of predictors of AIDS risk for sexually active young men and women found that high perceived susceptibility for risk, high self- and partner-risk histories, and low assertiveness skills for condom use, were significant predictors for both genders. However, gender risk differentials for predicting high risk behavior were found among other variables, where: (1) high AIDS misconceptions was significant for sexually active young men, but not for women; (2) low situational condom use efficacy was significant for young women, but not for men; (3) low partner communication assertiveness was significant for young women, but not for men; (4) high substance use and abuse were significant for young men, but not for women; and (5) high homophobia was significant for the sexually active group, but not for either gender, individually.

Hypothesis Three: Participants' Estimated AIDS Risk Level. In order to examine which factors adolescents take into account when rating their own level of AIDS risk, several potential predictors were examined within the third hypothesis. As hypothesized, high substance use and abuse, self- and partner-risk histories were significant contributors to adolescents' self-rated risk. Low homophobia was significant, although hypothesized to be contributory in the other direction. None of the four knowledge components (factual, perceived, transmission, and misconceptions) had a significant bearing on estimated risk 
level, nor did perceived susceptibility for risk, situational condom use efficacy, or sexual assertiveness.

Separate analyses by sexual activity and gender provided no additional insight to adolescents' reasoning for rating themselves in the manner in which they did. For sexually active young men, only low substance abuse contributed to their self-rated level of risk, and for sexually active young women, high self- and partner-risk histories and high substance use and abuse contributed to the same. Teasing out which variables contributed to level of risk seemed impossible due to several confounds. Confounds were mainly discrepancies with youths' reported unsafe sexual activity and level of risk; the associated accuracy or inaccuracy; and, over- versus underestimating self-rated risk levels.

Discrepancies between reported risky behavior and self-rated risk level prevailed as it did in recent findings (Lang, 1994). Over one-third of the entire sample indicated inaccuracies either by over- or underestimating their AIDS risk level. Of most concern were underestimaters, who claimed to be at "no risk," which comprised nearly 40 percent of the sexually active youth, reporting inconsistent or no condom use. One explanation may be an optimistic bias as noted by other researchers (van der Velde, van der Pligt, \& Hooykaas, 1994). Perceived risk for others was typically higher than perceived risk for self -- adolescents simply are not personalizing their risk of AIDS. Freimuth, Edgar and Hammond (1987) also found high risk adolescents in their sample rated themselves at the lowest end of the risk continuum. Yet, Neuwirth and Dunwoody (1989) found two groups of "high risk estimators" in their sample: those with low, and those with high personal efficacy. Both had low partner communication skills but differed in condom use. Ironically, 
those with high risk estimates, low efficacy, and low communication had the greatest increase in condom use (Neuwirth \& Dunwoody, 1989). Reasons for low risk ratings among another sample of behaviorally high risk teens were implicit trust in their partner(s), current monogamy, and lack of intravenous drug use (Overby \& Kegeles, 1994). These issues were discussed at length by focus group participants in this study, and may partially explain such discrepancies between reported risk behavior and perceived risk level. Yet more research is needed in this area.

Accuracy in one's self-rated risk level was examined as a contributory factor of one's perceived current level of AIDS risk. For sexually active adolescents who reported "unsafe" sexual activity, specifically inconsistent or no condom use, and who also rated themselves accordingly (other than "no risk"), significant predictors of their self-rated risk level included having low AIDS misconceptions and low homophobia. Therefore it was hypothesized that the reverse must hold true for sexually active, unsafe group with inaccurate self-rated risk levels. That is, the inaccurate group would have significantly higher misconceptions and homophobia than the accurate risk self-raters. However, this was not the case with self-rated level of risk comparisons of accurate versus inaccurate (or "underestimaters").

The accurate, unsafe group had significantly higher AIDS misconceptions than the underestimaters, and no significant difference between the two groups was found for homophobia. Thus misconceptions (such as believing AIDS can be cured if treated early, a vaccine exists to prevent AIDS, mosquitoes transmit AIDS, healthy looking people do not have AIDS, only gay and needle-users can get AIDS), may have no bearing on inaccurate 
perceptions of risk. This is contrary to some findings where the association between AIDS and homosexuality has resulted in inaccurate perceptions of risk (e.g., Paxton \& Susky, 1988). Nevertheless, whether these misconceptions have been challenged in school, home or other settings, and these youth choose to continue to believe them, or whether they hold these fallacies due to lack of informational resources, is unknown, and may be a significant factor worth pursuing in future research. It may be that accurate risk estimators are aware of their misconceptions and choose to consciously retain them, and underestimaters of risk may correct distortions as new information is provided, which would partially explain the higher AIDS misconceptions within the former group.

Regarding homophobia, although significantly low levels of homophobia were found for accurate self-raters which contributed to their self-rated risk level, the underestimaters also had low homophobia. Both groups reported slight uncomfortableness and stereotyping, but this had no bearing on underestimating level of risk. It may be that this slight homophobia is enough to contribute to unsafe, sexually active teens accurately placing themselves at some level of risk, following Burkholder's (1994) finding that individuals with homophobia falsely believe protection is unnecessary because AIDS is a "gay disease." Further, the unsafe, sexually active underestimaters simply ignore all or most factors, possibly feeling invulnerable to infection, evidenced by discrepancies between their unsafe behavior and level selection of "no risk." Examining differences between sexually active young men and women, discussed more under hypothesis four, sexually active young men significantly exceeded women, with higher levels of homophobia. Statistical analyses of gender differentials within the inaccurate, underestimaters subgroup were not possible 
due to the small sample size ( 21 young men and 26 young women). Thus it is possible that a gender confound exists; further research is needed in this area.

Further group comparisons also found that the accurate, unsafe group had perceived their susceptibility for risk as being significantly higher, had significantly higher substance use and abuse, had significantly higher risk histories for both partner and self, and had significantly lower sexual assertiveness skills for condom use than the unsafe underestimaters. Perhaps the accurate self-raters were simply more realistic in their choice level, having more areas of potential risk apparent, and being more aware of their personal risk, while the underestimaters have not yet become aware of, or deny potential risk habits, providing further support for the perceived invulnerability hypothesis.

Summarizing findings from the third hypothesis, it was found that determining which factors contribute to adolescents' perceptions of their present level of risk is extremely complex. Analyses of predictors of adolescents' self-rated level of risk are uninterpretable without taking into account subgroup classifications. Several factors must be considered in examining predictors of self-rated risk, including the accuracy versus inaccuracy of selfrated risk level, based on reported risk behavior, the direction of the inaccuracy, and the individual's gender.

Focusing on sexually active, unsafe youth, it was found that: (1) low levels of ADS misconceptions and homophobia contributed to accurate self-ratings of being at some level of risk; (2) the reverse, or high levels of AIDS misconceptions and homophobia, were not found among youth underestimating their risk as "no risk;" (3) accurate raters of self-rated risk had higher AIDS misconceptions; higher perceived susceptibility for risk; lower 
condom use sexual assertiveness; higher substance use and abuse; and higher risk histories for self and partner(s) than underestimaters of risk; and (4) given the above, it is likely that accurate raters of self-rated risk level are more aware of their knowledge, attitude and behavior, whereas underestimaters may ignore or deny the same and/or perceive themselves as being invulnerable to infection.

Hypothesis Four: Gender Differences in AIDS Risk Predictors. In the fourth hypothesis, it was predicted that among sexually active teens, young men would have higher substance use and abuse than young women, and report higher AIDS risk behavior, as defined by multiple partners and unprotected sex. Further, it was hypothesized that sexually active young women would report lower sexual assertiveness skills than their male counterparts. Consistent with previous findings (Lang, 1994), these gender differences were significant in the current study. Not only did the sexually active young men significantly exceed women in reported substance use and abuse, and sexual assertiveness skills, they also perceived their susceptibility for risk as higher, had higher homophobia, and reported their personal risk histories as higher, than sexually active women.

Although not predicted a priori within the above hypothesis, another potential risk factor for sexually active young men was a significantly lower AIDS knowledge base than young women. Other researchers have found young women to be more knowledgeable about AIDS than young men (e.g., St. Lawrence, 1993), although they are not insistent with partners about using condoms (Goodman \& Kohall, 1989). Sexually active young women exceeded young men in factual AIDS knowledge, perceived their AIDS knowledge as higher than young men did their knowledge, and had significantly lower AIDS 
misconceptions than young men. Despite conclusive findings that knowledge alone will not influence behavior change, knowledge and education, together with assertiveness skills, and empowerment to utilize these skills, backed up by health and social services and a supportive environment, are influential to safe sexual behavior (e.g., WHO, 1990, 1992). Thus sexually active young women's high AIDS knowledge may be one of many other reasons why their risky behavior is lower than their sexually active male counterparts.

Sexually active young men revealed differences in the attitudinal domain from young women, particularly with a higher perceived susceptibility for risk, and higher levels of homophobia. Gaines, et al. (1988) found many negative feelings toward persons with AIDS among older adolescents, less so among young women than young men; those findings were partially supported with the current study. Nevertheless, there is little research on theories accounting for gender differences in these areas pertaining to adolescents, thus only tentative assumptions can be made herein.

Adolescents must first feel personally vulnerable to acquiring AIDS, before steps toward reducing risk can be initiated (Boyer \& Kegeles, 1991). It may be that sexually active young men do not feel vulnerable. Invulnerability is associated with a lower frequency of avoiding risky situations, as measured by a high perceived susceptibility for risk. Based on focus group feedback, it may be that young men are less likely to avoid or remove themselves from risky situations than young women, or perhaps they simply lack the desire to avoid risky situations, based on perceptions of invulnerability. For instance, one 13-year-old young woman inquired whether it was true that women can contract HIV more easily than men. Approximately one half of group participants were familiar with this 
finding, but about a dozen youth erroneously assumed young men could not acquire AIDS from young women. Most of these young men believed they could only contract AIDS from another man, but could transmit it to a woman. Additionally, ample feedback was provided from focus group participants of both genders regarding the high likelihood of young men being untruthful about their risk histories, serostatus or STD infection, or even their emotions, "just to have sex." This untruthfulness among young men to gain sex has been noted elsewhere in the literature (Cochran \& Mays, 1990; Keeling, 1989). Hence one theory may be an association of invulnerability with inability to avoid risk situations; however, "inability" may be a misnomer for young men, in the typical context of a research variable. That is, ability may be present, but the situation is not recognized as being of potential risk, or the desire to avoid a potential risky situation is absent; both due to perceptions of invulnerability. On the other hand, estimating oneself to be at greater risk, and believing that safer sex is not an expected norm within one's peer reference group, are factors that have been strongly predictive of risk through unsafe sex (Kelly, et al., 1995), which also may account for the findings in the current study among young men.

Possible perceptions of personal invulnerability, together with findings of lower AIDS knowledge among sexually active young men, may also account for the gender differential for homophobia. Sexually active young men had significantly higher misconceptions statistically, and though infrequent, more often reported misperceptions of risk populations in focus groups than young women (e.g., a gay disease, a drug user disease, cannot be infected via sexual contact with women). Again, it is difficult to determine whether correct information has been provided to these young men, and they 
chose to ignore it in order to rationalize their invulnerability; whether incorrect information is being emphasized as being accurate (e.g., school, home, peers, media, etc.); or whether no information has been provided.

Given the potential explanations and qualitative support provided for the first and second hypotheses, it is not surprising to find that sexually active young men had significantly higher sexual assertiveness skills for both partner communication and condom use than women. As mentioned above, sexually active young women report condom use typically as being the man's responsibility, often feel constrained and pressured with various situational and emotional factors, perceive numerous negative consequences of assertiveness, and report a lack of trust and honesty, with reference to partner communication. Other researchers found the strongest predictor of condom use for young women was having asked a partner to use one, although the majority of young women were in need of acquiring the social skills necessary to negotiate condom use with partners (Weisman, et al., 1989). DuRant and Jay (1989) found that contraceptive behavior is poorest among heterosexual couples where the young man has higher self-esteem than the young woman, and the young woman perceives a great deal of pressure from her partner not to use condoms.

Sexually active young men had significantly higher substance use and abuse than young women, which was a predictor of high risk behavior in this study. A higher self-risk history was associated with substance use and abuse, particularly in use of substances prior to having sex, and young men had significantly higher self-risk histories, and used substances prior to sexual activity more often than young women. Significantly more young 
men reported alcohol and cannabis use prior to sex than young women (44 versus 34 percent for alcohol, and 22 versus 17 percent for cannabis, respectively). Additionally, as mentioned above in the second hypothesis, measures of self-risk histories for young men were increased significantly by having a lower age of sexual debut, and a higher number of sex partners, than young women. These results support other findings that correlated age of first alcohol use and age of first sexual intercourse (Flanigan, 1990; Leigh, Schafer, \& Temple, 1995; McLean \& Flanigan, 1993), with higher incidence among young men than women, especially in relation to their first sexual encounter (Kraft, Rise, \& Traeen, 1990; Robertson \& Plant, 1988).

Behaviorally, sexually active young men reported a significantly higher AIDS risk, more specifically, multiple partners and unprotected sexual activity, than sexually active young women. This finding logically follows suit, since sexually active young men had lower knowledge, more negative attitudes, and higher behavior risk predictors. The increasing number of teenagers engaging in sexual activity has a cumulative impact on the number of sex partners (CDC, 1990); and the earlier that sexual activity occurs, the longer the interval of exposure to different sex partners (Cates, 1991).

Although no hypotheses were made $a$ priori, analyses were conducted for gender differences among the sexually nonactive youth. As with the sexually active group, nonactive young women exceeded young men in factual AIDS knowledge, although both groups reported fairly high mean scores. Nonactive young men perceived their susceptibility for risk as higher than young women, and predicted their situational condom use to be lower than young women. Contrary findings were realized with sexual 
assertiveness in comparison to the sexually active group. Nonactive young men had predicted their sexual assertiveness skills for both partner communication and condom use to be significantly lower than nonactive young women, although for both genders, nonactive adolescents' predicted assertiveness scores were very high. Interpretation of these data is cautioned due to group inequality and low numbers of nonactive men. It is interesting, however, that sexually nonactive young women appear to be significantly more confident in their future sexual assertiveness skills, and condom use across varying situations compared to young men, but the reverse holds true once becoming active. Qualitative data provided in the first hypothesis support nonactive young women's interpretation of sexual situations as simplistic and concrete (e.g., "just say, "No,"” "just leave," "just dump him," etc.), while active women emphasize their behavior depend on numerous interpersonal and emotional conditions that nonactive youth cannot account for given limited experiences. Deiter (1994) found that sexual assertiveness decreased with a 5-week intervention among college women, which was attributed to an optimistic bias in women's initial reports. At postintervention, these women recognized numerous difficulties involved in assertiveness, which they were not cognizant of at pre-intervention, thus their lower assertiveness scores reflected a more realistic view.

Summarizing, the fourth hypothesis was supported. First, sexually active young men had higher AIDS risk than young women, significantly exceeding young women with: (1) lower factual AIDS knowledge; (2) lower perceived AIDS knowledge; (3) higher AIDS misconceptions; (4) higher perceived susceptibility for risk, or inability to avoid risky situations; (5) higher homophobia; (6) higher substance use and abuse; and, (7) higher self- 
risk history for AIDS. Second, sexually active young women had lower sexual assertiveness for both condom use and partner communication than active young men.

Additionally, data on gender differences among the nonactive reveal that young women have significantly: (1) higher factual AIDS knowledge; (2) lower perceived susceptibility for risk; (3) higher predicted situational condom use efficacy; and (4) higher predicted sexual assertiveness skills for both partner communication and condom use, than young men.

\section{Hypothesis Five: Sexually Active Versus Nonactive Group Differences. As} predicted in the fifth hypothesis, group differences were found between sexually active and nonactive adolescents across all domains of interest. Cognitively, nonactive teens reported significantly higher factual AIDS knowledge, and significantly lower misconceptions about ADDS. No group difference was found specific to ADS transmission knowledge, with both groups reporting moderate knowledge regarding AIDS transmission. Interestingly, perceived ADS knowledge also had a nonsignificant group difference, with both groups perceiving their AIDS knowledge as fairly high. Nonactive teens also reported significantly lower homophobia, and perceived their susceptibility for risk as lower than sexually active youth. Behaviorally, nonactive youth had significantly lower substance use and abuse and lower partner and self ADS risk histories than sexually active youth. These findings are not surprising, since the majority of these variables were found to be significant AIDS risk predictors within the second hypothesis, therefore it is understandable that sexually nonactive youth would have more desirable mean scores. 
As might be expected, when comparing sexually active youths' reported actual behavior to the nonactive youths' estimated or predicted behavior, the latter group fared well in all areas. Nonactive youth predicted their condom use efficacy as higher, and predicted their assertiveness skills for partner communication and condom use to be higher than actual behavior reported among sexually active teens. Such firm convictions of predicted behavior on the part of nonactive youth provided for interesting debates with focus group participants when challenged by sexually active teens. More often than not, sexually active youth denounced nonactive teens' seemingly innocent perceptions and suggestions, replying, "That's what I thought, too, until I was in that situation." Interestingly, such challenges enticed sexually nonactive focus group participants to come up with, as one participant put it, "more realistic," solutions to posed dilemmas, as they "never thought about" several confounding variables. Questionnaire responses might have been "more realistic," if sexually nonactive focus group participants completed measures after attending discussion groups, rather than before.

Analyses of group differences among the sexually active youth between "safe," or consistent condom users, and "unsafe," or inconsistent or no condom use, were undertaken, although no a priori predictions were made. Group differences were found only within the attitudinal domain, with all three subscales being significant. Safe youth perceived their susceptibility for risk as lower, had higher situational condom use efficacy, and had lower homophobia than the unsafe adolescents. No significant differences were realized with cognitive components, with both groups reporting high factual AIDS knowledge, moderate transmission ADS knowledge, moderately low AIDS misconceptions, and accurately 
perceiving their AIDS knowledge to be fairly high. Similarly, no group differences were found with sexual assertiveness skills, with both safe and unsafe youth having moderate indicators for both partner communication and condom use. Nor were group differences found with substance use and abuse, which were both moderately low. Both groups were similar with low partner-risk histories, and moderately low risk histories for self.

Nevertheless, given the extremely small number of youth in the "safe" category $(n=6)$, interpretation of safe versus unsafe results should be cautioned.

One insight provided with the attitude differences between the safe and unsafe sexually active youth is a potential linear association between consistent condom use, across varying circumstances, and low perceived susceptibility for risk. Specifically the avoidance of risky situations is likely increased and one's perceived susceptibility for risk is likely lowered based primarily on the consistency of using condoms. Thus these safe participants are less apt to be concerned about potential exposure experiences and hold the attitude that they can protect themselves given their consistent behavioral history. The lower homophobia among consistent condom users, compared with unsafe teens, might also be partially explained by the former group having a more accurate perception of personal vulnerability and potential risk with regard to sexual situations; thereby taking appropriate precautions. The safe youth would be less likely to attribute AIDS to be "a gay disease," and be less likely to feel a need to avoid homosexuals as a basis to reduce risk of contracting AIDS.

In summary, the fifth hypothesis was supported. Sexually nonactive youth had less risk for AIDS than active youth, evidenced by: (1) higher factual AIDS knowledge; (2) 
lower AIDS misconceptions; (3) lower perceived susceptibility for risk, or higher ability to avoid risky situations; (4) lower homophobia; (5) lower substance use and abuse; (6) lower self and partner-risk histories; (7) predicted their situational condom use efficacy to be higher; (8) and predicted their sexual assertiveness to be higher, than sexually active youth. Additionally, differences were found between the sexually active safe and unsafe groups, where the unsafe youth had: (1) higher perceived susceptibility for risk, (2) lower situational condom use efficacy; and (3) higher homophobia than the safe, or consistent condom users.

Interaction Effects Among Risk Predictors. Interaction effects between gender and sexual activity were found with several risk predictors. AIDS misconceptions appear to be highest for sexually active youth, particularly young men, and lowest for nonactive youth, again, more so for young men. Perceived susceptibility for risk was also a function of gender and sexual activity, being highest for sexually active youth, with young men reporting the highest perceived risk susceptibility. Likewise, homophobia was highest for young men, within both the sexually active and the nonactive subgroups. It may be that having fewer misconceptions about AIDS, low homophobia, adequate self-confidence to avoid risky situations, and low perceived risk susceptibility, all assist in the decision-making process of postponing sexual activity. Furthermore, young women may surpass young men in these abilities, having more desirable knowledge and attitudes.

Behaviorally, several interactions were found between gender and sexual activity. Young men typically had higher substance use and abuse than young women, particularly sexually active young men. Further, sexually active youth had significantly higher substance 
use and abuse than sexually nonactive teens. As pointed out in the second and fourth hypotheses, several associations have been made between substance use and sexual activity, and typically young men have higher frequencies and intensity of both behaviors.

Sexual assertiveness skills for both partner communication and condom use, as well as high risk histories for both partner and self, were a function of gender and sexual activity. Given that sexually nonactive youth had negative risk histories, the latter two variables seem rather immaterial. As mentioned briefly above, a complete reversal of the sexual assertiveness skills deserves some attention. It appears that sexually nonactive young women have clear perceptions of their abilities at a fairly young age, where their male counterparts are more hesitant in giving themselves full credit for predicted sexual behavior. Yet, once sexually active, young women appear to lose abilities they once were sure they would have, while young men maintain a relatively stable outlook from nonactivity to becoming active. One explanation may be the importance young women place on maintaining the relationship, mentioned in the first hypothesis. The marked change in the sense of self, confidence in beliefs and abilities in preadolescent young women in the context of relationships has been noted with regard to gender role stereotypes and the potential effects of differential socialization in the interpersonal realm (e.g., Gilligan, 1979, 1982; Miller, 1986; Stern, 1990). Developmental feminist theorists have described the extreme importance women place on relationships, particularly in adolescent development, and the major conflict women face when entering adulthood in Western culture, “...the deeply knotted dilemma of how to listen both to herself and to the tradition, how to care for herself as well as for others..." (Gilligan, Rogers, \& Brown, 1990, p. 328). Thus, sexually 
nonactive young women may easily believe their own welfare is primary, until faced with the dilemma of pleasing a partner at the risk of one's own safety.

Overall, gender and sexual activity interactions were found with: (1) AIDS

misconceptions; (2) perceived susceptibility for risk; (3) homophobia; (4) substance use and abuse; (5) risk history for self; and (6) sexual assertiveness for both partner communication and condom use.

Stages of Change for Current and Predicted Condom Use. Utilizing the Transtheoretical Model of behavior change (see Prochaska, \& DiClemente, 1983, 1984; Prochaska, DiClemente, \& Norcross, 1992; Prochaska, et al., 1994), youth were staged for their readiness for consistent condom use in the present, as well as predicted condom use six months in the future. The four stages of change, Precontemplation, Contemplation, Action and Maintenance, represented progressive consistent condom use, from "no use," "sometimes using," "started always using in the past six months," and "started always using over one year ago," respectively. Similarly, youth predicted their stage for condom use in the next six months, from "no use," "sometimes using," "plan to start always using," and "already always use condoms."

Half of the sexually active adolescents reported they were currently in the Contemplation stage for condom use, while over 30 percent claimed to be either in the Action or Maintenance stages. However, comparisons with sexual behavior reports during the past year in matrix format revealed that 41 percent of sexually active youth had inaccurately staged themselves in the more advanced stages, which were not reflective of their actual condom use behavior. Interestingly, when adolescents were asked to rate 
themselves for AIDS risk on a 5-point scale, the inconsistency and inaccuracy in risk and behavior prevailed, with nearly 39 percent of sexually active unsafe youth claiming to be "no risk" (see Table 34). Thirty sexually active youth incorrectly rated themselves in both areas. Other researchers have noted reported condom use among adolescents may be inflated by what is perceived to be socially acceptable answers (Cates, 1991), which is one possible explanation for this finding. It appears that sexually active youth may be more naive, less realistic or less accurate about assessing their behavior, and erroneously categorize themselves in more favorable light, or perhaps are simply providing socially acceptable responses.

With regard to predicted condom use in the next six months, the Action stage received the largest growth in figures with nearly a four-fold increase (12 to 47), according to adolescents' reports. Overall, 60 percent of sexually active youth reported they would be in either the Action or Maintenance stages within the next six months. However, these data are highly inflated due to adolescents' staging inaccuracies mentioned above. Adjusting for inflated predictions and stage-skipping, based on reported sexual behavior, the majority of sexually active youth would move only to the Contemplation stage in a six months period, if progression and not regression was realized. At best, only 40 percent of sexually active youth would move into the two advanced stages, joining the 5 percent already in Action and Maintenance.

No gender differences were found with staging inaccuracies for current condom use, where 27 young men and 25 young women placed themselves in stages which reflected more desirable behavior than what they reported in matrix format. On the extreme end, 
three young men, and one young woman whose behavior depicted "never" using condoms, placed themselves in the most desirable stage, Maintenance (see Table 48).

Summarizing findings for current and predicted condom use staging among sexually active adolescents: (1) an optimistic bias was realized with teens' reports, with 41 percent inaccurately staging themselves in more desirable stages than their reported behavior demonstrated; (2) no gender differences were found with staging inaccuracies for current condom use; (3) a four-fold increase in the Action stage was reported by youth in a six month period for predicted condom use; and (4) based on adjustments for inaccuracies and accommodating for stage-skipping, only 45 percent of the sexually active adolescents would be in the Action and Maintenance stages of condom use in six months time, if no stage regression was realized.

\section{Risk Variables in the Context of Stages of Change for Condom Use. Differential} risk by stage generally showed what might be expected, that is, higher stages earmarked the most adaptive behavior, and coincided with reported adaptive behavior among sexually active youth. Teens' reported levels were utilized in analyses of staging variables rather than adjusted figures mentioned above, due to inability to distinguish whether staging or behavior reports were inaccurate. However, it is important to keep in mind the 41 percent discrepancy between youth's staging and behavior.

For current condom use, participants in the Maintenance stage had significantly lower AIDS misconceptions, higher situational condom use efficacy and higher condom use assertiveness skills, than those in the Contemplation stage. These findings are consistent with the Transtheoretical Model. However, with respect to overall AIDS risk, based on 
number of sex partners and number of occasions of unprotected sex, results were contrary to the Transtheoretical Model's progression of stages. Sexually active youth rating themselves in the lowest stage, Precontemplation, for current condom use, fared significantly better than youth in the second stage, Contemplation, having a lower ADS risk composite score. Thus, youth reporting themselves in stage two in the Transtheoretical Model had riskier behavior than teens remaining in the first stage. This contrary finding may be explained simply with inflated figures by virtue of inaccurate staging, as nearly 15 percent of youth rating themselves in the Contemplation stage had reported "no condom use," which is more accurately defined as Precontemplation.

For AIDS misconceptions clear differences were noted between stages two and four; however, adolescents in Precontemplation had fewer misconceptions than adolescents in the middle stages (see Table 49). It appears as though misconceptions may temporarily rise with the progression of condom use, and then be dispelled as condom use becomes more consistent. Similarly, both situational condom use efficacy and condom use assertiveness followed the same pattern, with a temporary regression from stage one to stage two, and becoming more stable with condom use consistency. It may be that once teens decide to make a behavioral change, moving from "no condom use" to "sometimes using," they find it more difficult to use condoms in some situations, and have trouble successfully asserting themselves all the time. Another explanation for these temporary regression findings may well be youths' inaccurate staging of current condom use behavior.

Gender differences were found for predicted condom use staging, thus separate analyses were undertaken which are discussed more fully in the following section. 
In summary, staging for current condom use followed the Transtheoretical Model's successive stages for three risk variables, but was contradictory with one risk variable. Results among sexually active adolescents showed: (1) those rating themselves in the Maintenance stage had lower AIDS misconceptions; higher situational condom use efficacy; and higher condom use assertiveness skills than those rating themselves in the Contemplation stage; and (2) those in the self-rated Precontemplation stage had lower AIDS risk, as defined by number of sex partners and unprotected sex occasions during the past year, than those in the Contemplation stage.

Gender Differences in Stages of Change for Condom Use. Gender differences in staging variables were noted only with regard to predicting condom use, and not for current condom use stages. Findings conformed to the Transtheoretical Model's progressive stages of change for both sexually active young men and young women. Sexually active young men in Maintenance significantly exceeded those in Contemplation for situational condom use efficacy. Similarly, sexually active young women in the Maintenance stage exceeded those in the Action stage for situational condom use efficacy.

Sexually active men revealed a linear progression of situational condom use through the four stages (see Table 50). Young women, however, reported a stage regression pattern, from stage one to stage two, before predicted condom use improvement was realized (see Table 51). Thus, the instability of using condoms in various situations referred to above may be more persistent with young women than young men. This coincides with qualitative data reported by young women regarding condom use, that is, there are too 
many confounding variables (being the man's responsibility, partner pressure, emotional factors, negative consequences, etc.).

The coinciding of situational condom use efficacy with predicted condom use for behavioral change suggests that youth may be focusing more on mood, partner pressure, and substance use with regard to condom use, rather than on successful assertiveness skills. Sexual assertiveness skills for condom use across predicted stages in six months revealed moderate high, fairly consistent mean scores for young men, and an unexpected Udistribution for young women. Interestingly, some young women who reported moderately high current condom use assertiveness skills predicting themselves to be in either Precontemplation or Maintenance in six months, the two stages at each end of the behavioral change continuum. Young women with current mid-ranged assertiveness for condom use predicted themselves to be in the middle stages, or Contemplation and Action, in six months time. One explanation for the nonsignificance of condom use assertiveness may be young men's seemingly stagnant stance, and young women's stage regression which may be highly dependent on their partner's motivation for condom use.

Summarizing gender differentials for predicted condom use staging: (1) for sexually active young men, those in the Maintenance stage had higher situational condom use efficacy, than those in Contemplation; and, (2) for sexually active young women, those in the Maintenance stage had higher situational condom use efficacy than those in Action. Other Qualitative Topics Investigated

Adolescents provided feedback in discussion format regarding the psychosocial impact of societal dilemmas and suggestions for intervention and prevention for teenagers. 
These topics were not covered, or associated with risk predictors within the questionnaire constructs, but deserve some mention.

Psychosocial Dilemmas. Youth discussed and debated three societal issues with respect to AIDS: insurance, segregation, and pregnancy. The intent was to promote awareness of legal, ethical and moral repercussions of the AIDS pandemic. Adolescents were asked to generate potential solutions, although it was stated at the onset that it would not be an easy task. Whenever possible, role reversal and hypothetical personal factors were added to examine potential changes in perspectives and associated responses.

In the first dilemma, youth were asked about their position on whether insurance companies have the right to drop clientele infected with AIDS due to the high cost of maintenance. Nearly all replied that this would be unfair practice. However, when a personal stance was taken, and participants were asked to imagine themselves as president of an insurance company about to go bankrupt, youth continued to have difficulty justifying dropping infected clients but provided some solutions. Most solutions entailed raising rates for the rich and lowering rates for the poor, providing time-limited coverage, or having "the state help out." Others suggested recommending other insurance companies, or splitting coverage between two companies. One 14-year-old young man stated, "if they got a job they should be able to deduct it from their paycheck or something," and a 15-year-old young woman decided to move funds around, "what about all that money in research, why can't we take some of that money and put it into funding for people with AIDS... you can't spend all the money on trying to find a cure." These findings clearly reveal youth's unawareness of the financial burdens realized as a consequence of the AIDS pandemic. 
In the second dilemma, students were asked to provide positive and negative points about segregating infected individuals. When "individuals with AIDS" were strangers, many younger teenagers replied segregation was a good idea to reduce the spread of infection, although the majority immediately personalized the situation, and discussed the negative aspects of separating families, and "feelings." Some differentiated individuals by infection mode, those who acquired AIDS via blood transfusion "was not their fault," and therefore should not be segregated, but for others “it's their fault, so they should be isolated." Researchers have noted that knowledge about AIDS is improving among youth, but misconceptions and negative biases are still prevalent, particularly toward certain afflicted groups. In one study, 45 percent of the students believed that homosexuals and intravenous drug users were "getting what they deserve" (Steiner, Sorokin, Schiedermayer, \& Van Susteren, 1990). This finding was evident within focus groups conducted, with approximately 10 percent of verbal responses. However, information based on a national probability sample indicate that misconceptions are more common among the less educated and in areas where AIDS is more prevalent (McCaig, Hardy, \& Winn, 1991). Thus focus group participants' misconceptions may be based on less education, since AIDS prevalence was fairly low in their locale.

When "individuals with AIDS" were defined as close friends or family members, older participants held consistent opinions, typically discussing the unfairness of segregation, while younger teens changed their views. Youth 13- and 14-years-old stated they would "protest," "kidnap," "fight for," or "hide," friends and family members from segregation, with some of these teens being the same ones who were for segregation when 
the individual was identified as a stranger. Results from these discussions provide some insight to the progression of moral development. Older adolescents appeared to apply moral decisions consistently, in the name of "fairness," while younger counterparts ignored or had difficulty rationalizing differing solutions for strangers and personal acquaintances.

In the last posed dilemma, group participants debated the issue of pregnant women's rights versus rights of the unborn. Adolescents were briefed about new medications that have potential to greatly reduce the likelihood of infection for an unborn child to an infected mother (e.g., CDC, 1994h, 1994i). Adolescents were asked whether forced testing and medication was right or wrong, and based on whose rights. Inconsistencies were noted in youth's responses, particularly in the manner in which the question was asked. For instance, many group members stated the pregnant woman should be forced to be tested and take medications, and then responded that the woman's rights should be of utmost concern. In other groups the reverse was observed, women's rights were emphasized, but the baby should be priority. Several evaded confrontation of contrary responses, simply stating "well, I would," "it depends..." (on degree of sickness or how old the pregnant woman was) or brought in issues of denial, "everybody always thinks, 'Oh it would never happen to me." The "it could never happen to me" rationalization, has been noted elsewhere in the literature, as a particular denial characteristic of adolescence and risk taking behavior (Brookman, 1990; Cates, 1991; Irwin, 1990), and with risk-taking among emotionally disturbed adolescents (DiScipio, 1994; Katz, Mills, Singh, \& Best, 1995). One 13-year-old young woman who was adamant about women's rights during the onset of discussion, suggested towards the end, "if she doesn't want it or stuff like that, they should 
just do it like without telling her, life for the baby, and just tell her it's another test." Interestingly, in a survey of 200 hospital based pediatricians, most believed that high-risk newborns should be screened regardless of maternal consent, based primarily on increased pregnancies among adolescents (Bhushan \& Cushman, 1995). These findings revealed a distinct confusion for adolescents about women's rights, having a "firm but flexible" stance, often not realizing they were contradicting themselves in the process.

Adolescents' Suggestions. Focus group participants were eager to provide suggestions for intervention and prevention strategies targeted at their own age group. Providing education was most frequently discussed, primarily with extensive elaboration on giving youth information at younger ages. Several participants voiced dissatisfaction with the current health class curriculum, learning very little beyond basic physiology in junior high school. Not enough was being taught and what was being taught, was addressed too late. Other researchers note the pattern of barely addressing sex education in junior high, "Students may learn, for example, about the physiology of sex, but that knowledge seldom alters their sexual behavior and attitudes" (Flora \& Thoresen, 1988, p. 967). This appears to be typical for the majority of school systems; however topics covered tend to vary by grade, geographic location, parent involvement, school system, etc. (see Holtzman, et al., 1992, for a review). It has been argued that a developmentally sound, effective childhood education program should tailor its pace with children's developing sense of control and ability to understand and master healthy behaviors (Whitt, 1995).

Issues around accuracy of information were raised, as many teens stated they had received inaccurate information from parents, siblings and friends, due to ignorance, fear, or 
misinformation. Educating parents was also suggested, however the majority stated they could not or would not speak with parents about sexual issues. Brock and Beazley (1995) found that parents who were not involved in an "at-home" sexuality education program for youth were less confident their children wanted to do activities with them; less sure of their children's desire to talk with them about sex-related issues; and, were less certain that their AIDS knowledge was current, than parents who were involved. Shoop and Davidson (1994) found that youth who had discussed general sexual issues with parents felt more competent to talk about AIDS with sex partners. Thus, educating and involving parents may be crucial for early prevention methods, especially showing promise toward increasing the lines of communication about sexuality. Finally, some group participants desired optional discussion groups like the one they were currently involved in, because "classes are boring." All participants stated that providing information would not be perceived as condoning sexual activity, because "it's much worse not to tell kids," "they'll do it thave sex] anyway."

The second most popular suggestion was having a "real" person with AIDS talk with them, with strong emphasis on "real." Preferably, an individual their age, would have a significant impression, according to teens, and more so if the infected speaker was heterosexual. Ideally, the more the speaker was similar to the audience, the higher the impact. Along these lines, peer education was discussed positively, as most adolescents were currently receiving their information from friends, and were more likely to discuss personal, sexual matters with peers than adults. Use of peer educators has been suggested as a useful method of intervention and prevention for adolescents elsewhere (Black \& 
Jones, 1988; Mellanby, Phelps, \& Tripp, 1992; Rickert, Jay, \& Gottlieb, 1991; StevensSmith \& Remley, 1994; Sunwoo, et al., 1995).

The third most popular suggestion was distribution of condoms, which again, was emphasized as not condoning sexual activity. Adolescents admitted to embarrassment in purchasing condoms, but felt condoms would be used if available in bathroom dispensers, or the like. Fourth, teens thought use of media would be helpful, through pamphlets, billboards, television, books, radio and movies. Some data indicate that exposure to media information is associated with lower fears of homosexual persons (Ross \& Carson, 1988); however, another study reported only marginally effective changes in AIDS-related attitudes following an AIDS Awareness Week (Dommeyer, Marquard, Gibson, \& Taylor, 1989). Regarding television media, one study found that youth who viewed television shows with high sexual content were more likely to engage in sexual activity than those who did not view such shows (Brown \& Newcomer, 1991). Thus content of media presentation appears relevant. Utilization of famous people such as Earvin "Magic" Johnson, was viewed as helpful, and would capture the attention of adolescents, according to group discussants. This latter suggestion has been supported in the literature, where increased interest in AIDS resulted from celebrity self-disclosure, and was greatest among African-American men with reference to Magic Johnson (Kalichman, Russell, Hunter, \& Sarwer, 1993). In a similar study conducted in Maryland recently, the greatest behavioral impact found among 283 study participants after Magic Johnson's HIV-infection announcement, was on the number and type of sex partners rather than on condom use, with a trend toward fewer "one night stands" for young adolescents (CDC, 1993a). 
Finally, a small percentage discussed "finding a cure," counseling, mandatory testing, and "giving people more self-esteem..., to know what to do." The issue of acquiring self-esteem was most frequently raised in a separate discussion on how sexual assertiveness could be acquired. Young women, especially, associated self-esteem with the ability to assert oneself sexually. Other researchers found that assertiveness training with young women increased self-esteem scores with educational situations (Stake, DeVille, \& Pennell, 1983); but this finding may differ by ethnicity and adolescent development (Rotheram-Borus, 1990); thus more research is needed in relation to assertiveness and selfesteem.

\section{Suggested Intervention and Prevention Strategies}

The present findings indicate that most adolescents who are sexually active are not using condoms despite having fairly high knowledge, suggesting that educational efforts must move beyond solely conveying knowledge. Further, findings show that adolescents are in need of interventions that help to promote awareness of personal risk, as they reported many inconsistencies with their own behavior. The present study also confirms previously mentioned trends from longitudinal studies (DiClemente, 1989, 1990) of increased sexual activity, initiated at earlier ages, with increased number of sex partners. Youth need to increase their awareness of true risk; increase their abilities to obtain and use condoms properly; increase negotiation skills with sex partners; and increase personal commitment to use condoms consistently. Proper use of condoms is essential, as they are typically used incorrectly by adolescents (Werner \& Biro, 1990). Moreover, sexually active young women are at a higher risk for infection, than: (1) sexually active adult women, due 
to biological susceptibilities in the developing cervix (Bowler, Sheon, D'Angelo, \& Vermund, 1992; Brooks-Gunn, 1988; Cates, 1991; Ostergard, 1977; Moscicki, Winkler, Irwin, \& Schachter, 1989); and (2) men, in general, because HIV is presumed to be more prevalent among partners of heterosexual young women (Smith, McGraw, Crawford, Costa, \& McKinlay, 1993); and transmission is acquired more efficiently from man to woman than vice versa, due to biological constraints (Friedman \& Klein, 1987; Padian, et al., 1987; Padian, Shiboski, \& Jewell, 1991). Intervention and early prevention must address these concerns.

The Educational Domain. Evident from results in the current study, the majority of youth are sexually active, practicing risky behaviors, and only contemplating behavior change. From the perspective of the Transtheoretical Model, moving adolescents from Precontemplation to Contemplation, and from Contemplation to the Action stage of behavior change may require proper, thorough, education prior to building competence skills, in order to increase motivation. Educators should emphasize the growing susceptibility of adolescents to infection, and that HIV and some STDs can be asymptomatic. Additionally, educators should correct commonly held misconceptions, that HIV cannot be transmitted by insects, that healthy people cannot be identified by appearance, and that no cure exists although some drugs may be successful in prolonging the lives of those who are HIV-positive. A key concern of health education is to make adolescents aware that there are risks associated with many of the behaviors in which they engage (Jessor, 1991), and to provide accurate information, dispel myths, and avoid feararousing messages which can be counterproductive (DiClemente \& Houston-Hamilton, 
1989; Herold, Fisher, Smith, \& Yarber, 1990; Siegel, 1993; Sy, Richter, \& Copello, 1989; Visser \& Ketting, 1994). Fear-arousing messages, or scare tactics, and "just say no" campaigns have been found to increase alcohol consumption in sexually active teens (Harvey \& Spigner, 1995). It has been extensively noted that informational efforts alone will not change behavior, but some evidence exists that high school students who had high levels of HIV knowledge are less likely to have multiple lifetime sex partners or have injected illicit drugs (Holtzman, Lowry, Kann, Collins, \& Kolbe, 1994; Holtzman, et al., 1991), report lower intention to engage in such behavior (Jemmott, Jemmott, \& Fong, 1992), and have a greater tolerance of AIDS patients (Brown, Fritz, \& Barone, 1989), supporting the importance of acquiring an accurate knowledge base.

Additionally, use of peer-led, versus adult-led, AIDS education programs may prove to be highly beneficial in promoting knowledge gains and appropriate attitude changes (e.g., Rickert, Jay, \& Gottlieb, 1991; Remafedi, 1994; Stevens-Smith \& Remley, 1994).

Education and enlightenment from people with AIDS has also been noteworthy in capturing youth's attention, and personalizing the potential risk (Sunwoo, et al., 1995), and was suggested as being helpful among focus group participants. Health professionals have also been targeted as significant informants for AIDS (Braverman \& Strasburger, 1995; Gruber \& Changers, 1987; Kaplan \& Schonberg, 1994; Lyons, Sheridan, \& Larson, 1989; Nutbeam \& Blakey, 1990), and that teenagers overwhelmingly preferred to learn about AIDS from a physician (Manning \& Balson, 1989). Since adolescents are less likely to have access to health care, use of comprehensive, disease-prevention programs, especially school-based 
programs, which provide informational resources, counseling, support and reproductive health services, are sorely needed for youth (CDC, 1993d).

Beyond providing information, and encompassed in the educational domain, is the controversy of program emphasis, more particularly, abstinence versus safe sexual practices. This issue deserves special attention.

Promoting Abstinence Versus Safe Sexual Behaviors. Many parents assert that the promotion of condom use to prevent HIV infection appears to be condoning sexual intercourse outside of marriage among adolescents. Adolescents disagree. Research findings have also supported the contrary. This controversy deserves much attention, as school administrators, educators, and parents of school youth involved in the current study were not easily convinced, despite the amount of supportive evidence contrary to their beliefs. Recent data indicate that African-American adults in rural areas were 50 percent more likely than Caucasians to support public school-based, comprehensive adolescent health programs, including services for pregnancy testing and treatment of STDs; however, they were only half as likely as Caucasians to approve of sexual experimentation by youth (Horner, Kolasa, Irons, \& Wilson, 1994). It appears as though local community attitudes are important regarding health curriculums. Nonetheless, information is frequently requested.

Christopher and Roosa (1990) utilized a quasi-experimental pretest-posttest control group design with 191 participants and 129 controls (mean age 12.8 years). The "Success Express" abstinence promotion program was conducted at eight sites, with results finding participants increased their mean sexual interaction level between time 1 and time 2, while 
controls did not. To determine whether the abstinence program would work better for youth who have not yet experience sexual intercourse (as hypothesized by Olson, 1987, cited in Christopher \& Roosa, 1990), nonvirgins were removed from the analyses. Even after removal of nonvirgins, participants, but not controls, reported a significant increase in sexual behaviors in programs that stressed abstinence as the only alternative. These researchers concluded that sex education programs that rely on teaching refusal skills alone do not achieve their aims (Christopher \& Roosa, 1990). Goodson and Edmundson (1994) did a content evaluation on an abstinence-based sexuality education curriculum called "Sex Respect." Results showed that the program omits basic content, and included misinformation, especially in areas of reproductive health and human sexual response. These researchers concluded that the program fails to meet professional standards as a comprehensive sexuality education curriculum (Goodson \& Edmundson, 1994). Unfortunately, many states continue to promote abstinence as the single option, without any mention or emphasizing safe sex (Bowler, Sheon, D'Angelo, \& Vermund, 1992).

Two other programs in the United States showed sizable reductions in the pregnancy rates among urban, lower class, predominantly African-American youth (Zabin, Hirsch, Smith, Streett, \& Hardy, 1986; Zabin, et al., 1988). One program consisted of an in-school program, class presentations, discussion groups, individual counseling, and an after-school clinical which provided free education intervention, medical examinations, counseling and contraceptives. A 3-year evaluation revealed that participants delayed first intercourse, started using effective contraception earlier in sexual activity, and significantly reduced their pregnancy rate by 30 percent, while controls experienced a 57 percent 
increase. Vincent, et al. (1987) conducted a program with a similar population which provided teachers with courses in sex education, and implemented a community-wide curriculum that integrated sex education within present courses, kindergarten through grade 12. Secondary to emphasizing abstinence, was to promote consistent condom use to those who chose to be sexually active. Results showed pregnancy rates declined from 54.1 per 1000 young women to 25.1 within five years, while comparison data indicated controls experienced large increases in pregnancy rates (Vincent, Clearle, \& Schluchter, 1987).

Recent data from Switzerland suggest that public education campaigns that promote condom use can be effective without increasing the proportion of adolescents who are sexually active (Dubois-Arber, Jeannin, \& Zeugin, 1992; Hausser \& Michaud, 1992). From January, 1987 to October, 1991, self-reported consistent condom use among individuals aged 17 to 30 increased from 8 to 52 percent in association with the Switzerland campaign (Dubois-Arber, Jeannine, \& Zeugin, 1992); in contrast, the proportion of adolescents (aged 16 to 19 ) who had sexual intercourse did not increase over the 3-year study period (Hausser \& Michaud, 1992). Other researchers report success of a skills training group where participants had a reduction in unprotected sex, increased condom use, and displayed increased behavioral skills to a greater extent than youth who received information alone (St. Lawrence, et al., 1995).

The CDC (1994c) reported the WHO's findings regarding the same issue with reference to sex education, where "no study revealed evidence of sex education leading to earlier or increased sexual activity among young people" (p. 6). Furthermore, the WHO reported that: (1) in six studies, sex education either delayed the onset of sexual activity or 
caused a fall in its overall extent; (2) two studies showed that access to counseling and contraceptives did not encourage earlier or increased sexual activity; (3) ten studies showed that sex education increased the adoption of safer practices by sexually active youth; and (4) school programs which promoted both the postponement of sexual intercourse and the use of condoms were more effective than those which promoted abstinence alone, emphasizing that these latter programs were most effective when given before young people become sexually active, and when they emphasized skills and social norms rather than knowledge alone (CDC, 1994c).

Lohrmann (1988) provided three reasons why programs that promote only abstinence fail. First, youth do not "buy it," due to contradictions in behavior they observe in society. Second, a position which preaches only responsible sexual behavior within the context of marriage alienates the homosexual population. Third, a dogmatic position on what constitutes acceptable sexual behavior crosses the line into the perceived domain of the family, while usually acceptable to parents. Nevertheless, despite compelling evidence to the contrary, many school districts continue to emphasize abstinence as the most effective strategy toward combating HIV among adolescents (Kenney, Guardado, \& Brown, 1989); or worse, have not incorporated AIDS education into the curriculum at all (e.g., Calamidas, 1990). Parents of youth in public schools in New York have mixed feelings about condom availability in high schools. Sixty-nine percent of parents believed students should be able to receive condoms at school, although nearly half felt they should have the right to keep their children from doing so (Guttmacher, et al., 1995). 
The fear that sex education would promote early sexuality is largely unfounded (Furstenberg, Moore, \& Peterson, 1986; Zelnik \& Kim, 1982, both cited in Brooks-Gunn \& Furstenberg, 1989; see also, Marsiglio \& Mott, 1986; Moore, Furstenberg, \& Peterson, 1986, both cited in Brooks-Gunn \& Furstenberg, 1990). "Sex education leads not to more sex but to more responsible sex" (Des Jarlais, et al., 1990, cited in Hein, 1993, p. 4). U.S. Surgeon General Koop stated, "You can't teach kids about AIDS unless they know about their own sexuality" (Koop, 1990, p. 82). Despite the remote chance of sex education resulting in increased sexual activity (Olsen \& Weed, 1986; Weed \& Olsen, 1986), educators and parents must ask where the greater threat lies: in teaching adolescents how to avoid AIDS infection at the risk of promoting increased sexual activity, or in not teaching them about AIDS and how to avoid it, thus allowing a sizable number of teens to risk? Quantitative and qualitative evidence compiled in the current study support findings that educational efforts which promote both postponement of sexual activity and safe sex practices would be effective in combating adolescent HIV-infection.

Empowerment. Further efforts towards risk reduction include empowerment for assertiveness, and self-efficacy to exercise control in various sexual situations, such as communication about sexual matters and protective methods to ensure condom use. Importantly, the complexity of sexual situations, emotional states, and contextual factors which can potentially undermine HIV-preventive intentions, must be considered, especially for adolescent young women (Abraham \& Sheeran, 1994). Results of the present study support past research that self-efficacy may be linked to sex risk behaviors (e.g., Bandura, 1990, 1992; Jemmott \& Jemmott, 1992; O'Leary, 1985; Quina, Harlow, Morokoff, \& 
Saxon, in press; Stevens-Smith \& Remley, 1994), which may be important in empowering individuals to take control of their situation.

Globally, two thirds of the projects targeted at HIV preventive efforts emphasize explicitly or implicitly on the empowerment of women, often through assertiveness training, learning how to negotiate safe sex, and initiating use of the female condom (WHO, 1994b). At the heart of these activities is the growing awareness of the need to train women to negotiate safer sex with their partners; however to do so, often suggests a partner's infidelity, and may even invite violence. Results of workshops conducted by the WHO (1994b) have pointed out that adolescence is experienced differently by young women and men, and that women are typically placed in a subordinate role, emphasizing a need to teach negotiating skills, and safe options. Offering women the increased possibility of ensuring self-protection through use of the female condom is promising. The safety and effectiveness of the female condom, when used appropriately, have been established in several pre-clinical and clinical studies (e.g., Drew, Blair, Miner, \& Conant, 1990; Leeper \& Concardy, 1989; Soper, Brockwell, \& Dalton, 1991), and its acceptability to significant proportions of test groups of sexually active women and their partners has been repeatedly demonstrated (e.g., Bounds, Guillebaud, Stewart, \& Steele, 1988; Ortiz, Kauman, Uribe, Decaso, \& Hernandez-Avila, 1992; Tansathit, Thawatchai, \& Cheevakej, 1990).

Skills-Building. In addition to education, and accessible resources, youth need to acquire, practice, and generalize safe behaviors. Emphasis on condom use is crucial for those who are sexually active, and more importantly, instruction on communication skills and on how to negotiate safety with a potential sex partner. The issue of proper instruction 
has been raised in past research regarding interpersonal obstacles to condom use (e.g., Boyer \& Kegeles, 1991; Cohen, Dent, \& MacKinnon, 1991; Fisher \& Fisher, 1992; Franzini, Sideman, Dexter, \& Elder, 1990; Kann, et al., 1991). Other researchers have found that few adolescents report discussion on condom use prior to intercourse with sex partners, due to fears that discussion may raise questions about fidelity, moral values with the expectation of sexual activity, or that sex partners may assume that they are infected with HIV already (Hingson \& Strunin, 1992). Bandura (1992) has pointed out that young people tend to make their risk appraisals on the basis of social and physical appearances which can be highly misleading, and generally do not obtain knowledge of their sex partner's behavioral history and serostatus of past sexual partners. This was evident in the present study with low sexual assertiveness skills for sexually active women, and high frequencies of AIDS misconceptions, particularly judging serostatus by appearance of a potential sex partner. Nevertheless, numerous studies have reported successful outcomes with comprehensive programs, school-based or otherwise, that develop social and selfregulatory skills, self-esteem, problem-solving techniques, and promote assertiveness skills and peer-acceptable alternatives to resist peer group pressure, unwanted sexual advances and to negotiate safe sex practices (e.g., Coyle \& Loveless, 1995; Fisher, Fisher, Misovich, Kimble, \& Malloy, 1996; St. Lawrence, Jefferson, Alleyne, \& Brasfield, 1995; Kelly, Murphy, Sikkema, \& Kalichman, 1993; St. Lawrence, et al., 1994; Stevens-Smith \& Remley, 1994; Trad, 1994).

One impediment to sexual assertiveness and skills-building is the effect of substances on attitudes and behavior. Substance use and abuse in the present study were found to be 
significant predictors of AIDS risk, and may be related to risk, particularly by lowering condom use efficacy and sexual assertiveness abilities. Many findings in the literature indicate that substance use may impair judgment in sexual situations, interfere with selfcontrol and/or the ability or willingness to use condoms, and/or lower self-efficacy in adolescents (e.g., Bandura, 1990; Brooks-Gunn \& Furstenberg, 1990; Kelly \& St. Lawrence, 1988; Millstein, 1990). Youth need to be aware of the consequences of substance use, and the potential risks it poses on unsafe sexual practices. A high degree of parental nurturance has been found to be a significant deterrent to adolescent alcohol problems (Barnes, 1984), and high-risk sexual behavior was found to be more likely among adolescents with limited family availability, low parental support, and friends who engaged in problem behavior and alcohol use (Biglan, et al., 1988; Walter, et al., 1992). Both these findings suggest the importance of primary support systems in preventing substance use, substance abuse, and associated high-risk behaviors. Thus educators may need to address these issues by focusing on the potential effects of substance use and abuse on self-efficacy to negotiate condom use, and assist youth in viewing condom use as a symbol of caring for partners.

Further, educators may need to elaborate on reducing anxiety by dispelling negative attitudes about the costs associated with condom use, particularly with young women. Modeling techniques may be helpful to heighten self-esteem, efficacy and assertiveness, as well as to convey strategies for dealing effectively with coercions and peer pressure to engage in risk behavior. Aside from information and motivation, AIDS-preventive behavior involves acquiring the behavioral skills for initiating, performing and maintaining a safe 
status (Fisher, Fisher, Williams, \& Malloy, 1994; Schaalma, Kok, \& Peters, 1993). It seems clear that adolescents, especially young women, would greatly benefit in learning how to communicate with their partners about sexual matters and protection, how to deal with conflicts associated with such activities, and how to resist unwanted sexual advances. Such sexual assertiveness and empowerment in conjunction with educational efforts may be effective in assisting adolescents in the adoption or maintenance of HIV-preventive behaviors.

\section{Future Research Directions}

Through discussions with adolescents, it became evident that numerous potential variables are associated with risk behavior that were not thoroughly addressed in the current study. As mentioned above, young women made several references to situational contexts, affective needs, degree of attachment, potential personal consequences, gender role expectations, external pressures, social influence, and trust with regard to sexual assertiveness. Attitudes towards condoms and their use were also neglected. Was condom use low due to negative perceptions such as inconvenience, difficulty of use, discomfort, embarrassment in purchasing them, reduction in pleasure? Did condom use rely on self and partner satisfaction? What would sentiments be regarding use of the female condom, would a partner agree, who would have the final say? Would the female condom be viewed as a burden to women, or as empowering? Despite near-universal asymmetry in gender relations, women in different cultures have multiple roles and status, shaped not only by gender, but also by age, marital status, ethnicity and class. How do cultural parameters influence women's abilities to negotiate safe sex? 
Various personality states, traits, and emotions were also evident in focus group participants' responses, such as love, anger, motivation, nervousness and anxiety associated with partner communication in sexual matters. Guilt related to sex is postulated to be associated with self-reported safer sex practices (Rimberg \& Lewis, 1994), and was touched upon by focus group participants in relation to partner(s)' reactions to assertiveness. Affective reactions have been noted to affect attitudes toward safe behavior, responses to social pressure, and perceived behavior control toward safe or unsafe sexual behaviors (Richard, van der Pligt, \& deVries, 1995). To what extent does mood influence safe sexual behavior?

Social support as a factor potentially affecting attitude and behavior was mentioned in focus groups, suggesting another area for future research. The influence of social supports regarding availability, desire, type of relationship, trust, motivation, and ability to confide in peers, teachers, counselors, parents, siblings and others for advice, appears to be connected with self-efficacy and assertiveness. Positive relationships with teachers and educational aspirations have been noted to improve AIDS knowledge and attitude and to increase safe behaviors (Slonim-Nevo, Auslander, \& Ozawa, 1995), yet youth in the current study said they preferred to discuss such matters with peers. Perhaps they had not had a teacher or adult they felt they could talk to, or could trust. Stressful events may also influence HIV-related risk behaviors during adolescence and young adulthood (Stiffman, Dore, Cunningham, \& Earles, 1995), implying a need for support. Researchers found that youth with low social supports knew less about AIDS, had more negative attitudes toward condoms, were more likely to engage in casual sex, had more sex partners, and higher 
STDs, than teens with higher supports (St. Lawrence, Brasfield, Jefferson, Allyene, \& Shirley, 1994). Other issues likely requiring support systems may include knowing a person with AIDS, or having a family member with AIDS (e.g., Aronson, 1995; Levine, 1995).

Due to ethical concerns given the sample's age, this study did not address correlates of HIV-risk behavior that have been noted in the literature, particularly history of sexual abuse. Correlates of childhood sexual abuse and subsequent risk of HIV infection have also been noted among both genders, and a significant number of adolescents who are HIV positive have sexual abuse histories (Hein, Dell, Futterman, Rotheram-Borus, \& Shaffer, 1995; Johnsen \& Harlow, in press). Women who report childhood rape are three times more likely to become pregnant before the age of 18 ; while men who report childhood abuse have a twofold increase in HIV infection relative to unabused men (Zierler, et al., 1991; Gutman, et al., 1991). Additionally, age of first intercourse, a previous suicide attempt, and history of physical and sexual abuse have been found to be associated with substance use (Kirpke, Montgomery, \& MacKenzie, 1993). Appropriate methods of obtaining such personal and potentially sensitive information have been suggested to reduce uneasiness (e.g., Miller, Johnson, \& Johnson, 1991), and may need to be addressed in future research with adolescents.

\section{Study Limitations}

As mentioned above, there are some limitations with interpretation and use of the questionnaire with certain populations. Measures used with youth under age 15 for perceived susceptibility for risk and condom use assertiveness, with youth of color for perceived susceptibility for risk, substance abuse and condom use assertiveness, and with 
youth from lower-to-middle socioeconomic class, for homophobia and condom use assertiveness, may be relatively unstable. The construct of homophobia may be questionable as to content measured, as it contains items that do not pertain to sexual orientation.

Secondly, self-report measures that contain inquiries about highly personal behaviors are subject to both under-reporting and over-reporting. This was particularly evident with sexually active adolescents, for whom discrepancies were found in perceived risk level, condom use staging and associated reported sexual behavior. However, the questionnaire was designed to be anonymous to maximize accurate self-disclosure, and the majority of the focus group participants confirmed the anonymity was important in truthful responses. Further, the internal consistencies of most subscales were acceptable. Future researchers may need to assess adolescent sexual behaviors utilizing different or supplemental methods. For instance, it has been noted that self-reports of recent condom use are strongly related to condom possession (Smith, McGraw, Crawford, Costa, \& McKinlay, 1993), and the anonymity and confidential feedback afforded with computer assisted methods have been more successful with accuracy of adolescent responses (Schneider, Taylor, Prater, \& Wright, 1991).

Third, retrospective measures such as those in the current study are also susceptible to memory errors, although none of the participants indicated having much difficulty recalling behaviors during the past year anonymously on the survey or feedback sheets, or generally within discussion groups. Fourth, as mentioned with focus group participants with regard to sexual assertiveness, and noted by other researchers (e.g., Donovan \& 
McEwan, 1995), the concept of sexual risk-taking has to include acknowledgment of the context (e.g. degree of attachment in the relationship, perceived threat to lose the relationship, gender role expectations, peer or partner pressure to engage in sex, etc.) in which sexual behavior takes place, rather than defining risk only in terms of sexual acts.

Fifth, measurement error and attribution bias pose limitation especially with obtaining information relevant to HIV transmission (Catania, Gibson, Martin, Coates, \& Greenblatt, 1990), and substance use (e.g., McAllister \& Makkai, 1991; Mensch \& Kandel, 1988). For instance, the correlation between measures of substance use and sexual behavior could share common sources of bias (e.g., social desirability or bias with selfreport), thus the correlation could be artificially inflated due to error, or could compound random error and decrease the power of the analyses. It also should be noted that gender differentials found in attitude and behavior measures may be a reflection of gender differences in reporting tendencies regarding sexual attitudes and behaviors, rather than actual frequencies. Young men, for example, may have a tendency to exaggerate their sexual experiences while young women may under-report the same. However, feedback regarding truthfulness was positive, mainly due to anonymity of the responses.

Lastly the present study may not be representative of the general population and may be considered limited in terms of generalizability. For instance, behavior of over 1,000 adolescents who did not opt to participate, or whose parents did not grant permission for participation, cannot be taken into account. Nevertheless, findings in the current study appear to be consistent with the literature, and provide areas for future researchers to pursue with the adolescent population. 


\section{Conclusions}

This study provided psychometric information and validation with a reliable measure for youth that assesses AIDS knowledge, attitude and behavior risk predictors. The selection of predictors was guided by variables utilized in past research and by theorizing that sexual risk is caused by multiple factors within cognitive, attitudinal and behavioral domains. Thirteen psychometrically reliable scales were isolated and validated: factual, transmission, and perceived AIDS knowledge, AIDS misconceptions, perceived susceptibility for risk, self-efficacy for condom use, homophobia, substance use and abuse, sexual assertiveness for partner communication, condom use assertiveness, and self- and partner-risk histories. HIV-risk was assessed among 239 adolescents between the ages of 13-19, with a 163 -item self-administered questionnaire, and provided qualitative data from 28 focus groups. Gender differences for risk among teens were also examined utilizing a multivariate approach on a number of subscale variables, and supplemented with qualitative information with informal focus groups with feedback on: survey participation for future survey improvement; knowledge and concerns about the prevalence of HIV/AIDS; perceptions on the societal impact of HIV/AIDS; sexual assertiveness and potential gender roles; and suggestions for useful interventions for teens. These data were used to assess $a$ priori hypotheses, and behavioral change staging according to the Transtheoretical Model.

First, multiple regression analyses, showed high AIDS misconceptions, high perceived susceptibility for risk, and high substance use and abuse, were significantly related to potential risk, defined as low sexual assertiveness, for young women. Low perceived 
AIDS knowledge and high misconceptions were predictive of low sexual assertiveness for young men. However, it was discovered that sexual assertiveness, for young women, may be dependent on situational context, degree and intensity of emotions and attachment, potential threat to the relationship, gender role expectations, intensity of peer and/or partner pressures, positive connotations associated with sexual activity (popularity and/or a sense of belongingness), and the likelihood of untruthful responses from potential sex partners. Additionally, misperceptions, knowledge gaps and perceived invulnerability may be at least partially associated with barriers to sexual assertiveness. Nonactive youth may have difficulty in accurately predicting sexual behavior due their inability to appreciate emotional aspects of intimacy, comprehend potential interactional effects of personal behavior with a partner's, integrate potential external pressures with predicted responses, and consider possible negative consequences.

Second, predictors of AIDS risk, as defined by frequency of unprotected sexual activity and number of sex partners were differentiated by gender among the sexually active teens. Both genders perceived their AIDS risk as high, had high self and partner-risk histories, and low condom use assertiveness skills, all being predictive of risk. For sexually active young men, high AIDS misconceptions, and high substance use and abuse were also significant predictors of risk; and for women, low condom use efficacy, and low partner communication assertiveness were significant. High homophobia was also found to be a significant risk predictor but only in gender-combined group analysis.

Third, among sexually active, unsafe youth, low misconceptions and low homophobia contributed to accurate self-ratings AIDS risk. Accurate self-raters of risk had 
higher AIDS misconceptions, higher perceived susceptibility for risk, lower condom use sexual assertiveness, higher substance use and abuse, and higher risk histories for self and partners than underestimaters. Accurate self-raters appear more cognizant of their risk levels than underestimaters who may ignore, deny or perceive themselves as invulnerable to AIDS.

Fourth, sexually active young men had lower factual AIDS knowledge, lower perceived AIDS knowledge, higher AIDS misconceptions, higher perceived susceptibility for risk, higher homophobia, higher substance use and abuse, and higher self-risk histories, than sexually active young women. Sexually active young women had lower sexual assertiveness for both condom use and partner communication than active young men. Fifth, sexually nonactive youth had higher factual AIDS knowledge, lower AIDS misconceptions, lower perceived susceptibility for risk, lower homophobia, lower substance use and abuse, lower self and partner-risk histories, and predicted their condom use efficacy and their sexual assertiveness for both condom use and partner communication to be higher, than sexually active youth.

Interaction effects were found between gender and sexual activity for AIDS misconceptions, perceived susceptibility for risk, homophobia, substance use and abuse, self-risk history, and sexual assertiveness for both partner communication and condom use. Typically sexually nonactive youth had higher knowledge with more desirable attitudes and behavior than sexually active teens as did young women in comparison to young men. Complete reversals were noted with sexual assertiveness, where sexually nonactive young 
women predicted their assertiveness to be highest, however among sexually active youth, young men significantly exceeded women in areas of assertiveness.

Most sexually active adolescents reported their Transtheoretical Model stages for current condom use inaccurately, when compared to reported sexual behavior; placing themselves at higher stages in the model than their condom use behavior indicated. With adjustments made to account for reported condom use, 96 percent were in Precontemplation or Contemplation, with the remaining 4 percent in Action or Maintenance. Based on adjusted figures, fewer than half of the sexually active youth would be in the more desirable advanced stages in six months. Significant progression through stages of change for current condom use were found for AIDS misconceptions, condom use efficacy, and sexual assertiveness for condom use between the Contemplation and Maintenance stages, even with adolescent inaccurate staging. One contradictory finding placed those in Precontemplation as having a lower AIDS risk than youth in the Maintenance stage, which is likely due to adolescents' inaccurate self-staging.

The profiles identified here that may place youth at risk for AIDS may facilitate educational efforts towards providing interventions appropriate to specific age groups, and identifying various attitudinal and belief factors that are associated with high risk behavior. The present findings suggest that high substance use and abuse may be areas for intervention and prevention with young men, and there is a need to empower young women with sexual assertiveness. Most important, these findings emphasize that to understand the causes of AIDS risk, it is essential to consider the role of multiple factors, including gender, in order to develop appropriate intervention and prevention directives. 


\section{References}

Abraham, C. \& Sheeran, P. (1994). Modelling and modifying young heterosexuals' HIVpreventive behavior: A review of theories, findings and educational implications. Patient Education and Counseling, 23(3), 173-186.

AIDS plague expected to hit teenagers next. (1993, June 6). The Providence Sunday Journal, A-1, A-15.

Adame, D.D., Taylor-Nicholson, M.E., Wang, M., \& Abbas, M.A. (1991). Southern college freshman students: A survey of knowledge, attitudes and beliefs about AIDS. Journal of Sex Education and Therapy, 17(3), 196-206.

Aggleton, P. (1991). When will they ever learn? Young people, health promotion and HIV/AIDS social research. AIDS Care, 3 (3), 259-264.

Airhihenbuwa, C.O., DiClemente, R.J., Wingood, G.M., \& Lowe, A. (1992). HIV/AIDS education and prevention among African-Americans: A focus on culture. AIDS Education and Prevention, 4(3), 267-276.

Alexander, C.S., Ensminger, M.E., Kim, Y.J., Smith, B.J., Johnson, K.E., \& Dolan, L.J. (1989). Early sexual activity among adolescents in small towns and rural areas: Race and gender patterns. Family Planning Perspectives, 21(6), 261- 266.

Anderson, J.R., Amaro, H., Bailey, W.A., Barret, R.L., Boccellari, A.A., Bonde, L., BoydFranklin, N., Coates, T.J., Franks, P.E., Gornemann, I., Landry, C., Lo, B., McKusick, L., Shore, M.D., Steiner, G.L., \& Tafoya, T.N. (1991). Review of recent literature on the behavioral and psychosocial aspects of HIV disease. In J.R. Anderson, C.P. Landry, \& J.L. Kerby, (Eds.) (1991). AIDS Abstracts of the Psychological and Behavioral Literature, 1983-1991, Third Edition, (p. 24). Washington, DC: American Psychological Association.

Anderson, J.E., Kann, L., Holtzman, D., Arday, S., Truman, B., \& Kolbe, L. (1990). HIV/AIDS knowledge and sexual behavior among high school students. Family Planning Perspectives, 22(6), 252-255.

Arnett, J. (1990). Contraceptive use, sensation seeking, and adolescent egocentrism. Journal of Youth and Adolescence, 19, 171-180.

Aronson, S. (1995). Five girls in search of a group: A group experience for adolescents of parents with AIDS. International Journal of Group Psychotherapy, 45(2), 223-235.

Aruffo, J.F., Gottlieb, A, Webb, R., \& Neville, B. (1994). Adolescent psychiatric inpatients: Alcohol use and HIV risk-taking behavior. Special Issue: Serving people 
with psychiatric disability at risk for HIV/AIDS. Psychosocial Rehabilitation Journal, 17(4), 150-156.

Baldwin, J.D. \& Baldwin, J.I. (1988a). AIDS information and sexual behavior on a university campus. Journal of Sex Education and Therapy, 14, 24-28.

Baldwin, J.D. \& Baldwin, J.I. (1988b). Factors affecting AIDS-related sexual risktaking behavior among college students. Journal of Sex Research, 25, 197-218.

Baldwin, J.I., Whiteley, S., \& Baldwin, J.D. (1990). Changing AIDS- and fertility-related behavior: The effectiveness of sexual education. The Journal of Sex Research, 27(2), 245-262.

Bandura, A. (1977). Self-efficacy: Toward a unifying theory of behavioral change. Psychological Review, 84, 191-215.

Bandura, A. (1990). Perceived self-efficacy in the exercise of control over AIDS infection. Evaluation and Program Planning, 13, 9-17.

Bandura, A. (1992). A social cognitive approach to the exercise of control over AIDS infection. In R.J. DiClemente, (Ed.) Adolescents and AIDS: A Generation in Jeopardy, Newbury Park, CA: Sage Publications, pp. 89-116.

Barling, N.R. \& Moore, S.M. (1990). Adolescents' attitudes towards AIDS precautions and intention to use condoms. Psychological Reports, 67, 883-890.

Barnard, M. \& McKeganey, N. (1990). Adolescents, sex and injecting drug use: Risk for HIV infection. AIDS Care, 2(2), 103-116.

Barnes, G.M. (1984). Adolescent alcohol abuse and other problem behaviors: Their relationships and common parental influences. Journal of Youth and Adolescence, 13(4), 329-348.

Basch, C.E. (1987). Focus group interview: An underutilized research technique for improving theory and practice in health education. Health Education Quarterly, 14, 411-448.

Basch, C.E. (1989). Preventing AIDS through education: Concepts, strategies, and research priorities. Journal of School Health, 59(7), 296-300.

Basen-Engquist, K. \& Parcel, G.S. (1992). Attitudes, norms, and self-efficacy: A model of adolescents' HIV-related sexual risk behavior. Health Education Quarterly, 19(2), 263-277. 
Baum, A. \& Nesselhof, S.E. (1988). Psychological research and the prevention, etiology, and treatment of AIDS, Special Issue: Psychology and AIDS. American Psychologist, 43(11), 900-906.

Bauman, L.J. \& Siegel, K. (1987). Misperceptions among gay men of the risk for AIDS associated with their sexual behavior. Journal of Applied Social Psychology, 17, 329350 .

Becker, M.H. \& Joseph, J.G. (1988). AIDS and behavioral change to reduce risk: A review. American Journal of Public Health, 78(4), 394-410.

Belfer, M.L., Krener, P.K., \& Miller, F.B. (1988). AIDS in children and adolescents. Journal of the American Academy of Child and Adolescent Psychiatry, 27(2), 147-151.

Bell, D., Feraios, A., \& Bryan, T. (1990). Adolescent males' knowledge and attitudes about AIDS in the context of their social world. Journal of Applied Social Psychology, 20(5), 424-448.

Bertrand, J.T., Brown, L.F., Kinzonzi, M., Mansilu, M., \& Djunghu, B. (1992). AIDS knowledge in three sites in Bas-Zaire. AIDS Education and Prevention, 4(3), 251-266.

Bhushan, V. \& Cushman, L.F. (1995). Paediatric AIDS: Selected attitudes and behaviours of paediatricians in New York City hospitals. AIDS Care, 7(1), 27-34.

Biglan, A., Metzler, C.W., Wirt, R., Ary, D., Noell, J., Ochs, L., French, C., \& Hood, D. (1990). Social and behavioral factors associated with high-risk sexual behavior among adolescents. Journal of Behavioral Medicine, 13(3), 245-261.

Billy, J.O.G. \& Udry, J.R. (1985). The influence of male and female best friends on adolescent sexual behavior. Adolescence, 20(77), 21-32.

Bing, E.G., Nichols, S.E., Goldfinger, S.M., Fernandez, F., Cabaj, R., Dudley, R.G., Krener, P., Prager, M., \& Ruiz, P. (1990). The many faces of AIDS: Opportunities for intervention. New Directions for Mental Health Services, 48 , 69-81.

Black, J.L. \& Jones, L.H. (1988). HTV infection: Educational programs and policies for school personnel. Journal of School Health, 58(8), 317-322.

Boldero, J., Moore, S., \& Rosenthal, D. (1992). Intention, context and safe sex: Australian adolescents' responses to AIDS. Journal of Applied Social Psychology, 22(17), 1374-1396.

Bounds, W., Guillebaud, J., Stewart, L., \& Steele, S. (1988). A female condom (Femshield): A study of its user acceptability. British Journal of Family Planning, 14, 83-87. 
Bowen, A.M. \& Trotter, R. (1995). HIV risk in IV drug users and crack smokers: Predicting stage of change for condom use. Journal of Consulting and Clinical Psychology, 63(2), 238-248.

Bowler, S., Sheon, A.R., D'Angelo., L.J., \& Vermund, S.H. (1992). HIV and AIDS among adolescents in the United States: Increasing risk in the 1990s. Journal of Adolescence, $15,345-371$.

Boyer, C.B. \& Kegeles, S.M. (1991). AIDS risk and prevention among adolescents. Social Science and Medicine, 33, 11-23.

Brandt, A.M. (1988). AIDS in historical perspective: Four lessons from the history of sexually transmitted diseases. American Journal of Public Health, 78(4), 367-371.

Braverman, P.K. \& Strasburger, V.C. (1994). The practitioner's role. Clinical Pediatrics, 33(2), 100-109.

Brock, G.C. \& Beazley, R.P. (1995). Using the health belief model to explain parents' participation in adolescents' at-home sexuality education activities. Journal of School Health, 65(4), 124-128.

Broering, J., Moscicki, B., Millstein, S.G., Policar, M., \& Irwin, C.W. (1989). Sexual practices among adolescents. Journal of Adolescent Health Care, 10, 249.

Brookman, R.R. (1990). Adolescent sexual behavior. In K.K. Holmes, et al. (Eds). Sexually Transmitted Diseases, Second Edition. New York: McGraw-Hill, pp. 77-84.

Brooks-Gunn, J. (1988). Antecedents to and consequences of variations in girls' maturational timing. Journal of Adolescent Health Care, 9 , 1-11.

Brooks-Gunn, J., Boyer, C.B., \& Hein, K. (1988). Preventing HIV infection and AIDS in children and adolescents. Behavioral research and intervention strategies. American Psychologist, 43(11), 958-964.

Brooks-Gunn, J. \& Furstenberg, F.F. (1989). Adolescent sexual behavior. American Psychologist, 44(2), 249-257.

Brooks-Gunn, J. \& Furstenberg, F.F. (1990). Coming of age in the era of AIDS: Puberty, sexuality, and contraception. Milbank Quarterly, $\underline{68}, 59-84$.

Broverman, I., Vogel, S., Broverman, D., Clarkson, F., \& Rosenkrantz, P. (1972). Sexrole stereotypes: A current appraisal. Journal of Social Issues, $\underline{28}$, 59-78. 
Brown, J.D. \& Newcomer, S.F. (1991). Television viewing and adolescents' sexual behavior. Journal of Homosexuality, 21, 77-91.

Brown, L.K., DiClemente, R.J., \& Beausoleil, N.I. (1992). Comparison of human immunodeficiency virus related knowledge, attitudes, intentions, and behaviors among sexually active and abstinent young adolescents. Journal of Adolescent Health, $13(2)$, 140-145.

Brown, L.K., DiClemente, R.J., \& Park, T. (1992). Predictors of condom use in sexually active adolescents. Journal of Adolescent Health, 13(8), 651-657.

Brown, L.K. \& Fritz, G.K. (1988). Children's knowledge and attitudes about AIDS. Journal of the American Academy of Child and Adolescent Psychiatry, 27, 504-508.

Brown, L.K., Fritz, G.K., \& Barone, V.J. (1989). The impact of AIDS education on junior and senior high school students: A pilot study. Journal of Adolescent Health Care, 10, 386-392.

Brown, L.K., Nassau, J.H., \& Levy, V. (1990). "What upsets me the most about AIDS is ...": A survey of children and adolescents. AIDS Education and Prevention, 2(4), 296-304.

Brunswick, A.F., Aidala, A., Dobkin, J., Howard, J., Titus, S.P., \& Banaszak-Holl, J. (1993). HIV-1 seroprevalence and risk behaviors in an urban African-American community cohort. American Journal of Public Health, 83(10), 1390-1394.

Burger, J.M. \& Burns, L. (1988). The illusion of unique invulnerability and the use of effective contraception. Personality and Social Psychology Bulletin, 14(2), 264-270.

Burke, D.S., Brundage, J.F., Goldenbaum, M., Gardner, L.I., Peterson, M., Visintine, R., Redfield, R.R., \& the Walter Reed Retrovirus Research Group. (1990). Human Immunodeficiency Virus infections in teenagers: Seroprevalence among applicants for US military service. Journal of the American Medical Association, 263 (15), 20742077.

Burkholder, G.J. (1994). Education, attitudes toward gay and lesbian persons, anxiety and HIV/AIDS risk. Unpublished master's thesis, University of Rhode Island, Kingston, Rhode Island.

Calamidas, E.G. (1990). AIDS and STD education: What's really happening in our schools? Journal of Sex Education and Therapy, 16(1), 54-63.

Calder, J. (1977). Focus groups and the nature of qualitative marking. Journal of Marketing Research, 14, 353-364. 
Carnegie Council on Adolescent Development (1989). Turning Points: Preparing American Youth for the 21st Century. New York: Carnegie Corporation.

Caron, S.L., Davis, C.M., Halteman, W.A., \& Stickle, M. (1993). Predictors of condomrelated behaviors among first-year college students. The Journal of Sex Research, 30(3), 252-259.

Carpenter, C.C.J., Mayer, K.H., Stein, M.D., Leibman, B.D., Fisher, A., \& Fiore, T.C. (1991). Human Immunodeficiency Virus infection in North American women: Experience with 200 cases and a review of the literature. Medicine, 70(5), 307-325.

Carroll, L. (1991). Gender, knowledge about AIDS, reported behavioral change, and the sexual behavior of college students. Journal of American College Health, 40, 5-12.

Catania, J.A., Coates, T.J., Greenblatt, R.M., Dolcini, M.M., Kegeles, S.M., Pucket, S., Corman, M., \& Miller, J. (1989a). Predictors of condom use and multiple partnered sex among sexually-active adolescent women: Implications for AIDS-related health interventions. The Journal of Sex Research, 26(4), 514-524.

Catania, J.A., Coates, T.J., \& Kegeles, S. (1994). A test of the AIDS risk reduction model: Psychosocial correlates of condom use in the AMEN cohort survey. Health Psychology, 13(6), 548-555.

Catania, J.A., Coates, T.J., Stall, R., Turner, H., Peterson, J., Hearst, N., Dolcini, M.M., Hudes, E., Gagnon, J., Wiley, J., \& Groves, R. (1992). Prevalence of AIDS-related risk factors and condom use in the United States. Science, 258, 1101-1106.

Catania, J.A., Dolcini, M.M., Coates, T.J., Kegeles, S.M., Greenblatt, R.M., Puckett, S., Corman, M., \& Miller, J. (1989b). Predictors of condom use and multiple partnered sex among sexually-active adolescent women: Implications for AIDS-related health interventions. The Journal of Sex Research, 26(4), 514-524.

Catania, J.A., Gibson, D.R., Chitwood, D.D., \& Coates, T.J. (1990). Methodological problems in AIDS behavioral research: Influences on measurement error and participation bias in studies of sexual behavior. Psychological Bulletin, 108(3), 339362.

Catania, J.A., Gibson, D.R., Marin, B., Coates, T.M., \& Greenblatt, R.M. (1990). Response bias in assessing sexual behaviors relevant to HIV transmission. Evaluation and Program Planning, 13, 19-29.

Catania, J.A., Kegeles, S.M., \& Coates, T.J. (1990). Towards an understanding of risk behavior: An AIDS risk reduction model (ARRM). Health Education Quarterly, 17(1), 53-72. 
Cates. W. (1990). Acquired Immunodeficiency Syndrome, sexually transmitted diseases, and epidemiology: Past lessons, present knowledge, and future opportunities. American Journal of Epidemiology, 131(5), 749-758.

Cates, W. (1991). Teenagers and sexual risk taking: The best of times and the worst of times. Journal of Adolescent Health, 12, 84-94.

Centers for Disease Control. (1990). Premarital sexual experience among adolescent women, United States, 1970-1988. Morbidity and Mortality Weekly Report, 39, $929-$ 932.

Centers for Disease Control. (1991). Premarital sexual experience among adolescent women -- United States, 1970-1988. Morbidity and Mortality Weekly Report, $\underline{39}$, 929932.

Centers for Disease Control and Prevention. (1992, October). Preventing risk behaviors among students. HIV/AIDS Prevention Newsletter, $\underline{3}(3), 1-2$.

Centers for Disease Control and Prevention (1993a, January 3). Sexual risk behaviors of STD clinic patients before and after Earvin "Magic" Johnson's HIV-infection announcement -- Maryland, 1991-1992. Morbidity and Mortality Weekly Report, $\underline{42}(3), 45-48$.

Centers for Disease Control and Prevention (1993b, May 28). Sexual behavior and condom use -- District of Columbia, January-February, 1992. Morbidity and Mortality Weekly Report, 42 (20), 390-398.

Centers for Disease Control and Prevention (1993c, July 2). Update: Mortality attributable to HIV Infection/AIDS among persons aged 25-44 years -- United States, 1990 and 1991. Morbidity and Mortality Weekly Report, 42(25), 481-486.

Centers for Disease Control and Prevention (1993d, July 9). Availability of comprehensive adolescent health services -- United States, 1990. Morbidity and Mortality Weekly Report, 42(26), 507-515.

Centers for Disease Control and Prevention (1993e, July 23). Update: Acquired Immunodeficiency Syndrome -- United States, 1992. Morbidity and Mortality Weekly Report, 42 (28), 447-557.

Centers for Disease Control and Prevention (1993f, August 6). Update: Barrier protection against HIV infection and other sexually transmitted diseases. Morbidity and Mortality Weekly Report, 42(30), 589-597. 
Centers for Disease Control and Prevention. (1993g, August 13). Surveillance for gonorrhea and primary and secondary syphilis among adolescents, United States--19811991. Morbidity and Mortality Weekly Report, 42(SS-3), 1-11.

Centers for Disease Control and Prevention. (1993h, October 1). Teenage pregnancy and birth rates - United States, 1990. Morbidity and Mortality Weekly Report, $\underline{42}$ (38), 733737.

Centers for Disease Control and Prevention. (1993i, November 19). World AIDS Day December 1, 1993; Update: Mortality Attributable to HIV infection among persons aged 25-44 years -- United States, 1991 and 1992; Distribution of STD clinic patients along a stages-of-behavioral-change continuum -- selected sites, 1993. Morbidity and Mortality Weekly Report, 42(45), 869; 869-872; 880-883.

Centers for Disease Control and Prevention (1993j, November 26). Mortality patterns -United States, 1991. Morbidity and Mortality Weekly Report, 42(46), 891-900.

Centers for Disease Control and Prevention (1993k, December 17). Surveillance for pregnancy and birth rates among teenagers, by state -- United States, 1980 and 1990. Morbidity and Mortality Weekly Report, 4 (SS-6), 1-27.

Centers for Disease Control and Prevention (1994a). HIV/AIDS Surveillance Report. Year-end edition, $\underline{6}(2), 5-35$.

Centers for Disease Control and Prevention (1994b, Spring). HIV infection among young people. HIV/AIDS Prevention Newsletter, 5(1), 2-3.

Centers for Disease Control and Prevention (1994c, Spring). Summary of data on AIDS cases reported in 1993. HIV/AIDS Prevention Newsletter, $\underline{5}(1), 6$.

Centers for Disease Control and Prevention (1994d, Spring). WHO: Sex education leads to safer behavior. HIV/AIDS Prevention Newsletter, $\underline{5}(1), 9$.

Centers for Disease Control and Prevention (1994e, March 4). Health risk behaviors among adolescents who do and do not attend school -- United States, 1992. Morbidity and Mortality Weekly Report, 43(8), 129-132.

Centers for Disease Control and Prevention (1994f, March 11). Heterosexually acquired AIDS -- United States, 1993; Update: Impact of the expanded AIDS surveillance case definition for adolescents and adults on case reporting -- United States, 1993. Morbidity and Mortality Weekly Report, 43(9), 155-160; 160-170.

Centers for Disease Control and Prevention (1994g, April 8). Health risk behaviors among persons aged 12-21 years -- United States, 1992. Morbidity and Mortality Weekly Report, $\underline{43}$ (13), 231--235. 
Centers for Disease Control and Prevention (1994h, April 29). Zidovudine for the prevention of HIV transmission from mother to infant. Morbidity and Mortality Weekly Report, 43 (16), 285-287.

Centers for Disease Control and Prevention (1994i, August 5). Recommendations of the U.S. Public Health Services Task Force on the use of Zidovudine to reduce perinatal transmission of Human Immunodeficiency. Morbidity and Mortality Weekly Report, 43(RR-11), 1-20.

Centers for Disease Control and Prevention (1994j, November 18). World AIDS Day -December 1, 1994. Morbidity and Mortality Weekly Report, 43(45), 825-831.

Centers for Disease Control and Prevention (1994k, December 16). Mortality patterns -United States, 1992. Morbidity and Mortality Weekly Report, 43(49), 916-920.

Centers for Disease Control and Prevention (19941, Winter). HIV/AIDS Advances as leading cause of death for people aged 25-44 in the United States. HIV/AIDS Prevention Newsletter, $\underline{5}(3), 2$.

Centers for Disease Control and Prevention (1995a, February 10). Update: AIDS among women -- United States, 1994. Morbidity and Mortality Weekly Report, 44(5), 81-84.

Centers for Disease Control and Prevention (1995b, February 24). Trends in sexual risk behavior among high school students -- United States, 1990, 1991, and 1993. Morbidity and Mortality Weekly Report, 44(7), 124-132.

Centers for Disease Control and Prevention (1995c, March 24). Youth risk behavior surveillance -- United States, 1993. Morbidity and Mortality Weekly Report, 44(SS-1), 1-58.

Centers for Disease Control and Prevention (1995d, September 22). State-specific pregnancy and birth rates among teenagers -- United States, 1991-1192. Morbidity and Mortality Weekly Report, 44(37), 677-684.

Centers for Disease Control and Prevention (1995e, November 24). First 500,000 AIDS cases --- United States, 1995. Morbidity and Mortality Weekly Report, 44(46), 849853.

Centers for Disease Control and Prevention (1995f). HIV/AIDS Surveillance Report. Mid-year edition, $7(1), 8$.

Centers for Disease Control and Prevention (1996, February 16). Update: Mortality attributable to HIV infection among persons aged 25-44 years -- United States, 1994. Morbidity and Mortality Weekly Report, 44(6), 121-125. 
Christopher, F.S. \& Roosa, M.W. (1990). An evaluation of an adolescent pregnancy prevention program: Is "Just Say No" enough. Family Relations, $\underline{39}, 68-72$.

Clapper, R.L. \& Lipsitt, L.P. (1991). A retrospective study of risk-taking and alcoholmediated unprotected intercourse. Journal of Substance Abuse, 3 , 91-96.

Clark, S.D., Zabin, L.S., \& Hardy, J.B. (1984). Sex contraception and parenthood. Family Planning Perspectives, 15 , 77-84.

Clarke, J., Abram, R., \& Monteiro, E.F. (1990). The sexual behaviour and knowledge about AIDS in a group of young adolescent girls in Leeds. Genitourinary Medicine, $\underline{66}$, 189-192.

Coates, T.J. (1990). Strategies for modifying sexual behavior for primary and secondary prevention of HIV disease. Journal of Consulting and Clinical Psychology, 58, 57-69.

Cochran, S.D. \& Mays, V.M. (1990). Sex, lies and HIV. New England Journal of Medicine, $322,774-775$.

Cochran, S.D. \& Peplau, L.A. (1991). Sexual risk reduction behaviors among young heterosexual adults. Social Science Medicine, 1, 25-36.

Cohen, D., Dent, C., \& MacKinnon, D. (1991). Condom skills education and sexually transmitted disease reinfection. The Journal of Sex Research, 28(1), 139-144.

Cohn, L.D., Macfarlane, S., Yanez, C., \& Imai, W.K. (1995). Risk-perception: Differences between adolescents and adults. Health Psychology, 14(3), 217-222.

Conant, M., Hardy, D., Sernatinger, J., Spicer, D., \& Levy, J.A. (1986). Condoms prevent transmission of AIDS-associated retrovirus. Journal of the American Medical Association, 255(13), 1706.

Corea, G. (1992). The Invisible Epidemic: The Story of Women and AIDS. New York: Harper Collins.

Coyle, A. \& Loveless. L. (1995). A study of youth workers as health promoters on sexrelated issues and HIV/AIDS in a British city. International Journal of Adolescence and Youth, $5(3), 157-171$.

Crawford, I. \& Robinson, W.L. (1991). Adolescents and AIDS: Knowledge and attitudes of African-American, Latino and Caucasian midwestern U.S. high school seniors. Journal of Psychology and Human Sexuality, $\underline{3}$ (2), 25-32. 
Crawford, J., Turtle, A., \& Kippax, S. (1990). Student-favoured strategies for AIDS avoidance. Australian Journal of Psychology, 42(2), 123-137.

Cullari, S., \& Mikus, R. (1990). Correlates of adolescent sexual behavior. Psychological Reports, $66,1179-1184$.

Curran, J.W., Jaffe, H.W., Hardy, A.M., Morgan, W.M., Selik, R.M., \& Dondero, T.J. (1988). Epidemiology of HIV infection and AIDS in the United States. Science, $\underline{239}(4840), 610-616$.

Damond, M.E., Breuer, N.L., \& Pharr, A.E. (1993). The evaluation of setting and a culturally specific HIV/AIDS curriculum: HIV/AIDS knowledge and behavioral intent of African American adolescents, Journal of Black Psychology, 19(2), 169-189.

Dancy, B.L. (1991). The development of an ethnically sensitive and gender-specific AIDS questionnaire for African-American Women. Health Values, 15(6), 49-54.

D'Angelo, L.J. (1994). Adolescents and HIV infection: A clinician's perspective. Acta Pediatric Supplement, 400 , 88-94.

D’Angelo, L.J., Brown, R. English, A., Hein, K., \& Remafedi, G. (1994). HIV infection and AIDS in adolescents: A position paper of the Society for Adolescent Medicine. Journal of Adolescent Health, 15(5), 427-434.

D'Angelo, L.J., Getson, P.R., Luban, N.L.C., \& Gayle, H.D. (1991). Human Immunodeficiency Virus infection in urban adolescents: Can we predict who is at risk? Pediatrics, $\underline{88}(5)$, 982-986.

Dawson, D.A. (1986). The effects of sex education on adolescent behavior. Family Planning Perspectives, $18,162-170$.

Deiter, P. (1994). Sexual assertiveness training for college women. Unpublished doctoral dissertation, University of Rhode Island, Kingston, Rhode Island.

Demb, J. (1990). Black, inner-city, female adolescents and condoms: What the girls say. Family Systems Medicine, 8(4), 401-406.

Des Jarlais, D., Ehrhardt, A., Fullilove, M., Hein, K., Menken, J., Mensch, B.S., Miller, H., \& Turner, C. (1990). AIDS and adolescents. In H. Miller, C. Turner, \& L. Moses, (Eds.), AIDS: The Second Decade. Washington, DC: National Academy Press, $p p$. 147-252.

Desvousges, W.H. \& Frey, J.H. (1989). Integrating focus groups and surveys: Examples from environmental risk studies. Journal of Official Statistics, $\underline{5}, 349-363$. 
deVries, H., Weijts, W., Dijkstra, M., \& Kok, G. (1992). The utilization of qualitative and quantitative data for health education program planning, implementation, and evaluation: A spiral approach. Health Education Quarterly, 19, 101-115.

DiClemente, C.C., Prochaska, J.O., Fairhurst, S.K., Velicer, W.F., Velasquez, M.M., \& Rossi, J.S. (1991). The process of smoking cessation: An analyses of precontemplation, contemplation, and preparation stages of change. Joumal of Consulting and Clinical Psychology, 59, 295-304.

DiClemente, R.J. (1989). Prevention of Human Immunodeficiency Virus infection among adolescents: The interplay of health education and public policy in the development and implementation of school-based AIDS education programs. AIDS Education and Prevention, 1 , 70-78.

DiClemente, R.J. (1990). The emergence of adolescents as a risk group for human immunodeficiency virus infection. Journal of Adolescent Research, 5, 7-17.

DiClemente, R.J. (1991). Predictors of HIV-Preventive sexual behavior in a high-risk adolescent population: The influence of perceived peer norms and sexual communication on incarcerated adolescents' consistent use of condoms. Journal of Adolescent Health, 12, 385-390.

DiClemente, R.J. (1992). Epidemiology of AIDS, HIV Prevalence, and HIV incidence among adolescents. Journal of School Health, 62(7), 325-330.

DiClemente, R.J. (1993). Confronting the challenge of AIDS among adolescents: Directions for future research. Journal of Adolescent Research, $\underline{8}(2), 156-166$.

DiClemente, R.J., Boyer, C.B., \& Mills, S.J. (1987). Prevention of AIDS among adolescents: Strategies for the development of comprehensive risk-reduction health education programs. Health Education Research, 2(3), 287-291.

DiClemente, R.J., Boyer, C.J., \& Morales, E.S. (1988). Minorities and AIDS: Knowledge, attitudes, and misconceptions among Black and Latino adolescents. American Journal of Public Health, $\underline{78}$, 55-57.

DiClemente, R.J., Brown, L.K., Beausoleil, N.I., \& Lodico, M. (1993). Comparison of AIDS knowledge and HIV-related sexual risk behaviors among adolescents in low and high AIDS prevalence communities. Journal of Adolescent Health, 14(3), 231-236.

DiClemente, F.J., Durbin, M., Siegel, D., Krasnovsky, F., Lazarus, N., \& Comacho, T. (1992). Determinants of condom use among junior high school students in a minority, inner-city school district. Pediatrics, 89(2), 197-201. 
DiClemente, R.J., Forrest, K.A., \& Mickler, S. (1990). College students' knowledge and attitudes about AIDS and changes in HIV-preventive behaviors. AIDS Education and Prevention, 2(3) 201-212.

DiClemente, R.J. \& Houston-Hamilton, A. (1989). Health promotion strategies for prevention of Human Immunodeficiency Virus Infection among minority adolescents. Health Education, 20(5), 39-43.

DiClemente, R.J., Zorn, J, \& Temoshok, L. (1986). Adolescents and AIDS: A survey of knowledge, attitudes and beliefs about AIDS in San Francisco. American Journal of $\underline{\text { Public Health, }} \underline{76}$, 1443-1445.

DiClemente, R.J., Zorn, J., \& Temoshok, L. (1987). The association of gender, ethnicity, and length of residence in the Bay Area to adolescents' knowledge and attitudes about Acquired Immune Deficiency Syndrome. Journal of Applied Social Psychology, 17, 216-230.

DiScipio, W.J. (1994). Sex, drugs, and AIDS: Issues for hospitalized emotionally disturbed youth. Special Issue: Brief and intermediate treatment for psychiatrically disturbed youth. Psychiatric Quarterly, 65(2), 149-155.

Dolcini, M.M., Coates, T.J., Catania, J.A., Kegeles, S.M., \& Hauck, W.W. (1995). Multiple sex partners and their psychosocial correlates: The population-based AIDS in multiethnic neighborhoods (AMEN) study. Health Psychology, 14(1), 22-31.

Dolcini, P., Adler, N.E., Irwin, C.E., Jr., Millstein S., Kegeles, S.M., Cohn, L., \& Stone G. (1988). Perceptions of susceptibility to AIDS among adolescents. Presented at the Fourth International Conference on AIDS, Stockholm, Sweden.

Dommeyer, C.J., Marquard, J.L., Gibson, J.E., \& Taylor, R.L. (1989). The effectiveness of an AIDS education campaign on a college campus. Journal of American College Health, $\underline{38}, 131-135$.

Donovan, C. \& McEwan, R. (1995). A review of the literature examining the relationship between alcohol use and HIV-related sexual risk-taking in young people. Addiction, 90(3), 319-328.

Donovan, J.E., Jessor, R., \& Costa, F.M. (1991). Adolescent health behavior and conventionality-unconventionality. An extension of problem-behavior theory. Health Psychology, 10(1), 52-61.

Drew, W.L., Blair, M., Miner, R.C., \& Conant, M. (1990). Evaluation of the virus permeability of a new condom for women. Sexually Transmitted Diseases, 17, 110 112. 
du Guerny J. \& Sjöberg, E. (1992, July 8). Inter-relationship between gender relations and the HIV/AIDS-epidemic: Some possible considerations for policies and programs. (Prepublication draft), Vienna: Centre for Social Development and Humanitarian Affairs, Division for the Advancement of Women, p. 7.

Dubois-Arber, F., Jeannin, A., \& Zeugin, P. (1992, July 19-24). Evaluation of AIDS prevention in Switzerland: Behavioral change in the general population. Presented at the Eighth International Conference on AIDS, Amsterdam, The Netherlands. Abstract PoD5140.

DuRant, R.H., Ashworth, C.S., Newman, C., \& Gaillard, G. (1992). High school students' knowledge of HIV/AIDS and perceived risk of currently having AIDS. Journal of School Health, 62(2), 59-63.

DuRant, R.H. \& Jay, S. (1989). The adolescent heterosexual relationship and its association with the sexual and contraceptive behavior of Black females. American Journal of Diseases of Children, $143,1467-1472$.

DuRant, R.H., Sanders, J.M, Jay, S., \& Levinson, R. (1988). Analyses of contraceptive behavior of sexually active female adolescents in the United States. The Journal of Pediatrics, 113, 930-936.

Durbin, M., DiClemente, R.J., Siegel, D., Krasnovsky, F., Lazarus, N., \& Camacho, T. (1993). Factors associated with multiple sex partners among junior high school students. Journal of Adolescent Health, 14, 202-207.

Dusenbury, L., Botvin, G.J., Baker, E., \& Laurence, J. (1991). AIDS risk knowledge, attitudes, and behavioral intentions among multi-ethnic adolescents. AIDS Education and Prevention, 3 (4), 367-375.

Eiser, C., Eiser, R., \& Lang, J. (1990). How adolescents compare AIDS with other diseases: Implications for prevention. Journal of Pediatric Psychology, 15, 97-103.

Ekstrand, M.L. \& Coates, T.J. (1990). Maintenance of safer sexual behaviors and predictors of risky sex: The San Francisco Men's Health Study. American Journal of Public Health, 80(8), 973-977.

Epstein, J.A, Dusenbury, L., Botvin, G.J., \& Diaz, T. (1994). Determinants of intentions of junior high school students to become sexually active and use condoms: Implications for reduction and prevention of AIDS risk. Psychological Reports, 75(2), 1043-1053.

European Study Group on Heterosexual Transmission of HIV (1992). Comparison of female to male and male to female transmission of HIV in 563 stable couples. British Medical Journal, 304, 809-813. 
Fennell, R. \& Beyrer, M.K. (1989). AIDS: Some ethical considerations for the Health educator. Journal of American College Health, 38(3), 145-147.

Fergusson, D.M., Lynskey, M.T., \& Horwood, L.J. (1994). AIDS knowledge and condom use in a birth cohort of 16-year olds. New Zealand Medical Journal, 107(990), 480-483.

Fineberg, H.V. (1988). Education to prevent AIDS: Prospects and obstacles. Science, $\underline{239}, 592-596$.

Fisher, J.D. \& Fisher, W.A. (1992). Changing AIDS-risk behavior. Psychological Bulletin, 111(3), 455-474.

Fisher, J.D., Fisher, W.A., Misovich, S.J., Kimble, D.L., \& Malloy, T.E. (1996). Changing AIDS risk behavior: Effects of an intervention emphasizing AIDS risk reduction information, motivation, and behavioral skills in a college student population. Health Psychology, 15(2), 114-123.

Fisher, J.D., Fisher, W.A., Williams, S.S., \& Malloy, T.E. (1994). Empirical tests of an information-motivation-behavioral skills model of AIDS-preventive behavior with gay men and heterosexual university students. Health Psychology, 13(3), 238-250.

Fisher, W.A., Fisher, J.D, \& Rye, B.J. (1995). Understanding and promoting AIDSpreventive behavior: Insights from the theory of reasoned action. Health Psychology, 14(3), 255-264.

Flanigan, B.J. (1990). The social context of alcohol consumption prior to female sexual intercourse. Journal of Alcohol and Drug Education, 36(1), 97-113.

Flanigan, B.J., McLean, A., Hall, C., \& Propp, V. (1990). Alcohol use as a situational influence on young women's pregnancy risk-taking behaviors. Adolescence, 25(97), 205-214.

Flora, J.A. \& Thoresen, C.E. (1988). Reducing the risk of AIDS in adolescents. American Psychologist, 43(11), 965-970.

Folkman, S., Chesney, M.A., Cooke, M., Boccellari, A., \& Collette, L. (1994). Caregiver burden in HIV-positive and HIV-negative partners of men with AIDS. Journal of Consulting and Clinical Psychology, 62(4), 746-756.

Ford, K., Rubinstein, S., \& Norris, A. (1994). Sexual behavior and condom use among urban, low-income, African American and Hispanic youth. AIDS Education and Prevention, 6(3), 219-229. 
Frances, R.J. \& Miller, S.I. (Eds.). (1991). Clinical Textbook of Addictive Disorders.

New York: Guilford Press, p. 342.

Franzini, L.R., Sideman, L.M., Dexter, K.E., \& Elder, J.P. (1991). Promoting AIDS risk reduction via behavioral training. AIDS Education and Prevention, 2(4), 313-321.

Freeman, A., Cohn, D., Corby, N., \& Wood, R. (1991). Patterns of sexual behavior change among homosexual/bisexual men: Selected U.S. Sites, 1987-1990. Morbidity and Mortality Weekly Report, 40(46), 792-794.

Freimuth, V.S., Edgar, T., \& Hammond, S.L. (1987). College students' awareness and interpretation of the AIDS risk. Science, Technology, and Human Values, 12, 37-40.

Friedland, G.H. \& Klein, M.D. (1987). Transmission of the Human Immunodeficiency Virus. The New England Journal of Medicine, 317(18), 1125-1135.

Friedman, H.L. (1993). Reproductive health in adolescence. World Health Statistics Quarterly, 47(1), 31-35.

Fuller, T.D., Edwards, J.N., Vorakiphokatorn, S., \& Sermski, S. (1990). Using focus groups to adapt survey instruments to new populations. In D.L. Morgan (Ed.) (1993). Successful Focus Groups: Advancing the State of the Art. Newbury Park, CA: Sage Publications, $p p$. 89-104.

Fullilove, M.T., Fullilove, R.E., Haynes, K., \& Gross, S. (1990). Black women and AIDS prevention: A view towards understanding the gender rules. Special Issue: Feminist perspectives on sexuality. Journal of Sex Research, 27, 47-64.

Furstenberg, F.F., Jr., Moore, K.A., \& Peterson, J.L. (1986). Sex education and sexual experience among adolescents. American Journal of Public Health, 75, 1221-1222.

Gaines, J., Iglar, A.F., Michal, M.L., \& Patton, R.D. (1988). Attitudes towards AIDS. Health Values, 12(4), 53-59.

Galavotti, C., Cabral, R.J., Lansky, A., Grimley, D.M., Riley, G.E., \& Prochaska, J.O. (1995). Validation of measures of condom and other contraceptive use among women at high risk for HIV infection and unintended pregnancy. Health Psychology, 14(6), 570-578.

Gallup, B. \& Gallup, A. (1988). AIDS: We worry about the wrong things. American Health, 6(June), 50-52.

Gardner, W. \& Wilcox, B.L. (1993). Political intervention in scientific peer review: Research on adolescent sexual behavior. American Psychologist, 48(9), 972-983. 
Gayle, H.D. \& D’Angelo, L.J. (1991). Epidemiology of Acquired Immunodeficiency Syndrome and Human Immunodeficiency Virus infection in adolescents. The Pediatric Infectious Disease Journal, 10(4), 322-328.

Gayle, H.D., Keeling, R.P., Garcia-Tunon, M., Kilbourne, B.W., Narkunas, J.P., Ingram, F.R., Rogers, M.F., \& Curran, J.W. (1990). Prevalence of the Human Immunodeficiency Virus among university students. The New England Journal of Medicine, $\underline{323}(22), 1538-1541$.

Gilligan, C. (1979). Woman's place in man's life cycle. Harvard Educational Review, 49(4), 431-446.

Gilligan, C. (1982). In a Different Voice: Psychological Theory and Women's Development. Cambridge, MA: Harvard University Press, p. 30.

Gilligan, C., Rogers, A., \& Brown, L.M. (1990). Soundings into development. In C. Gilligan, N.P. Lyons, \& T.J. Hanmer, (Eds). Making Connections: The Relational Worlds of Adolescent Girls at Emma Willard School. Cambridge: Harvard University Press, pp. 314-334.

Gladis, M.M., Michela, J.L., Walter, H.J., \& Vaughan, R.D. (1992). High school students' perceptions of AIDS risk: Realistic appraisal or motivated denial? Health Psychology, 11(5), 307-316.

Gold, R.S., Skinner, M.J., \& Ross., M.W. (1994). Unprotected anal intercourse in HIVinfected and non-HIV-infected gay men. The Journal of Sex Research, 31(1), 59-77.

Goodman, E. \& Berecochea, J.E. (1994). Predictors of HIV testing among runaway and homeless adolescents. Journal of Adolescent Health, 15(7), 566-572.

Goodman, E. \& Cohall, A.T. (1989). Acquired Immune Deficiency Syndrome and adolescence: Knowledge, attitudes, beliefs and behaviors in a New York City adolescent minority population. Pediatrics, $84,36-42$.

Goodson, P. \& Edmundson, E. (1994). The problematic promotion of abstinence: An overview of Sex Respect. Journal of School Health, 64(5), 205-210.

Graham, C.A. (1994). AIDS and the adolescent [Editorial]. International Journal of STDs/AIDS, 5(5), 305-309.

Grant, M. \& Johnstone, B.M. (1990). Research priorities for drug and alcohol studies: The next 25 years. The International Journal of the Addictions, 25(2A), 201-219.

Gray, L.A. \& Saracino, M. (1991). College students' attitudes, beliefs, and behaviors about AIDS: Implications for family life educators. Family Relations, $\underline{40}$, 258-263. 
Grimley, D.M., Harlow, L.L., Morokoff, P., \& Quina, K. (1992, August). Predicting sexual assertiveness in college-age women. American Psychological Association, Washington, DC.

Grimley, D.M., Riley, G.E., Bellis, J.M., \& Prochaska, J.O. (1993). Assessing the stages of change and decision-making for contraceptive use for the prevention of pregnancy, sexually transmitted diseases, and acquired immunodeficiency syndrome. Health Education Quarterly, 20, 455-470.

Gruber, E. \& Chambers, C.V. (1987). Cognitive development and adolescent contraception: Integrating theory and practice. Adolescence, 22(87), 661-670.

Gutman, L.T., St. Claire, K.K., Weedy, C., Herman-Giddens, M.E., Lane, B.A., Niemeyer, J.G., \& McKinney, R.E. (1991). Human Immunodeficiency Virus transmission by child sexual abuse. American Journal of Diseases of Children, 145, 137-141.

Guttmacher, S., Lieberman, L., Ward, D., Radosh, A., Rafferty, Y., \& Freundenberg, N. (1995). Parents' attitudes and beliefs about HIV/AIDS prevention with condom availability in New York City public high schools. Journal of School Health, 65(3), 101-106.

Gwadz, M. \& Rotheram-Borus, M.J. (1992). Tracking high-risk adolescents longitudinally. AIDS Education and Prevention, Supplement, 69-82.

Hankins, C. (1993). Women and HIV infection. In L. Sherr, (Ed.), AIDS and the Heterosexual Population, Langhorne, PA: Harwood Academic Publishers, pp. 31-38.

Hardy, A.M. (1990). National health interview survey data on adult knowledge of AIDS in the United States. Public Health Reports, 105(6), 629-634.

Harvey, S.M., \& Spigner, C. (1995). Factors associated with sexual behavior among adolescents: A multivariate analysis. Adolescence, 30(118), 253-264.

Harlow, L.L., Quina, K., \& Morokoff, P. (1991). Lifestyle Survey. University of Rhode Island. Unpublished manuscript.

Harlow, L.L., Quina, K., Morokoff, P.J., Grimley, D.M., \& Rose, J.S. (1993). HIV Risk in women: A multifaceted model. Journal of Applied Biobehavioral Research, 1, 3-38.

Hausser, D. \& Michaud, P.A. (1992, July 19-24). Condom promotion does not increase sexual intercourse among adolescents. Presented at the Eighth International Conference on AIDS, Amsterdam, The Netherlands. Abstract TuDo575. 
Hausser, D. \& Michaud, P.A. (1994). Does a condom-promoting strategy (the Swiss STOP-AIDS campaign) modify sexual behavior among adolescents? Pediatrics, 93(4), $580-585$.

Hays, R.B., Turner, H., \& Coates, T.J. (1992). Social support, AIDS-related symptoms, and depression among gay men. Journal of Consulting and Clinical Psychology, 60(3), 463-469.

Healy, R. \& Coleman, T. (1988/December, 1989/January). A primer on AIDS for health professionals. Health Education, 19(6), 4-10.

Hearst, N. \& Hulley, S.B. (1988). Preventing the heterosexual spread of AIDS: Are we giving our patients the best advice? Journal of the American Medical Association, $\underline{259}(16), 2428-2432$.

Hein, K. (1988). AIDS in adolescents: A rationale for concern. New York State Journal of Medicine, 87(5), 290-295.

Hein, K. (1990). Lessons from New York City on HIV/AIDS in adolescents. Special Issue: Acquired Immunodeficiency Syndrome. New York State Journal of Medicine, 90(3), 143-145.

Hein, K. (1991a). Fighting AIDS in adolescents. Issues in Science and Technology, $\underline{7}(3)$, 67-72.

Hein, K. (1991b). Mandatory HIV testing of youth: A lose-lose proposition. Journal of the American Medical Association, 266(17), 2430-2431.

Hein, K. (1991c). Risky business: Adolescents and Human Immunodeficiency Virus. Pediatrics, 88(5), 1052-1054.

Hein, K. (1992). Adolescents at risk for HIV infection. In R.J. DiClemente, (Ed.) Adolescents and AIDS: A Generation in Jeopardy, Newbury Park, CA: Sage Publications, pp. 3-16.

Hein, K. (1993). "Getting real" about HIV in adolescents. American Journal of Public Health, $\underline{83}(4), 492-494$.

Hein, K., Dell, R., Futterman, D., Rotheram-Borus, M.J., \& Shaffer, N. (1995). Comparison of HIV+ and HIV-adolescents: Risk factors and psychosocial determinants. Pediatrics, 95(1), 96-104.

Helgerson, S.D., Petersen, L.R., \& The AIDS Education Study Group. (1988). Acquired Immunodeficiency Syndrome and secondary school students: Their knowledge is limited and they want to learn more. Pediatrics, 81(3), 350-355. 
Helweg-Larsen, M. \& Collins, B.E. (1994). The UCLA multidimensional condom attitudes scale: Documenting the complex determinants of condom use in college students. Health Psychology, 13(3), 224-237.

Henderson, E.B., Weinman, M.L., \& Smith, P.B. (1994). The behavioral implications of myths and medical knowledge about AIDS among indigent female adolescents. Journal of Sex Education and Therapy, 20(2), 79-91.

Hernandez, J.T. \& Smith, F.J. (1990). Inconsistencies and misperceptions putting college students at risk of HIV infection. Journal of Adolescent Health Care, 11(4), 295-297.

Herold, E.S., Fisher, W.A., Smith, E.A., \& Yarber, W.A. (1990). Sex education and the prevention of STD/AIDS and pregnancy among youths. Canadian Journal of Public Health $\underline{81}, 141-145$.

Hingson, R. \& Strunin L. (1992). Monitoring adolescents' response to the AIDS epidemic: Changes in knowledge, attitudes, beliefs, and behaviors. In R.J. DiClemente, (Ed) Adolescents and AIDS: A Generation in Jeopardy, Newbury Park, CA: Sage Publications, $p p$. 17-33.

Hingson, R, Strunin, L., \& Berlin, B. (1990). Acquired Immunodeficiency Syndrome transmission: Changes in knowledge and behaviors among teenagers, Massachusetts statewide surveys, 1986-1988. Pediatrics, $\underline{85}$ (1), 24-29.

Hingson, R.W., Strunin, L., Berlin, B.M., \& Heeren, T. (1990). Beliefs about AIDS, use of alcohol and drugs, and unprotected sex among Massachusetts adolescents. American Journal of Public Health, 80(3), 295-299.

Hochhauser, M. (1989). AIDS and chemical dependency: Prevention needs of adolescents. Journal of Psychoactive Drugs, 21(4), 381-385.

Hofferth, S.L., Kahn, J.R., \& Baldwin, W. (1987). Premarital sexual activity among U.S. teenage women over the past three decades. Family Planning Perspectives, 19(2), 4653.

Holtzman, D., Anderson, J.E., Kann, L., Arday, S.L., Truman, B.I., \& Kolbe, L.J. (1991). HIV instruction, HIV knowledge, and drug injection among high school students in the United States. American Journal of Public Health, 81(12), 1596-1601.

Holtzman, D., Green, B.Z., Ingraham, G.C., Daily, L.A., Demchuk, D.G., \& Kolbe, L.J. (1992). HIV education and health education in the United States: A national survey of local school district policies and practices. Journal of School Health, 62(9), 421-427. 
Holtzman, D., Lowry, R., Kann, L., Collins, J.L., \& Kolbe, L.J. (1994). Changes in HIVrelated information sources, instruction, knowledge, and behaviors among US high

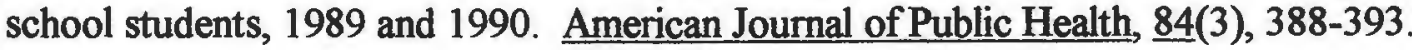

Horner, R.D., Kolasa, K.M., Irons, T.G., \& Wilson, K. (1994). Racial differences in rural adults' attitudes toward issues of adolescent sexuality. American Journal of Public Health, $\underline{84}(3), 456-459$.

Irwin, C.E. (1990). The theoretical concept of at-risk adolescents. Adolescent Medicine: State of the Art Reviews, 1, 1-14.

Irwin, C.E. \& Millstein, S.G. (1986). Biopsychosocial correlates of risk-taking behaviors during adolescence. Journal of Adolescent Health Care, I(6), 582-596.

Jaffe, L.R., Seehaus, M., Wagner, C., \& Leadbeater, B.J. (1988). Anal intercourse and knowledge of acquired immunodeficiency syndrome among minority-group female adolescents. The Journal of Pediatrics, $112,1005-1007$.

Janke, J. (1989). Dealing with AIDS and the adolescent population. The Nurse Practitioner, 4(3), 5-36.

Janz, N.K. \& Becker, M.H. (1984). The health belief model: A decade later. Health Education Quarterly, 11, 1-47.

Jemmott, J.B., Jemmott, L.S., \& Fong, G.T. (1992). Reductions in HIV risk -Associated sexual behaviors among Black male adolescents: Effects of an AIDS prevention intervention. American Journal of Public Health, 82(3), 372-377.

Jemmott, L.S. \& Jemmott, J.B. (1991). Applying the theory of reasoned action to AIDS risk behavior: Condom use among Black women. Nursing Research, 40, 228-234.

Jemmott, L.S. \& Jemmott, J.B. (1992). Increasing condom-use intentions among sexually active black adolescent women. Nursing Research, 41(5), 273-279.

Jessor, R. (1991). Risk behavior in adolescence: A psychosocial framework for understanding and action. Society for Adolescent Medicine, 12, 597-605.

Jessor, S.L. \& Jessor, R. (1975). Transition from virginity to nonvirginity among youth: A social-psychological study over time. Developmental Psychology, 11(4), 473-484.

Johnsen, L.W. \& Harlow, L.L. (in press). Childhood sexual abuse linked with adult substance use, victimization, and AIDS-risk. AIDS Education and Prevention.

Johnson, J.L. (1991). Preventive interventions for children at risk: Introduction. The International Journal of the Addictions, 25(4A), 429-434. 
Johnston, J. (1993). The sexual enlightenment of children in the modern cultural context: Care or corruption? Sexual and Marital Therapy, $\underline{8}(1), 53-74$.

Joseph, J.G., Emmons, C.A., Kessler, R.C., Wortman, C.B., O'Brien, K., Hocker, W.T., \& Schaefer, C. (1984). Coping with the threat of AIDS: An approach to psychosocial assessment. American Psychologist, 39(11), 1297-1302.

Joseph, S.C. (1991). AIDS and adolescents: A challenge to both treatment and prevention. Journal of Adolescent Health, 12, 614-618.

Kaemingk, K.L. \& Bootzin, R.R. (1990). Behavior change strategies for increasing condom use. Evaluation and Program Planning, 13, 47-54.

Kahn, J.R., Kalsbeek, W.D., \& Hofferth, S.L. (1988). National estimates of teenage sexual activity: Evaluating the comparability of three national surveys. Demography, $\underline{25}(2), 189-204$.

Kain, C. (Ed.). (1989). No Longer Immune: A Counselors Guide to AIDS. Alexandria, VA: American Association for Counseling and Development.

Kalichman, S.C. (1995). Understanding AIDS: A Guide for Mental Health Professionals. Washington, DC: American Psychological Association.

Kalichman, S.C. (1996). Answering Your Questions About AIDS. Washington, DC: American Psychological Association.

Kalichman, S.C. \& Coley, B. (1995). Context framing to enhance HIV-antibody-testing messages targeted to African American women. Health Psychology, 14(3), 247-254.

Kalichman, S.C., Hunter, T.L., \& Kelly, J.A. (1992). Perceptions of AIDS susceptibility among minority and nonminority women at risk for HIV infection. Journal of Consulting and Clinical Psychology, 60(5), 725-732.

Kalichman, S.C., Kelly, J.A., Hunter, T.L., Murphy, D.A., \& Tyler, R. (1993). Culturally tailored HIV-AIDS risk-reduction messages targeted to African-American urban women: Impact on risk sensitization and risk reduction. Journal of Consulting and Clinical Psychology, 61(2), 291-295.

Kalichman, S.C., Russell, R.L., Hunter, T.L., \& Sarwer, D.B. (1993). Earvin "Magic" Johnson's HIV serostatus disclosure: Effects on men's perceptions of AIDS. Journal of Consulting and Clinical Psychology, 61(5), 887-891.

Kamachi, K.L., Irwin, C.E., Shafer, M.B., Wong, V. (1990). "Understanding AIDS": Did female adolescents read it? Journal of Adolescent Health Care, 11(3), 254. 
Kann, L., Anderson, J.E., Holtzman, D., Ross, J., Truman, B.I., Collins, J., \& Kolbe, L.J. (1991). HIV-related knowledge, beliefs, and behaviors among high school students in the United States: Results from a national survey. Journal of School Health, 61(9), 397-401.

Kaplan, M.E. \& Schonberg, S.K. (1994). HIV in adolescents. Clinical Perinatology, 21(1), 75-84.

Katz, R.C., Mills, K., Singh, N.N., \& Best, A.M. (1995). Knowledge and attitudes about AIDS: A comparison of public high school students, incarcerated delinquents, and emotionally disturbed adolescents. Journal of Youth and Adolescence, 24(1), 117-131.

Keeling, R.P. (1989). (Ed.) AIDS on the College Campus, Second Edition. Rockville, MD: American College Health Association.

Kegeles, S.M., Adler, N.E., \& Irwin, C.E. (1988). Sexually active adolescents and condoms: Changes over one year in knowledge, attitudes and use. American Journal of Public Health, 78, 460-461.

Kegeles, S.M., Adler, N.E., \& Irwin, C.E. (1989). Adolescents and condoms: Associations of beliefs with intentions to use. American Journal of Diseases of Children, 143, 911-915.

Keller, S.E., Bartlett, J.A., Schleifer, S.J., Johnson, R.L., Pinner, E., \& Delaney, B. (1991). HIV-relevant sexual behavior among a healthy inner-city heterosexual adolescent population in an endemic area of HIV. Journal of Adolescent Health 12 , 44-48.

Keller, S.E., Schleifer, S.J., Bartlett, J.A., \& Johnson, R.L. (1988). The sexual behavior of adolescents and risk of AIDS. Journal of the American Medical Association, $\underline{260}(24), 3586$.

Kelly, J.A. \& Kalichman, S.C. (1995). Increased attention to human sexuality can improve HIV-AIDS prevention efforts: Key research issues and directions. Journal of Consulting and Clinical Psychology, 63(6), 907-918

Kelly, J.A., \& Murphy, D.A. (1992). Psychological interventions with AIDS and HIV: Prevention and treatment. Journal of Consulting and Clinical Psychology, 60(4), 576585.

Kelly, J.A., Murphy, D.A., Bahr, G.R, Koob, J.J., Morgan, M.G., Kalichman, S.C., Stevenson, L.Y., Brasfield, T.L., Bernstein, B.M., \& St. Lawrence, J.S. (1993). Factors associated with severity of depression and high-risk sexual behavior among 
persons diagnosed with Human Immunodeficiency Virus (HIV) infection. Health Psychology, 12(3), 215-219.

Kelly, J.A., Murphy, D.A., Sikkema, K.J., \& Kalichman, S.C. (1993). Psychological interventions to prevent HIV infection are urgently needed. American Psychologist, 48(10), 1023-1034.

Kelly, J.A. \& St. Lawrence, J.S. (1988). AIDS prevention and treatment: Psychology's role in the health crisis. Clinical Psychology Review, $\underline{8}, 255-284$.

Kelly, J.A. \& St. Lawrence, J.S. (1990). The impact of community-based groups to help persons reduce HIV infection risk behaviours. AIDS Care, $\underline{2}, 25-36$.

Kelly, J.A, St. Lawrence, J.S., Brasfield, T.L. (1991). Predictors of vulnerability to AIDS risk behavior relapse. Journal of Consulting and Clinical Psychology, 59(1), 163-166.

Kelly, J.A., St. Lawrence, J., Brasfield, T., Lemke, A., Amidei, T., Roffman, R.E., Hood, H.V., Smith, J.E., Kilgore, H., \& McNeill, C. (1990). Psychological factors that predict AIDS high-risk versus AIDS precautionary behavior. Journal of Consulting and Clinical Psychology, 58, 117-120.

Kelly, J.A., St. Lawrence, J., Hood, H., \& Brasfield T. (1989). Behavioral intervention to reduce AIDS risk activities. Journal of Consulting and Clinical Psychology, 57, 60-67.

Kelly, J.A., Sikkema, K.J., Winett, R.A., Solomon, L.J., Roffman, R.A., Heckman, T.G., Stevenson, L.Y., Perry, M.J., Norman, A.D., \& Desiderato, L.J. (1995). Factors predicting continued high-risk behavior among gay men in small cities: Psychological, behavioral, and demographic characteristics related to unsafe sex. Journal of Consulting and Clinical Psychology, 63(1), 101-107.

Kenney, A.M. Guardado, S., \& Brown, L. (1989). Sex education and AIDS education in the Schools: What states and large school districts are doing. Family Planning Perspectives, 21(2), 56-64.

Kilbourne, B.W., Buehler, J.W., \& Rogers, M.F. (1990). AIDS as a cause of death in children, adolescents, and young adults. American Journal of Public Health, 80, 499500 .

King, N.J. \& Gullone, E. (1990). Fear of AIDS: Self-reports of Australian children and adolescents. Psychological Reports, 66, 245-246.

Kipke, M.D., Futterman, D., \& Hein, K. (1990). HIV infection and AIDS during adolescence. Adolescent Medicine, 74(5), 1149-1167. 
Kipke, M.D., Montgomery, S, \& MacKenzie, R.G. (1993). Substance use among youth seen at a community-based health clinic, Journal of Adolescent Health, 14(4), 289-294.

Klassen, A.D., Williams, C.J., \& Levitt, E.E. (1989). Sex and Morality in the U.S.: An Empirical Inquiry under the Auspices of The Kinsey Institute. Middletown, CT: Wesleyan University Press.

Kline, A., Kline, E., \& Oken, E. (1992). Minority women and sexual choice in the age of AIDS. Social Science and Medicine, 34(4), 447-457.

Kolbe, L.J. (1990). An epidemiological surveillance system to monitor the prevalence of youth behaviors that most affect health. Health Education, 21(6), 44-48.

Koop, C.E. (1990, April). Do the right thing. The Washingtonian, 81-87.

Koopman, C., Rosario, M., \& Rotheram-Borus, M.J. (1994). Alcohol and drug use and sexual behaviors placing runaways at risk for HIV infection. Addictive Behaviors, 19(1), 95-103.

Kraft, P., Rise, J., \& Traeen, B. (1990). The HIV epidemic and changes in the use of contraception among Norwegian adolescents. AIDS, 4, 673-678.

Ku, L.C., Sonenstein, F.L., \& Pleck, J.H. (1992). The association of AIDS education and sex education with sexual behavior and condom use among teenage men. Family Planning Perspectives, 24, 100-106.

Laga, M., Taelman, H., van der Stuyft, P., Bonneux, L., Vercauteren, G., Piot., P. (1989). Advanced immunodeficiency as a risk factor for heterosexual transmission of HIV. AIDS, $3(6), 361-366$.

Landefeld, C.S., Chren, M.M., Shega, J., Speroff, T., \& McGuire, E. (1988). Students' sexual behavior, knowledge, and attitudes relating to the Acquired Immunodeficiency Syndrome. Journal of General Internal Medicine, 3(March/April), 161-165.

Lang, M. (1994). Cognitive, attitudinal and behavioral risk predictors for AIDS among heterosexual adolescents. Unpublished master's thesis, University of Rhode Island, Kingston, Rhode Island.

Lang, M.A., Harlow, L.L., Quina, K., \& Morokoff, P.J. (1994, May 11-14). Age group comparisons of heterosexual HIV risk predictors for women. Poster presentation for the American Psychological Association's Psychosocial and Behavioral Factors in Women's Health Conference, Washington, DC. 
Lang, M.A., Quina, K., Harlow, L.L., \& Morokoff, P.J. (1994, August 12-16). AIDS risk predictors among heterosexual adolescents. Poster presentation at the 102nd Annual Convention for the American Psychological Association, Los Angeles, CA.

Langer, L.M., Zimmerman, R.S., \& Katz, J.A. (1995). Virgins' expectations and nonvirgins' reports: How adolescents feel about themselves. Journal of Adolescent Research, 10(2), 291-306.

Langer, L.M., Zimmerman, R.S., Warheit, G.J., \& Duncan, R.C. (1993). Decision-making orientation and AIDS-related knowledge, attitudes, and behaviors of Hispanic, AfricanAmerican, and white adolescents. Health Psychology, 12(3), 277-234.

Larzelere, R.E. \& Mulaik, S.A. (1977). Single-sample test for many correlations. Psychological Bulletin, 84(3), 557-569.

Laumann, E.O., Gagnon, J.H., Michaels, S., Michael, R.T., \& Coleman, J.S. (1989). Monitoring the AIDS epidemic in the United States: A network approach. Science, 244, 1186-1189.

Lauritsen, J. (1993). The AIDS War: Propaganda, Profiteering and Genocide from the Medical-Industrial Complex. New York: Asklepios/Pagan Press, pp. 18-19.

Ledergerber, B., von Overbeck, J., Egger, M., \& Luthy, R. (1994). The Swiss HIV Cohort Study: Rationale, organization and selected baseline characteristics. Soz Praventivmed, $\underline{39}(6), 387-394$.

Leeper, M.A. \& Concardy, M. (1989). Preliminary evaluation of reality, a condom for women to wear. Advances in Contraception, 5 , 229-235.

Leigh, B.C. (1990). The relationship of substance use during sex to high-risk sexual behavior. Journal of Sex Research, 27, 199-213.

Leigh, B.C., Morrison, D.M., Trocki, K., \& Temple, M.T. (1994). Sexual behavior of American adolescents: Results from a U.S. national survey. Journal of Adolescent Health, 15(2), 117-125.

Leigh, B.C., Schafer, J., \& Temple, M.T. (1995). Alcohol use and contraception in first sexual experiences. Journal of Behavioral Medicine, 18(1), 81-95.

Leigh, B.C. \& Stall, R. (1993). Substance use and risky sexual behavior for exposure to HIV: Issues in methodology, interpretation, and prevention. American Psychologist, 48(10), 1035-1045.

Leishman, K. (1987, February). Heterosexuals and AIDS. The Atlantic Monthly, 39-58. 
Leite, R.M. Buocompagno, E.M., Leite, A.C., Mergulhao, E.A., \& Battistoni, M.M. (1995). Psychosexual characteristics of male university students in Brazil. Adolescence, $\underline{30}$ (118), 363-80.

Levine, C. (1995). Orphans of the HIV epidemic: Unmet needs in six U.S. cities. Special Issue: AIDS' impact Second International Conference on Biopsychosocial aspects of HIV, \& AIDS (1994, Brighton, England). AIDS Care, 7(Suppl 1), S57-S62.

Levy, S.R., Handler, A.S., Weeks, K.A., Lampman, C., Flay, B.R., \& Rashid, J. (1994). Adolescent risk for HIV as viewed by youth and their parents. Family Community Health, 17(1), 30-41.

Lewis, C. Battistich, V., \& Schaps, E. (1990). School-based primary prevention: What is an effective program? New Directions for Child Development, $\underline{50}, 35-59$.

Livingston, I.L. (1991). Perceived control, knowledge and fear of AIDS among college students: An exploratory study. Journal of Health and Social Policy, 2(2), 47-66.

Lohrmann, D.K. (1988). AIDS education at the local level: The pragmatic issues. Journal of School Health, 58(8), 330-334.

Long, J.D., Williamson, R.L., Gaynor, P., \& Clark, D. (1988). Relationship of locus of control to life style habits. Journal of Clinical Psychology, 44, 209-214.

Longshore, D. (1990). AIDS education for three high-risk populations. Evaluation and Program Planning, 13 , 67-72.

Lott, A. (1991). Attitudes toward gay male and lesbians scale. Unpublished, draft survey. University of Rhode Island, Department of Psychology, Kingston, Rhode Island.

Lowry, R., Holtzman, D., Truman, B.I., Kann, L., Collins, J.L., \& Kolbe, L.J. (1994). Substance use and HIV-related sexual behaviors among United States high school students: Are they related? American Journal of Public Health, 84(7), 1116-1120.

Lui, K.J., Darrow, W.W., \& Rutherford, W. (1988). A model-based estimate of the mean incubation period for AIDS in homosexual men. Science, 249(4857), 1333-1335.

Lyons, J.S., Sheridan, K., \& Larson, D.B. (1988/December, 1989/January). A model for AIDS education for health professionals. Health Education, 19(6), 12-15.

MacDonald, N.E., Wells, G.A., Fisher, W.A., Warren, W.K., King, M.A., Doherty, J.A., \& Bowie, W.R. (1990). High-risk STD/HIV behavior among college students. Journal of the American Medical Association, 263(23), 3155-3159. 
MacGregor, R.R. (1988). Alcohol and drugs as co-factors for AIDS. Advances in Alcohol and Substance Abuse, 77(2), 47-71.

Magura, S., Kang, S.Y., \& Shapiro, J.L. (1994). Outcomes of intensive AIDS education for male adolescent drug users in jail. Journal of Adolescent Health, 15(6), 457-463.

Mann, J.M., Tarantola, D.J., \& Netter, T.W. (Eds.) (1992). AIDS in the World, Cambridge, MA: Harvard University Press.

Manning, D.T. \& Balson, P.M. (1989). Teenagers' beliefs about AIDS education and physicians' perceptions about them. The Journal of Family Practice, 29(2), 173-177.

Manning, D.T., Balson, P.M., Brenberg, N., \& Moore, T.M. (1989). Susceptibility to AIDS: What college students do and don't believe. Journal of American College Health, $\underline{38}(2), 67-73$.

Manoff, R.E. (1985). Social Marketing. New York: Praeger.

Manoff, S.B., Gayle, H.D., Mays, M.A., \& Rogers, M.F. (1989). Acquired immunodeficiency syndrome in adolescents: Epidemiology, prevention and public health issues. The Pediatric Infectious Disease Journal, $8(5), 309-314$.

Mansfield, C.J., Conroy, M.E., Emans, S.J., \& Woods, E.R. (1993). A pilot study of AIDS education and counseling of high-risk adolescents in an office setting. Journal of Adolescent Health, 14, 115-119.

Marsiglio, W. \& Mott, F. (1986). The impact of sex education on sexual activity, contraception use and premarital pregnancy among American teenagers. Family Planning Perspectives, 18(4), 151-161.

Martin, J.L. (1990). Drug use and unprotected anal intercourse among gay men. Health Psychology, $9,450-465$.

Mason, J.O. (1989). Forging working partnerships for school health education. Journal of School Health, 59(1), 18-20.

Maticka-Tyndale, E. (1991). Sexual scripts and AIDS prevention: Variations in adherence to safer-sex guidelines by heterosexual adolescents. The Journal of Sex Research, 28(1), 45-66.

Maxwell, A.E., Bastani, R., \& Yan, K.X. (1995). AIDS risk behaviors and correlates in teenagers attending sexually transmitted diseases clinics in Los Angeles. Genitourinary Medicine, 71(2), 82-87. 
McAllister, I. \& Makkai, T. (1991). Correcting for the underreporting of drug use in opinion surveys. The International Journal of the Addictions, 26(9), 945-961.

McCaig, L.F., Hardy, A.M., \& Winn, D.M. (1991). Knowledge about AIDS and HIV in the US adult population: Influence of the local incidence of AIDS. American Journal of Public Health, 81(12), 1591-1595.

McCoy, C.B. \& Khoury, E. (1990). Drug use and the risk of AIDS. American Behavioral Scientist, $33(4), 419-431$.

McCoy, H.V., McKay, C.Y., Hermanns, L., \& Lai, S. (1990). Sexual behavior and the risk of HIV infection. American Behavioral Scientist, 33(4), 432-450.

McDermott, R.J., Hawkins, M.J., Moore, J.R., \& Cittadino, S.K. (1987). AIDS awareness and information sources among selected university students. Journal of American College of Health, 35(5), 222-226.

McGrath, J.W., Strasburger, V.C., \& Cushing, A.H. (1994). Secretory IgA in the cervical mucus. Journal of Adolescent Health, 15(5), 423-425.

McLean, A.L. \& Flanigan, B.J. (1993). Transition-making behaviors of adolescent males at first intercourse, Adolescence, 28(111), 579-595.

Melchert, T. \& Bumett, K.F. (1990). Attitudes, knowledge, and sexual behavior of highrisk adolescents: Implications for counseling and sexuality education. Journal of Counseling, and Development, 68, 293-298.

Mellanby, A., Phelps, F., \& Tripp, J. (1992). Sex education: More is not enough. Journal of Adolescence, $15,449-466$.

Melton, G.B. (1989). Ethical and legal issues in research and intervention. Journal of Adolescent Health Care, 10, 36-44.

Memon, A. (1990). Young people's knowledge, beliefs and attitudes about HIV/AIDS: A review of research. Health Education Research, 5(3), 327-335.

Mensch, B.S. \& Kandel, D.B. (1988). Underreporting of substance use in a national longitudinal youth cohort: Individual and interviewer effects. Public Opinion Quarterly, 52(1), 100-124.

Mickler, S.E. (1993). Perceptions of vulnerability: Impact on AIDS-preventive behavior among college adolescents. AIDS Education and Prevention, 5 (1), 43-53.

Miller, J.B. (1986). Toward a New Psychology of Women, Second Edition. Boston, MA: Beacon Press, p. 83. 
Miller, R.S., Johnson, J.A., \& Johnson, J.K. (1991). Assessing the prevalence of unwanted childhood sexual experiences. Journal of Psychology and Human Sexuality, $4(3), 43-53$.

Millstein, S.B. (1990). Risk factors for AIDS among adolescents. New Directions for Child Development, 50, 3-15.

Millstein, S.G., Moscicki, A.B., \& Broering, J.M. (1994). Female adolescents at high, moderate, and low risk of exposure to HIV: Differences in knowledge, beliefs, and behavior. Journal of Adolescent Health, 15(2), 133-141.

Molgaard, C.A., Nakamura, C., Hovell, M., \& Elder, J.P. (1988). Assessing alcoholism as a risk factor for acquired immunodeficiency syndrome (AIDS). Social Science and Medicine, 27(11), 1147-1152.

Mondanaro, J. (1987). Strategies for AIDS prevention: Motivating health behavior in drug dependent women. Journal of Psychoactive Drugs, 19(2), 143-149.

Moore, K.A., Furstenberg, F.F., \& Peterson, J.L. (1986). Parental attitudes and the occurrence of early sexual activity: A research note. Journal of Marriage and the Family, 48(4), 777-782.

Moore, K.A. \& Stief, T.M. (1991). Changes in marriage and fertility behavior: Behavior versus attitudes of young adults. Youth and Society, 22, 362-386.

Moore, S. \& Rosenthal, D.A. (1991a). Adolescent invulnerability and perceptions of AIDS risk. Journal of Adolescent Research, 6 (2), 164-180.

Moore, S. \& Rosenthal, D.A. (1991b). Condoms and coitus: Adolescents' attitudes to AIDS and safe sex behavior. Journal of Adolescence, 14(3), 211-227.

Morgan, D.L. \& Krueger, R.A. (1993). When to use focus groups and why. In D.L. Morgan, (Ed), Successful Focus Groups: Advancing the State of the Art, (pp. 3-19). Newbury, CA: Sage Publications.

Morrison, D.M. (1985). Adolescent contraceptive behavior: A review. Psychological Bulletin, 98(3), 538-568.

Morrison, D.M., Baker, S.A., \& Gillmore, M.R. (1994). Sexual risk behavior, knowledge, and condom use among adolescents in juvenile detention. Journal of Youth and Adolescence, 23(2), 271-288. 
Moscicki, A.B., Winkler, B., Irwin, C.E., \& Schachter, J. (1989). Differences in biologic maturation, sexual behavior, and sexually transmitted disease between adolescents with and without cervical intraepithelial neoplasia. Journal of Pediatrics, 115, 487-493.

Nader, P.R., Wexler, D.B., Patterson, T.L., McKusick, L., \& Coates, T. (1989). Comparisons of beliefs about AIDS among urban, suburban, incarcerated, and gay adolescents. Journal of Adolescent Health Care, 10, 413-418.

Nangle, D.W. \& Hansen, D.J. (1993). Relations between social skills and high-risk sexual interactions among adolescents. Behavior Modification, 17(2), 113-135.

Ndeki, S.S., Klepp, K.I., Seha, A.M., \& Leshabari, M.T. (1994). Exposure to HIV/AIDS information, AIDS knowledge, perceived risk and attitudes toward people with AIDS among primary school-children in northern Tanzania. AIDS Care, $6(2), 183-191$.

Nelkin, D., Willis, D.P., \& Parris, S.V., (Eds.). (1991). A Disease of Society: Cultural and Institutional Responses to AIDS. Cambridge, MA: Cambridge University Press.

Neuwirth, K. \& Dunwoody, S. (1989). The complexity of AIDS-related behavioral change: The interaction between communication and noncommunication variables. AIDS \& Public Policy Journal, 4(1), 20-30.

Newton, M. (1995). Adolescence: Guiding Youth Through the Perilous Ordeal. New York: Norton.

Nguyet, N.T.M., Maheux, B., Béland, F., \& Pica, L.A. (1994). Sexual behaviors and condom use: A study of suburban male adolescents. Adolescence, 29(113), 37-48.

Niven, R.G. (1987). The impact of AIDS on the chemical dependency field. Advances in Alcohol and Substance Abuse, 7(2) 3-14.

Noell, J., Biglan, A., Berendt, J., Ochs, L., Metzler, C.W., Ary, D., \& Smolkowski, K. (1993). Problematic sexual situations for adolescents: Alcohol and unsafe sex. Health Values, $17(6), 40-49$.

Norusis, M.J. (1990). SPSS/PC+ Statistics Version 4.0, Statistical Package for the Social Sciences, Chicago: SPSS, Inc.

Nutbeam, D. \& Blakey, V. (1990). The concept of health promotion and AIDS prevention: A comprehensive and integrated basis for action in the 1990s. Health Promotion International, 5(3), 233-242.

Nyamathi, A. \& Vasquez, R. (1989). Impact of poverty, homelessness, and drugs on Hispanic women at risk for HIV infection. Hispanic Journal of Behavioral Sciences, 11(4), 299-314. 
O'Brien, K. (1990). Improving survey questionnaires through focus groups. In D.L. Morgan (Ed.) (1993). Successful Focus Groups: Advancing the State of the Art. Newbury Park, CA: Sage Publications, pp. 105-117.

O'Connell, D. \& Velicer, W.F. (1988). A decisional balance measure and the stages of change model for weight loss. The International Journal of Addictions, 23 , 729-750.

O'Leary, A. (1985). Self-efficacy and health. Behavior Research Therapy, 23, 437-451.

Olsen, J.A. \& Weed, S.E. (1986). The effects of family planning for teenagers on adolescent birth and pregnancy rates. Family Perspective, 20(3), 153-170.

Olsen, T.D. (1987, September). Adolescent pregnancy and abstinence: How far have we come? Paper presented at an Office of Adolescent Pregnancy Program technical workshop, Washington, DC.

Olson, C.L. (1970). Practical considerations in choosing a MANOVA test statistic: A rejoinder to Stevens. Psychological Bulletin, $\underline{86}, 1350-1352$.

O'Reilly, K.R. \& Aral, S.O. (1985). Adolescence and sexual behavior. Journal of Adolescent Health Care, $\underline{6}, 262-270$.

Orr, D.P., Langefeld, C.D., Katz, BP., Caine, V.A., Dias, P., Blythe, M., \& Jones, R.B. (1992). Factors associated with condom use among sexually active female adolescents. Adolescent Medicine, 120, 311-317.

Ortiz, V., Kauman, J., Uribe, P., Decaso, L.E., Hernandez-Avila, M. (1992). Acceptability of female condoms among female prostitutes in Mexico City: Preliminary findings. Conasida, Mexico: Instituto Nacional de Salud Publica, in World Health Organization, (1993c). Sexual Negotiation, The Empowerment of Women and the Female Condom: General Protocal, Geneva, Switzerland: WHO.

Osborn, J.E. (1988). AIDS: Politics and science. New England Journal of Medicine, $\underline{318}, 444-447$.

Osborn, J.E. (1991). Prevention: Can we mobilize what has been learned? In N.F. McKenzie, (Ed.) The AIDS Reader: Social, Political, Ethical Issues. New York: Meridian.

Ostergard, D.R. (1977). The effect of age, gravity, and parody on the location of the cervical squamocolumnar junction as determined by colposcopy. American Journal of Obstetrics and Gynecology, 129, 59-63. 
Otten, M.W., Zaidi, A.A., Peterman, T.A., Rolfs, R.T., \& Witte, J.J. (1994). High rate of HIV seroconversion among patients attending urban sexually transmitted disease clinics. AIDS, $8(4), 549-553$.

Overby, K.J. \& Kegeles, S.M. (1994). The impact of AIDS on an urban population of high-risk female minority adolescents: Implications for intervention. Journal of Adolescent Health, 15(3), 216-227.

Padian, N., Marquis, L., Francis, D.P., Anderson, R.E., Rutherford, G.W., O'Malley, P.M., \& Winkelstein, W. (1987). Male-to-female transmission of Human Immunodeficiency Virus. Journal of the American Medical Association, 258(6), 788790.

Padian, N.S., Shiboski, S.C., \& Jewell, N.P. (1991). Female-to-male transmission of Human Immunodeficiency Virus. Journal of the American Medical Association, 266(12), 1664-1667.

Patton, M.J. (1991). Qualitative research on college students: Philosophical and methodological comparisons with the quantitative approach. Journal of College Student Development, 32, 389-396.

Paxson, M.A. \& Quina, K. (1989). Psychological factors related to contraceptive use by unmarried college women. Paper presented at the meetings of the Eastern Psychological Association, March 31, 1989, Boston, Massachusetts.

Paxton, C., \& Susky, D. (1988). AIDS, homophobia, and sexual attitudes. Health Values, 12(4), 39-43.

Pendergrast, R.A., DuRant, R.H., \& Gaillard, G.L. (1992). Attitudinal and behavioral correlates of condom use in urban adolescent males. Journal of Adolescent Health, $\underline{13}(2), 133-139$.

Peruga, A. \& Celentano, D.D. (1993). Correlates of AIDS knowledge in samples of the general population. Social Science Medicine, 16(4), 509-524.

Petosa, R. \& Wessinger, J. (1990). The AIDS education needs of adolescents: A theorybased approach. AIDS Education and Prevention, 2(2), 127-136.

Piercy, F.P., Trepper, T., \& Jurich, J. (1993). The role of family therapy in decreasing HIV high-risk behaviors among adolescents. AIDS Education and Prevention, 5(1), 71-86.

Pino, R. (1993). The Transtheoretical Model: An assessment of readiness to change HIV-risking behaviors in a sample of high-risk adolescents. Unpublished master's thesis, University of Rhode Island, Kingston, Rhode Island. 
Plant, M.A. (1990). Alcohol, sex and AIDS. Alcohol and Alcoholism, 25(2), 293-301.

Pleck, J.H., Sonenstein, F.L., \& Ku, L.C. (1991). Adolescent males' condom use: Relationships between perceived cost-benefits and consistency. Journal of Marriage and the Family, 53, 731-745.

Ponton, L.E., DiClemente, R.J., \& McKenna, S. (1991). An AIDS education and prevention program for hospitalized adolescents. Journal of the American Academy of Child and Adolescent Psychiatry, 30(5), 729-734.

Poppen, P.J. \& Reisen, C.A. (1994). Heterosexual behaviors and risk of exposure to HIV: Current status and prospects for change. Applied and Preventive Psychology, $\underline{3}(2), 75-90$.

Prewitt, V.R. (1989). Health beliefs and AIDS educational materials. Family and Community Health, 12(2), 65-76.

Price, J.H., Desmond, S.M., Hallinan, C., \& Griffin, T.B. (1988). College students' perceived risk and seriousness of AIDS. Health Education, 19(4), 16-19.

Price, J.H., Desmond, S., \& Kakulka, G. (1985). High school students' perceptions and misperceptions of AIDS. Journal of School Health, 55(3), 107-109.

Price-Greathouse, J. \& Trice, A.D. (1986). Chance health-orientation and AIDS information seeking. Psychological Reports, 59, 10.

Prochaska, J.O. (1979). Systems of Psychotherapy: A Transtheoretical Analysis. Pacific Grove, CA: Brooks/Cole Publishing.

Prochaska, J.O. (1984). Systems of Psychotherapy: A Transtheoretical Analysis, Second Edition. Pacific Grove, CA: Brooks/Cole Publishing.

Prochaska, J.O. \& DiClemente, C.C. (1983). Stages and processes of self-change of smoking: Toward an integrative model of change. Journal of Consulting and Clinical Psychology, 51, 390-395.

Prochaska, J.O. \& DiClemente, C.C. (1984). The Transtheoretical Approach: Crossing Traditional Boundaries of Therapy. Homewood, IL: Dow Jones-Irwin.

Prochaska, J.O., DiClemente, C.C., \& Norcross, J.C. (1992). In search of how people change: Applications to the addictive behaviors. American Psychologist, 47, 11021114. 
Prochaska, J.O., Harlow, L.L., Redding, C.A., Snow, M.G., Rossi, J.S., \& Velicer, W.F. (1990). Stages of change, self efficacy, and decisional balance of condom use in a high HIV risk sample. Technical Report to the Centers for Disease Control Contract Grant \#0-4115-002.

Prochaska, J.O., Harlow, L.L., Redding, C.A., Snow, M.G., Rossi, J.S., Velicer, W.F., Rhodes, F., Schnell, D., Gavabotti, C. (1992). Stages of change, self-efficacy, and decision balance of condom use in a high-risk HIV sample. Unpublished Manuscript.

Prochaska, J.O., Velicer, W.F., Rossi, J.S., Goldstein, M.G., Marus, B., H., Rakowski, W., Fiore, C., Harlow, L.L., Redding, C.A., Rosenbloom, D., \& Rossi, S.R. (1994). Stages of change and decisional balance for 12 problem behaviors. Health Psychology, 13(1), 39-46.

Pulford, J.S.W. (1991). North American college and university responses to the AIDS threat. Journal of College Student Psychotherapy, 5(2) 101-107.

Quadrel, M.J., Fischhoff, B., \& Davis, W. (1993). Adolescent (in)vulnerability. American Psychologist, 48 (2), 102-116.

Quina, K., Harlow, L.L., Gibson, P., \& Morokoff, P. (1990, August). Psychometric investigation of a sexual assertiveness scale. Paper presented at the annual meeting of the American Psychological Association, Boston, MA.

Quina, K., Harlow, L.L., Morokoff, P.J., \& Saxon, S.E. (in press). Interpersonal power and women's HIV risk. In J. Manlowe \& N. Goldstein, Gender and Politics, New York, New York University Press.

Quinn, T.C., Glasser, D., Cannon, R.O., Matuszak, D.L., Dunning, R.W., Kline, R.L., Campbell, C.H., Israel, E., Fauci, A.S., \& Hook, E.W. (1988). Human Immunodeficiency Virus infection among patients attending clinics for sexually transmitted diseases. The New England Journal of Medicine, 318(4), 197-203.

Redding, C.A. (1993). The Transtheoretical Model applied to safer sex behavior among university students: A cross-sectional study. Unpublished doctoral dissertation, University of Rhode Island, Kingston, Rhode Island.

Redding, C.A. \& Rossi, J.S. (1993a, March). The processes of safer sex adoption. Paper presentation at the Society of Behavioral Medicine, San Francisco, CA.

Redding, C.A. \& Rossi, J.S. (1993b, November). Self efficacy for safer sex: Stage and gender-based differences on situational subscales. Paper presentation at the 27th annual convention of Association for Advancement of Behavior Therapy, Atlanta, GA. 
Redding, C.A., Rossi, J.S., Velicer, W.F., \& Prochaska, J. (1989, August). The pros and cons of safer sex: A measurement model. Paper presented at the 97th annual convention of the American Psychological Association, New Orleans, LA.

Rehmet, S., Staszewski, S., Muller, R., Doerr, H.W., Bergmann, L., von Wangenheim, G., Helm, E.G., \& Stille, W. (1992). Transmission rates and co-factors of heterosexual HIV transmission. Presented at the VIII International Conference on AIDS/III STD World Congress, Amsterdam, 1992 (PoC 4165).

Reiss, I.L. \& Leik, R.K. (1989). Evaluating strategies to avoid AIDS: Number of partners vs. use of condoms. The Journal of Sex Research, 26(4), 411-433.

Remafedi, G.J. (1988) Preventing the sexual transmission of AIDS during adolescence. Journal of Adolescent Health Care, 9 , 139-143.

Remafedi, G.J. (1994). Cognitive and behavioral adaptations to HIV/AIDS among gay and bisexual adolescents. Journal of Adolescent Health, 15(2), 142-148.

Reuben, N., Hein, K., \& Drucker, E. (1988). The relationship of high risk behaviors to AIDS knowledge in adolescent high school students. Presented at the Annual Conference of the Society for Adolescent Medicine, New York, NY.

Reulbach, W. (1991). Counseling chemically dependent HIV positive adolescents. Journal of Chemical Dependency Treatment, 4 (2), 31-43..

Rhode Island Department of Health (1994, December). AIDS/STD Morbidity Mortality Report. Office of AIDS/STD: Author.

Richard, R. \& van der Pligt, J. (1991). Factors affecting condom use among adolescents. Journal of Community and Applied Social Psychology, 1, 105-116.

Richard, R., van der Pligt, J., \& deVries, N. (1995). Anticipated affective reactions and prevention of AIDS. Special Issue: Social psychology and health. British Journal of Social Psychology, 34(1), 9-21.

Rickert, V.I., Gottlieb, A., \& Jay, S.M. (1990). A comparison of three clinic-based AIDS education programs on female adolescents' knowledge, attitudes, and behavior. Journal of Adolescent Health Care, 11(4), 298-303.

Rickert, V.I., Jay, S.M., \& Gottlieb, A. (1991). Effects of a peer-counseled AIDS education program on knowledge, attitudes, and satisfaction of adolescents. Journal of Adolescent Health, 12, 38-43. 
Rickert, V.I., Jay, S.M., Gottlieb, A., \& Bridges, C. (1989). Adolescents and AIDS: Females' attitudes and behaviors toward condom purchase and use. Journal of Adolescent Health Care, 10(4), 313-316.

Rickman, R.L., Lodico, M., DiClemente, R.J., Morris, R., Baker, C., \& Huscroft, S. (1994). Sexual communication is associated with condom use by sexually active incarcerated adolescents. Journal of Adolescent Health, 15(5), 383-388.

Rimberg, H.M. \& Lewis, R.J. (1994). Older adolescents and AIDS: Correlates of selfreported safer sex practices. Journal of Research on Adolescence, 4(3), 453-464.

Robertson, J.A. \& Plant, M.A. (1988). Alcohol, sex and risks of HIV infection. Drug and Alcohol Dependence, 22, 75-78.

Romanowski, B. \& Piper, G. (1988). Sexually transmitted diseases -- An overview. Journal of Social Work and Human Sexuality, 6(2), 7-20.

Roosa, M.W. \& Christopher, F.S. (1990). An evaluation of an adolescent pregnancy prevention program: Is "Just say no" enough? Family Relations, $\underline{39}, 68-72$.

Roscoe, B. \& Kruger, T.L. (1990). AIDS: Late adolescents' knowledge and its influence on sexual behavior. Adolescence, 25(97), 39-48.

Rosenthal, D., Moore, S., \& Brumen, I. (1990). Ethnic group differences in adolescents' responses to AIDS. Australian Journal of Social Issues, 25(3), 220-239.

Rosenthal, D., Moore, S., \& Buzwell, S. (1994). Homeless youths: Sexual and drugrelated behavior, sexual beliefs and HIV/AIDS risk. AIIDS Care, 6(1), 83-94.

Rosenthal, D., Moore, S., Flynn, I. (1991). Adolescent self-efficacy, self-esteem and sexual risk-taking. Special Issue: Social dimensions of AIDS. Journal of Community and Applied Social Psychology, 1(2), 77-88.

Rosenthal, D.A. \& Shepherd, H. (1993). A six-month follow-up of adolescents' sexual risk-taking, HIV/AIDS knowledge, and attitudes to condoms. Journal of Community and Applied Social Psychology, 3 (1), 53-56.

Ross, M.W. \& Carson, J.A. (1988). Effectiveness of distribution of information on AIDS: A national study of six media in Australia. New York State Journal of Medicine, $\underline{88}$, 239-241.

Rotheram-Borus, M.J. (1990). Adolescents' reference-group choices, self-esteem, and adjustment. Journal of Personality and Social Psychology, 59(5), 1075-1081. 
Rotheram-Borus, M.J., Becker, J.V., Koopman, C., \& Kaplan, M.S. (1991). AIDS knowledge and beliefs, and sexual behavior of sexually delinquent and non-delinquent (runaway) adolescents. Journal of Adolescents, 14(3), 229-244.

Rotheram-Borus, M.J. \& Koopman, C. (1991). Sexual risk behavior, AIDS knowledge, and beliefs about AIDS among predominantly minority gay and bisexual male adolescents. AIDS Education and Prevention, 3(4), 305-312.

Rotheram-Borus, M.J., Meyer-Bahlburg, H.F.L., Koopman, C., Rosario, M., Exner, T.M., Henderson, R., Matthieu, M., \& Gruen, R.S. (1992). Lifetime sexual behaviors among runaway males and females. The Journal of Sex Research, 29(1), 15-29.

Rotheram-Borus, M.J., Rosario, M., Meyer-Bahlburg, H.F.L., Koopman, C., Dopkins, S.C., \& Davies, M. (1994). Sexual and substance use acts of gay and bisexual male adolescents in New York City. The Journal of Sex Research, 31(1), 47-57.

Rothspan, S. \& Read, S.J. (1996). Present versus future time perspective and HIV risk among heterosexual college students. Health Psychology, 15(2), 131-134.

Rubinson, L. \& deRubertis, L. (1991). Trends in sexual attitudes and behaviors of a college population over a 15-year period. Journal of Sex Education and Therapy, 17, $32-41$.

Sacco, W.P., Levine, B, Reed, D.L., \& Thompson, K. (1991). Attitudes about condom use as an AIDS-relevant behavior: Their factor structure and relation to condom use. Journal of Consulting and Clinical Psychology, 3(2) 265-272.

St. Lawrence, J.S. (1993). African-American adolescents' knowledge, health-related attitudes, sexual behavior, and contraceptive decisions: Implications for the prevention of adolescent HIV infection. Journal of Consulting and Clinical Psychology, 61(1), 104-112.

St. Lawrence, J.S., Brasfield, T.L., Jefferson, K.W., Alleyne, E., \& Shirley, A. (1994). Social support as a factor in African-American adolescents' sexual risk behavior. Journal of Adolescent Research, 9(3), 292-310.

St. Lawrence, J.S., Brasfield, T.L., Shirley, A., Jefferson, K.W., Alleyne, E., \& O’Bannon, R.E., III (1995). Cognitive-behavioral intervention to reduce African American adolescents' risk for HIV infection. Journal of Consulting and Clinical Psychology, $\underline{63}(2), 221-237$.

St. Lawrence, J.S., Jefferson, K.W., Alleyne, E., \& Brasfield, T.L. (1995). Comparison of education versus behavioral skills training interventions in lowering sexual HIV-risk behavior of substance-dependent adolescents. Journal of Consulting and Clinical Psychology, 63(1), 154-157. 
St. Lawrence, J.S., Jefferson, K.W., Banks, P.G., Cline, T.R., Alleyne, E., \& Brasfield, T.L. (1994). Cognitive-behavioral group intervention to assist substance-dependent adolescents in lowering HIV infection risk. AIDS Education and Prevention, 6(5), 425435.

Sandberg, D.E., Rotheram-Borus, M.J., Bradley, J., \& Martin, J. (1988). Methodological issues in assessing ADS prevention programs. Special Issue: Adolescent sexual behavior. Journal of Adolescent Research, $\underline{3}, 413-418$.

Schaalma, H., Kok, G., \& Peters, L. (1993). Determinants of consistent condom use by adolescents: The impact of experience of sexual intercourse. Health Education Research, $8(2), 255-269$.

Schinke, S.P., Botvin, G.J., Orlandi, M.A., Schilling, R.F., \& Gordon, A.N. (1990). African-American and Hispanic-American adolescents, HIV infection and preventive intervention. AIDS Education and Prevention, 2(4), 305-312.

Schneider, D.J., Taylor, E.L., Prater, L.M., \& Wright, M.P. (1991). Risk assessment for HIV infection: Validation study of a computer-assisted preliminary screen. AIDS Education and Prevention, 3(3), 215-229.

Schnell, D.J., Galavotti, C., \& O'Rielly, K.R. (1993). An evaluation of sexual behavior change using statistical and cognitive models. Statistics in Medicine, 12, 219-228.

Schuster, C.R. (1988). Intravenous drug use and AIDS prevention. Public Health Reports, 103(3), 261-266.

Schwarcz, S.K. \& Rutherford, G.W. (1989). Acquired Immunodeficiency Syndrome in infants, children, and adolescents. The Journal of Drug Issues, 19, 75-92.

Sears, D.O. (1986). College sophomores in the laboratory: Influences of a narrow data base on social psychology's view of human nature. Journal of Personality and Social Psychology, 51(3), 515-530.

Segest, E., Mygind, O., Harris, C.N., \& Bay, H. (1991). The correlation between general disease prevention and prevention of HIV-contagion among adolescents. Journal of Adolescence, 14(4), 389-396.

Seidman, S.N. \& Rieder, R.O. (1994). A review of sexual behavior in the United States. American Journal of Psychiatry, 151(3), 330-341.

Shafer, M.A. \& Boyer, C.B. (1991). Psychosocial and behavioral factors associated with risk of sexually transmitted diseases, including Human Immunodeficiency Virus infection, among urban high school students. Journal of Pediatrics, $119,826-833$. 
Shoop, D.M. \& Davidson, P.M. (1994). AIDS and adolescents: The relation of parent and partner communication to adolescent condom use. Journal of Adolescence, 17(2), 137-148.

Siegel, D., Larazus, N., Krashovsky, F., Durbin, M., \& Chesney, M. (1991). AIDS knowledge, attitudes, and behavior among inner city, junior high school students. Journal of School Health, 61, 160-165.

Siegel, K. \& Gibson, W.C. (1988). Barriers to the modification of sexual behavior among heterosexuals at risk for Acquired Immunodeficiency Syndrome. New York State Journal of Medicine, 87, 66-70.

Siegel, L.J. (1993). Editorial: Children's understanding of AIDS: Implications for preventive interventions. Journal of Pediatric Psychology, 18(2), 173-176.

Simoni, J.M., Mason, H.R.C., Marks, G., Ruiz, M.S., Reed, D., \& Richardson, J.L. (1995). Women's self-disclosure of HIV infection: Rates, reasons, and reactions. Journal of Consulting and Clinical Psychology, 63(3), 474-478.

Singh, A.N., Zemitzsch, A.A., Ellis, C.R., Best, A.M., \& Singh, N.N. (1994). Seriously emotionally disturbed students' knowledge and attitudes about AIDS. Journal of Emotional and Behavioral Disorders, 2(3), 156-163.

Slater, B.R. (1989). Pediatric and adolescent AIDS in the schools: The need for educative programs. Professional School Psychology, 4(4), 233-244.

Slonim-Nevo, V., Auslander, W.F., \& Ozawa, M.N. (1995). Educational options and AIDS-related behaviors among troubled adolescents. Journal of Pediatric Psychology, 20(1), 41-60.

Smith, K.W., McGraw, S.A., Crawford, S.L., Costa, L.A., \& McKinlay, J.B. (1993). HIV risk among Latino adolescents in two New England cities. American Journal of Public Health, 83(10), 1395-1399.

Sonenstein, F.L., Pleck, J.H., \& Ku, L.C. (1989). Sexual activity, condom use, and AIDS awareness among adolescent males. Family Planning Perspectives, 4 , 152-158.

Sonenstein, F.L., Pleck, J.H., \& Ku, L.C. (1991). Levels of sexual activity among adolescent males in the United States. Family Planning Perspectives, 23(4), 162-167.

Soper, D.E., Brockwell, N.J., \& Dalton, H.P. (1991). Evaluation of the effects of a female condom on the female lower genital tract. Contraception, 44(1), 21-29. 
Sorenson, J.L., Gibson, D.R., Heitzmann, C., Dumontet, R., \& Acampora, A. (1988, August). AIDS prevention with drug abusers in residential treatment: Preliminary results. Paper presented at the annual meeting of the American Psychological Association, Atlanta, GA.

Stake, J.E., DeVille, C.J., \& Pennell, C.L. (1983). The effects of assertive training on the performance self-esteem of adolescent girls. Journal of Youth and Adolescence, 12(5), 435-442.

Stall, R. (1988). The prevention of HIV infection associated with drug and alcohol use during sexual activity. Advances in Alcohol and Substance Abuse, $7,73-88$.

Stall, R.D., Coates, T.J., , \& Hoff, C. (1988). Behavioral risk reduction for HIV infection among gay and bisexual men. American Psychologist, 43(11), 878-885.

Stall, R.D., McKusick, L., Wiley, J., Coates, T., \& Ostrow, D. (1986). Alcohol and drug use during sexual activity and compliance with safe sex guidelines for AIDS: The AIDS Behavioral Research Project. Health Education Quarterly, 13, 359-371.

Stanton, B.F., Black, M., Kaljee, L., \& Ricardo, I. (1993). Perceptions of sexual behavior among urban early adolescents: Translating theory through focus groups. Journal of Early Adolescence, 13(1), 44-66.

Stanton, B.F., Black, M., Keane, V., \& Feigelman, S. (1990). HIV risk behaviors in young people: Can we benefit from 30 years of research experience? AIDS and Public Policy Journal, 5(1), 17-23.

Steckler, A., McLeroy, K.R., Goodman, R.M., Bird, S.T., \& McCormick, L. (1992). Toward integrating qualitative and quantitative methods: An introduction. Health Education Quarterly, 19, 1-8.

Stein, G.J. (1995). AIDS Update: 1994 - 1995. Englewood Cliffs, NJ: Prentice-Hall, Inc.

Stein, J.A., Newcomb, M.D., \& Bentler, P.M. (1994). Psychosocial correlates and predictors of AIDS risk behaviors, abortion, and drug use among a community sample of young adult women. Health Psychology, 13(4), 308-318.

Steiner, J.D., Sorokin, G., Schiedermayer, D.L., \& Van Susteren, T.J. (1990). Are adolescents getting smarter about Acquired Immunodeficiency Syndrome? American Journal of Diseases of Children, 144, 302-306.

Stern, L. (1990). Conceptions of separation and connection in female adolescents. In C. Gilligan, N.P. Lyons, \& T.J. Hanmer, (Eds.) Making connections: The relational 
worlds of adolescent girls at Emma Willard School. Cambridge: Harvard University Press, $p p$. 73-87.

Stevens-Smith, P. \& Remley, T.P. (1994). Drugs, AIDS, and teens: Intervention and the school counselor. The School Counselor, 41, 181-184.

Stevenson, H.C. \& Davis, G. (1994). Impact of culturally sensitive AIDS video education on the AIDS risk knowledge of African-American adolescents. AIDS Education and Prevention, $6(1), 40-52$.

Stevenson, H.C., Davis, G., Weber, E., Weiman, D., \& Abdul-Kabir, S. (1995). HIV prevention beliefs among urban African-American youth. Journal of Adolescent Health, $\underline{16}(4), 316-323$.

Stevenson, H.C., Gay, K.M., \& Josar, L. (1995). Culturally sensitive AIDS education and perceived AIDS risk knowledge: Reaching the "know-it-all" teenager. AIDS Education and Prevention, 7(2), 134-144.

Stevenson, M.R. \& Stevenson, D.M. (1990). Beliefs about AIDS among entering college students. Journal of Sex Education and Therapy, 16(3), 201-204.

Stiffman, A.R., Dore, P., Cunningham, R.M., \& Earls, F. (1995). Person and environment in HIV risk behavior changes between adolescence and young adulthood. Health Education Quarterly, 22(2), 211-226.

Stiffman, A.R. \& Earls, F. (1990). Behavioral risks for Human Immunodeficiency Virus infection in adolescent medical patients. Pediatrics, 85(3), 303-310.

Stiffman, A.R., Earls, F., Dore, P., \& Cunningham, R. (1992). Changes in Acquired Immunodeficiency Syndrome-related risk behavior after adolescence: Relationships to knowledge and experience concerning Human Immunodeficiency Virus Infection. Pediatrics, 89(5), 950-956.

Strickland, B.R. (1978). Internal-external expectancies of health-related behavior. Journal of Consulting and Clinical Psychology, 46, 1192-1211.

Strunin L. (1991). Adolescents' perceptions of risk for HIV infection: Implications for future research. Social Science and Medicine, 32(2), 221-228.

Strunin, L. \& Hingson, R. (1987). Acquired immune deficiency syndrome and adolescents: Knowledge, beliefs, attitudes and behaviors. Pediatrics, 79, 825-828.

Strunin, L. \& Hingson, R. (1993). Adolescents. In L. Sherr, (Ed), AIDS and the Heterosexual Population, Langhorne, PA: Harwood Academic Publishers, pp. 125137. 
Stuber, M.L. (1990). Psychiatric consultation in pediatric HIV and AIDS. Journal of the American Academy of Child and Adolescent Psychiatry, 29(3), 463-467.

Sugerman, S.T., Hergenroeder, A.C., Chacko, M.R., \& Parcel, G.S. (1991). Acquired Immunodeficiency Syndrome and adolescents: Knowledge, attitudes, and behaviors of runaway and homeless youths. American Journal of Diseases of Children, 145, 431436.

Sunwoo, J., Brenman, A, Escobedo, J., Philpott, T., Allman, K., Mueller, J., Jaeger, J., Brown, L.K., \& Cole, F.S. (1995). School-based AIDS education for adolescents. Journal of Adolescent Health, 16(4), 309-315.

Surgeon General of U.S. Public Health Service (1987). Special Report: From the Surgeon General, US Public Health Service. Journal of Substance Abuse Treatment, 4 , 5-13.

Sweeney, P., Lindegren, M.L., Buehler, J.W., Onorato, I.M., \& Janssen, R.S. (1995). Teenagers at risk of human immunodeficiency virus type 1 infection: Results from seroprevalence surveys in the United States. Archives of Pediatric Adolescent Medicine, $149(5), 521-528$.

Sy, F.S., Richter, D.I., \& Copello, A.G. (1989). Innovative educational strategies and recommendations for AIDS prevention and control. AIDS Education and Prevention, $1(1), 53-56$.

Tabachnick, B.G. \& Fidell, L.S. (1989). Using Multivariate Statistics, Second Edition, New York: Harper Collins.

Takanishi, R. (1993). The opportunities of adolescence -- Research, interventions, and policy: Introduction to the special issue. American Psychologist, 48(2), 85-87.

Tansathit, Thawatchi, \& Cheevakej S. (1990). Femshield accptability study among family planning accepters (unpublished report). Chiang Mai University, Thailand. Family Planning Unit, Obstetrics and Gynaecology Department, Faculty of Medicine, cited in World Health Organization, (1993c). Sexual Negotiation, The Empowerment of Women and the Female Condom: General Protocal, Geneva, Switzerland: WHO.

Temoshok, L., Sweet, D.M., \& Zich, J. (1987). A three city comparison of the public's knowledge and attitudes about AIDS. Psychology and Health, 1, 43-60.

Trad, P.V. (1994). A developmental model for risk avoidance in adolescents confronting AIDS. AIDS Education and Prevention, 6 (4), 322-328. 
Trice, A.D. \& Price-Greathouse, J. (1987). Locus of control and AIDS informationseeking in college women. Psychological Reports, $\underline{60}(2), 665-666$.

U.S. Department of Health and Human Services (1993). AIDS Research: An NIMH Blueprint for the Second Decade. Atlanta: National Institutes of Mental Health, pp. 13

Valdiserri, R.O., Lyter, D., Leviton, L.C., Callahan, C.M., Kingsley, L.A., \& Rinaldo, C.R. (1988). Variables influencing condom use in a cohort of gay and bisexual men. American Journal of Public Health, 78, 801-805.

van de Wigert, H. \& Padian, N.S. (1993). Heterosexual transmission of HIV. In L. Sherr, (Ed.), AIDS and the Heterosexual Population, Langhorne, PA: Harwood Academic Publishers, pp. 1-19.

van der Pligt, J. \& Richard, R. (1994). Changing adolescents' sexual behavior: Perceived risk, self-efficacy and anticipated regret. Patient Education and Counseling, 23(3), 187196.

van der Velde, F.W., van der Pligt, J., \& Hooykaas, C. (1994). Perceiving AIDS-related risk: Accuracy as a function of differences in actual risk. Health Psychology, 13(1), 25-33.

Vanderschmidt, H.F., Lang, J.M., Knight-Williams, V., \& Vanderschmidt, G.F. (1993). Risks among inner-city young teens: The prevalence of sexual activity, violence, drugs, and smoking. Journal of Adolescent Health, 14, 282-288.

Vermund, S.H. Hein, K., Gayle, H.D., Cary, J.M., Thomas, P.A., \& Drucker, E. (1989). Acquired Immunodeficiency Syndrome among adolescents. American Journal of Diseases of Children, 143, 1220-1224.

Vincent, M.L., Clearle, A.F., \& Schluchter, M.D. (1987). Reducing adolescent pregnancy through school and community based education. Journal of the American Medical Association, 257, 3382-3386.

Visser, A.P. \& Ketting, E. (1994). Sexual health: Education and counseling perspectives on contraceptive use, HIV and sexuality. Patient Education and Counseling, $\underline{23}(3)$, 141-145.

Wagner, H.U., Van Dyck, E., Roggen, E., Nunn, A.J., Kamali, A., Schmid, D.S., Dobbins, J.G., \& Mulder, D.W. (1994). Seroprevalence and incidence of sexually transmitted diseases in a rural Ugandan population. International Journal of STDs/AIDS, 5(5), 332-337. 
Walter, H.J., Vaughn, R.D., \& Cohall, A.T. (1991). Psychosocial influences on Acquired Immunodeficiency Syndrome-risk behaviors among high school students. Pediatrics, $\underline{88}(4), 846-852$.

Walter, H.J., Vaughan, R.D., Gladis, M.M., Ragin, D.F., Kasen, S., \& Cohall, A.T. (1993). Factors associated with AIDS-related behavioral intentions among high school students in an AIDS epicenter. Health Education Quarterly, 20(3), 409-420.

Weed, S.E. \& Olsen, J.A. (1986). Effects of family planning programs and teenage pregnancy: Replication and extension. Family Perspective, 20(3), 173-195.

Weinstein, N.D. \& Nicolich, M. (1993). Correct and incorrect interpretations of correlations between risk perceptions and risk behaviors. Health Psychology, 12(3), 235-245.

Weisman, C.S., Nathanson, C.A., Ensminger, M., Teitelbaum, M.A., Robinson, J.C., \& Plichta, S. (1989). AIDS knowledge, perceived risk and prevention among adolescent clients of a family planning clinic. Family Planning Perspectives, 21(5), 213-217.

Werner, M.J. \& Biro, F.M. (1990). Contraception and sexually transmitted diseases in adolescent females. Adolescent Pediatric Gynecology, $\underline{3}$, 127-136.

Westerman, P.L. \& Davidson, P.M. (1993). Homophobic attitudes and AIDS risk behavior of adolescents. Journal of Adolescent Health, 14(3), 208-213.

Whalen, C.K, Henker, B., O'Neil, R., Hollingshead, J., Holman, A., \& Moore, B. (1994). Optimism in children's judgments of health and environmental risks. Health Psychology, 13(4), 319-325.

Whitley, B.E. (1990). College student contraceptive use: A multivariate analysis. The Journal of Sex Research, 27(2), 305-313.

Whitt, J.K. (1995). Current research on children's and adolescents' HIV/AIDS-related knowledge, attitudes, and behavior. Journal of Pediatric Psychology, 20(1), 37-40.

Wilcox, B.L. (1990). Federal policy and adolescent AIDS. New Directions for Child Development, $50,61-70$.

Wilkins, R. \& Lewis, C. (1993). Sex and drugs and nuclear war: Secular, developmental and Type A influences upon adolescents' fears of the nuclear threat, AIDS and drug addiction. Journal of Adolescence, $16,23-41$.

Windle, M. (1989). High-risk behaviors for AIDS among heterosexual alcoholics: A pilot study. Journal of Studies on Alcohol, 50(6), 503-507. 
Wingood, G.M. \& DiClemente, R.J. (1992). Cultural, gender, and psychosocial influences on HIV-related behavior of African-American female adolescents:

Implications for the development of tailored prevention programs. Ethnicity \& Disease, $\underline{2}, 381-388$.

Witt, L.A. (1989). Authoritarianism, knowledge of AIDS, and affect toward persons with AIDS: Implications for health education. Journal of Applied Social Psychology, 19(7), 599-607.

World Health Organization (1989). The health of youth: Young people and adults face the issues together. A summary of national and regional activities in preparation for the technical discussions and beyond. Facts for action: Youth and sexually transmitted diseases. Geneva, Switzerland: Author.

World Health Organization (1990, November 19-20). Report of the meeting on research priorities relating to women and HIV/AIDS. Geneva, Switzerland: Author, GPA/DIR/91.2, p. 4.

World Health Organization (1992). The global AIDS strategy, WHO AIDS Series 11, Geneva, Switzerland: Author, pp. 10-11.

World Health Organization (1993a). Global Programme on AIDS: The HIV/AIDS Pandemic: 1993 Overview. Geneva, Switzerland: Author, WHO/GPA/CNP/ $\mathrm{EVA} / 93.1, p .16$.

World Health Organization (1993b, February 3-5). Global Programme on AIDS: Women and AIDS: Research Priorities. First meeting of the Advisory Council on HIV and AIDS, Global Programme on AIDS/ACA(1)93.8, Geneva, Switzerland: Author, p. 5.

World Health Organization (1993c, May 27). Sexual Negotiation, The Empowerment of Women and the Female Condom: General Protocol. Geneva, Switzerland: World Health Organization, and the Global Programme on AIDS Office of Intervention Development and Support; Social and Behavioral Studies and Support Unit, SNPROTL.

World Health Organization (1993d, September 7). 13 million HIV positive women by 2000. Press release published in Edinburgh, 7 September, 1993 during The Second International Conference on HIV in Children and Mothers (7 - 10 September 1993).

World Health Organization (1993e, November 1). Women and AIDS: Proposed research priorities for Global Programme on AIDS. Presented at the Second meeting of the Advisory Council on HIV and AIDS, Geneva, Switzerland, 9 - 11 November 1993, GPA/ACA(2)/93.7. Rev1. 
World Health Organization (1993f, November 16). New approach to fighting AIDS. Press release WHO/90, Geneva, Switzerland: Author.

World Health Organization (1994a, July). Women and AIDS: Agenda for Action.

Geneva, Switzerland, World Health Organization and the United Nations Development Programme in Consultation with the United Nations Division for the Advancement of Women.

World Health Organization (1994b, April 12). Report of a consultation on women and HIV/AIDS GPA's action agenda in support of countries (draft). Geneva, Switzerland: World Health Organization, Global Programme on AIDS, 6 - 8 December 1993.

World Health Organization. (1995a). The current global situation of the HIV/AIDS pandemic. Geneva, Switzerland: Author; In Centers for Disease Control and Prevention, Morbidity and Mortality Weekly Report, 44(46), 849-853.

World Health Organization. (1995b). Global AIDS News. The Newsletter of the World Health Organization Global Programme on AIDS, 1,5 .

Wynder, E.L. (1994). Child Health Day task forces report: Introduction: Toward national comprehensive school health education. Preventive Medicine An International Journal Devoted to Practice and Theory, 23(1), 106-118.

Zabin, L.S., Hirsch, M.B., Smith, E.A., Streett, R., \& Hardy, J.B. (1986). Evaluation of a pregnancy prevention program for urban teenagers. Family Planning Perspectives, $\underline{18}$, 119-126.

Zabin, L.S., Hirsch, M.B., Streett, R., Emerson, M.R., Smith, M., Hardy, J.B., \& King, T.M. (1988). The Baltimore pregnancy prevention program for urban teenagers: How did it work? Family Planning Perspectives, 20, 182-187.

Zelnick, M. \& Kantner, J.F. (1977). Sexual and contraceptive experience of young unmarried women in the United States, 1976 and 1971. Family Planning Perspectives, 9, 55-77.

Zelnick, M. \& Kantner, J.F. (1980). Sexual activity, contraceptive use, and pregnancy among metropolitan teenagers; 1971-1979. Family Planning Perspectives, 12, 230-237.

Zelnick, M. \& Kim, Y.J. (1982). Sex education and its association with teenage sexual activity, pregnancy and contraceptive use. Family Planning Perspectives, 14(3), 117126.

Zelnik, M. \& Shah, F.K. (1983). First intercourse among young Americans. Family Planning Perspectives, 15 , 64-70. 
Zierler, S., Feingold, L., Laufer, D., Velentgas, P., Kantrowitz-Gordon, I., \& Mayer, K. (1991). Adult survivors of childhood sexual abuse and subsequent risk of HIV infection. American Journal of Public Health, 81(5), 572-575.

Zimet, G.D., Bunch, D.L., Anglin, T.M., Lazebnik, R., Williams, P., \& Krowchuck, D.P. (1992). Relationship of AIDS-related attitudes to sexual behavior changes in adolescents. Journal of Adolescent Health, 13, 493-498.

Zimet, G.D., DiClemente, R.J., Lazebnik, R., Anglin, T.M., Ellick, E.M, \& Williams, P. (1993). Changes in adolescents' knowledge and attitudes about AIDS over the course of the AIDS epidemic. Journal of Adolescent Health, 14(2), 85-90.

Zimet, G.D., Hillier, S.A., Anglin, T.M., Ellick, E.M., Krowchuck, D.P., \& Williams, P. (1991). Journal of Pediatric Psychology, 16 (3), 287-294. 
Table 1

Demographic Profiles for Conference Youth and School Youth by Gender

Conference Youth

Young M
$(n=49)$
$n \quad \%$
School Youth

Young Men Young Women

$$
n \quad \%
$$$$
\begin{gathered}
(n=239) \\
n \quad \%
\end{gathered}
$$

Age:

$\begin{array}{lrrrrrrrrrr}13 \text { years old } & 4 & 8.2 \% & 8 & 10.0 \% & 14 & 42.4 \% & 21 & 27.3 \% & 47 & 19.7 \% \\ 14 \text { years old } & 9 & 18.4 & 15 & 18.8 & 9 & 27.3 & 12 & 15.6 & 45 & 18.8 \\ 15 \text { years old } & 13 & 26.5 & 18 & 22.5 & 5 & 15.2 & 29 & 37.7 & 65 & 27.2 \\ 16 \text { years old } & 17 & 34.7 & 15 & 18.8 & 2 & 6.1 & 11 & 14.3 & 45 & 18.8 \\ 17 \text { years old } & 5 & 10.2 & 12 & 15.0 & 3 & 9.1 & 4 & 5.2 & 24 & 10.0 \\ 18 \text { years old } & 1 & 2.0 & 4 & 5.0 & 0 & 0.0 & 0 & 0.0 & 5 & 2.1 \\ 19 \text { years old } & 0 & 0.0 & 8 & 10.0 & 0 & 0.0 & 0 & 0.0 & 8 & 3.3\end{array}$

Ethnicity:

$\begin{array}{lrrrrrrrrrr}\text { Caucasian } & 25 & 51.0 & 67 & 83.8 & 31 & 93.9 & 76 & 98.7 & 199 & 83.3 \\ \text { African-Am. } & 17 & 34.7 & 6 & 7.5 & 2 & 6.1 & 0 & 0.0 & 25 & 10.5 \\ \text { Hispanic-Am. } & 3 & 6.1 & 1 & 1.3 & 0 & 0.0 & 1 & 1.3 & 5 & 2.1 \\ \text { Asian-Am. } & 0 & 0.0 & 4 & 5.0 & 0 & 0.0 & 0 & 0.0 & 4 & 1.7 \\ \text { Native-Am. } & 4 & 8.2 & 2 & 2.5 & 0 & 0.0 & 0 & 0.0 & 6 & 2.5\end{array}$

Residence:

$\begin{array}{lrrrrrrrrrr}\text { RI } & 13 & 26.5 & 26 & 32.5 & 0 & 0.0 & 0 & 0.0 & 39 & 16.3 \\ \text { CT } & 2 & 4.1 & 2 & 2.5 & 33 & 100.0 & 77 & 100.0 & 114 & 47.7 \\ \mathrm{MA} & 4 & 8.2 & 7 & 8.8 & 0 & 0.0 & 0 & 0.0 & 11 & 4.6 \\ \mathrm{NH} & 1 & 2.0 & 2 & 2.5 & 0 & 0.0 & 0 & 0.0 & 3 & 1.3 \\ \mathrm{NJ} & 12 & 24.5 & 12 & 15.0 & 0 & 0.0 & 0 & 0.0 & 24 & 10.0 \\ \mathrm{ME} & 2 & 4.1 & 2 & 2.5 & 0 & 0.0 & 0 & 0.0 & 4 & 1.7 \\ \mathrm{NY} & 1 & 2.0 & 3 & 3.8 & 0 & 0.0 & 0 & 0.0 & 4 & 1.7 \\ \mathrm{PA} & 5 & 10.2 & 14 & 17.5 & 0 & 0.0 & 0 & 0.0 & 19 & 7.9 \\ \mathrm{VT} & 1 & 2.0 & 2 & 2.5 & 0 & 0.0 & 0 & 0.0 & 3 & 1.3 \\ \mathrm{DE} & 0 & 0.0 & 3 & 3.8 & 0 & 0.0 & 0 & 0.0 & 3 & 1.3 \\ \text { OH } & 3 & 6.1 & 2 & 2.5 & 0 & 0.0 & 0 & 0.0 & 5 & 2.1 \\ \text { Bermuda } & 1 & 2.0 & 3 & 3.8 & 0 & 0.0 & 0 & 0.0 & 4 & 1.7 \\ \text { Jamaica } & 2 & 4.1 & 0 & 0.0 & 0 & 0.0 & 0 & 0.0 & 2 & 0.8 \\ \text { Cayman Islands } & 2 & 4.1 & 2 & 2.5 & 0 & 0.0 & 0 & 0.0 & 4 & 1.7\end{array}$

(continued on next page) 
Grade:

$\begin{array}{lrrrrrrrrrr}\text { Sixth } & 2 & 4.1 \% & 0 & 0.0 \% & 0 & 0.0 \% & 0 & 0.0 \% & 2 & 0.8 \% \\ \text { Seventh } & 4 & 8.2 & 1 & 1.3 & 11 & 33.3 & 18 & 23.4 & 34 & 14.2 \\ \text { Eighth } & 3 & 6.1 & 12 & 15.0 & 7 & 21.2 & 6 & 7.8 & 28 & 11.7 \\ \text { Ninth } & 11 & 22.4 & 10 & 12.5 & 7 & 21.2 & 13 & 16.9 & 41 & 17.2 \\ \text { Tenth } & 12 & 24.5 & 15 & 18.8 & 4 & 12.1 & 23 & 29.9 & 54 & 22.6 \\ \text { Eleventh } & 11 & 22.4 & 18 & 22.5 & 2 & 6.1 & 13 & 16.9 & 44 & 18.4 \\ \text { Twelfth } & 5 & 10.2 & 11 & 13.8 & 2 & 6.1 & 4 & 5.2 & 22 & 9.2 \\ \text { 1st/yr college } & 1 & 2.0 & 7 & 8.8 & 0 & 0.0 & 0 & 0.0 & 8 & 3.3 \\ \text { 2nd/yr + college } & 0 & 0.0 & 6 & 7.5 & 0 & 0.0 & 0 & 0.0 & 6 & 2.5\end{array}$

Religion:

$\begin{array}{lrrrrrrrrrr}\text { Catholic } & 13 & 26.5 & 37 & 46.3 & 14 & 42.4 & 45 & 58.4 & 109 & 45.6 \\ \text { Protestant } & 3 & 6.1 & 16 & 20.0 & 3 & 9.1 & 6 & 7.8 & 28 & 11.7 \\ \text { Jewish } & 2 & 4.1 & 2 & 2.5 & 0 & 0.0 & 0 & 0.0 & 4 & 1.7 \\ \text { None } & 12 & 24.5 & 8 & 10.0 & 8 & 24.2 & 12 & 15.6 & 40 & 16.7 \\ \text { Other } & 19 & 38.8 & 17 & 21.3 & 8 & 24.2 & 14 & 18.2 & 58 & 24.3\end{array}$

Religiosity:

$\begin{array}{lrrrrrrrrrr}\text { Not at all } & 13 & 26.5 & 8 & 10.0 & 6 & 18.2 & 14 & 18.2 & 41 & 17.2 \\ \text { Slightly } & 11 & 22.4 & 12 & 15.0 & 9 & 27.3 & 19 & 24.7 & 51 & 21.3 \\ \text { Somewhat } & 8 & 16.3 & 17 & 21.3 & 11 & 33.3 & 27 & 35.1 & 63 & 26.4 \\ \text { Fairly } & 14 & 28.6 & 35 & 43.7 & 6 & 18.2 & 12 & 15.6 & 67 & 28.0 \\ \text { Very } & 3 & 6.1 & 8 & 10.0 & 1 & 3.0 & 5 & 6.5 & 17 & 7.1\end{array}$

Living with:

$\begin{array}{lrrrrrrrrrr}\text { Both parents } & 27 & 55.1 & 63 & 78.8 & 23 & 69.7 & 53 & 68.8 & 166 & 69.5 \\ \text { Mother only } & 20 & 40.8 & 14 & 17.5 & 5 & 15.2 & 18 & 23.4 & 57 & 23.8 \\ \text { Father only } & 1 & 2.0 & 0 & 0.0 & 4 & 12.1 & 2 & 2.6 & 7 & 2.9 \\ \text { Other } & 1 & 2.0 & 3 & 3.8 & 1 & 3.0 & 4 & 5.2 & 9 & 3.8\end{array}$

Mother's Ed:

$\begin{array}{lrrrrrrrrrr}\text { 8th grade or less } & 2 & 4.1 & 0 & 0.0 & 1 & 3.0 & 1 & 1.3 & 4 & 1.7 \\ \text { 8th-1 lth grade } & 5 & 10.2 & 3 & 3.8 & 4 & 12.1 & 7 & 9.1 & 19 & 7.9 \\ \text { High sch. grad } & 19 & 38.8 & 23 & 28.8 & 10 & 30.3 & 35 & 45.5 & 87 & 36.4 \\ \text { Some college } & 14 & 28.6 & 25 & 31.3 & 4 & 12.1 & 13 & 16.9 & 56 & 23.4 \\ \text { College grad } & 9 & 18.4 & 28 & 35.0 & 14 & 42.4 & 21 & 27.3 & 72 & 30.1 \\ \text { Missing data } & 0 & 0.0 & 1 & 1.3 & 0 & 0.0 & 0 & 0.0 & 1 & 0.4\end{array}$

Father's Ed.:

$\begin{array}{lrrrrrrrrrr}\text { 8th grade or less } & 1 & 2.0 & 0 & 0.0 & 1 & 3.0 & 3 & 3.9 & 5 & 2.1 \\ \text { 8th-11th grade } & 8 & 16.3 & 3 & 3.8 & 2 & 6.1 & 5 & 6.5 & 18 & 7.5 \\ \text { High sch. grad } & 10 & 20.4 & 12 & 15.0 & 14 & 42.4 & 41 & 53.2 & 77 & 32.2 \\ \text { Some college } & 20 & 40.8 & 25 & 31.3 & 4 & 12.1 & 11 & 14.3 & 60 & 25.1 \\ \text { College grad } & 10 & 20.4 & 38 & 47.5 & 12 & 36.4 & 16 & 20.8 & 76 & 31.8 \\ \text { Missing data } & 0 & 0.0 & 2 & 2.5 & 0 & 0.0 & 1 & 1.3 & 3 & 1.3\end{array}$




\section{Table 2}

Exploratory Factor Analysis Pattern Matrix and Factor Correlation Matrix for Cognitive Subscales Utilizing 4-Sample Pooled Data $(n=457)$

Item

AIDS is a medical condition in which your body cannot fight off diseases.

AIDS is caused by a virus.

If a pregnant woman has AIDS, there is a chance it may harm her unborn baby.

Most people who get AIDS usually die from it.

Using a condom (rubber) during sex can lower the risk of getting AIDS.

Receiving a blood transfusion with infected blood can give a person AIDS.

A woman who has unprotected sex only with men can get AIDS.

A man who has unprotected sex only with women can get AIDS.

A woman who has unprotected sex only with women can get AIDS.

A man who has unprotected sex only with men can get AIDS.

If someone asked me a question about how to prevent getting HIV/AIDS, I can give them the right answer.

If someone asked me what things people do that can cause HIV/AIDS, I can tell them the right answer.

If someone asked me how HIV/AIDS is transmitted, I can give them the right answer.

AIDS can be cured if treated early.

A new vaccine has been developed which can prevent AIDS.

You can have AIDS and not know it. (R)

Mosquitoes can transmit AIDS.

I can avoid getting AIDS by having sex only with healthy looking persons.

Only gay people and people who use needle drugs can get AIDS.

$(\mathrm{R})=$ Item reverse coded.

\section{Factor Correlation Matrix}

Factor 1 (FACTUAL AIDS KNOWLEDGE)

Factor 2 (TRANSMISSION KNOWLEDGE)

Factor 3 (PERCEIVED AIDS KNOWLEDGE)

Factor 4 (MISCONCEPTIONS)
(F-KNOWL) (T-KNOWL) (P-KNOWL) (MISCON)

$(\alpha=.74) \quad(\alpha=.94) \quad(\alpha=.85) \quad(\alpha=.79)$

Factor 1 Factor 2 Factor 3 Factor 4

$\begin{array}{rrrr}\mathbf{- . 5 4 4 5 3} & -.00251 & .07986 & .14419 \\ -\mathbf{5 4 0 9 0} & .02651 & .06632 & .13172\end{array}$

$\begin{array}{rrrr}\mathbf{- . 7 1 8 5 3} & .01241 & .04378 & .06978 \\ \mathbf{- . 6 0 2 3 6} & .07897 & .04196 & -.12267 \\ & & & \\ \mathbf{- . 7 0 4 6 9} & -.07819 & .09537 & -.06951 \\ \mathbf{- . 7 7 1 2 9} & -.07044 & -.20348 & -.04027 \\ & & & \\ .00507 & \mathbf{- . 9 2 5 5 9} & -.00730 & .12532 \\ -.00277 & \mathbf{- . 9 3 7 8 3} & -.01271 & .11373 \\ .02128 & \mathbf{- . 9 1 8 4 4} & .00062 & -.00940 \\ .00363 & \mathbf{- . 8 6 9 9 1} & .07088 & -.21113 \\ & & & \\ -.03494 & .01360 & .82336 & .04094\end{array}$

$\begin{array}{llll}-.00574 & -.03964 & \mathbf{. 9 1 7 2 4} & -.05293\end{array}$

$\begin{array}{lllll}-.01058 & -.02765 & \mathbf{. 8 6 6 6 6} & .00175\end{array}$

$\begin{array}{llll}.01362 & -.01383 & .00514 & \mathbf{. 8 0 5 1 9}\end{array}$

$\begin{array}{llll}.11385 & .00724 & .01154 & \mathbf{. 7 5 5 1 9}\end{array}$

$\begin{array}{llll}-.17548 & -.07462 & .00481 & \mathbf{. 3 9 9 6 9}\end{array}$

$\begin{array}{llll}.03986 & .07173 & .08043 & \mathbf{6 6 5 3 7}\end{array}$

$\begin{array}{lllll}.00826 & .02107 & -.06973 & \mathbf{8 4 4 0 9}\end{array}$

$\begin{array}{lllll}-.10076 & -.06245 & -.04181 & \mathbf{. 6 5 4 4 1}\end{array}$

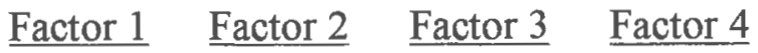
1.00000

$\begin{array}{ll}.16608 & 1.00000\end{array}$

$\begin{array}{lll}-.25707 & -.09913 & 1.00000\end{array}$

$\begin{array}{lll}-.20825 & -.09861 & .07390\end{array}$ 


\section{Table 3}

Confirmatory Factor Analysis Pattern Matrix and Factor Correlation Matrix for Cognitive Subscales Utilizing a Random 50 Percent Sample from 4-Sample Pooled Data ( $n=228)$

Item

AIDS is a medical condition in which your body cannot fight off diseases.

AIDS is caused by a virus.

If a pregnant woman has AIDS, there is a chance it may harm her unborn baby.

Most people who get AIDS usually die from it.

Using a condom (rubber) during sex can lower the risk of getting AIDS.

Receiving a blood transfusion with infected blood can give a person AIDS.

A woman who has unprotected sex only with men can get AIDS.

A man who has unprotected sex only with women can get AIDS.

A woman who has unprotected sex only with women can get AIDS.

A man who has unprotected sex only with men can get AIDS.

If someone asked me a question about how to prevent getting HIV/AIDS, I can give them the right answer.

If someone asked me what things people do that can cause HIV/AIDS, I can tell them the right answer.

If someone asked me how HIV/AIDS is transmitted, I can give them the right answer. AIDS can be cured if treated early.

A new vaccine has been developed which can prevent AIDS.

You can have AIDS and not know it. (R)

Mosquitoes can transmit AIDS.

I can avoid getting AIDS by having sex only with healthy looking persons.

Only gay people and people who use needle drugs can get AIDS.

$(\mathrm{R})=$ Item reverse coded.

Factor Correlation Matrix

Factor 1 (FACTUAL AIDS KNOWLEDGE)

Factor 2 (TRANSMISSION KNOWLEDGE)

Factor 3 (PERCEIVED AIDS KNOWLEDGE)

Factor 4 (MISCONCEPTIONS)
(F-KNOWL) (T-KNOWL) (P-KNOWL) (MISCON) Factor 1 Factor 2 Factor 3 Factor 4

\begin{tabular}{|c|c|c|c|}
\hline .50439 & -.07972 & .12791 & -.15747 \\
\hline .61584 & -.06225 & .15402 & -.03728 \\
\hline .76900 & .02749 & .02965 & -.06989 \\
\hline .59574 & .09910 & .01230 & .12496 \\
\hline .75863 & -.04539 & .00498 & .0390 \\
\hline .80041 & -.01854 & -19971 & -.0044 \\
\hline 01787 & -.92441 & -.01034 & -.1396 \\
\hline .01418 & -.92893 & -.02009 & -.1345 \\
\hline-.02008 & -.90685 & -.02690 & .0196 \\
\hline-.02058 & -.83815 & .03369 & .2454 \\
\hline-.01716 & .02403 & .86781 & -.0070 \\
\hline-.00091 & .00198 & .94211 & .02951 \\
\hline .01721 & -.00626 & .89685 & .00348 \\
\hline-.02088 & -.00210 & -.01230 & -.84175 \\
\hline-.06506 & -.01099 & -.02141 & -.74869 \\
\hline 19991 & -.04727 & .07550 & -.36302 \\
\hline-.06252 & .05900 & .06347 & -.68187 \\
\hline-.01780 & .06383 & -.06538 & -.889 \\
\hline .07060 & -.08323 & -.02382 & -.72844 \\
\hline
\end{tabular}

Factor 1 Factor 2 Factor 3 Factor 4 1.00000 $-.15151 \quad 1.00000$ $.25633-.05755$

1.00000

$-.17285$ 
Table 4

Response Frequencies for Cognitive Scale Items ( $n=239$, current sample only)

$\underline{\text { Item }}$

Definitely Probably Not Probably Definitely

AIDS is a medical condition in which your body cannot fight off diseases.

AIDS is caused by a virus.

If a pregnant woman has AIDS, there is a chance it may harm her unborn baby.

Most people who get AIDS usually die from it.

Using a condom (rubber) during sex can lower the risk of getting AIDS.

Receiving a blood transfusion with infected blood can give a person AIDS.

A woman who has unprotected sex only with men can get AIDS.

A man who has unprotected sex only with women can get AIDS.

A woman who has unprotected sex only with women can get AIDS.

A man who has unprotected sex only with men can get AIDS.

AIDS can be cured if treated early

A new vaccine has been developed which can prevent AIDS.

You can have AIDS and not know it.

Mosquitoes can transmit AIDS.

I can avoid getting AIDS by having sex only with healthy looking persons.

Only gay people and people who use needle drugs can get AIDS.

$\begin{array}{lllcl}5.4 \% & 1.3 \% & 7.9 \% & 15.1 \% & 70.3 \% \\ 5.9 & 1.3 & 9.2 & 8.8 & 74.9\end{array}$

$\begin{array}{lllll}2.1 & 1.7 & 7.5 & 19.2 & 69.5\end{array}$

$\begin{array}{lllll}3.8 & 2.5 & 5.4 & 25.5 & 62.8\end{array}$

$\begin{array}{lllll}2.5 & 2.1 & 2.9 & 27.6 & 64.9\end{array}$

$\begin{array}{lllll}3.3 & 0.4 & 2.9 & 23.0 & 70.3\end{array}$

$\begin{array}{lllll}20.9 & 8.8 & 7.9 & 18.0 & 44.4\end{array}$

$\begin{array}{lllll}20.1 & 8.4 & 8.8 & 18.0 & 44.8\end{array}$

$\begin{array}{lllll}20.5 & 8.8 & 11.3 & 18.4 & 41.0\end{array}$

$\begin{array}{rrrrr}16.7 & 3.8 & 8.8 & 16.3 & 54.4 \\ 56.5 & 12.1 & 13.0 & 12.1 & 6.3\end{array}$

$\begin{array}{lllll}44.4 & 16.7 & 23.0 & 10.0 & 5.9\end{array}$

$\begin{array}{lllll}16.7 & 6.7 & 6.7 & 16.3 & 53.6\end{array}$

$\begin{array}{lllll}43.1 & 15.5 & 18.8 & 13.0 & 9.6\end{array}$

$\begin{array}{lllll}72.4 & 7.5 & 4.6 & 10.0 & 5.4\end{array}$

$\begin{array}{lllll}74.1 & 3.8 & 5.4 & 8.8 & 7.9\end{array}$

Definitely Not really Somewhat Mostly Definitely

If someone asked me a question about how to not true true true true true prevent getting HIV/AIDS, I can give them the right answer.

If someone asked me what things people do that can cause HIV/AIDS, I can tell them the right answer.

If someone asked me how HIV/AIDS is transmitted, I can give them the right answer.

$\begin{array}{lllll}2.1 & 2.5 & 10.5 & 34.3 & 50.6\end{array}$

$\begin{array}{lllll}2.9 & 2.5 & 7.1 & 31.8 & 55.6\end{array}$




\section{Table 5}

Exploratory Factor Analysis Pattern Matrix and Factor Correlation Matrix for Attitudinal Subscales Utilizing 4-Sample Pooled Data $(n=457)$

Item

I feel that I am at risk of getting AIDS at this time in my life.

I sometimes think that I may have been exposed to AIDS.

I have had sex with someone who could have given me AIDS.

I believe I can do things to help protect myself from getting AIDS. (R)

I try not to get into situations where I might get AIDS. (R)

I would use a condom when...

...I am really turned on.

...my partner gets mad about using a condom.

...I am depressed.

...I have been drinking or doing drugs.

...I feel angry.

...I feel pressured to have sex.

...I'm afraid I might get AIDS.

I think people with HIV/AIDS should be in a special classroom at school.

I would not like it if someone in my gym class has HIV/AIDS.

A gay person should not be allowed to teach school.

A gay person should not be allowed to run for public office.

If my roommate told me he or she was gay, I would probably ask them to move out.

A gay person should not be allowed to be a coach.

I would stay away from people who are gay, because if I didn't, I would have a higher chance of getting HIV/AIDS.

It's okay for people who have HIV/AIDS to teach school. (R)

I would feel nervous in a group of gay people.

If I saw two men or two women holding hands, I would feel uncomfortable.

I would feel uncomfortable sitting next to a gay person.

I am afraid that someone around me has HIV/AIDS and I do not know it.

I am afraid of making new friends because of HIV/AIDS.

If my brother/sister told me he/she was gay, I would be upset.

$(R)=$ Item reverse coded.

Factor Correlation Matrix

Factor 1 (PERCEIVED SUSCEPTIBILITY FOR RISK)

Factor 2 (CONDOM USE EFFICACY)

Factor 3 (HOMOPHOBIA)
(SUSCEP) (EFFICACY) (HOMOPH)

$(\alpha=.73) \quad(\alpha=.92) \quad(\alpha=.91)$

Factor 1 Factor 2 Factor 3

$\begin{array}{lll}.56544 & -.01283 & .06379\end{array}$

$\begin{array}{lll}.63588 & -.04986 \quad .03263\end{array}$

$.57249 \quad-.14147 \quad .13246$

$\begin{array}{lll}.70533 & -.00900 & -.10044 \\ .65528 & -.01355 & -.11421\end{array}$

$\begin{array}{lll}.65528 & -.01355 & -.11421\end{array}$

$\begin{array}{lll}-.05656 & \mathbf{8 6 9 7 0} & -.02520\end{array}$

$\begin{array}{llll}-.07258 & \mathbf{8 4 6 5 6} & -.03238\end{array}$

$\begin{array}{lll}.00362 & \mathbf{8 9 9 3 0} & .07637\end{array}$

$\begin{array}{lll}.01810 & \mathbf{8 2 0 0 6} & -.00344\end{array}$

$\begin{array}{lll}-.00788 & \mathbf{8 9 8 1 7} & .02745\end{array}$

$\begin{array}{lll}.05651 & \mathbf{8 6 1 7 9} & .01240\end{array}$

$\begin{array}{lll}.02169 & \mathbf{5 8 1 1 6} & -.03275\end{array}$

$\begin{array}{lll}.20718 \quad .00605 & \mathbf{5 8 0 2 6}\end{array}$

$\begin{array}{lll}.21293 & .06785 & .64455\end{array}$

$\begin{array}{lll}.03435 & -.02760 & \mathbf{8 0 3 4 4}\end{array}$

$.07444 \quad .01621 \quad .79385$

$\begin{array}{lll}.26092 & -.02722 & \mathbf{5 8 7 6 7}\end{array}$

$\begin{array}{lll}-.02038 & -.03966 \quad .79446\end{array}$

$\begin{array}{lll}.16562 & -.08751 & \mathbf{6 6 6 4 5 7}\end{array}$

$\begin{array}{llll}.21347 & .02899 & .39478\end{array}$

$\begin{array}{lll}-.19657 & .00108 \quad .74676\end{array}$

$\begin{array}{lll}-.19787 & .01604 \quad .75916\end{array}$

$\begin{array}{lll}-.19751 & -.09804 & .78237\end{array}$

$\begin{array}{rrr}.21551 & .02923 & \mathbf{. 5 0 8 5 0} \\ .21402 & -.06123 & \mathbf{. 4 8 0 3 5} \\ -.14442 & .03558 & \mathbf{. 6 6 8 6 5}\end{array}$

Factor 1 Factor 2 Factor 3

1.00000

$-.18162 \quad 1.00000$

$.25956-.18053 \quad 1.00000$ 
Confirmatory Factor Analysis Pattern Matrix and Factor Correlation Matrix for Attitudinal Subscales Utilizing a Random 50 Percent Sample from 4-Sample Pooled Data $(n=228)$

Item

I feel that I am at risk of getting AIDS at this time in my life.

I sometimes think that I may have been exposed to AIDS.

I have had sex with someone who could have given me AIDS.

I believe I can do things to help protect myself from getting AIDS. (R)

I try not to get into situations where I might get AIDS. (R)

I would use a condom when...

...I am really turned on.

...my partner gets mad about using a condom.

...I am depressed.

...I have been drinking or doing drugs.

...I feel angry.

...I feel pressured to have sex.

...I'm afraid I might get AIDS.

I think people with HIV/AIDS should be in a special

classroom at school.

I would not like it if someone in my gym class has HIV/AIDS.

A gay person should not be allowed to teach school.

A gay person should not be allowed to run for public office.

If my roommate told me he or she was gay, I would probably ask them to move out.

A gay person should not be allowed to be a coach.

I would stay away from people who are gay, because if I didn't, I would have a higher chance of getting HIV/AIDS.

It's okay for people who have HIV/AIDS to teach school. (R)

I would feel nervous in a group of gay people.

If I saw two men or two women holding hands, I

would feel uncomfortable.

I would feel uncomfortable sitting next to a gay person.

I am afraid that someone around me has HIV/AIDS and

I do not know it.

I am afraid of making new friends because of HIV/AIDS.

If my brother/sister told me he/she was gay, I would be upset.

$(\mathrm{R})=$ Item reverse coded.

Factor Correlation Matrix

Factor 1 (PERCEIVED SUSCEPTIBILITY FOR RISK)

Factor 2 (CONDOM USE EFFICACY)

Factor 3 (HOMOPHOBIA)
(SUSCEP) (EFFICACY) (HOMOPH)

Factor 1 Factor 2 Factor 3

$\begin{array}{lll}.59966 & -.02207 & .11251\end{array}$

$.65704 \quad-.10191 \quad .00749$

$\begin{array}{lll}.57196 & -.17799 & .16378\end{array}$

$\begin{array}{lll}.71136 & .00902 & -.08753\end{array}$

$\begin{array}{llll}.70370 & .00231 & -.10438\end{array}$

$\begin{array}{lll}-.12357 & \mathbf{8 5 3 6 8} & -.01513\end{array}$

$\begin{array}{llll}-.08065 & \mathbf{8 3 6 0 3} & -.05417\end{array}$

$\begin{array}{lll}-.04538 & \mathbf{8 9 7 4 5} & .05224\end{array}$

$\begin{array}{lll}-.11842 & \mathbf{8 1 9 3 3} \quad .03080\end{array}$

$\begin{array}{llll}-.05001 & \mathbf{8 7 8 9 6} & -.00543\end{array}$

$\begin{array}{lll}.07042 & \mathbf{8 3 3 1 6} & .04544\end{array}$

$\begin{array}{lll}.09106 & .54179 & -.10689\end{array}$

$\begin{array}{lll}.00468 & .0502 \quad .70254\end{array}$

$\begin{array}{lll}.13081 & .09511 \quad .68821\end{array}$

$\begin{array}{lll}-.12371 & -.06629 & \mathbf{8 3 4 0 5}\end{array}$

$\begin{array}{lll}-.03059 & .00834 & \mathbf{8 1 5 0 2}\end{array}$

$\begin{array}{lll}.11658 & .04133 & \mathbf{6 8 0 6 8}\end{array}$

$\begin{array}{lll}-.10263 & -.06413 \quad .81172\end{array}$

$\begin{array}{lll}.03572 & -.01579 & .74411\end{array}$

$\begin{array}{lll}.039 & .35066\end{array}$

$\begin{array}{lll}-.07015 & -.10287 & .65777\end{array}$

$\begin{array}{lll}-.06203 & -.10348 \quad .64958\end{array}$

$\begin{array}{lll}-.12029 & -.17660 \quad .72090\end{array}$

$\begin{array}{lll}.08904 & .08495 & \mathbf{5 5 2 0 6}\end{array}$

$\begin{array}{lll}.05063 & -.03987 & \mathbf{5 4 3 0 6}\end{array}$

$\begin{array}{lll}.00655 & .01546 \quad \mathbf{5 4 8 8 2}\end{array}$

Factor 1 Factor 2 Factor 3

1.00000

$-.08704 \quad 1.00000$

$.24492-.20781$

1.00000 
Table 7

Response Frequencies for Attitudinal Scale Items ( $n=239$, current sample only)

Item

Definitely Probably Not Probably Definitely

I feel that I am at risk of getting AIDS at this time in my life.

I sometimes think that I may have been exposed to AIDS.

I have had sex with someone who could have given me AIDS.

I believe I can do things to help protect

myself from getting AIDS.

I try not to get into situations where I might get AIDS.

\begin{tabular}{ccccc} 
no & no & sure & yes & yes \\
\hline $43.1 \%$ & $27.2 \%$ & $18.8 \%$ & $7.9 \%$ & $2.9 \%$ \\
51.5 & 18.8 & 16.1 & 8.8 & 4.6 \\
68.6 & 8.4 & 13.4 & 4.6 & 5.0 \\
4.2 & 0.4 & 7.1 & 13.4 & 74.9 \\
6.3 & 2.1 & 7.5 & 12.1 & 72.0 \\
$\begin{array}{c}\text { Not at all } \\
\text { sure }\end{array}$ & $\begin{array}{c}\text { A little } \\
\text { sure }\end{array}$ & $\begin{array}{c}\text { Kind of } \\
\text { sure }\end{array}$ & $\begin{array}{c}\text { Fairly } \\
\text { sure }\end{array}$ & $\begin{array}{c}\text { Very } \\
\text { sure }\end{array}$ \\
\hline
\end{tabular}

I would use a condom...

...when I am really turned on.

...when my partner gets mad about using a condom.

...when I am depressed.

$12.1 \% \quad 15.5 \% \quad 13.4 \% \quad 19.2 \% \quad 39.7 \%$

$\begin{array}{lllll}11.3 & 14.2 & 15.1 & 17.2 & 42.3\end{array}$

$\begin{array}{lllll}19.7 & 11.7 & 12.6 & 14.6 & 41.4\end{array}$

...when I have been drinking or doing drugs.

22.2

$\begin{array}{llll}10.0 & 14.5 & 13.8 & 39.3\end{array}$

...when I feel angry.

$\begin{array}{lllll}20.5 & 8.4 & 13.8 & 15.1 & 42.3\end{array}$

...when I feel pressured to have sex.

15.1
4.2

$\begin{array}{ll}7.5 & 10.0\end{array}$

$13.4 \quad 54.0$

...when I'm afraid I might get AIDS.

(continued on next page) 
Definitely Not really Somewhat Mostly Definitely

I think people with HIV/AIDS should be in a special classroom at school.

\begin{tabular}{|c|c|c|c|c|}
\hline not true & true & true & true & true \\
\hline $62.3 \%$ & $18.8 \%$ & $8.8 \%$ & $5.0 \%$ & $5.0 \%$ \\
\hline 52.3 & 23.0 & 10.9 & 6.3 & 7.5 \\
\hline 70.3 & 10.9 & 6.7 & 5.0 & 7.1 \\
\hline 72.4 & 13.0 & 5.0 & 4.2 & 5.4 \\
\hline 59.8 & 18.0 & 7.5 & 7.5 & 7.1 \\
\hline 66.9 & 15.1 & 5.4 & 5.4 & 7.1 \\
\hline 67.4 & 15.1 & 7.1 & 5.4 & 5.0 \\
\hline 14.2 & 3.3 & 12.1 & 14.2 & 56.1 \\
\hline 28.5 & 19.2 & 26.4 & 9.2 & 16.7 \\
\hline 24.3 & 25.1 & 25.1 & 12.6 & 13.0 \\
\hline 39.3 & 23.8 & 16.3 & 10.0 & 10.5 \\
\hline 55.6 & 21.3 & 15.1 & 3.3 & 4.6 \\
\hline 75.7 & 13.8 & 5.9 & 2.1 & 2.5 \\
\hline 29.7 & 19.2 & 18.4 & 12.1 & 20.5 \\
\hline
\end{tabular}

I would not like it if someone in my gym class has HIV/AIDS.

A gay person should not be allowed to teach.

A gay person should not be allowed to run for public office.

If my roommate told me he or she was gay, I would probably ask them to move out.

A gay person should not be allowed to be a coach.

I would stay away from people who are gay, because if I didn't, I would have a higher chance of getting HIV/AIDS.

It's ok for people with HIV/AIDS to teach school.

I would feel nervous in a group of gay people.

If I saw two men or two women holding hands, I would feel uncomfortable.

I'd feel uncomfortable sitting next to a gay person.

I am afraid that someone around me has HIV/AIDS and I do not know it.

I'm afraid of making new friends because of AIDS.

If my brother/sister told me he/she was gay, I would be upset. 


\section{Table 8}

Exploratory Factor Analysis Pattern Matrix and Factor Correlation Matrix for Substance Use and Abuse Behavior Subscales Utilizing 4-Sample Pooled Data of Substance Using Adolescents ( $n=308$ )

Item

In the past year, how often have you had a drink of beer, wine, or liquor?

In the past year, how many drinks did you have when you drank alcohol?

In the past year, how often did you have 3 or more drinks in one day?

In the past year, how often did you have 6 or more drinks in one day?

In the past year, how many times did you use marijuana or hashish (grass, pot, hash, hash oil) without a doctor's orders?

In the past year, how many times did you use other drugs without a doctor's orders?

In the past year, how many times did you share a needle to shoot drugs?

In the past year, how often have these things happened because of your drinking or drug use:

I had a hangover.

I messed up on something important.

I was in trouble with police or other authority.

I broke or messed up someone else's things.

I got into an argument or fight.

I got sick/threw up.

I drove a car after having alcohol or drugs.

I was hurt/injured.

Someone I know talked to me about how I was acting.

I thought I might have a drinking/drug problem.

I couldn't remember (blacked out).

I did something I wished I hadn't.

I was arrested for drunk driving.

I had sex that I wouldn't have had if sober.

I tried to stop using but didn't.

I thought about or tried to kill myself.

Factor Correlation Matrix

Factor 1 (SUBSTANCE USE)

Factor 2 (SUBSTANCE ABUSE)
(S-USE) (S-ABUSE)

$(\alpha=.87) \quad(\alpha=.90)$

Factor 1 Factor 2

$\begin{array}{ll}-.77090 & .01447\end{array}$

$\begin{array}{ll}-.80099 & -.14616\end{array}$

$\begin{array}{ll}-.80152 & .04499\end{array}$

$\begin{array}{ll}-.75042 & .05171\end{array}$

$\begin{array}{rl}-.61760 & .03643\end{array}$

$-.40677 \quad .18644$

$\begin{array}{ll}-.34251 & .0107\end{array}$

$-.77994-.11756$

$-.19189 \quad \mathbf{5 0 9 0 3}$

$-.10151 \quad \mathbf{. 5 0 4 7 0}$

-.02294 $\mathbf{. 5 4 4 1 5}$

$.00332 \quad \mathbf{. 6 2 5 8 7}$

$-.13077 \quad \mathbf{5 9 8 0 2}$

-.32989 $\mathbf{. 3 3 1 1 9}$

$.24779 \quad .75076$

$-.05948 \quad \mathbf{5 7 6 4 3}$

$.09643 \quad .67803$

$-.11045 \quad \mathbf{4 7 9 4 9}$

$.05297 \quad \mathbf{. 6 7 4 5 2}$

$.09881 \quad \mathbf{. 5 8 4 7 4}$

$-.27579 \quad .41168$

$.00740 \quad \mathbf{. 5 5 0 5 1}$

. $.02783 \quad .29784$

Factor 1 Factor 2

1.00000

$-.36870 \quad 1.00000$ 


\section{Table 9}

Confirmatory Factor Analysis Pattern Matrix and Factor Correlation Matrix for Substance Use and Abuse Behavior Subscales Utilizing a Random 50 Percent Sample from 4-Sample Pooled Data of Substance Using Adolescents $(n=154)$

$\underline{\text { Item }}$

In the past year, how often have you had a drink of beer, wine, or liquor?

In the past year, how many drinks did you have when you drank alcohol?

In the past year, how often did you have 3 or more drinks in one day?

In the past year, how often did you have 6 or more drinks in one day?

In the past year, how many times did you use marijuana or hashish (grass, pot, hash, hash oil) without a doctor's orders?

In the past year, how many times did you use other drugs without a doctor's orders?

In the past year, how many times did you share a needle to shoot drugs?

In the past year, how often have these things happened because of your drinking or drug use:

I had a hangover.

I messed up on something important.

I was in trouble with police or other authority.

I broke or messed up someone else's things.

I got into an argument or fight.

I got sick/threw up.

I drove a car after having alcohol or drugs.

I was hurt/injured.

Someone I know talked to me about how I was acting.

I thought I might have a drinking/drug problem.

I couldn't remember (blacked out).

I did something I wished I hadn't.

I was arrested for drunk driving.

I had sex that I wouldn't have had if sober.

I tried to stop using but didn't.

I thought about or tried to kill myself.

Factor Correlation Matrix

Factor 1 (SUBSTANCE USE)

Factor 2 (SUBSTANCE ABUSE)
(S-USE) (S-ABUSE)

\section{Factor 1 Factor 2}

$\begin{array}{lll}-.79005 & -.03420\end{array}$

$\begin{array}{ll}-.78787 & -.11404\end{array}$

$\begin{array}{ll}-.81939 & .02851\end{array}$

$-.72242 \quad .06747$

$-.69316 \quad .01507$

$-.56005 \quad .17248$

$-.24158 \quad .00402$

$\begin{array}{ll}-.71583 & -.04047\end{array}$

$-.04248 \quad \mathbf{5 3 8 9 0}$

-.20691 $\mathbf{. 4 9 8 0 7}$

-.01710 $\mathbf{. 4 5 7 3 5}$

$.15565 \quad \mathbf{6 7 8 5 4}$

-.07482 $\mathbf{. 6 5 6 9 4}$

-.23339 $\mathbf{. 3 5 9 2 9}$

$.32575 \quad \mathbf{. 7 5 0 4 1}$

$.06771 \quad \mathbf{6 9 4 9 4}$

$.04329 \quad \mathbf{6 3 6 8 9}$

$-.10831 \quad . \mathbf{4 4 7 1 7}$

$.08139 \quad \mathbf{7 2 1 9 6}$

$.00551 \quad \mathbf{. 5 0 5 4 4}$

-.34931 .45590

$-.10951 \quad .56913$

-.16003 $\quad .30377$

Factor 1 Factor 2

1.00000

$-.28401 \quad 1.00000$ 


\section{Table 10}

Exploratory Factor Analysis Pattern Matrix and Factor Correlation Matrix for Sexual Assertiveness Behavior Subscales Utilizing 4-Sample Pooled Data of Sexually Active Adolescents $(n=268)$

Item

I would ask if I want to know if my partner ever had an HIV test.

I would ask my partner about the AIDS risk of his/her past partners, if I want to know.

I would ask if I want to know if my partner ever had a STD.

I would ask if I want to know if my partner ever had sex with someone who shoots drugs with a needle.

I would ask if I want to know if my partner ever used needles to take drugs.

I would have sex without a condom if my partner doesn't like them, even if I want to use one. (R)

I would make sure my partner and I use a condom when we have sex.

I would insist on using a condom if I want to, even if my partner doesn't like them.

I would have sex without using a condom if my partner insists, even if I don't want to. (R)

I would refuse to have sex if my partner refuses to use a condom.

$(\mathrm{R})=$ Item reverse coded.

Factor Correlation Matrix

Factor 1 (SEXUAL ASSERT. PARTNER COMMUNIC.)

Factor 2 (SEXUAL ASSERT. CONDOM USE)
(SAPC) (SACU)

$(\alpha=.93) \quad(\alpha=.85)$

Factor 1 Factor 2

$\mathbf{8 7 4 5 4}-.08503$

$.58113 \quad .38036$

$.56868 \quad .38914$

$.87026-.11064$

$.55066 \quad .38596$

$-.05039 \quad .91368$

$-.08668 \quad \mathbf{9 2 6 3 6}$

$.00636 \quad \mathbf{9 2 0 0 3}$

$.01538 \quad \mathbf{8 6 5 9 0}$

$.13245 \quad .74067$

Factor 1 Factor 2

1.00000

$.48284 \quad 1.00000$ 


\section{Table 11}

Confirmatory Factor Analysis Pattern Matrix and Factor Correlation Matrix for Sexual Assertiveness Behavior Subscales Utilizing a Random 50 Percent Sample from 4-Sample Pooled Data of Sexually Active Adolescents ( $n=134)$

Item

I would ask if I want to know if my partner ever had an HIV test.

I would ask my partner about the AIDS risk of his/her past partners, if I want to know.

I would ask if I want to know if my partner ever had a STD.

I would ask if I want to know if my partner ever had sex with someone who shoots drugs with a needle.

I would ask if I want to know if my partner ever used needles to take drugs.

I would have sex without a condom if my partner doesn't like them, even if I want to use one. (R)

I would make sure my partner and I use a condom when we have sex.

I would insist on using a condom if I want to, even if my partner doesn't like them.

I would have sex without using a condom if my partner insists, even if I don't want to. (R)

I would refuse to have sex if my partner refuses to use a condom.

$(\mathrm{R})=$ Item reverse coded.

Factor Correlation Matrix

Factor 1 (SEXUAL ASSERT. PARTNER COMMUNIC.)

Factor 2 (SEXUAL ASSERT. CONDOM USE)
(SAPC) (SACU)

Factor 1 Factor 2

$\mathbf{8 4 9 0 7}-.12596$

$.78173 \quad .09418$

$\mathbf{8 0 9 0 7} .09365$

$\begin{array}{ll}.73822 & -.02172\end{array}$

$\begin{array}{ll}.76367 & .11683\end{array}$

$.01109 \quad \mathbf{9 0 0 2 0}$

$.00254 \quad \mathbf{8 8 5 0 2}$

$.01702 \quad \mathbf{9 2 6 5 9}$

$-.03506 \quad .92131$

$.05320 \quad \mathbf{8 6 9 2 7}$

Factor 1 Factor 2

1.00000

$.59362 \quad 1.00000$ 


\section{Exploratory Factor Analysis Pattern Matrix and Factor Correlation Matrix for Behavior Risk History Subscales Utilizing 4-Sample Pooled Data of Sexually Active Adolescents ( $n=268$ )}

\section{$\underline{\text { Item }}$}

About how long have you been involved with your most recent sex partner?

How old were you the first time you willingly had intercourse?

About how many sex partners have you had in your life?

About how many sex partners have you had in the past year?

In the past year, did you use alcohol before having sex?

In the past year, how many drinks did you normally have before having sex?

In the past year, did you use marijuana or other drugs before having sex?

How sure are you that you have not been exposed to AIDS?

How sure are you that your sex partner(s) have not been exposed to AIDS?

Have you ever been tested for HIV?

How many sex partners have you had who shoot drugs with a needle?

How many sex partners have you had who tested positive for HIV or had AIDS?

About how many sex partners has your most recent partner had?

How sure are you about your answer to the above question?

Factor Correlation Matrix

Factor 1 (SELF RISK HISTORY)

Factor 2 (PARTNER RISK HISTORY)
(SELF-RISK) (PART-RISK)

$(\alpha=.93) \quad(\alpha=.68)$

Factor 1 Factor 2

$.41011-.29817$

$\begin{array}{ll}.57322 & -.04779\end{array}$

$\begin{array}{ll}.73832 & .15924\end{array}$

$.63739 \quad .12693$

$.82604 \quad .05163$

$.76655 \quad .11869$

$\begin{array}{ll}.59278 & .01217\end{array}$

$.08360 \quad \mathbf{. 7 2 0 5 8}$

$.09124 \quad \mathbf{8 4 0 5 8}$

$-.00564 \quad .29020$

$-.09466 \quad \mathbf{4 0 6 5 5}$

$-.01608 \quad \mathbf{2 9 5 4 5}$

$\begin{array}{ll}.46507 & \mathbf{4 2 3 8 5}\end{array}$

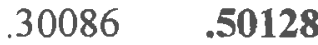

Factor 1 Factor 2

1.00000

$.17223 \quad 1.00000$ 
Confirmatory Factor Analysis Pattern Matrix and Factor Correlation Matrix for Behavior Risk History Subscales Utilizing a Random 50 Percent Sample from 4-Sample Pooled Data of Sexually Active Adolescents $(n=134)$

Item

About how long have you been involved with your most recent sex partner?

How old were you the first time you willingly had intercourse?

About how many sex partners have you had in your life?

About how many sex partners have you had in the past year?

In the past year, did you use alcohol before having sex?

In the past year, how many drinks did you normally have before having sex?

In the past year, did you use marijuana or other drugs before having sex?

How sure are you that you have not been exposed to AIDS?

How sure are you that your sex partner(s) have not been exposed to AIDS?

Have you ever been tested for HIV?

How many sex partners have you had who shoot drugs with a needle?

How many sex partners have you had who tested positive for HIV or had AIDS?

About how many sex partners has your most recent partner had?

How sure are you about your answer to the above question?

\section{Factor Correlation Matrix}

Factor 1 (SELF RISK HISTORY)

Factor 2 (PARTNER RISK HISTORY)
(SELF-RISK) (PART-RISK)

Factor 1 Factor 2

$\mathbf{4 0 0 6 5}-.29839$

$\mathbf{5 9 0 7 9}-.04797$

$.74369 \quad .14339$

$.65956 \quad .08457$

$\mathbf{8 2 8 8 9} \quad .09955$

$.76552 \quad .14833$

$.62817 \quad .06058$

.68999

$.11355 \quad \mathbf{. 7 8 2 4 2}$

$.02913 \quad .34214$

$-.15518 \quad \mathbf{4 0 7 1 2}$

$-.00852 \quad .29629$

.64999

$.02913 \quad \mathbf{3 4 2 1 4}$

Factor 1 Factor 2

1.00000

$.15775 \quad 1.00000$ 
Exploratory Factor Analysis Pattern Matrix and Factor Correlation Matrix for Three Major Areas of Behavior Utilizing 4-Sample Pooled Data $(n=457)$

Subscale

Substance Use

Substance Abuse

Sexual Assertiveness Condom Use

Sexual Assertiveness Partner Communication Self Risk History

Partner Risk History

Factor Correlation Matrix

Factor 1 (SUBSTANCE USE/ABUSE)

Factor 2 (SEXUAL ASSERTIVENESS)

Factor 3 (BEHAVIOR RISK HISTORY)
(SUBSTANCE) (ASSERT) (RISK)

$(\alpha=.92) \quad(\alpha=.92) \quad(\alpha=.89)$

Factor 1 Factor 2 Factor 3

$\begin{array}{lll}\mathbf{- . 7 8 2 1 0} & .00678 & .18945\end{array}$

$\begin{array}{lll}-.96524 & -.02096 & -.08856\end{array}$

$\begin{array}{lll}-.00923 & \mathbf{. 7 9 7 0 1} & -.18312\end{array}$

$\begin{array}{lll}.01890 & \mathbf{. 9 6 4 9 4} & .09994\end{array}$

$\begin{array}{lll}-.13249 & .00777 & \mathbf{. 8 3 9 8 2}\end{array}$

$\begin{array}{lll}.05002 & -.03798 & \mathbf{9 3 5 2 7}\end{array}$

\begin{tabular}{rrr}
$\underline{\text { Factor 1 }}$ & Factor 2 & Factor 3 \\
\hline 1.00000 & & \\
.28600 & 1.00000 & \\
-.51615 & -.45538 & 1.00000
\end{tabular}


Confirmatory Factor Analysis Pattern Matrix and Factor Correlation Matrix for Three Major Areas of Behavior Utilizing a Random 50 Percent Sample from 4-Sample Pooled Data $(n=229)$

$\underline{\text { Subscale }}$

Substance Use

Substance Abuse

Sexual Assertiveness Condom Use

Sexual Assertiveness Partner Communication

Self Risk History

Partner Risk History

Factor Correlation Matrix

Factor 1 (SUBSTANCE USE/ABUSE)

Factor 2 (SEXUAL ASSERTIVENESS)

Factor 3 (BEHAVIOR RISK HISTORY)
(SUBSTANCE) (ASSERT) (RISK)

Factor 1 Factor 2 Factor 3

$\begin{array}{lll}-.78120 & .02103 & .20295\end{array}$

$\begin{array}{lll}-.96908 & -.02824 & -.09824\end{array}$

$\begin{array}{lll}-.00931 & \mathbf{7 7 7 9 5} & -.20726\end{array}$

$\begin{array}{lll}.02090 & \mathbf{9 6 8 8 1} & .09923\end{array}$

$\begin{array}{lll}-.07004 & .00118 & \mathbf{. 9 2 6 1 5}\end{array}$

$\begin{array}{lll}.01354 & -.05293 & \mathbf{. 9 1 2 5 4}\end{array}$

$\begin{array}{rrr}\frac{\text { Factor 1 }}{1.00000} & \text { Factor 2 } & \text { Factor 3 } \\ .28152 & 1.00000 & \\ -.49914 & -.47029 & 1.00000\end{array}$


Table 16

Response Frequencies for Behavior Scale Items ( $n=239$, current sample only)

Substance Items:

In the past year, how often have you had a drink of beer, wine, or liquor?

In the past year, how many drinks did you have when you drank alcohol?

In the past year, how often did you have 3 or more drinks in one day?

In the past year, how often did you have 6 or more drinks in one day?

In the past year, how many times did you use marijuana or hashish (grass, pot, hash, hash oil) without a doctor's orders?

In the past year, how many times did you use other drugs without a doctor's orders?

In the past year, how many times did you share a needle to shoot drugs?

the past year, how often have these things happened because of your drinking/drug use:

I had a hangover.

I messed up on something important.

I was in trouble with police or other authority.

I broke or messed up someone else's things.

I got into an argument or fight.

I got sick/threw up.

I drove a car after having alcohol or drugs.

I was hurt/injured.

Someone I know talked to me about how I was acting.

I thought I might have a drinking/drug problem.

I couldn't remember (blacked out).

I did something I wished I hadn't.

I was arrested for drunk driving.

I had sex that I wouldn't have had if sober.

I tried to stop using but didn't.

I thought about or tried to kill myself.

(continued on next page)
1-2 times 1-2 times 3-4 times Almost

Never mo. or less week week every day

$\begin{array}{lllll}35.6 \% & 49.4 \% & 13.0 \% & 1.3 \% & 0.8 \%\end{array}$

\begin{tabular}{ccccc} 
None & $\begin{array}{c}1-2 \\
\text { drinks }\end{array}$ & $\begin{array}{c}3-5 \\
\text { drinks }\end{array}$ & $\begin{array}{c}6-10 \\
\text { drinks }\end{array}$ & $\begin{array}{c}11 \text { or more } \\
\text { drinks }\end{array}$ \\
\hline $36.4 \%$ & $44.4 \%$ & $15.1 \%$ & $2.5 \%$ & $1.7 \%$ \\
& $\begin{array}{l}1-2 \text { times } \\
\text { Never }\end{array}$ & $\begin{array}{c}1-2 \text { times } \\
\text { mo less }\end{array}$ & $\begin{array}{c}3-4 \text { times } \\
\text { week }\end{array}$ & $\begin{array}{c}\text { Almost } \\
\text { every day }\end{array}$ \\
\hline
\end{tabular}

$\begin{array}{lllll}62.3 \% & 30.5 \% & 6.7 \% & 0.0 \% & 0.4 \%\end{array}$

$\begin{array}{lllll}77.4 & 18.8 & 2.9 & 0.4 & 0.4\end{array}$

$\begin{array}{lllll}81.2 & 12.6 & 2.9 & 2.5 & 0.8\end{array}$

$\begin{array}{lllll}87.9 & 7.5 & 4.2 & 0.0 & 0.4\end{array}$

$\begin{array}{lllll}98.3 & 0.4 & 1.3 & 0.0 & 0.0\end{array}$

\begin{tabular}{|c|c|c|c|c|}
\hline Never & Once & $\begin{array}{l}2-3 \\
\text { times }\end{array}$ & $\begin{array}{l}4-6 \\
\text { time }\end{array}$ & $\begin{array}{c}\text { More than } \\
6 \text { times }\end{array}$ \\
\hline
\end{tabular}

$\begin{array}{lllll}66.1 \% & 18.0 \% & 10.5 \% & 2.5 \% & 2.9 \%\end{array}$

$\begin{array}{lllll}66.5 & 17.2 & 10.0 & 2.1 & 4.2\end{array}$

$\begin{array}{lllll}88.3 & 6.3 & 3.3 & 0.4 & 1.7\end{array}$

$\begin{array}{lllll}73.6 & 14.6 & 8.4 & 2.5 & 0.8\end{array}$

$\begin{array}{lllll}61.9 & 17.6 & 9.2 & 4.2 & 7.1\end{array}$

$\begin{array}{lllll}63.6 & 19.2 & 8.8 & 2.9 & 5.4\end{array}$

$\begin{array}{lllll}92.5 & 5.4 & 1.7 & 0.4 & 0.0\end{array}$

$\begin{array}{lllll}79.1 & 12.6 & 4.6 & 2.1 & 1.7\end{array}$

$\begin{array}{lllll}69.0 & 15.9 & 9.2 & 3.3 & 2.5\end{array}$

$\begin{array}{lllll}93.3 & 5.0 & 0.4 & 0.8 & 0.4\end{array}$

$\begin{array}{lllll}85.8 & 8.8 & 2.9 & 1.7 & 0.8\end{array}$

$\begin{array}{lllll}73.6 & 14.6 & 5.0 & 3.3 & 3.3\end{array}$

$\begin{array}{lllll}96.7 & 2.1 & 0.4 & 0.8 & 0.0\end{array}$

$\begin{array}{lllll}81.6 & 13.8 & 2.1 & 1.3 & 1.3\end{array}$

$\begin{array}{lllll}94.1 & 3.8 & 1.3 & 0.0 & 0.8\end{array}$

$\begin{array}{lllll}86.2 & 9.6 & 2.5 & 0.4 & 1.3\end{array}$ 
About half

Assertiveness Items:

Never Sometimes the time Usually Always

I would ask if I want to know if my partner ever had an HIV test.

I would ask my partner about the AIDS risk of his/her past partners, if I want to know.

I would ask if I want to know if my partner ever had a STD.

I would ask if I want to know if my partner ever had sex with someone who shoots drugs with a needle.

I would ask if I want to know if my partner ever used needles to take drugs.

$3.3 \% \quad 16.3 \% \quad 13.8 \% \quad 19.2 \% \quad 47.3 \%$

$\begin{array}{lllll}3.3 & 18.0 & 17.2 & 15.9 & 45.6\end{array}$

$\begin{array}{lllll}3.3 & 15.5 & 17.2 & 15.1 & 49.0\end{array}$

$\begin{array}{lllll}5.0 & 18.0 & 16.3 & 15.5 & 45.2\end{array}$

$\begin{array}{lllll}2.5 & 22.6 & 14.6 & 10.9 & 49.4\end{array}$

I would have sex without a condom if my partner doesn't like them, even if I want to use one.

I would make sure my partner and I use a condom when we have sex.

I would insist on using a condom if I want to, even if my partner doesn't like them.

I would have sex without using a condom if my partner insists, even if I don't want to.

I would refuse to have sex if my partner refuses to use a condom.

$\begin{array}{lllll}56.5 & 13.8 & 14.6 & 9.6 & 5.4\end{array}$

$\begin{array}{lllll}4.2 & 15.1 & 15.1 & 9.6 & 56.1\end{array}$

$\begin{array}{lllll}7.1 & 13.4 & 13.8 & 7.9 & 57.7\end{array}$

$\begin{array}{lllll}54.0 & 14.2 & 13.0 & 10.5 & 8.4\end{array}$

$\begin{array}{lllll}5.9 & 18.8 & 15.3 & 6.3 & 53.6\end{array}$

(continued on next page) 


\section{Risk Items:}

About how long have you been involved with your most recent sex partner?

How old were you the first time you willingly had intercourse?

About how many sex partners have you had in your life?

About how many sex partners have you had in the past year?

About how many partners has you most recent partner had?

How many sex partners have you had who shoot drugs with a needle?

How many sex partners have you had who tested positive for HIV or had AIDS?

In the past year, did you use alcohol before having sex?

In the past year, did you use marijuana or other drugs before having sex?

In the past year, how many drinks did you normally have before having sex?

How sure are you that you have not been exposed to AIDS?

How sure are you that your sex partner(s) have not been exposed to AIDS?

How sure are you about your estimate of number of partners your most recent partner has had?
No sex Less than 1-6 6-12 Over partners 1 month months months 1 year

$46.4 \% \quad 7.9 \% \quad 5.0 \% \quad 10.5 \% \quad 30.1 \%$

No sex 12 years or $13-14 \quad 15-16 \quad 17$ years

partners younger years years or older

$46.4 \% \quad 12.1 \% \quad 18.8 \% \quad 11.7 \% \quad 10.9 \%$

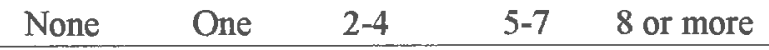

$46.4 \% \quad 23.0 \% \quad 15.1 \% \quad 6.3 \% \quad 9.2 \%$

$\begin{array}{lllll}46.4 & 32.6 & 15.9 & 2.9 & 2.1\end{array}$

$\begin{array}{lllll}46.4 & 30.1 & 13.8 & 3.3 & 6.3\end{array}$

$\begin{array}{lllll}98.3 & 0.4 & 0.4 & 0.4 & 0.4\end{array}$

$\begin{array}{lllll}99.2 & 0.4 & 0.4 & 0.0 & 0.0\end{array}$

About half

Never Sometimes the time Usually Always

$68.2 \% \quad 20.9 \% \quad 6.7 \% \quad 3.8 \% \quad 0.4 \%$

$\begin{array}{lllll}87.4 & 10.5 & 0.4 & 1.7 & 0.0\end{array}$ 1-2 3-5 6-10 11 or more

None drinks drinks drinks drinks

$68.6 \% \quad 24.7 \% \quad 6.7 \% \quad 0.0 \% \quad 0.0 \%$

Absolutely Very Somewhat Slightly Not at all

$59.4 \% \quad 20.1 \% \quad 16.3 \% \quad 2.1 \% \quad 2.1 \%$

$\begin{array}{lllll}64.9 & 18.8 & 11.7 & 1.3 & 3.3\end{array}$

$\begin{array}{lllll}66.1 & 19.2 & 9.6 & 0.8 & 4.2\end{array}$

Yes, Yes,

No negative positive

$90.0 \% \quad 9.2 \% \quad 0.8 \%$


Started Started Sometimes

Complex items examined individually:

Do you always use condoms right now?

If you were to make a guess, how much risk do you have for getting HIV/AIDS at this time in your life?
No sex $1 \mathrm{yr}$ ago+ $<1$ yr ago not always Never

$\begin{array}{lllll}46.4 \% & 11.3 \% & 5.0 \% & 27.2 \% & 10.0 \%\end{array}$

Already Plan to Maybe

No sex use start sometimes No

$40.2 \% \quad 13.4 \% \quad 24.3 \% \quad 20.5 \% \quad 1.7 \%$

No risk Slight Some Moderate High

$54.4 \% \quad 29.3 \% \quad 9.6 \% \quad 5.9 \% \quad 0.8 \%$ 
Exploratory Factor Analysis Pattern Matrix and Factor Correlation Matrix for Environment Subscales Utilizing 4-Sample Pooled Data $(n=457)$

$\underline{\text { Item }}$

Most of my friends have had sex.

One of my close friends does things that could lead to them getting AIDS.

My close friends insist on using condoms when they have sex with a risky partner. (R)

My close friends think that their partner would not mind using a condom when they have sex. $(\mathrm{R})$

My close friends are afraid to ask their partner to use a condom when they have sex.

Some of my close friends think that they are at risk of getting AIDS.

My close friends think that it's okay for a woman to ask a partner to use condoms. ( $R$ )

My close friends ask a sex partner to use a condom. (R)

If you know someone with HIV/AIDS, how old were you when you first knew this person with HIV/AIDS? (R)

A family member or close friend has HIV/AIDS.

I know someone with HIV/AIDS who is not a close friend or a member of my family.

I know a gay person who has HIV/AIDS.

I know a person who is not gay who has HIV/AIDS.

I know a woman who has HIV/AIDS.

I know a person who shoots drugs with a needle who has HIV/AIDS.

I know someone who was tested for AIDS.

For me, watching TV has been an important way to learn about HIV/AIDS.

For me, listening to the radio has been an important way to learn about HIV/AIDS.

For me, seeing movies in the theater, or on video has been an important way to learn about HIV/AIDS.

For me, reading books or magazines has been an important way to learn about HIV/AIDS.

For me, reading newspapers has been an important way to learn about HIV/AIDS.

(continued on next page)
(PEER-RISK) (PROXIM) (MEDIA-R) (MEDIA-I) $(\alpha=.69) \quad(\alpha=.82) \quad(\alpha=.77) \quad(\alpha=.95)$

Factor 1 Factor 2 Factor 3 Factor 4 $\begin{array}{llll}-.30674 & .21057 & .02710 & .05297\end{array}$

$\begin{array}{llll}-.31362 & .06479 & .05257 & -.13959\end{array}$

$\begin{array}{llll}-.81977 & .14322 & .07535 & .00526\end{array}$

$\begin{array}{llll}-.80749 & .08031 & .06454 & .05284\end{array}$

$\begin{array}{llll}.38101 & .09473\end{array}$

$\begin{array}{llll}.29955 & .17064 & .01426 & .00415\end{array}$

$\begin{array}{llll}-.79124 & .03829 & -.02111 & .04985\end{array}$

$\begin{array}{llll}-.80164 & .09717 & .04190 & .03370\end{array}$

$\begin{array}{llll}-.00524 & \mathbf{. 5 7 3 0 2} & -.06607 & .03736\end{array}$

$\begin{array}{llll}-.20666 & .43867 & -.12384 & .05945\end{array}$

$\begin{array}{llll}.08436 & \mathbf{. 7 8 7 3 0} & -.11135 & .06590\end{array}$

$\begin{array}{llll}-.02603 & \mathbf{. 7 0 3 8 9} & .00225 & -.05701\end{array}$

$\begin{array}{llll}.03536 & \mathbf{. 8 3 3 0 4} & -.09525 & .05446\end{array}$

$\begin{array}{llll}.10242 & \mathbf{7 7 8 0 5} & .05928 & .01110\end{array}$

$\begin{array}{llll}-.12529 & \mathbf{. 6 2 8 4 9} & .04741 & -.02630\end{array}$

$\begin{array}{llll}.05993 & \mathbf{. 4 8 7 4 3} & .09688 & -.09572\end{array}$

$\begin{array}{llll}-.12929 & -.09341 & \mathbf{. 6 5 6 6 1} & .10599\end{array}$

$\begin{array}{llll}-.21888 & -.06439 & .67706 & .06995\end{array}$

$\begin{array}{llll}-.03944 & -.01958 & \mathbf{. 6 5 7 5 5} & .18152\end{array}$

$\begin{array}{lllll}.14269 & .04761 & \mathbf{. 7 5 2 8 7} & -.10381\end{array}$

$\begin{array}{llll}.09810 & .07217 & \mathbf{. 7 7 3 1 8} & -.04600\end{array}$ 
Item

Knowing that "Magic Johnson," a famous basketball player, has HIV/AIDS has made me want to learn more about HIV/AIDS.

Knowing that a famous singer I really like has died of AIDS would make me want to learn more about how I can avoid getting it.

Knowing that a famous movie or TV star I really like has died of AIDS would make me want to learn more about how I can avoid getting it.

Knowing a famous movie or TV star I really like has HIV/AIDS would make me want to learn more about how I can avoid getting it.

Knowing a famous singer I really like has HIV/ AIDS would make me want to learn more about how I can avoid getting it.

$(\mathrm{R})=$ Item reverse coded.

Factor Correlation Matrix

Factor 1 (PEER RISK)

Factor 2 (AIDS PROXIMITY)

Factor 3 (MEDIA RESOURCES)

Factor 4 (MEDIA INFLUENCE)
(PEER-RISK) (PROXIM) (MEDIA-R) (MEDIA-I)

Factor 1 Factor 2 Factor 3 Factor 4

$\begin{array}{llll}-.11507 & -.01313 & .07517 & .79047\end{array}$

$\begin{array}{llll}-.04378 & -.03217 & .02461 & \mathbf{. 9 3 5 2 1}\end{array}$

$\begin{array}{llll}-.00251 & -.02769 & .01924 & \mathbf{9 5 7 2 3}\end{array}$

$\begin{array}{llll}.00105 & -.04071 & .02419 & \mathbf{9 5 5 5 6}\end{array}$

$\begin{array}{lllll}-.00625 & -.02648 & .02557 & \mathbf{9 4 3 3 2}\end{array}$

Factor 1 Factor 2 Factor 3 Factor 4 1.00000

$-.06495 \quad 1.00000$

$\begin{array}{lll}-.06287 & .06376 & 1.00000\end{array}$

$\begin{array}{llll}-.09634 & .03554 & .21866 & 1.00000\end{array}$ 


\section{Confirmatory Factor Analysis Pattern Matrix and Factor Correlation Matrix for} Environment Subscales Utilizing a Random 50 Percent Sample from 4-Sample Pooled Data $(n=228)$

$\underline{\text { Item }}$

Most of my friends have had sex.

One of my close friends does things that could lead to them getting AIDS.

My close friends insist on using condoms when they have sex with a risky partner. (R)

My close friends think that their partner would not mind using a condom when they have sex. $(R)$

My close friends are afraid to ask their partner to use a condom when they have sex.

Some of my close friends think that they are at risk of getting AIDS.

My close friends think that it's okay for a woman to ask a partner to use condoms. (R)

My close friends ask a sex partner to use a condom. (R)

If you know someone with HIV/AIDS, how old were you when you first knew this person with HIV/AIDS? (R)

A family member or close friend has HIV/AIDS

I know someone with HIV/AIDS who is not a close friend or a member of my family.

I know a gay person who has HIV/AIDS.

I know a person who is not gay who has HIV/AIDS.

I know a woman who has HIV/AIDS.

I know a person who shoots drugs with a needle who has HIV/AIDS.

I know someone who was tested for AIDS.

For me, watching TV has been an important way to learn about HIV/AIDS.

For me, listening to the radio has been an important way to learn about HIV/AIDS.

For me, seeing movies in the theater, or on video has been an important way to learn about HIV/AIDS.

For me, reading books or magazines has been an important way to learn about HIV/AIDS.

For me, reading newspapers has been an important way to learn about HIV/AIDS.
(PEER-RISK) (PROXIM) (MEDIA-R) (MEDIA-I)

$\begin{array}{llll}\frac{\text { Factor 1 }}{\mathbf{3 8 1 5 7}} & \frac{\text { Factor 2 }}{.20810} & \frac{\text { Factor 3 }}{.02902} & \text { Factor 4 } \\ -.02064\end{array}$

\begin{tabular}{llll}
\hline 34926 & .08238 & .02243 & -.09558
\end{tabular}

$\begin{array}{llll}.82542 & .06719 & .07737 & .00961\end{array}$

$\begin{array}{llll}\mathbf{8 0 5 2 0} & .04618 & .07737 & -.00186\end{array}$

$\begin{array}{llll}-.34955 & .06158 & -.00381 & .00460\end{array}$

$\begin{array}{llll}-.29871 & .20058 & .08749 & -.09351\end{array}$

$\begin{array}{llll}.76127 & -.01455 & .00187 & -.02063\end{array}$

$\begin{array}{llll}\mathbf{8 3 5 0 7} & -.09317 & .07542 & -.01354\end{array}$

$\begin{array}{llll}-.00791 & \mathbf{. 6 6 7 0 4} & -.03514 & .07012\end{array}$

$\begin{array}{llll}.14993 & \mathbf{4 5 7 2 8} & -.08810 & .08066\end{array}$

$\begin{array}{llll}-.02456 & \mathbf{6 9 8 2 0} & -.02093 & .07128\end{array}$

$\begin{array}{llll}.03112 & \mathbf{6 5 7 3 5} & .00048 & -.06580\end{array}$

$\begin{array}{lllll}-.02663 & \mathbf{. 8 1 0 8 2} & -.00971 & .00786\end{array}$

$\begin{array}{llll}-.09457 & \mathbf{. 7 8 9 0 0} & .06755 & .00888\end{array}$

$\begin{array}{lllll}.12199 & \mathbf{5 6 8 8 9} & .00487 & .00977\end{array}$

$\begin{array}{llll}-.15828 & .49733 & -.02799 & .05522\end{array}$

$\begin{array}{llll}.09733 & -.10452 & \mathbf{7 1 7 1 2} & .04566\end{array}$

$\begin{array}{llll}.27946 & -.11486 & .60528 & .09281\end{array}$

$\begin{array}{lllll}.04256 & .01332 & .66516 & .13148\end{array}$

$\begin{array}{llll}-.12033 & .01133 & .74643 & -.07762\end{array}$

$\begin{array}{llll}-.06503 & .08042 & .74895 & .01386\end{array}$

(continued on next page) 
$\underline{\text { Item }}$

Knowing that "Magic Johnson," a famous basketball player, has HIV/AIDS has made me want to learn more about HIV/AIDS.

Knowing that a famous singer I really like has died of AIDS would make me want to learn more about how I can avoid getting it.

Knowing that a famous movie or TV star I really like has died of AIDS would make me want to learn more about how I can avoid getting it. Knowing a famous movie or TV star I really like has HIV/AIDS would make me want to learn more about how I can avoid getting it.

Knowing a famous singer I really like has HIV/ AIDS would make me want to learn more about how I can avoid getting it. $(\mathrm{R})=$ Item reverse coded.

Factor Correlation Matrix

Factor 1 (PEER RISK)

Factor 2 (AIDS PROXIMITY)

Factor 3 (MEDIA RESOURCES)

Factor 4 (MEDIA INFLUENCE)
(PEER-RISK) (PROXIM) (MEDIA-R) (MEDIA-I)

Factor 1 Factor 2 Factor 3 Factor 4

$\begin{array}{llll}.05507 & .04578 & .10454 & \mathbf{. 7 8 6 9 7}\end{array}$

$\begin{array}{lllll}.01001 & .01008 & -.02988 & \mathbf{9 3 5 9 3}\end{array}$

$\begin{array}{llll}-.04793 & .02794 & -.00457 & \mathbf{9 5 9 6 8}\end{array}$

$\begin{array}{llll}-.04474 & .01858 & .05246 & .95501\end{array}$

$\begin{array}{llll}-.05816 & .02039 & .04668 & \mathbf{9 4 5 8 6}\end{array}$

Factor 1 Factor 2 Factor 3 Factor 4 1.00000

$11966 \quad 1.00000$

$\begin{array}{lll}.09021 & .02871 & 1.00000\end{array}$

$\begin{array}{llll}.16882 & -.03772 & .17884 & 1.00000\end{array}$ 


\section{Table 19}

Response Frequencies for Environment Scale Items ( $n=239$, current sample only)

Items:

Most of my friends have had sex.

One of my close friends does things that could lead to them getting AIDS.

My close friends insist on using condoms when they have sex with a risky partner.

My close friends think that their partner would not mind using a condom when they have sex. My close friends are afraid to ask their partner to use a condom when they have sex.

Some of my close friends think that they are at risk of getting AIDS.

My close friends think that it's okay for a woman to ask a partner to use condoms.

My close friends ask a sex partner to use a condom.

If you know someone with HIV/AIDS, how old were you when you first knew this person with HIV/AIDS?

A family member or close friend has HIV/AIDS.

I know someone with HIV/AIDS who is not a close friend or a member of my family.

I know a gay person who has HIV/AIDS.

I know a person who is not gay who has HIV/AIDS.

I know a woman who has HIV/AIDS.

I know a person who shoots drugs with a needle who has HIV/AIDS.

I know someone who was tested for AIDS.
Definitely Not really Somewhat Mostly Definitely

\begin{tabular}{|c|c|c|c|c|}
\hline not true & true & true & true & true \\
\hline $23.8 \%$ & $19.7 \%$ & $20.1 \%$ & $18.4 \%$ & $18.0 \%$ \\
\hline $\begin{array}{c}\text { Definitely } \\
\text { no }\end{array}$ & $\begin{array}{c}\text { Probably } \\
\text { no }\end{array}$ & $\begin{array}{l}\text { Not } \\
\text { sure }\end{array}$ & $\begin{array}{l}\text { Probably } \\
\text { yes }\end{array}$ & $\begin{array}{l}\text { Definitely } \\
\text { yes }\end{array}$ \\
\hline $31.4 \%$ & $15.5 \%$ & $18.0 \%$ & $13.4 \%$ & $21.8 \%$ \\
\hline $\begin{array}{c}\text { Definitely } \\
\text { no }\end{array}$ & $\begin{array}{c}\text { Probably } \\
\text { no }\end{array}$ & $\begin{array}{c}\text { Probably } \\
\text { yes }\end{array}$ & $\begin{array}{c}\text { Definitely } \\
\text { yes }\end{array}$ & \\
\hline $6.3 \%$ & $22.2 \%$ & $35.6 \%$ & $36.0 \%$ & \\
\hline 3.8 & 25.1 & 41.8 & 29.3 & \\
\hline 25.9 & 41.8 & 22.6 & 9.6 & \\
\hline 24.7 & 44.4 & 24.3 & 6.7 & \\
\hline 2.5 & 12.6 & 32.6 & 52.3 & \\
\hline 7.5 & 22.6 & 31.8 & 38.1 & \\
\hline $\begin{array}{l}12 \text { yrs or } \\
\text { younger }\end{array}$ & $13-14$ yrs & $15-16$ yrs & $\begin{array}{c}17 \text { yrs or } \\
\text { older }\end{array}$ & N/A \\
\hline
\end{tabular}

\section{$\begin{array}{lllll}9.2 \% & 12.6 \% & 4.2 \% & 2.9 \% & 71.1 \%\end{array}$}

Definitely Probably Not Probably Definitely

no no sure yes yes

$54.0 \% \quad 22.2 \% \quad 17.2 \% \quad 1.7 \% \quad 5.0 \%$

$\begin{array}{lllll}49.8 & 11.3 & 13.8 & 7.5 & 17.6\end{array}$

$\begin{array}{lllll}62.8 & 13.0 & 13.0 & 2.5 & 8.8\end{array}$

$\begin{array}{rrrrr}56.5 & 12.1 & 13.0 & 5.4 & 13.0 \\ 62.3 & 12.1 & 14.2 & 4.2 & 7.1 \\ & & & & \\ 67.8 & 13.8 & 12.1 & 0.4 & 5.9 \\ 36.8 & 12.1 & 12.6 & 7.9 & 30.5\end{array}$


Definitely Not really Somewhat Mostly Definitely

For me, watching TV has been an important way to learn about HIV/AIDS.

For me, listening to the radio has been an important way to learn about HIV/AIDS.

For me, seeing movies in the theater, or on

not true true true true true video has been an important way to learn about HIV/AIDS.

For me, reading books or magazines has been an important way to learn about HIV/ADS.

For me, reading newspapers has been an important way to learn about HIV/AIDS.

Knowing that "Magic Johnson," a famous basketball player, has HIV/AIDS has made me want to learn more about HIV/AIDS.

Knowing that a famous singer I really like has died of AIDS would make me want to learn more about how I can avoid getting it.

$15.9 \% \quad 18.0 \% \quad 36.0 \% \quad 15.5 \% \quad 14.6 \%$

$\begin{array}{lllll}37.2 & 31.8 & 18.8 & 7.1 & 5.0\end{array}$

$\begin{array}{lllll}17.6 & 20.9 & 32.2 & 17.6 & 11.7\end{array}$

$\begin{array}{lllll}15.9 & 18.8 & 36.8 & 17.6 & 10.9\end{array}$

$\begin{array}{lllll}15.5 & 26.4 & 33.9 & 15.1 & 9.2\end{array}$

$\begin{array}{lllll}15.9 & 25.5 & 23.8 & 17.6 & 17.2\end{array}$

Knowing that a famous movie or TV star I really like has died of AIDS would make me want $\begin{array}{llllll}\text { to learn more about how I can avoid getting it. } & 14.2 & 21.3 & 23.8 & 22.2 & 18.4\end{array}$ Knowing a famous movie or TV star I really like has HIV/AIDS would make me want to learn more about how I can avoid getting it.

$\begin{array}{lllll}13.0 & 20.1 & 28.9 & 20.9 & 17.2\end{array}$

Knowing a famous singer I really like has HIV/ AIDS would make me want to learn more about how I can avoid getting it.

$\begin{array}{lllll}12.6 & 22.2 & 26.8 & 20.9 & 17.6\end{array}$




\section{Table 20}

Exploratory Factor Analysis Pattern Matrix and Factor Correlation Matrix for School Education Subscales Utilizing 4-Sample Pooled Data $(n=457)$

$\underline{\text { Item }}$

How old were you when you had your first class in school that talked about HIV/AIDS? (R)

1 learned a lot about HIV/AIDS in classes I took in grade school.

I learned a lot about HIV/AIDS in classes I took in junior high school.

I learned a lot about HIV/AIDS in classes I took in high school.

Most of what I learned about HIV/AIDS in my classes was from listening to my teacher talk about it.

Most of what I learned about HIV/AIDS in my classes was from movies, books, and/or slides.

Most of what I learned about HIV/AIDS in my classes was from asking the teacher to answer my questions about it.

Most of what I learned about HIV/AIDS in my classes was from doing assignments or projects.

I learned a lot in my classes about the things people do that can cause them to get HIV/AIDS.

I learned a lot in my classes about what to do if I do not want to have sex.

Most of what I learned about how to practice safer sex was from my health or sex education classes.

I was taught how to keep myself from getting into a situation that would put me at risk for getting HIV/AIDS.

It would be easy for me to talk about sex with a teacher or school counselor.

$(\mathrm{R})=$ Item reverse coded.

Factor Correlation Matrix

Factor 1 (SCHOOL EDUCATION)

Factor 2 (SCHOOL INFLUENCE)
(SCHOOL-ED) (SCHOOL-INF)

$$
(\alpha=.72) \quad(\alpha=.75)
$$

Factor 1 Factor 2

\begin{tabular}{ll}
$\mathbf{. 5 3 3 2 8}$ & -.04741 \\
$\mathbf{. 7 5 6 0 8}$ & -.12732 \\
$\mathbf{. 5 6 9 8 6}$ & .31153 \\
\hline $\mathbf{6 9 6 5 0}$ & -.08703 \\
\hline $\mathbf{. 6 5 7 8}$ & .30420 \\
\hline $\mathbf{5 2 4 8 9}$ & .20376
\end{tabular}

$\begin{array}{ll}.58387 & .07037\end{array}$

$\mathbf{5 9 6 6 6}-.03092$

. $\mathbf{6 5 7 1 5}$

$.22174 \quad \mathbf{7 0 4 8 9}$

$\begin{array}{ll}.67993 & .693\end{array}$

$.11607 \quad .72191$

$-.19229 \quad \mathbf{3 8 8 5 8}$

Factor 1 Factor 2

1.00000

$.28814 \quad 1.00000$ 


\section{Table 21}

Confirmatory Factor Analysis Pattern Matrix and Factor Correlation Matrix for School Education Subscales Utilizing a Random 50 Percent Sample from 4-Sample Pooled Data $(n=228)$

Item

How old were you when you had your first class in school that talked about HIV/AIDS? (R)

I learned a lot about HIV/AIDS in classes I took in grade school.

I learned a lot about HIV/AIDS in classes I took in junior high school.

I learned a lot about HIV/AIDS in classes I took in high school.

Most of what I learned about HIV/AIDS in my classes was from listening to my teacher talk about it.

Most of what I learned about HIV/ADS in my classes was from movies, books, and/or slides.

Most of what I learned about HIV/AIDS in my classes was from asking the teacher to answer my questions about it.

Most of what I learned about HIV/ADSS in my classes was from doing assignments or projects.

I learned a lot in my classes about the things people do that can cause them to get HIV/AIDS.

I learned a lot in my classes about what to do if I do not want to have sex.

Most of what I learned about how to practice safer sex was from my health or sex education classes.

I was taught how to keep myself from getting into a situation that would put me at risk for getting HIV/AIDS.

It would be easy for me to talk about sex with a teacher or school counselor.

$(\mathrm{R})=$ Item reverse coded.

\section{Factor Correlation Matrix}

Factor 1 (SCHOOL EDUCATION)

Factor 2 (SCHOOL INFLUENCE)
(SCHOOL-ED) (SCHOOL-INF)

Factor 1 Factor 2

$.61496-.06546$

$.76211-.13476$

$.58709 \quad .31461$

$.68337-.06029$

$.38131 \quad .17463$

$.49231 \quad .22621$

$.56666 \quad .06862$

$\mathbf{5 3 7 2 3}-\mathbf{0}-01013$

$.30068 \quad \mathbf{6 5 8 4 0}$

$.18601 \quad \mathbf{. 7 2 6 2 4}$

$.16250 \quad \mathbf{6 8 7 3 6}$

$.09334 \quad \mathbf{. 7 3 1 7 5}$

$-.18660 \quad .37729$

Factor 1 Factor 2

1.00000

$.29420 \quad 1.00000$ 
Exploratory Factor Analysis Pattern Matrix and Factor Correlation Matrix for Home Education Subscales Utilizing 4-Sample Pooled Data $(n=457)$

Item

It would be easy for me to talk to my mother or father(or guardian) about sex.

Most of what I know about HIV/AIDS I learned from my mother or father (or guardian).

It would be easy to talk about sex with my brother or sister (if you don't have brothers or sisters, consider cousins, or other relatives).

Most of what I know about HIV/AIDS I learned from my brother(s) and/or sister(s).

Factor Correlation Matrix

Factor 1 (PARENT EDUCATION \& INFLUENCE)

Factor 2 (SIBLING EDUCATION \& INFLUENCE)
(PARENT) (SIB)

$(\alpha=.68) \quad(\alpha=.69)$

Factor 1 Factor 2

$\mathbf{8 9 3 6 2}-.05837$

$.84418 \quad .07245$

$-.03927 \quad \mathbf{8 0 9 3 3}$

$.04822 \quad \mathbf{. 7 8 0 5 0}$

Factor $1 \quad$ Factor 2

1.00000

$.32250 \quad 1.00000$ 
Confirmatory Factor Analysis Pattern Matrix and Factor Correlation Matrix for Home Education Subscales Utilizing a Random 50 Percent Sample from 4-Sample Pooled Data $(n=228)$

\section{$\underline{\text { Item }}$}

It would be easy for me to talk to my mother or father(or guardian) about sex.

Most of what I know about HIV/AIDS I learned from my mother or father (or guardian).

It would be easy to talk about sex with my brother or sister (if you don't have brothers or sisters, consider cousins, or other relatives).

Most of what I know about HIV/AIDS I learned from my brother(s) and/or sister(s).

Factor Correlation Matrix

Factor 1 (PARENT EDUCATION \& INFLUENCE)

Factor 2 (SIBLING EDUCATION \& INFLUENCE)
(PARENT) (SIB)

Factor 1 Factor 2

$\mathbf{8 7 0 8 9}-.03082$

$\mathbf{8 4 9 2 4} .03510$

$-.02954 \quad \mathbf{8 2 7 9 4}$

$.03353 \quad \mathbf{8 0 4 3 6}$

Factor 1 Factor 2

1.00000

$.33558 \quad 1.00000$ 


\section{Table 24}

Exploratory Factor Analysis Pattern Matrix and Factor Correlation Matrix for Peer Education Subscales Utilizing 4-Sample Pooled Data $(n=457)$

$\underline{\text { Item }}$

It would be easy for me to talk about sex with my best friend(s).

Most of what I know about HIV/AIDS I learned from my friends.

When my best friend(s) talk about HIV/AIDS, I believe that what they say is true.

Most of what I know about sex I learned from my friends.

Factor Correlation Matrix

Factor 1 (PEER EDUCATION)

Factor 2 (PEER INFLUENCE)

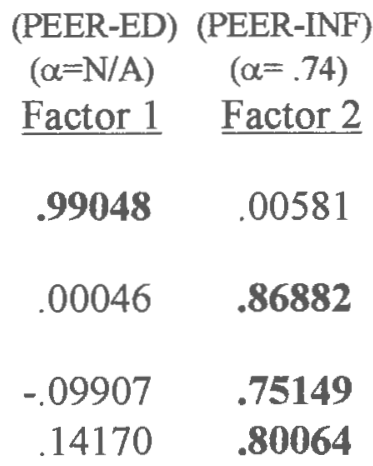

Factor $1 \quad$ Factor 2

1.00000

$.17987 \quad 1.00000$ 


\section{Table 25}

Confirmatory Factor Analysis Pattern Matrix and Factor Correlation Matrix for Peer Education Subscales Utilizing a Random 50 Percent Sample from 4-Sample Pooled Data $(n=228)$

$\underline{\text { Item }}$

It would be easy for me to talk about sex with my best friend(s).

Most of what I know about HIV/AIDS I learned from my friends.

When my best friend(s) talk about HIV/AIDS,

I believe that what they say is true.

Most of what I know about sex I learned from my friends.

Factor Correlation Matrix

Factor 1 (PEER EDUCATION)

Factor 2 (PEER INFLUENCE)
(PEER-ED) (PEER-INF)

Factor 1 Factor 2

$.99634 \quad .00457$

$-.09674 \quad \mathbf{8 8 1 4 0}$

$.04200 \quad \mathbf{. 7 1 6 9 0}$

$.05677 \quad \mathbf{8 3 9 4 4}$

Factor 1 Factor 2

1.00000

$17989 \quad 1.00000$ 


\section{Table 26}

Exploratory Factor Analysis Pattern Matrix and Factor Correlation Matrix for Three Major Areas of Education Utilizing 4-Sample Pooled Data $(n=457)$

Subscale

School Education

School Influence

Home Education

Home Influence

Peer Education

Peer Influence

Factor Correlation Matrix

Factor 1 (SCHOOL EDUC. \& INFLUENCE)

Factor 2 (HOME EDUC. \& INFLUENCE)

Factor 3 (PEER EDUC. \& INFLUENCE)
(SCHOOL) (HOME) (PEERS)

$(\alpha=.82) \quad(\alpha=.66) \quad(\alpha=.68)$

Factor 1 Factor 2 Factor 3

$\begin{array}{lll}\mathbf{8 1 7 9 9} & -.06643 & .09330\end{array}$

$\begin{array}{lll}.91164 & -.06818 & -.19472\end{array}$

$\begin{array}{lll}.09295 & \mathbf{- . 8 3 8 6 4} & -.20417\end{array}$

$\begin{array}{lll}-.01232 & \mathbf{- . 8 1 0 8 4} \quad .31421\end{array}$

$\begin{array}{lll}.20842 & .07800 \quad \mathbf{4 6 2 1 0}\end{array}$

$\begin{array}{lll}-.06957 & -.08251 \quad .94727\end{array}$

Factor 1 Factor 2 Factor 3

1.00000

$-.22151 \quad 1.00000$

$\begin{array}{lll}.18966 & -.06256 & 1.00000\end{array}$ 
Confirmatory Factor Analysis Pattern Matrix and Factor Correlation Matrix for Three Major Areas for Education Utilizing a Random 50 Percent Sample from 4-Sample Pooled Data $(n=228)$

$\underline{\text { Subscale }}$

School Education

School Influence

Home Education

Home Influence

Peer Education

Peer Influence
(SCHOOL) (HOME) (PEERS)

Factor 1 Factor 2 Factor 3

$\begin{array}{rrr}\mathbf{. 9 0 2 6 0} & -.05114 & .12717 \\ \mathbf{. 9 0 7 4 6} & -.04554 & -.14408 \\ -.01592 & \mathbf{- . 9 3 4 7 9} & -.16811 \\ .06602 & -.71240 & .29909 \\ .01203 & -.12718 & \mathbf{. 4 9 6 8 7} \\ -.01814 & .16301 & \mathbf{. 9 3 6 5 9}\end{array}$

Factor 1 Factor 2 Factor 3 1.00000

$\begin{array}{lrr}\text { Factor } 2 \text { (HOME EDUC. \& INFLUENCE) } & -.22000 & 1.00000 \\ \text { Factor } 3 \text { (PEER EDUCATION \& INFLUENCE) } & 10119 & -.14770\end{array}$

Factor Correlation Matrix

Factor 1 (SCHOOL EDUC. \& INFLUENCE)

.10119 
Response Frequencies for Education Scale Items ( $n=239$, current sample only)

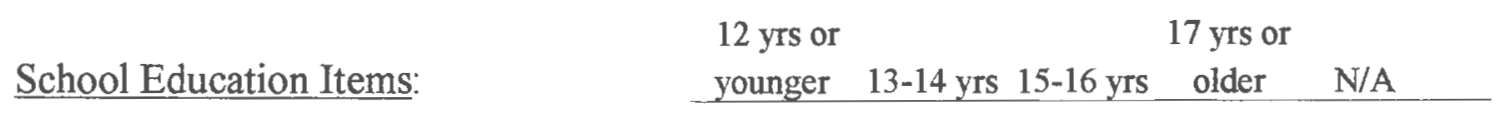

How old were you when you had your first class in school that talked about HIV/AIDS?
$2.9 \%$
$0.8 \%$
$5.4 \%$
$40.2 \% \quad 50.6 \%$

I learned a lot about HIV/AIDS in classes Definitely Not really Somewhat Mostly Definitely I took in grade school.

I learned a lot about HIV/AIDS in classes I took in junior high school.

I learned a lot about HIV/AIDS in classes I took in high school.

Most of what I learned about HIV/AIDS in my classes was from listening to my teacher talk about it.

Most of what I learned about HIV/AIDS in my classes was from movies, books, and/or slides.

Most of what I learned about HIV/AIDS in my classes was from asking the teacher to answer my questions about it.

Most of what I learned about HIV/AIDS in my classes was from doing assignments/projects.

I learned a lot in my classes about things people do that can cause them to get HIV/AIDS.

I learned a lot in my classes about what to do if I do not want to have sex.

\begin{tabular}{rrrrr} 
not true & true & true & true & true \\
\hline $17.6 \%$ & $22.6 \%$ & $31.8 \%$ & $17.6 \%$ & $10.5 \%$ \\
15.1 & 15.5 & 29.7 & 24.7 & 15.1 \\
27.6 & 7.5 & 16.3 & 18.8 & 29.7 \\
7.1 & 8.8 & 25.1 & 35.6 & 23.4 \\
9.2 & 16.3 & 31.0 & 28.0 & 15.5 \\
21.3 & 27.2 & 29.7 & 13.8 & 7.9 \\
33.5 & 30.5 & 18.4 & 11.3 & 6.3 \\
5.4 & 11.7 & 15.9 & 25.9 & 41.0 \\
15.5 & 11.7 & 19.2 & 24.3 & 29.3 \\
13.4 & 11.7 & 20.5 & 26.4 & 28.0 \\
7.9 & 10.0 & 17.2 & 23.8 & 41.0 \\
38.9 & 29.3 & 18.4 & 7.9 & 5.4
\end{tabular}

Most of what I learned about how to practice safer sex was from my health/sex education classes.

I was taught how to keep myself from getting into a situation that would put me at risk for getting HIV/AIDS.

It would be easy for me to talk about sex with a teacher or school counselor.

\section{Home Education Items:}

Definitely Not really Somewhat Mostly Definitely

It would be easy for me to talk to my mother or father(or guardian) about sex.

Most of what I know about HIV/AIDS I learned from my mother or father (or guardian).

It would be easy to talk about sex with my brother or sister (if you don't have brothers or sisters, consider cousins, or other relatives).

Most of what I know about HIV/AIDS I learned from my brother(s) and/or sister(s).

\begin{tabular}{ccccc} 
not true & true & true & true & true \\
\hline $20.9 \%$ & $24.7 \%$ & $22.6 \%$ & $13.4 \%$ & $18.4 \%$ \\
27.2 & 33.9 & 24.7 & 10.9 & 3.3 \\
& & & & \\
20.5 & 20.5 & 23.8 & 18.4 & 16.7 \\
56.9 & 25.5 & 13.4 & 3.8 & 0.4
\end{tabular}

(continued on next page) 
Definitely Not really Somewhat Mostly Definitely

Peer Education Items:

not true true true true true

It would be easy for me to talk about sex with my best friend(s).

$6.7 \% \quad 5.4 \% \quad 14.2 \% \quad 22.6 \% \quad 51.0 \%$

Most of what I know about HIV/AIDS I learned from my friends.

$\begin{array}{lllll}31.0 & 26.4 & 20.1 & 12.1 & 10.5\end{array}$

When my best friend(s) talk about HIV/AIDS, I believe that what they say is true.

$\begin{array}{lllll}18.4 & 21.8 & 34.7 & 18.4 & 6.7\end{array}$

Most of what I know about sex I learned from my friends.

$\begin{array}{lllll}21.3 & 24.3 & 20.9 & 22.6 & 10.9\end{array}$




\section{Table 29}

Group Comparisons by Demographic and Risk Variables Across Four Samples

$\begin{array}{ccccc}\begin{array}{c}\text { Community } \\ \text { Youth } \\ (n=113)\end{array} & \begin{array}{c}\text { Conference '93 } \\ \text { Youth } \\ (n=105)\end{array} & \begin{array}{c}\text { Conference '94 } \\ \text { Youth } \\ (n=129)\end{array} & \begin{array}{c}\text { School } \\ \text { Youth } \\ (n=110)\end{array} & \\ \text { Mean SD } & \text { Mean SD } & \text { Mean } S D & \text { Mean } S D & \text { Significance }\end{array}$

Demographics:

MANOVA, Pillais:

\section{$\%$}

$\%$ Participation $57.0 \%$

Gender (\% M/F) 40/60

$15.9 \quad 1.1$

$\%$ Living in

RI, CT, MA

$\%$ Caucasian

Grade

Religion

Religiosity

$\%$ Living with

both parents

Mother's educ.

(approx 90\%)
$72.6 \% \quad--$

$35.0 \% \quad--$

$43.0 \% \quad-$

$41 / 59$

$38 / 62 \quad--$

$\begin{array}{llll}15.3 & 1.3 & 15.5 & 1.6\end{array}$

$\begin{array}{ll}12.0 \% & -- \\ 30 / 70 & --\end{array}$

$F(30,1323)=18.32 *$

Post hoc ANOVA:

Father's educ.

$10.4 \quad 1.9$

(approx 65\%)

$86.7 \% \quad--$

$41.9 \% \quad--\quad 100.0 \% \quad-$

$71.3 \% \quad--$

$97.3 \%$

$2.3 \quad 1.6$

9.3

1.4

10.3

1.8

9.0

2.6

1.6

2.8

1.7

2.4

2.4

2.7

1.6

1.7

$-$

$\begin{array}{ll}2.6 & 1.1\end{array}$

$\begin{array}{lll}2.8 & 1.3 & 3.1\end{array}$

1.3

$69.8 \%$

$69.1 \%$

1.1

$\mathrm{n} / \mathrm{s}$

$\begin{array}{cccccccc}59.3 \% & - & 75.2 \% & -- & 69.8 \% & -- & 69.1 \% & -- \\ 3.8 & 1.2 & 4.1 & 0.9 & 3.8 & 1.0 & 3.7 & 1.1 \\ 3.9 & 1.2 & 4.3 & 0.9 & 4.0 & 1.0 & 3.5 & 1.1\end{array}$

$\mathrm{n} / \mathrm{s}$

$\underline{F}(3,452)=3.62 * *$

$\underline{F}(3,448)=9.61^{*}$

MANOVA, Pillais:

\section{Cognitive:}

$\begin{array}{lllllll}\text { Factual Knowl } & 4.6 & 0.5 & 4.5 & 0.8 & 4.4 & 0.7\end{array}$

$\begin{array}{llll}\text { Transm Knowl } \quad \text { (subscale not yet developed) } & 3.7 & 1.3\end{array}$

$\begin{array}{ll}4.6 & 0.5\end{array}$

$\underline{F}(12,1356)=12.00^{*}$

Post hoc ANOVA:

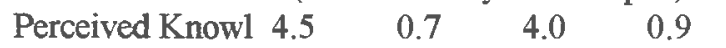

$4.3 \quad 0.8$

3.6

1.6

$\mathrm{n} / \mathrm{s}$

Misconceptions 2.7

$\begin{array}{lll}1.0 & 2.5 & 0.6\end{array}$

2.3

4.2

0.9

$\mathrm{n} / \mathrm{s}$

$\begin{array}{llll}2.3 & 1.2 & 1.6 & 0.6\end{array}$

$\underline{F}(3,453)=6.95^{*}$

$\vec{F}(3,453)=27.80^{*}$

MANOVA, Pillais:

Attitudinal:

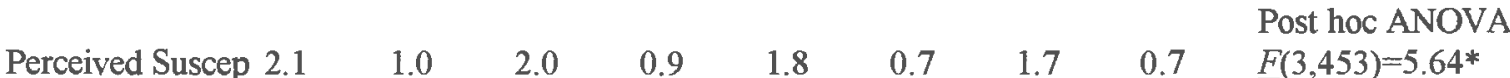

Condom Efficacy 3.8

$\begin{array}{llll}1.3 & 3.8 & 1.2 & 3.8\end{array}$

1.3

3.7

1.2

$\mathrm{n} / \mathrm{s}$

Homophobia 2.1

0.4

2.2

$0.4 \quad 2.1$

1.0

1.9

0.7

$\mathrm{n} / \mathrm{s}$

MANOVA, Pillais:

Behavioral:

\begin{tabular}{|c|c|c|c|c|c|c|c|c|c|}
\hline Assert Communic & 3.8 & 1.2 & 4.0 & 1.1 & 3.9 & 1.2 & 3.8 & 1.2 & $\mathrm{n} / \mathrm{s}$ \\
\hline "Condom use & 3.8 & 1.1 & 3.9 & 0.9 & 3.9 & 1.1 & 4.0 & 1.2 & $\mathrm{n} / \mathrm{s}$ \\
\hline Substance Use & 1.7 & 0.6 & 1.3 & 0.3 & 1.4 & 0.5 & 1.5 & 0.5 & $\underline{F}(3,453)=12.39 *$ \\
\hline Substance Abuse & 1.5 & 0.5 & 1.5 & 0.6 & 1.4 & 0.5 & 1.3 & 0.5 & $\mathrm{n} / \mathrm{s}$ \\
\hline Partner Risk Hx & 1.5 & 0.5 & 1.4 & 0.5 & 1.4 & 0.5 & 1.2 & 0.5 & 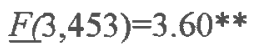 \\
\hline Self Risk Hx & 2.1 & 0.9 & 1.8 & 0.9 & 1.9 & 1.0 & 1.6 & 0.8 & $\underline{F}(3,453)=6.69^{*}$ \\
\hline
\end{tabular}

(continued on next page)

${ }^{*} p<.001 ;{ }^{* *} p<.01$.

Note: All subscales are based on a 5-point scale, with 5 indicating highest endorsement of scale. 


$\begin{array}{cccc}\text { Community } & \text { Conference '93 } & \text { Conference'94 } & \text { School } \\ \text { Youth } & \text { Youth } & \text { Youth } & \text { Youth } \\ (n=113) & (n=105) & (n=129) & (n=110)\end{array}$

Mean $S D$ Mean $S D$ Mean $S D$ Mean $S D$ Significance

Sexual Behavior:

$\begin{array}{cccccccccc}\text { Ss' estim risk } & 1.8 & 0.9 & 2.2 & 0.9 & 1.7 & 0.8 & 1.7 & 1.1 & \underline{F}(3,453)=6.52^{*}\end{array}$

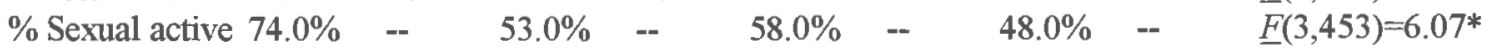

Sexually active only:

\begin{tabular}{lrrrrrrrrl}
\hline Age sex onset & 13.3 & 2.0 & 12.8 & 1.5 & 13.8 & 1.4 & 13.2 & 1.8 & $\underline{F}(3,264)=3.97 * *$ \\
\# Part/past yr & 2.5 & 2.1 & 1.6 & 1.1 & 2.3 & 2.1 & 2.1 & 2.4 & $\mathrm{n} / \mathrm{s}$ \\
\# Part/lifetime & 4.3 & 3.1 & 3.4 & 2.5 & 4.3 & 3.2 & 2.5 & 2.3 & $\underline{F}(3,264)=5.58^{*}$ \\
\% Consistent & & & & & & & & &
\end{tabular}

$\%$ Consistent

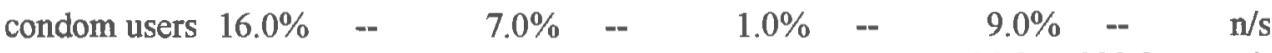

$\begin{array}{llllllllll}\text { "Sex risk" } & 158.4 & 571.6 & 41.4 & 51.2 & 277.4 & 648.2 & 94.8 & 289.8 & \mathrm{n} / \mathrm{s}\end{array}$

$\begin{array}{llllllllll}\text { "Log sex risk" } & 1.4 & 0.9 & 1.3 & 0.6 & 1.4 & 1.1 & 0.9 & 0.9 & \underline{F}(3,264)=3.73^{* *}\end{array}$

Environment:

$\begin{array}{llllllllll}\text { Peer Risk } & 2.4 & 0.7 & 2.4 & 0.6 & 2.6 & 0.7 & 2.2 & 0.8 & \underline{F}(3,453)=5.00^{* *} \\ \text { AIDS Proximity } & 1.9 & 0.8 & 1.8 & 0.7 & 2.1 & 0.9 & 2.0 & 1.0 & \mathrm{n} / \mathrm{s} \\ \text { Media Resources 2.7 } & 0.8 & 2.8 & 0.9 & 2.8 & 0.9 & 2.6 & 0.9 & \mathrm{n} / \mathrm{s} \\ \text { Media Influence 22.9 } & 1.1 & 2.6 & 1.1 & 3.1 & 1.3 & 3.0 & 1.1 & \underline{F}(3,453)=3.68^{* *} \\ & & & & & & & & & \text { MANOVA, Pillais } \\ \text { Education: } & & & & & & & & & \underline{F}(24,1344)=5.03^{*} \\ \text { School Education 2.9 } & 0.7 & 3.3 & 0.7 & 3.1 & 0.8 & 3.2 & 0.6 & \underline{F}(3,453)=4.89^{* *} \\ \text { School Influence 3.3 } & 0.9 & 3.3 & 0.7 & 3.3 & 1.0 & 3.3 & 1.0 & \mathrm{n} / \mathrm{s} \\ \text { Parent Education 2.8 } & 1.4 & 3.0 & 1.3 & 2.8 & 1.3 & 2.9 & 1.5 & \mathrm{n} / \mathrm{s} \\ \text { Parent Influence 2.1 } & 1.1 & 2.5 & 1.2 & 2.2 & 1.1 & 2.4 & 1.1 & \mathrm{n} / \mathrm{s} \\ \text { Sibling Education 3.3 } & 1.3 & 3.3 & 1.3 & 2.9 & 1.2 & 2.9 & 1.5 & \mathrm{n} / \mathrm{s} \\ \text { Sibling Influence 1.9 } & 1.1 & 2.4 & 1.3 & 1.9 & 1.0 & 1.4 & 0.6 & \underline{F}(3,453)=17.82^{*} \\ \text { Peer Education } 4.3 & 0.9 & 4.0 & 1.0 & 4.2 & 1.1 & 4.0 & 1.4 & \mathrm{n} / \mathrm{s} \\ \text { Peer Influence } & 2.7 & 1.0 & 2.8 & 1.0 & 3.0 & 1.0 & 2.3 & 0.9 & \underline{F}(3,453)=10.60^{*}\end{array}$

MANOVA, Pillais: $\underline{F}(12,1356)=3.55^{*}$

Post hoc ANOVA:

$* p<.001 ; * * p<.01$.

Note: All subscales are based on a 5-point scale, with 5 indicating highest endorsement of scale; with exception to the "sex risk" composite score computed as number of partners in the past year multiplied by the number of unprotected vaginal, oral and anal sex occasions, and "log sex risk" which is the logarithmic transformation of the "sex risk" composite score. 
นำ

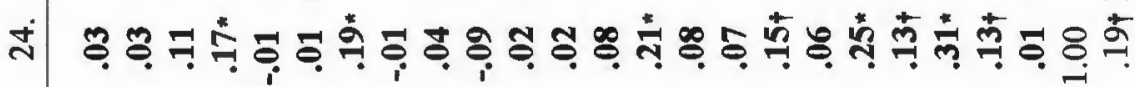

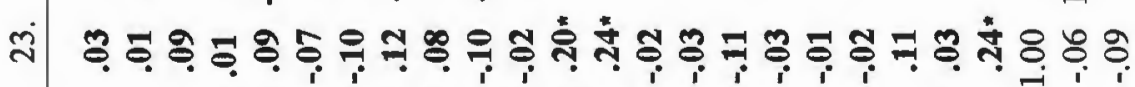

ส่

ส น้ำ

¿ 寺草

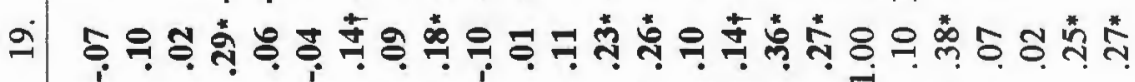

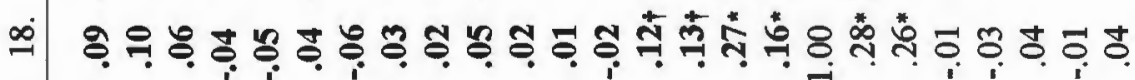

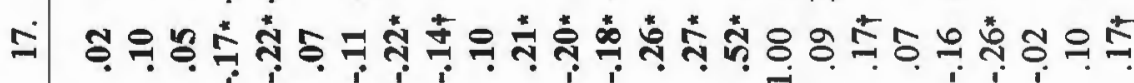

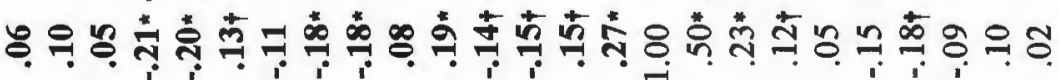

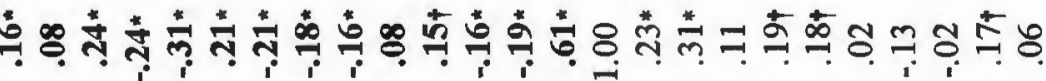

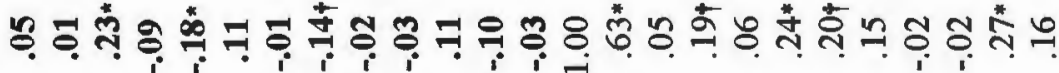

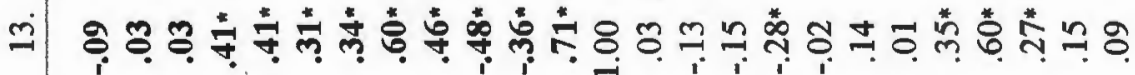

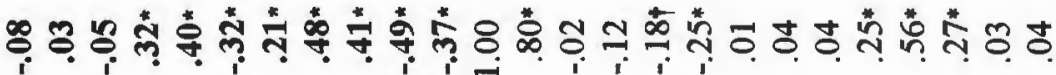

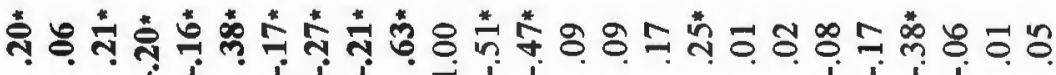

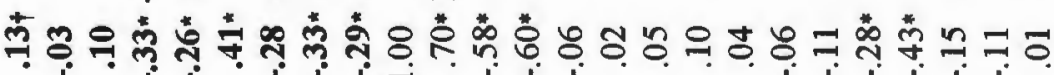

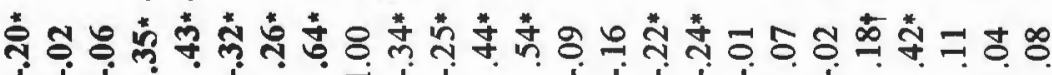

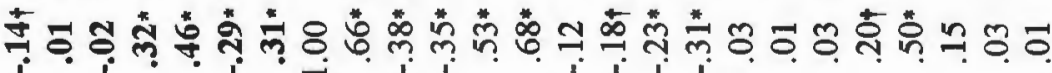

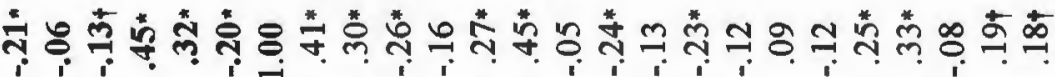

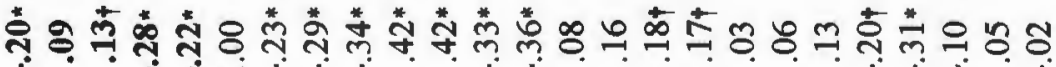

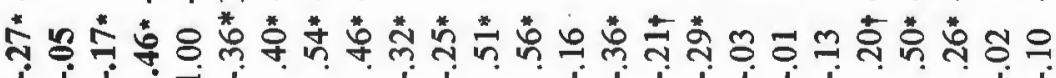

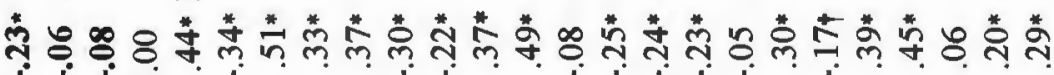
คิ่

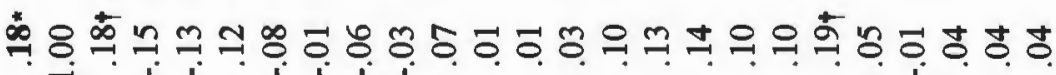

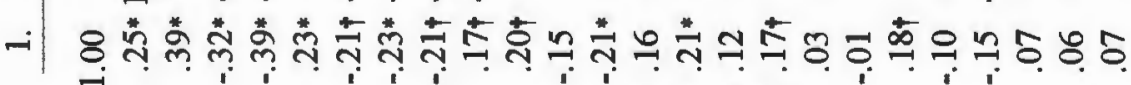

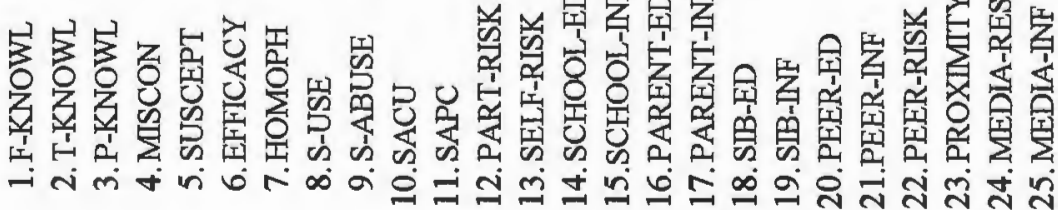


Summary Table of 88 Confirmatory Factor Analyses for Scale and Subscale Structures for Differences on Dichotomized Demographic Variables

Variable: Age $=\leq 15$ years $(n=146)$

$\%$ Factor

No. of items No. of No. of consistency

Scale/ Variance Concordant varying from inconsistent complex w/original

Subscale Accounted Alpha Factor(s) original structure items

\begin{tabular}{|c|c|c|c|c|c|c|c|}
\hline Cognitive: & $56.9 \%$ & .68 & Yes & $0 / 19$ & $0 / 19$ & $0 / 19$ & $100.0 \%$ \\
\hline F-KNOWL & -- & .77 & Yes & $0 / 6$ & $0 / 6$ & $0 / 6$ & 100.0 \\
\hline T-KNOWL & -- & .91 & Yes & $0 / 4$ & $0 / 4$ & $0 / 4$ & 100.0 \\
\hline P-KNOWL & -- & .80 & Yes & $0 / 3$ & $0 / 3$ & $0 / 3$ & 100.0 \\
\hline MISCONCEP & -- & .69 & Yes & $0 / 6$ & $0 / 6$ & $0 / 6$ & 100.0 \\
\hline Attitudinal: & 52.3 & .83 & No & $6 / 26$ & $1 / 26$ & $5 / 26$ & 76.9 \\
\hline SUSCEPTIBILITY & -- & .69 & No & $2 / 5$ & $1 / 5$ & $1 / 5$ & 60.0 \\
\hline CU EFFICACY & -- & .90 & Yes & $0 / 7$ & $0 / 7$ & $0 / 7$ & 100.0 \\
\hline HOMOPHOBIA & - & .90 & No & $4 / 14$ & $0 / 14$ & $4 / 14$ & 71.4 \\
\hline Behavioral: & 82.7 & .88 & No & $6 / 47$ & $1 / 47$ & $5 / 47$ & 87.2 \\
\hline ASSERTIVENESS** & 74.6 & .91 & No & $2 / 10$ & $0 / 10$ & $2 / 10$ & 80.0 \\
\hline SACU & -- & .86 & No & $2 / 5$ & $0 / 5$ & $2 / 5$ & 60.0 \\
\hline SAPC & -- & .94 & Yes & $0 / 5$ & $0 / 5$ & $0 / 5$ & 100.0 \\
\hline SUBSTANCE* & 41.7 & .87 & No & $1 / 23$ & $0 / 23$ & $1 / 23$ & 95.6 \\
\hline S-USE & -- & .87 & Yes & $0 / 8$ & $0 / 8$ & $0 / 8$ & 100.0 \\
\hline S-ABUSE & -- & .85 & No & $1 / 15$ & $0 / 15$ & $1 / 15$ & 93.3 \\
\hline SEXUAL RISK** & 40.0 & .73 & No & $3 / 14$ & $1 / 14$ & $2 / 14$ & 78.6 \\
\hline PART-RISK HX & -- & .60 & No & $2 / 7$ & $0 / 7$ & $2 / 7$ & 71.4 \\
\hline SELF-RISK HX & -- & .77 & No & $1 / 7$ & $1 / 7$ & $0 / 7$ & 85.7 \\
\hline Environment: & 53.8 & .76 & No & $2 / 26$ & $1 / 26$ & $1 / 26$ & 92.3 \\
\hline PEER-RISK & -- & .63 & No & $2 / 8$ & $1 / 8$ & $1 / 8$ & 75.0 \\
\hline PROXTMITY & -- & .82 & Yes & $0 / 8$ & $0 / 8$ & $0 / 8$ & 100.0 \\
\hline MEDIA-RES & -- & .80 & Yes & $0 / 5$ & $0 / 5$ & $0 / 5$ & 100.0 \\
\hline MEDIA-INF & -- & .94 & Yes & $0 / 5$ & $0 / 5$ & $0 / 5$ & 100.0 \\
\hline Education: & 73.0 & .82 & No & $7 / 21$ & $0 / 21$ & $7 / 21$ & 66.7 \\
\hline SCHOOL & 42.5 & .78 & No & $7 / 13$ & $0 / 13$ & $7 / 13$ & 46.2 \\
\hline SCHOOL-ED & -- & .66 & No & $6 / 8$ & $0 / 8$ & $6 / 8$ & 25.0 \\
\hline SCHOOL-INF & -- & .72 & No & $1 / 5$ & $0 / 5$ & $1 / 5$ & 80.0 \\
\hline HOME & 70.0 & .60 & Yes & $0 / 4$ & $0 / 4$ & $0 / 4$ & 100.0 \\
\hline PARENT-ED & -- & single item & Yes & $0 / 1$ & $0 / 1$ & $0 / 1$ & 100.0 \\
\hline PARENT-INF & -- & single item & Yes & $0 / 1$ & $0 / 1$ & $0 / 1$ & 100.0 \\
\hline SIB-ED & -- & single item & Yes & $0 / 1$ & $0 / 1$ & $0 / 1$ & 100.0 \\
\hline SIB-INF & -- & single item & Yes & $0 / 1$ & $0 / 1$ & $0 / 1$ & 100.0 \\
\hline PEERS & 72.3 & .70 & Yes & $0 / 4$ & $0 / 4$ & $0 / 4$ & 100.0 \\
\hline PEER-ED & -- & single item & Yes & $0 / 1$ & $0 / 1$ & $0 / 1$ & 100.0 \\
\hline PEER-INF & -- & .70 & Yes & $0 / 3$ & $0 / 3$ & $0 / 3$ & 100.0 \\
\hline
\end{tabular}

(continued on next page)

Total: $21 / 139=84.9 \%$ overall consistency

* 75/146 substance users utilized in analyses.

** 58/146 sexually active utilized in analyses. 
Variable: Age $=15$ years and older $(n=311)$

\begin{tabular}{|c|c|c|c|c|c|c|c|}
\hline $\begin{array}{l}\text { Scale/ } \\
\text { Subscale }\end{array}$ & $\begin{array}{l}\text { Variance } \\
\text { Accounted }\end{array}$ & Alpha & $\begin{array}{c}\text { Concordant } \\
\text { Factor(s) }\end{array}$ & $\begin{array}{l}\text { No. of items } \\
\text { varying from } \\
\text { original structure }\end{array}$ & $\begin{array}{c}\text { No. of } \\
\text { inconsistent } \\
\text { items }\end{array}$ & $\begin{array}{l}\text { No. of } \\
\text { complex } \\
\text { items }\end{array}$ & $\begin{array}{c}\% \text { Factor } \\
\text { consistency } \\
\text { w/original } \\
\text { structure } \\
\end{array}$ \\
\hline Cognitive: & $61.9 \%$ & .69 & Yes & $0 / 19$ & $0 / 19$ & $0 / 19$ & $100.0 \%$ \\
\hline F-KNOWL & -- & .71 & Yes & $0 / 6$ & $0 / 6$ & $0 / 6$ & 100.0 \\
\hline T-KNOWL & - & .95 & Yes & $0 / 4$ & $0 / 4$ & $0 / 4$ & 100.0 \\
\hline P-KNOWL & -- & .89 & Yes & $0 / 3$ & $0 / 3$ & $0 / 3$ & 100.0 \\
\hline MISCONCEP & -- & .80 & Yes & $0 / 6$ & $0 / 6$ & $0 / 6$ & 100.0 \\
\hline Attitudinal: & 56.5 & .82 & No & $1 / 26$ & $0 / 26$ & $1 / 26$ & 96.2 \\
\hline SUSCEPTIBILITY & Y -- & .75 & Yes & $0 / 5$ & $0 / 5$ & $0 / 5$ & 100.0 \\
\hline CU EFFICACY & - & .93 & Yes & $0 / 7$ & $0 / 7$ & $0 / 7$ & 100.0 \\
\hline HOMOPHOBIA & -- & .91 & No & $1 / 14$ & $0 / 14$ & $1 / 14$ & 92.9 \\
\hline Behavioral: & 84.5 & .88 & No & $9 / 47$ & $1 / 47$ & $8 / 47$ & 83.0 \\
\hline ASSERTIVENESS** & $* * 71.7$ & .91 & Yes & $0 / 10$ & $0 / 10$ & $0 / 10$ & 100.0 \\
\hline SACU & -- & .86 & Yes & $0 / 5$ & $0 / 5$ & $0 / 5$ & 100.0 \\
\hline SAPC & - & .93 & Yes & $0 / 5$ & $0 / 5$ & $0 / 5$ & 100.0 \\
\hline SUBSTANCE* & 38.2 & .87 & No & $6 / 23$ & $1 / 23$ & $5 / 23$ & 73.9 \\
\hline S-USE & -- & .80 & No & $2 / 8$ & $1 / 8$ & $1 / 8$ & 75.0 \\
\hline S-ABUSE & -- & .84 & No & $4 / 15$ & $0 / 15$ & $4 / 15$ & 73.3 \\
\hline SEXUAL RISK** & 41.5 & .77 & No & $3 / 14$ & $0 / 14$ & $3 / 14$ & 78.6 \\
\hline PART-RISK HX & X & .69 & No & $2 / 7$ & $0 / 7$ & $2 / 7$ & 71.4 \\
\hline SELF-RISK HX & - & .80 & No & $1 / 7$ & $0 / 7$ & $1 / 7$ & 85.7 \\
\hline Environment: & 52.6 & .80 & No & $3 / 26$ & $3 / 26$ & $0 / 26$ & 88.5 \\
\hline$\overline{\text { PEER-RISK }}$ & -- & .69 & No & $3 / 8$ & $3 / 8$ & $0 / 8$ & 62.5 \\
\hline PROXIMITY & - & .82 & Yes & $0 / 8$ & $0 / 8$ & $0 / 8$ & 100.0 \\
\hline MEDIA-RES & -- & .76 & Yes & $0 / 5$ & $0 / 5$ & $0 / 5$ & 100.0 \\
\hline MEDIA-INF & -- & .96 & Yes & $0 / 5$ & $0 / 5$ & $0 / 5$ & 100.0 \\
\hline Education: & 72.5 & .81 & No & $4 / 21$ & $0 / 21$ & $4 / 21$ & 81.0 \\
\hline$\overline{\mathrm{SCHOOL}}$ & 48.5 & .85 & No & $4 / 13$ & $0 / 14$ & $4 / 14$ & 71.4 \\
\hline SCHOOL-ED & -- & .77 & No & $3 / 8$ & $0 / 8$ & $3 / 8$ & 62.5 \\
\hline SCHOOL-INF & -- & .76 & No & $1 / 5$ & $0 / 5$ & $1 / 5$ & 80.0 \\
\hline HOME & 70.9 & .64 & Yes & $0 / 4$ & $0 / 4$ & $0 / 4$ & 100.0 \\
\hline PARENT-ED & -- & ingle item & Yes & $0 / 1$ & $0 / 1$ & $0 / 1$ & 100.0 \\
\hline PARENT-INF & -- & ingle item & Yes & $0 / 1$ & $0 / 1$ & $0 / 1$ & 100.0 \\
\hline SIB-ED & - & ingle item & Yes & $0 / 1$ & $0 / 1$ & $0 / 1$ & 100.0 \\
\hline SIB-INF & -- & ingle item & Yes & $0 / 1$ & $0 / 1$ & $0 / 1$ & 100.0 \\
\hline PEERS & 75.0 & .60 & Yes & $0 / 4$ & $0 / 4$ & $0 / 4$ & 100.0 \\
\hline PEER-ED & -- & ingle item & Yes & $0 / 1$ & $0 / 1$ & $0 / 1$ & 100.0 \\
\hline PEER-INF & -- & .74 & Yes & $0 / 3$ & $0 / 3$ & $0 / 3$ & 100.0 \\
\hline
\end{tabular}

Total: $\quad 17 / 139=87.8 \%$ overall consistency

(continued on next page)

\footnotetext{
* 186/311 substance users utilized in analyses.

** $210 / 311$ sexually active utilized in analyses.
} 
Variable: Geographic Location $=$ RI, MA, \& CT $(n=382)$

\begin{tabular}{|c|c|c|c|c|c|c|c|}
\hline $\begin{array}{l}\text { Scale/ } \\
\text { Subscale }\end{array}$ & $\begin{array}{l}\text { Variance } \\
\text { Accounted }\end{array}$ & Alpha & $\begin{array}{c}\text { Concordant } \\
\text { Factor(s) }\end{array}$ & $\begin{array}{l}\text { No. of items } \\
\text { varying from } \\
\text { original structure }\end{array}$ & $\begin{array}{l}\text { No. of } \\
\text { inconsistent } \\
\text { items }\end{array}$ & $\begin{array}{l}\text { No. of } \\
\text { complex } \\
\text { items }\end{array}$ & $\begin{array}{c}\% \text { Factor } \\
\text { consistency } \\
\text { w/original } \\
\text { structure }\end{array}$ \\
\hline Cognitive: & $59.7 \%$ & .71 & Yes & $0 / 19$ & $0 / 19$ & $0 / 19$ & $100.0 \%$ \\
\hline$\overline{\text { F-KNOWL }}$ & -- & .73 & Yes & $0 / 6$ & $0 / 6$ & $0 / 6$ & 100.0 \\
\hline T-KNOWL & -- & .95 & Yes & $0 / 4$ & $0 / 4$ & $0 / 4$ & 100.0 \\
\hline P-KNOWL & - & .86 & Yes & $0 / 3$ & $0 / 3$ & $0 / 3$ & 100.0 \\
\hline MISCONCEP & -- & .74 & Yes & $0 / 6$ & $0 / 6$ & $0 / 6$ & 100.0 \\
\hline Attitudinal: & 50.0 & .80 & No & $4 / 26$ & $0 / 26$ & $4 / 26$ & 84.6 \\
\hline SUSCEPTIBILITY & Y - & .72 & Yes & $0 / 5$ & $0 / 5$ & $0 / 5$ & 100.0 \\
\hline CU EFFICACY & - & .92 & Yes & $0 / 7$ & $0 / 7$ & $0 / 7$ & 100.0 \\
\hline HOMOPHOBIA & -- & .88 & No & $4 / 14$ & $0 / 14$ & $4 / 14$ & 71.4 \\
\hline Behavioral: & 82.5 & .88 & No & $3 / 47$ & $2 / 47$ & $1 / 47$ & 93.6 \\
\hline ASSERTIVENESS* & $* * 70.3$ & .91 & Yes & $0 / 10$ & $0 / 10$ & $0 / 10$ & 100.0 \\
\hline SACU & -- & .84 & Yes & $0 / 5$ & $0 / 5$ & $0 / 5$ & 100.0 \\
\hline SAPC & -- & .93 & Yes & $0 / 5$ & $0 / 5$ & $0 / 5$ & 100.0 \\
\hline SUBSTANCE* & 36.7 & .86 & No & $3 / 23$ & $2 / 23$ & $1 / 23$ & 87.0 \\
\hline S-USE & -- & .81 & No & $1 / 8$ & $1 / 8$ & $0 / 8$ & 87.5 \\
\hline S-ABUSE & -- & .83 & No & $2 / 15$ & $1 / 15$ & $1 / 15$ & 86.7 \\
\hline SEXUAL RISK** & * 36.1 & .71 & Yes & $0 / 14$ & $0 / 14$ & $0 / 14$ & 100.0 \\
\hline PART-RISK HX & X -- & .69 & Yes & $0 / 7$ & $0 / 7$ & $0 / 7$ & 100.0 \\
\hline SELF-RISK HX & K - & .72 & Yes & $0 / 7$ & $0 / 7$ & $0 / 7$ & 100.0 \\
\hline Environment: & 51.2 & .78 & No & $2 / 26$ & $2 / 26$ & $0 / 26$ & 92.3 \\
\hline PEER-RISK & -- & .68 & No & $2 / 8$ & $2 / 8$ & $0 / 8$ & 75.0 \\
\hline PROXIMITY & - & .81 & Yes & $0 / 8$ & $0 / 8$ & $0 / 8$ & 100.0 \\
\hline MEDIA-RES & - & .76 & Yes & $0 / 5$ & $0 / 5$ & $0 / 5$ & 100.0 \\
\hline MEDIA-INF & -- & .94 & Yes & $0 / 5$ & $0 / 5$ & $0 / 5$ & 100.0 \\
\hline Education: & 72.2 & .80 & No & $1 / 21$ & $0 / 21$ & $1 / 21$ & 95.3 \\
\hline$\overline{\mathrm{SCHOOL}}$ & 43.7 & .81 & No & $1 / 13$ & $0 / 13$ & $1 / 13$ & 92.3 \\
\hline SCHOOL-ED & -- & .71 & No & $1 / 8$ & $0 / 8$ & $1 / 8$ & 87.5 \\
\hline SCHOOL-INF & -- & .72 & Yes & $0 / 5$ & $0 / 5$ & $0 / 5$ & 100.0 \\
\hline HOME & 69.3 & .61 & Yes & $0 / 4$ & $0 / 4$ & $0 / 4$ & 100.0 \\
\hline PARENT-ED & -- & single item & Yes & $0 / 1$ & $0 / 1$ & $0 / 1$ & 100.0 \\
\hline PARENT-INF & -- & single item & Yes & $0 / 1$ & $0 / 1$ & $0 / 1$ & 100.0 \\
\hline SIB-ED & -- & single item & Yes & $0 / 1$ & $0 / 1$ & $0 / 1$ & 100.0 \\
\hline SIB-INF & -- & single item & Yes & $0 / 1$ & $0 / 1$ & $0 / 1$ & 100.0 \\
\hline PEERS & 73.8 & .67 & Yes & $0 / 4$ & $0 / 4$ & $0 / 4$ & 100.0 \\
\hline PEER-ED & -- & single item & Yes & $0 / 1$ & $0 / 1$ & $0 / 1$ & 100.0 \\
\hline PEER-INF & -- & .73 & Yes & $0 / 3$ & $0 / 3$ & $0 / 3$ & 100.0 \\
\hline
\end{tabular}

Total: $\quad 10 / 139=92.8 \%$ overall consistency

(continued on next page)

* 219/382 substance users utilized in analyses.

** 218/382 sexually active utilized in analyses. 
Variable: Geographic Location $=$ NH, NJ, ME, NY, PA, OH, DE, VT,

Bermuda, Jamaica, Cayman Islands $(n=75)$

\begin{tabular}{|c|c|c|c|c|c|c|c|}
\hline $\begin{array}{l}\text { Scale/ } \\
\text { Subscale }\end{array}$ & $\begin{array}{l}\text { Variance } \\
\text { Accounted }\end{array}$ & Alpha & $\begin{array}{c}\text { Concordant } \\
\text { Factor(s) }\end{array}$ & $\begin{array}{l}\text { No. of items } \\
\text { varying from } \\
\text { original structure }\end{array}$ & $\begin{array}{l}\text { No. of } \\
\text { inconsistent } \\
\text { items }\end{array}$ & $\begin{array}{l}\text { No. of } \\
\text { complex } \\
\text { items }\end{array}$ & $\begin{array}{r}\% \text { Factor } \\
\text { consistenc } \\
\text { w/origina } \\
\text { structure }\end{array}$ \\
\hline Cognitive: & 66.9 & .70 & No & $1 / 19$ & $0 / 19$ & $1 / 19$ & $94.7 \%$ \\
\hline$\overline{\text { F-KNOWL }}$ & -- & .80 & No & $1 / 6$ & $0 / 6$ & $1 / 6$ & 83.3 \\
\hline T-KNOWL & -- & .88 & Yes & $0 / 4$ & $0 / 4$ & $0 / 4$ & 100.0 \\
\hline P-KNOWL & -- & .81 & Yes & $0 / 3$ & $0 / 3$ & $0 / 3$ & 100.0 \\
\hline MISCONCEP & -- & .87 & Yes & $0 / 6$ & $0 / 6$ & $0 / 6$ & 100.0 \\
\hline Attitudinal: & 69.2 & .87 & No & $1 / 26$ & $0 / 26$ & $1 / 26$ & 96.2 \\
\hline SUSCEPTIBILITY & Y - & .81 & Yes & $0 / 5$ & $0 / 5$ & $0 / 5$ & 100.0 \\
\hline CU EFFICACY & -- & .94 & No & $1 / 7$ & $0 / 7$ & $1 / 7$ & 85.7 \\
\hline HOMOPHOBIA & -- & .96 & Yes & $0 / 14$ & $0 / 14$ & $0 / 14$ & 100.0 \\
\hline Behavioral: & 88.8 & .90 & No & $8 / 47$ & $0 / 47$ & $8 / 47$ & 83.0 \\
\hline ASSERTIVENESS* & $* 81.7$ & .93 & No & $1 / 10$ & $0 / 10$ & $1 / 10$ & 90.0 \\
\hline SACU & -- & .90 & No & $1 / 5$ & $0 / 5$ & $1 / 5$ & 80.0 \\
\hline SAPC & - & .96 & Yes & $0 / 5$ & $0 / 5$ & $0 / 5$ & 100.0 \\
\hline SUBSTANCE* & 52.0 & .90 & No & $4 / 23$ & $0 / 23$ & $4 / 23$ & 82.6 \\
\hline S-USE & -- & .87 & Yes & $0 / 8$ & $0 / 8$ & $0 / 8$ & 100.0 \\
\hline S-ABUSE & -- & .89 & No & $4 / 15$ & $0 / 15$ & $4 / 15$ & 73.3 \\
\hline SEXUAL RISK** & 48.9 & .82 & No & $3 / 14$ & $0 / 14$ & $3 / 14$ & 78.6 \\
\hline PART-RISK HX & X -- & .67 & No & $2 / 7$ & $0 / 7$ & $2 / 7$ & 71.4 \\
\hline SELF-RISK HX & K - & .87 & No & $1 / 7$ & $0 / 7$ & $1 / 7$ & 85.7 \\
\hline Environment: & 60.9 & .71 & No & $2 / 26$ & $0 / 26$ & $2 / 26$ & 92.3 \\
\hline PEER-RISK & -- & .80 & No & $2 / 8$ & $0 / 8$ & $2 / 8$ & 75.0 \\
\hline PROXIMITY & -- & .85 & Yes & $0 / 8$ & $0 / 8$ & $0 / 8$ & 100.0 \\
\hline MEDIA-RES & -- & .65 & Yes & $0 / 5$ & $0 / 5$ & $0 / 5$ & 100.0 \\
\hline MEDIA-INF & - & .99 & Yes & $0 / 5$ & $0 / 5$ & $0 / 5$ & 100.0 \\
\hline Education: & 75.4 & .85 & No & $4 / 21$ & $0 / 21$ & $4 / 21$ & 81.0 \\
\hline SCHOOL & 55.0 & .87 & No & $4 / 13$ & $0 / 13$ & $4 / 13$ & 69.2 \\
\hline SCHOOL-ED & -- & .78 & No & $4 / 8$ & $0 / 8$ & $4 / 8$ & 50.0 \\
\hline SCHOOL-INF & -- & .86 & Yes & $0 / 5$ & $0 / 5$ & $0 / 5$ & 100.0 \\
\hline HOME & 75.2 & .69 & Yes & $0 / 4$ & $0 / 4$ & $0 / 4$ & 100.0 \\
\hline PARENT-ED & -- & ingle item & Yes & $0 / 1$ & $0 / 1$ & $0 / 1$ & 100.0 \\
\hline PARENT-INF & -- & ingle item & Yes & $0 / 1$ & $0 / 1$ & $0 / 1$ & 100.0 \\
\hline SIB-ED & -- & ingle item & Yes & $0 / 1$ & $0 / 1$ & $0 / 1$ & 100.0 \\
\hline SIB-INF & -- & ingle item & Yes & $0 / 1$ & $0 / 1$ & $0 / 1$ & 100.0 \\
\hline PEERS & 74.7 & .66 & Yes & $0 / 4$ & $0 / 4$ & $0 / 4$ & 100.0 \\
\hline PEER-ED & -- & ingle item & Yes & $0 / 1$ & $0 / 1$ & $0 / 1$ & 100.0 \\
\hline PEER-INF & - & .71 & Yes & $0 / 3$ & $0 / 3$ & $0 / 3$ & 100.0 \\
\hline
\end{tabular}

$\underline{\text { Total }:}: 16 / 139=88.5 \%$ overall consistency

(continued on next page)

* 42/75 substance users utilized in analyses.

** 50/75 sexually active utilized in analyses. 
Variable: Ethnicity $=$ Caucasian $(n=372)$

\begin{tabular}{|c|c|c|c|c|c|c|c|}
\hline $\begin{array}{l}\text { Scale/ } \\
\text { Subscale }\end{array}$ & $\begin{array}{l}\text { Variance } \\
\text { Accounted }\end{array}$ & Alpha & $\begin{array}{c}\text { Concordant } \\
\text { Factor(s) }\end{array}$ & $\begin{array}{l}\text { No. of items } \\
\text { varying from } \\
\text { original structure }\end{array}$ & $\begin{array}{l}\text { No. of } \\
\text { inconsistent } \\
\text { items }\end{array}$ & $\begin{array}{l}\text { No. of } \\
\text { complex } \\
\text { items }\end{array}$ & $\begin{array}{c}\% \text { Factor } \\
\text { consistency } \\
\text { w/original } \\
\text { structure } \\
\end{array}$ \\
\hline Cognitive: & $59.7 \%$ & .73 & Yes & $0 / 19$ & $0 / 19$ & $0 / 19$ & $100.0 \%$ \\
\hline$\overline{\text { F-KNOWL }}$ & - & .73 & Yes & $0 / 6$ & $0 / 6$ & $0 / 6$ & 100.0 \\
\hline T-KNOWL & -- & .92 & Yes & $0 / 4$ & $0 / 4$ & $0 / 4$ & 100.0 \\
\hline P-KNOWL & - & .86 & Yes & $0 / 3$ & $0 / 3$ & $0 / 3$ & 100.0 \\
\hline MISCONCEP & -- & .75 & Yes & $0 / 6$ & $0 / 6$ & $0 / 6$ & 100.0 \\
\hline Attitudinal: & 51.4 & .82 & No & $1 / 26$ & $0 / 26$ & $1 / 26$ & 96.2 \\
\hline SUSCEPTIBILITY & Y -- & .75 & Yes & $0 / 5$ & $0 / 5$ & $0 / 5$ & 100.0 \\
\hline CU EFFICACY & - & .92 & Yes & $0 / 7$ & $0 / 7$ & $0 / 7$ & 100.0 \\
\hline HOMOPHOBIA & -- & .89 & No & $1 / 14$ & $0 / 14$ & $1 / 14$ & 93.6 \\
\hline Behavioral: & 82.9 & .87 & No & $8 / 47$ & $1 / 47$ & $7 / 47$ & 83.0 \\
\hline ASSERTIVENESS** & $* * 72.6$ & .91 & Yes & $0 / 10$ & $0 / 10$ & $0 / 10$ & 100.0 \\
\hline SACU & -- & .87 & Yes & $0 / 5$ & $0 / 5$ & $0 / 5$ & 100.0 \\
\hline SAPC & -- & .94 & Yes & $0 / 5$ & $0 / 5$ & $0 / 5$ & 100.0 \\
\hline SUBSTANCE* & 35.7 & .85 & No & $5 / 23$ & $1 / 23$ & $4 / 23$ & 82.6 \\
\hline S-USE & -- & .81 & No & $1 / 8$ & $1 / 8$ & $0 / 8$ & 87.5 \\
\hline S-ABUSE & -- & .81 & No & $4 / 15$ & $0 / 15$ & $4 / 15$ & 73.3 \\
\hline SEXUAL RISK** & * 38.9 & .74 & No & $3 / 14$ & $0 / 14$ & $3 / 14$ & 78.6 \\
\hline PART-RISK HX & X -- & .65 & No & $2 / 7$ & $0 / 7$ & $2 / 7$ & 71.4 \\
\hline SELF-RISK HX & $K \quad-$ & .75 & No & $1 / 7$ & $0 / 7$ & $1 / 7$ & 85.7 \\
\hline Environment: & 52.2 & .78 & No & $2 / 26$ & $0 / 26$ & $2 / 26$ & 92.3 \\
\hline PEER-RISK & -- & .67 & No & $2 / 8$ & $0 / 8$ & $2 / 8$ & 75.0 \\
\hline PROXIMITY & -- & .82 & Yes & $0 / 8$ & $0 / 8$ & $0 / 8$ & 100.0 \\
\hline MEDIA-RES & -- & .77 & Yes & $0 / 5$ & $0 / 5$ & $0 / 5$ & 100.0 \\
\hline MEDIA-INF & -- & .96 & Yes & $0 / 5$ & $0 / 5$ & $0 / 5$ & 100.0 \\
\hline Education: & 71.5 & .80 & No & $4 / 21$ & $0 / 21$ & $4 / 21$ & 81.0 \\
\hline$\overline{\mathrm{SCHOOL}}$ & 43.1 & .80 & No & $4 / 13$ & $0 / 13$ & $4 / 13$ & 69.2 \\
\hline SCHOOL-ED & -- & .70 & No & $3 / 8$ & $0 / 8$ & $3 / 8$ & 62.5 \\
\hline SCHOOL-INF & - & .74 & No & $1 / 5$ & $0 / 5$ & $1 / 5$ & 80.0 \\
\hline HOME & 70.1 & .60 & Yes & $0 / 4$ & $0 / 4$ & $0 / 4$ & 100.0 \\
\hline PARENT-ED & - & single item & Yes & $0 / 1$ & $0 / 1$ & $0 / 1$ & 100.0 \\
\hline PARENT-INF & - & single item & Yes & $0 / 1$ & $0 / 1$ & $0 / 1$ & 100.0 \\
\hline SIB-ED & - & single item & Yes & $0 / 1$ & $0 / 1$ & $0 / 1$ & 100.0 \\
\hline SIB-INF & -- & single item & Yes & $0 / 1$ & $0 / 1$ & $0 / 1$ & 100.0 \\
\hline PEERS & 71.9 & .67 & Yes & $0 / 4$ & $0 / 4$ & $0 / 4$ & 100.0 \\
\hline PEER-ED & -- & single item & Yes & $0 / 1$ & $0 / 1$ & $0 / 1$ & 100.0 \\
\hline PEER-INF & -- & .70 & Yes & $0 / 3$ & $0 / 3$ & $0 / 3$ & 100.0 \\
\hline
\end{tabular}

Total: $\quad 15 / 139=89.2 \%$ overall consistency

(continued on next page)

* 198/372 substance users utilized in analyses.
$* * 199 / 372$ sexually active utilized in analyses. 
Variable: Ethnicity = African-, Asian-, Hispanic-Americans \& "other" $(n=85)$

\begin{tabular}{|c|c|c|c|c|c|c|c|}
\hline $\begin{array}{l}\text { Scale/ } \\
\text { Subscale }\end{array}$ & $\begin{array}{l}\text { Variance } \\
\text { Accounted }\end{array}$ & Alpha & $\begin{array}{l}\text { Concordant } \\
\text { Factor(s) }\end{array}$ & $\begin{array}{l}\text { No. of items } \\
\text { varying from } \\
\text { original structure }\end{array}$ & $\begin{array}{l}\text { No. of } \\
\text { inconsistent } \\
\text { items }\end{array}$ & $\begin{array}{l}\text { No. of } \\
\text { complex } \\
\text { items }\end{array}$ & $\begin{array}{c}\% \text { Factor } \\
\text { consistenc } \\
\text { w/original } \\
\text { structure }\end{array}$ \\
\hline Cognitive: & $61.4 \%$ & .69 & No & $2 / 19$ & $0 / 19$ & $2 / 19$ & $89.5 \%$ \\
\hline F-KNOWL & -- & .78 & Yes & $0 / 6$ & $0 / 6$ & $0 / 6$ & 100.0 \\
\hline T-KNOWL & - & .80 & No & $1 / 4$ & $0 / 4$ & $1 / 4$ & 75.0 \\
\hline P-KNOWL & -- & .85 & Yes & $0 / 3$ & $0 / 3$ & $0 / 3$ & 100.0 \\
\hline MISCONCEP & - & .80 & No & $1 / 6$ & $0 / 6$ & $1 / 6$ & 83.3 \\
\hline Attitudinal: & 60.7 & .83 & No & $5 / 26$ & $0 / 26$ & $5 / 26$ & 80.8 \\
\hline$\overline{\text { SUSCEPTIBILITY }}$ & Y -- & .63 & No & $2 / 5$ & $0 / 5$ & $2 / 5$ & 60.0 \\
\hline CU EFFICACY & -- & .95 & Yes & $0 / 7$ & $0 / 7$ & $0 / 7$ & 100.0 \\
\hline HOMOPHOBIA & - & .93 & No & $3 / 14$ & $0 / 14$ & $3 / 14$ & 78.6 \\
\hline Behavioral: & 83.9 & .90 & No & $9 / 47$ & $1 / 47$ & $8 / 47$ & 80.9 \\
\hline ASSERTIVENESS* & $* 75.3$ & .91 & No & $2 / 10$ & $0 / 10$ & $2 / 10$ & 80.0 \\
\hline SACU & -- & .83 & No & $2 / 5$ & $0 / 5$ & $2 / 5$ & 60.0 \\
\hline SAPC & -- & .93 & Yes & $0 / 5$ & $0 / 5$ & $0 / 5$ & 100.0 \\
\hline SUBSTANCE* & 49.1 & .91 & No & $6 / 23$ & $1 / 23$ & $5 / 23$ & 73.9 \\
\hline S-USE & - & .81 & No & $1 / 8$ & $1 / 8$ & $0 / 8$ & 87.5 \\
\hline S-ABUSE & -- & .90 & No & $5 / 15$ & $0 / 15$ & $5 / 15$ & 66.7 \\
\hline SEXUAL RISK** & * 43.1 & .76 & No & $1 / 14$ & $0 / 14$ & $1 / 14$ & 92.9 \\
\hline PART-RISK HX & $x-$ & .68 & Yes & $0 / 7$ & $0 / 7$ & $0 / 7$ & 100.0 \\
\hline SELF-RISK HX & -- & .82 & No & $1 / 7$ & $0 / 7$ & $1 / 7$ & 85.7 \\
\hline Environment: & 56.9 & .80 & No & $1 / 25$ & $0 / 25$ & $1 / 25$ & 96.0 \\
\hline PEER-RISK & - & .67 & No & $1 / 8$ & $0 / 8$ & $1 / 8$ & 87.5 \\
\hline PROXIMITY & -- & .83 & Yes & $0 / 8$ & $0 / 8$ & $0 / 8$ & 100.0 \\
\hline MEDIA-RES & - & .79 & Yes & $0 / 5$ & $0 / 5$ & $0 / 5$ & 100.0 \\
\hline MEDIA-INF & - & .95 & Yes & $0 / 5$ & $0 / 5$ & $0 / 5$ & 100.0 \\
\hline Education: & 78.6 & .86 & No & $4 / 21$ & $1 / 21$ & $3 / 21$ & 81.0 \\
\hline SCHOOL & 52.9 & .87 & No & $4 / 13$ & $1 / 13$ & $3 / 13$ & 71.4 \\
\hline SCHOOL-ED & - & .78 & No & $3 / 8$ & $0 / 8$ & $3 / 8$ & 62.5 \\
\hline SCHOOL-INF & -- & .80 & No & $1 / 5$ & $1 / 5$ & $0 / 5$ & 80.0 \\
\hline HOME & 75.3 & .70 & No & $1 / 4$ & $0 / 4$ & $1 / 4$ & 75.0 \\
\hline PARENT-ED & -- & ingle item & Yes & $0 / 1$ & $0 / 1$ & $0 / 1$ & 100.0 \\
\hline PARENT-INF & -- & ingle item & Yes & $0 / 1$ & $0 / 1$ & $0 / 1$ & 100.0 \\
\hline SIB-ED & -- & ingle item & Yes & $0 / 1$ & $0 / 1$ & $0 / 1$ & 100.0 \\
\hline SIB-INF & -- & ingle item & No & $1 / 1$ & $0 / 1$ & $1 / 1$ & 0.0 \\
\hline PEERS & 82.3 & .70 & Yes & $0 / 4$ & $0 / 4$ & $0 / 4$ & 100.0 \\
\hline PEER-ED & -- & ingle item & Yes & $0 / 1$ & $0 / 1$ & $0 / 1$ & 100.0 \\
\hline PEER-INF & - & .85 & Yes & $0 / 3$ & $0 / 3$ & $0 / 3$ & 100.0 \\
\hline
\end{tabular}

Total: $21 / 139=84.9 \%$ overall consistency

(continued on next page)

* 63/85 substance users utilized in analyses.

** 69/85 sexually active utilized in analyses. 
Variable: Socioeconomic Estimate $=$ Lower to Middle Class

(father's education high school graduate or less) $(n=168)$

\begin{tabular}{|c|c|c|c|c|c|c|c|}
\hline $\begin{array}{l}\text { Scale/ } \\
\text { Subscale }\end{array}$ & $\begin{array}{l}\text { Variance } \\
\text { Accounted }\end{array}$ & Alpha & $\begin{array}{c}\text { Concordant } \\
\text { Factor(s) }\end{array}$ & $\begin{array}{l}\text { No. of items } \\
\text { varying from } \\
\text { original structure }\end{array}$ & $\begin{array}{l}\text { No. of } \\
\text { inconsistent } \\
\text { items }\end{array}$ & $\begin{array}{l}\text { No. of } \\
\text { complex } \\
\text { items }\end{array}$ & $\begin{array}{c}\% \text { Factor } \\
\text { consistency } \\
\text { w/original } \\
\text { structure }\end{array}$ \\
\hline Cognitive: & $58.1 \%$ & .69 & Yes & $0 / 19$ & $0 / 19$ & $0 / 19$ & $100.0 \%$ \\
\hline F-KNOWL & -- & .70 & Yes & $0 / 6$ & $0 / 6$ & $0 / 6$ & 100.0 \\
\hline T-KNOWL & -- & .92 & Yes & $0 / 4$ & $0 / 4$ & $0 / 4$ & 100.0 \\
\hline P-KNOWL & -- & .84 & Yes & $0 / 3$ & $0 / 3$ & $0 / 3$ & 100.0 \\
\hline MISCONCEP & -- & .77 & Yes & $0 / 6$ & $0 / 6$ & $0 / 6$ & 100.0 \\
\hline Attitudinal: & 54.5 & .81 & No & $5 / 26$ & $0 / 26$ & $5 / 26$ & 80.8 \\
\hline SUSCEPTIBILITY & Y -- & .73 & Yes & $0 / 5$ & $0 / 5$ & $0 / 5$ & 100.0 \\
\hline CU EFFICACY & -- & .92 & Yes & $0 / 7$ & $0 / 7$ & $0 / 7$ & 100.0 \\
\hline HOMOPHOBIA & -- & .91 & No & $5 / 14$ & $0 / 14$ & $5 / 14$ & 64.3 \\
\hline Behavioral: & 84.8 & .89 & No & $5 / 47$ & $2 / 47$ & $3 / 47$ & 89.4 \\
\hline ASSERTIVENESS*: & $* 74.0$ & .92 & No & $2 / 10$ & $0 / 10$ & $2 / 10$ & 80.0 \\
\hline SACU & -- & .86 & No & $2 / 5$ & $0 / 5$ & $2 / 5$ & 60.0 \\
\hline SAPC & - & .94 & Yes & $0 / 5$ & $0 / 5$ & $0 / 5$ & 100.0 \\
\hline SUBSTANCE* & 41.5 & .88 & No & $2 / 23$ & $1 / 23$ & $1 / 23$ & 91.3 \\
\hline S-USE & -- & .83 & No & $1 / 8$ & $1 / 8$ & $0 / 8$ & 87.5 \\
\hline S-ABUSE & -- & .86 & No & $1 / 15$ & $0 / 15$ & $1 / 15$ & 93.3 \\
\hline SEXUAL RISK** & k 41.2 & .76 & No & $1 / 14$ & $1 / 14$ & $0 / 14$ & 93.6 \\
\hline PART-RISK HX & X -- & .67 & No & $1 / 7$ & $1 / 7$ & $0 / 7$ & 85.7 \\
\hline SELF-RISK HX & r - & .81 & Yes & $0 / 7$ & $0 / 7$ & $0 / 7$ & 100.0 \\
\hline Environment: & 55.1 & .80 & No & $2 / 26$ & $0 / 26$ & $2 / 26$ & 92.3 \\
\hline PEER-RISK & -- & .73 & No & $2 / 8$ & $0 / 8$ & $2 / 8$ & 75.0 \\
\hline PROXIMITY & -- & .83 & Yes & $0 / 8$ & $0 / 8$ & $0 / 8$ & 100.0 \\
\hline MEDIA-RES & -- & .78 & Yes & $0 / 5$ & $0 / 5$ & $0 / 5$ & 100.0 \\
\hline MEDIA-INF & -- & .95 & Yes & $0 / 5$ & $0 / 5$ & $0 / 5$ & 100.0 \\
\hline Education: & 73.4 & .80 & No & $5 / 21$ & $0 / 21$ & $5 / 21$ & 76.2 \\
\hline$\overline{\mathrm{SCHOOL}}$ & 46.7 & .82 & No & $5 / 13$ & $0 / 13$ & $5 / 13$ & 61.5 \\
\hline SCHOOL-ED & -- & .71 & No & $4 / 8$ & $0 / 8$ & $4 / 8$ & 50.0 \\
\hline SCHOOL-INF & -- & .72 & No & $1 / 5$ & $0 / 5$ & $1 / 5$ & 80.0 \\
\hline HOME & 69.0 & .61 & Yes & $0 / 4$ & $0 / 4$ & $0 / 4$ & 100.0 \\
\hline PARENT-ED & -- & ngle item & Yes & $0 / 1$ & $0 / 1$ & $0 / 1$ & 100.0 \\
\hline PARENT-INF & -- & ngle item & Yes & $0 / 1$ & $0 / 1$ & $0 / 1$ & 100.0 \\
\hline SIB-ED & -- & ngle item & Yes & $0 / 1$ & $0 / 1$ & $0 / 1$ & 100.0 \\
\hline SIB-INF & -- & ngle item & Yes & $0 / 1$ & $0 / 1$ & $0 / 1$ & 100.0 \\
\hline PEERS & 77.5 & .68 & Yes & $0 / 4$ & $0 / 4$ & $0 / 4$ & 100.0 \\
\hline PEER-ED & -- & ngle item & Yes & $0 / 1$ & $0 / 1$ & $0 / 1$ & 100.0 \\
\hline PEER-INF & - & .78 & Yes & $0 / 3$ & $0 / 3$ & $0 / 3$ & 100.0 \\
\hline
\end{tabular}

Total: $\quad 17 / 139=87.8 \%$ overall consistency

(continued on next page)

* $108 / 168$ substance users utilized in analyses

** 101/168 sexually active utilized in analyses. 
Variable: Socioeconomic Estimate $=$ Middle to Upper Class

(father's education greater than high school graduate) $(n=284)$

\begin{tabular}{|c|c|c|c|c|c|c|c|}
\hline $\begin{array}{l}\text { Scale/ } \\
\text { Subscale }\end{array}$ & $\begin{array}{l}\text { Variance } \\
\text { Accounted }\end{array}$ & Alpha & $\begin{array}{c}\text { Concordant } \\
\text { Factor(s) }\end{array}$ & $\begin{array}{l}\text { No. of items } \\
\text { varying from } \\
\text { original structure }\end{array}$ & $\begin{array}{l}\text { No. of } \\
\text { inconsistent } \\
\text { items }\end{array}$ & $\begin{array}{l}\text { No. of } \\
\text { complex } \\
\text { items }\end{array}$ & $\begin{array}{c}\% \text { Factor } \\
\text { consistency } \\
\text { w/original } \\
\text { structure }\end{array}$ \\
\hline Cognitive: & $62.3 \%$ & .76 & Yes & $0 / 19$ & $0 / 19$ & $0 / 19$ & $100.0 \%$ \\
\hline F-KNOWL & -- & .77 & Yes & $0 / 6$ & $0 / 6$ & $0 / 6$ & 100.0 \\
\hline T-KNOWL & - & .95 & Yes & $0 / 4$ & $0 / 4$ & $0 / 4$ & 100.0 \\
\hline P-KNOWL & -- & .86 & Yes & $0 / 3$ & $0 / 3$ & $0 / 3$ & 100.0 \\
\hline MISCONCEP & - & .78 & Yes & $0 / 6$ & $0 / 6$ & $0 / 6$ & 100.0 \\
\hline Attitudinal: & 53.8 & .83 & No & $1 / 26$ & $1 / 26$ & $0 / 26$ & 96.2 \\
\hline$\overline{\text { SUSCEPTIBILITY }}$ & Y -- & .73 & Yes & $0 / 5$ & $0 / 5$ & $0 / 5$ & 100.0 \\
\hline CU EFFICACY & -- & .92 & Yes & $0 / 7$ & $0 / 7$ & $0 / 7$ & 100.0 \\
\hline HOMOPHOBIA & - & .91 & No & $1 / 14$ & $1 / 14$ & $0 / 14$ & 92.9 \\
\hline Behavioral: & 83.6 & .87 & No & $6 / 47$ & $1 / 47$ & $5 / 47$ & 87.2 \\
\hline ASSERTIVENESS** & $* 71.8$ & .91 & Yes & $0 / 10$ & $0 / 10$ & $0 / 10$ & 100.0 \\
\hline SACU & - & .86 & Yes & $0 / 5$ & $0 / 5$ & $0 / 5$ & 100.0 \\
\hline SAPC & - & .93 & Yes & $0 / 5$ & $0 / 5$ & $0 / 5$ & 100.0 \\
\hline SUBSTANCE* & 41.5 & .86 & No & $2 / 23$ & $1 / 23$ & $1 / 23$ & 91.3 \\
\hline S-USE & -- & .81 & No & $1 / 8$ & $1 / 8$ & $0 / 8$ & 87.5 \\
\hline S-ABUSE & - & .83 & No & $1 / 15$ & $0 / 15$ & $1 / 15$ & 93.3 \\
\hline SEXUAL RISK** & 39.0 & .74 & No & $4 / 14$ & $0 / 14$ & $4 / 14$ & 71.4 \\
\hline PART-RISK HX & X -- & .63 & No & $2 / 7$ & $0 / 7$ & $2 / 7$ & 71.4 \\
\hline SELF-RISK HX & -- & .76 & No & $2 / 7$ & $0 / 7$ & $2 / 7$ & 71.4 \\
\hline Environment: & 53.1 & .79 & No & $2 / 26$ & $0 / 26$ & $2 / 26$ & 92.3 \\
\hline$\overline{\text { PEER-RISK }}$ & -- & .67 & No & $2 / 8$ & $0 / 8$ & $2 / 8$ & 75.0 \\
\hline PROXIMITY & -- & .83 & Yes & $0 / 8$ & $0 / 8$ & $0 / 8$ & 100.0 \\
\hline MEDIA-RES & - & .76 & Yes & $0 / 5$ & $0 / 5$ & $0 / 5$ & 100.0 \\
\hline MEDIA-INF & -- & .96 & Yes & $0 / 5$ & $0 / 5$ & $0 / 5$ & 100.0 \\
\hline Education: & 71.6 & .82 & No & $1 / 21$ & $0 / 21$ & $1 / 21$ & 95.2 \\
\hline$\overline{\mathrm{SCHOOL}}$ & 45.0 & .83 & No & $1 / 13$ & $0 / 13$ & $1 / 13$ & 92.3 \\
\hline SCHOOL-ED & -- & .73 & No & $1 / 8$ & $0 / 8$ & $1 / 8$ & 87.5 \\
\hline SCHOOL-INF & -- & .77 & Yes & $0 / 5$ & $0 / 5$ & $0 / 5$ & 100.0 \\
\hline HOME & 70.8 & .65 & Yes & $0 / 4$ & $0 / 4$ & $0 / 4$ & 100.0 \\
\hline PARENT-ED & -- & ingle item & Yes & $0 / 1$ & $0 / 1$ & $0 / 1$ & 100.0 \\
\hline PARENT-INF & -- & ingle item & Yes & $0 / 1$ & $0 / 1$ & $0 / 1$ & 100.0 \\
\hline SIB-ED & - & ingle item & Yes & $0 / 1$ & $0 / 1$ & $0 / 1$ & 100.0 \\
\hline SIB-INF & -- & ingle item & Yes & $0 / 1$ & $0 / 1$ & $0 / 1$ & 100.0 \\
\hline PEERS & 74.7 & .69 & Yes & $0 / 4$ & $0 / 4$ & $0 / 4$ & 100.0 \\
\hline PEER-ED & -- & ingle item & Yes & $0 / 1$ & $0 / 1$ & $0 / 1$ & 100.0 \\
\hline PEER-INF & -- & .71 & Yes & $0 / 3$ & $0 / 3$ & $0 / 3$ & 100.0 \\
\hline
\end{tabular}

Total: $10 / 139=92.8 \%$ overall consistency

\footnotetext{
* 150/284 substance users utilized in analyses.

** $164 / 284$ sexually active utilized in analyses.
} 


\section{Table 32}

Descriptive Statistics for Subscale Variables by Gender and Sexual Activity

\begin{tabular}{|c|c|c|c|c|c|c|c|c|c|c|c|c|c|c|c|c|c|c|}
\hline & \multicolumn{6}{|c|}{ Sexually Active Youth } & \multicolumn{6}{|c|}{ Sexually Nonactive Youth } & \multicolumn{6}{|c|}{ All Participants } \\
\hline & \multicolumn{2}{|c|}{$\begin{array}{c}\text { Young } \\
\text { Men } \\
(n=63)\end{array}$} & \multicolumn{2}{|c|}{$\begin{array}{l}\text { Young } \\
\text { Women } \\
(n=65)\end{array}$} & \multicolumn{2}{|c|}{$\begin{array}{l}\text { Total } \\
(n=128)\end{array}$} & \multicolumn{2}{|c|}{$\begin{array}{l}\text { Young } \\
\text { Men } \\
(n=19)\end{array}$} & \multicolumn{2}{|c|}{$\begin{array}{l}\text { Young } \\
\text { Women } \\
(n=92)\end{array}$} & \multicolumn{2}{|c|}{$\begin{array}{l}\text { Total } \\
(n=111)\end{array}$} & \multicolumn{2}{|c|}{$\begin{array}{c}\text { Young } \\
\text { Men } \\
(n=82)\end{array}$} & \multicolumn{2}{|c|}{$\begin{array}{l}\text { Young } \\
\text { Women } \\
(n=157)\end{array}$} & \multicolumn{2}{|c|}{$\begin{array}{l}\text { Total } \\
(n=239)\end{array}$} \\
\hline & $M$ & $S D$ & $M$ & $S D$ & $M$ & $S D$ & $M$ & $S D$ & $M$ & $S D$ & $M$ & $S D$ & $M$ & $S D$ & $M$ & $S D$ & $M$ & $S D$ \\
\hline & & & & & & & & & & & & & & & & & & \\
\hline & 4.2 & 0.9 & 4.6 & 0.4 & 4.4 & 0.7 & 4.4 & 0.6 & 4.6 & 0.5 & 4.6 & 0.5 & 4.2 & 0.8 & 4.6 & 0.5 & 4.5 & 0.6 \\
\hline$几$ & & 1.2 & & & & 1.4 & 3.4 & 1.3 & 3.6 & 1.6 & & 1.5 & 3.5 & 1.2 & 3.7 & 1.5 & 3.6 & 1.4 \\
\hline WL & 4.1 & 0.9 & 4.5 & & & 0.8 & 0 & 1.1 & 4.3 & 0.8 & & 0.9 & & 0.9 & 4.4 & 0.8 & 4.3 & 0.8 \\
\hline MISC & 2.8 & 1.1 & 1.9 & & & 1.1 & 1.5 & 0.5 & 1.7 & 0.7 & 1.7 & 0.7 & 2.5 & 1.2 & 1.8 & 0.8 & 2.0 & 1.0 \\
\hline \multicolumn{19}{|l|}{ Attitudinal: } \\
\hline$\overline{\text { SUSCEPT }}$ & 2.4 & 0.8 & & & & & 1.7 & 0.6 & 1.4 & & & 0.6 & 2.2 & 0.8 & 1.5 & 0.6 & 1.7 & 0.7 \\
\hline EFFI & & 1.1 & & & & & 6 & 1.4 & 4.2 & & 1 & 1.2 & .3 & 1.2 & 3.9 & 1.2 & .7 & 1.2 \\
\hline HOMOPH & 2.7 & 1.1 & 1.7 & & 2.2 & 1.0 & 1.8 & 0.6 & 1.7 & 0.7 & 1.7 & 0.7 & 2.5 & 1.1 & 1.7 & 0.7 & 2.0 & 0.9 \\
\hline \multicolumn{19}{|l|}{ Behavioral: } \\
\hline$\overline{\mathrm{SACU}}$ & 3.6 & 1.0 & & & & & 4.3 & 0.9 & 4.8 & & & & & 1.0 & 4.1 & & & 1.1 \\
\hline $\mathrm{AF}$ & & 1.0 & & & & & & & 4 & & & 0.8 & 8 & 1.0 & 3.9 & 3 & & 1.2 \\
\hline -IUS & 1.8 & 0.6 & 1 . & & & 0.6 & 3 & 0.3 & 1.2 & 0.3 & 1 & 0.3 & 1.7 & 0.6 & 1.3 & 0.4 & .4 & 0.5 \\
\hline $\mathrm{Al}$ & 1.7 & 0.7 & & & & 0.6 & & 0.4 & 1.2 & & & 0.3 & 1.6 & 0.6 & 1.2 & 0.4 & .3 & 0.5 \\
\hline SK & 6 & 0.4 & & & & & & & & & & & & 0.5 & 1.2 & 0.5 & 3 & 0.5 \\
\hline SELF & 2.8 & 0.7 & & & & & & 0.0 & 0.9 & & 0.9 & 0.0 & 2.4 & 1.0 & 1.4 & 0.7 & 1.8 & 1.0 \\
\hline \multicolumn{19}{|c|}{ Environment: } \\
\hline PEER-RISK & 2.9 & 0.7 & & & & & & & & & & & & 0.7 & 2.3 & & & \\
\hline TTY & 2.4 & 0.9 & 2.2 & & & & 1.6 & 0.7 & 1.7 & 0.9 & 7 & 0.9 & 2.2 & 0.9 & 1.9 & 1.0 & .0 & 1.0 \\
\hline & 2.8 & 1.0 & 2.8 & & & & 2.2 & 0.8 & 2.7 & 0.9 & 2.6 & 0.9 & 2.7 & 1.0 & 2.7 & 0.9 & .7 & 0.9 \\
\hline MEDIA-INF & 3.1 & 1.3 & 2.9 & 1.2 & 3.0 & 1.2 & 2.9 & 1.4 & 3.2 & 1.1 & 3.1 & 1.2 & 3.1 & 1.3 & 3.1 & 1.2 & 3.1 & 1.2 \\
\hline \multicolumn{19}{|l|}{ Education: } \\
\hline$\overline{\text { SCHOOL-ED }}$ & 3.1 & 0.7 & 3.3 & & & & 2.8 & & 3.1 & & & & 3.0 & 0.7 & 3.2 & 0.7 & 3.1 & 0.7 \\
\hline $\mathrm{CH}$ & 3.0 & 0.9 & 3.6 & & & & & 1.2 & 3.5 & 0 . & & 1 . & 3.0 & 1.0 & 3.5 & 0.9 & 3.3 & 1.0 \\
\hline PAR & 2.7 & 1.3 & 2.8 & & & & & 1.6 & 3.0 & 1.4 & 2.9 & & 2.7 & 1.4 & 2.9 & 4 & 2.8 & 1.4 \\
\hline PARENT-INF & 1.9 & 1.0 & 2.3 & 1.1 & 2.1 & 1.1 & 2.2 & 1.2 & 2.6 & 1.0 & 2.5 & 1.1 & 2.0 & 1.0 & 2.5 & 1.1 & 2.3 & 1.1 \\
\hline SIB-ED & 2.9 & 1.4 & 3.0 & & 2.9 & 1.4 & 2.5 & 1.7 & 3.0 & 1.3 & 2.9 & 1.4 & 2.8 & 1.5 & 3.0 & 1.3 & 2.9 & 1.4 \\
\hline SIB- & 1.8 & 1.0 & 1.7 & 0 . & 1. & 0 . & 1.3 & 0.8 & 1.6 & 0 . & 1.6 & 0.8 & 1.7 & 0.9 & 1.6 & 0.9 & 1.7 & 0.9 \\
\hline PEER-ED & 3.8 & 1.4 & 4.4 & 1. & 4.1 & 1. & 3.5 & 1.4 & 4.1 & 1.1 & 4.0 & 1.2 & 3.8 & 1.4 & 4.2 & 1.1 & 4.1 & 1.2 \\
\hline PEER-INF & 3.0 & 1.2 & 2.7 & 0.9 & 2.9 & 1.1 & 2.1 & 0.9 & 2.4 & 0.8 & 2.4 & 0.8 & 2.8 & 1.2 & 2.6 & 0.9 & 2.7 & 1.0 \\
\hline
\end{tabular}




\section{Table 33}

Age of Sexual Debut by Gender Reported by the Sexually Active Participants ( $n=128$ )

\begin{tabular}{|c|c|c|c|c|c|c|}
\hline & \multicolumn{2}{|c|}{ Young Men } & \multicolumn{2}{|c|}{ Young Women } & \multicolumn{2}{|c|}{ Total } \\
\hline & $n$ & $\%$ & $n$ & $\%$ & $n$ & $\%$ \\
\hline 10 years old & 7 & ( 11.1) & 1 & $(1.5)$ & 8 & $(6.3)$ \\
\hline 11 years old & 4 & $(6.3)$ & 2 & $(3.1)$ & 6 & $(4.7)$ \\
\hline 12 years old & 8 & $(12.7)$ & 4 & $(6.4)$ & 12 & $(9.4)$ \\
\hline 13 years old & 18 & $(28.6)$ & 10 & ( 15.4$)$ & 28 & (21.9) \\
\hline 14 years old & 18 & $(28.6)$ & 27 & $(41.5)$ & 45 & $(35.2)$ \\
\hline 15 years old & 5 & $(7.9)$ & 12 & $(18.5)$ & 17 & (13.3) \\
\hline 16 years old & 2 & $(3.2)$ & 6 & $(9.2)$ & 8 & $(6.3)$ \\
\hline 17 years old & 1 & $(1.6)$ & 3 & $(4.6)$ & 4 & $(3.1)$ \\
\hline Total active: & 63 & $(100.0)$ & 65 & $(100.0)$ & 128 & $(100.0)$ \\
\hline Mean age: & 13.0 & $(\underline{S D}=1.6)$ & 14.1 & $(\underline{S D}=1.4)$ & 13.6 & $(\underline{S D}=1.6)$ \\
\hline Nonactive & 19 & $(17.1)$ & 92 & ( 82.9) & 111 & $(100.0)$ \\
\hline
\end{tabular}




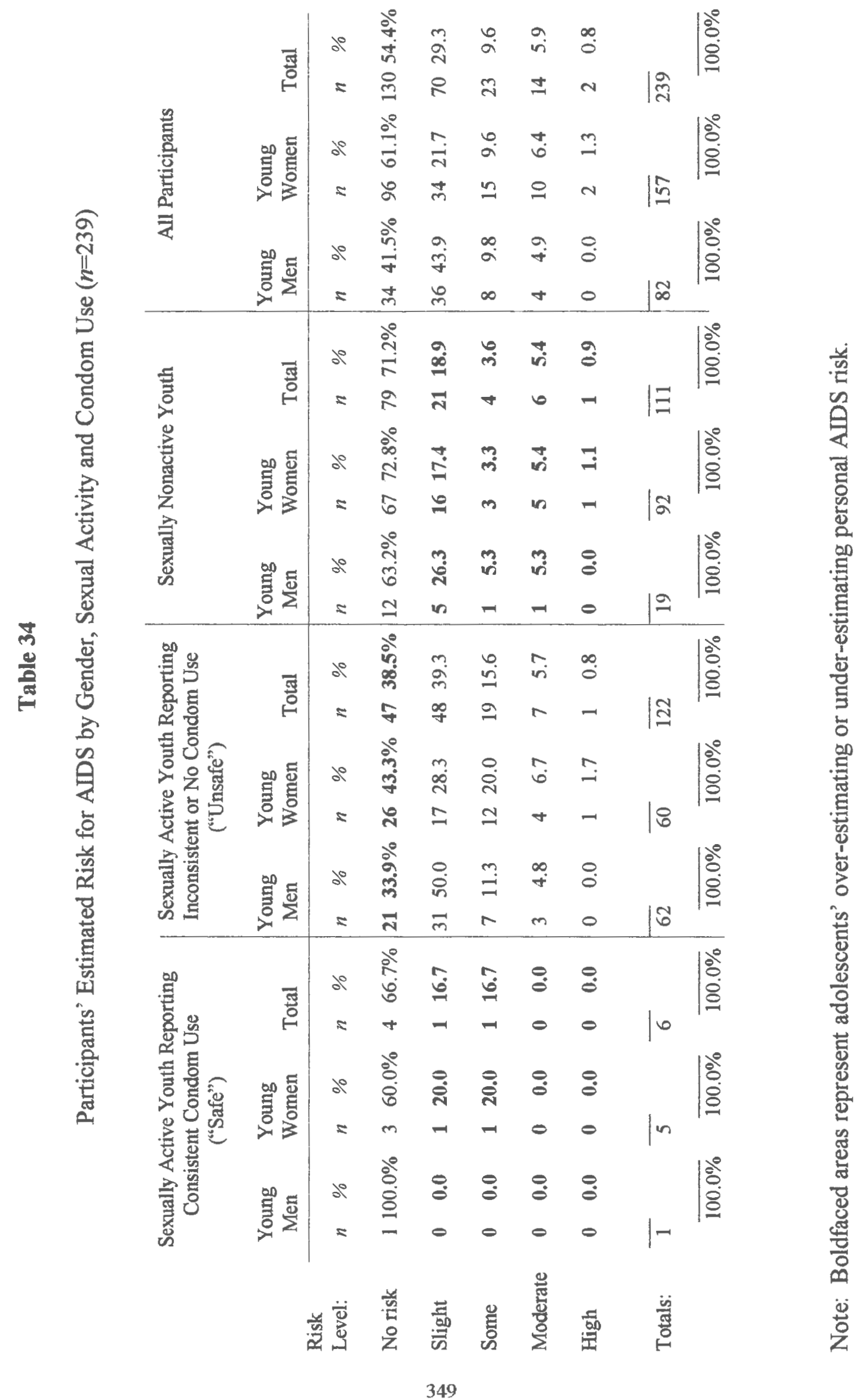


Multiple Regression $\beta$ Weights, and $s r^{2}$ Predictors of Sexual Assertiveness Among all Participants by Gender

\section{Young Men ( $n=82)$}

\begin{tabular}{|c|c|c|c|c|c|c|}
\hline Predictor Variable & Mean & $\begin{array}{l}\text { Standard } \\
\text { Deviation }\end{array}$ & $\begin{array}{l}\text { Correlation } \\
\text { (with DV) }\end{array}$ & n & $\underline{T}$ & $\begin{array}{c}s r^{2} \\
\text { (unique) }\end{array}$ \\
\hline Factual AIDS Knowledge & 4.23 & .79 & .35 & .033645 & .25 & .00 \\
\hline Transmission AIDS Knowledge & 3.49 & 1.23 & .23 & .037278 & .34 & .05 \\
\hline Perceived AIDS Knowledge & 4.06 & .92 & .34 & .318960 & $2.50 * *$ & .10 \\
\hline Misconceptions $^{\dagger}$ & 2.46 & 1.16 & $-.44^{* *}$ & -.242744 & -1.76 & .03 \\
\hline Perceived Susceptibility for Risk & 2.23 & .77 & -.34 & .085210 & .62 & .00 \\
\hline Homophobia & 2.50 & 1.05 & -.32 & -.081172 & -.63 & .03 \\
\hline Substance Use & 1.70 & .61 & -.34 & -.069336 & -.47 & .00 \\
\hline Substance Abuse & 1.56 & .63 & -.36 & -.231186 & -1.78 & .13 \\
\hline
\end{tabular}

\section{Young Women $(n=157)$}

\section{Predictor Variable}

Factual ADSS Knowledge

Transmission ADDS Knowledge

Perceived AIDS Knowledge

Misconceptions

Perceived Susceptibility for Risk

Homophobia

Substance Use

Substance Abuse

\section{Standard Correlation}

Mean

4.62

3.71

4.36

1.77

1.48

1.71

1.31

1.23

Standard Correlation

.10

$-.06$ (with DV) $\underline{\beta}$

.041823

$-.057033$

.004083

$-.82$

$\underline{\text { (unique) }}$

$\underline{R}=.56, \underline{R^{2}}=.31$, Adjusted $\underline{R^{2}}=.27, \underline{F}(8,148)=8.26, p<.001$.

${ }^{*} p<.001 ;{ }^{* *} p<.05$.

${ }^{\dagger}$ Post hoc significance only. 
Multiple Regression $\beta$ Weights, and $s r^{2}$ Predictors of AIDS Risk by Cognitive, Attitudinal and Behavioral Area, Among the Sexually Active Young Men $(n=63)$

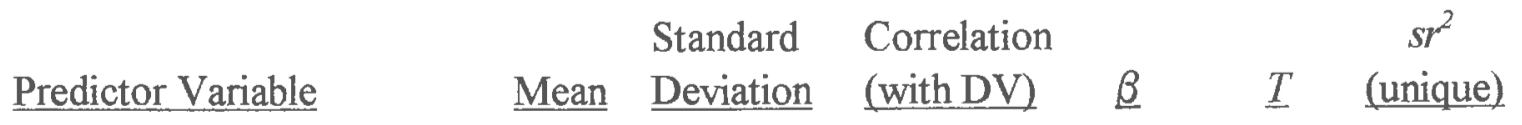

Cognitive MRA Results:

Factual AIDS Knowledge

$$
-.02
$$

092753

$.53 \quad .00$

Transmission AIDS Knowledge

3.52

1.20

.04

.044268

$.32 \quad .00$

Perceived AIDS Knowledge

4.01

.87

.05

.012953

$.08 \quad .01$

Misconceptions

2.75

1.14

.39

.429577

$3.29 * * \quad .15$

$\underline{R}=.40, \underline{R^{2}}=.16$, Adjusted $\underline{R^{2}}=.11, \underline{F}(4,58)=2.83, p<.05$.

Attitudinal MRA Results:

Perceived Susceptibility for Risk

Condom Use Efficacy

Homophobia

2.38

.76

.48

.440586

$3.46^{*}$

.15

$3.26 \quad 1.07$

2.70

1.07

$-.15$

.091375

.74

.196211

1.57

.02

$\underline{R}=.52, \underline{R^{2}}=.27$, Adjusted $\underline{R^{2}}=.23, \underline{F}(3,59)=7.17, p<.001$.

Behavioral MRA Results:

Sexual Assertiveness

$\begin{array}{lrrrrrr}\text { Partner Communication } & 3.72 & 1.01 & -.20 & -.034411 & -.37 & .02 \\ \text { Condom Use }^{\dagger} & 3.59 & .98 & -.48^{* *} & -.180178 & -1.75 & .06 \\ \text { Substance Use }^{\dagger} & 1.83 & .63 & .54^{* *} & -.125973 & -.97 & .04 \\ \text { Substance Abuse }^{\dagger} & 1.68 & .65 & .40^{* * *} & -.132459 & -1.33 & .16 \\ \text { Partner Risk History } & 1.63 & .41 & .66 & .302724 & 3.10^{* *} & .28 \\ \text { Self Risk History } & 2.80 & .70 & .76 & .667984 & 4.51^{*} & .12\end{array}$

$\underline{R}=.82, \underline{R^{2}}=.68$, Adjusted $\underline{R^{2}}=.64, \underline{F}(6,56)=19.35, p<.001$.

${ }^{*} p<.001 ; * * p<.01 ; * * * p<.05$.

${ }^{\dagger}$ Post hoc significance only. 
Multiple Regression $\beta$ Weights, and $s r^{2}$ Predictors of AIDS Risk by Cognitive, Attitudinal and Behavioral Area, Among the Sexually Active Young Women $(n=65)$

\section{Predictor Variable}

\begin{tabular}{|c|c|c|c|c|c|}
\hline & Standard & Correlation & & & $s r^{2}$ \\
\hline Mean & Deviation & (with DV) & $\underline{\beta}$ & $\underline{T}$ & (unique) \\
\hline
\end{tabular}

Cognitive MRA Results:

Factual ADS Knowledge

.41

3.83

1.47

$-.11$

$\begin{array}{rrr}-.105126 & -.83 & .01 \\ .128740 & 1.02 & .01 \\ .149557 & 1.20 & .02 \\ .151776 & 1.20 & .03\end{array}$

Perceived ADS Knowledge

4.51

.65

.19

.93

.15

.16

$.151776 \quad 1.20$

$\underline{R}=.27, \underline{R^{2}}=.07$, Adjusted $\underline{R^{2}}=.01, \underline{F}(4,60)=1.18, p=\mathrm{n} / \mathrm{s}$.

Attitudinal MRA Results:

$\begin{array}{lrrrrrr}\text { Perceived Susceptibility for Risk } & 1.62 & .61 & .39 & .315591 & 2.89^{* *} & .09 \\ \text { Condom Use Efficacy } & 3.58 & 1.19 & -.46 & -.381632 & -3.41^{*} & .21 \\ \text { Homophobia } & 1.71 & .61 & .14 & .044420 & .41 & .00 \\ \underline{R}=.55, \underline{R^{2}=.30, \text { Adjusted } \underline{R^{2}}=.27, \underline{F}(3,61)=8.87, p<.001 .} & \end{array}$

Behavioral MRA Results:

Sexual Assertiveness

$\begin{array}{lrrrrrr}\quad \text { Partner Communication } & 2.84 & 1.14 & -.25 & -.258856 & 1.81^{* * *} & .02 \\ \quad \text { Condom Use } & 3.09 & 1.24 & -.47 & -.448680 & -3.24^{* *} & .15 \\ \text { Substance Use } & 1.48 & .48 & .35 & .129751 & .90 & .01 \\ \text { Substance Abuse } & 1.32 & .45 & .35 & .087409 & .61 & .13 \\ \text { Partner Risk History }^{\dagger} & 1.66 & .54 & .45^{* *} & .218115 & 1.66 & .06 \\ \text { Self Risk History }^{\dagger} & 2.25 & .45 & .45^{* *} & .141667 & .98 & .02\end{array}$

$\underline{R}=.62, \underline{R^{2}}=.39$, Adjusted $\underline{R^{2}}=.32, \underline{F}(6,58)=6.05, p<.001$.

${ }^{*} p<.001 ;{ }^{* *} p<.01 ;{ }^{* * *} p<.05$.

${ }^{\dagger}$ Post hoc significance only. 


\section{Table 38}

Multiple Regression $\beta$ Weights, and $s r^{2}$ Predictors of Participants'

Self-rated Level of AIDS Risk by Cognitive, Attitudinal and Behavioral Area,

Among the Sexually Active Young Men $(n=63)$

\section{Predictor Variable}

Standard Correlation

$s r^{2}$

Mean Deviation (with DV) $\underline{\beta} \quad \underline{T} \quad \underline{\text { (unique) }}$

Cognitive MRA Results:

$\begin{array}{lrrrrrr}\text { Factual AIDS Knowledge } & 4.19 & .85 & .10 & .206869 & 1.12 & .02 \\ \text { Transmission AIDS Knowledge } & 3.52 & 1.20 & -.10 & -.158385 & -1.08 & .01 \\ \text { Perceived ADS Knowledge } & 4.06 & .87 & .08 & .006856 & .04 & .01 \\ \text { Misconceptions } & 2.75 & 1.14 & .10 & .120900 & .87 & .01\end{array}$

$\underline{R}=.22, \underline{R^{2}}=.05$, Adjusted $\underline{R^{2}}=-.02, \underline{F}(4,58)=.75, p=\mathrm{n} / \mathrm{s}$.

Attitudinal MRA Results:

Perceived Susceptibility for Risk

$2.38 \quad .76$

$\begin{array}{llll}.24 & .238400 & 1.65 & .05\end{array}$

Condom Use Efficacy

$3.26 \quad 1.07$

$-.10$

$-.015458-.11$

.00

Homophobia

$2.70 \quad 1.07$

$\begin{array}{lll}.09 & -.009695 & -.07\end{array}$

.01

$\underline{R}=.24, \underline{R^{2}}=.06$, Adjusted $\underline{R^{2}}=.01, \underline{F}(3,59)=1.21, p=\mathrm{n} / \mathrm{s}$.

Behavioral MRA Results:

Sexual Assertiveness

$\begin{array}{lrrrrrr}\text { Partner Communication } & 3.72 & 1.01 & .06 & .236393 & 1.63 & .00 \\ \text { Condom Use } & 3.59 & .98 & -.19 & -.279765 & -1.77 & .09 \\ \text { ubstance Use } & 1.83 & .63 & .26 & .261937 & 1.31 & .06 \\ \text { Substance Abuse } & 1.68 & .65 & -.03 & -.390664 & -2.56^{*} & .03 \\ \text { Partner Risk History } & 1.63 & .41 & .28 & .212333 & 1.42 & .03 \\ \text { Self Risk History } & 1.86 & .80 & .29 & .095951 & .42 & .02\end{array}$

$\underline{R}=.48, \underline{R^{2}}=.23$, Adjusted $\underline{R^{2}}=.15, \underline{F}(6,56)=2.84, p<.05$.

${ }^{*} p<.01$ 
Multiple Regression $\beta$ Weights, and $s r^{2}$ Predictors of Participants' Self-rated Level of AIDS Risk by Cognitive, Attitudinal and Behavioral Area, Among the Sexually Active Young Women $(n=65)$

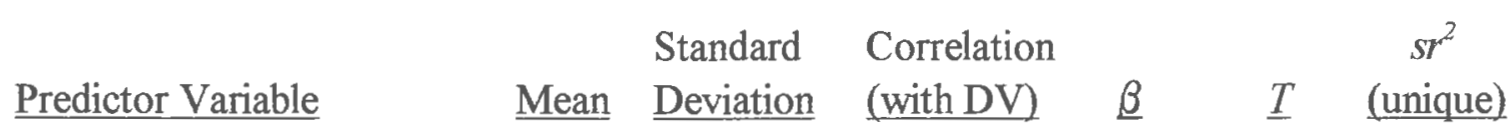

Cognitive MRA Results:

$\begin{array}{lrrrrrr}\text { Factual AIDS Knowledge } & 4.61 & .41 & -.04 & -.071436 & -.55 & .01 \\ \text { Transmission AIDS Knowledge } & 3.83 & 1.47 & .08 & .083721 & .65 & .00 \\ \text { Perceived ADS Knowledge } & 4.51 & .65 & -.02 & -.031089 & -.24 & .00 \\ \text { Misconceptions } & 1.85 & .93 & -.13 & -.139640 & -1.08 & .02\end{array}$

$\underline{R}=.17, \underline{R^{2}}=.03$, Adjusted $\underline{R^{2}}=-.04, \underline{F}(4,60)=.44, p=\mathrm{n} / \mathrm{s}$.

Attitudinal MRA Results:

$\begin{array}{lrrrrrr}\text { Perceived Susceptibility for Risk } & 1.62 & .61 & .22 & .207618 & 1.64 & .00 \\ \text { Condom Use Efficacy } & 3.58 & 1.19 & -.11 & -.086310 & -.66 & .06 \\ \text { Homophobia } & 1.71 & .61 & -.06 & -.079194 & -.62 & .00\end{array}$

$\underline{R}=.24, \underline{R^{2}}=.06$, Adjusted $\underline{R^{2}}=.01, \underline{F}(3,59)=1.21, p=\mathrm{n} / \mathrm{s}$.

Behavioral MRA Results:

Sexual Assertiveness

\begin{tabular}{|c|c|c|c|c|c|}
\hline Partner Communication & 2.84 & 1.14 & -.31 & -.041084 & -.28 \\
\hline Condom Use & 3.09 & 1.24 & -.25 & .010955 & .08 \\
\hline Substance Use ${ }^{\dagger}$ & 1.48 & .48 & $.50^{*}$ & .256720 & 1.76 \\
\hline Substance Abuse ${ }^{\dagger}$ & 1.32 & .45 & $.48^{*}$ & .163052 & 1.13 \\
\hline artner Risk History & 1.66 & .54 & .47 & .274513 & $2.06 * *$ \\
\hline Self Risk History ${ }^{\dagger}$ & 2.25 & .45 & $.43^{*}$ & .048231 & .33 \\
\hline
\end{tabular}

$\underline{R}=.61, \underline{R^{2}}=.37$, Adjusted $\underline{R^{2}}=.31, \underline{F}(6,58)=5.70, p<.001$.

${ }^{*} p<.01 ;{ }^{* *} p<.05$.

${ }^{\dagger}$ Post hoc significance only. 


\section{Table 40}

Multiple Regression $\beta$ Weights, and $s r^{2}$ Predictors of Participants'

Self-rated Level of AIDS Risk by Cognitive, Attitudinal and Behavioral Area,

Among the Sexually Active Participants Reporting

an Accurate Level of "Unsafe" Risk ( $n=75)$

Predictor Variable

Standard Correlation

$s r^{2}$

Mean Deviation (with DV) $\quad \underline{\beta} \quad \underline{T}$ (unique)

Cognitive MRA Results:

Factual ADS Knowledge

4.38

.59

3.71

1.27

$\begin{array}{lll}.16 & -.044925 & -.33\end{array}$

.03

Transmission AlDS Knowledge

4.33

.76

.13

$.055921 \quad .48$

.01

Perceived Alls Knowledge

2.50

1.20

$-.01$

$\begin{array}{ll}-.062345 & -.54\end{array}$

.00

Misconceptions

$-.40$

$-.415160-3.29 *$

.13

$\underline{R}=.41, \underline{R^{2}}=.17$, Adjusted $\underline{R^{2}}=.12, \underline{F}(4,70)=3.48, p<.01$.

Attitudinal MRA Results:

Perceived Susceptibility for Risk

Condom Use Efficacy

2.17

.78

$-.03$

$\begin{array}{lll}.125560 & .96 & .00\end{array}$

Homophobia

$3.28 \quad 1.09$

.01

$\begin{array}{ll}-.057187 & -.47\end{array}$

.00

$2.37 \quad 1.05$

$-.29$

$-.369956$

$-2.86^{* *} .10$

$\underline{R}=.32, \underline{R^{2}}=.10$, Adjusted $\underline{R^{2}}=.07, \underline{F}(3,71)=2.76, p<.05$.

Behavioral MRA Results:

Sexual Assertiveness

$\begin{array}{lrrrrrr}\quad \text { Partner Communication } & 3.20 & 1.12 & -.16 & .041956 & .27 & .02 \\ \text { Condom Use } & 3.17 & 1.11 & -.16 & -.126801 & -.79 & .00 \\ \text { Substance Use } & 1.85 & .59 & .08 & .329497 & 1.84 & .01 \\ \text { Substance Abuse } & 1.61 & .57 & .01 & -.042584 & -.27 & .01 \\ \text { Partner Risk History } & 1.81 & .48 & .16 & .203254 & 1.52 & .01 \\ \text { Self Risk History } & 2.76 & .66 & -.14 & -.438266 & -2.58 & .08\end{array}$

$\underline{R}=.36, \underline{R^{2}}=.13$, Adjusted $\underline{R^{2}}=.06, \underline{F}(6,68)=1.72, p=\mathrm{n} / \mathrm{s}$.

${ }^{*} p<.001 ; * * p<.01$. 


\section{Table 41}

MANOVA and Post Hoc ANOVA Results Across Cognitive, Attitudinal and Behavioral Areas by Gender and Accuracy Versus Underestimation in Participants' Self-rated Level of AIDS Risk Among the Sexually Active "Unsafe" Participants $(n=122)$

Gender

Pillais $\underline{F}(13,106)=9.48, p<.001 \quad$ Pillais $\underline{F}(13,106)=3.27, p<.001$
Self-rated Level of Risk

$\begin{array}{lccrrrrrrrr}\text { Factual ADS Knowledge } & 12.17^{*} & 4.06 & .87 & 4.53 & .64 & .07 & 4.33 & .76 & 4.23 & .86 \\ \text { Transmission AIDS Knowledge } & 1.72 & 4.18 & .86 & 4.59 & .42 & .14 & 4.38 & .59 & 4.39 & .86 \\ \text { Perceived AIDS Knowledge } & 12.70^{*} & 3.50 & 1.19 & 3.88 & 1.45 & 1.15 & 3.71 & 1.27 & 3.65 & 1.43 \\ \text { Misconceptions } & 18.08^{*} & 2.77 & 1.14 & 1.85 & .97 & 3.92^{* * *} 2.50 & 1.20 & 2.03 & 1.01 \\ & & & & & & & & & & \\ & & & & & & & & & & \\ \text { Perceived Susceptibility } & 30.71^{*} & 2.39 & .76 & 1.65 & .62 & 5.97^{* *} & 2.17 & .78 & 1.79 & .74 \\ \text { Condom Use Efficacy } & 2.16 & 3.23 & 1.05 & 3.52 & 1.20 & 1.16 & 3.28 & 1.09 & 3.54 & 1.19 \\ \text { Homophobia } & 30.64^{*} & 2.72 & 1.07 & 1.74 & .62 & 2.47 & 2.37 & 1.05 & 2.03 & .89 \\ & & & & & & & & & & \\ \text { Sexual Assertiveness } & & & & & & & & & & \\ \quad \text { Partner Communication } & 16.31^{*} & 3.70 & 1.01 & 2.84 & 1.14 & 1.97 & 3.20 & 1.12 & 3.40 & 1.21 \\ \quad \text { Condom Use } & 6.02^{* *} & 3.57 & .97 & 3.07 & 1.27 & 4.94^{* * *} 3.17 & 1.11 & 3.58 & 1.17 \\ \text { Substance Use } & 8.25^{* *} & 1.82 & .63 & 1.50 & .49 & 19.47^{*} & 1.85 & .59 & 1.38 & .46 \\ \text { Substance Abuse } & 12.52^{*} & 1.69 & .65 & 1.34 & .46 & 4.09^{* * *} 1.61 & .57 & 1.37 & .59 \\ \text { Partner Risk History } & .28 & 1.63 & .42 & 1.67 & .56 & 25.72^{*} & 1.81 & .48 & 1.39 & .40 \\ \text { Self Risk History } & 22.14^{*} & 2.80 & .71 & 2.26 & .45 & 26.84^{*} & 2.76 & .66 & 2.19 & .47\end{array}$

${ }^{*} p<.001 ;{ }^{* *} p<.01 ;{ }^{* * *} p<.05$. 


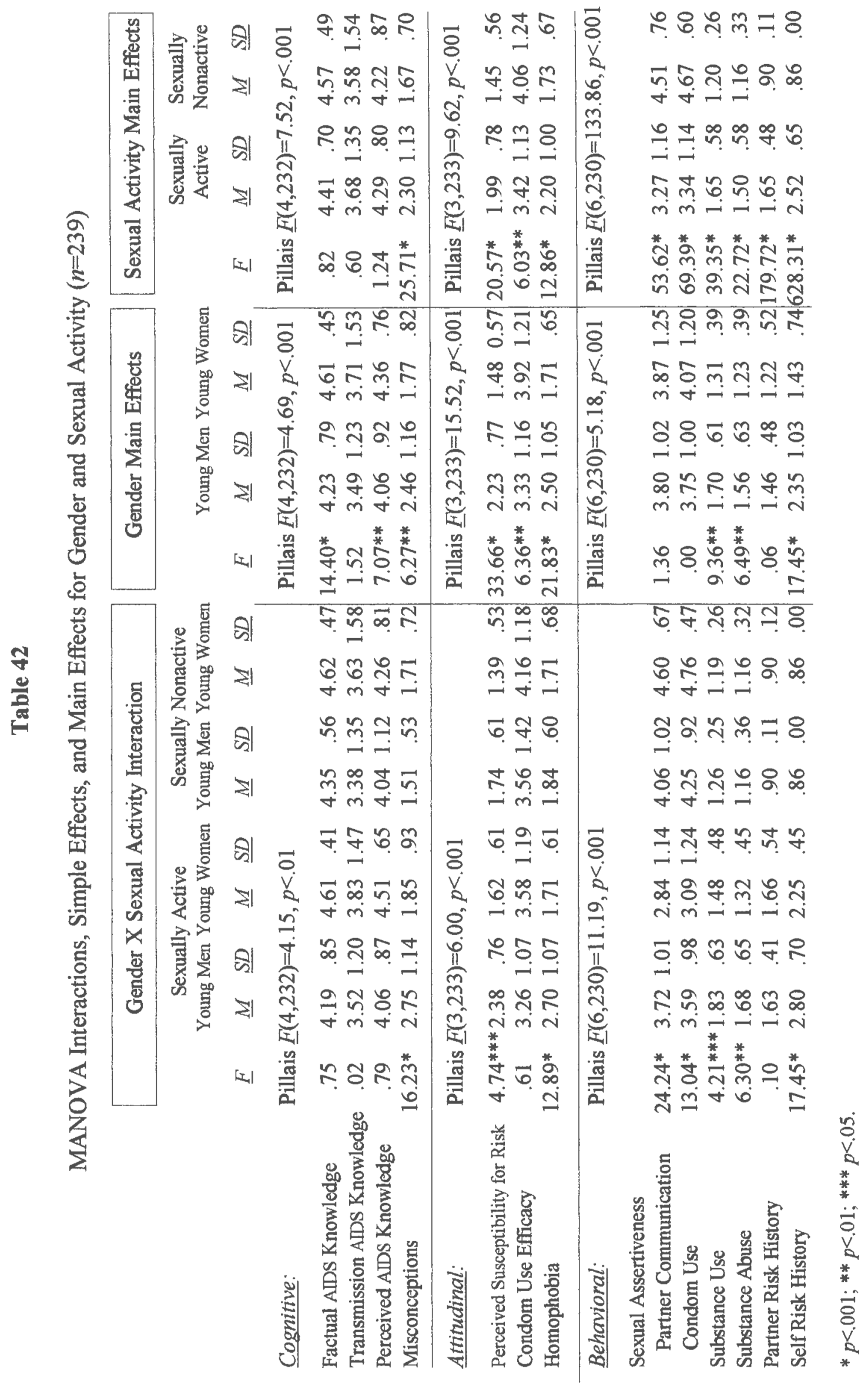




\section{Table 43}

MANOVA and Post Hoc ANOVA Results Across Cognitive, Attitudinal and Behavioral Areas by Gender Among the Sexually Active Participants $(n=128)$

$\begin{array}{cl}\begin{array}{c}\text { Young Men } \\ (n=63)\end{array} & \begin{array}{c}\text { Young Women } \\ (n=65)\end{array} \\ \text { Mean } S D & \text { Mean } S D\end{array}$

Cognitive:

Factual AIDS Knowledge

Transmission AIDS Knowledge

Perceived AIDS Knowledge

Misconceptions

\section{Attitudinal:}

Perceived Susceptibility for Risk

Condom Use Efficacy

Homophobia

\section{Behavioral:}

Sexual Assertiveness

Partner Communication

Condom Use

Substance Use

Substance Abuse

Partner Risk History

Self Risk History

$\begin{array}{rrrr}4.19 & .85 & 4.61 & .41 \\ 3.52 & 1.20 & 3.83 & 1.47 \\ 4.06 & .87 & 4.51 & .65 \\ 2.75 & 1.14 & 1.85 & .93\end{array}$

$2.38 \quad .76$

$3.26 \quad 1.07$

$2.70 \quad 1.07$
1.62

3.58

1.71

1.19

.61

MANOVA, Pillais: $\underline{F}(4,123)=8.39$ * Post hoc ANOVA: $\underline{F}(1,126)=12.89^{*}$ $\mathrm{n} / \mathrm{s}$ $\underline{F}(1,126)=10.75^{*}$ $\underline{F}(1,126)=24.05^{*}$

MANOVA, Pillais: $F(3,124)=21.68$ * Post hoc ANOVA: $\underline{F}(1,126)=39.96^{*}$ $\mathrm{n} / \mathrm{s}$ $\underline{F}(1,126)=41.95$ * MANOVA, Pillais: $\underline{F}(6,121)=13.21^{*}$ Post hoc ANOVA:

$\underline{F}(1,126)=6.50 * *$ $\underline{F}(1,126)=21.14$ * $\underline{F}(1,126)=12.59^{*}$ $\underline{F}(1,126)=13.54^{*}$ $\mathrm{n} / \mathrm{s}$ $\underline{F}(1,126)=28.36^{*}$

ANOVA only:

Sex Risk 320.18723 .75 $87.05 \quad 188.67$

Log Sex Risk

$$
\begin{array}{ll}
1.40 \quad 1.08
\end{array}
$$

$\underline{F}(1,126)=6.30^{* *}$ $\underline{F}(1,126)=3.71^{* * *}$

${ }^{*} p<.001 ;{ }^{* *} p<.01 ;{ }^{* * *} p<.05$. 
MANOVA and Post Hoc ANOVA Results Across Cognitive, Attitudinal and Behavioral Areas by Gender Among the Sexually Nonactive Participants ( $n=111)$

$$
\begin{array}{cc}
\begin{array}{c}
\text { Young Men } \\
(n=19)
\end{array} & \begin{array}{c}
\text { Young Women } \\
(n=92) \\
\text { Mean SD }
\end{array} \\
\text { Mean } S D
\end{array}
$$

Cognitive:

Factual AIDS Knowledge

$\begin{array}{ll}4.35 & .56\end{array}$

Transmission AIDS Knowledge

Perceived AIDS Knowledge

Misconceptions

\section{Attitudinal:}

Perceived Susceptibility for Risk

Condom Use Efficacy

Homophobia

\section{Behavioral:}

Sexual Assertiveness

Partner Communication Condom Use

Substance Use

Substance Abuse

Partner Risk History

Self Risk History

${ }^{*} p<.001 ;{ }^{* *} p<.01 ; * * * p<.05$.

$3.38 \quad 1.35$

4.04

1.51

1.12

.53

$\begin{array}{rrrr}1.74 & .61 & 1.39 & .53 \\ 3.56 & 1.42 & 4.16 & 1.18 \\ 1.84 & .60 & 1.71 & .68\end{array}$

4.62 .47

$3.63 \quad 1.58$

$4.26 \quad .81$

$1.71 \quad .72$

1.71

68

$\begin{array}{rrrr}4.06 & 1.02 & 4.60 & .67 \\ 4.25 & .92 & 4.76 & .47 \\ 1.26 & .25 & 1.19 & .26 \\ 1.16 & .36 & 1.16 & .32 \\ .90 & .11 & .90 & .12 \\ .86 & .00 & .86 & .00\end{array}$

$\underline{F}(1,109)=12.28^{*}$ $\underline{F}(1,109)=8.50 * *$ $\mathrm{n} / \mathrm{s}$ $\mathrm{n} / \mathrm{s}$ $\mathrm{n} / \mathrm{s}$ $\mathrm{n} / \mathrm{s}$

Significance

MANOVA, Pillais:

$\mathrm{n} / \mathrm{s}$

Post hoc ANOVA:

$\underline{F}(1,109)=4.71 * * *$

$\mathrm{n} / \mathrm{s}$

$\mathrm{n} / \mathrm{s}$

$\mathrm{n} / \mathrm{s}$

MANOVA, Pillais: $\underline{F}(3,107)=2.87 * * *$ Post hoc ANOVA: $E(1,109)=6.44^{* *}$ $\underline{F(1,109)}=3.73 * * *$ $\mathrm{n} / \mathrm{s}$

MANOVA, Pillais: $\underline{F}(5,105)=3.32 * *$ Post hoc ANOVA:

$F(1,109)=12.28 *$
$F(1,109)=8.50 * *$
$\mathrm{n} / \mathrm{s}$
$\mathrm{n} / \mathrm{s}$
$\mathrm{n} / \mathrm{s}$
$\mathrm{n} / \mathrm{s}$




\section{Table 45}

MANOVA and Post Hoc ANOVA Results Across Cognitive, Attitudinal and Behavioral Areas by Safe Versus Unsafe Sexual Activity

Among the Sexually Active Participants ( $n=128$ )

$\begin{array}{ccc}\begin{array}{c}\text { Safe } \\ (n=6)\end{array} & \begin{array}{c}\text { Unsafe } \\ (n=122)\end{array} & \\ \text { Mean } S D & \text { Mean } S D & \text { Significance }\end{array}$

Cognitive:

$\begin{array}{lrr}\text { Factual AIDS Knowledge } & 4.86 & .16 \\ \text { Transmission AIDS Knowledge } & 3.50 & 1.76 \\ \text { Perceived AIDS Knowledge } & 4.28 & .74 \\ \text { Misconceptions } & 1.83 & .28\end{array}$

\section{Attitudinal:}

Perceived Susceptibility for Risk $\quad 1.30 \quad .33$

Condom Use Efficacy

Homophobia

\section{Behavioral:}

Sexual Assertiveness

Partner Communication

Condom Use

Substance Use

Substance Abuse

Partner Risk History

Self Risk History $\begin{array}{ll}4.33 & .78\end{array}$

$\begin{array}{ll}1.32 & .19\end{array}$

2.03

3.38

$2.24 \quad 1.00$

$4.38 \quad .70$

$3.69 \quad 1.33$

$\begin{array}{ll}4.29 & .80\end{array}$

$2.32 \quad 1.15$

MANOVA, Pillais:

$\mathrm{n} / \mathrm{s}$

Post hoc ANOVA:

$\mathrm{n} / \mathrm{s}$

$\mathrm{n} / \mathrm{s}$

$\mathrm{n} / \mathrm{s}$

$\mathrm{n} / \mathrm{s}$

MANOVA, Pillais: $F(3,124)=2.78^{*}$ Post hoc ANOVA: $\underline{F}(1,126)=5.08^{*}$ $\underline{F}(1,126)=4.18^{*}$ $F(1,126)=4.99 *$

MANOVA, Pillais: $\mathrm{n} / \mathrm{s}$

Post hoc ANOVA:

$\begin{array}{rrrrr}3.17 & 1.48 & 3.28 & 1.15 & \mathrm{n} / \mathrm{s} \\ 3.57 & 1.13 & 3.33 & 1.15 & \mathrm{n} / \mathrm{s} \\ 1.31 & .45 & 1.67 & .59 & \mathrm{n} / \mathrm{s} \\ 1.12 & .20 & 1.52 & .59 & \mathrm{n} / \mathrm{s} \\ 1.67 & .34 & 1.65 & .49 & \mathrm{n} / \mathrm{s} \\ 2.12 & .42 & 2.54 & .65 & \mathrm{n} / \mathrm{s}\end{array}$

${ }^{*} p<.05$. 
MANOVA and Post Hoc ANOVA Results Across Cognitive, Attitudinal and Behavioral Areas by Sexual Activity $(n=239)$

$\begin{array}{cc}\begin{array}{c}\text { Sexually Active } \\ (n=128)\end{array} & \text { Sexually Nonactive } \\ (n=111) \\ \text { Mean SD } & \text { Mean } S D\end{array}$

Cognitive:

Factual AIDS Knowledge

Transmission AIDS Knowledge

Perceived AIDS Knowledge

Misconceptions

\section{Attitudinal:}

Perceived Susceptibility for Risk

Condom Use Efficacy

Homophobia

\section{Behavioral:}

Sexual Assertiveness

Partner Communication

Condom Use

Substance Use

Substance Abuse

Partner Risk History

Self Risk History

${ }^{*} p<.001 ; * * p<.01 ; * * * p<.05$.

$\begin{array}{rrrr}4.41 & .70 & 4.57 & .49 \\ 3.68 & 1.35 & 3.58 & 1.54 \\ 4.29 & .80 & 4.22 & .87 \\ 2.30 & 1.13 & 1.67 & .70\end{array}$

$\begin{array}{rrrr}1.99 & .78 & 1.45 & .56 \\ 3.42 & 1.13 & 4.06 & 1.24 \\ 2.20 & 1.00 & 1.73 & .67\end{array}$

$3.27 \quad 1.16$

$3.34 \quad 1.14$

$1.65 \quad .58$

$1.50 \quad .58$

$\begin{array}{ll}1.65 & .48\end{array}$

$2.52 \quad .65$
MANOVA, Pillais:

$\underline{F}(4,234)=7.70^{*}$

Post hoc ANOVA:

$\underline{F}(1,237)=4.38^{* * *}$

$\mathrm{n} / \mathrm{s}$

$\mathrm{n} / \mathrm{s}$

$\underline{F(1,237)}=25.41^{*}$

MANOVA, Pillais:

$\underline{F}(3,235)=15.62 *$

Post hoc ANOVA:

$F(1,237)=37.18^{*}$

$\underline{F}(1,237)=17.20^{*}$

$F(1,237)=17.48^{*}$

MANOVA, Pillais:

$\underline{F}(6,232)=176.64^{*}$

Post hoc ANOVA:

$\underline{F}(1,237)=121.75^{*}$

$\underline{F}(1,237)=92.10^{*}$

$\underline{F}(1,237)=56.36^{*}$

$F(1,237)=29.47^{*}$

$F(1,237)=255.34$ *

$\underline{F}(1,237)=724.65^{*}$ 
Current and Predicted Condom Use by Transtheoretical Model Staging Among the Sexually Active Adolescents by Gender $(n=128)$

\begin{tabular}{|c|c|c|c|c|c|c|c|c|c|c|}
\hline \multirow[t]{3}{*}{ Stage } & \multicolumn{5}{|c|}{ Current Reported Condom Use } & \multicolumn{5}{|c|}{ Predicted Condom Use Next 6-Mos } \\
\hline & \multicolumn{2}{|c|}{ Young men $Y$} & \multicolumn{2}{|c|}{ Young women } & \multirow{2}{*}{$\begin{array}{l}\text { Total } \\
n \quad \%\end{array}$} & \multicolumn{2}{|c|}{ Young men } & \multicolumn{2}{|c|}{ Young women } & Total \\
\hline & $n$ & $\%$ & $n$ & $\%$ & & $n$ & $\%$ & $n$ & $\%$ & \\
\hline Precontemplation & 9 & $14.3 \%$ & 15 & $23.1 \%$ & $2418.8 \%$ & 12 & $19.0 \%$ & 1 & $1.5 \%$ & $3.1 \%$ \\
\hline Contemplation & 36 & 57.1 & 29 & 44.6 & 6550.8 & 20 & 31.7 & 19 & 29.2 & 4736.7 \\
\hline Action & 6 & 9.5 & 6 & 9.2 & 129.4 & 28 & 44.4 & 27 & 41.5 & 4736.7 \\
\hline \multirow[t]{2}{*}{ Maintenance } & 12 & 19.0 & 15 & 23.1 & 2721.1 & 3 & 4.8 & 18 & 27.7 & 3023.4 \\
\hline & - & - & - & - & -- & - & - & - & $\longrightarrow$ & $-\longrightarrow$ \\
\hline Totals & 63 & 100.0 & 65 & 100.0 & 128100.0 & 63 & 100.0 & 65 & 100.0 & 128100.0 \\
\hline
\end{tabular}


过

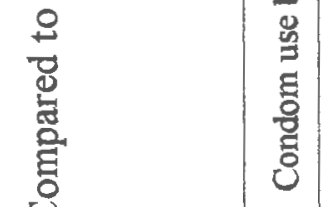

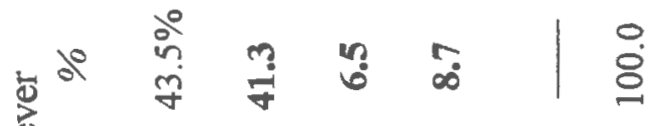

总 $=$ त $9 m+1$ 字

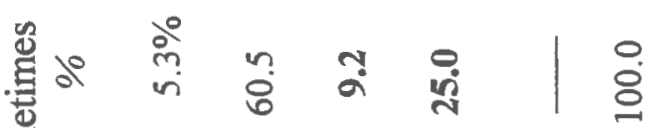

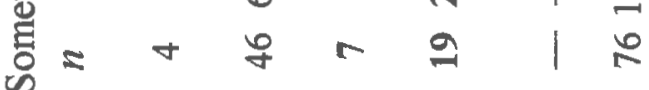

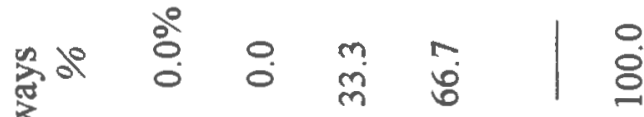

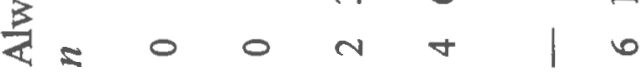

:

تี

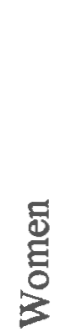

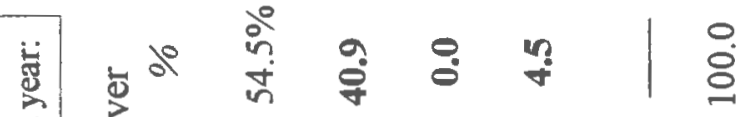

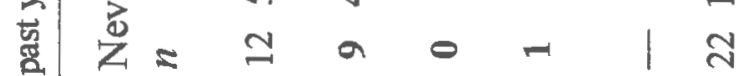

苋

을

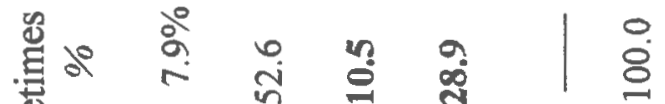

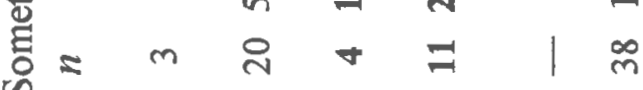

\&

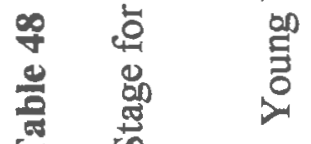

호

들

8

惫

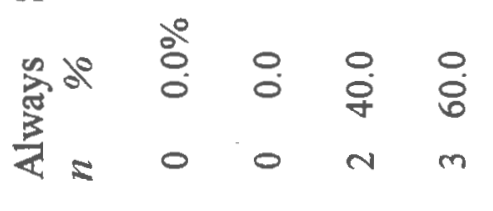

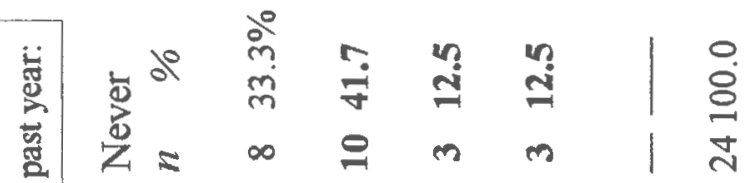

0
8
in

. 률

올

E

莺 焉

东

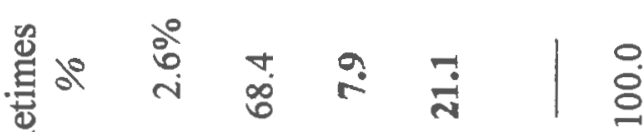

宓

is

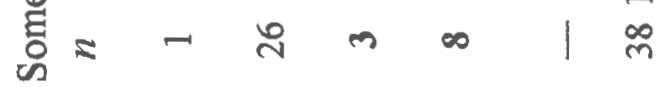

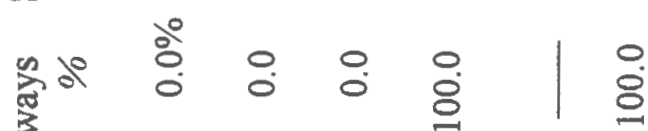

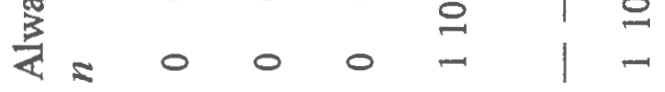

总

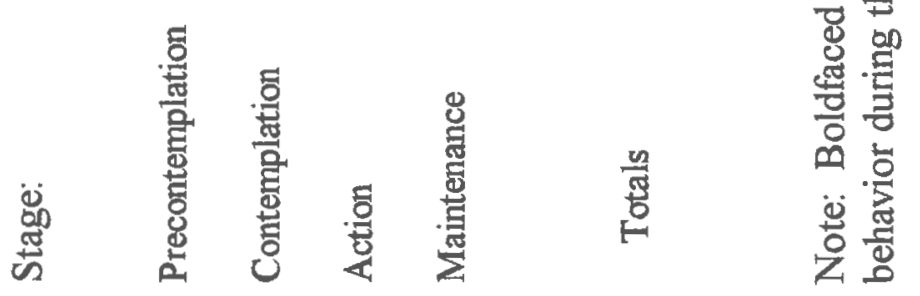




\section{Current Condom Use by Transtheoretical Model Staging Comparisons by Cognitive, Attitudinal and Behavioral Risk Variables \\ Across the Sexually Active Participants $(n=128)$}

$\begin{array}{cccc}\begin{array}{c}\text { Precontemplation } \\ (n=24)\end{array} & \begin{array}{c}\text { Contemplation } \\ (n=65)\end{array} & \begin{array}{c}\text { Action } \\ (n=12)\end{array} & \begin{array}{c}\text { Maintenance } \\ (n=27)\end{array} \\ \text { Mean } S D & \text { Mean SD } & \text { Mean } S D & \text { Mean } S D\end{array}$

\section{Cognitive:}

Factual Knowl

Transm Knowl

$$
4.49 \quad .53
$$

$\begin{array}{ll}4.26 & .70\end{array}$

4.53

$3.72 \quad 1.12$

$3.25 \quad 1.56$

Perceived Knowl

$3.22 \quad 1.55$

$4.28 \quad .73$

$4.19 \quad .69$

Misconceptions

$1.86 \quad .90$

$2.25 \quad 1.05$

\section{Attitudinal:}

$\begin{array}{lll}\text { Perceived Suscept } & 1.76 \quad .95\end{array}$

$\begin{array}{lll}\text { Condom Efficacy } & 3.37 \quad 1.27\end{array}$

Homophobia

$2.15 \quad .84$

$2.15 \quad .70$

$\begin{array}{ll}1.78 \quad .65 \\ 3.69 & .84\end{array}$

$\begin{array}{llll}3.01 & 1.02 & 3.69 & .84\end{array}$

$\begin{array}{llll}2.44 & 1.10 & 1.91 & .86\end{array}$

Behavioral:

Sexual Assert.

\begin{tabular}{lrr}
\hline P Communicat'n & 3.31 & 1.28 \\
Condom Use & 3.53 & 1.27 \\
Substance Use & 1.43 & .60 \\
Substance Abuse & 1.27 & .48 \\
Partner Risk Hx & 1.46 & .50 \\
Self Risk Hx & 2.32 & .45
\end{tabular}

$\begin{array}{ll}3.05 & 1.09\end{array}$

$3.18 \quad 1.18$

$3.02 \quad 1.05$

$3.27 \quad .96$

$1.75 \quad .56$

1.58

$1.57 \quad .52$

1.66

$1.71 \quad .49$

1.60

$2.71 \quad .71$

\section{Sex Risk}

$$
\begin{array}{lllll}
116.96 & 380.33 & 316.40 & 687.81
\end{array}
$$

$\begin{array}{llll}.70 & .97 & 1.56 & 1.03\end{array}$

Log Sex Risk

$37.92 \quad 83.77$

$.93 \quad .81$
1.93

$4.33 \quad .84 \quad \underline{F}(3,124)=11.00^{*}$

$1.78 \quad .76 \quad \underline{F}(3,124)=3.41^{* * *}$

MANOVA, Pillais: $\underline{F}(18,363)=2.33 * *$ Post hoc ANOVA:
3.821 .09

$3.97 \quad 1.08$

$\begin{array}{ll}1.61 & .67\end{array}$

$1.46 \quad .67$

$1.68 \quad .47$

$2.26 \quad .61$
MANOVA, Pillais: $\underline{F}(12,369)=2.96^{*}$ Post hoc ANOVA: $\mathrm{n} / \mathrm{s}$ $\underline{F}(3,124)=2.64^{* * *}$ $\mathrm{n} / \mathrm{s}$ $\underline{F}(3,124)=7.44^{*}$

MANOVA, Pillais: $\underline{F}(9,372)=4.11^{*}$ Post hoc ANOVA: $\mathrm{n} / \mathrm{s}$ $F(3,124)=2.92^{* * *}$ $\underline{F}(3,124)=5.19^{* *}$ $\mathrm{n} / \mathrm{s}$ $\mathrm{n} / \mathrm{s}$ $\mathrm{n} / \mathrm{s}$ $\underline{F}(3,124)=4.28^{* *}$

ANOVA only:

$74.11198 .32 \mathrm{n} / \mathrm{s}$ $.99 \quad .89 \quad \underline{F}(3,124)=5.83^{*}$

${ }^{*} p<.001 ;{ }^{* *} p<.01 ;{ }^{* * *} p<.05$. 


\section{Table 50}

Predicted Future Condom Use by Transtheoretical Model Staging Comparisons by Cognitive, Attitudinal and Behavioral Risk Variables

Across the Sexually Active Young Men $(n=63)$

$\begin{array}{cccc}\begin{array}{c}\text { Precontemplation } \\ (n=12)\end{array} & \begin{array}{c}\text { Contemplation } \\ (n=20)\end{array} & \begin{array}{c}\text { Action } \\ (n=28)\end{array} & \begin{array}{c}\text { Maintenance } \\ (n=3)\end{array} \\ \text { Mean SD } & \text { Mean SD } & \text { Mean SD } & \text { Mean } S D\end{array}$

Mean $S D$ Mean $S D$ Mean $S D$ Mean $S D$ Significance

Cognitive:

MANOVA, Pillais:

$\underline{F}(12,174)=1.85^{* * *}$

Post hoc ANOVA:

$\begin{array}{ll}3.83 & .87\end{array}$

$4.23 \quad .58$

$4.10 \quad 1.00$

$4.35 \quad 1.15$

$\mathrm{n} / \mathrm{s}$

Transm Knowl $\quad 2.42 \quad 1.67$

$3.37 \quad 1.81$

$3.39 \quad 1.05$

$4.40 \quad .99 \quad \underline{F}(3,59)=3.64^{* * *}$

Perceived Knowl $3.11 \quad 1.26$

$4.19 \quad .76$

$3.98 \quad .75$

$\begin{array}{lll}4.14 & 1.11 \mathrm{n} / \mathrm{s}\end{array}$

$\begin{array}{lll}\text { Misconceptions } \quad 3.00 & 1.61\end{array}$

$\begin{array}{llll}2.82 & 1.56 & 3.13 & 1.03\end{array}$

$1.92 \quad .80$

$\underline{F}(3,59)=3.32^{* * *}$

MANOVA, Pillais:

Atritudinal:

$F(9,177)=2.45^{* *}$

Post hoc ANOVA:

Perceived Suscept $3.20 \quad 1.51$

$\begin{array}{llll}2.29 & .80 & 2.47 & .52\end{array}$

2.23

$\mathrm{n} / \mathrm{s}$

Condom Efficacy $2.52 \quad 1.09$

$\begin{array}{llll}2.93 & .98 & 3.29 & .91\end{array}$

$4.18 \quad 1.02$

$F(3,59)=5.26^{* *}$

Homophobia

$2.62 \quad 1.28$

$\begin{array}{llll}2.91 & 1.12 & 2.71 & 1.06\end{array}$

$2.23 \quad .88 \mathrm{n} / \mathrm{s}$

MANOVA, Pillais: $F(18,168)=2.43 * *$

Post hoc ANOVA:

Sexual Assert.

$\begin{array}{lrrrrrrrrl}\text { P Communicat'n } & 3.00 & 1.25 & 3.59 & 1.10 & 3.81 & .90 & 4.05 & .90 & \mathrm{n} / \mathrm{s} \\ \text { Condom Use } & 3.87 & .12 & 3.64 & .86 & 3.26 & 1.08 & 3.98 & 1.06 & \mathrm{n} / \mathrm{s} \\ \text { Substance Use } & 2.08 & .96 & 1.89 & .64 & 1.81 & .53 & 1.65 & .73 & \mathrm{n} / \mathrm{s} \\ \text { Substance Abuse } & 1.80 & .50 & 1.72 & .65 & 1.57 & .47 & 1.76 & .95 & \mathrm{n} / \mathrm{s} \\ \text { Partner Risk Hx } & 2.21 & .81 & 1.54 & .34 & 1.58 & .31 & 1.80 & .50 & \underline{F}(3,59)=3.74^{* *} \\ \text { Self Risk Hx } & 3.14 & 1.29 & 2.80 & .70 & 2.90 & .64 & 2.54 & .67 & \mathrm{n} / \mathrm{s}\end{array}$

$\begin{array}{lllllllll}\text { Sex Risk } \quad 1320.67 & 1094.00 & 304.82 & 788.08 & 304.70 & 686.86 & 131.67 & 287.48 & \mathrm{n} / \mathrm{s}\end{array}$

ANOVA only:

$\begin{array}{llllllllll}\log \text { Sex Risk } & 2.81 & .83 & 1.28 & 1.06 & 1.42 & 1.15 & 1.29 & .92 & \mathrm{n} / \mathrm{s}\end{array}$

${ }^{*} p<.001 ;{ }^{* *} p<.01 ;{ }^{* * *} p<.05$. 


\section{Table 51}

Predicted Future Condom Use by Transtheoretical Model Staging Comparisons by Cognitive, Attitudinal and Behavioral Risk Variables

Across the Sexually Active Young Women $(n=65)$

$\begin{array}{cccc}\begin{array}{c}\text { Precontemplation } \\ (n=1)\end{array} & \begin{array}{c}\text { Contemplation } \\ (n=19)\end{array} & \begin{array}{c}\text { Action } \\ (n=27)\end{array} & \begin{array}{c}\text { Maintenance } \\ (n=18)\end{array} \\ \text { Mean } S D & \text { Mean } S D & \text { Mean } S D & \text { Mean } S D\end{array}$

Cognitive:

MANOVA, Pillais:

$\mathrm{n} / \mathrm{s}$

Post hoc ANOVA:

$\begin{array}{lrrrrrrrrr}\text { Factual Knowl } & 5.00 & .00 & 4.58 & .36 & 4.54 & .43 & 4.74 & .43 & \mathrm{n} / \mathrm{s} \\ \text { Transm Knowl } & 4.00 & .00 & 3.97 & 1.30 & 3.83 & 1.41 & 3.65 & 1.81 & \mathrm{n} / \mathrm{s} \\ \text { Perceived Knowl } & 4.00 & .00 & 4.53 & .76 & 4.47 & .53 & 4.57 & .74 & \mathrm{n} / \mathrm{s} \\ \text { Misconceptions } & 1.67 & .00 & 1.83 & .96 & 2.03 & 1.04 & 1.63 & .75 & \mathrm{n} / \mathrm{s}\end{array}$

\section{Attitudinal:}

Perceived Suscept $2.20 \quad .00$

$\begin{array}{llll}1.65 & .57 & 1.60 & .58\end{array}$

$1.57 \quad .71$

$\begin{array}{llll}3.53 & 1.16 & 3.11 & 1.28\end{array}$

$4.29 \quad .65$

Condom Efficacy

$4.14 \quad .00$

$\begin{array}{llll}1.59 & .48 & 1.93 & .66\end{array}$

$1.52 \quad .58$

\section{Behavioral:}

$.14 \quad .00$

Sexual Assert.

\begin{tabular}{lrrrrrrrrr}
\multicolumn{1}{|c}{ P Communicat'n } & 4.40 & .00 & 2.73 & .94 & 2.59 & 1.21 & 3.24 & 1.15 & $\mathrm{n} / \mathrm{s}$ \\
Condom Use & 4.00 & .00 & 2.78 & 1.17 & 2.93 & 1.29 & 3.61 & 1.16 & $\mathrm{n} / \mathrm{s}$ \\
Substance Use & 1.50 & .00 & 1.47 & .50 & 1.48 & .49 & 1.48 & .49 & $\mathrm{n} / \mathrm{s}$ \\
Substance Abuse & 1.00 & .00 & 1.32 & .48 & 1.33 & .42 & 1.32 & .49 & $\mathrm{n} / \mathrm{s}$ \\
Partner Risk Hx & 1.86 & .00 & 1.64 & .63 & 1.81 & .56 & 1.46 & .36 & $\mathrm{n} / \mathrm{s}$ \\
Self Risk Hx & 2.18 & .00 & 2.22 & .44 & 2.39 & .47 & 2.06 & .40 & $\mathrm{n} / \mathrm{s}$
\end{tabular}

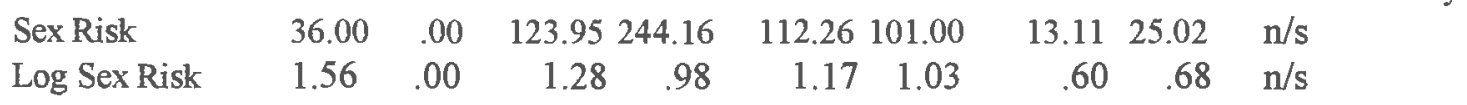

${ }^{*} p<.001 ;{ }^{* *} p<.01 ;{ }^{* * *} p<.05$. 


\section{Table 52}

Predicted Condom Use by Transtheoretical Model Staging Among the Sexually Nonactive Adolescents by Gender $(n=111)$

Stage

Predicted Condom Use Next 6-Mos.

\begin{tabular}{lccccccc} 
& \multicolumn{2}{c}{ Young men } & \multicolumn{2}{c}{ Young women } & \multicolumn{2}{c}{ Total } \\
& $n$ & $\%$ & $n$ & $\%$ & $n$ & $\%$ \\
Not applicable * & 14 & $73.7 \%$ & 82 & $89.1 \%$ & 96 & $86.5 \%$ \\
Precontemplation & 0 & 0.0 & 2 & 2.2 & 2 & 1.8 \\
Contemplation & 5 & 26.4 & 8 & 8.7 & 13 & 11.7 \\
Action & 0 & 0.0 & 0 & 0.0 & 0 & 0.0 \\
Maintenance & 0 & 0.0 & 0 & 0.0 & 0 & 0.0 \\
\cline { 2 - 3 } & 19 & 100.0 & 92 & 100.0 & 111 & 100.0
\end{tabular}

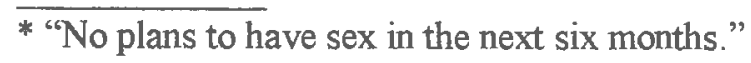




\section{Table 53}

Focus Group Tally for Study Participation with School Sample $(n=600)$

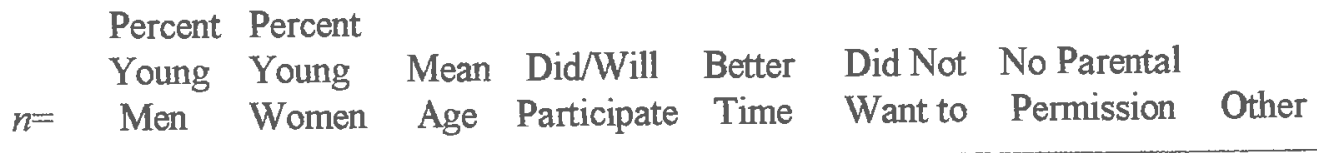

$\begin{array}{llllllllll}\text { Grade 7: } & 116 & 47.4 \% & 52.6 \% & 12.8 & 24 & 20 & 45 & 8 & 35\end{array}$

$\begin{array}{llllllllll}\text { Grade } 8: & 78 & 52.6 & 47.4 & 13.1 & 13 & 13 & 34 & 7 & 21\end{array}$

$\begin{array}{llllllllll}\text { Grade 9: } & 136 & 48.5 & 51.5 & 14.1 & 18 & 31 & 72 & 3 & 17\end{array}$

$\begin{array}{llllllllll}\text { Grade 10: } & 119 & 43.7 & 56.3 & 15.0 & 34 & 21 & 35 & 8 & 23\end{array}$

$\begin{array}{lllllllll}\text { Grade 11: } & 119 & 49.6 & 50.4 & 16.1 & 31 & 25 & 48 & 2\end{array}$

$\begin{array}{llllllllll}\text { Grade 12: } & 32 & 40.6 & 59.4 & 17.1 & 4 & 9 & 17 & 1 & 2\end{array}$

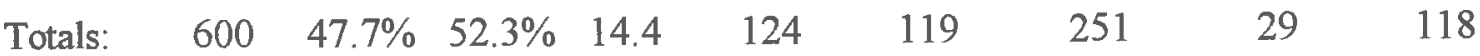




\section{Table 54}

Focus Group Demographics and

Participant Feedback for School Youth Sample ( $n=121)$

\begin{tabular}{lcccc} 
& Day 1 & Day 2 & Day 3 & Total \\
\cline { 2 - 5 } & 8 & 7 & 7 & 22 \\
Number of groups held & 46 & 46 & 29 & 121 \\
Total number of participants & 5.75 & 6.57 & 4.14 & 5.5 \\
Mean number of participants per group & $1-18$ & $1-18$ & $1-7$ & $1-18$ \\
Range of participants per group & $13-17$ & $13-18$ & $13-16$ & $13-18$ \\
Age range & 14.1 & 14.1 & 14.0 & 14.1 \\
Mean age & $13.0 \%$ & $19.6 \%$ & $24.1 \%$ & $18.2 \%$ \\
Percent participants young men & 87.0 & 80.4 & 75.9 & 81.8 \\
Percent participants young women & 100.0 & 23.9 & 3.4 & - \\
Percent first group attended & -- & 76.1 & 20.7 & - \\
Percent second group attended & - & -- & 75.9 & - \\
Percent third group attended & 37.0 & 45.7 & 48.3 & 43.0 \\
Percent grade 7 & 8.7 & 6.5 & 10.3 & 8.3 \\
Percent grade 8 & 19.6 & 4.3 & 10.3 & 11.6 \\
Percent grade 9 & 23.9 & 37.0 & 20.7 & 28.1 \\
Percent grade 10 & 8.7 & 4.3 & 6.9 & 6.6 \\
Percent grade 11 & 2.2 & 2.2 & 3.4 & 2.5 \\
Percent grade 12 & & & &
\end{tabular}

Focus Group Checklist Feedback (percent checked):

$\begin{array}{lcccc}\text { Interesting } & 80.4 \% & 80.4 \% & 82.8 \% & 81.0 \% \\ \text { Boring } & 2.2 & 0.0 & 0.0 & 0.8 \\ \text { Worthwhile } & 43.5 & 60.9 & 62.1 & 54.5 \\ \text { Helpful } & 52.2 & 45.7 & 48.3 & 48.8 \\ \text { Upsetting } & 2.2 & 8.7 & 0.0 & 4.1 \\ \text { Dumb } & 0.0 & 0.0 & 0.0 & 0.0 \\ \text { Easy } & 13.9 & 10.9 & 13.8 & 12.4 \\ \text { Difficult } & 2.2 & 2.2 & 13.8 & 5.0 \\ \text { Fun } & 23.9 & 34.8 & 17.2 & 26.4 \\ \text { Depressing } & 0.0 & 4.3 & 0.0 & 1.7 \\ \text { Important } & 47.8 & 65.2 & 55.2 & 56.2 \\ \text { Silly } & 0.0 & 2.2 & 3.4 & 1.7 \\ \text { Just Okay } & 15.2 & 13.0 & 3.4 & 11.6 \\ \text { Other } & 21.7 & 17.4 & 6.9 & 21.5\end{array}$




\section{Table 55}

Survey Feedback Checklist Responses for School Youth Sample $(n=92)$

Survey Feedback Participants:

Young Young Mean

Men Women Age Grade 7 Grade 8 Grade 9 Grade 10 Grade 11 Grade 12

$\begin{array}{lllllllll}16.3 \% & 83.7 \% & 14.1 & 41.3 \% & 7.6 \% & 12.0 \% & 30.4 \% & 6.5 \% & 2.2 \%\end{array}$

Survey Checklist Indicators: $\quad$ Percent (checked)

Interesting

Boring

Worthwhile

Helpful

Upsetting

Dumb

Easy

Difficult

Fun

Depressing

Important

Silly

Just Okay

Other
$71.7 \%$

3.3

43.5

39.1

1.1

3.3

35.9

5.4

18.5

2.2

46.7

5.4

12.0

18.5 
Figure 1

Questionnaire Scale and Subscale Breakdown by Exploratory and Confirmatory Factor Analyses Results

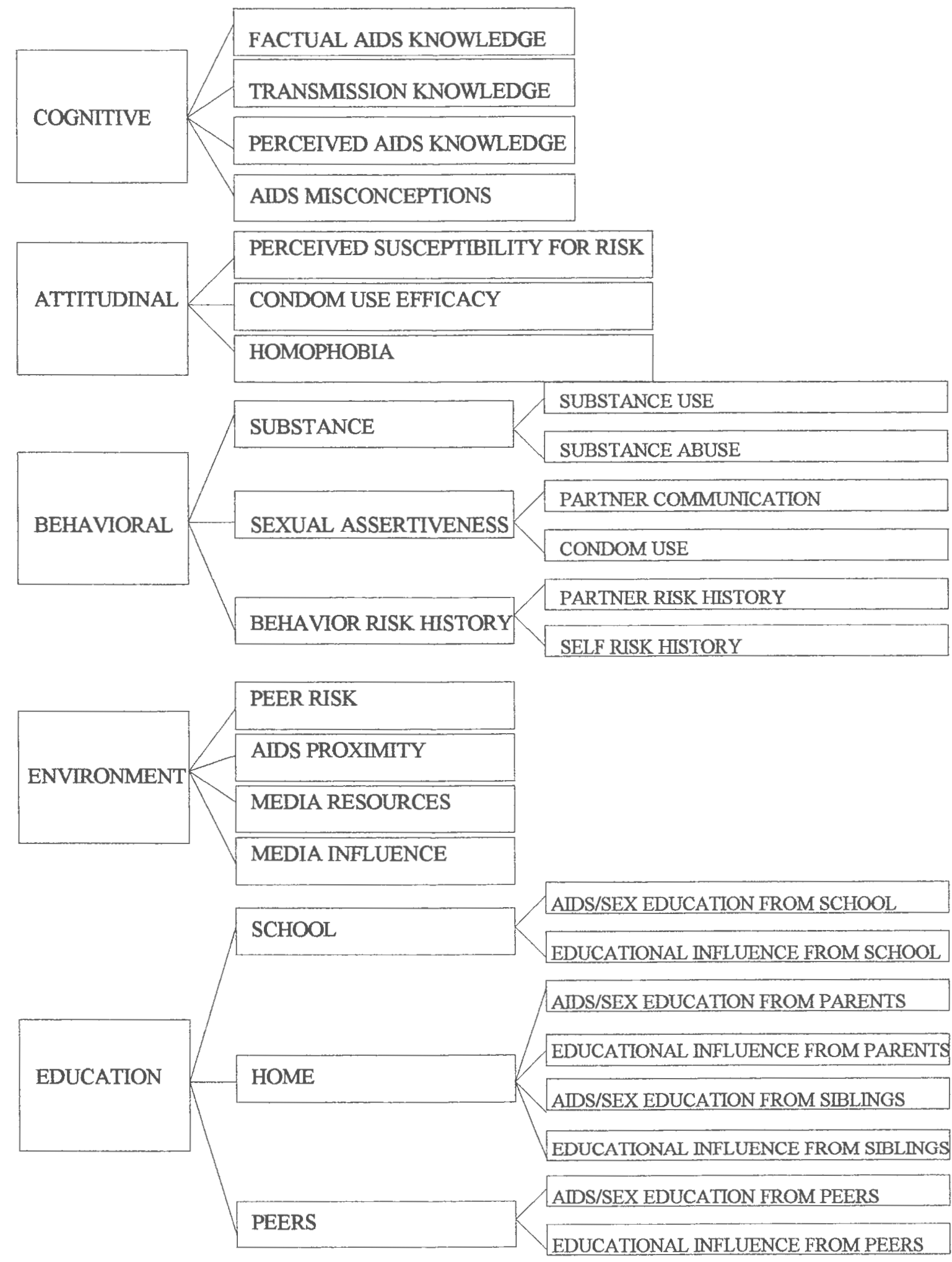




\section{Figure 2}

Sample Characteristics by Gender, Sexual Activity (Active Versus Nonactive), and Condom Use Behavior ("Safe" Consistent Use, or

"Unsafe" No Use or Inconsistent Use)

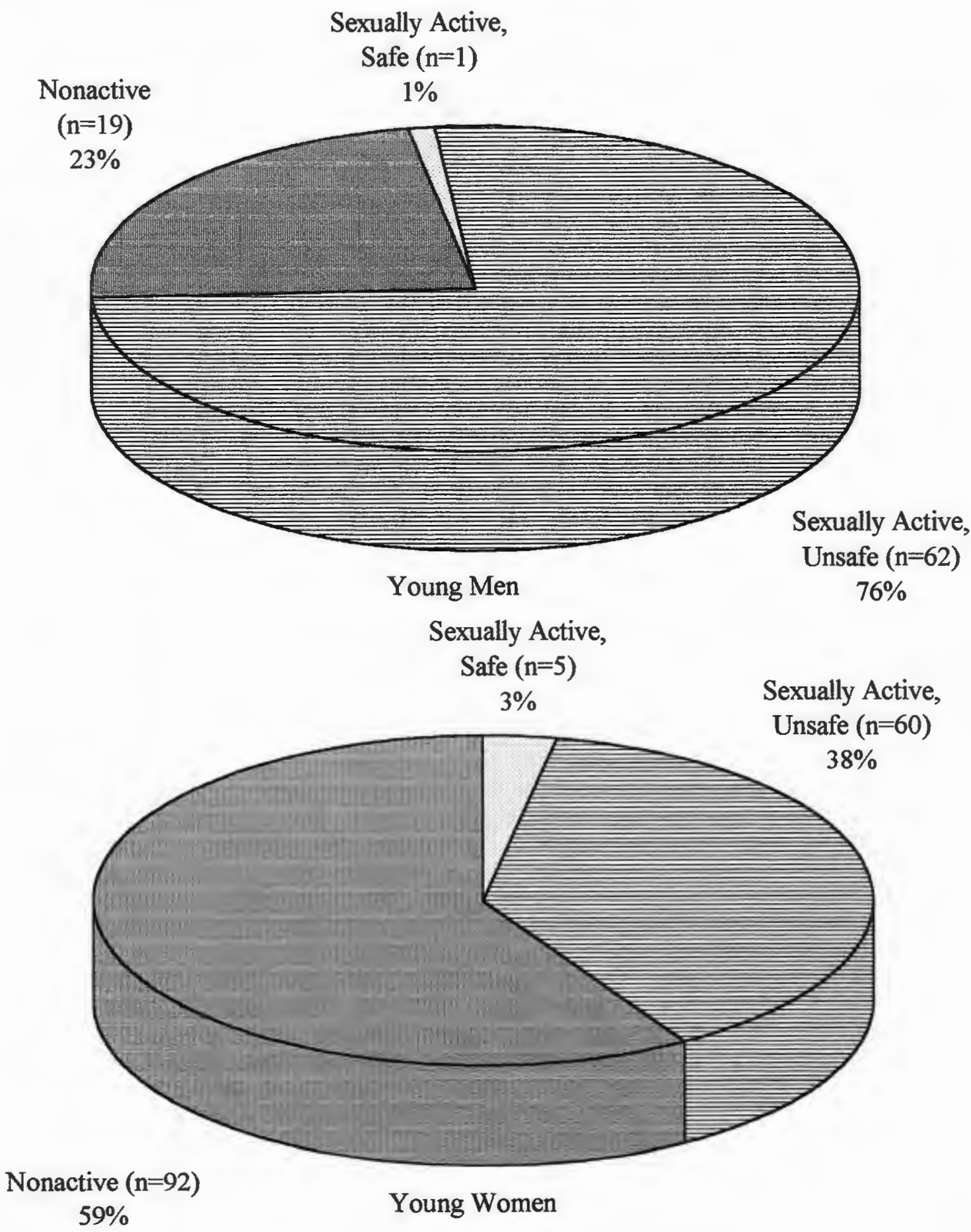


Figure 3

Mean Scores for Dependent Variables by Gender Among the Sexually Active "Unsafe" (Inconsistent or No Condom Use)

Participants

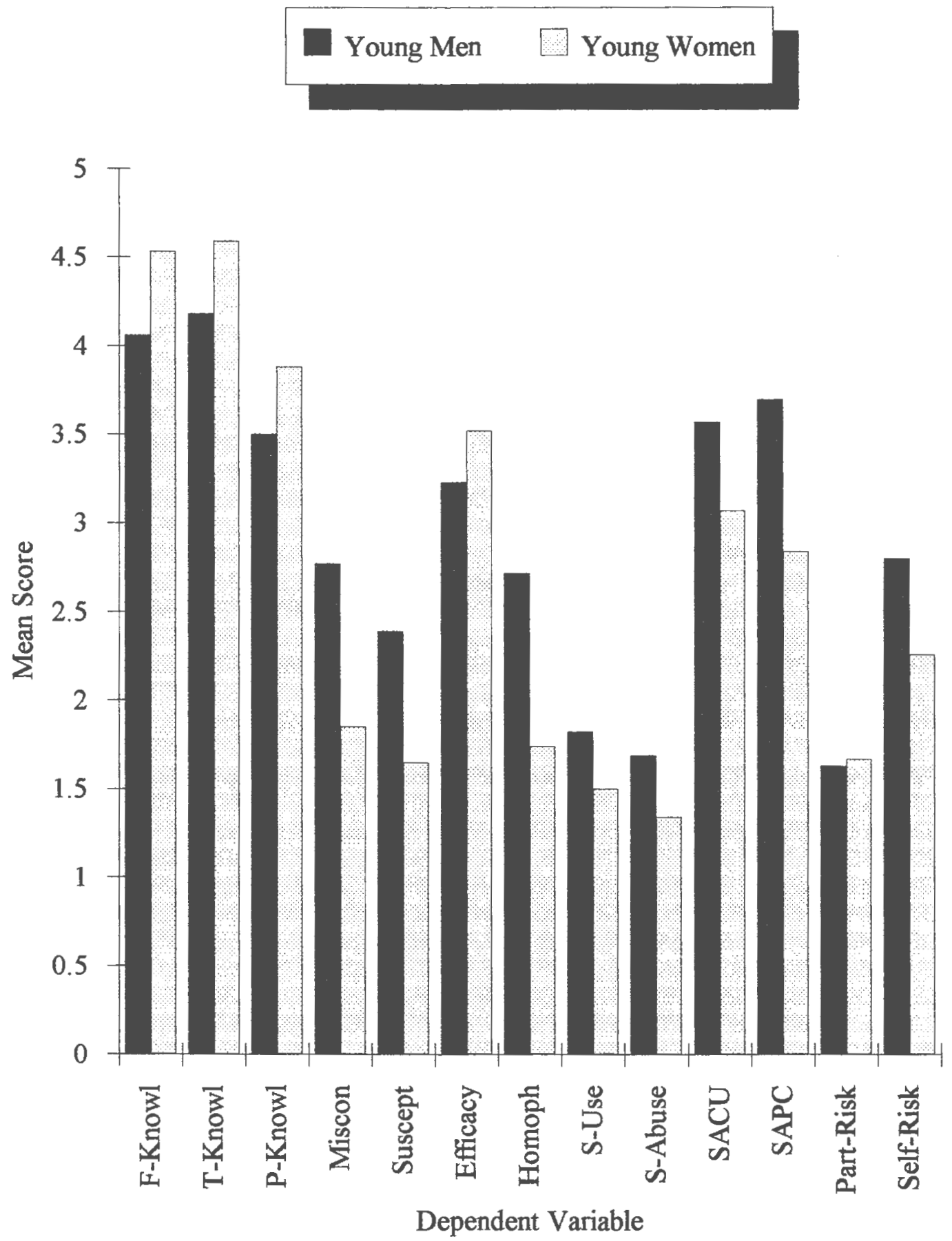


Figure 4

Mean Scores for Dependent Variables by Self-rated Level of AIDS Risk Among the Sexually Active "Unsafe" (Inconsistent or No Condom Use) Participants

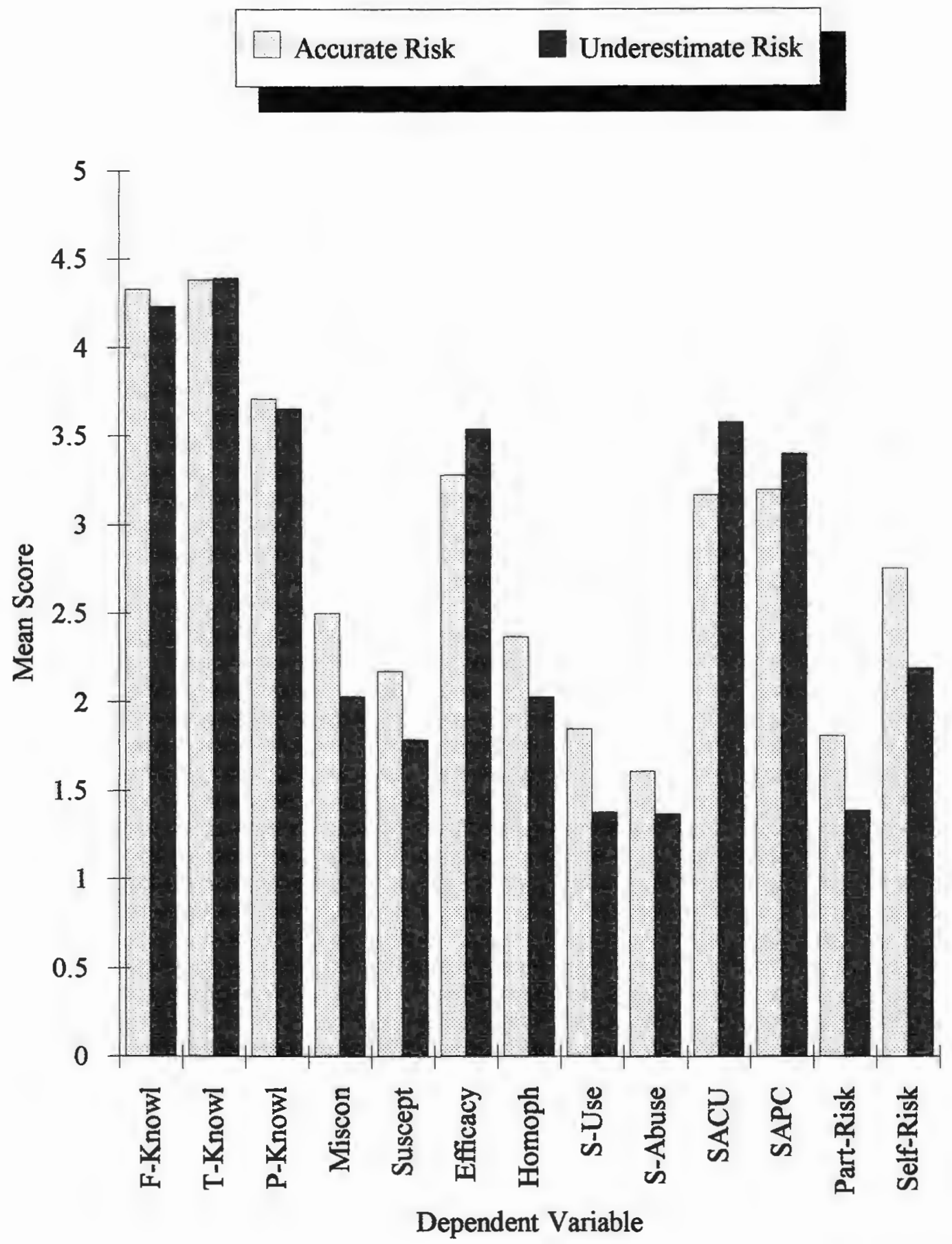


Figure 5

2X2 MANOVA Interaction for Gender and Sexual Activity with

Dependent Variable: Misconceptions

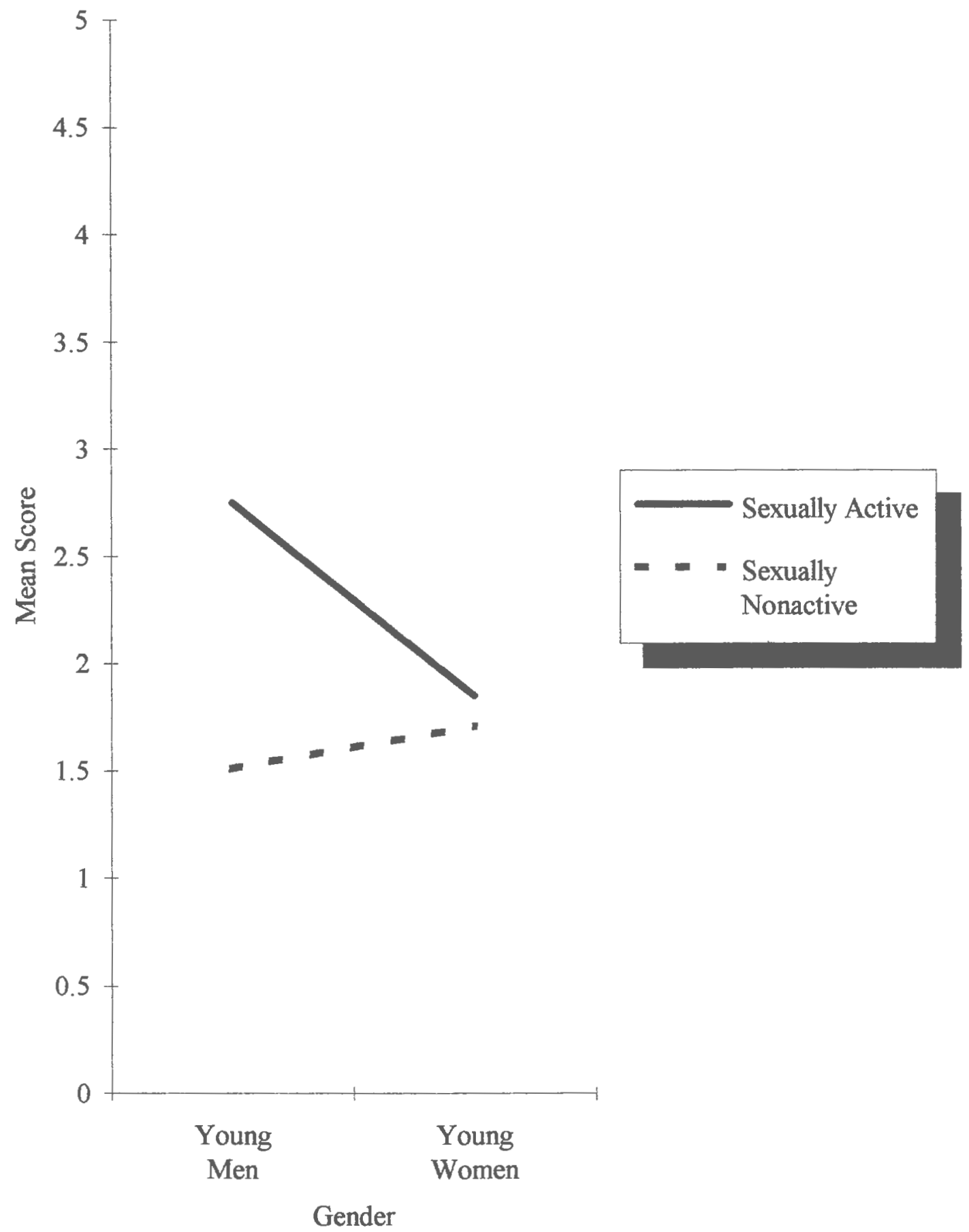


Figure 6

2X2 MANOVA Interaction for Gender and Sexual Activity with

Dependent Variable: Perceived Susceptibility for Risk

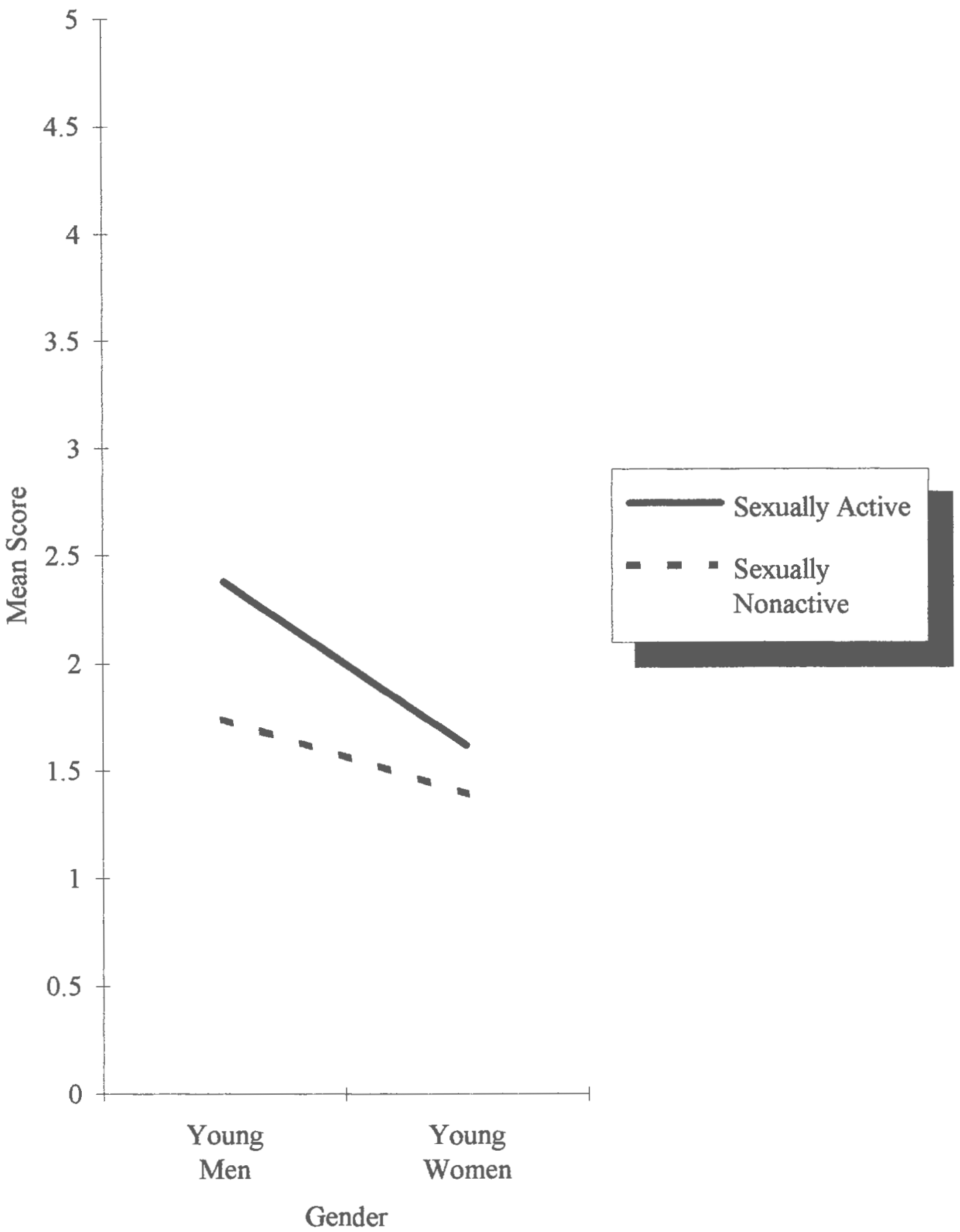


Figure 7

2X2 MANOVA Interaction for Gender and Sexual Activity with Dependent Variable: Homophobia

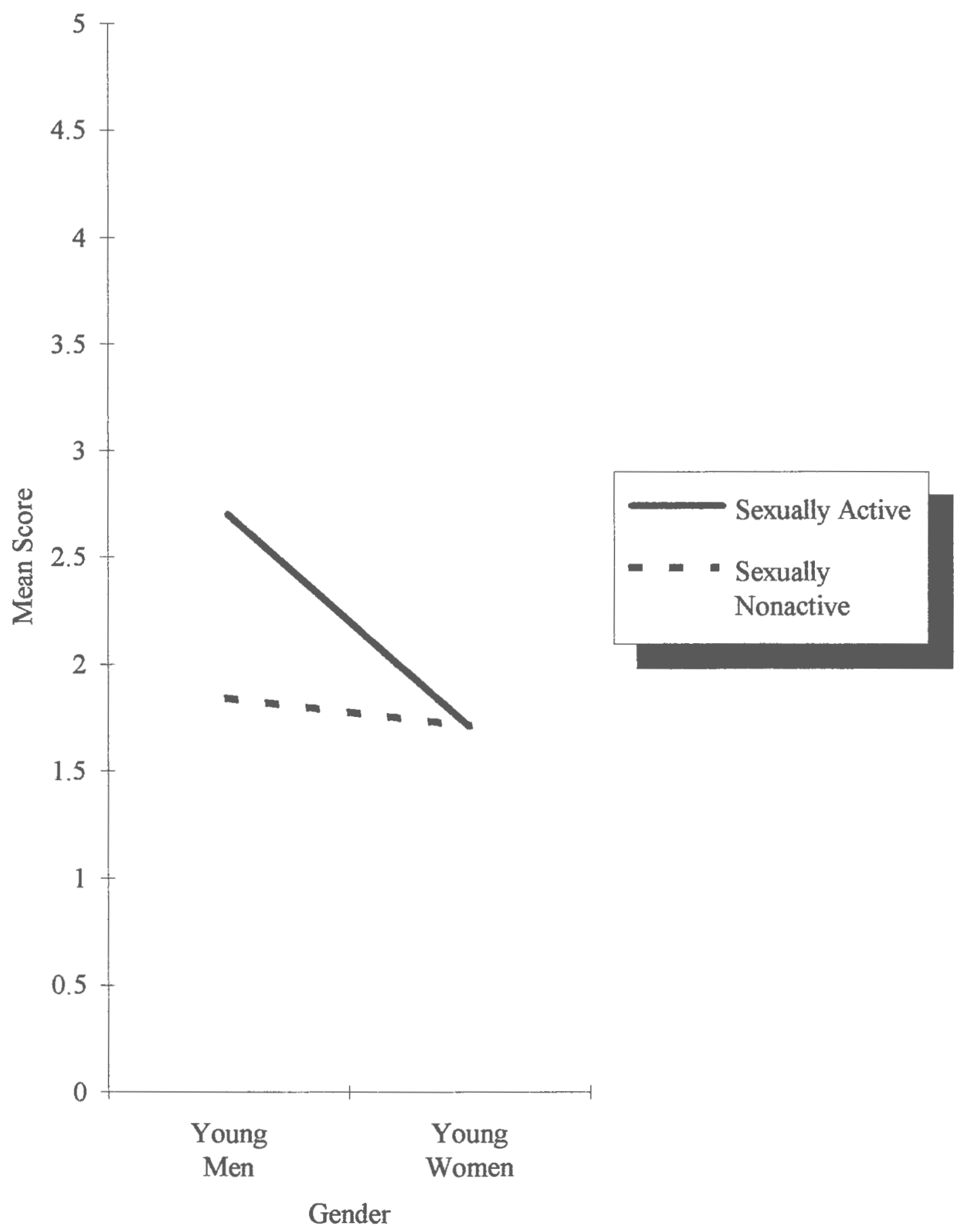


Figure 8

2X2 MANOVA Interaction for Gender and Sexual Activity with Dependent Variable: Sexual Assertiveness Partner Communication (Actual Versus Predicted)

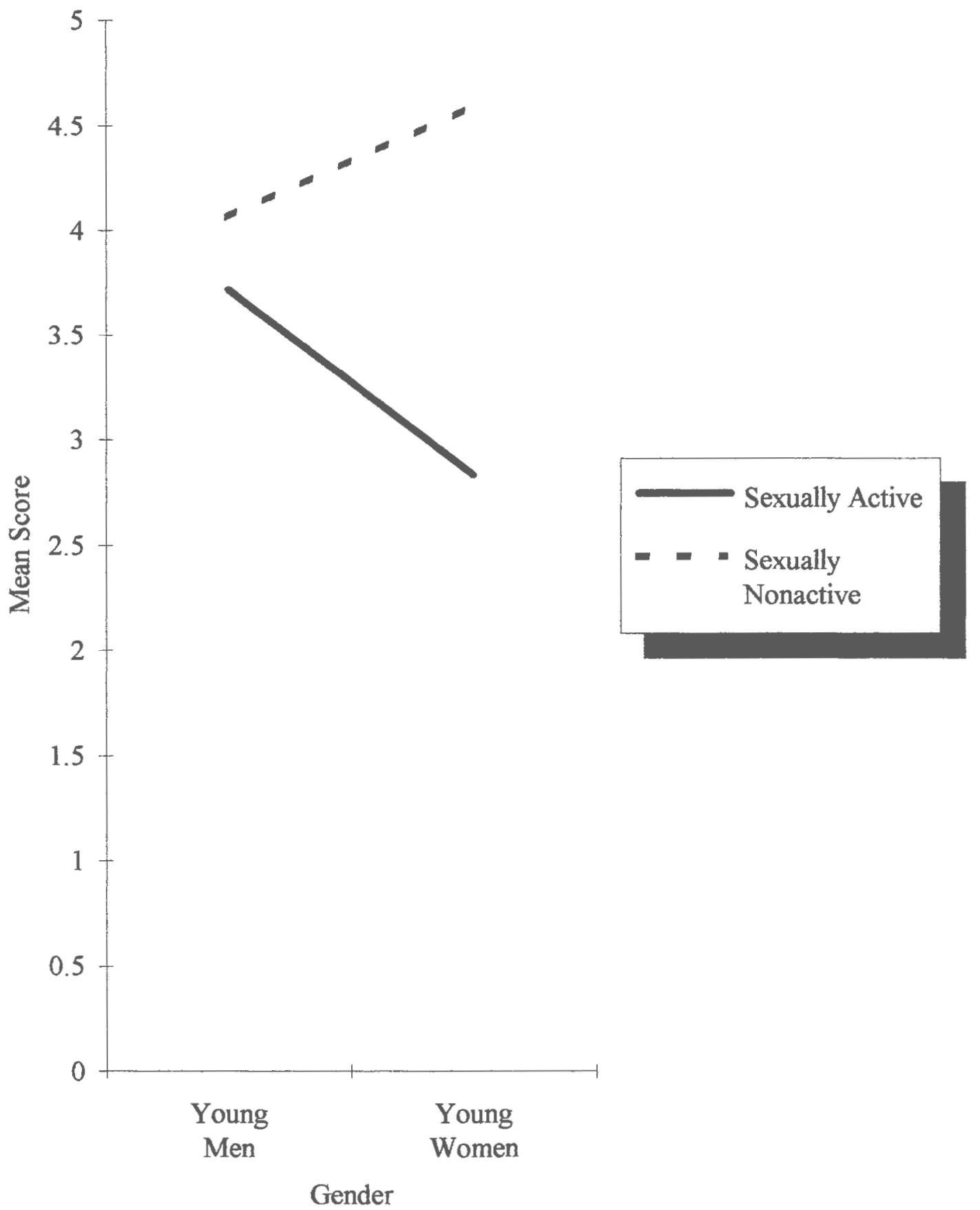


Figure 9

2X2 MANOVA Interaction for Gender and Sexual Activity with Dependent Variable: Sexual Assertiveness Condom Use (Actual

Versus Predicted)

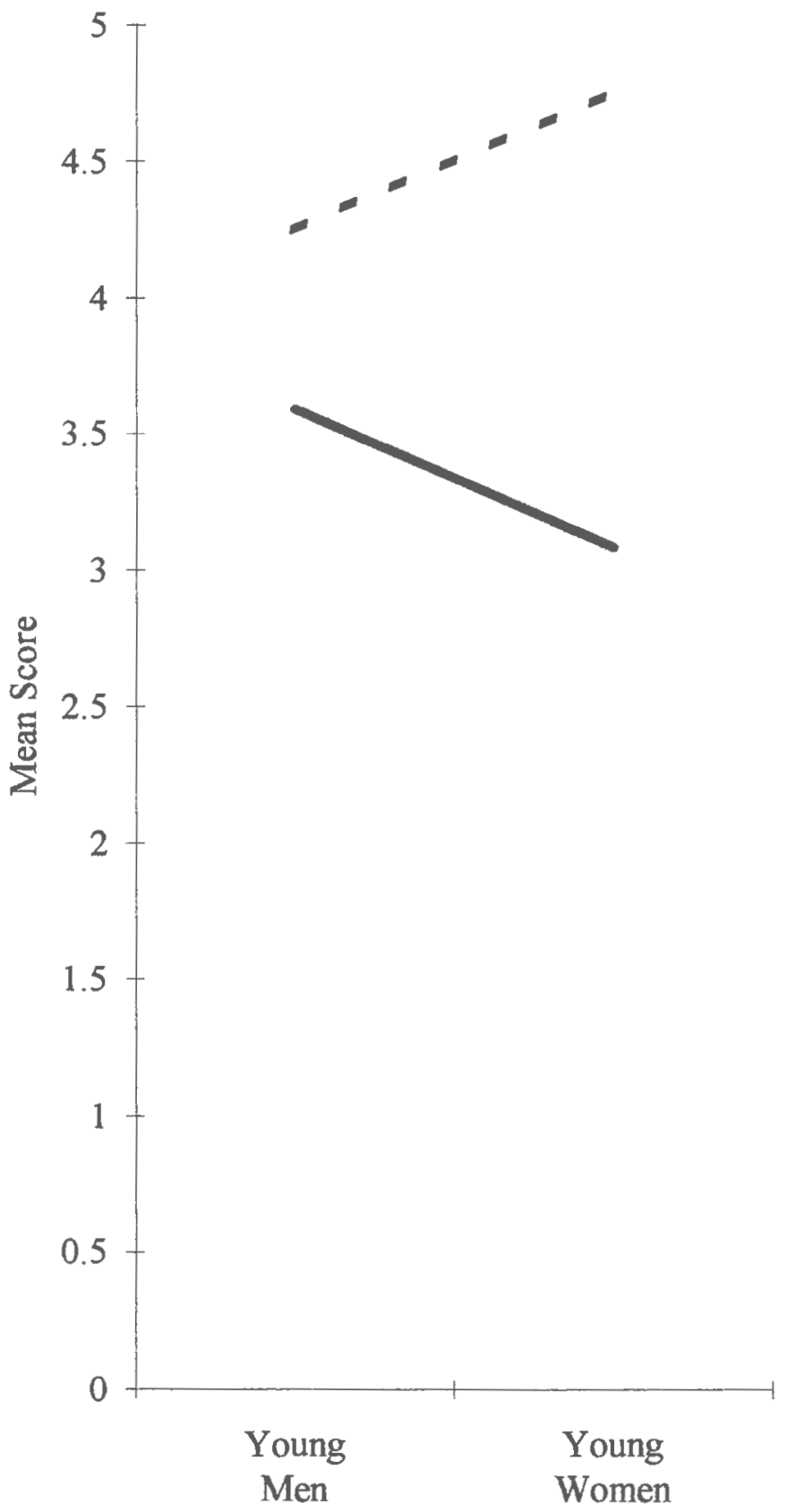

Gender 
Figure 10

2X2 MANOVA Interaction for Gender and Sexual Activity with

Dependent Variable: Substance Use

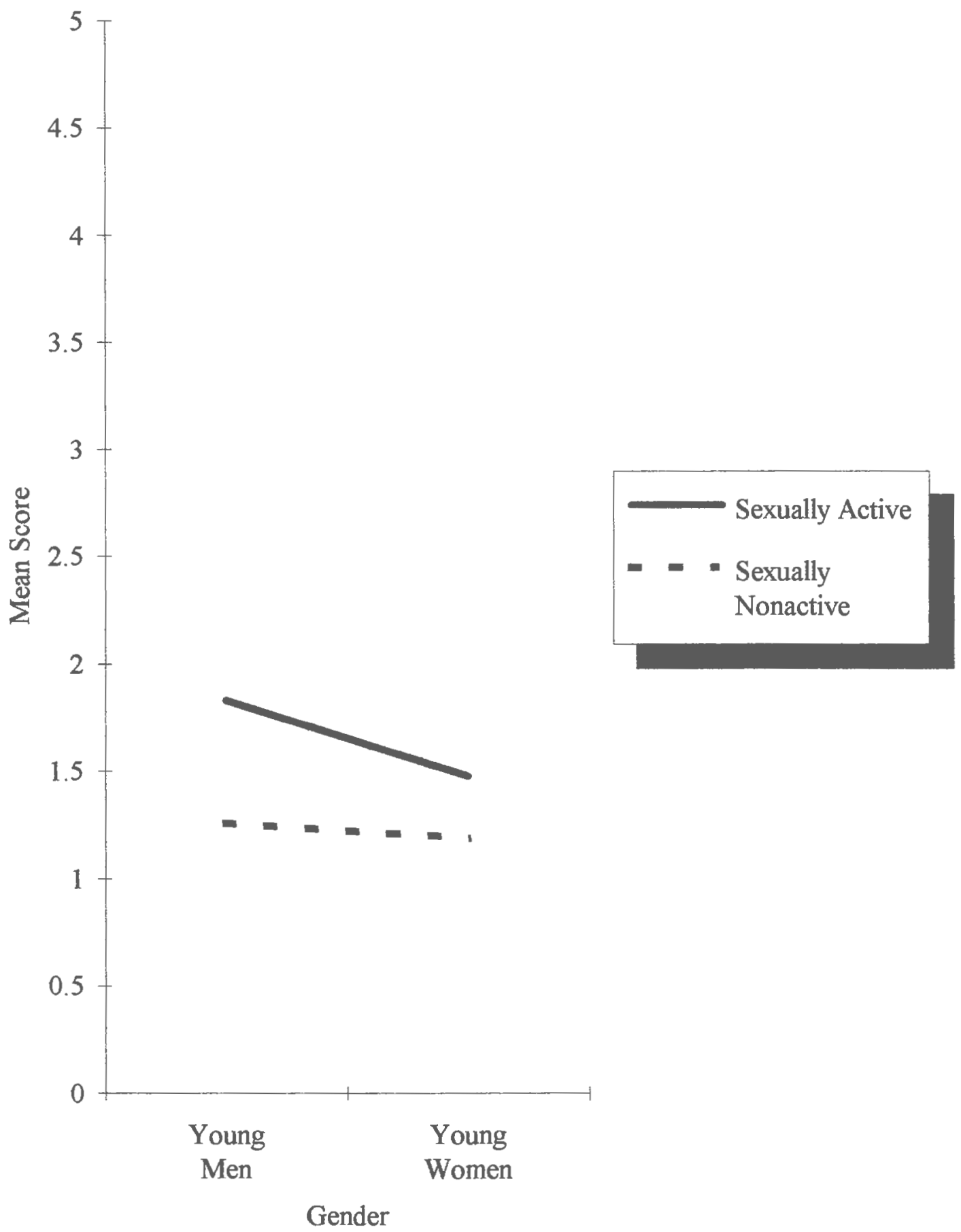




\section{Figure 11}

2X2 MANOVA Interaction for Gender and Sexual Activity with

Dependent Variable: Substance Abuse

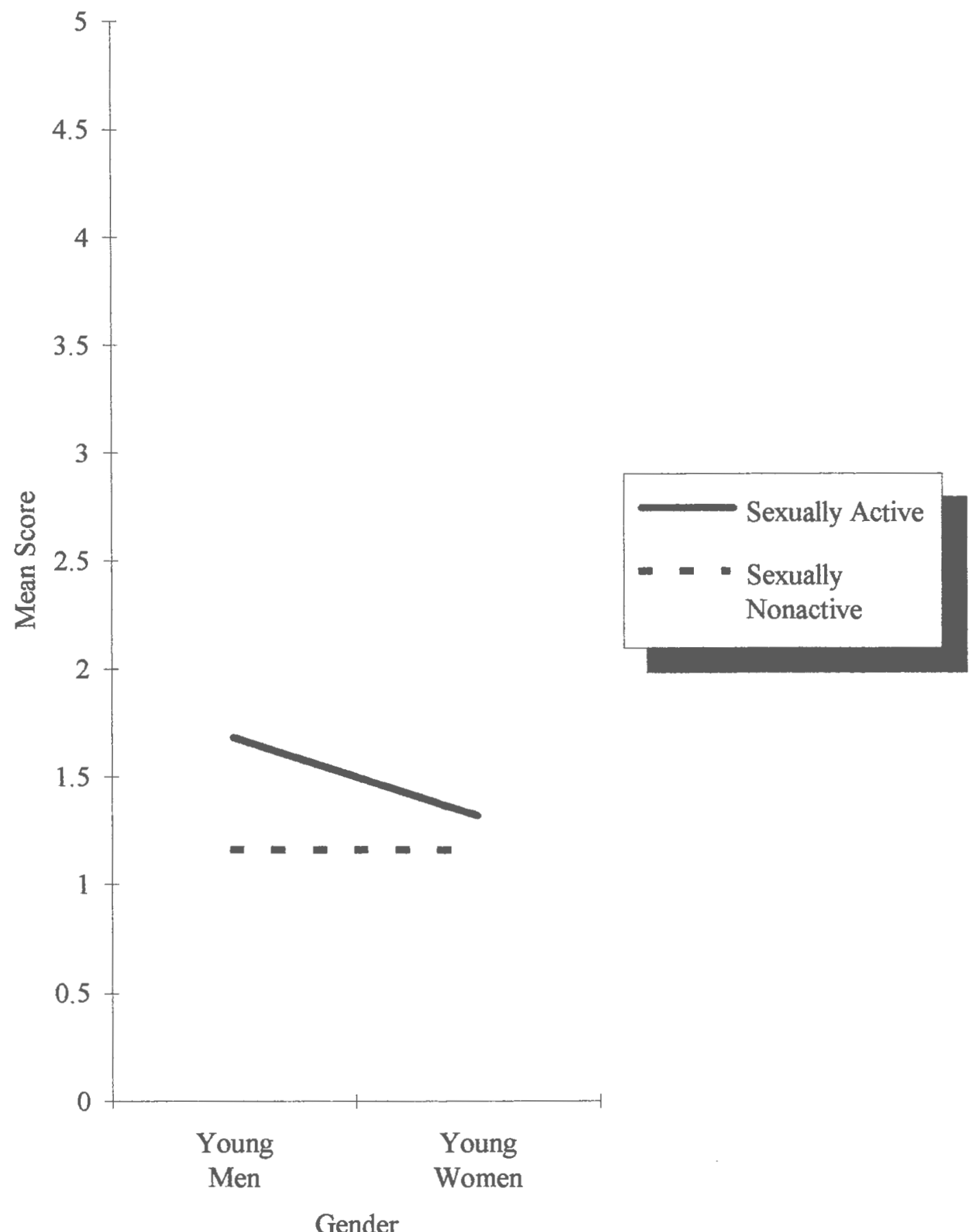


Figure 12

2X2 MANOVA Interaction for Gender and Sexual Activity with

Dependent Variable: Self Risk History

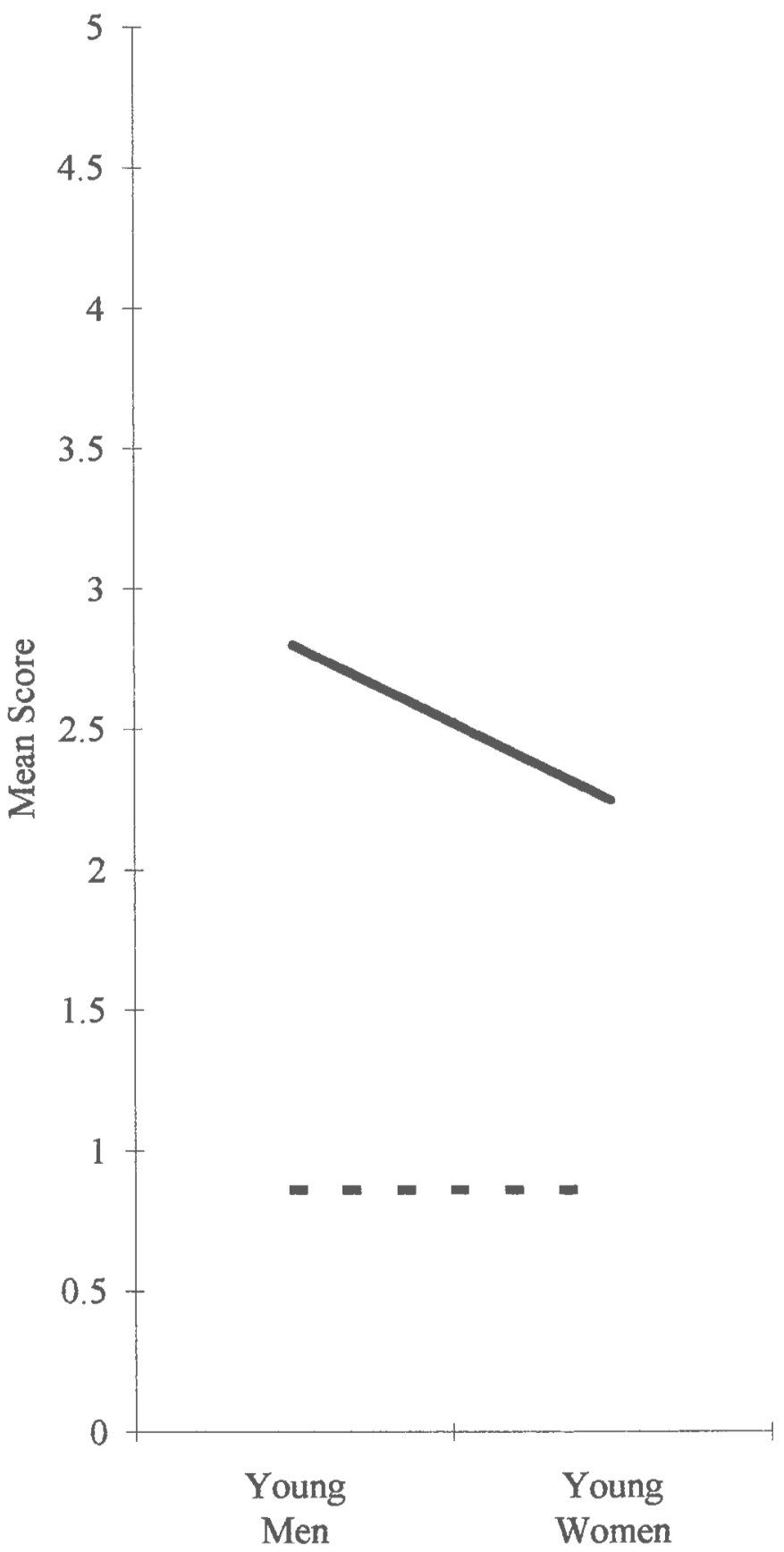

Gender 
Figure 13

Mean Scores for Dependent Variables for Sexually Active Youth by Gender

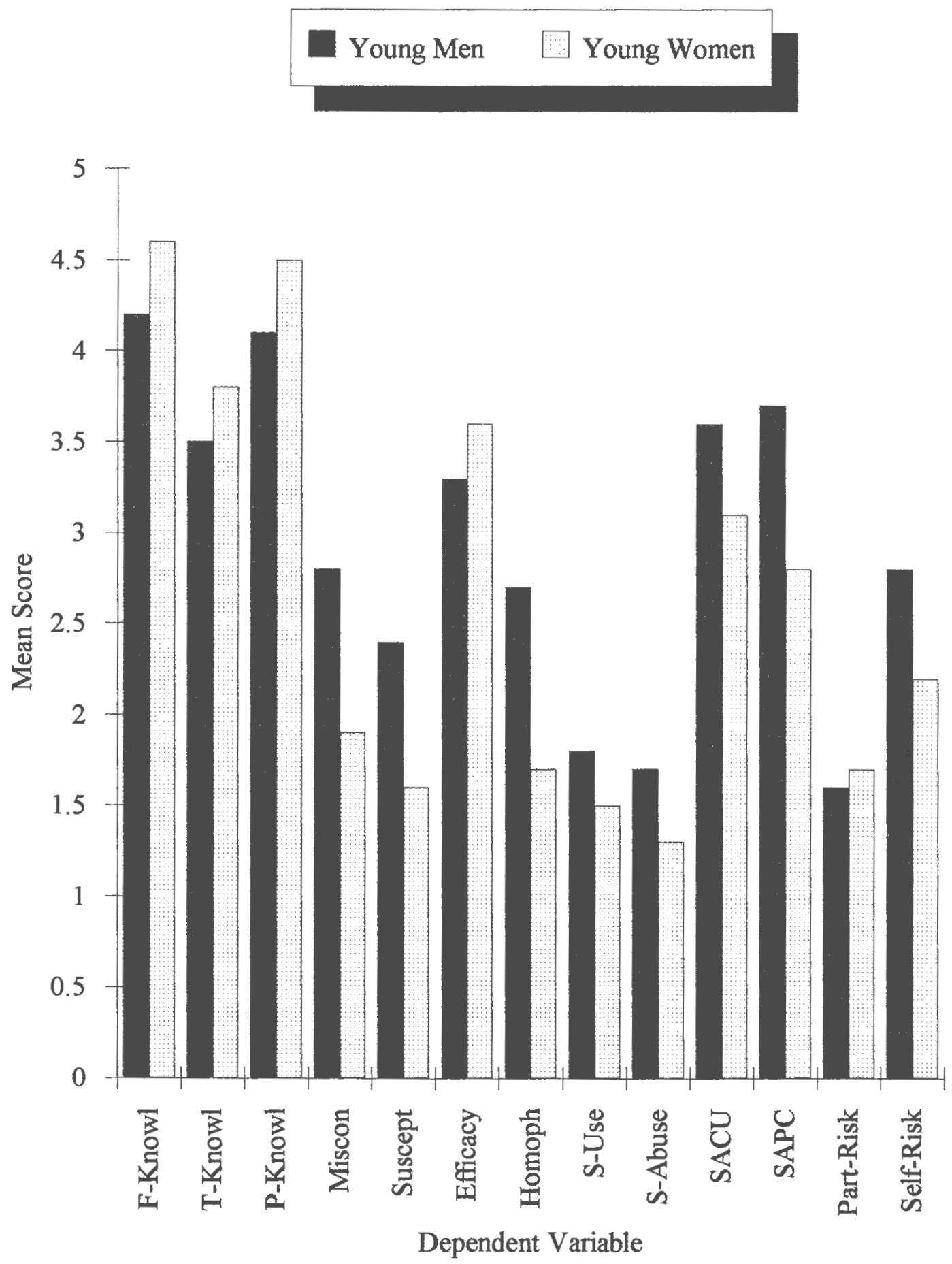


Figure 14

Mean Scores for Dependent Variables (with Predicted Behavioral) for Sexually Nonactive Youth by Gender

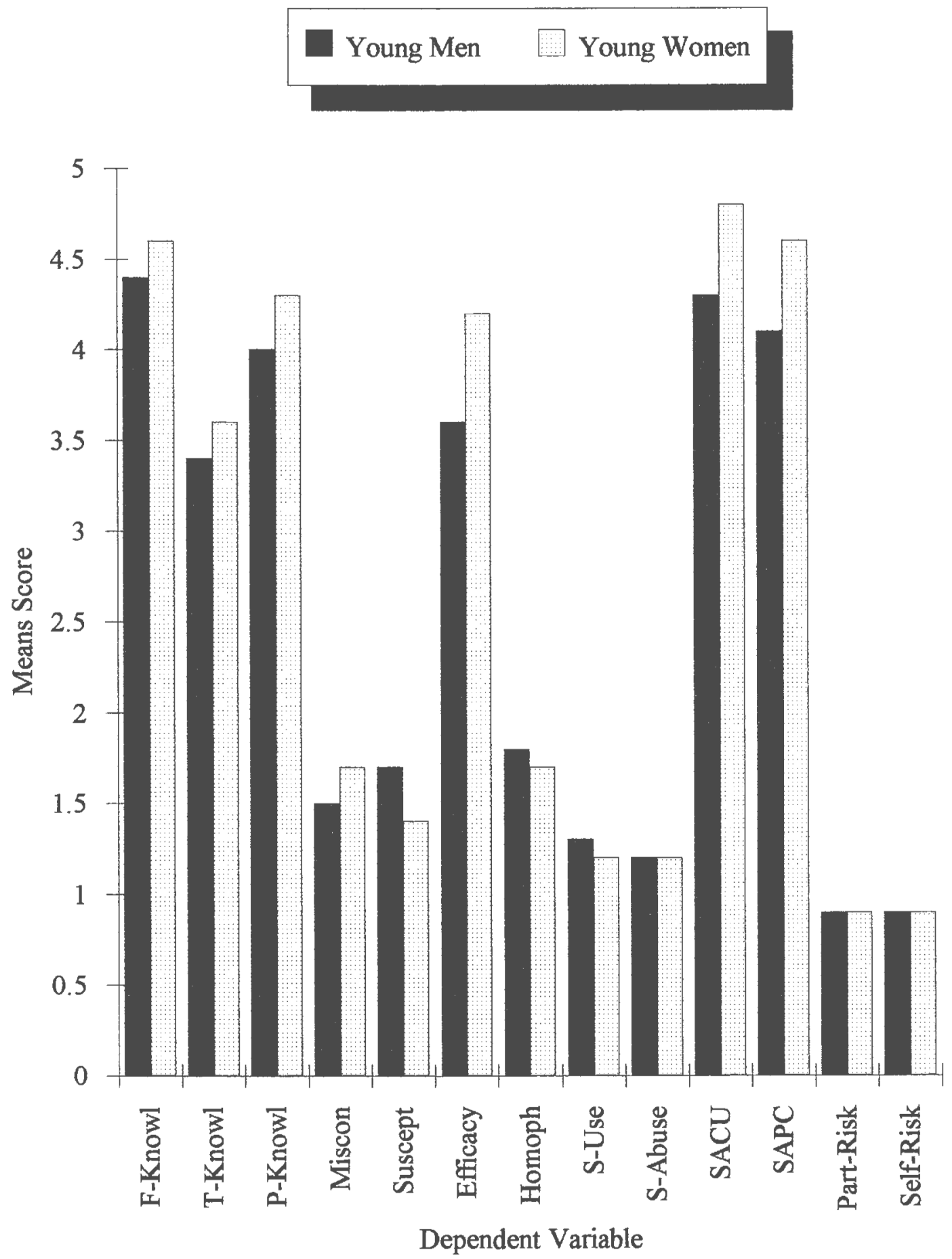


Figure 15

Mean Scores for Dependent Variables by Sexual Activity (Actual Versus Predicted Behavioral)
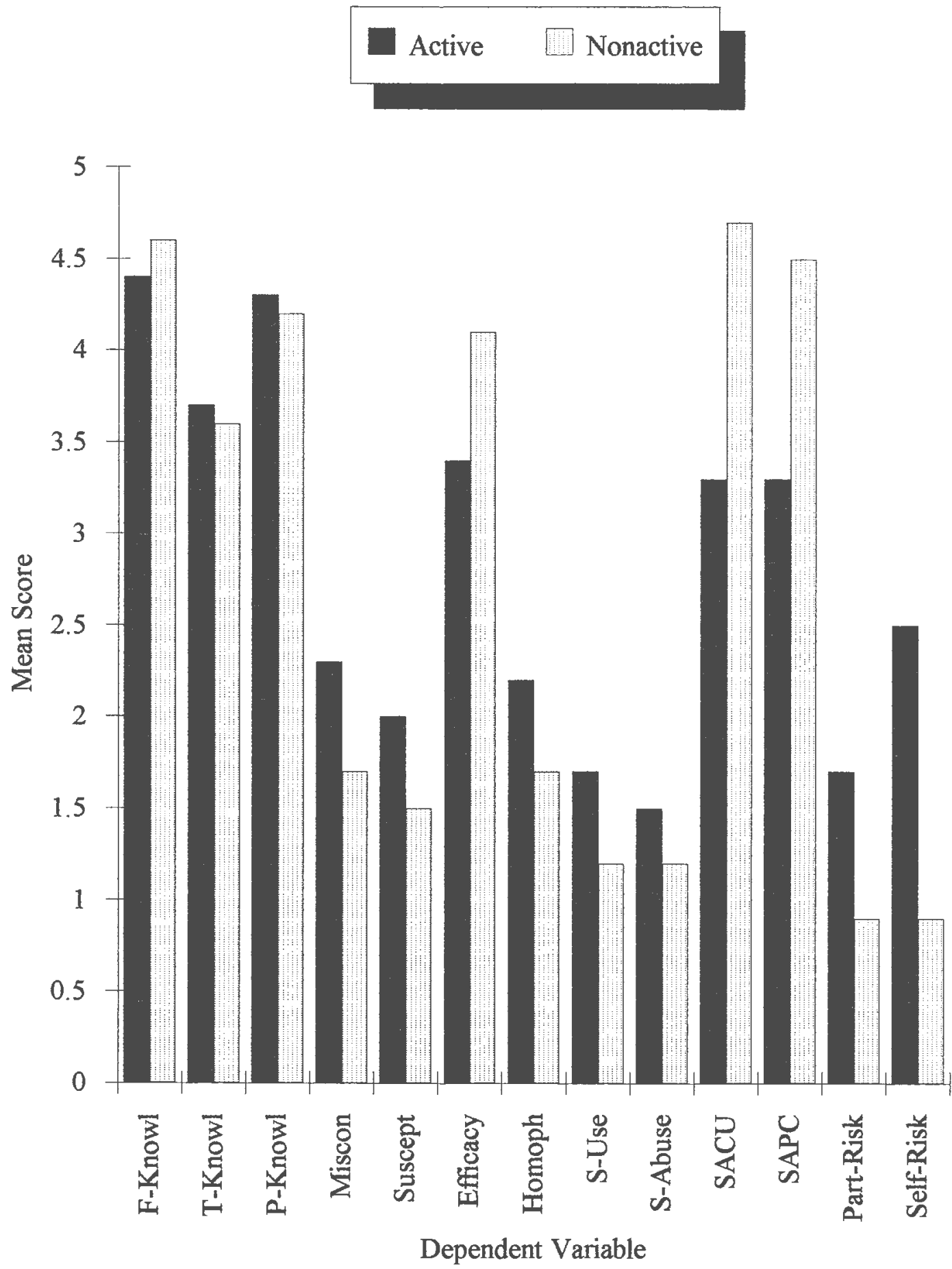
Figure 16

Sexually Active Adolescents' Reported Current and Six-Month

Predicted Condom Use by Transtheoretical Model Stage

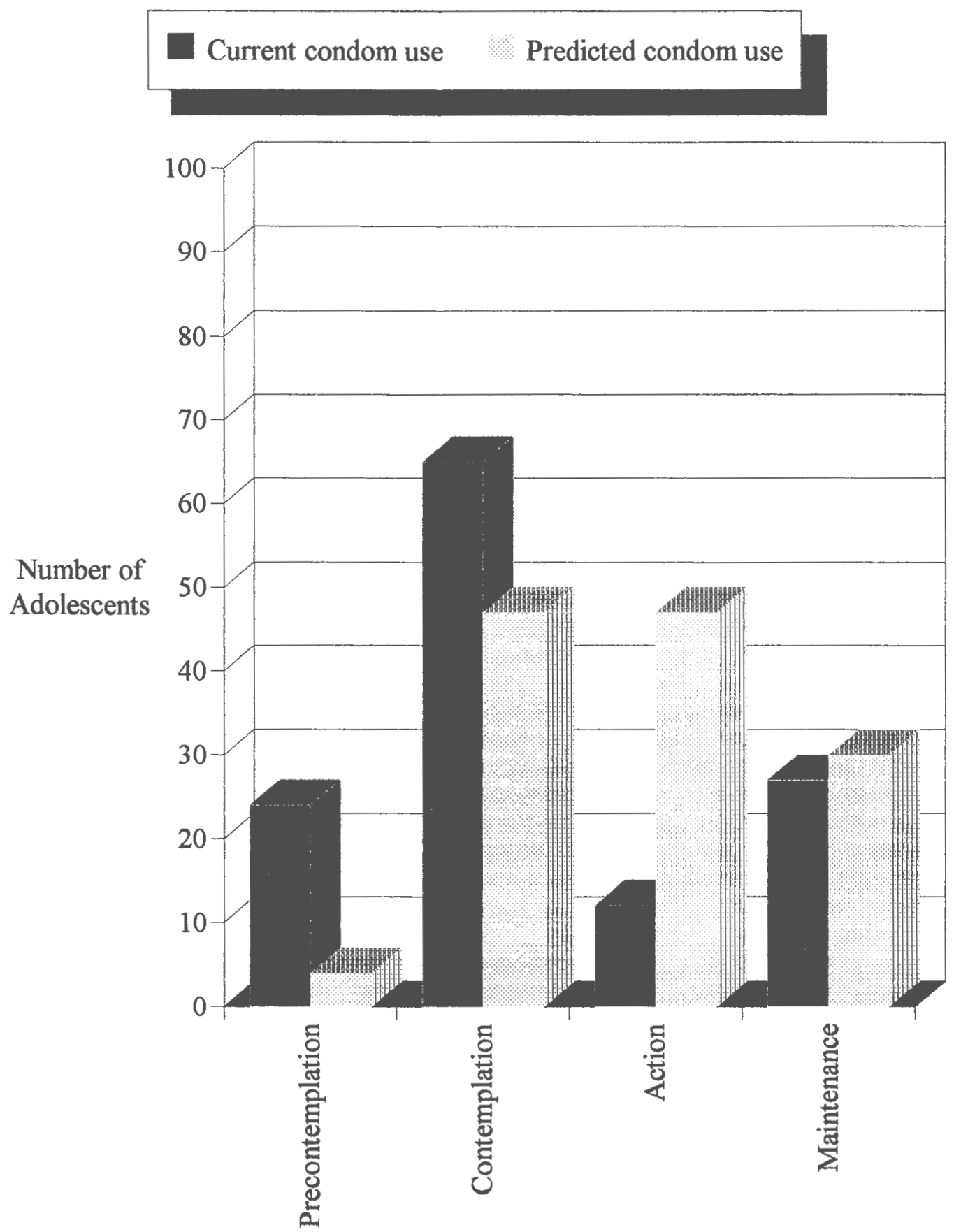

Stage 
Figure 17

Sexually Active Adolescents' Current and Six-Month Predicted

Condom Use Adjusted by Reported Sexual Behavior by

Transtheoretical Model Stage

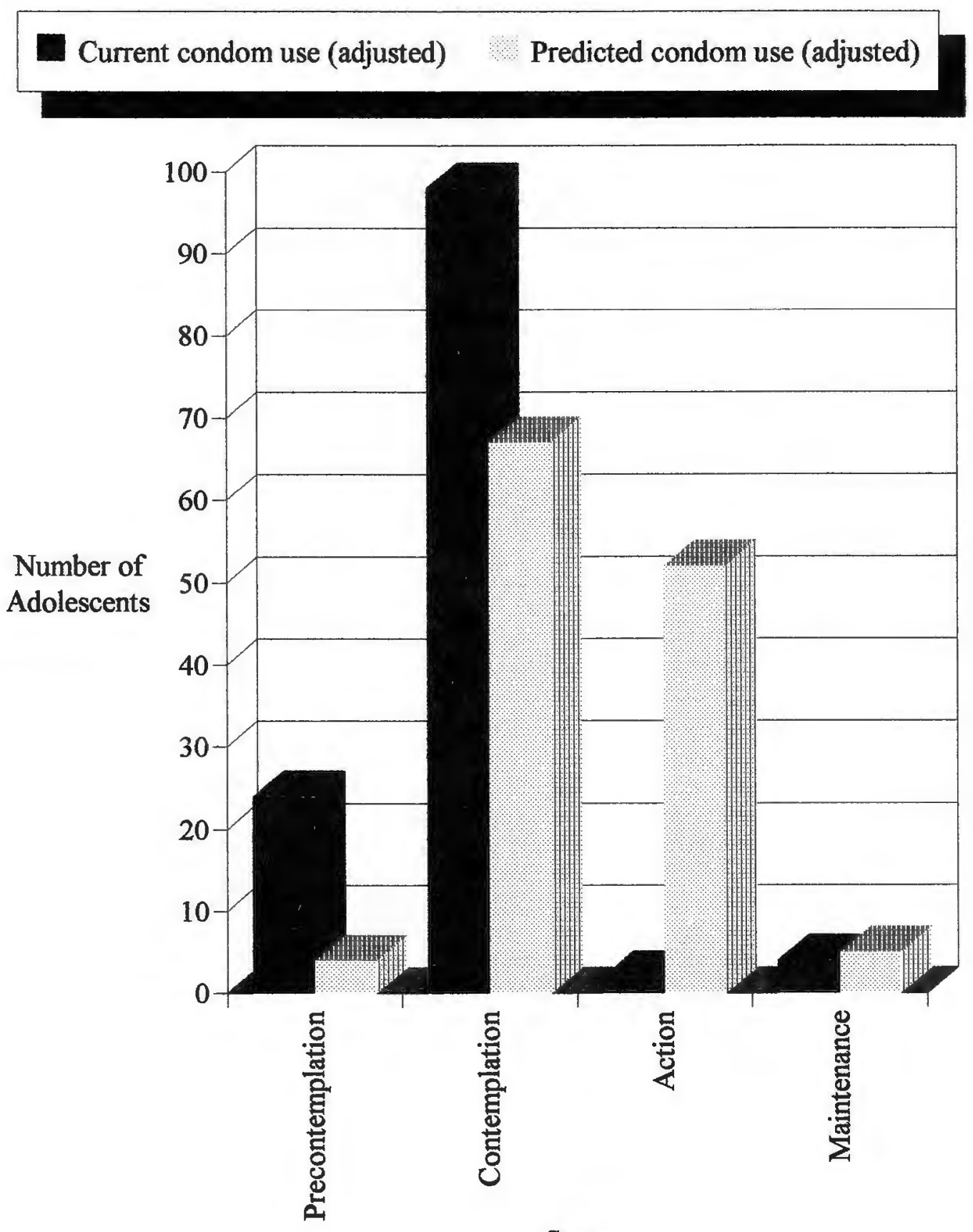

Stage 


\section{Appendix A}

\section{Demographics}

Please circle the letter next to the answer that is best for you. Remember, all of your answers are completely anonymous. The answers you give are private, no one will know that they are yours.

1. I am

2. What is your age?

3. Which state do you live in? (or country if outside USA)

4. What is your race or cultural group?

5. What grade are you in?

6. Are you single or married now?

7. What is your religion?

8. How religious or spiritual are you?

9. Who do you presently live with?

10. To the best of your knowledge, how much education has your mother completed?

11. ...has your father completed?
A. Male
B. Female

$\overline{\text { (please fill in age) }}$

$\overline{\text { (please fill in state or country) }}$
A. White
B. African-American
C. Asian-American
D. Hispanic-American
E. Other:
A. Single
B. Married
A. Catholic
B. Protestant
C. Jewish
D. None
E. Other:
A. Not at all
B. Slightly
C. Somewhat
D. Fairly
E. Very
A. Both Mother and Father
B. Mother Only
C. Father Only
D. Someone else
E. Live alone

(answer selection for $10 \& 11$ )
A. 8th grade or less
B. Some High School
C. High School Graduate
D. Some College
E. College Graduate 


\section{Appendix B}

\section{Cognitive Subscales}

Hypothesized Subscales:

FAK=Factual AIDS Knowledge

TAK=Transmission AIDS Knowledge

PAK $=$ Perceived AIDS Knowledge

M=Misconceptions

(answer selection for questions 1 through 16)
A. Definitely no
B. Probably no
C. Not sure
D. Probably yes
E. Definitely yes

1. AIDS is a medical condition in which your body cannot fight off diseases. (FAK)

2. AIDS is caused by a virus. (FAK)

3. If a pregnant woman has AIDS, there is a chance it may harm her unborn baby. (FAK)

4. Most people who get AIDS usually die from it. (FAK)

5. Using a condom during sex can lower the risk of getting AIDS. (FAK)

6. Receiving a blood transfusion with infected blood can give a person AIDS. (FAK)

7. Mosquitoes can transmit AIDS. (M)

8. AIDS can be cured if treated early. (M)

9. A new vaccine has recently been developed which can prevent AIDS. (M)

10. You can have AIDS and not know it. (M)

11. I can avoid getting AIDS by having sex only with healthy looking persons. (M)

12. Only gay people and people who use needle drugs can get AIDS. (M)

13. A woman who has unprotected sex only with men can get AIDS. (TAK)

14. A man who has unprotected sex only with women can get AIDS. (TAK)

15. A woman who has unprotected sex only with women can get AIDS. (TAK)

16. A man who has unprotected sex only with men can get AIDS. (TAK)

(answer selection for questions 17 through 19)
A. Definitely not true
B. Not really true
C. Somewhat true
D. Mostly true
E. Definitely true

17. If someone asked me a question about how to prevent getting HIV/AIDS, I can give them the right answer. (PAK)

18. If someone asked me what things people do that can cause HIV/AIDS, I can tell them the right answer. (PAK)

19. If someone asked me how HIV/AIDS is transmitted, I can give them the right answer. (PAK) 


\section{Appendix C}

\section{Attitudinal Subscales}

Hypothesized Subscales:

CUE= Condom Use Efficacy

PSR $=$ Perceived Susceptibility for Risk

$\mathrm{H}=$ Homophobia

$\mathrm{FA}=$ Fear of AIDS

(answer selection for questions 1 through 7)
A. Not at all sure
B. A little sure
C. Kind of sure
D. Fairly sure
E. Very sure

\section{I would use a condom ...}

1. ...when I am really turned on. (CUE)

2. ...when my partner gets mad about using a condom. (CUE)

3. ...when I am depressed. (CUE)

4. ...when I am drunk or high. (CUE)

5. ...when I feel angry. (CUE)

6. ...when I feel pressured to have sex. (CUE)

7. ...when I'm afraid I might get AIDS. (CUE)

(answer selection for questions 8 through 12)
A. Definitely No
B. Probably No
C. Not sure
D. Probably Yes
E. Definitely Yes

8. I feel that I am at risk of getting AIDS at this time in my life. (PSR)

9. I sometimes think that I may have been exposed to AIDS. (PSR)

10. I have had sex with someone who could have given me AIDS. (PSR)

11. I believe I can do things to help protect myself from getting AIDS. (PSR)

12. I try not to get into situations where I might get AIDS. (PSR)

(answer selection for questions 13 through 26)
A. Definitely not true
B. Not really true
C. Somewhat true
D. Mostly true
E. Definitely true

13. I think people with HIV/AIDS should be in a special classroom at school. (H) 
14. I would not like it if someone in my gym class has HIV/AIDS. (H)

15. A gay person should not be allowed to teach school. $(\mathrm{H})$

16. A gay person should not be allowed to run for public office. $(\mathrm{H})$

17. If my roommate told me he or she had HIV/AIDS, I would ask them to move out. (H)

18. A gay person should not be allowed to be a coach. $(\mathrm{H})$

19. I would stay away from people who are gay, because if I didn't, I would have a higher chance of getting HIV/AIDS. (H)

20. It's okay for people who have HIV/AIDS to teach school. (H)

21. I would feel nervous in a group of gay people. $(\mathrm{H})$

22. If I saw two men or two women holding hands, I would feel uncomfortable. $(\mathrm{H})$

23. I would feel uncomfortable sitting next to a person who is gay. $(\mathrm{H})$

24. I am afraid that someone around me has HIV/AIDS and I do not know it. (FA)

25. I am afraid of making new friends because of HIV/AIDS. (FA)

26. If my brother or sister told me he or she was gay, I would be upset. $(\mathrm{H})$ 


\section{Appendix D}

\section{Behavior Subscales}

Hypothesized Subscales:

$\mathrm{SAPC}=$ Sexual Assertiveness Partner Communication

$\mathrm{SACU}=$ Sexual Assertiveness Condom Use

$\mathrm{SU}=$ Substance Use

$\mathrm{SA}=$ Substance Abuse

SRH=Self Risk History

PRH=Partner Risk History

In this survey, a "sex partner" is any person that you have willingly had sexual intercourse with, or a person you have willingly had oral or anal sex with. "Having sex" is doing any of these things with a sex partner. Please remember that no one will know that these answers are yours.

Think about what you would do even if you have never done some of these things, and circle the letter that is best for you. Otherwise, think about a person you have sex with or someone you used to have sex with when you answer these questions.

(answer selection for questions 1 through 10)
A. Never
B. Sometimes
C. About half of the time
D. Usually
E. Always

1. I would ask if I wanted to know if my partner ever had an HIV test. (SAPC)

2. I'd have sex without a condom if my partner doesn't like them, even if I want to use one. (SACU)

3. I would ask my partner about the AIDS risk of his or her past partners, if I want to know. (SAPC)

4. I would make sure my partner and I use a condom when we have sex. (SACU)

5. I would ask if I want to know if my partner ever had a sexually transmitted disease (STD). (SAPC)

6. I would insist on using a condom if I want to, even if my partner doesn't like them. (SACU)

7. I would ask if I want to know if my partner ever had sex with someone who shoots drugs with a needle. (SAPC)

8. I would have sex without using a condom if my partner insists, even if I don't want to. (SACU)

9. I would ask if I want to know if my partner ever used needles to take drugs. (SAPC)

10. I would refuse to have sex if my partner refuses to use a condom. (SACU)

Next, here are some questions that ask about your possible use of alcohol and other drugs. Remember your answers will be added to the answers of many other people filling out this survey. No one will know which answers are yours.

11. In the past year, how often have you had a drink of beer, wine, or liquor? (SU)
A. Never
B. 1-2 times a month or less
C. 1-2 times a week
D. 3-4 times a week
E. Almost every day

(continued on next page) 
12. In the past year, how many drinks did you normally have when you drank alcohol? (SU)
A. None, I don't drink
B. 1-2 drinks
C. 3-5 drinks
D. 6-10 drinks
E. 11 or more drinks

(answer selection for questions $13 \& 14$ )
A. Never
B. 1-2 times a month or less
C. 1-2 times a week
D. $3-4$ times a week
E. Almost every day

13. In the past year, how often did you have 3 or more drinks in one day? (SU)

14. In the past year, how often did you have 6 or more drinks in one day? (SU)

(answer selection for questions $15 \& 16$ )
A. Never
B. Once a month or less
C. About once a week
D. About twice a week
E. Almost every day, or every day

15. In the past year, how many times did you use marijuana or hashish (grass, pot, hash, hash oil) without a doctor's orders? (SU)

16. In the past year, how many times did you use other drugs, without a doctor's orders? (SU)

17. In the past year, how many times did you share a needle to shoot drugs? (SU)
A. Never
B. 1 time
C. 2-5 times
D. 6-10 times
E. 11 or more times

How often have these things happened to you in the past year because of your drinking or drug use? (answer selection for questions 18 through 32 )
A. Never
B. Once
C. 2-3 times
D. 4-6 times
E. More than 6 times

18. I had a hangover. (SU)

19. I messed up on something important. (SA)

20. I was in trouble with the police or other authority. (SA)

21. I broke or messed up someone else's things. (SA)

22. I got into an argument or fight. (SA)

(continued on next page) 
23. I got sick or threw up. (SA)

24. I drove a car after having alcohol or drugs. (SA)

25. I was hurt or injured. (SA)

26. Someone I know talked to me about how I was acting. (SA)

27. I thought I might have a drinking or drug problem. (SA)

28. I couldn't remember for a while (blacked out). (SA)

29. I did something I wished I hadn't. (SA)

30. I was arrested for drunk driving. (SA)

31. I had sex that I wouldn't have had if I was sober. (SA)

32. I tried to stop using but didn't. (SA)

33. I thought about or tried to kill myself. (SA)

34. Is there a partner or partners you have (or had) sex with? (Independent item)
A. No
B. Yes

35. Which do you prefer as your sex partner(s)? (Independent item)
A. I have never had a sex partner
B. Only males
C. Only females
D. Both males and females

36. About how long have you been involved with your most recent sex partner? (SRH)
A. I have never had a sex partner
B. Less than a month
C. Between 1 and 6 months
D. Six months to a year
E. Over a year

37. How old were you the first time you willingly had sexual intercourse? (SRH)
A. I have never had sex
B. years old

38. Do you use condoms (rubbers)? (SRH)
A. I have never had a sex partner
B. Started always using 6 or more months ago
C. Started always using 1-6 months ago
D. Sometimes, but not always
E. Never

39. In the next 6 months, do you plan to start always using condoms? (SRH)
A. I have never had a sex partner and don't plan to in the next 6 months
B. I already use condoms
C. I plan to start using condoms
D. Maybe sometimes, not always
E. No

(continued on next page) 
(answer selection for questions $40 \& 41$ )
A. Absolutely sure, or never had a sex partner
B. Very sure
C. Somewhat sure
D. Slightly sure
E. Not at all sure

40. How sure are you that you have NOT been exposed to AIDS? (PRH)

41. How sure are you that your sex partner(s) have NOT been exposed to AIDS? (PRH)

42. Have you ever been tested for HIV? (PRH)
A. No, I have never been tested
B. Yes, I have been tested, and I do not have HIV
C. Yes, I have been tested, and I do have HIV

(answer selection for questions 43 through 47)
A. None or I have never had a sex partner
B. One
C. Two to four
D. Five to seven
E. Eight or more

43. How many sex partners have you had who shoot drugs with a needle? (PRH)

44. How many sex partners have you had who tested positive or told they had AIDS? (PRH)

45. About how many sex partners have you had in your life? (SRH)

46. About how many sex partners have you had in the past year? (SRH)

47. About how many sex partners has your most recent partner had? (include self). (PRH)

48. How sure are you of your answer to question \#47? (PRH)
A. Absolutely sure, or I have never had a sex partner
B. Very sure
C. Somewhat sure
D. Slightly sure
E. Not at all sure

49. In the past year, did you use alcohol before having sex? (SRH)
A. I have never had a sex partner, OR, never used alcohol before sex
B. Sometimes
C. About half the time
D. Usually
E. Always

50. In the past year, how many drinks did you normally have before having sex? (SRH)
A. I have never had a sex partner, $O R$, never used alcohol before sex
B. 1-2 drinks
C. 3-5 drinks
D. 6-10 drinks
E. 11 or more 
51. In the past year, did you use marijuana or other drugs before having sex? (SRH)
A. I have never had a sex partner, $O R$, never used drugs before sex
B. Sometimes
C. About half the time
D. Usually
E. Always

52. If you were to make a guess, how much risk do you have for getting HIV/AIDS at this time in your life? (SRH)
A. No risk
B. Slight risk
C. Some risk
D. Moderate risk
E. High risk

If you have never had a sex partner, you may cross this chart out with a large "X."

If you had sex partner(s) this past year, these directions may help. Boxes under "Partner 1" are about your and Partner 1, the boxes under "Partner 2" are about Partner 2 and so on. It may help to put your partner's initials in each column if you want. We know that it's hard to put exact numbers but please make a guess. If you have not done some of these things, please fill in those boxes with a zero. Remember, no one will know the these answers are yours, and there are no "right" or "wrong" answers to these questions.

\begin{tabular}{|c|c|c|c|c|c|c|}
\hline In the past 12 months: & Example & Partner 1 & Partner 2 & Partner 3 & Partner 4 & Partner 5 \\
\hline $\begin{array}{l}\text { If you want to put } \\
\text { partner's initials: }\end{array}$ & J.S. & & & & & \\
\hline $\begin{array}{l}\text { 53. Vaginal sex with } \\
\text { a condom }\end{array}$ & 10 & & & & & \\
\hline $\begin{array}{l}\text { 54. Vaginal sex without } \\
\text { a condom }\end{array}$ & 7 & & & & & \\
\hline 55. Oral sex with condom & 6 & & & & & \\
\hline $\begin{array}{l}\text { 56. Oral sex without } \\
\text { a condom }\end{array}$ & 0 & & & & & \\
\hline 57. Anal sex with condom & 1 & & & & & \\
\hline $\begin{array}{l}\text { 58. Anal sex without } \\
\text { a condom }\end{array}$ & 2 & & & & & \\
\hline
\end{tabular}

59. Have you had more than five sex partners in the past year?
A. No
B. Yes

60. If yes, how many sex partners have you had in the past year? partners 


\section{Appendix E}

\section{Psychosocial Subscales}

Hypothesized Subscales:

$\mathrm{ED}=$ Education

$\mathrm{M}=$ Media

$\mathrm{AP}=\mathrm{AIDS}$ Proximity

$\mathrm{PR}=$ Peer Risk

1. How old were you when you had your first class in school that talked about HIV/AIDS? (ED)
A. 12 years old or younger
B. About 13-14 years old
C. About 15-16 years old
D. About 17 years old or older
E. I never had a class that talked about HIV/AIDS

(answer selection for questions 2 through 30 )
A. Definitely not true
B. Not really true
C. Somewhat true
D. Mostly true
E. Definitely true

2. I learned a lot about HIV/AIDS in classes I took in grade school. (ED)

3. I learned a lot about HIV/AIDS in classes I took in junior high school. (ED)

4. I learned a lot about HIV/AIDS in classes I took in high school. (ED)

5. Most of what I learned about HIV/AIDS in my classes was from listening to my teacher talk about it. (ED)

6. Most of what I learned about HIV/AIDS in my classes was from movies, books, and/or slides. (ED)

7. Most of what I learned about HIV/AIDS in my classes was from asking the teacher to answer my questions about it. (ED)

8. Most of what I learned about HIV/AIDS in my classes was from doing assignments or projects. (ED)

9. I learned a lot in my classes about the things people do that can cause them to get HIV/AIDS. (ED)

10. I learned a lot in my classes about what to do if I do not want to have sex. (ED)

11. Most of what I learned about how to practice safer sex was from health/sex education classes. (ED)

12. I was taught how to keep myself from getting into a situation that would put me at risk for getting HIV/AIDS. (ED)

13. It would be easy for me to talk to my mother or father (or guardian) about sex. (ED)

14. It would be easy to talk about sex with my brother or sister (if you do not have brothers or sisters, consider cousins, or other relatives). (ED)

15. It would be easy for me to talk about sex with a teach or school counselor. (ED) 
16. Most of what I know about HIV/AIDS I learned from my mother or father (or guardian). (ED)

17. Most of what I know about HIV/AIDS I learned from my brother(s) and/or sister(s). (ED)

18. Most of what I know about HIV/AIDS I learned from my friends. (ED)

19. When my best friend(s) talk about HIV/AIDS, I believe that what they say is true. (ED)

20. Most of what I know about sex I learned from my friends. (ED)

21. Knowing that "Magic Johnson," a famous basketball player, has HIV/AIDS has made me want to learn more about HIV/AIDS. (M)

22. Knowing that a famous singer I really like has died of AIDS would make me want to learn more about how I can avoid getting it. (M)

23. Knowing that a famous movie or TV star I really like has died of AIDS would make me want to learn more about how I can avoid getting it. (M)

24. Knowing a famous movie or TV star I really like has HIV/AIDS would make me want to learn more about how I can avoid getting it. (M)

25. Knowing a famous singer I really like has HIV/AIDS would make me want to learn more about how I can avoid getting it. (M)

26. For me, watching TV has been an important way to learn about HIV/AIDS. (M)

27. For me, listening to the radio has been an important way to learn about HIV/AIDS. (M)

28. For me, watching movies in the theater or videos has been an important way to learn about HIV/AIDS. (M)

29. For me, reading books or magazines has been an important way to learn about HIV/AIDS. (M)

30. For me, reading newspapers has been an important way to learn about HIV/AIDS. (M)

31. If you do know someone with HIV/AIDS, how old were you when you first knew this person with HIV/ADS? (AP)
A. 12 years old or younger
B. About 13-14 years old
C. About $15-16$ years old
D. About 17 years old or older
E. I do not know anyone with HIV/AIDS

(answer selection for questions 32 through 38 )
A. Definitely no
B. Probably no
C. Not sure
D. Probably yes
E. Definitely yes

32. A family member or a close friend has HIV/AIDS. (AP)

33. I know someone with HIV/AIDS who is not a close friend or member of my family. (AP)

34. I know a gay person who has HIV/AIDS. (AP)

35. I know a person who is not gay who has HIV/AIDS. (AP)

(continued on next page) 
36. I know a woman who has HIV/AIDS. (AP)

37. I know a person who shoots drugs with a needle who has HIV/AIDS. (AP)

38. I know someone who was tested for AIDS. (AP)

39. One of my close friends does things that could lead to them getting AIDS. (PR)
A. Definitely no
B. Probably no
C. Not sure
D. Probably yes
E. Definitely yes

(answer selection for questions 40 through 45)
A. Definitely no
B. Probably no
C. Probably yes
D. Definitely yes

40. My close friends insist on using condoms when they have sex with a risky partner. (PR)

41. My close friends think their partner wouldn't mind using a condom when they have sex. (PR)

42. My close friends are afraid to ask their partner to use a condom when they have sex. (PR)

43. Some of my close friends think that they are at risk of getting AIDS. (PR)

44. My close friends think that it's okay for a woman to ask a partner to use condoms. (PR)

45. My close friends ask a sex partner to use a condom. (PR)

46. Most of my friends have had sex. (PR)
A. Definitely not true
B. Not really true
C. Somewhat true
D. Mostly true
E. Definitely true 


\section{Appendix F}

\section{Codebook}

Items Grouped by Scales and Subscales

\section{Demographics}

Item

Gender

Age

Geographic location

Cultural group

Current grade

Marital status

Religion

Religiosity

Presently live with

Mother's education \& Father's education $\underline{\text { Raw Score Coding }}$

$\mathrm{A}=0$-Male; $\mathrm{B}=1$-Female

(Age)

$0=$ unknown; $1=\mathrm{RI} ; 2=\mathrm{CT} ; 3=\mathrm{MA} ; 4=\mathrm{NH} ; 5=\mathrm{NJ}$; $6=\mathrm{ME} ; 7=\mathrm{NY} ; 8=\mathrm{PA} ; 9=\mathrm{OH} ; 10=$ Bermuda; $11=$ Jamaica; $12=$ Cayman Islands; $13=\mathrm{DE} ; 14=\mathrm{VT}$

$\mathrm{A}=1$-White; $\mathrm{B}=2$-African-American;

$\mathrm{C}=3$-Asian-American; $\mathrm{D}=4$-Hispanic-American; $\mathrm{E}=5$-Other

Grade; 13=freshman; 14=sophomore+

$\mathrm{A}=0$-Single; $\mathrm{B}=1$-Married

$\mathrm{A}=1$-Catholic; $\mathrm{B}=2$-Protestant; $\mathrm{C}=3$-Jewish; $\mathrm{D}=4$-None; $\mathrm{E}=5$-Other

$\mathrm{A}=1$-Not at all; $\mathrm{B}=2$-Slightly; $\mathrm{C}=3$-Somewhat; $\mathrm{D}=4$-Fairly; $\mathrm{E}=5$-Very

$\mathrm{A}=1$-Both parents; $\mathrm{B}=2$-Mother; $\mathrm{C}=3$-Father;

$\mathrm{D}=4$-Someone else; $\mathrm{E}=5$-Alone

$\mathrm{A}=1$-8th grade or less; $\mathrm{B}=2$-some high school; $\mathrm{C}=3$-high school graduate; $\mathrm{D}=4$-some college; $\mathrm{E}=5$-college graduate 
Scale Range: 1-5; high score indicative of high factual knowledge

Item

AIDS is a medical condition in which your body cannot fight off diseases.

AIDS is caused by a virus.

If a pregnant woman has AIDS, there is a chance it may harm her unborn baby.

Most people who get AIDS usually die from it.

Using a condom (rubber) during sex can lower the risk of getting AIDS.

Receiving a blood transfusion with infected blood can give a person AIDS.
Raw Score Coding

A=1-Definitely no;

$\mathrm{B}=2$-Probably no;

$\mathrm{C}=3$-Not sure;

$\mathrm{D}=4$-Probably yes;

$\mathrm{E}=5$-Definitely yes
Coding Adjustment for Scale/Subscale

\section{Cognitive Subscales: Transmission AIDS Knowledge}

Scale Range: 1-5; high score indicative of high transmission knowledge

\section{$\underline{\text { Item }}$}

A woman who has unprotected sex only with men can get AIDS.

A man who has unprotected sex only with women can get AIDS.

A woman who has unprotected sex only with women can get AIDS.

A man who has unprotected sex only with men can get AIDS.
Coding Adjustment

$\underline{\text { Raw Score Coding }}$ for Scale/Subscale

$\mathrm{A}=1$-Definitely no; $\mathrm{B}=2$-Probably no;

$\mathrm{C}=3$-Not sure; $\mathrm{D}=4$-Probably yes; $\mathrm{E}=5$-Definitely yes

\section{Cognitive Subscales: Perceived AIDS Knowledge}

Scale Range: $1-5$; high score indicative of high perceived knowledge

\section{$\underline{\text { Item }}$}

If someone asked me a question about how to prevent getting HIV/AIDS, I can give them the right answer.

If someone asked me what things people do that can cause HIV/AIDS, I can tell them the right answer.

If someone asked me how HIV/AIDS is transmitted, I can give them the right answer.

\section{Raw Score Coding}

$\mathrm{A}=1$-Definitely not true;

$\mathrm{B}=2-$ Not really true;

$\mathrm{C}=3$-Somewhat true;

$\mathrm{D}=4$-Mostly true;

$\mathrm{E}=5$-Definitely true

(continued on next page)

Coding Adjustment for Scale/Subscale

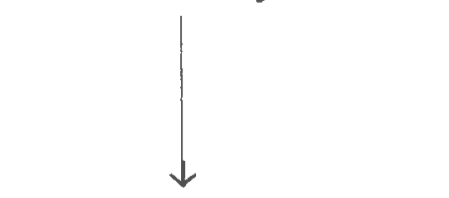


Cognitive Subscales: Misconceptions

Scale Range: 1-5; high score indicative of high misconception

Item

AIDS can be cured if treated early

A new vaccine has been developed which can prevent AIDS.

You can have AIDS and not know it.

Mosquitoes can transmit AIDS.

I can avoid getting AIDS by having sex only with healthy looking persons.

Only gay people and people who use needle drugs can get AIDS.
Coding Adjustment for Scale/Subscale

$\underline{\text { Raw Score Coding }}$

$\mathrm{A}=1$-Definitely no;

$\mathrm{B}=2$-Probably no;

$\mathrm{C}=3$-Not sure;

$\mathrm{D}=4$-Probably yes;

$\mathrm{E}=5$-Definitely yes

Reverse coding

\section{Attitudinal Subscales: Perceived Susceptibility for Risk}

Scale Range: $1-5$; high score indicative of high perceived susceptibility

Item

I feel that I am at risk of getting AIDS at this time in my life.

I sometimes think that I may have been exposed to AIDS.

I have had sex with someone who could have given me AIDS.

I believe I can do things to help protect myself from getting AIDS.

I try not to get into situations where I might get AIDS.
Raw Score Coding

Coding Adjustment for Scale/Subscale

$\mathrm{A}=1$-Definitely no;

$\mathrm{B}=2$-Probably no;

$\mathrm{C}=3$-Not sure;

$\mathrm{D}=4$-Probably yes;

$\mathrm{E}=5$-Definitely yes

Reverse coding

Reverse coding

\section{Attitudinal Subscales: Condom Use Efficacy}

Scale Range: $1-5$; high score indicative of high condom use efficacy

Item

I would use a condom when...

...I am really turned on.

...my partner gets mad about using

a condom.

...I am depressed.

...I have been drinking or doing drugs.

...I feel angry.

...I feel pressured to have sex.

...I'm afraid I might get AIDS.
Coding Adjustment

Raw Score Coding

$A=1-$ Not at all sure;

$B=2-A$ little sure;

$\mathrm{C}=3-$ Kind of sure;

$\mathrm{D}=4$-Fairly sure;

$E=5$-Very sure

(continued on next page) 
Scale Range: 1-5; high score indicative of high homophobia

\section{$\underline{\text { Item }}$}

I think people with HIV/AIDS should be in a special classroom at school.

I would not like it if someone in my gym class has HIV/AIDS.

A gay person should not be allowed to teach school.

A gay person should not be allowed to run for public office.

If my roommate told me he or she was gay, I would probably ask them to move out.

A gay person should not be allowed to be a coach.

I would stay away from people who are gay, because if I didn't, I would have a higher chance of getting HIV/AIDS.

It's okay for people who have HIV/AIDS to teach school.

I would feel nervous in a group of gay people.

If I saw two men or two women holding hands, I would feel uncomfortable.

I would feel uncomfortable sitting next to a gay person.

I am afraid that someone around me has HIV/AIDS and I do not know it.

I am afraid of making new friends because of HIV/AIDS.

If my brother/sister told me he/she was gay, I would be upset.

\section{Raw Score Coding}

$\mathrm{A}=1$-Definitely not true; $\mathrm{B}=2-$ Not really true;

$\mathrm{C}=3$-Somewhat true; $\mathrm{D}=4$-Mostly true; $\mathrm{E}=5$-Definitely true
Coding Adjustment for Scale/Subscale

Reverse coding

(continued on next page) 
Scale Range: 1-5; high score indicative of high substance use

\section{Item}

In the past year, how often have you had a drink of beer, wine, or liquor?

In the past year, how many drinks did you have when you drank alcohol?

In the past year, how often did you have

3 or more drinks in one day?

In the past year, how often did you have 6 or more drinks in one day?

In the past year, how many times did you use marijuana or hashish (grass, pot, hash, hash oil) without a doctor's orders?

In the past year, how many times did you use other drugs without a doctor's orders? In the past year, how many times did you share a needle to shoot drugs?

In the past year, how often did you have a hangover from your drinking/drug use?

$A=1-N e v e r ;$

$\mathrm{B}=2$-Once;

C=3-2-3 times;

$\mathrm{D}=4-4-6$ times;

$\mathrm{E}=5$-More than 6 times

$A=1-N e v e r ;$

$\mathrm{B}=2-1-2$ times/mo or less;

$\mathrm{C}=3-1-2$ times/wk;

$\mathrm{D}=4-3-4$ times/wk;

$\mathrm{E}=5$-Almost every day

$\mathrm{B}=2-1-2$ drinks;

$\mathrm{C}=3-3-5$ drinks;

$\mathrm{D}=4-6-10$ drinks;

$\mathrm{E}=5-11$ or more drinks

$\mathrm{A}=1$-Never;

$\mathrm{B}=2-1-2$ times/mo. or less;

$\mathrm{C}=3-1-2$ times/wk;

$\mathrm{D}=4-3-4$ times/wk;

$\mathrm{E}=5$-Almost every day

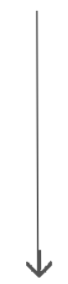

Coding Adjustment for Scale/Subscale 
Scale Range: 1-5; high score indicative of high substance abuse

$\underline{\text { Item }}$

In the past year, how often have these things happened because of your drinking or drug use:

I messed up on something important.

I was in trouble with police or other authority.

I broke or messed up someone else's things.

I got into an argument or fight.

I got sick/threw up.

I drove a car after having alcohol or drugs.

I was hurt/injured.

Someone I know talked to me about how I was acting.

I thought I might have a drinking or drug problem.

I couldn't remember (blacked out).

I did something I wished I hadn't.

I was arrested for drunk driving.

I had sex that I wouldn't have had if I was sober.

I tried to stop using but didn't.

I thought about or tried to kill myself.
Raw Score Coding

Coding Adjustment for Scale/Subscale
$A=1-N e v e r ;$

$\mathrm{B}=2$-Once;

$\mathrm{C}=3-2-3$ times;

$\mathrm{D}=4-4-6$ times;

$\mathrm{E}=5$-More than 6 times 


\section{Behavior Subscales: Sexual Assertiveness Partner Communication}

Scale Range: 1-5; high score indicative of high assertiveness for partner communication

\section{Item}

I would ask if I want to know if my partner ever had an HIV test.

I would ask my partner about the AIDS risk his or her past partners, if I want to know.

I would ask if I want to know if my partner ever had a STD.

I would ask if I want to know if my partner ever had sex with someone who shoots drugs with a needle.

I would ask if I want to know if my partner ever used needles to take drugs.

\section{Raw Score Coding}

$\mathrm{A}=1$-Never;

$\mathrm{B}=2$-Sometimes;

$\mathrm{C}=3$-About half the time;

$\mathrm{D}=4$-Usually;

$\mathrm{E}=5$-Always

\section{Behavior Subscales: Sexual Assertiveness Condom Use}

Scale Range: 1-5; high score indicative of high assertiveness for condom use

\section{Item}

I would have sex without a condom if my partner doesn't like them, even if I want to use one.

I would make sure my partner and I use a condom when we have sex.

I would insist on using a condom if I want to, even if my partner doesn't like them.

I would have sex without using a condom if my partner insists, even if I don't want to.

I would refuse to have sex if my partner refuses to use a condom.
Coding Adjustment for Scale/Subscale
Coding Adjustment

Raw Score Coding for Scale/Subscale

$A=1$-Never;

$\mathrm{B}=2$-Sometimes;

$\mathrm{C}=3$-About half the time; $\quad$ Reverse coding

$\mathrm{D}=4$-Usually;

$\mathrm{E}=5$-Always

Reverse coding

(continued on next page) 
Scale Range: 1-5; high score indicative of high self risk history

$\underline{\text { Item }}$

About how long have you been involved with your most recent sex partner?

How old were you the first time you willingly had intercourse?

About how many sex partners have you had in your life?

About how many sex partners have you had in the past year?

In the past year, did you use alcohol before having sex?

In the past year, did you use marijuana or other drugs before having sex?

In the past year, how many drinks did you normally have before having sex?

\section{Raw Score Coding}

$A=1$-No sex partners;

$\mathrm{B}=2$-Less than one mo;

$\mathrm{C}=3-1-6$ months;

$\mathrm{D}=4-6-12$ months; $E=5$-Over one year

(Age)

$\mathrm{A}=1$-None;

$\mathrm{B}=2$-One;

$C=3-2-4$;

$\mathrm{D}=4-5-7$;

$E=5-8$ or more

$A=1-N e v e r$;

$\mathrm{B}=2$-Sometimes;

$\mathrm{C}=3$-About half the time;

$\mathrm{D}=4$-Usually;

$\mathrm{E}=5$-Always

$A=1-N o n e ;$

$\mathrm{B}=2-1-2$ drinks;

C=3-3-5 drinks;

$\mathrm{D}=4-6-10$ drinks;

$E=5-11$ or more
Coding Adjustment for Scale/Subscale

Recode $(1=1),(2=5)$, $(3=4),(4=3),(5=2)$

Recode $(10-12=5)$, $(13=3.75),(14=2.50)$, $(15-18=1.25),(\mathrm{N} / \mathrm{A}=0)$

(continued on next page)

\footnotetext{
" Age range for point spread derived from sample distribution.
} 


\section{Behavior Subscales: Partner Risk History}

Scale Range: 1-5; high score indicative of high partner risk history

\section{Item}

How sure are you that you have not been exposed to AIDS?

How sure are you that your sex partner(s) have not been exposed to AIDS?

How sure are you about your estimate of number of partners your most recent partner has had?

Have you ever been tested for HIV?

About how many partners has you most recent partner had?

How many sex partners have you had who shoot drugs with a needle?

How many sex partners have you had who tested positive for HIV or had AIDS?

\section{Raw Score Coding}

$\mathrm{A}=1$-Absolutely;

$\mathrm{B}=2$-Very;

$\mathrm{C}=3$-Somewhat;

$\mathrm{D}=4$-Slightly;

$\mathrm{E}=5$-Not at all

$\mathrm{A}=1-\mathrm{No}$;

$\mathrm{B}=2$-Yes, negative;

$\mathrm{C}=3-\mathrm{Yes}$, positive

Coding Adjustment for Scale/Subscale

$\mathrm{A}=1$-None;

$\mathrm{B}=2$-One;

$\mathrm{C}=3-2-4$;

$\mathrm{D}=4-5-7$;

$\mathrm{E}=5-8$ or more
Recode $(1=1),(2=3)$, $(3=5)$

\section{Behavior Items}

(complex items examined individually)

\section{Item}

Do you always use condoms right now?

In the next 6 months, do you plan to start using condoms if you have sex?

If you were to make a guess, how much risk do you have for getting HIV/AIDS at this time in your life?
Raw Score Coding

Coding Adjustment for Scale/Subscale

$A=1$-No sex partners;

$\mathrm{B}=2$-Started using $1 \mathrm{yr}$ ago + ;

$\mathrm{C}=3$-Started using < yr ago;

$\mathrm{D}=4$-Sometimes, not always;

$\mathrm{E}=5$-Never

$A=1-$ No sex partners;

$\mathrm{B}=2$-Already always use;

$\mathrm{C}=3$-Plan to start using;

$\mathrm{D}=4$-Maybe, sometimes;

$\mathrm{E}=5-\mathrm{No}$

$\mathrm{A}=1$-No risk;

$\mathrm{B}=2$-Slight risk;

$\mathrm{C}=3$-Some risk;

$\mathrm{D}=4$-Moderate risk;

$\mathrm{E}=5$-High risk 
$\underline{\text { Item }}$

Vaginal sex/with condom

Vaginal sex/without condom

Oral sex/with condom

Oral sex/without condom

Anal sex/with condom

Anal sex/without a condom

More than five sex partners, past year

If yes, how many sex partners
Coding Adjustment

Raw Score Coding for Scale/Subscale

\section{Environment Subscales: Peer Risk}

Scale Range: 1-5; high score indicative of high peer risk

\section{Item}

Most of my friends have had sex.

One of my close friends does things that could lead to them getting AIDS.

My close friends insist on using condoms when they have sex with a risky partner. My close friends think that their partner would not mind using a condom when they have sex.

My close friends are afraid to ask a partner to use a condom when they have sex.

Some of my close friends think that they are at risk of getting AIDS.

My close friends think that it's okay for a woman to ask a partner to use condoms.

My close friends ask a sex partner to use a condom.

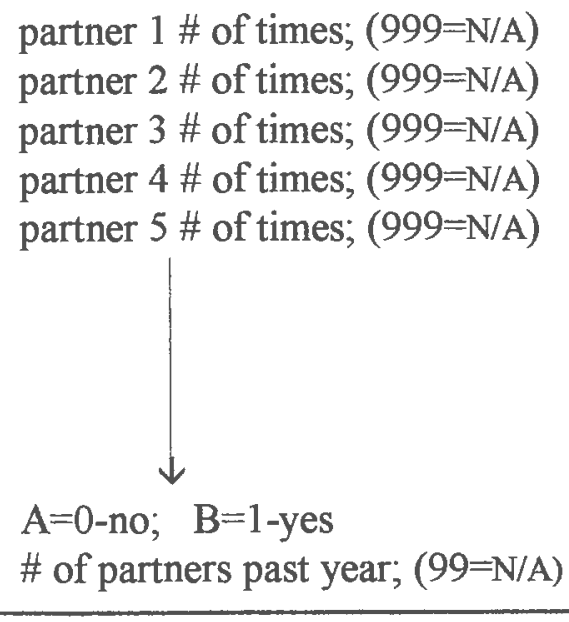

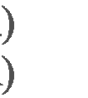

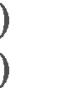


Scale Range: 1-5; high score indicative of high AIDS proximity

Item

If you know someone with HIV/AIDS, how old were you when you first knew this person with HIV/AIDS?

A family member or close friend has HIV/AIDS.

I know someone with HIV/AIDS who is not a close friend or a member of my family.

I know a gay person who has HIV/AIDS.

I know a person who is not gay who has HIV/AIDS.

I know a woman who has HIV/AIDS.

I know a person who shoots drugs with a needle who has HIV/AIDS.

I know someone who was tested for AIDS.
Coding Adjustment

Raw Score Coding

$A=1-12$ yrs or younger;

Reverse coding

$\mathrm{B}=2-13-14$ years;

$\mathrm{C}=3-15-16$ years;

$\mathrm{D}=4-17$ yrs or older;

$\mathrm{E}=5-\mathrm{N} / \mathrm{A}$

$\mathrm{A}=1$-Definitely no;

$\mathrm{B}=2$-Probably no;

$\mathrm{C}=3$-Not sure;

$D=4$-Probably yes;

$\mathrm{E}=5$-Definitely yes

\section{Environment Subscales: Media Resources}

Scale Range: 1-5; high score indicative of high use of media resources

$\underline{\text { Item }}$

For me, watching TV has been an important way to learn about HIV/AIDS

For me, listening to the radio has been an important way to learn about HIV/AIDS.

For me, seeing movies in the theater, or on video has been an important way to learn about HIV/AIDS.

For me, reading books or magazines has been an important way to learn about HIV/AIDS.

For me, reading newspapers has been an important way to learn about HIV/AIDS.
Raw Score Coding

Coding Adjustment for Scale/Subscale

$A=1$-Definitely not true;

$\mathrm{B}=2$-Not really true;

$\mathrm{C}=3$-Somewhat true;

$\mathrm{D}=4-$ Mostly true;

$\mathrm{E}=5$-Definitely true 
Scale Range: $1-5$; high score indicative of high media influence

Item

Knowing that "Magic Johnson," a famous basketball player, has HIV/ AIDS has made me want to learn more about HIV/AIDS.

Knowing that a famous singer I really like has died of AIDS would make me want to learn more about how I can avoid getting it.

Knowing that a famous movie or TV star I really like has died of AIDS would make me want to learn more about how I can avoid getting it.

Knowing a famous movie or TV star I really like has HIV/AIDS would make me want to learn more about how I can avoid getting it.

Knowing a famous singer I really like has HIV/AIDS would make me want to learn more about how I can avoid getting it.

\section{Raw Score Coding}

$A=1-$ Definitely not true;

$\mathrm{B}=2$-Not really true;

$\mathrm{C}=3$-Somewhat true;

$\mathrm{D}=4-$ Mostly true;

$\mathrm{E}=5$-Definitely true
Coding Adjustment for Scale/Subscale

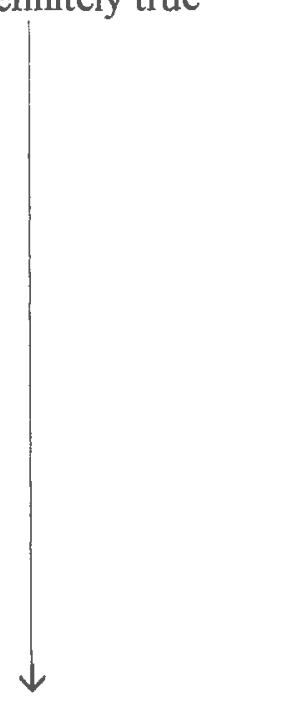

(continued on next page) 
Scale Range: 1-5; high score indicative of high education derived from school

$\underline{\text { Item }}$

How old were you when you had your first class in school that talked about HIV/AIDS?

I learned a lot about HIV/AIDS in classes I took in grade school.

I learned a lot about HIV/AIDS in classes I took in junior high school.

I learned a lot about HIV/AIDS in classes I took in high school.

Most of what I learned about HIV/AIDS in my classes was from listening to my teacher talk about it.

Most of what I learned about HIV/AIDS in classes was from movies, books, and/or slides.

Most of what I learned about HIV/AIDS in my classes was from asking the teacher to answer my questions about it.

Most of what I learned about HIV/AIDS in my classes was from doing assignments.
Coding Adjustment for Scale/Subscale

Reverse coding $B=2-13-14$ years; $C=3-15-16$ years; $\mathrm{D}=4-17$ yrs or older; $E=5-N / A$

$A=1$-Definitely not true; $B=2-$ Not really true; $\mathrm{C}=3$-Somewhat true; $\mathrm{D}=4-$ Mostly true; $\mathrm{E}=5$-Definitely true

\section{Education Subscales: School Influence}

Scale Range: 1-5; high score indicative of high influence derived from school

$\underline{\text { Item }}$

I learned a lot in my classes about the things people do that can cause them to get HIV/AIDS.

I learned a lot in my classes about what to do if I do not want to have sex.

Most of what I learned about how to practice safer sex was from my health or sex education classes.

I was taught how to keep myself from getting into a situation that would put me at risk for getting HIV/AIDS.

It would be easy for me to talk about sex with a teacher or school counselor.
Coding Adjustment

Raw Score Coding for Scale/Subscale

(continued on next page)

$\mathrm{A}=1$-Definitely true;

$\mathrm{B}=2$-Not really true;

$\mathrm{C}=3$-Somewhat true;

$\mathrm{D}=4$-Mostly true;

$\mathrm{E}=5$-Definitely true 
Education Subscales: Peer Education (single item)

Scale Range: $1-5$; high score indicative of high education derived from peers

$\underline{\text { Item }}$

It would be easy for me to talk about sex with my best friend(s). $\underline{\text { Raw Score Coding }}$

$A=1-$ Definitely not true;

$B=2-$ Not really true;

$\mathrm{C}=3$-Somewhat true;

$\mathrm{D}=4-$ Mostly true;

$\mathrm{E}=5$-Definitely true

\section{Education Subscales: Peer Influence}

Scale Range: $1-5$; high score indicative of high influence derived from peers

Item

Most of what I know about HIV/AIDS

I learned from my friends.

When my best friend(s) talk about HIV/
Raw Score Coding

$\mathrm{A}=1$-Definitely not true;

$\mathrm{B}=2$-Not really true;

$\mathrm{C}=3$-Somewhat true;

AIDS, I believe that what they say is true. $\mathrm{D}=4$-Mostly true;

Most of what I know about sex I learned from my friends.

Coding Adjustment for Scale/Subscale

$\mathrm{E}=5$-Definitely true

$\downarrow$

\section{Education Subscales: Parent Education (single item)}

Scale Range: 1-5; high score indicative of high education derived from parents

$\underline{\text { Item }}$

It would be easy for me to talk to my mother or father(or guardian) about sex.
Raw Score Coding

$A=1$-Definitely not true;

$B=2-N o t$ really true;

$\mathrm{C}=3$-Somewhat true;

$\mathrm{D}=4-$ Mostly true;

$\mathrm{E}=5$-Definitely true
Coding Adjustment for Scale/Subscale

\section{Education Subscales: Parent Influence (single item)}

Scale Range: 1-5; high score indicative of high influence derived from parents

Item

Most of what I know about HIV/AIDS I learned from my mother or father (or guardian).
Raw Score Coding Coding Adjustment for Scale/Subscale

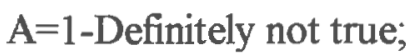

$\mathrm{B}=2-$ Not really true;

$\mathrm{C}=3$-Somewhat true;

$\mathrm{D}=4-$ Mostly true;

$\mathrm{E}=5$-Definitely true

(continued on next page) 
Education Subscales: Sibling Education (single item)

Scale Range: 1-5; high score indicative of high education derived from siblings

Item

It would be easy to talk about sex with my brother or sister (if you don't have brothers or sisters, consider cousins, or other relatives). $\underline{\text { Raw Score Coding }}$

$\mathrm{A}=1$-Definitely not true;

$\mathrm{B}=2-$ Not really true;

$\mathrm{C}=3$-Somewhat true;

$\mathrm{D}=4$-Mostly true;

$E=5$-Definitely true

Coding Adjustment for Scale/Subscale

Education Subscales: Sibling Influence (single item)

Scale Range: 1-5; high score indicative of high influence derived from siblings

$\underline{\text { Item }}$

Most of what I know about HIV/AIDS I learned from my brother(s) and $/$ or sister(s).
Coding Adjustment

$\underline{\text { Raw Score Coding }}$ for Scale/Subscale

$\mathrm{A}=1$-Definitely not true;

$\mathrm{B}=2-$ Not really true;

$\mathrm{C}=3$-Somewhat true;

$\mathrm{D}=4$-Mostly true;

$\mathrm{E}=5$-Definitely true 


\section{Appendix G}

Focus Group Feedback Sheets and

Semi-Structured Interview Questions

Feedback Sheets

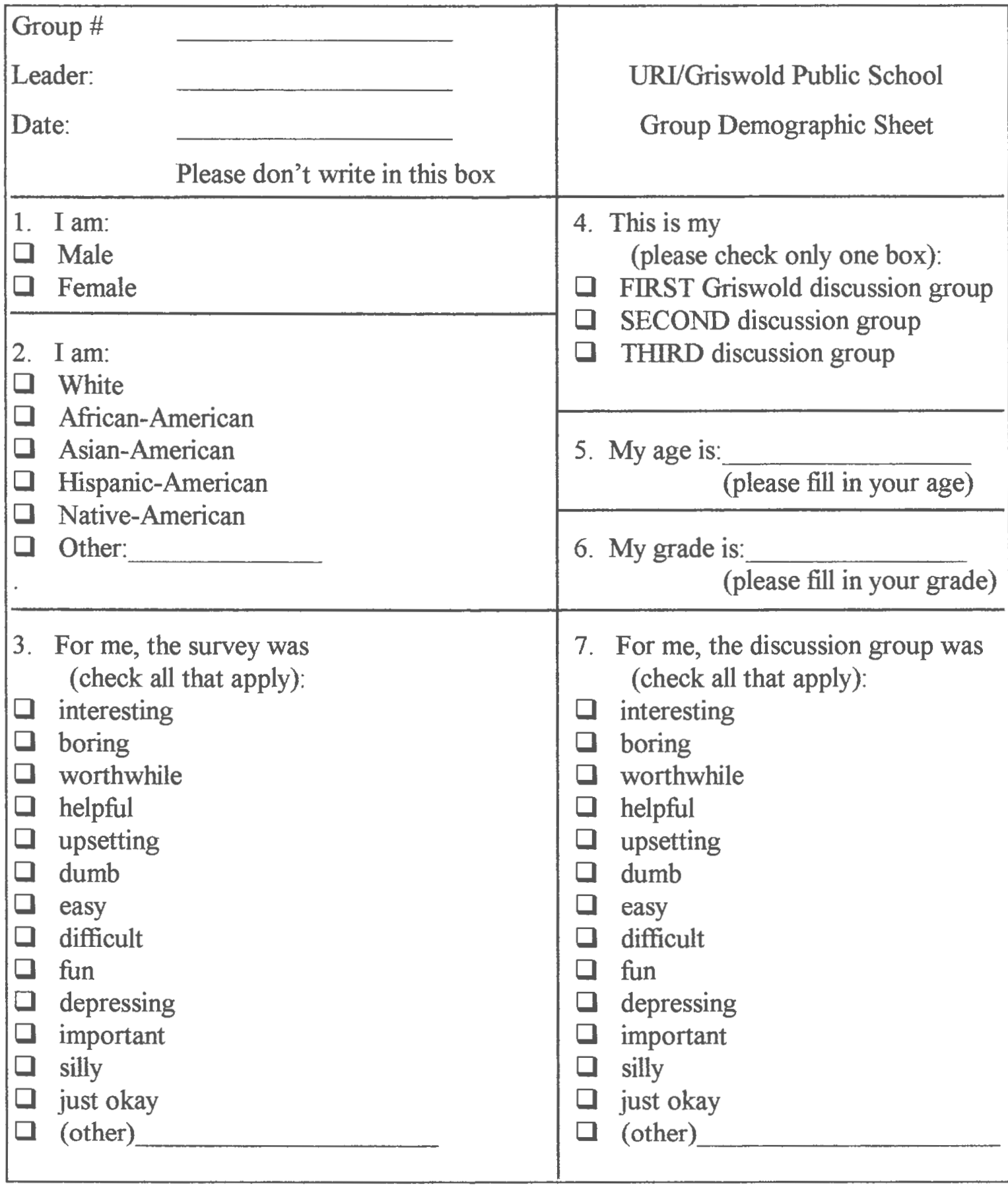


Overview [repeated before each group]: I'd like to thank you all for participating in this important research. Before we begin, it is important that we understand that anything discussed in this group is confidential. And we should not talk about what other people say outside of the group. So that nobody is embarrassed about talking about personal things, I'd like to establish a format. You can talk about yourself, your friends, your family, or anyone you know, but you cannot give their name or identify who you are talking about. To do that, you can say, 'I know somebody who...' This way nobody knows if you're talking about yourself or somebody else. Is that okay with everybody?

First some simple questions. Did you find the survey easy to understand? Were there parts you liked or didn't like? Were there parts that you would have taken out, or things you think should have been added that weren't?

Do you think surveys like this can help researchers learn more about HIV/AIDS? Do you think they will help researchers help other people from getting HIV/AIDS? Why or why not? What do you think would help?

Here's your chance, pretend you have the power. There is no cure for AIDS how would you help people from getting it, and what would you do for all those people who already have it?

Do you think that HIV/AIDS is a problem for teenagers? How about for teenagers, let's say, in Connecticut?

What other things about HIV/AIDS would you have liked to discuss but we didn't have time?

Okay today we will be discussing moral and ethical dilemmas people may face regarding AIDS, both as a society and individually. We talked in some groups about some places that separate people with AIDS to reduce spreading, what do you think about putting people with AIDS in a separate area to live and no one could go in and no one could come out? What are the good points and bad points about that?

What if your best friend had HIV or AIDS and you couldn't see them anymore? What if it were your brother or sister? What if it were your parents?

What about gay people? Do you think that AIDS is only a problem for them? What if you found out someone in your school is gay, would you feel differently about them? How would you feel? What if you found out your best friend was gay, what if anything, would be different? What if you found out your best friend's parents were gay, what if anything, would be different? What if you found out your brother or sister was gay, what 
if anything, would be different? What if your parents were getting a divorce and you found out one of them was gay, what, if anything, would be different?

Other than a gay person's choice in a sexual partner, how are they different? Can you tell if someone is gay without them telling you?

Recently scientists have discovered that one medication can help pregnant women who have AIDS not give it to their unborn child. Do you think that all pregnant women should be forced to be tested for AIDS and forced to take medication for their baby? What about rights? Should society consider the pregnant woman's rights or the rights of the unborn baby? What if she doesn't want testing, what if she doesn't want to know if she has AIDS? Does she have the right to refuse to be tested, and still be a good mother? Why might a pregnant woman not want to be tested? What might change in her life if she found out she did have HIV or AIDS?

Can someone with HIV have sex without giving it to someone else? Should people with AIDS refrain from having sex for the rest of their lives?

Okay generally, most people said that AIDS is a problem for teens. How much risk do you think teenagers would take in different situations. If someone's parents said they couldn't go out for some reason, and a friend comes to the window after the parents went to bed, what do you think it would take to make someone go out anyway and risk getting in trouble? (Prompt: The first thing that came to mind?) What if the parents weren't going to be home until late and friends show up with alcohol, how risky is that situation? What if it were drugs instead of alcohol? What if a boyfriend or girlfriend showed up and the parents were out until late, what are the risks if they decided to have sex? What would make that person decide it was okay to have sex? What if the guy or the girl was really popular? What if the guy or the girl was really, really cute?

Do you think teens are comfortable talking with friends about sex? Why or why not? How about parents? Who do you think teenagers should talk to about sex and why? Who would be the best person to talk to if they could? Do you think parents talk to their children about AIDS? In general, what might be good about sex and what might be bad about sex? Do you think those ideas reflect how people are brought up?

What other things about HIV/AIDS would you have liked to discuss but we didn't have time? 


\section{Appendix H}

Passive Consent (Youth-to-Youth)

[URI Psychology Department Letterhead]

July, 1994

\section{* * * IMPORTANT * * *}

Adolescent Health and Lifestyle Project, The University of Rhode Island Department of Psychology, Kingston, Rhode Island 02881

\section{PARENTAL CONSENT FORM FOR RESEARCH}

(To be returned only if you DO NOT want your child to participate in a University of Rhode Island survey study)

Dear Parent/Guardian:

I am a doctoral student in the Clinical Psychology Program at the University of Rhode Island and a Youth-to-Youth adult volunteer, and I am requesting your permission to have your son or daughter participate in a study on Acquired Immune Deficiency Syndrome (AIDS) knowledge, attitudes and awareness among adolescents. If you agree, your child, and all other teens attending the 1994 Youth-to-Youth Conference in Bristol, RI will be asked to fill out an ANONYMOUS questionnaire measuring knowledge, attitude and behavior regarding AIDS. The study has been approved by the University of Rhode Island Institutional Review Board which protects research participants' rights in such projects. The paper-and-pencil survey will take about 45 minutes to complete and will be conducted during the conference registration.

Participation is voluntary. If your child decides to take part in this study, he or she may quit at any time. The possible risks or discomforts of the study are minimal, some degree of initial awkwardness about sensitive information relating to sexual and drug behavior may occur. The possible benefits from this study include an increased knowledge and awareness for your child about his or her personal behavior. In addition, the researcher hopes to learn valuable information about prevention of AIDS, toward designing future health education intervention/prevention programs for youth. Your child's survey answers in this study are anonymous. None of the responses to the survey will identify your child by name, even the researcher will not be able to match surveys with participants. The only place your child's name will appear is on similar assent form to verify that your child has agreed to volunteer. The assent form will NOT be attached to any responses and will be collected separately to ensure confidentiality. Data from this study will be coded and reported in grouped data only and will not reveal specifics about any individual. 
Participation is not expected to be harmful to your child. However, if completing the survey is upsetting, phone numbers are provided on the assent form for assistance. If either you or your child has questions or want more information on the study or about AIDS, please contact me at URI (401-792-2741). If you are not satisfied with the way this study is performed, you may discuss your complaints with me or my supervisor, Kathryn Quina, Ph.D. (401-277-3832) anonymously, if you choose. Also, you may contact the office of the Vice Provost for Research, URI, 70 Lower College Road, Kingston, RI (401-792-2635).

\section{IF YOU CONSENT TO YOUR CHILD'S PARTICIPATION, PLEASE DO NOT RETURN THIS FORM, KEEP IT FOR YOUR INFORMATION.}

If you have questions, please call me or Dr. Quina at the telephone numbers above, we will be happy to discuss the project with you and answer any questions. Thank you for your assistance, and I hope this study will lead to better understanding of adolescent behavior and AIDS awareness.

Sincerely,

Michelle A. Lang, M.A.

University of Rhode Island

If you DO NOT want your child to participate in this project, please sign, date, and have your child return this form at the Youth-to-Youth Conference registration.

My child is not allowed to participate in

(please print your child's name)

the URI Health and Lifestyle Project for adolescents. I understand that my decision does not affect my child's participation in the Youth-to-Youth conference in any way.

Signature of Parent/Guardian

Printed Name of Parent/Guardian

Date: 


\section{Appendix I}

\section{Parent Cover Letter (Griswold)}

[URI Psychology Department Letterhead]

September, 1994

Dear Parent/Guardian:

I am a doctoral student in the Clinical Psychology Program at the University of Rhode Island. I am writing to request your permission to have your son or daughter participate in a study related to Acquired Immune Deficiency Syndrome (AIDS) knowledge and awareness among adolescents at Griswold Public School. The study has been approved by the School Board and faculty for the junior and senior high school students at Griswold, in an effort to assess the overall knowledge and awareness of the students attending the school. The study has also received approval from the University of Rhode Island Institutional Review Board safeguarding the rights and welfare of research participants, and ensuring ethical practices of researchers.

As you may know, the number of AIDS cases continues to increase every year, and Human Immunodeficiency Virus (HIV) and AIDS has extended into the heterosexual adolescent population. The importance of understanding risk factor and promoting behavioral awareness is an essential component in successful prevention of the spread of AIDS. It is our hope to extend existing research efforts to include younger members of the community, specifically, adolescents. For instance, the number of AIDS cases among teens is doubling each year, yet very little research has included the adolescent population.

With your permission, participation will involve completion of a paper and pencil survey at Griswold lasting about 40 minutes measuring your son or daughter's knowledge, attitude and behavior regarding AIDS. The survey answers will be completely anonymous. The questionnaire has been administered to 500 teenagers in the Rhode Island, Connecticut and Massachusetts area during 1993. With parental and participant feedback, an improved, shortened version was distributed to an additional 500 teens attending a youth conference during the summer of 1994. Findings from these earlier studies show that many teens in our area obtain the majority of their information from friends, and still hold several misconceptions about the transmission of AIDS.

Consequently many of these teens underrated or overrated their personal risk for AIDS. However, many adolescents reported positive feedback in their increased awareness of their personal behavior with the survey taking process.

Surveys will be provided at Griswold in the Fall 1994 semester, during health or study class, or activity period. Only those students who have obtained parental or guardian permission, indicated by the return of the enclosed parental consent form, will participate. The names of all survey participants will be kept in the strictest confidence, and personal identification of all individual survey responses will be impossible to obtain even for the researcher. Absolutely no names or individual profiles will be used in the study report. Informal discussion groups will take place after survey completion at a later 
date for those students who wish to participate by providing their feedback about the survey, such as readability, and discussing any general concerns or ideas they may have about helping teenagers in the intervention and prevention of HIV/AIDS. This will also provide an opportunity for students to participate in an informal question and answer session. Absolutely no questions about personal behavior will be asked in the discussion groups.

Two $\$ 50$ cash incentive bonuses will be offered to the participants, one drawn from the names of those completing the survey, and a second drawn from those participating in a discussion group. Numbered bonus tickets will be distributed to survey and discussion participants, and a two winners will be drawn later during the semester.

Participation is voluntary. If any individual wishes to withdraw from the study, his or her wish will be respected. I believe all participants will receive direct benefits from this study in an increased knowledge and awareness about his or her personal behavior in this area. Additionally, along with other researchers, I hope to learn valuable information about intervention and prevention of AIDS from the information derived from the adolescent population, and assist Griswold in an assessment of their potential health curriculum needs.

Enclosed with this letter are two copies of a "Parental Consent Form for Research." The rather formal style of the consent form is required by Federal regulations. Please read the consent form. If you approve and your child agrees to participate, please sign both copies and have your child return one copy to Griswold. The second copy is for you to keep.

If you have any questions, or if you would like to see the survey in advance, please feel free to call me, or my supervisor, Dr. Kathryn Quina, University of Rhode Island Psychology Department at the College of Continuing Education Campus in Providence (401) 277-3832 or the AIDS Research Center at University of Rhode Island in Kingston, Rhode Island (401) 792-2741. Additionally, a copy of the survey will be available at Griswold for parent or guardian review. The results of this study will be offered to the faculty at Griswold in an effort to help develop a health curriculum tailored for the population at the school. Please contact me at either of the numbers listed on the enclosed card if you would like a copy of the results at the conclusion of the study in the Spring of 1995 , or if you have any questions or concerns regarding this study.

Thank you for your cooperation. I hope that you will give permission to let your son or daughter take part in this very important study. Further, I hope this study will lead to a better understanding of adolescent behavior and AIDS awareness toward more effective intervention and prevention strategies with young people.

Sincerely,

Michelle A. Lang, M.A.

University of Rhode Island 


\section{The University of Rhode Island \\ Department of Psychology, Kingston, RII 02881 Health and Lifestyle Project}

\section{PARENTAL CONSENT FORM FOR RESEARCH}

I have been asked to grant my son or daughter permission to take part in a research project described below. The researcher will explain the project to me in detail and I should feel free to ask questions. If I have more questions later, Michelle Lang, the person mainly responsible for this study, will discuss them with me (401-792-2741 or 401792-2193).

I have been asked to grant my son or daughter permission to take part in a study of adolescent knowledge, attitudes and health-related behaviors concerning AIDS by anonymously completing a pencil and paper survey. Additionally, informal confidential discussion groups will be offered to my son or daughter to participate, with the opportunity to ask questions and/or provide feedback about the survey, and concerns or ideas about HIV/AIDS prevention for teenagers. Because of the nature of some of the survey questions, my child must be 18 years of age or acquire parental consent to participate.

If I decide to allow my child to take part in this study, his or her participation will involve filling out a survey at Griswold Public School of approximately 160 items pertaining to his or her knowledge, attitudes and behavior. This will take about 40 minutes. Upon completion of the survey, my child will return it to the researcher at Griswold. Only subject code numbers will be attached to my child's responses at a later date for data entry purposes only and will be assigned after all surveys are completed to ensure complete anonymity of responses. My child will have the opportunity to participate in small, informal group discussions about HIV/AIDS after completing the survey if he or she likes. My child will be eligible to win a cash bonus incentive of $\$ 50$ for survey completion, and $\$ 50$ for discussion group participation.

The possible risks or discomforts of the study are minimal, some degree of initial awkwardness about sensitive information relating to sexual and drug behavior may occur.

The possible benefits from this study include an increased knowledge and awareness for my child about his or her personal behavior in this area. In addition, the researcher hopes to learn valuable information about prevention of AIDS, and listen to feedback and ideas that adolescents have regarding intervention and prevention.

My child's part in this study is confidential. None of the actual responses to the survey will identify my child by name. All records will be collected anonymously in a 
manner in which the researcher will not be able to match the surveys with the participants. The only place my name is used is at the bottom of this form to verify that I agreed to allow my child to participate. The only place my child's name is used is at the bottom of a similar assent form to verify that my child has agreed to volunteer for this study. This form and the assent form will not, however, be attached with any of the survey responses, and both will be collected independently of the survey to ensure confidentiality. Information from this study will be reported only in grouped data that does not reveal specifics regarding any individual.

Participation in this study is not expected to be harmful to my child. However, if completing this survey is upsetting to my child telephone numbers for assistance are provided on the enclosed card. All survey and discussion group participants will also receive this information resource card. If either myself or my child has any questions, concerns or would like more information about this study or about AIDS, I can contact the researcher, Michelle Lang. If I am not satisfied with the way this study is performed, I may discuss my complaints with Michelle Lang, or her supervisor Kathryn Quina, Ph.D. (401-277-3832) anonymously, if I choose. In addition, I may contact the office of the Vice Provost for Research, 70 Lower College Road, University of Rhode Island, Kingston, Rhode Island (401-792-2635).

With my permission, the decision whether or not to take part in this study is up to my child. In giving my child permission to participate, I am giving my child the choice whether to participate. My child does not have to participate. If my child decides to take part in this study, he or she may quit at any time. If my child wishes to quit, he or she simply informs the researcher of his or her decision. I have read this Consent Form and my questions have been answered. My signature on this form means that I understand the information and I grant my child permission to participate.

Signature of parent $\&$ date

Please print child's name

Signature of researcher \& date
Typed/printed name

Parents/guardians may be contacted to verify permission. Please include telephone number where you can be reached:

Michelle A. Lang

Typed/printed name

\section{Please retain one of the two consent forms for your records}




\section{Appendix K}

\section{Information Card}

\section{Telephone Numbers for Assistance or Information}

For more information about this project:

Michelle Lang, URI, toll free: 1-800-287-1823 John Cormier, School Psychologist, Griswold or $(401) 792-2741 ;(401) 792-2193$ (203) 376-7027

For more information or assistance about AIDS or AIDS-related issues:

RI Project AIDS Hotline: 1-800-726-3010

Alcoholics Anonymous: 1-800-439-8860

or (9-9 M-F, 12-4 Sat): (401) 831-5522

The Samaritans (RI): (401) 272-4044

The Samaritans (CT): (203) 232-2121

AIDS Information Lines of Eastern Connecticut:

Hartford: (203) 249-6850

Old Saybrook area: (203) 388-9941

New London County: (203) 886-0516
Middletown area: (203) 346-6691

Putnam/Thompson area: (203) 928-6577

Danielson/Plainfield/Willimantic: (203) 456-8886 


\section{Appendix L}

\section{Participant Cover Letter}

[URI Psychology Department Letterhead]

, 1994

Dear Participant:

Thank you for agreeing to fill out this survey on teenagers and health. It will take you about 40 minutes or less to fill it out. Please read and sign the "Informed Assent" Form attached, and return one copy to me, apart from the survey. The second copy is for you to keep. Please remember that all of your answers on the survey are completely ANONYMOUS. Your name will never be matched to your answers. ONLY YOU WILL KNOW WHAT YOU ANSWER TO THE SURVEY QUESTIONS! If you would like to participate in a small discussion group in the next few weeks, please sign the list located next to the survey return area. You will receive a $\$ 50$ bonus ticket after you complete the survey. If you decide to join a discussion group, you will receive another $\$ 50$ bonus ticket after you participate. Winners will be drawn [at the closing ceremonies of the Youth-toYouth Conference/later this semester].

If you have any questions about HIV/AIDS or if you have any feelings that you want to talk about privately after you answer the survey, phone numbers have been provided on the cards attached to this letter. Please consider using the phone numbers to find someone who could help you. Please make as many calls as you feel you need to.

Your answers are very important to myself and other researchers. It is important to learn how to protect ourselves and others from AIDS, and other sexually transmitted diseases, and from other things that harm us. Your answers will help us learn about how to do that. Please remember that no one will know what YOUR answers are. Your name will not be on any survey. No one can learn your private answers to these questions. We hope this survey will not make you feel embarrassed or judged. There are no "right" or "wrong" answers to these questions. No answers are "good" answers or "bad" answers.

Your answers will help us to understand more and we are very grateful for your help. Thank you again for participating.

Sincerely,

Michelle A. Lang

University of Rhode Island 
Participant Assent (Youth-to-Youth)

\section{The University of Rhode Island Department of Psychology, Kingston, RI o2881 Health and Lifestyle Project}

\section{PARTICIPANT ASSENT FORM FOR RESEARCH}

A researcher at the University of Rhode Island has asked me to be a part of a research project at the Youth-to-Youth Conference described below. The researcher will explain the project to me and answer any questions that I may have about this study. If I have more questions later, Michelle Lang, the person mainly responsible for the study will discuss them with me (401-792-2741 or 401-792-2193).

I have been asked to take part in a study about teenagers, knowledge attitudes and other health-related behaviors concerning AIDS by filling out a survey that will be anonymous at the 1994 Youth-to-Youth Conference. Because some of the survey questions are very sensitive and personal, my parents have given me permission to participate by not returning the Parental Consent Form stating that they do not want me to participate.

If I decide to take part in this study, my participation will involve filling out a survey of about 160 questions regarding my knowledge, my attitudes and my behavior about AIDS. This will take about 40 minutes. When I finish the survey, I will return it to Michelle Lang at the Youth-to-Youth Conference. I will not put my name on the survey. All of my answers will be anonymous, no one will know which survey is mine, and I understand that my parents or anyone at the conference will not have access to any of my personal answers

The possible risks or discomforts of this study are small, I might feel awkward about answering some of the survey questions about sexual and drug behavior.

The possible benefits from this study include an increased knowledge and awareness about my personal behavior. In addition, the researcher hopes to learn valuable information about prevention of AIDS, by listening to what teenagers have to say. Additionally, when I return the completed survey I will be eligible to win a \$50 bonus prize for participation.

My involvement in this study is confidential. None of the actual answers to the survey will identify me by name. Everything will be collected anonymously in a manner in which even the researcher will not be able to match up the surveys with the people filling them out. The only place my name is used is at the bottom of this form to verify that I agree to volunteer in this study. This form will not be attached to any of the completed surveys. Everything will be collected separately. Information from my survey will be 
added together with information from many other people completing the survey in this study. No specific or individual answers will be reported, only totals as a group.

Participation in this study is not expected to be harmful to me. If I feel uncomfortable after completing the survey, I can call Michelle Lang or the telephone numbers on this form or on the questionnaire for someone to talk to. I can make as many calls as I feel I need to. If I want some additional information about this study or about AIDS, I can call Michelle Lang and she will answer my questions or send me some information that will help me. If I am not satisfied with the way this study is performed, I may discuss my complaints with Michelle Lang, or her supervisor, Dr. Kathryn Quina, (401-277-3832) privately or anonymously, if I choose, or I can contact the office of the Vice Provost for Research, 70 Lower College Road, University of Rhode Island, Kingston, Rhode Island (401-792-2635).

The decision whether or not to take part in this study is up to me. I have permission from my parents or guardian to be a part of this study, but I do not have to participate if I do not want to. If I decide that I want to quit, I may do so at any time. If I want to quit, I will tell Michelle Lang that I do not want to be a part of this study. I have read this Assent Form and my questions have been answered. My signature on this form means that I understand the information and I agree to participate in this study.

Signature of participant \& date

Typed/printed name

Michelle A. Lang

Signature of researcher \& date

Typed/printed name

Telephone Numbers for Assistance or Information

Michelle Lang, URI: (401) 792-2741

RI Project AIDS Hotline: 1-800-726-3010

Alcoholics Anonymous: 1-800-439-8860

The Samaritans (RI): (401) 272-4044

Please retain one of the two assent forms for your records 


\section{The University of Rhode Island Department of Psychology, Kingston, RI o2881 Health and Lifestyle Project}

\section{PARTICIPANT ASSENT FORM FOR RESEARCH}

A researcher at the University of Rhode Island has asked me to be a part of a research project described below. The researcher will explain the project to me and answer any questions that I may have about this study. If I have more questions later, Michelle Lang, the person mainly responsible for the study will discuss them with me (401-792-2741 or 401-792-2193).

I have been asked to take part in a study about teenagers, knowledge attitudes and other health-related behaviors concerning AIDS by filling out a survey that will be anonymous. I also have the option to take part in a small discussion group to ask any questions that I may have, talk about whether the survey was easy or hard to read, and to provide the researcher with any ideas that I may have about preventing AIDS with teenagers. Because some of the survey questions are very sensitive and personal, my parents have given me permission to participate (or I am 18 years old or older).

If I decide to take part in this study, my participation will involve filling out a survey of about 160 questions at Griswold Public School regarding my knowledge, attitudes and behavior about AIDS. This will take about 40 minutes. When I finish the survey, I will return it to Michelle Lang at Griswold. I will not put my name on the survey. All of my answers will be anonymous, no one will know which survey is mine, and I understand that my parents or my teachers will not have access to any of my personal answers. If I want to, I can participate in a small discussion group after filling out the survey at a later date, if I have any questions, comments about the survey, or ideas about AIDS prevention.

The possible risks or discomforts of this study are small, I might feel awkward about answering some of the survey questions about sexual and drug behavior.

The possible benefits from this study include an increased knowledge and awareness about my personal behavior. In addition, the researcher hopes to learn valuable information about prevention of AIDS, by listening to what teenagers have to say.

My involvement in this study is confidential. None of the actual survey answers will identify me by name. Everything will be collected anonymously in a manner in which even the researcher will not be able to match up the surveys with the people filling them out. The only place my name is used is at the bottom of this form to verify that I agree to 
volunteer in the study. This form will also be used for a $\$ 50$ cash bonus that I will be eligible to win after completing the survey. If I decide to participate in a discussion group later, a similar form will be used for a separate $\$ 50$ cash bonus. This form and my parents' permission form will not be attached to any completed surveys, everything will be collected separately. Information from my survey will be added together with information from many other people completing the survey. No specific or individual answers will be reported, only totals as a group. If I decide to participate in a discussion group, none of my comments will identify my name, and I will not be asked to talk about my own behavior.

Participation in this study is not expected to be harmful to me. If I feel uncomfortable after completing the survey, or participating in a discussion group, I can call Michelle Lang or any of the other telephone numbers on the attached card for someone to talk to. I can make as many calls as I feel I need to. If I want some additional information about this study or about AIDS, I can call Michelle Lang and she will answer my questions or send me some information that will help me. If I am not satisfied with the way this study is performed, I may discuss my complaints with Michelle Lang, or her supervisor, Dr. Kathryn Quina, (401-277-3832) privately or anonymously, if I choose, or I can contact the office of the Vice Provost for Research, 70 Lower College Road, University of Rhode Island, Kingston, Rhode Island (401-792-2635).

The decision whether or not to take part in this study is up to me. I have permission from my parents or guardian to be a part of this study (or I am 18 years old or older), but I do not have to participate if I do not want to. If I decide that I want to quit, I may do so at any time. If I want to quit, I will call Michelle Lang and inform her that I do not want to be a part of this study. I have read this Assent Form and my questions have been answered. My signature on this form means that I understand the information and I agree to participate in this study.

Signature of participant $\&$ date

Typed/printed name

Signature of researcher \& date

Michelle A. Lang

Typed/printed name

Please retain one of the two assent forms for your records 


\section{Bibliography}

Abraham, C. \& Sheeran, P. (1994). Modelling and modifying young heterosexuals' HIVpreventive behavior: A review of theories, findings and educational implications. Patient Education and Counseling, 23(3), 173-186.

AIDS plague expected to hit teenagers next. (1993, June 6). The Providence Sunday Journal, A-1, A-15.

Adame, D.D., Taylor-Nicholson, M.E., Wang, M., \& Abbas, M.A. (1991). Southern college freshman students: A survey of knowledge, attitudes and beliefs about AIDS. Journal of Sex Education and Therapy, 17(3), 196-206.

Aggleton, P. (1991). When will they ever learn? Young people, health promotion and HIV/AIDS social research. AIDS Care, 3(3), 259-264.

Airhihenbuwa, C.O., DiClemente, R.J., Wingood, G.M., \& Lowe, A. (1992). HIV/AIDS education and prevention among African-Americans: A focus on culture. AIDS Education and Prevention, 4(3), 267-276.

Alexander, C.S., Ensminger, M.E., Kim, Y.J., Smith, B.J., Johnson, K.E., \& Dolan, L.J. (1989). Early sexual activity among adolescents in small towns and rural areas: Race and gender patterns. Family Planning Perspectives, 21(6), 261- 266.

Anderson, J.R., Amaro, H., Bailey, W.A., Barret, R.L., Boccellari, A.A., Bonde, L., BoydFranklin, N., Coates, T.J., Franks, P.E., Gornemann, I., Landry, C., Lo, B., McKusick, L., Shore, M.D., Steiner, G.L., \& Tafoya, T.N. (1991). Review of recent literature on the behavioral and psychosocial aspects of HIV disease. In J.R. Anderson, C.P. Landry, \& J.L. Kerby, (Eds.) (1991). AIDS Abstracts of the Psychological and Behavioral Literature, 1983-1991, Third Edition, (p. 24). Washington, DC: American Psychological Association.

Anderson, J.E., Kann, L., Holtzman, D., Arday, S., Truman, B., \& Kolbe, L. (1990). HIV/AIDS knowledge and sexual behavior among high school students. Family Planning Perspectives, 22(6), 252-255.

Arnett, J. (1990). Contraceptive use, sensation seeking, and adolescent egocentrism. Journal of Youth and Adolescence, 19, 171-180.

Aronson, S. (1995). Five girls in search of a group: A group experience for adolescents of parents with AIDS. International Journal of Group Psychotherapy, 45(2), 223-235.

Aruffo, J.F., Gottlieb, A, Webb, R., \& Neville, B. (1994). Adolescent psychiatric inpatients: Alcohol use and HIV risk-taking behavior. Special Issue: Serving people 
with psychiatric disability at risk for HIV/AIDS. Psychosocial Rehabilitation Journal, 17(4), 150-156.

Baldwin, J.D. \& Baldwin, J.I. (1988a). AIDS information and sexual behavior on a university campus. Journal of Sex Education and Therapy, 14, 24-28.

Baldwin, J.D. \& Baldwin, J.I. (1988b). Factors affecting AIDS-related sexual risktaking behavior among college students. Journal of Sex Research, $\underline{25}, 197-218$.

Baldwin, J.I., Whiteley, S., \& Baldwin, J.D. (1990). Changing AIDS- and fertility-related behavior: The effectiveness of sexual education. The Journal of Sex Research, 27(2), 245-262.

Bandura, A. (1977). Self-efficacy: Toward a unifying theory of behavioral change. Psychological Review, 84, 191-215.

Bandura, A. (1990). Perceived self-efficacy in the exercise of control over AIDS infection. Evaluation and Program Planning, 13, 9-17.

Bandura, A. (1992). A social cognitive approach to the exercise of control over AIDS infection. In R.J. DiClemente, (Ed.) Adolescents and AIDS: A Generation in Jeopardy, Newbury Park, CA: Sage Publications, pp. 89-116.

Barling, N.R. \& Moore, S.M. (1990). Adolescents' attitudes towards AIDS precautions and intention to use condoms. Psychological Reports, $\underline{67}, 883-890$.

Barnard, M. \& McKeganey, N. (1990). Adolescents, sex and injecting drug use: Risk for HIV infection. AIDS Care, 2(2), 103-116.

Barnes, G.M. (1984). Adolescent alcohol abuse and other problem behaviors: Their relationships and common parental influences. Journal of Youth and Adolescence, $\underline{13}(4), 329-348$.

Basch, C.E. (1987). Focus group interview: An underutilized research technique for improving theory and practice in health education. Health Education Quarterly, 14, 411-448.

Basch, C.E. (1989). Preventing AIDS through education: Concepts, strategies, and research priorities. Journal of School Health, 59(7), 296-300.

Basen-Engquist, K. \& Parcel, G.S. (1992). Attitudes, norms, and self-efficacy: A model of adolescents' HIV-related sexual risk behavior. Health Education Quarterly, 19(2), 263-277. 
Baum, A. \& Nesselhof, S.E. (1988). Psychological research and the prevention, etiology, and treatment of AIDS, Special Issue: Psychology and AIDS. American Psychologist, 43(11), 900-906.

Bauman, L.J. \& Siegel, K. (1987). Misperceptions among gay men of the risk for AIDS associated with their sexual behavior. Journal of Applied Social Psychology, 17, 329350 .

Becker, M.H. \& Joseph, J.G. (1988). AIDS and behavioral change to reduce risk: A review. American Journal of Public Health, 78(4), 394-410.

Belfer, M.L., Krener, P.K., \& Miller, F.B. (1988). AIDS in children and adolescents. Journal of the American Academy of Child and Adolescent Psychiatry, 27(2), 147-151.

Bell, D., Feraios, A., \& Bryan, T. (1990). Adolescent males' knowledge and attitudes about AIDS in the context of their social world. Journal of Applied Social Psychology, 20(5), 424-448.

Bertrand, J.T., Brown, L.F., Kinzonzi, M., Mansilu, M., \& Djunghu, B. (1992). AIDS knowledge in three sites in Bas-Zaire. AIDS Education and Prevention, 4(3), 251-266.

Bhushan, V. \& Cushman, L.F. (1995). Paediatric AIDS: Selected attitudes and behaviours of paediatricians in New York City hospitals. AIDS Care, 7(1), 27-34.

Biglan, A., Metzler, C.W., Wirt, R., Ary, D., Noell, J., Ochs, L., French, C., \& Hood, D. (1990). Social and behavioral factors associated with high-risk sexual behavior among adolescents. Journal of Behavioral Medicine, 13(3), 245-261.

Billy, J.O.G. \& Udry, J.R. (1985). The influence of male and female best friends on adolescent sexual behavior. Adolescence, 20(77), 21-32.

Bing, E.G., Nichols, S.E., Goldfinger, S.M., Fernandez, F., Cabaj, R., Dudley, R.G., Krener, P., Prager, M., \& Ruiz, P. (1990). The many faces of AIDS: Opportunities for intervention. New Directions for Mental Health Services, $\underline{48}, 69-81$.

Black, J.L. \& Jones, L.H. (1988). HIV infection: Educational programs and policies for school personnel. Journal of School Health, 58(8), 317-322.

Boldero, J., Moore, S., \& Rosenthal, D. (1992). Intention, context and safe sex: Australian adolescents' responses to AIDS. Journal of Applied Social Psychology, 22(17), 1374-1396.

Bounds, W., Guillebaud, J., Stewart, L., \& Steele, S. (1988). A female condom (Femshield): A study of its user acceptability. British Journal of Family Planning, 14, 83-87. 
Bowen, A.M. \& Trotter, R. (1995). HIV risk in IV drug users and crack smokers: Predicting stage of change for condom use. Journal of Consulting and Clinical Psychology, 63(2), 238-248.

Bowler, S., Sheon, A.R., D'Angelo., L.J., \& Vermund, S.H. (1992). HIV and AIDS among adolescents in the United States: Increasing risk in the 1990s. Journal of Adolescence, 15 , 345-371.

Boyer, C.B. \& Kegeles, S.M. (1991). AIDS risk and prevention among adolescents. Social Science and Medicine, 33 , 11-23.

Brandt, A.M. (1988). AIDS in historical perspective: Four lessons from the history of sexually transmitted diseases. American Journal of Public Health, 78(4), 367-371.

Braverman, P.K. \& Strasburger, V.C. (1994). The practitioner's role. Clinical Pediatrics, 33(2), 100-109.

Brock, G.C. \& Beazley, R.P. (1995). Using the health belief model to explain parents' participation in adolescents' at-home sexuality education activities. Journal of School Health, 65(4), 124-128.

Broering, J., Moscicki, B., Millstein, S.G., Policar, M., \& Irwin, C.W. (1989). Sexual practices among adolescents. Journal of Adolescent Health Care, 10, 249.

Brookman, R.R. (1990). Adolescent sexual behavior. In K.K. Holmes, et al. (Eds). Sexually Transmitted Diseases, Second Edition. New York: McGraw-Hill, pp. 77-84.

Brooks-Gunn, J. (1988). Antecedents to and consequences of variations in girls' maturational timing. Journal of Adolescent Health Care, $9,1-11$.

Brooks-Gunn, J., Boyer, C.B., \& Hein, K. (1988). Preventing HIV infection and AIDS in children and adolescents. Behavioral research and intervention strategies. American Psychologist, $\underline{43}(11), 958-964$.

Brooks-Gunn, J. \& Furstenberg, F.F. (1989). Adolescent sexual behavior. American Psychologist, 44(2), 249-257.

Brooks-Gunn, J. \& Furstenberg, F.F. (1990). Coming of age in the era of AIDS: Puberty, sexuality, and contraception. Milbank Quarterly, 68, 59-84.

Broverman, I., Vogel, S., Broverman, D., Clarkson, F., \& Rosenkrantz, P. (1972). Sexrole stereotypes: A current appraisal. Journal of Social Issues, $\underline{28}, 59-78$. 
Brown, J.D. \& Newcomer, S.F. (1991). Television viewing and adolescents' sexual behavior. Journal of Homosexuality, 21, 77-91.

Brown, L.K., DiClemente, R.J., \& Beausoleil, N.I. (1992). Comparison of human immunodeficiency virus related knowledge, attitudes, intentions, and behaviors among sexually active and abstinent young adolescents. Journal of Adolescent Health, 13(2), 140-145.

Brown, L.K., DiClemente, R.J., \& Park, T. (1992). Predictors of condom use in sexually active adolescents. Journal of Adolescent Health 13 (8), 651-657.

Brown, L.K. \& Fritz, G.K. (1988). Children's knowledge and attitudes about AIDS. Journal of the American Academy of Child and Adolescent Psychiatry, 27, 504-508.

Brown, L.K., Fritz, G.K., \& Barone, V.J. (1989). The impact of AIDS education on junior and senior high school students: A pilot study. Journal of Adolescent Health Care, 10, 386-392.

Brown, L.K., Nassau, J.H., \& Levy, V. (1990). "What upsets me the most about AIDS is ...": A survey of children and adolescents. AIDS Education and Prevention, 2(4), 296-304.

Brunswick, A.F., Aidala, A., Dobkin, J., Howard, J., Titus, S.P., \& Banaszak-Holl, J. (1993). HIV-1 seroprevalence and risk behaviors in an urban African-American community cohort. American Journal of Public Health, 83(10), 1390-1394.

Burger, J.M. \& Burns, L. (1988). The illusion of unique invulnerability and the use of effective contraception. Personality and Social Psychology Bulletin, 14(2), 264-270.

Burke, D.S., Brundage, J.F., Goldenbaum, M., Gardner, L.I., Peterson, M., Visintine, R., Redfield, R.R., \& the Walter Reed Retrovirus Research Group. (1990). Human Immunodeficiency Virus infections in teenagers: Seroprevalence among applicants for US military service. Journal of the American Medical Association, 263(15), 20742077.

Burkholder, G.J. (1994). Education, attitudes toward gay and lesbian persons, anxiety and HIV/AIDS risk. Unpublished master's thesis, University of Rhode Island, Kingston, Rhode Island.

Calamidas, E.G. (1990). AIDS and STD education: What's really happening in our schools? Journal of Sex Education and Therapy, 16(1), 54-63.

Calder, J. (1977). Focus groups and the nature of qualitative marking. Journal of Marketing Research, 14, 353-364. 
Carnegie Council on Adolescent Development (1989). Turning Points: Preparing American Youth for the 21st Century. New York: Carnegie Corporation.

Caron, S.L., Davis, C.M., Halteman, W.A., \& Stickle, M. (1993). Predictors of condomrelated behaviors among first-year college students. The Journal of Sex Research, 30(3), 252-259.

Carpenter, C.C.J., Mayer, K.H., Stein, M.D., Leibman, B.D., Fisher, A., \& Fiore, T.C. (1991). Human Immunodeficiency Virus infection in North American women: Experience with 200 cases and a review of the literature. Medicine, 70(5), 307-325.

Carroll, L. (1991). Gender, knowledge about AIDS, reported behavioral change, and the sexual behavior of college students. Journal of American College Health, 40, 5-12.

Catania, J.A., Coates, T.J., Greenblatt, R.M., Dolcini, M.M., Kegeles, S.M., Pucket, S., Corman, M., \& Miller, J. (1989a). Predictors of condom use and multiple partnered sex among sexually-active adolescent women: Implications for AIDS-related health interventions. The Journal of Sex Research, 26(4), 514-524.

Catania, J.A., Coates, T.J., \& Kegeles, S. (1994). A test of the AIDS risk reduction model: Psychosocial correlates of condom use in the AMEN cohort survey. Health Psychology, 13(6), 548-555.

Catania, J.A., Coates, T.J., Stall, R., Turner, H., Peterson, J., Hearst, N., Dolcini, M.M., Hudes, E., Gagnon, J., Wiley, J., \& Groves, R. (1992). Prevalence of AIDS-related risk factors and condom use in the United States. Science, $\underline{258}, 1101-1106$.

Catania, J.A., Dolcini, M.M., Coates, T.J., Kegeles, S.M., Greenblatt, R.M., Puckett, S., Corman, M., \& Miller, J. (1989b). Predictors of condom use and multiple partnered sex among sexually-active adolescent women: Implications for AIDS-related health interventions. The Journal of Sex Research, 26(4), 514-524.

Catania, J.A., Gibson, D.R., Chitwood, D.D., \& Coates, T.J. (1990). Methodological problems in AIDS behavioral research: Influences on measurement error and participation bias in studies of sexual behavior. Psychological Bulletin, 108(3), 339362.

Catania, J.A., Gibson, D.R., Marin, B., Coates, T.M., \& Greenblatt, R.M. (1990). Response bias in assessing sexual behaviors relevant to HIV transmission. Evaluation and Program Planning, 13, 19-29.

Catania, J.A., Kegeles, S.M., \& Coates, T.J. (1990). Towards an understanding of risk behavior: An AIDS risk reduction model (ARRM). Health Education Quarterly, 17(1), 53-72. 
Cates. W. (1990). Acquired Immunodeficiency Syndrome, sexually transmitted diseases, and epidemiology: Past lessons, present knowledge, and future opportunities. American Journal of Epidemiology, 131(5), 749-758.

Cates, W. (1991). Teenagers and sexual risk taking: The best of times and the worst of times. Journal of Adolescent Health, 12, 84-94.

Centers for Disease Control. (1990). Premarital sexual experience among adolescent women, United States, 1970-1988. Morbidity and Mortality Weekly Report, 39, 929932.

Centers for Disease Control. (1991). Premarital sexual experience among adolescent women -- United States, 1970-1988. Morbidity and Mortality Weekly Report, 39, 929932.

Centers for Disease Control and Prevention. (1992, October). Preventing risk behaviors among students. HIV/AIDS Prevention Newsletter, $\underline{3}(3), 1-2$.

Centers for Disease Control and Prevention (1993a, January 3). Sexual risk behaviors of STD clinic patients before and after Earvin "Magic" Johnson's HIV-infection announcement -- Maryland, 1991-1992. Morbidity and Mortality Weekly Report, $\underline{42}(3), 45-48$.

Centers for Disease Control and Prevention (1993b, May 28). Sexual behavior and condom use -- District of Columbia, January-February, 1992. Morbidity and Mortality Weekly Report, $\underline{42}$ (20), 390-398.

Centers for Disease Control and Prevention (1993c, July 2). Update: Mortality attributable to HIV Infection/AIDS among persons aged 25-44 years -- United States, 1990 and 1991. Morbidity and Mortality Weekly Report, 42 (25), 481-486.

Centers for Disease Control and Prevention (1993d, July 9). Availability of comprehensive adolescent health services -- United States, 1990. Morbidity and Mortality Weekly Report, 42 (26), 507-515.

Centers for Disease Control and Prevention (1993e, July 23). Update: Acquired Immunodeficiency Syndrome -- United States, 1992. Morbidity and Mortality Weekly Report, $42(28), 447-557$.

Centers for Disease Control and Prevention (1993f, August 6). Update: Barrier protection against HIV infection and other sexually transmitted diseases. Morbidity and Mortality Weekly Report, 42(30), 589-597. 
Centers for Disease Control and Prevention. (1993g, August 13). Surveillance for gonorrhea and primary and secondary syphilis among adolescents, United States--19811991. Morbidity and Mortality Weekly Report, 42(SS-3), 1-11.

Centers for Disease Control and Prevention. (1993h, October 1). Teenage pregnancy and birth rates - United States, 1990. Morbidity and Mortality Weekly Report, 42(38), 733737.

Centers for Disease Control and Prevention. (1993i, November 19). World AIDS Day December 1, 1993; Update: Mortality Attributable to HIV infection among persons aged 25-44 years -- United States, 1991 and 1992; Distribution of STD clinic patients along a stages-of-behavioral-change continuum -- selected sites, 1993. Morbidity and Mortality Weekly Report, 42 (45), 869; 869-872; 880-883.

Centers for Disease Control and Prevention (1993j, November 26). Mortality patterns -United States, 1991. Morbidity and Mortality Weekly Report, 42(46), 891-900.

Centers for Disease Control and Prevention (1993k, December 17). Surveillance for pregnancy and birth rates among teenagers, by state -- United States, 1980 and 1990. Morbidity and Mortality Weekly Report, 42(SS-6), 1-27.

Centers for Disease Control and Prevention (1994a). HIV/AIDS Surveillance Report. Year-end edition, $\underline{6}(2), 5-35$.

Centers for Disease Control and Prevention (1994b, Spring). HIV infection among young people. HIV/AIDS Prevention Newsletter, $\underline{5}(1), 2-3$.

Centers for Disease Control and Prevention (1994c, Spring). Summary of data on AIDS cases reported in 1993. HIV/AIDS Prevention Newsletter, $5(1), 6$.

Centers for Disease Control and Prevention (1994d, Spring). WHO: Sex education leads to safer behavior. HIV/AIDS Prevention Newsletter, 5(1), 9 .

Centers for Disease Control and Prevention (1994e, March 4). Health risk behaviors among adolescents who do and do not attend school -- United States, 1992. Morbidity and Mortality Weekly Report, 43(8), 129-132.

Centers for Disease Control and Prevention (1994f, March 11). Heterosexually acquired AIDS -- United States, 1993; Update: Impact of the expanded AIDS surveillance case definition for adolescents and adults on case reporting -- United States, 1993. Morbidity and Mortality Weekly Report, 43(9), 155-160; 160-170.

Centers for Disease Control and Prevention (1994g, April 8). Health risk behaviors among persons aged 12-21 years -- United States, 1992. Morbidity and Mortality Weekly Report, 43(13), 231--235. 
Centers for Disease Control and Prevention (1994h, April 29). Zidovudine for the prevention of HIV transmission from mother to infant. Morbidity and Mortality Weekly Report, 43(16), 285-287.

Centers for Disease Control and Prevention (1994i, August 5). Recommendations of the U.S. Public Health Services Task Force on the use of Zidovudine to reduce perinatal transmission of Human Immunodeficiency. Morbidity and Mortality Weekly Report, 43(RR-11), 1-20.

Centers for Disease Control and Prevention (1994j, November 18). World AIDS Day -December 1, 1994. Morbidity and Mortality Weekly Report, 43(45), 825-831.

Centers for Disease Control and Prevention (1994k, December 16). Mortality patterns -United States, 1992. Morbidity and Mortality Weekly Report, 43(49), 916-920.

Centers for Disease Control and Prevention (19941, Winter). HIV/AIDS Advances as leading cause of death for people aged 25-44 in the United States. HIV/AIDS Prevention Newsletter, $5(3), 2$.

Centers for Disease Control and Prevention (1995a, February 10). Update: AIDS among women -- United States, 1994. Morbidity and Mortality Weekly Report, 44(5), 81-84

Centers for Disease Control and Prevention (1995b, February 24). Trends in sexual risk behavior among high school students -- United States, 1990, 1991, and 1993.

Morbidity and Mortality Weekly Report, 44(7), 124-132.

Centers for Disease Control and Prevention (1995c, March 24). Youth risk behavior surveillance -- United States, 1993. Morbidity and Mortality Weekly Report, 44(SS-1), $1-58$.

Centers for Disease Control and Prevention (1995d, September 22). State-specific pregnancy and birth rates among teenagers -- United States, 1991-1192. Morbidity and Mortality Weekly Report, 44(37), 677-684.

Centers for Disease Control and Prevention (1995e, November 24). First 500,000 AIDS cases --- United States, 1995. Morbidity and Mortality Weekly Report, 44(46), 849853.

Centers for Disease Control and Prevention (1995f). HIV/AIDS Surveillance Report. Mid-year edition, $7(1), 8$.

Centers for Disease Control and Prevention (1996, February 16). Update: Mortality attributable to HIV infection among persons aged 25-44 years -- United States, 1994. Morbidity and Mortality Weekly Report, 44(6), 121-125. 
Christopher, F.S. \& Roosa, M.W. (1990). An evaluation of an adolescent pregnancy prevention program: Is "Just Say No" enough. Family Relations, 39, 68-72.

Clapper, R.L. \& Lipsitt, L.P. (1991). A retrospective study of risk-taking and alcoholmediated unprotected intercourse. Journal of Substance Abuse, $\underline{3}, 91-96$.

Clark, S.D., Zabin, L.S., \& Hardy, J.B. (1984). Sex contraception and parenthood. Family Planning Perspectives, $\underline{15}, 77-84$.

Clarke, J., Abram, R., \& Monteiro, E.F. (1990). The sexual behaviour and knowledge about AIDS in a group of young adolescent girls in Leeds. Genitourinary Medicine, $\underline{66}$, 189-192.

Coates, T.J. (1990). Strategies for modifying sexual behavior for primary and secondary prevention of HIV disease. Journal of Consulting and Clinical Psychology, 58, 57-69.

Cochran, S.D. \& Mays, V.M. (1990). Sex, lies and HIV. New England Journal of Medicine, $\underline{322}, 774-775$.

Cochran, S.D. \& Peplau, L.A. (1991). Sexual risk reduction behaviors among young heterosexual adults. Social Science Medicine, 1, 25-36.

Cohen, D., Dent, C., \& MacKinnon, D. (1991). Condom skills education and sexually transmitted disease reinfection. The Journal of Sex Research, 28(1), 139-144.

Cohn, L.D., Macfarlane, S., Yanez, C., \& Imai, W.K. (1995). Risk-perception:

Differences between adolescents and adults. Health Psychology, 14(3), 217-222.

Conant, M., Hardy, D., Sernatinger, J., Spicer, D., \& Levy, J.A. (1986). Condoms prevent transmission of AIDS-associated retrovirus. Journal of the American Medical Association, 255(13), 1706.

Corea, G. (1992). The Invisible Epidemic: The Story of Women and ADS. New York: Harper Collins.

Coyle, A. \& Loveless. L. (1995). A study of youth workers as health promoters on sexrelated issues and HIV/AIDS in a British city. International Journal of Adolescence and Youth, $\underline{5}(3), 157-171$.

Crawford, I. \& Robinson, W.L. (1991). Adolescents and AIDS: Knowledge and attitudes of African-American, Latino and Caucasian midwestern U.S. high school seniors. Journal of Psychology and Human Sexuality, $\underline{3}$ (2), 25-32. 
Crawford, J., Turtle, A., \& Kippax, S. (1990). Student-favoured strategies for AIDS avoidance. Australian Journal of Psychology, 42(2), 123-137.

Cullari, S., \& Mikus, R. (1990). Correlates of adolescent sexual behavior. Psychological Reports, 66 , 1179-1184.

Curran, J.W., Jaffe, H.W., Hardy, A.M., Morgan, W.M., Selik, R.M., \& Dondero, T.J. (1988). Epidemiology of HIV infection and AIDS in the United States. Science, $\underline{239}(4840), 610-616$.

Damond, M.E., Breuer, N.L., \& Pharr, A.E. (1993). The evaluation of setting and a culturally specific HIV/AIDS curriculum: HIV/AIDS knowledge and behavioral intent of African American adolescents, Journal of Black Psychology, 19(2), 169-189.

Dancy, B.L. (1991). The development of an ethnically sensitive and gender-specific AIDS questionnaire for African-American Women. Health Values, 15(6), 49-54.

D'Angelo, L.J. (1994). Adolescents and HIV infection: A clinician's perspective. Acta Pediatric Supplement, $400,88-94$.

D’Angelo, L.J., Brown, R. English, A., Hein, K., \& Remafedi, G. (1994). HIV infection and AIDS in adolescents: A position paper of the Society for Adolescent Medicine. Journal of Adolescent Health, 15(5), 427-434.

D'Angelo, L.J., Getson, P.R., Luban, N.L.C., \& Gayle, H.D. (1991). Human Immunodeficiency Virus infection in urban adolescents: Can we predict who is at risk? Pediatrics, $\underline{88(5), 982-986 .}$

Dawson, D.A. (1986). The effects of sex education on adolescent behavior. Family Planning Perspectives, 18, 162-170.

Deiter, P. (1994). Sexual assertiveness training for college women. Unpublished doctoral dissertation, University of Rhode Island, Kingston, Rhode Island.

Demb, J. (1990). Black, inner-city, female adolescents and condoms: What the girls say. Family Systems Medicine, 8 (4), 401-406.

Des Jarlais, D., Ehrhardt, A., Fullilove, M., Hein, K., Menken, J., Mensch, B.S., Miller, H., \& Turner, C. (1990). AIDS and adolescents. In H. Miller, C. Turner, \& L. Moses, (Eds.), AIDS: The Second Decade. Washington, DC: National Academy Press, $p p$. 147-252.

Desvousges, W.H. \& Frey, J.H. (1989). Integrating focus groups and surveys: Examples from environmental risk studies. Journal of Official Statistics, $\underline{5}, 349-363$. 
deVries, H., Weijts, W., Dijkstra, M., \& Kok, G. (1992). The utilization of qualitative and quantitative data for health education program planning, implementation, and evaluation: A spiral approach. Health Education Quarterly, 19, 101-115.

DiClemente, C.C., Prochaska, J.O., Fairhurst, S.K., Velicer, W.F., Velasquez, M.M., \& Rossi, J.S. (1991). The process of smoking cessation: An analyses of precontemplation, contemplation, and preparation stages of change. Journal of Consulting and Clinical Psychology, 59, 295-304.

DiClemente, R.J. (1989). Prevention of Human Immunodeficiency Virus infection among adolescents: The interplay of health education and public policy in the development and implementation of school-based AIDS education programs. AIDS Education and Prevention, 1 , 70-78.

DiClemente, R.J. (1990). The emergence of adolescents as a risk group for human immunodeficiency virus infection. Journal of Adolescent Research, $\underline{5}, 7-17$.

DiClemente, R.J. (1991). Predictors of HIV-Preventive sexual behavior in a high-risk adolescent population: The influence of perceived peer norms and sexual communication on incarcerated adolescents' consistent use of condoms. Journal of Adolescent Health, 12, 385-390.

DiClemente, R.J. (1992). Epidemiology of AIDS, HIV Prevalence, and HIV incidence among adolescents. Journal of School Health, 62(7), 325-330.

DiClemente, R.J. (1993). Confronting the challenge of AIDS among adolescents: Directions for future research. Journal of Adolescent Research, $\underline{8}(2), 156-166$.

DiClemente, R.J., Boyer, C.B., \& Mills, S.J. (1987). Prevention of AlDS among adolescents: Strategies for the development of comprehensive risk-reduction health education programs. Health Education Research, 2(3), 287-291.

DiClemente, R.J., Boyer, C.J., \& Morales, E.S. (1988). Minorities and AIDS: Knowledge, attitudes, and misconceptions among Black and Latino adolescents. American Journal of Public Health, 78, 55-57.

DiClemente, R.J., Brown, L.K., Beausoleil, N.I., \& Lodico, M. (1993). Comparison of AIDS knowledge and HIV-related sexual risk behaviors among adolescents in low and high AIDS prevalence communities. Journal of Adolescent Health, 14(3), 231-236.

DiClemente, F.J., Durbin, M., Siegel, D., Krasnovsky, F., Lazarus, N., \& Comacho, T. (1992). Determinants of condom use among junior high school students in a minority,

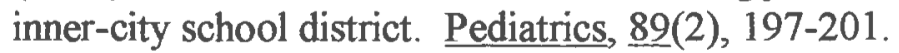


DiClemente, R.J., Forrest, K.A., \& Mickler, S. (1990). College students' knowledge and attitudes about AIDS and changes in HIV-preventive behaviors. AIDS Education and Prevention, 2(3) 201-212.

DiClemente, R.J. \& Houston-Hamilton, A. (1989). Health promotion strategies for prevention of Human Immunodeficiency Virus Infection among minority adolescents. Health Education, 20(5), 39-43.

DiClemente, R.J., Zorn, J, \& Temoshok, L. (1986). Adolescents and AIDS: A survey of knowledge, attitudes and beliefs about AIDS in San Francisco. American Journal of Public Health, 76, 1443-1445.

DiClemente, R.J., Zorn, J., \& Temoshok, L. (1987). The association of gender, ethnicity, and length of residence in the Bay Area to adolescents' knowledge and attitudes about Acquired Immune Deficiency Syndrome. Journal of Applied Social Psychology, 17, 216-230.

DiScipio, W.J. (1994). Sex, drugs, and AIDS: Issues for hospitalized emotionally disturbed youth. Special Issue: Brief and intermediate treatment for psychiatrically disturbed youth. Psychiatric Quarterly, 65(2), 149-155.

Dolcini, M.M., Coates, T.J., Catania, J.A., Kegeles, S.M., \& Hauck, W.W. (1995). Multiple sex partners and their psychosocial correlates: The population-based AIDS in multiethnic neighborhoods (AMEN) study. Health Psychology, 14(1), 22-31.

Dolcini, P., Adler, N.E., Irwin, C.E., Jr., Millstein S., Kegeles, S.M., Cohn, L., \& Stone G. (1988). Perceptions of susceptibility to AIDS among adolescents. Presented at the Fourth International Conference on AIDS, Stockholm, Sweden.

Dommeyer, C.J., Marquard, J.L., Gibson, J.E., \& Taylor, R.L. (1989). The effectiveness of an AIDS education campaign on a college campus. Journal of American College Health, $\underline{38}, 131-135$.

Donovan, C. \& McEwan, R. (1995). A review of the literature examining the relationship between alcohol use and HIV-related sexual risk-taking in young people. Addiction, 90(3), 319-328.

Donovan, J.E., Jessor, R., \& Costa, F.M. (1991). Adolescent health behavior and conventionality-unconventionality: An extension of problem-behavior theory. Health Psychology, 10(1), 52-61.

Drew, W.L., Blair, M., Miner, R.C., \& Conant, M. (1990). Evaluation of the virus permeability of a new condom for women. Sexually Transmitted Diseases, 17,110 112. 
du Guerny J. \& Sjöberg, E. (1992, July 8). Inter-relationship between gender relations and the HIV/AIDS-epidemic: Some possible considerations for policies and programs. (Prepublication draft), Vienna: Centre for Social Development and Humanitarian Affairs, Division for the Advancement of Women, p. 7.

Dubois-Arber, F., Jeannin, A., \& Zeugin, P. (1992, July 19-24). Evaluation of AIDS prevention in Switzerland: Behavioral change in the general population. Presented at the Eighth International Conference on AIDS, Amsterdam, The Netherlands. Abstract PoD5140.

DuRant, R.H., Ashworth, C.S., Newman, C., \& Gaillard, G. (1992). High school students' knowledge of HIV/AIDS and perceived risk of currently having AIDS. Journal of School Health, 62(2), 59-63.

DuRant, R.H. \& Jay, S. (1989). The adolescent heterosexual relationship and its association with the sexual and contraceptive behavior of Black females. American Journal of Diseases of Children, 143, 1467-1472.

DuRant, R.H., Sanders, J.M, Jay, S., \& Levinson, R. (1988). Analyses of contraceptive behavior of sexually active female adolescents in the United States. The Journal of Pediatrics, 113, 930-936.

Durbin, M., DiClemente, R.J., Siegel, D., Krasnovsky, F., Lazarus, N., \& Camacho, T. (1993). Factors associated with multiple sex partners among junior high school students. Joumal of Adolescent Health, 14, 202-207.

Dusenbury, L., Botvin, G.J., Baker, E., \& Laurence, J. (1991). AIDS risk knowledge, attitudes, and behavioral intentions among multi-ethnic adolescents. AIDS Education and Prevention, 3(4), 367-375.

Eiser, C., Eiser, R., \& Lang, J. (1990). How adolescents compare AIDS with other diseases: Implications for prevention. Journal of Pediatric Psychology, 15, 97-103.

Ekstrand, M.L. \& Coates, T.J. (1990). Maintenance of safer sexual behaviors and predictors of risky sex: The San Francisco Men's Health Study. American Journal of Public Health, $\underline{80}(8), 973-977$.

Epstein, J.A., Dusenbury, L., Botvin, G.J., \& Diaz, T. (1994). Determinants of intentions of junior high school students to become sexually active and use condoms: Implications for reduction and prevention of AIDS risk. Psychological Reports, 75(2), 1043-1053.

European Study Group on Heterosexual Transmission of HIV (1992). Comparison of female to male and male to female transmission of HIV in 563 stable couples. British Medical Journal, $\underline{304}$, 809-813. 
Fennell, R. \& Beyrer, M.K. (1989). AIDS: Some ethical considerations for the Health educator. Journal of American College Health, 38(3), 145-147.

Fergusson, D.M., Lynskey, M.T., \& Horwood, L.J. (1994). AIDS knowledge and condom use in a birth cohort of 16-year olds. New Zealand Medical Journal, 107(990), 480-483.

Fineberg, H.V. (1988). Education to prevent AIDS: Prospects and obstacles. Science, $\underline{239}, 592-596$.

Fisher, J.D. \& Fisher, W.A. (1992). Changing AIDS-risk behavior. Psychological Bulletin, 111(3), 455-474.

Fisher, J.D., Fisher, W.A., Misovich, S.J., Kimble, D.L., \& Malloy, T.E. (1996).

Changing AIDS risk behavior: Effects of an intervention emphasizing AIDS risk reduction information, motivation, and behavioral skills in a college student population. Health Psychology, 15(2), 114-123.

Fisher, J.D., Fisher, W.A., Williams, S.S., \& Malloy, T.E. (1994). Empirical tests of an information-motivation-behavioral skills model of AIDS-preventive behavior with gay men and heterosexual university students. Health Psychology, 13(3), 238-250.

Fisher, W.A., Fisher, J.D, \& Rye, B.J. (1995). Understanding and promoting AIDSpreventive behavior: Insights from the theory of reasoned action. Health Psychology, 14(3), 255-264.

Flanigan, B.J. (1990). The social context of alcohol consumption prior to female sexual intercourse. Journal of Alcohol and Drug Education, 36(1), 97-113.

Flanigan, B.J., McLean, A., Hall, C., \& Propp, V. (1990). Alcohol use as a situational influence on young women's pregnancy risk-taking behaviors. Adolescence, 25(97), 205-214.

Flora, J.A. \& Thoresen, C.E. (1988). Reducing the risk of AIDS in adolescents. American Psychologist, 43(11), 965-970.

Folkman, S., Chesney, M.A., Cooke, M., Boccellari, A., \& Collette, L. (1994). Caregiver burden in HIV-positive and HIV-negative partners of men with AIDS. Journal of Consulting and Clinical Psychology, 62(4), 746-756.

Ford, K., Rubinstein, S., \& Norris, A. (1994). Sexual behavior and condom use among urban, low-income, African American and Hispanic youth. AIDS Education and Prevention, 6 (3), 219-229. 
Frances, R.J. \& Miller, S.I. (Eds.). (1991). Clinical Textbook of Addictive Disorders. New York: Guilford Press, p. 342.

Franzini, L.R., Sideman, L.M., Dexter, K.E., \& Elder, J.P. (1991). Promoting AIDS risk reduction via behavioral training. AIDS Education and Prevention, 2(4), 313-321.

Freeman, A., Cohn, D., Corby, N., \& Wood, R. (1991). Patterns of sexual behavior change among homosexual/bisexual men: Selected U.S. Sites, 1987-1990. Morbidity and Mortality Weekly Report, 40(46), 792-794.

Freimuth, V.S., Edgar, T., \& Hammond, S.L. (1987). College students' awareness and interpretation of the AIDS risk. Science, Technology, and Human Values, 12 , 37-40.

Friedland, G.H. \& Klein, M.D. (1987). Transmission of the Human Immunodeficiency Virus. The New England Journal of Medicine, 317(18), 1125-1135.

Friedman, H.L. (1993). Reproductive health in adolescence. World Health Statistics Quarterly, 47(1), 31-35.

Fuller, T.D., Edwards, J.N., Vorakiphokatorn, S., \& Sermski, S. (1990). Using focus groups to adapt survey instruments to new populations. In D.L. Morgan (Ed.) (1993). Successful Focus Groups: Advancing the State of the Art. Newbury Park, CA: Sage Publications, $p p$. 89-104.

Fullilove, M.T., Fullilove, R.E., Haynes, K., \& Gross, S. (1990). Black women and AIDS prevention: A view towards understanding the gender rules. Special Issue: Feminist perspectives on sexuality. Journal of Sex Research, 27, 47-64.

Furstenberg, F.F., Jr., Moore, K.A., \& Peterson, J.L. (1986). Sex education and sexual experience among adolescents. American Journal of Public Health, 75, 1221-1222.

Gaines, J., Iglar, A.F., Michal, M.L., \& Patton, R.D. (1988). Attitudes towards AIDS. Health Values, $12(4), 53-59$.

Galavotti, C., Cabral, R.J., Lansky, A., Grimley, D.M., Riley, G.E., \& Prochaska, J.O. (1995). Validation of measures of condom and other contraceptive use among women at high risk for HIV infection and unintended pregnancy. Health Psychology, 14(6), 570-578.

Gallup, B. \& Gallup, A. (1988). AIDS: We worry about the wrong things. American Health, 6 (June), 50-52.

Gardner, W. \& Wilcox, B.L. (1993). Political intervention in scientific peer review: Research on adolescent sexual behavior. American Psychologist, 48(9), 972-983. 
Gayle, H.D. \& D'Angelo, L.J. (1991). Epidemiology of Acquired Immunodeficiency Syndrome and Human Immunodeficiency Virus infection in adolescents. The Pediatric Infectious Disease Journal, 10(4), 322-328.

Gayle, H.D., Keeling, R.P., Garcia-Tunon, M., Kilbourne, B.W., Narkunas, J.P., Ingram, F.R., Rogers, M.F., \& Curran, J.W. (1990). Prevalence of the Human Immunodeficiency Virus among university students. The New England Journal of Medicine, $\underline{323}(22), 1538-1541$.

Gilligan, C. (1979). Woman's place in man's life cycle. Harvard Educational Review, 49(4), 431-446.

Gilligan, C. (1982). In a Different Voice: Psychological Theory and Women's Development. Cambridge, MA: Harvard University Press, p. 30.

Gilligan, C., Rogers, A., \& Brown, L.M. (1990). Soundings into development. In C. Gilligan, N.P. Lyons, \& T.J. Hanmer, (Eds). Making Connections: The Relational Worlds of Adolescent Girls at Emma Willard School. Cambridge: Harvard University Press, pp. 314-334.

Gladis, M.M., Michela, J.L., Walter, H.J., \& Vaughan, R.D. (1992). High school students' perceptions of AIDS risk: Realistic appraisal or motivated denial? Health Psychology, 11(5), 307-316.

Gold, R.S., Skinner, M.J., \& Ross., M.W. (1994). Unprotected anal intercourse in HIVinfected and non-HIV-infected gay men. The Journal of Sex Research, 31(1), 59-77.

Goodman, E. \& Berecochea, J.E. (1994). Predictors of HIV testing among runaway and homeless adolescents. Journal of Adolescent Health, 15(7), 566-572.

Goodman, E. \& Cohall, A.T. (1989). Acquired Immune Deficiency Syndrome and adolescence: Knowledge, attitudes, beliefs and behaviors in a New York City adolescent minority population. Pediatrics, $\underline{84}, 36-42$.

Goodson, P. \& Edmundson, E. (1994). The problematic promotion of abstinence: An overview of Sex Respect. Journal of School Health 64(5), 205-210.

Graham, C.A. (1994). AIDS and the adolescent [Editorial]. International Journal of STDs/AIDS, 5(5), 305-309.

Grant, M. \& Johnstone, B.M. (1990). Research priorities for drug and alcohol studies: The next 25 years. The International Journal of the Addictions, 25(2A), 201-219.

Gray, L.A. \& Saracino, M. (1991). College students' attitudes, beliefs, and behaviors about AIDS: Implications for family life educators. Family Relations, $\underline{40}$, 258-263. 
Grimley, D.M., Harlow, L.L., Morokoff, P., \& Quina, K. (1992, August). Predicting sexual assertiveness in college-age women. American Psychological Association, Washington, DC.

Grimley, D.M., Riley, G.E., Bellis, J.M., \& Prochaska, J.O. (1993). Assessing the stages of change and decision-making for contraceptive use for the prevention of pregnancy, sexually transmitted diseases, and acquired immunodeficiency syndrome. Health Education Quarterly, 20, 455-470.

Gruber, E. \& Chambers, C.V. (1987). Cognitive development and adolescent contraception: Integrating theory and practice. Adolescence, 22(87), 661-670.

Gutman, L.T., St. Claire, K.K., Weedy, C., Herman-Giddens, M.E., Lane, B.A., Niemeyer, J.G., \& McKinney, R.E. (1991). Human Immunodeficiency Virus transmission by child sexual abuse. American Journal of Diseases of Children, $145,137-141$.

Guttmacher, S., Lieberman, L., Ward, D., Radosh, A., Rafferty, Y., \& Freundenberg, N. (1995). Parents' attitudes and beliefs about HIV/AIDS prevention with condom availability in New York City public high schools. Journal of School Health, 65(3), 101-106.

Gwadz, M. \& Rotheram-Borus, M.J. (1992). Tracking high-risk adolescents longitudinally. AIDS Education and Prevention, Supplement, 69-82.

Hankins, C. (1993). Women and HIV infection. In L. Sherr, (Ed.), AIDS and the Heterosexual Population, Langhorne, PA: Harwood Academic Publishers, pp. 31-38.

Hardy, A.M. (1990). National health interview survey data on adult knowledge of AIDS in the United States. Public Health Reports, 105(6), 629-634.

Harvey, S.M., \& Spigner, C. (1995). Factors associated with sexual behavior among adolescents: A multivariate analysis. Adolescence, 30(118), 253-264.

Harlow, L.L., Quina, K., \& Morokoff, P. (1991). Lifestyle Survey. University of Rhode Island. Unpublished manuscript.

Harlow, L.L., Quina, K., Morokoff, P.J., Grimley, D.M., \& Rose, J.S. (1993). HIV Risk in women: A multifaceted model. Journal of Applied Biobehavioral Research, 1, 3-38.

Hausser, D. \& Michaud, P.A. (1992, July 19-24). Condom promotion does not increase sexual intercourse among adolescents. Presented at the Eighth International Conference on AIDS, Amsterdam, The Netherlands. Abstract TuDo575. 
Hausser, D. \& Michaud, P.A. (1994). Does a condom-promoting strategy (the Swiss STOP-AIDS campaign) modify sexual behavior among adolescents? Pediatrics, 93(4), 580-585.

Hays, R.B., Turner, H., \& Coates, T.J. (1992). Social support, AIDS-related symptoms, and depression among gay men. Journal of Consulting and Clinical Psychology, 60(3), 463-469.

Healy, R. \& Coleman, T. (1988/December, 1989/January). A primer on AIDS for health professionals. Health Education, 19(6), 4-10.

Hearst, N. \& Hulley, S.B. (1988). Preventing the heterosexual spread of AIDS: Are we giving our patients the best advice? Journal of the American Medical Association, 259(16), 2428-2432.

Hein, K. (1988). AIDS in adolescents: A rationale for concern. New York State Journal of Medicine, 87(5), 290-295.

Hein, K. (1990). Lessons from New York City on HIV/AIDS in adolescents. Special Issue: Acquired Immunodeficiency Syndrome. New York State Journal of Medicine, 90(3), 143-145.

Hein, K. (1991a). Fighting AIDS in adolescents. Issues in Science and Technology, $\underline{7}(3)$, 67-72.

Hein, K. (1991b). Mandatory HIV testing of youth: A lose-lose proposition. Journal of the American Medical Association, 266(17), 2430-2431.

Hein, K. (1991c). Risky business: Adolescents and Human Immunodeficiency Virus. Pediatrics, $\underline{88}(5), 1052-1054$.

Hein, K. (1992). Adolescents at risk for HIV infection. In R.J. DiClemente, (Ed.) Adolescents and AIDS: A Generation in Jeopardy, Newbury Park, CA: Sage Publications, $p p$. 3-16.

Hein, K. (1993). "Getting real" about HIV in adolescents. American Journal of Public $\underline{\text { Health }}, \underline{83}(4), 492-494$.

Hein, K., Dell, R., Futterman, D., Rotheram-Borus, M.J., \& Shaffer, N. (1995). Comparison of HIV+ and HIV- adolescents: Risk factors and psychosocial determinants. Pediatrics, 95(1), 96-104.

Helgerson, S.D., Petersen, L.R., \& The AIDS Education Study Group. (1988). Acquired Immunodeficiency Syndrome and secondary school students: Their knowledge is limited and they want to learn more. Pediatrics, 81(3), 350-355. 
Helweg-Larsen, M. \& Collins, B.E. (1994). The UCLA multidimensional condom attitudes scale: Documenting the complex determinants of condom use in college students. Health Psychology, 13(3), 224-237.

Henderson, E.B., Weinman, M.L., \& Smith, P.B. (1994). The behavioral implications of myths and medical knowledge about AIDS among indigent female adolescents. Journal of Sex Education and Therapy, 20(2), 79-91.

Hernandez, J.T. \& Smith, F.J. (1990). Inconsistencies and misperceptions putting college students at risk of HIV infection. Journal of Adolescent Health Care, 11 (4), $295-297$.

Herold, E.S., Fisher, W.A., Smith, E.A., \& Yarber, W.A. (1990). Sex education and the prevention of STD/AIDS and pregnancy among youths. Canadian Journal of Public Health, 81, 141-145.

Hingson, R. \& Strunin L. (1992). Monitoring adolescents' response to the AIDS epidemic: Changes in knowledge, attitudes, beliefs, and behaviors. In R.J. DiClemente, (Ed.) Adolescents and AIDS: A Generation in Jeopardy, Newbury Park, CA: Sage Publications, pp. 17-33.

Hingson, R, Strunin, L., \& Berlin, B. (1990). Acquired Immunodeficiency Syndrome transmission: Changes in knowledge and behaviors among teenagers, Massachusetts statewide surveys, 1986-1988. Pediatrics, 85(1), 24-29.

Hingson, R.W., Strunin, L., Berlin, B.M., \& Heeren, T. (1990). Beliefs about AIDS, use of alcohol and drugs, and unprotected sex among Massachusetts adolescents. American Journal of Public Health, 80(3), 295-299.

Hochhauser, M. (1989). AIDS and chemical dependency: Prevention needs of adolescents. Journal of Psychoactive Drugs, 21(4), 381-385.

Hofferth, S.L., Kahn, J.R., \& Baldwin, W. (1987). Premarital sexual activity among U.S. teenage women over the past three decades. Family Planning Perspectives, 19 (2), 4653.

Holtzman, D., Anderson, J.E., Kann, L., Arday, S.L., Truman, B.I., \& Kolbe, L.J. (1991). HIV instruction, HIV knowledge, and drug injection among high school students in the United States. American Journal of Public Health, 81(12), 1596-1601.

Holtzman, D., Green, B.Z., Ingraham, G.C., Daily, L.A., Demchuk, D.G., \& Kolbe, L.J. (1992). HIV education and health education in the United States: A national survey of local school district policies and practices. Journal of School Health, 62(9), 421-427. 
Holtzman, D., Lowry, R., Kann, L., Collins, J.L., \& Kolbe, L.J. (1994). Changes in HIVrelated information sources, instruction, knowledge, and behaviors among US high school students, 1989 and 1990. American Journal of Public Health, 84(3), 388-393.

Horner, R.D., Kolasa, K.M., Irons, T.G., \& Wilson, K. (1994). Racial differences in rural adults' attitudes toward issues of adolescent sexuality. American Journal of Public Health, $\underline{84}(3), 456-459$.

Irwin, C.E. (1990). The theoretical concept of at-risk adolescents. Adolescent Medicine: State of the Art Reviews, $1,1-14$.

Irwin, C.E. \& Millstein, S.G. (1986). Biopsychosocial correlates of risk-taking behaviors during adolescence. Journal of Adolescent Health Care, $7(6), 582-596$.

Jaffe, L.R., Seehaus, M., Wagner, C., \& Leadbeater, B.J. (1988). Anal intercourse and knowledge of acquired immunodeficiency syndrome among minority-group female adolescents. The Journal of Pediatrics, 112, 1005-1007.

Janke, J. (1989). Dealing with AIDS and the adolescent population. The Nurse Practitioner, 4(3), 5-36..

Janz, N.K. \& Becker, M.H. (1984). The health belief model: A decade later. Health Education Quarterly, 11, 1-47.

Jemmott, J.B., Jemmott, L.S., \& Fong, G.T. (1992). Reductions in HIV risk -Associated sexual behaviors among Black male adolescents: Effects of an AIDS prevention intervention. American Journal of Public Health, 82 (3), 372-377.

Jemmott, L.S. \& Jemmott, J.B. (1991). Applying the theory of reasoned action to AIDS risk behavior: Condom use among Black women. Nursing Research, 40, 228-234.

Jemmott, L.S. \& Jemmott, J.B. (1992). Increasing condom-use intentions among sexually active black adolescent women. Nursing Research, 41(5), 273-279.

Jessor, R. (1991). Risk behavior in adolescence: A psychosocial framework for understanding and action. Society for Adolescent Medicine, 12, 597-605.

Jessor, S.L. \& Jessor, R. (1975). Transition from virginity to nonvirginity among youth: A social-psychological study over time. Developmental Psychology, 11(4), 473-484.

Johnsen, L.W. \& Harlow, L.L. (in press). Childhood sexual abuse linked with adult substance use, victimization, and AIDS-risk. AIDS Education and Prevention.

Johnson, J.L. (1991). Preventive interventions for children at risk: Introduction. The International Journal of the Addictions, 25(4A), 429-434. 
Johnston, J. (1993). The sexual enlightenment of children in the modern cultural context: Care or corruption? Sexual and Marital Therapy, $\underline{8}(1), 53-74$.

Joseph, J.G., Emmons, C.A., Kessler, R.C., Wortman, C.B., O'Brien, K., Hocker, W.T., \& Schaefer, C. (1984). Coping with the threat of AIDS: An approach to psychosocial assessment. American Psychologist, 39(11), 1297-1302.

Joseph, S.C. (1991). AIDS and adolescents: A challenge to both treatment and prevention. Journal of Adolescent Health, $\underline{12}$, 614-618.

Kaemingk, K.L. \& Bootzin, R.R. (1990). Behavior change strategies for increasing condom use. Evaluation and Program Planning, 13, 47-54.

Kahn, J.R., Kalsbeek, W.D., \& Hofferth, S.L. (1988). National estimates of teenage sexual activity: Evaluating the comparability of three national surveys. Demography, $\underline{25}(2), 189-204$.

Kain, C. (Ed.). (1989). No Longer Immune: A Counselors Guide to AIDS. Alexandria, VA: American Association for Counseling and Development.

Kalichman, S.C. (1995). Understanding AIDS: A Guide for Mental Health Professionals. Washington, DC: American Psychological Association.

Kalichman, S.C. (1996). Answering Your Questions About AIDS. Washington, DC: American Psychological Association.

Kalichman, S.C. \& Coley, B. (1995). Context framing to enhance HIV-antibody-testing messages targeted to African American women. Health Psychology, 14(3), 247-254.

Kalichman, S.C., Hunter, T.L., \& Kelly, J.A. (1992). Perceptions of AIDS susceptibility among minority and nonminority women at risk for HIV infection. Journal of Consulting and Clinical Psychology, 60(5), 725-732.

Kalichman, S.C., Kelly, J.A., Hunter, T.L., Murphy, D.A., \& Tyler, R. (1993). Culturally tailored HIV-AIDS risk-reduction messages targeted to African-American urban women: Impact on risk sensitization and risk reduction. Journal of Consulting and Clinical Psychology, 61(2), 291-295.

Kalichman, S.C., Russell, R.L., Hunter, T.L., \& Sarwer, D.B. (1993). Earvin "Magic" Johnson's HIV serostatus disclosure: Effects on men's perceptions of AIDS. Journal of Consulting and Clinical Psychology, 61(5), 887-891.

Kamachi, K.L., Irwin, C.E., Shafer, M.B., Wong, V. (1990). "Understanding AIDS": Did female adolescents read it? Journal of Adolescent Health Care, 11(3), 254. 
Kann, L., Anderson, J.E., Holtzman, D., Ross, J., Truman, B.I., Collins, J., \& Kolbe, L.J. (1991). HIV-related knowledge, beliefs, and behaviors among high school students in the United States: Results from a national survey. Journal of School Health, 61(9), 397-401.

Kaplan, M.E. \& Schonberg, S.K. (1994). HIV in adolescents. Clinical Perinatology, 21(1), 75-84.

Katz, R.C., Mills, K., Singh, N.N., \& Best, A.M. (1995). Knowledge and attitudes about AIDS: A comparison of public high school students, incarcerated delinquents, and emotionally disturbed adolescents. Journal of Youth and Adolescence, 24(1), 117-131.

Keeling, R.P. (1989). (Ed.) AIDS on the College Campus, Second Edition. Rockville, MD: American College Health Association.

Kegeles, S.M., Adler, N.E., \& Irwin, C.E. (1988). Sexually active adolescents and condoms: Changes over one year in knowledge, attitudes and use. American Journal of Public Health, $\underline{78}, 460-461$.

Kegeles, S.M., Adler, N.E., \& Irwin, C.E. (1989). Adolescents and condoms: Associations of beliefs with intentions to use. American Journal of Diseases of Children, $\underline{143}, 911-915$.

Keller, S.E., Bartlett, J.A., Schleifer, S.J., Johnson, R.L., Pinner, E., \& Delaney, B. (1991). HIV-relevant sexual behavior among a healthy inner-city heterosexual adolescent population in an endemic area of HIV. Journal of Adolescent Health, $\underline{12}$, 44-48.

Keller, S.E., Schleifer, S.J., Bartlett, J.A., \& Johnson, R.L. (1988). The sexual behavior of adolescents and risk of AIDS. Journal of the American Medical Association, $\underline{260}(24), 3586$.

Kelly, J.A. \& Kalichman, S.C. (1995). Increased attention to human sexuality can improve HIV-AIDS prevention efforts: Key research issues and directions. Journal of Consulting and Clinical Psychology, 63(6), 907-918.

Kelly, J.A., \& Murphy, D.A. (1992). Psychological interventions with AIDS and HIV: Prevention and treatment. Journal of Consulting and Clinical Psychology, 60(4), 576585.

Kelly, J.A., Murphy, D.A., Bahr, G.R., Koob, J.J., Morgan, M.G., Kalichman, S.C., Stevenson, L.Y., Brasfield, T.L., Bernstein, B.M., \& St. Lawrence, J.S. (1993). Factors associated with severity of depression and high-risk sexual behavior among 
persons diagnosed with Human Immunodeficiency Virus (HIV) infection. Health Psychology, 12(3), 215-219.

Kelly, J.A., Murphy, D.A., Sikkema, K.J., \& Kalichman, S.C. (1993). Psychological interventions to prevent HIV infection are urgently needed. American Psychologist, $\underline{48}(10), 1023-1034$.

Kelly, J.A. \& St. Lawrence, J.S. (1988). AIDS prevention and treatment: Psychology's role in the health crisis. Clinical Psychology Review, $\underline{8}, 255-284$.

Kelly, J.A. \& St. Lawrence, J.S. (1990). The impact of community-based groups to help persons reduce HIV infection risk behaviours. AIDS Care, 2 , 25-36.

Kelly, J.A., St. Lawrence, J.S., Brasfield, T.L. (1991). Predictors of vulnerability to AIDS risk behavior relapse. Journal of Consulting and Clinical Psychology, 59(1), 163-166.

Kelly, J.A., St. Lawrence, J., Brasfield, T., Lemke, A., Amidei, T., Roffman, R.E., Hood, H.V., Smith, J.E., Kilgore, H., \& McNeill, C. (1990). Psychological factors that predict AIDS high-risk versus AIDS precautionary behavior. Journal of Consulting and Clinical Psychology, 58, 117-120.

Kelly, J.A., St. Lawrence, J., Hood, H., \& Brasfield T. (1989). Behavioral intervention to reduce AIDS risk activities. Journal of Consulting and Clinical Psychology, 57, 60-67.

Kelly, J.A., Sikkema, K.J., Winett, R.A., Solomon, L.J., Roffman, R.A., Heckman, T.G., Stevenson, L.Y., Perry, M.J., Norman, A.D., \& Desiderato, L.J. (1995). Factors predicting continued high-risk behavior among gay men in small cities: Psychological, behavioral, and demographic characteristics related to unsafe sex. Journal of Consulting and Clinical Psychology, 63(1), 101-107.

Kenney, A.M. Guardado, S., \& Brown, L. (1989). Sex education and AIDS education in the Schools: What states and large school districts are doing. Family Planning Perspectives, 21(2), 56-64.

Kilbourne, B.W., Buehler, J.W., \& Rogers, M.F. (1990). AIDS as a cause of death in children, adolescents, and young adults. American Journal of Public Health, $\underline{80}, 499$ 500 .

King, N.J. \& Gullone, E. (1990). Fear of AIDS: Self-reports of Australian children and adolescents. Psychological Reports, 66 , 245-246.

Kipke, M.D., Futterman, D., \& Hein, K. (1990). HIV infection and AIDS during adolescence. Adolescent Medicine, 74(5), 1149-1167. 
Kipke, M.D., Montgomery, S, \& MacKenzie, R.G. (1993). Substance use among youth seen at a community-based health clinic, Journal of Adolescent Health, 14(4), 289-294.

Klassen, A.D., Williams, C.J., \& Levitt, E.E. (1989). Sex and Morality in the U.S.: An Empirical Inquiry under the Auspices of The Kinsey Institute. Middletown, CT: Wesleyan University Press.

Kline, A., Kline, E., \& Oken, E. (1992). Minority women and sexual choice in the age of AIDS. Social Science and Medicine, 34(4), 447-457.

Kolbe, L.J. (1990). An epidemiological surveillance system to monitor the prevalence of youth behaviors that most affect health. Health Education, 21(6), 44-48.

Koop, C.E. (1990, April). Do the right thing. The Washingtonian, 81-87.

Koopman, C., Rosario, M., \& Rotheram-Borus, M.J. (1994). Alcohol and drug use and sexual behaviors placing runaways at risk for HIV infection. Addictive Behaviors, $\underline{19}(1), 95-103$.

Kraft, P., Rise, J., \& Traeen, B. (1990). The HIV epidemic and changes in the use of contraception among Norwegian adolescents. AIDS, $\underline{4}, 673-678$.

Ku, L.C., Sonenstein, F.L., \& Pleck, J.H. (1992). The association of AIDS education and sex education with sexual behavior and condom use among teenage men. Family Planning Perspectives, $\underline{24}, 100-106$.

Laga, M., Taelman, H., van der Stuyft, P., Bonneux, L., Vercauteren, G., Piot., P. (1989). Advanced immunodeficiency as a risk factor for heterosexual transmission of HIV. AIDS, $\underline{3}(6), 361-366$.

Landefeld, C.S., Chren, M.M., Shega, J., Speroff, T., \& McGuire, E. (1988). Students' sexual behavior, knowledge, and attitudes relating to the Acquired Immunodeficiency Syndrome. Journal of General Internal Medicine, 3 (March/April), 161-165.

Lang, M. (1994). Cognitive, attitudinal and behavioral risk predictors for AIDS among heterosexual adolescents. Unpublished master's thesis, University of Rhode Island, Kingston, Rhode Island.

Lang, M.A., Harlow, L.L., Quina, K., \& Morokoff, P.J. (1994, May 11-14). Age group comparisons of heterosexual HIV risk predictors for women. Poster presentation for the American Psychological Association's Psychosocial and Behavioral Factors in Women's Health Conference, Washington, DC. 
Lang, M.A., Quina, K., Harlow, L.L., \& Morokoff, P.J. (1994, August 12-16). AIDS risk predictors among heterosexual adolescents. Poster presentation at the 102nd Annual Convention for the American Psychological Association, Los Angeles, CA.

Langer, L.M., Zimmerman, R.S., \& Katz, J.A. (1995). Virgins' expectations and nonvirgins' reports: How adolescents feel about themselves. Journal of Adolescent Research, 10(2), 291-306.

Langer, L.M., Zimmerman, R.S., Warheit, G.J., \& Duncan, R.C. (1993). Decision-making orientation and AIDS-related knowledge, attitudes, and behaviors of Hispanic, AfricanAmerican, and white adolescents. Health Psychology, 12(3), 277-234.

Larzelere, R.E. \& Mulaik, S.A. (1977). Single-sample test for many correlations. Psychological Bulletin, 84(3), 557-569.

Laumann, E.O., Gagnon, J.H., Michaels, S., Michael, R.T., \& Coleman, J.S. (1989). Monitoring the AIDS epidemic in the United States: A network approach. Science, $\underline{244}, 1186-1189$.

Lauritsen, J. (1993). The AIDS War: Propaganda, Profiteering and Genocide from the Medical-Industrial Complex. New York: Asklepios/Pagan Press, pp. 18-19.

Ledergerber, B., von Overbeck, J., Egger, M., \& Luthy, R. (1994). The Swiss HIV Cohort Study: Rationale, organization and selected baseline characteristics. $\underline{\text { Soz }}$ Praventivmed, $\underline{39}$ (6), 387-394.

Leeper, M.A. \& Concardy, M. (1989). Preliminary evaluation of reality, a condom for women to wear. Advances in Contraception, 5 , 229-235.

Leigh, B.C. (1990). The relationship of substance use during sex to high-risk sexual behavior. Journal of Sex Research, 27, 199-213.

Leigh, B.C., Morrison, D.M., Trocki, K., \& Temple, M.T. (1994). Sexual behavior of American adolescents: Results from a U.S. national survey. Journal of Adolescent Health, 15 $(2), 117-125$.

Leigh, B.C., Schafer, J., \& Temple, M.T. (1995). Alcohol use and contraception in first sexual experiences. Journal of Behavioral Medicine, 18(1), 81-95.

Leigh, B.C. \& Stall, R. (1993). Substance use and risky sexual behavior for exposure to HIV: Issues in methodology, interpretation, and prevention. American Psychologist, 48(10), 1035-1045.

Leishman, K. (1987, February). Heterosexuals and AIDS. The Atlantic Monthly, 39-58. 
Leite, R.M. Buocompagno, E.M., Leite, A.C., Mergulhao, E.A., \& Battistoni, M.M. (1995). Psychosexual characteristics of male university students in Brazil. Adolescence, $\underline{30}$ (118), 363-80.

Levine, C. (1995). Orphans of the HIV epidemic: Unmet needs in six U.S. cities. Special Issue: AIDS' impact Second International Conference on Biopsychosocial aspects of HIV, \& AIDS (1994, Brighton, England). AIDS Care, 7(Suppl 1), S57-S62.

Levy, S.R., Handler, A.S., Weeks, K.A., Lampman, C., Flay, B.R., \& Rashid, J. (1994). Adolescent risk for HIV as viewed by youth and their parents. Family Community Health, 17(1), 30-41.

Lewis, C. Battistich, V., \& Schaps, E. (1990). School-based primary prevention: What is an effective program? New Directions for Child Development, 50, 35-59.

Livingston, I.L. (1991). Perceived control, knowledge and fear of AIDS among college students: An exploratory study. Journal of Health and Social Policy, 2(2), 47-66.

Lohrmann, D.K. (1988). AIDS education at the local level: The pragmatic issues. Journal of School Health, 58 (8), 330-334.

Long, J.D., Williamson, R.L., Gaynor, P., \& Clark, D. (1988). Relationship of locus of control to life style habits. Journal of Clinical Psychology, 44, 209-214.

Longshore, D. (1990). AIDS education for three high-risk populations. Evaluation and Program Planning, 13, 67-72.

Lott, A. (1991). Attitudes toward gay male and lesbians scale. Unpublished, draft survey. University of Rhode Island, Department of Psychology, Kingston, Rhode Island.

Lowry, R., Holtzman, D., Truman, B.I., Kann, L., Collins, J.L., \& Kolbe, L.J. (1994). Substance use and HIV-related sexual behaviors among United States high school students: Are they related? American Journal of Public Health, 84(7), 1116-1120.

Lui, K.J., Darrow, W.W., \& Rutherford, W. (1988). A model-based estimate of the mean incubation period for AIDS in homosexual men. Science, 249(4857), 1333-1335.

Lyons, J.S., Sheridan, K., \& Larson, D.B. (1988/December, 1989/January). A model for AIDS education for health professionals. Health Education, 19(6), 12-15.

MacDonald, N.E., Wells, G.A., Fisher, W.A., Warren, W.K., King, M.A., Doherty, J.A., \& Bowie, W.R. (1990). High-risk STD/HIV behavior among college students. Journal of the American Medical Association, 263(23), 3155-3159. 
MacGregor, R.R. (1988). Alcohol and drugs as co-factors for AIDS. Advances in Alcohol and Substance Abuse, 77(2), 47-71.

Magura, S., Kang, S.Y., \& Shapiro, J.L. (1994). Outcomes of intensive AIDS education for male adolescent drug users in jail. Journal of Adolescent Health, 15(6), 457-463.

Mann, J.M., Tarantola, D.J., \& Netter, T.W. (Eds.) (1992). AIDS in the World, Cambridge, MA: Harvard University Press.

Manning, D.T. \& Balson, P.M. (1989). Teenagers' beliefs about AIDS education and physicians' perceptions about them. The Journal of Family Practice, 29(2), 173-177.

Manning, D.T., Balson, P.M., Brenberg, N., \& Moore, T.M. (1989). Susceptibility to AIDS: What college students do and don't believe. Journal of American College Health, $\underline{38}(2), 67-73$.

Manoff, R.E. (1985). Social Marketing. New York: Praeger.

Manoff, S.B., Gayle, H.D., Mays, M.A., \& Rogers, M.F. (1989). Acquired immunodeficiency syndrome in adolescents: Epidemiology, prevention and public health issues. The Pediatric Infectious Disease Journal, $\underline{8}(5), 309-314$.

Mansfield, C.J., Conroy, M.E., Emans, S.J., \& Woods, E.R. (1993). A pilot study of AIDS education and counseling of high-risk adolescents in an office setting. Journal of Adolescent Health, 14, 115-119.

Marsiglio, W. \& Mott, F. (1986). The impact of sex education on sexual activity, contraception use and premarital pregnancy among American teenagers. Family Planning Perspectives, 18(4), 151-161.

Martin, J.L. (1990). Drug use and unprotected anal intercourse among gay men. Health Psychology, $\underline{9}, 450-465$.

Mason, J.O. (1989). Forging working partnerships for school health education. Journal of School Health, 59(1), 18-20.

Maticka-Tyndale, E. (1991). Sexual scripts and AIDS prevention: Variations in adherence to safer-sex guidelines by heterosexual adolescents. The Journal of Sex Research, $\underline{28}(1), 45-66$.

Maxwell, A.E., Bastani, R., \& Yan, K.X. (1995). AIDS risk behaviors and correlates in teenagers attending sexually transmitted diseases clinics in Los Angeles. Genitourinary Medicine, $71(2), 82-87$. 
McAllister, I. \& Makkai, T. (1991). Correcting for the underreporting of drug use in opinion surveys. The International Journal of the Addictions, 26(9), 945-961.

McCaig, L.F., Hardy, A.M., \& Winn, D.M. (1991). Knowledge about AIDS and HIV in the US adult population: Influence of the local incidence of AIDS. American Journal of Public Health, 81(12), 1591-1595.

McCoy, C.B. \& Khoury, E. (1990). Drug use and the risk of AIDS. American Behavioral Scientist, 33(4), 419-431.

McCoy, H.V., McKay, C.Y., Hermanns, L., \& Lai, S. (1990). Sexual behavior and the risk of HIV infection. American Behavioral Scientist, 33(4), 432-450.

McDermott, R.J., Hawkins, M.J., Moore, J.R., \& Cittadino, S.K. (1987). AIDS awareness and information sources among selected university students. Journal of American College of Health, 35(5), 222-226.

McGrath, J.W., Strasburger, V.C., \& Cushing, A.H. (1994). Secretory IgA in the cervical mucus. Journal of Adolescent Health, 15(5), 423-425.

McLean, A.L. \& Flanigan, B.J. (1993). Transition-making behaviors of adolescent males at first intercourse, Adolescence, 28(111), 579-595.

Melchert, T. \& Burnett, K.F. (1990). Attitudes, knowledge, and sexual behavior of highrisk adolescents: Implications for counseling and sexuality education. Journal of Counseling, and Development, 68 , 293-298.

Mellanby, A., Phelps, F., \& Tripp, J. (1992). Sex education: More is not enough. Journal of Adolescence, $15,449-466$.

Melton, G.B. (1989). Ethical and legal issues in research and intervention. Journal of Adolescent Health Care, 10, 36-44.

Memon, A. (1990). Young people's knowledge, beliefs and attitudes about HIV/AIDS: A review of research. Health Education Research, 5(3), 327-335.

Mensch, B.S. \& Kandel, D.B. (1988). Underreporting of substance use in a national longitudinal youth cohort: Individual and interviewer effects. Public Opinion Quarterly, 52 (1), 100-124.

Mickler, S.E. (1993). Perceptions of vulnerability: Impact on AIDS-preventive behavior among college adolescents. AIDS Education and Prevention, 5(1), 43-53.

Miller, J.B. (1986). Toward a New Psychology of Women, Second Edition. Boston, MA: Beacon Press, p. 83. 
Miller, R.S., Johnson, J.A., \& Johnson, J.K. (1991). Assessing the prevalence of unwanted childhood sexual experiences. Journal of Psychology and Human Sexuality, $\underline{4}(3), 43-53$.

Millstein, S.B. (1990). Risk factors for AIDS among adolescents. New Directions for Child Development, 50, 3-15.

Millstein, S.G., Moscicki, A.B., \& Broering, J.M. (1994). Female adolescents at high, moderate, and low risk of exposure to HIV: Differences in knowledge, beliefs, and behavior. Journal of Adolescent Health, 15(2), 133-141.

Molgaard, C.A., Nakamura, C., Hovell, M., \& Elder, J.P. (1988). Assessing alcoholism as a risk factor for acquired immunodeficiency syndrome (AIDS). Social Science and Medicine, 27(11), 1147-1152.

Mondanaro, J. (1987). Strategies for AIDS prevention: Motivating health behavior in drug dependent women. Journal of Psychoactive Drugs, 19(2), 143-149.

Moore, K.A., Furstenberg, F.F., \& Peterson, J.L. (1986). Parental attitudes and the occurrence of early sexual activity: A research note. Journal of Marriage and the Family, 48(4), 777-782.

Moore, K.A. \& Stief, T.M. (1991). Changes in marriage and fertility behavior: Behavior versus attitudes of young adults. Youth and Society, 22, 362-386.

Moore, S. \& Rosenthal, D.A. (1991a). Adolescent invulnerability and perceptions of AIDS risk. Journal of Adolescent Research, 6(2), 164-180.

Moore, S. \& Rosenthal, D.A. (1991b). Condoms and coitus: Adolescents' attitudes to AIDS and safe sex behavior. Journal of Adolescence, 14(3), 211-227.

Morgan, D.L. \& Krueger, R.A. (1993). When to use focus groups and why. In D.L. Morgan, (Ed), Successful Focus Groups: Advancing the State of the Art, (pp. 3-19). Newbury, CA: Sage Publications.

Morrison, D.M. (1985). Adolescent contraceptive behavior: A review. Psychological Bulletin, 98(3), 538-568.

Morrison, D.M., Baker, S.A., \& Gillmore, M.R. (1994). Sexual risk behavior, knowledge, and condom use among adolescents in juvenile detention. Journal of Youth and Adolescence, 23(2), 271-288. 
Moscicki, A.B., Winkler, B., Irwin, C.E., \& Schachter, J. (1989). Differences in biologic maturation, sexual behavior, and sexually transmitted disease between adolescents with and without cervical intraepithelial neoplasia. Journal of Pediatrics, $115,487-493$.

Nader, P.R., Wexler, D.B., Patterson, T.L., McKusick, L., \& Coates, T. (1989). Comparisons of beliefs about AIDS among urban, suburban, incarcerated, and gay adolescents. Journal of Adolescent Health Care, 10, 413-418.

Nangle, D.W. \& Hansen, D.J. (1993). Relations between social skills and high-risk sexual interactions among adolescents. Behavior Modification, 17(2), 113-135.

Ndeki, S.S., Klepp, K.I., Seha, A.M., \& Leshabari, M.T. (1994). Exposure to HIV/AIDS information, AIDS knowledge, perceived risk and attitudes toward people with AIDS among primary school-children in northern Tanzania. AIDS Care, 6(2), 183-191.

Nelkin, D., Willis, D.P., \& Parris, S.V., (Eds.). (1991). A Disease of Society: Cultural, and Institutional Responses to AIDS. Cambridge, MA: Cambridge University Press.

Neuwirth, K. \& Dunwoody, S. (1989). The complexity of AIDS-related behavioral change: The interaction between communication and noncommunication variables. AIDS \& Public Policy Journal, 4(1), 20-30.

Newton, M. (1995). Adolescence: Guiding Youth Through the Perilous Ordeal. New York: Norton.

Nguyet, N.T.M., Maheux, B., Béland, F., \& Pica, L.A. (1994). Sexual behaviors and condom use: A study of suburban male adolescents. Adolescence, $\underline{29}(113), 37-48$.

Niven, R.G. (1987). The impact of AIDS on the chemical dependency field. Advances in Alcohol and Substance Abuse, 7(2) 3-14.

Noell, J., Biglan, A., Berendt, J., Ochs, L., Metzler, C.W., Ary, D., \& Smolkowski, K. (1993). Problematic sexual situations for adolescents: Alcohol and unsafe sex. Health Values, 17(6), 40-49.

Norusis, M.J. (1990). SPSS/PC+ Statistics Version 4.0, Statistical Package for the Social Sciences, Chicago: SPSS, Inc.

Nutbeam, D. \& Blakey, V. (1990). The concept of health promotion and AIDS prevention: A comprehensive and integrated basis for action in the 1990s. Health Promotion International, $\underline{5}(3), 233-242$.

Nyamathi, A. \& Vasquez, R. (1989). Impact of poverty, homelessness, and drugs on Hispanic women at risk for HIV infection. Hispanic Journal of Behavioral Sciences, 11(4), 299-314. 
O'Brien, K. (1990). Improving survey questionnaires through focus groups. In D.L. Morgan (Ed.) (1993). Successful Focus Groups: Advancing the State of the Art. Newbury Park, CA: Sage Publications, $p p$. 105-117.

O'Connell, D. \& Velicer, W.F. (1988). A decisional balance measure and the stages of change model for weight loss. The International Journal of Addictions, $\underline{23}, 729-750$.

O'Leary, A. (1985). Self-efficacy and health. Behavior Research Therapy, 23, 437-451.

Olsen, J.A. \& Weed, S.E. (1986). The effects of family planning for teenagers on adolescent birth and pregnancy rates. Family Perspective, 20(3), 153-170.

Olsen, T.D. (1987, September). Adolescent pregnancy and abstinence: How far have we come? Paper presented at an Office of Adolescent Pregnancy Program technical workshop, Washington, DC.

Olson, C.L. (1970). Practical considerations in choosing a MANOVA test statistic: A rejoinder to Stevens. Psychological Bulletin, $\underline{86}, 1350-1352$.

O'Reilly, K.R. \& Aral, S.O. (1985). Adolescence and sexual behavior. Journal of Adolescent Health Care, 6 , 262-270.

Orr, D.P., Langefeld, C.D., Katz, BP., Caine, V.A., Dias, P., Blythe, M., \& Jones, R.B. (1992). Factors associated with condom use among sexually active female adolescents. Adolescent Medicine, 120, 311-317.

Ortiz, V., Kauman, J., Uribe, P., Decaso, L.E., Hernandez-Avila, M. (1992). Acceptability of female condoms among female prostitutes in Mexico City: Preliminary findings. Conasida, Mexico: Instituto Nacional de Salud Publica, in World Health Organization, (1993c). Sexual Negotiation, The Empowerment of Women and the Female Condom: General Protocal, Geneva, Switzerland: WHO.

Osborn, J.E. (1988). AIDS: Politics and science. New England Journal of Medicine, $\underline{318}, 444-447$.

Osborn, J.E. (1991). Prevention: Can we mobilize what has been learned? In N.F. McKenzie, (Ed.) The AIDS Reader: Social, Political, Ethical Issues. New York: Meridian.

Ostergard, D.R. (1977). The effect of age, gravity, and parody on the location of the cervical squamocolumnar junction as determined by colposcopy. American Journal of Obstetrics and Gynecology, 129, 59-63. 
Otten, M.W., Zaidi, A.A., Peterman, T.A., Rolfs, R.T., \& Witte, J.J. (1994). High rate of HIV seroconversion among patients attending urban sexually transmitted disease clinics. $\underline{\text { AIDS }}, \underline{8}(4), 549-553$.

Overby, K.J. \& Kegeles, S.M. (1994). The impact of AIDS on an urban population of high-risk female minority adolescents: Implications for intervention. Journal of Adolescent Health, 15(3), 216-227.

Padian, N., Marquis, L., Francis, D.P., Anderson, R.E., Rutherford, G.W., O'Malley, P.M., \& Winkelstein, W. (1987). Male-to-female transmission of Human Immunodeficiency Virus. Journal of the American Medical Association, 258(6), 788790.

Padian, N.S., Shiboski, S.C., \& Jewell, N.P. (1991). Female-to-male transmission of Human Immunodeficiency Virus. Journal of the American Medical Association, $\underline{266}(12), 1664-1667$

Patton, M.J. (1991). Qualitative research on college students: Philosophical and methodological comparisons with the quantitative approach. Journal of College Student Development, 32, 389-396.

Paxson, M.A. \& Quina, K. (1989). Psychological factors related to contraceptive use by unmarried college women. Paper presented at the meetings of the Eastern Psychological Association, March 31, 1989, Boston, Massachusetts.

Paxton, C., \& Susky, D. (1988). AIDS, homophobia, and sexual attitudes. Health Values, 12(4), 39-43.

Pendergrast, R.A., DuRant, R.H., \& Gaillard, G.L. (1992). Attitudinal and behavioral correlates of condom use in urban adolescent males. Journal of Adolescent Health, $\underline{13}(2), 133-139$.

Peruga, A. \& Celentano, D.D. (1993). Correlates of AIDS knowledge in samples of the general population. Social Science Medicine, 16(4), 509-524.

Petosa, R. \& Wessinger, J. (1990). The AIDS education needs of adolescents: A theorybased approach. AIDS Education and Prevention, 2(2), 127-136.

Piercy, F.P., Trepper, T., \& Jurich, J. (1993). The role of family therapy in decreasing HIV high-risk behaviors among adolescents. AIDS Education and Prevention, $\underline{5}(1)$, 71-86.

Pino, R. (1993). The Transtheoretical Model: An assessment of readiness to change HIV-risking behaviors in a sample of high-risk adolescents. Unpublished master's thesis, University of Rhode Island, Kingston, Rhode Island. 
Plant, M.A. (1990). Alcohol, sex and AIDS. Alcohol and Alcoholism, 25(2), 293-301.

Pleck, J.H., Sonenstein, F.L., \& Ku, L.C. (1991). Adolescent males' condom use: Relationships between perceived cost-benefits and consistency. Journal of Marriage and the Family, $\underline{53}, 731-745$.

Ponton, L.E., DiClemente, R.J., \& McKenna, S. (1991). An AIDS education and prevention program for hospitalized adolescents. Journal of the American Academy of Child and Adolescent Psychiatry, $\underline{30}$ (5), 729-734.

Poppen, P.J. \& Reisen, C.A. (1994). Heterosexual behaviors and risk of exposure to HIV: Current status and prospects for change. Applied and Preventive Psychology, $\underline{3}(2), 75-90$.

Prewitt, V.R. (1989). Health beliefs and AIDS educational materials. Family and Community Health, 12(2), 65-76.

Price, J.H., Desmond, S.M., Hallinan, C., \& Griffin, T.B. (1988). College students' perceived risk and seriousness of AIDS. Health Education, 19(4), 16-19.

Price, J.H., Desmond, S., \& Kakulka, G. (1985). High school students' perceptions and misperceptions of AIDS. Journal of School Health, 55(3), 107-109.

Price-Greathouse, J. \& Trice, A.D. (1986). Chance health-orientation and AIDS information seeking. Psychological Reports, 59, 10.

Prochaska, J.O. (1979). Systems of Psychotherapy: A Transtheoretical Analysis. Pacific Grove, CA: Brooks/Cole Publishing.

Prochaska, J.O. (1984). Systems of Psychotherapy: A Transtheoretical Analysis, Second Edition. Pacific Grove, CA: Brooks/Cole Publishing.

Prochaska, J.O. \& DiClemente, C.C. (1983). Stages and processes of self-change of smoking: Toward an integrative model of change. Journal of Consulting and Clinical Psychology, 51, 390-395.

Prochaska, J.O. \& DiClemente, C.C. (1984). The Transtheoretical Approach: Crossing Traditional Boundaries of Therapy. Homewood, IL: Dow Jones-Irwin.

Prochaska, J.O., DiClemente, C.C., \& Norcross, J.C. (1992). In search of how people change: Applications to the addictive behaviors. American Psychologist, 47, 11021114. 
Prochaska, J.O., Harlow, L.L., Redding, C.A., Snow, M.G., Rossi, J.S., \& Velicer, W.F. (1990). Stages of change, self efficacy, and decisional balance of condom use in a high HIV risk sample. Technical Report to the Centers for Disease Control Contract Grant \#0-4115-002.

Prochaska, J.O., Harlow, L.L., Redding, C.A., Snow, M.G., Rossi, J.S., Velicer, W.F., Rhodes, F., Schnell, D., Gavabotti, C. (1992). Stages of change, self-efficacy, and decision balance of condom use in a high-risk HIV sample. Unpublished Manuscript.

Prochaska, J.O., Velicer, W.F., Rossi, J.S., Goldstein, M.G., Marus, B., H., Rakowski, W., Fiore, C., Harlow, L.L., Redding, C.A., Rosenbloom, D., \& Rossi, S.R. (1994). Stages of change and decisional balance for 12 problem behaviors. Health Psychology, $\underline{13}(1), 39-46$.

Pulford, J.S.W. (1991). North American college and university responses to the AIDS threat. Journal of College Student Psychotherapy, 5(2) 101-107.

Quadrel, M.J., Fischhoff, B., \& Davis, W. (1993). Adolescent (in)vulnerability. American Psychologist, 48 (2), 102-116.

Quina, K., Harlow, L.L., Gibson, P., \& Morokoff, P. (1990, August). Psychometric investigation of a sexual assertiveness scale. Paper presented at the annual meeting of the American Psychological Association, Boston, MA.

Quina, K., Harlow, L.L., Morokoff, P.J., \& Saxon, S.E. (in press). Interpersonal power and women's HIV risk. In J. Manlowe \& N. Goldstein, Gender and Politics, New York, New York University Press.

Quinn, T.C., Glasser, D., Cannon, R.O., Matuszak, D.L., Dunning, R.W., Kline, R.L., Campbell, C.H., Israel, E., Fauci, A.S., \& Hook, E.W. (1988). Human Immunodeficiency Virus infection among patients attending clinics for sexually transmitted diseases. The New England Journal of Medicine, 318(4), 197-203.

Redding, C.A. (1993). The Transtheoretical Model applied to safer sex behavior among university students: A cross-sectional study. Unpublished doctoral dissertation, University of Rhode Island, Kingston, Rhode Island.

Redding, C.A. \& Rossi, J.S. (1993a, March). The processes of safer sex adoption. Paper presentation at the Society of Behavioral Medicine, San Francisco, CA.

Redding, C.A. \& Rossi, J.S. (1993b, November). Self efficacy for safer sex: Stage and gender-based differences on situational subscales. Paper presentation at the 27th annual convention of Association for Advancement of Behavior Therapy, Atlanta, GA. 
Redding, C.A., Rossi, J.S., Velicer, W.F., \& Prochaska, J. (1989, August). The pros and cons of safer sex: A measurement model. Paper presented at the 97th annual convention of the American Psychological Association, New Orleans, LA.

Rehmet, S., Staszewski, S., Muller, R., Doerr, H.W., Bergmann, L., von Wangenheim, G., Helm, E. G., \& Stille, W. (1992). Transmission rates and co-factors of heterosexual HIV transmission. Presented at the VIII International Conference on AIDS/III STD World Congress, Amsterdam, 1992 (PoC 4165).

Reiss, I.L. \& Leik, R.K. (1989). Evaluating strategies to avoid AIDS: Number of partners vs. use of condoms. The Journal of Sex Research, 26(4), 411-433.

Remafedi, G.J. (1988) Preventing the sexual transmission of AIDS during adolescence. Journal of Adolescent Health Care, 9, 139-143.

Remafedi, G.J. (1994). Cognitive and behavioral adaptations to HIV/AIDS among gay and bisexual adolescents. Journal of Adolescent Health, 15(2), 142-148.

Reuben, N., Hein, K., \& Drucker, E. (1988). The relationship of high risk behaviors to AIDS knowledge in adolescent high school students. Presented at the Annual Conference of the Society for Adolescent Medicine, New York, NY.

Reulbach, W. (1991). Counseling chemically dependent HIV positive adolescents. Journal of Chemical Dependency Treatment, 4(2), 31-43.

Rhode Island Department of Health (1994, December). AIDS/STD Morbidity Mortality Report. Office of AIDS/STD: Author.

Richard, R. \& van der Pligt, J. (1991). Factors affecting condom use among adolescents. Journal of Community and Applied Social Psychology, 1, 105-116.

Richard, R., van der Pligt, J., \& deVries, N. (1995). Anticipated affective reactions and prevention of AIDS. Special Issue: Social psychology and health. British Journal of Social Psychology, 34(1), 9-21.

Rickert, V.I., Gottlieb, A., \& Jay, S.M. (1990). A comparison of three clinic-based AIDS education programs on female adolescents' knowledge, attitudes, and behavior. Journal of Adolescent Health Care, 11(4), 298-303.

Rickert, V.I., Jay, S.M., \& Gottlieb, A. (1991). Effects of a peer-counseled AIDS education program on knowledge, attitudes, and satisfaction of adolescents. Journal of Adolescent Health, 12, 38-43. 
Rickert, V.I., Jay, S.M., Gottlieb, A., \& Bridges, C. (1989). Adolescents and AIDS: Females' attitudes and behaviors toward condom purchase and use. Journal of Adolescent Health Care, 10(4), 313-316.

Rickman, R.L., Lodico, M., DiClemente, R.J., Morris, R., Baker, C., \& Huscroft, S. (1994). Sexual communication is associated with condom use by sexually active incarcerated adolescents. Journal of Adolescent Health, 15(5), 383-388.

Rimberg, H.M. \& Lewis, R.J. (1994). Older adolescents and AIDS: Correlates of selfreported safer sex practices. Journal of Research on Adolescence, 4(3), 453-464.

Robertson, J.A. \& Plant, M.A. (1988). Alcohol, sex and risks of HIV infection. Drug and Alcohol Dependence, 22, 75-78.

Romanowski, B. \& Piper, G. (1988). Sexually transmitted diseases -- An overview. Journal of Social Work and Human Sexuality, 6(2), 7-20.

Roosa, M.W. \& Christopher, F.S. (1990). An evaluation of an adolescent pregnancy prevention program: Is "Just say no" enough? Family Relations, 39, 68-72.

Roscoe, B. \& Kruger, T.L. (1990). AIDS: Late adolescents' knowledge and its influence on sexual behavior. Adolescence, 25(97), 39-48.

Rosenthal, D., Moore, S., \& Brumen, I. (1990). Ethnic group differences in adolescents' responses to AIDS. Australian Journal of Social Issues, 25(3), 220-239.

Rosenthal, D., Moore, S., \& Buzwell, S. (1994). Homeless youths: Sexual and drugrelated behavior, sexual beliefs and HIV/AIDS risk. AIDS Care, 6 (1), 83-94.

Rosenthal, D., Moore, S., Flynn, I. (1991). Adolescent self-efficacy, self-esteem and sexual risk-taking. Special Issue: Social dimensions of AIDS. Journal of Community and Applied Social Psychology, 1(2), 77-88.

Rosenthal, D.A. \& Shepherd, H. (1993). A six-month follow-up of adolescents' sexual risk-taking, HIV/AIDS knowledge, and attitudes to condoms. Journal of Community and Applied Social Psychology, 3(1), 53-56.

Ross, M.W. \& Carson, J.A. (1988). Effectiveness of distribution of information on AIDS: A national study of six media in Australia. New York State Journal of Medicine, $\underline{88}$, 239-241.

Rotheram-Borus, M.J. (1990). Adolescents' reference-group choices, self-esteem, and adjustment. Journal of Personality and Social Psychology, 59(5), 1075-1081. 
Rotheram-Borus, M.J., Becker, J.V., Koopman, C., \& Kaplan, M.S. (1991). AIDS knowledge and beliefs, and sexual behavior of sexually delinquent and non-delinquent (runaway) adolescents. Journal of Adolescents, 14(3), 229-244.

Rotheram-Borus, M.J. \& Koopman, C. (1991). Sexual risk behavior, ADS knowledge, and beliefs about AIDS among predominantly minority gay and bisexual male adolescents. AIDS Education and Prevention, $\underline{3}$ (4), 305-312.

Rotheram-Borus, M.J., Meyer-Bahlburg, H.F.L., Koopman, C., Rosario, M., Exner, T.M., Henderson, R., Matthieu, M., \& Gruen, R.S. (1992). Lifetime sexual behaviors among runaway males and females. The Journal of Sex Research, 29(1), 15-29.

Rotheram-Borus, M.J., Rosario, M., Meyer-Bahlburg, H.F.L., Koopman, C., Dopkins, S.C., \& Davies, M. (1994). Sexual and substance use acts of gay and bisexual male adolescents in New York City. The Journal of Sex Research, 31(1), 47-57.

Rothspan, S. \& Read, S.J. (1996). Present versus future time perspective and HIV risk among heterosexual college students. Health Psychology, 15(2), 131-134.

Rubinson, L. \& deRubertis, L. (1991). Trends in sexual attitudes and behaviors of a college population over a 15-year period. Journal of Sex Education and Therapy, 17, $32-41$.

Sacco, W.P., Levine, B, Reed, D.L., \& Thompson, K. (1991). Attitudes about condom use as an ADS-relevant behavior: Their factor structure and relation to condom use. Journal of Consulting and Clinical Psychology, 3(2) 265-272.

St. Lawrence, J.S. (1993). African-American adolescents' knowledge, health-related attitudes, sexual behavior, and contraceptive decisions: Implications for the prevention of adolescent HIV infection. Journal of Consulting and Clinical Psychology, 61(1), 104-112.

St. Lawrence, J.S., Brasfield, T.L., Jefferson, K.W., Alleyne, E., \& Shirley, A. (1994). Social support as a factor in African-American adolescents' sexual risk behavior. Journal of Adolescent Research, 9(3), 292-310.

St. Lawrence, J.S., Brasfield, T.L., Shirley, A., Jefferson, K.W., Alleyne, E., \& O'Bannon, R.E., III (1995). Cognitive-behavioral intervention to reduce African American adolescents' risk for HIV infection. Journal of Consulting and Clinical Psychology, $\underline{63}(2), 221-237$.

St. Lawrence, J.S., Jefferson, K.W., Alleyne, E., \& Brasfield, T.L. (1995). Comparison of education versus behavioral skills training interventions in lowering sexual HIV-risk behavior of substance-dependent adolescents. Journal of Consulting and Clinical Psychology, 63(1), 154-157. 
St. Lawrence, J.S., Jefferson, K.W., Banks, P.G., Cline, T.R, Alleyne, E., \& Brasfield, T.L. (1994). Cognitive-behavioral group intervention to assist substance-dependent adolescents in lowering HIV infection risk. AIDS Education and Prevention, 6(5), 425435 .

Sandberg, D.E., Rotheram-Borus, M.J., Bradley, J., \& Martin, J. (1988). Methodological issues in assessing AIDS prevention programs. Special Issue: Adolescent sexual behavior. Journal of Adolescent Research, 3, 413-418.

Schaalma, H., Kok, G., \& Peters, L. (1993). Determinants of consistent condom use by adolescents: The impact of experience of sexual intercourse. Health Education Research, $\underline{8}(2), 255-269$.

Schinke, S.P., Botvin, G.J., Orlandi, M.A., Schilling, R.F., \& Gordon, A.N. (1990). African-American and Hispanic-American adolescents, HIV infection and preventive intervention. AIDS Education and Prevention, 2(4), 305-312.

Schneider, D.J., Taylor, E.L., Prater, L.M., \& Wright, M.P. (1991). Risk assessment for HIV infection: Validation study of a computer-assisted preliminary screen. AIDS Education and Prevention, 3(3), 215-229.

Schnell, D.J., Galavotti, C., \& O'Rielly, K.R. (1993). An evaluation of sexual behavior change using statistical and cognitive models. Statistics in Medicine, 12, 219-228.

Schuster, C.R. (1988). Intravenous drug use and AIDS prevention. Public Health Reports, 103(3), 261-266.

Schwarcz, S.K. \& Rutherford, G.W. (1989). Acquired Immunodeficiency Syndrome in infants, children, and adolescents. The Journal of Drug Issues, 19, 75-92.

Sears, D.O. (1986). College sophomores in the laboratory: Influences of a narrow data base on social psychology's view of human nature. Journal of Personality and Social Psychology, 51(3), 515-530.

Segest, E., Mygind, O., Harris, C.N., \& Bay, H. (1991). The correlation between general disease prevention and prevention of HIV-contagion among adolescents. Journal of Adolescence, 14(4), 389-396.

Seidman, S.N. \& Rieder, R.O. (1994). A review of sexual behavior in the United States. American Journal of Psychiatry, 151(3), 330-341.

Shafer, M.A. \& Boyer, C.B. (1991). Psychosocial and behavioral factors associated with risk of sexually transmitted diseases, including Human Immunodeficiency Virus infection, among urban high school students. Journal of Pediatrics, $119,826-833$. 
Shoop, D.M. \& Davidson, P.M. (1994). AIDS and adolescents: The relation of parent and partner communication to adolescent condom use. Journal of Adolescence, 17(2), $137-148$.

Siegel, D., Larazus, N., Krashovsky, F., Durbin, M., \& Chesney, M. (1991). AIDS knowledge, attitudes, and behavior among inner city, junior high school students. Journal of School Health, 61, 160-165.

Siegel, K. \& Gibson, W.C. (1988). Barriers to the modification of sexual behavior among heterosexuals at risk for Acquired Immunodeficiency Syndrome. New York State Journal of Medicine, $\underline{87}, 66-70$.

Siegel, L.J. (1993). Editorial: Children's understanding of AIDS: Implications for preventive interventions. Journal of Pediatric Psychology, 18(2), 173-176.

Simoni, J.M., Mason, H.R.C., Marks, G., Ruiz, M.S., Reed, D., \& Richardson, J.L. (1995). Women's self-disclosure of HIV infection: Rates, reasons, and reactions. Journal of Consulting and Clinical Psychology, 63 (3), 474-478.

Singh, A.N., Zemitzsch, A.A., Ellis, C.R., Best, A.M., \& Singh, N.N. (1994). Seriously emotionally disturbed students' knowledge and attitudes about AIDS. Journal of Emotional and Behavioral Disorders, 2(3), 156-163.

Slater, B.R. (1989). Pediatric and adolescent AIDS in the schools: The need for educative programs. Professional School Psychology, 4(4), 233-244.

Slonim-Nevo, V., Auslander, W.F., \& Ozawa, M.N. (1995). Educational options and AIDS-related behaviors among troubled adolescents. Journal of Pediatric Psychology, 20(1), 41-60.

Smith, K.W., McGraw, S.A., Crawford, S.L., Costa, L.A., \& McKinlay, J.B. (1993). HIV risk among Latino adolescents in two New England cities. American Journal of Public Health, 83(10), 1395-1399.

Sonenstein, F.L., Pleck, J.H., \& Ku, L.C. (1989). Sexual activity, condom use, and AIDS awareness among adolescent males. Family Planning Perspectives, $\underline{4}, 152-158$.

Sonenstein, F.L., Pleck, J.H., \& Ku, L.C. (1991). Levels of sexual activity among adolescent males in the United States. Family Planning Perspectives, 23 (4), 162-167.

Soper, D.E., Brockwell, N.J., \& Dalton, H.P. (1991). Evaluation of the effects of a female condom on the female lower genital tract. Contraception, 44(1), 21-29. 
Sorenson, J.L., Gibson, D.R., Heitzmann, C., Dumontet, R., \& Acampora, A. (1988, August). AIDS prevention with drug abusers in residential treatment: Preliminary results. Paper presented at the annual meeting of the American Psychological Association, Atlanta, GA.

Stake, J.E., DeVille, C.J., \& Pennell, C.L. (1983). The effects of assertive training on the performance self-esteem of adolescent girls. Journal of Youth and Adolescence, 12(5), 435-442.

Stall, R. (1988). The prevention of HIV infection associated with drug and alcohol use during sexual activity. Advances in Alcohol and Substance Abuse, 7, 73-88.

Stall, R.D., Coates, T.J., , \& Hoff, C. (1988). Behavioral risk reduction for HIV infection among gay and bisexual men. American Psychologist, 43(11), 878-885.

Stall, R.D., McKusick, L., Wiley, J., Coates, T., \& Ostrow, D. (1986). Alcohol and drug use during sexual activity and compliance with safe sex guidelines for AIDS: The AIDS Behavioral Research Project. Health Education Quarterly, 13, 359-371.

Stanton, B.F., Black, M., Kaljee, L., \& Ricardo, I. (1993). Perceptions of sexual behavior among urban early adolescents: Translating theory through focus groups. Journal of Early Adolescence, 13(1), 44-66.

Stanton, B.F., Black, M., Keane, V., \& Feigelman, S. (1990). HIV risk behaviors in young people: Can we benefit from 30 years of research experience? AIDS and Public Policy Journal, $\underline{5}(1), 17-23$.

Steckler, A., McLeroy, K.R., Goodman, R.M., Bird, S.T., \& McCormick, L. (1992). Toward integrating qualitative and quantitative methods: An introduction. Health Education Quarterly, 19, 1-8.

Stein, G.J. (1995). AIDS Update: 1994 -- 1995. Englewood Cliffs, NJ: Prentice-Hall, Inc.

Stein, J.A., Newcomb, M.D., \& Bentler, P.M. (1994). Psychosocial correlates and predictors of AIDS risk behaviors, abortion, and drug use among a community sample of young adult women. Health Psychology, 13(4), 308-318.

Steiner, J.D., Sorokin, G., Schiedermayer, D.L., \& Van Susteren, T.J. (1990). Are adolescents getting smarter about Acquired Immunodeficiency Syndrome? American Journal of Diseases of Children, 144, 302-306.

Stern, L. (1990). Conceptions of separation and connection in female adolescents. In C. Gilligan, N.P. Lyons, \& T.J. Hanmer, (Eds.) Making connections: The relational 
worlds of adolescent girls at Emma Willard School. Cambridge: Harvard University Press, pp. 73-87.

Stevens-Smith, P. \& Remley, T.P. (1994). Drugs, AIDS, and teens: Intervention and the school counselor. The School Counselor, 41, 181-184.

Stevenson, H.C. \& Davis, G. (1994). Impact of culturally sensitive AIDS video education on the AIDS risk knowledge of African-American adolescents. AIDS Education and Prevention, $\underline{6}(1), 40-52$.

Stevenson, H.C., Davis, G., Weber, E., Weiman, D., \& Abdul-Kabir, S. (1995). HIV prevention beliefs among urban African-American youth. Journal of Adolescent Health, $\underline{16}(4), 316-323$.

Stevenson, H.C., Gay, K.M., \& Josar, L. (1995). Culturally sensitive AIDS education and perceived AIDS risk knowledge: Reaching the "know-it-all" teenager. AIDS Education and Prevention, 7(2), 134-144.

Stevenson, M.R. \& Stevenson, D.M. (1990). Beliefs about AIDS among entering college students. Journal of Sex Education and Therapy, 16(3), 201-204.

Stiffman, A.R., Dore, P., Cunningham, R.M., \& Earls, F. (1995). Person and environment in HIV risk behavior changes between adolescence and young adulthood. Health Education Quarterly, 22(2), 211-226.

Stiffman, A.R. \& Earls, F. (1990). Behavioral risks for Human Immunodeficiency Virus infection in adolescent medical patients. Pediatrics, $\underline{85}(3), 303-310$.

Stiffman, A.R., Earls, F., Dore, P., \& Cunningham, R. (1992). Changes in Acquired Immunodeficiency Syndrome-related risk behavior after adolescence: Relationships to knowledge and experience concerning Human Immunodeficiency Virus Infection.

Pediatrics, 89(5), 950-956.

Strickland, B.R. (1978). Internal-external expectancies of health-related behavior. Journal of Consulting and Clinical Psychology, 46, 1192-1211.

Strunin L. (1991). Adolescents' perceptions of risk for HIV infection: Implications for future research. Social Science and Medicine, 32(2), 221-228.

Strunin, L. \& Hingson, R. (1987). Acquired immune deficiency syndrome and adolescents: Knowledge, beliefs, attitudes and behaviors. Pediatrics, 79, 825-828.

Strunin, L. \& Hingson, R. (1993). Adolescents. In L. Sherr, (Ed.), AIDS and the Heterosexual Population, Langhorne, PA: Harwood Academic Publishers, pp. 125137. 
Stuber, M.L. (1990). Psychiatric consultation in pediatric HIV and AIDS. Journal of the American Academy of Child and Adolescent Psychiatry, 29(3), 463-467.

Sugerman, S.T., Hergenroeder, A.C., Chacko, M.R., \& Parcel, G.S. (1991). Acquired Immunodeficiency Syndrome and adolescents: Knowledge, attitudes, and behaviors of runaway and homeless youths. American Journal of Diseases of Children, 145,431 436.

Sunwoo, J., Brenman, A., Escobedo, J., Philpott, T., Allman, K., Mueller, J., Jaeger, J., Brown, L.K., \& Cole, F.S. (1995). School-based AIDS education for adolescents. Journal of Adolescent Health, 16(4), 309-315.

Surgeon General of U.S. Public Health Service (1987). Special Report: From the Surgeon General, US Public Health Service. Journal of Substance Abuse Treatment, $\underline{4}$, 5-13.

Sweeney, P., Lindegren, M.L., Buehler, J.W., Onorato, I.M., \& Janssen, R.S. (1995). Teenagers at risk of human immunodeficiency virus type 1 infection: Results from seroprevalence surveys in the United States. Archives of Pediatric Adolescent Medicine, 149(5), 521-528.

Sy, F.S., Richter, D.I., \& Copello, A.G. (1989). Innovative educational strategies and recommendations for AIDS prevention and control. AIDS Education and Prevention, $1(1), 53-56$.

Tabachnick, B.G. \& Fidell, L.S. (1989). Using Multivariate Statistics, Second Edition, New York: Harper Collins.

Takanishi, R. (1993). The opportunities of adolescence -- Research, interventions, and policy: Introduction to the special issue. American Psychologist, $\underline{48(2), 85-87 . ~}$

Tansathit, Thawatchi, \& Cheevakej S. (1990). Femshield accptability study among family planning accepters (unpublished report). Chiang Mai University, Thailand. Family Planning Unit, Obstetrics and Gynaecology Department, Faculty of Medicine, cited in World Health Organization, (1993c). Sexual Negotiation. The Empowerment of Women and the Female Condom: General Protocal, Geneva, Switzerland: WHO.

Temoshok, L., Sweet, D.M., \& Zich, J. (1987). A three city comparison of the public's knowledge and attitudes about AIDS. Psychology and Health, 1, 43-60.

Trad, P.V. (1994). A developmental model for risk avoidance in adolescents confronting AIDS. AIDS Education and Prevention, $\underline{6}(4), 322-328$. 
Trice, A.D. \& Price-Greathouse, J. (1987). Locus of control and AIDS informationseeking in college women. Psychological Reports, 60(2), 665-666.

U.S. Department of Health and Human Services (1993). AIDS Research: An NIMH Blueprint for the Second Decade. Atlanta: National Institutes of Mental Health, $p p$. 13

Valdiserri, R.O., Lyter, D., Leviton, L.C., Callahan, C.M., Kingsley, L.A., \& Rinaldo, C.R. (1988). Variables influencing condom use in a cohort of gay and bisexual men. American Journal of Public Health, 78, 801-805.

van de Wigert, H. \& Padian, N.S. (1993). Heterosexual transmission of HIV. In L. Sherr, (Ed.), AIDS and the Heterosexual Population, Langhorne, PA: Harwood Academic Publishers, pp. 1-19.

van der Pligt, J. \& Richard, R. (1994). Changing adolescents' sexual behavior: Perceived risk, self-efficacy and anticipated regret. Patient Education and Counseling, 23 (3), 187196.

van der Velde, F.W., van der Pligt, J., \& Hooykaas, C. (1994). Perceiving AIDS-related risk: Accuracy as a function of differences in actual risk. Health Psychology, 13(1), 25-33.

Vanderschmidt, H.F., Lang, J.M., Knight-Williams, V., \& Vanderschmidt, G.F. (1993). Risks among inner-city young teens: The prevalence of sexual activity, violence, drugs, and smoking. Journal of Adolescent Health, 14, 282-288.

Vermund, S.H. Hein, K., Gayle, H.D., Cary, J.M., Thomas, P.A., \& Drucker, E. (1989). Acquired Immunodeficiency Syndrome among adolescents. American Journal of Diseases of Children, 143, 1220-1224.

Vincent, M.L., Clearle, A.F., \& Schluchter, M.D. (1987). Reducing adolescent pregnancy through school and community based education. Journal of the American Medical Association, 257, 3382-3386.

Visser, A.P. \& Ketting, E. (1994). Sexual health: Education and counseling perspectives on contraceptive use, HIV and sexuality. Patient Education and Counseling, 23(3), 141-145.

Wagner, H.U., Van Dyck, E., Roggen, E., Nunn, A.J., Kamali, A., Schmid, D.S., Dobbins, J.G., \& Mulder, D.W. (1994). Seroprevalence and incidence of sexually transmitted diseases in a rural Ugandan population. International Journal of STDs/AIDS, 5(5), 332-337. 
Walter, H.J., Vaughn, R.D., \& Cohall, A.T. (1991). Psychosocial influences on Acquired Immunodeficiency Syndrome-risk behaviors among high school students. Pediatrics, $\underline{88}(4), 846-852$.

Walter, H.J., Vaughan, R.D., Gladis, M.M., Ragin, D.F., Kasen, S., \& Cohall, A.T. (1993). Factors associated with AIDS-related behavioral intentions among high school students in an AIDS epicenter. Health Education Quarterly, 20(3), 409-420.

Weed, S.E. \& Olsen, J.A. (1986). Effects of family planning programs and teenage pregnancy: Replication and extension. Family Perspective, 20(3), 173-195.

Weinstein, N.D. \& Nicolich, M. (1993). Correct and incorrect interpretations of correlations between risk perceptions and risk behaviors. Health Psychology, 12(3), 235-245.

Weisman, C.S., Nathanson, C.A., Ensminger, M., Teitelbaum, M.A., Robinson, J.C., \& Plichta, S. (1989). AIDS knowledge, perceived risk and prevention among adolescent clients of a family planning clinic. Family Planning Perspectives, 21 (5), 213-217.

Werner, M.J. \& Biro, F.M. (1990). Contraception and sexually transmitted diseases in adolescent females. Adolescent Pediatric Gynecology, $\underline{3}$, 127-136.

Westerman, P.L. \& Davidson, P.M. (1993). Homophobic attitudes and AIDS risk behavior of adolescents. Journal of Adolescent Health, 14(3), 208-213.

Whalen, C.K., Henker, B., O'Neil, R., Hollingshead, J., Holman, A., \& Moore, B. (1994). Optimism in children's judgments of health and environmental risks. Health Psychology, 13(4), 319-325.

Whitley, B.E. (1990). College student contraceptive use: A multivariate analysis. The Journal of Sex Research, 27(2), 305-313.

Whitt, J.K. (1995). Current research on children's and adolescents' HIV/AIDS-related knowledge, attitudes, and behavior. Journal of Pediatric Psychology, 20(1), 37-40.

Wilcox, B.L. (1990). Federal policy and adolescent AIDS. New Directions for Child Development, 50 , 61-70.

Wilkins, R. \& Lewis, C. (1993). Sex and drugs and nuclear war: Secular, developmental and Type A influences upon adolescents' fears of the nuclear threat, AIDS and drug addiction. Journal of Adolescence, $16,23-41$.

Windle, M. (1989). High-risk behaviors for AIDS among heterosexual alcoholics: A pilot study. Journal of Studies on Alcohol, 50(6), 503-507. 
Wingood, G.M. \& DiClemente, R.J. (1992). Cultural, gender, and psychosocial influences on HIV-related behavior of African-American female adolescents: Implications for the development of tailored prevention programs. Ethnicity \& Disease, $2,381-388$.

Witt, L.A. (1989). Authoritarianism, knowledge of AIDS, and affect toward persons with AIDS: Implications for health education. Journal of Applied Social Psychology, 19(7), 599-607.

World Health Organization (1989). The health of youth: Young people and adults face the issues together. A summary of national and regional activities in preparation for the technical discussions and beyond. Facts for action: Youth and sexually transmitted diseases. Geneva, Switzerland: Author.

World Health Organization (1990, November 19-20). Report of the meeting on research priorities relating to women and HIV/AIDS. Geneva, Switzerland: Author, GPA/DIR/91.2, p. 4.

World Health Organization (1992). The global AIDS strategy, WHO AIDS Series 11, Geneva, Switzerland: Author, pp. 10-11.

World Health Organization (1993a). Global Programme on AIDS: The HIV/AIDS Pandemic: 1993 Overview. Geneva, Switzerland: Author, WHO/GPA/CNP/ EVA/93.1, p. 16.

World Health Organization (1993b, February 3-5). Global Programme on AIDS: Women and AIDS: Research Priorities. First meeting of the Advisory Council on HIV and AIDS, Global Programme on AIDS/ACA(1)93.8, Geneva, Switzerland: Author, p. 5.

World Health Organization (1993c, May 27). Sexual Negotiation. The Empowerment of Women and the Female Condom: General Protocol. Geneva, Switzerland: World Health Organization, and the Global Programme on AIDS Office of Intervention Development and Support; Social and Behavioral Studies and Support Unit, SNPROTL.

World Health Organization (1993d, September 7). 13 million HIV positive women by 2000. Press release published in Edinburgh, 7 September, 1993 during The Second International Conference on HIV in Children and Mothers (7 - 10 September 1993).

World Health Organization (1993e, November 1). Women and AIDS: Proposed research priorities for Global Programme on AIDS. Presented at the Second meeting of the Advisory Council on HIV and AIDS, Geneva, Switzerland, 9 - 11 November 1993, GPA/ACA(2)/93.7. Rev1. 
World Health Organization (1993f, November 16). New approach to fighting AIDS Press release WHO/90, Geneva, Switzerland: Author.

World Health Organization (1994a, July). Women and AIDS: Agenda for Action. Geneva, Switzerland, World Health Organization and the United Nations Development Programme in Consultation with the United Nations Division for the Advancement of Women.

World Health Organization (1994b, April 12). Report of a consultation on women and HIV/AIDS GPA's action agenda in support of countries (draft). Geneva, Switzerland: World Health Organization, Global Programme on AIDS, 6 - 8 December 1993.

World Health Organization. (1995a). The current global situation of the HIV/AIDS pandemic. Geneva, Switzerland: Author; In Centers for Disease Control and Prevention, Morbidity and Mortality Weekly Report, 44(46), 849-853.

World Health Organization. (1995b). Global AIDS News. The Newsletter of the World Health Organization Global Programme on AIDS, $1,5$.

Wynder, E.L. (1994). Child Health Day task forces report: Introduction: Toward national comprehensive school health education. Preventive Medicine An International Journal Devoted to Practice and Theory, 23(1), 106-118.

Zabin, L.S., Hirsch, M.B., Smith, E.A., Streett, R., \& Hardy, J.B. (1986). Evaluation of a pregnancy prevention program for urban teenagers. Family Planning Perspectives, $\underline{18}$, 119-126.

Zabin, L.S., Hirsch, M.B., Streett, R., Emerson, M.R., Smith, M., Hardy, J.B., \& King, T.M. (1988). The Baltimore pregnancy prevention program for urban teenagers: How did it work? Family Planning Perspectives, 20, 182-187.

Zelnick, M. \& Kantner, J.F. (1977). Sexual and contraceptive experience of young unmarried women in the United States, 1976 and 1971. Family Planning Perspectives, $\underline{9}$, 55-77.

Zelnick, M. \& Kantner, J.F. (1980). Sexual activity, contraceptive use, and pregnancy among metropolitan teenagers; 1971-1979. Family Planning Perspectives, 12, 230-237.

Zelnick, M. \& Kim, Y.J. (1982). Sex education and its association with teenage sexual activity, pregnancy and contraceptive use. Family Planning Perspectives, 14(3), 117126.

Zelnik, M. \& Shah, F.K. (1983). First intercourse among young Americans. Family Planning Perspectives, 15 , 64-70. 
Zierler, S., Feingold, L., Laufer, D., Velentgas, P., Kantrowitz-Gordon, I., \& Mayer, K. (1991). Adult survivors of childhood sexual abuse and subsequent risk of HIV infection. American Journal of Public Health, 81(5), 572-575.

Zimet, G.D., Bunch, D.L., Anglin, T.M., Lazebnik, R., Williams, P., \& Krowchuck, D.P. (1992). Relationship of AIDS-related attitudes to sexual behavior changes in adolescents. Journal of Adolescent Health, 13, 493-498.

Zimet, G.D., DiClemente, R.J., Lazebnik, R., Anglin, T.M., Ellick, E.M, \& Williams, P. (1993). Changes in adolescents' knowledge and attitudes about AIDS over the course of the AIDS epidemic. Journal of Adolescent Health, 14(2), 85-90.

Zimet, G.D., Hillier, S.A., Anglin, T.M., Ellick, E.M., Krowchuck, D.P., \& Williams, P. (1991). Journal of Pediatric Psychology, 16(3), 287-294. 